Cochrane Database of Systematic Reviews

\title{
Vaccines for preventing influenza in healthy adults (Review)
}

Demicheli V, Jefferson T, Ferroni E, Rivetti A, Di Pietrantonj C

Demicheli V, Jefferson T, Ferroni E, Rivetti A, Di Pietrantonj C.

Vaccines for preventing influenza in healthy adults.

Cochrane Database of Systematic Reviews 2018, Issue 2. Art. No.: CD001269.

DOI: 10.1002/14651858.CD001269.pub6.

www.cochranelibrary.com 
TABLE OF CONTENTS

HEADER

ABSTRACT

PLAIN LANGUAGE SUMMARY

SUMMARY OF FINDINGS

2

BACKGROUND

OBJECTIVES

METHODS

RESULTS

Figure 1.

Figure 2.

Figure 3.

DISCUSSION

AUTHORS' CONCLUSIONS

ACKNOWLEDGEMENTS

REFERENCES

CHARACTERISTICS OF STUDIES

DATA AND ANALYSES

Analysis 1.1. Comparison 1 Inactivated parenteral influenza vaccine versus placebo or 'do nothing', Outcome 1 Influenza. .....

Analysis 1.2. Comparison 1 Inactivated parenteral influenza vaccine versus placebo or 'do nothing', Outcome 2 Influenza-like illness.

Analysis 1.3. Comparison 1 Inactivated parenteral influenza vaccine versus placebo or 'do nothing', Outcome 3 Physician visits.

Analysis 1.4. Comparison 1 Inactivated parenteral influenza vaccine versus placebo or 'do nothing', Outcome 4 Days ill. .......... Analysis 1.5. Comparison 1 Inactivated parenteral influenza vaccine versus placebo or 'do nothing', Outcome 5 Times any drugs were prescribed.

Analysis 1.6. Comparison 1 Inactivated parenteral influenza vaccine versus placebo or 'do nothing', Outcome 6 Times antibiotic was prescribed.

Analysis 1.7. Comparison 1 Inactivated parenteral influenza vaccine versus placebo or 'do nothing', Outcome 7 Working days lost.

Analysis 1.8. Comparison 1 Inactivated parenteral influenza vaccine versus placebo or 'do nothing', Outcome 8 Hospitalisations.

Analysis 1.9. Comparison 1 Inactivated parenteral influenza vaccine versus placebo or 'do nothing', Outcome 9 Clinical cases (clinically defined without clear definition).

Analysis 1.10. Comparison 1 Inactivated parenteral influenza vaccine versus placebo or 'do nothing', Outcome 10 Local harms.

Analysis 1.11. Comparison 1 Inactivated parenteral influenza vaccine versus placebo or 'do nothing', Outcome 11 Systemic harms.

Analysis 2.1. Comparison 2 Live aerosol influenza vaccine versus placebo or 'do nothing', Outcome 1 Influenza. ..................... Analysis 2.2. Comparison 2 Live aerosol influenza vaccine versus placebo or 'do nothing', Outcome 2 Influenza-like illness. ..... Analysis 2.3. Comparison 2 Live aerosol influenza vaccine versus placebo or 'do nothing', Outcome 3 Influenza cases (clinically defined without clear definition).

Analysis 2.4. Comparison 2 Live aerosol influenza vaccine versus placebo or 'do nothing', Outcome 4 Local harms. .................. Analysis 2.5. Comparison 2 Live aerosol influenza vaccine versus placebo or 'do nothing', Outcome 5 Systemic harms. ........... Analysis 3.1. Comparison 3 Inactivated aerosol influenza vaccine versus placebo or 'do nothing', Outcome 1 Influenza. .......... Analysis 3.2. Comparison 3 Inactivated aerosol influenza vaccine versus placebo or 'do nothing', Outcome 2 Local harms. ..... Analysis 3.3. Comparison 3 Inactivated aerosol influenza vaccine versus placebo or 'do nothing', Outcome 3 Systemic harms. .. Analysis 4.1. Comparison 4 Inactivated parenteral influenza vaccine versus placebo or 'do nothing' administered during pregnancy, Outcome 1 Influenza in mothers.

Analysis 4.2. Comparison 4 Inactivated parenteral influenza vaccine versus placebo or 'do nothing' administered during pregnancy, Outcome 2 Influenza-like illness in mothers.

Analysis 4.3. Comparison 4 Inactivated parenteral influenza vaccine versus placebo or 'do nothing' administered during pregnancy, Outcome 3 Influenza in newborn.

Analysis 4.4. Comparison 4 Inactivated parenteral influenza vaccine versus placebo or 'do nothing' administered during pregnancy, Outcome 4 Influenza-like illness in newborn. 
Analysis 5.1. Comparison 5 Inactivated parenteral influenza vaccine versus placebo - cohort studies, Outcome 1 Seasonal inactivated vaccine effectiveness in mothers - pregnant women.

Analysis 5.2. Comparison 5 Inactivated parenteral influenza vaccine versus placebo - cohort studies, Outcome 2 Seasonal inactivated vaccine effectiveness in newborns - pregnant women.

Analysis 5.3. Comparison 5 Inactivated parenteral influenza vaccine versus placebo - cohort studies, Outcome 3 Seasonal inactivated vaccine effectiveness in newborns - pregnant women.

Analysis 5.4. Comparison 5 Inactivated parenteral influenza vaccine versus placebo - cohort studies, Outcome 4 H1N1 vaccine - safety - pregnancy-related outcomes - pregnant women.

Analysis 5.5. Comparison 5 Inactivated parenteral influenza vaccine versus placebo - cohort studies, Outcome 5 Seasonal vaccine - safety - pregnancy-related outcomes - pregnant women.

Analysis 5.6. Comparison 5 Inactivated parenteral influenza vaccine versus placebo - cohort studies, Outcome 6 Seasonal vaccine containing $\mathrm{H} 1 \mathrm{~N} 1$.

Analysis 6.1. Comparison 6 Inactivated parenteral influenza vaccine versus placebo - case-control studies, Outcome 1 Effectiveness in newborns - pregnant women (adjusted data).

Analysis 6.2. Comparison 6 Inactivated parenteral influenza vaccine versus placebo - case-control studies, Outcome 2 Seasonal vaccine safety - pregnancy-related outcomes (adjusted data).

Analysis 7.1. Comparison 7 Serious adverse events: Guillain-Barré syndrome - cohort studies, Outcome 1 Seasonal influenza vaccination and Guillain-Barré syndrome.

Analysis 8.1. Comparison 8 Serious adverse events: Guillain-Barré syndrome - case-control studies, Outcome 12009 to $2010 \mathrm{~A} /$ H1N1 - general population (unadjusted data).

Analysis 8.2. Comparison 8 Serious adverse events: Guillain-Barré syndrome - case-control studies, Outcome 22009 to $2010 \mathrm{~A} /$ H1N1 - general population (adjusted data).

Analysis 8.3. Comparison 8 Serious adverse events: Guillain-Barré syndrome - case-control studies, Outcome 3 Seasonal influenza vaccination general population (adjusted data).

Analysis 9.1. Comparison 9 Serious adverse events: demyelinating diseases (multiple sclerosis, optic neuritis) - cohort studies, Outcome 1 Influenza vaccination (seasonal) - demyelinating diseases (unadjusted data).

Analysis 9.2. Comparison 9 Serious adverse events: demyelinating diseases (multiple sclerosis, optic neuritis) - cohort studies, Outcome 2 Influenza vaccination (H1N1) - demyelinating diseases (unadjusted).

Analysis 10.1. Comparison 10 Serious adverse events: demyelinating diseases (multiple sclerosis, optic neuritis) - case-control studies, Outcome 1 Influenza vaccination (seasonal) - general population - demyelinating diseases (unadjusted data).

Analysis 10.2. Comparison 10 Serious adverse events: demyelinating diseases (multiple sclerosis, optic neuritis) - case-control studies, Outcome 2 Influenza vaccination (seasonal) - general population - multiple sclerosis (adjusted data).

Analysis 10.3. Comparison 10 Serious adverse events: demyelinating diseases (multiple sclerosis, optic neuritis) - case-control studies, Outcome 3 Influenza vaccination (seasonal) - general population - optic neuritis (adjusted data).

Analysis 11.1. Comparison 11 Serious adverse events: immune thrombocytopenic purpura - cohort studies, Outcome 1 Seasonal influenza vaccine - HR (adjusted data).

Analysis 11.2. Comparison 11 Serious adverse events: immune thrombocytopenic purpura - cohort studies, Outcome 2 Seasonal influenza vaccine (unadjusted data).

Analysis 12.1. Comparison 12 Serious adverse events: immune thrombocytopenic purpura - case-control studies, Outcome 1 Seasonal influenza vaccine - general population (adjusted data).

Analysis 12.2. Comparison 12 Serious adverse events: immune thrombocytopenic purpura - case-control studies, Outcome 2 Seasonal influenza vaccine - general population (unadjusted data).

Analysis 13.1. Comparison 131968 to 1969 pandemic: inactivated polyvalent parenteral influenza vaccine versus placebo, Outcome 1 Influenza-like illness.

Analysis 13.2. Comparison 131968 to 1969 pandemic: inactivated polyvalent parenteral influenza vaccine versus placebo, Outcome 2 Influenza.

Analysis 13.3. Comparison 131968 to 1969 pandemic: inactivated polyvalent parenteral influenza vaccine versus placebo, Outcome 3 Hospitalisations.

Analysis 13.4. Comparison 131968 to 1969 pandemic: inactivated polyvalent parenteral influenza vaccine versus placebo, Outcome 4 Pneumonia.

Analysis 14.1. Comparison 141968 to 1969 pandemic: inactivated monovalent parenteral influenza vaccine versus placebo, Outcome 1 Influenza-like illness.

Analysis 14.2. Comparison 141968 to 1969 pandemic: inactivated monovalent parenteral influenza vaccine versus placebo, Outcome 2 Influenza.

Analysis 14.3. Comparison 141968 to 1969 pandemic: inactivated monovalent parenteral influenza vaccine versus placebo, Outcome 3 Hospitalisations. 
Analysis 14.4. Comparison 141968 to 1969 pandemic: inactivated monovalent parenteral influenza vaccine versus placebo, Outcome 4 Pneumonia.

Analysis 14.5. Comparison 141968 to 1969 pandemic: inactivated monovalent parenteral influenza vaccine versus placebo, Outcome 5 Working days lost.

Analysis 14.6. Comparison 141968 to 1969 pandemic: inactivated monovalent parenteral influenza vaccine versus placebo, Outcome 6 Days ill.

Analysis 15.1. Comparison 151968 to 1969 pandemic: inactivated polyvalent aerosol influenza vaccine versus placebo, Outcome 1 Influenza-like illness.

Analysis 16.1. Comparison 161968 to 1969 pandemic: inactivated monovalent aerosol influenza vaccine versus placebo, Outcome 1 Influenza-like illness.

Analysis 17.1. Comparison 171968 to 1969 pandemic: live aerosol influenza vaccine versus placebo, Outcome 1 Influenza cases (clinically defined without clear definition).

Analysis 17.2. Comparison 171968 to 1969 pandemic: live aerosol influenza vaccine versus placebo, Outcome 2 Complications (bronchitis, otitis, pneumonia).

ADDITIONAL TABLES

APPENDICES

FEEDBACK

WHAT'S NEW

HISTORY

CONTRIBUTIONS OF AUTHORS 
[Intervention Review]

\section{Vaccines for preventing influenza in healthy adults}

Vittorio Demicheli ${ }^{1}$, Tom Jefferson², Eliana Ferroni ${ }^{3}$, Alessandro Rivetti ${ }^{4}$, Carlo Di Pietrantonj 5

1Servizio Regionale di Riferimento per l'Epidemiologia, SSEpi-SeREMI, Azienda Sanitaria Locale ASL AL, Alessandria, Italy. ${ }^{2}$ Centre for Evidence Based Medicine, University of Oxford, Oxford, UK. ${ }^{3}$ Epidemiological System of the Veneto Region, Regional Center for Epidemiology, Veneto Region, Padova, Italy. ${ }^{4}$ Dipartimento di Prevenzione - S.Pre.S.A.L, ASL CN2 Alba Bra, Alba, Italy. ${ }^{5}$ Regional Epidemiology Unit SeREMI, Local Health Unit Alessandria- ASL AL, Alessandria, Italy

Contact address: Vittorio Demicheli, Servizio Regionale di Riferimento per l'Epidemiologia, SSEpi-SeREMI, Azienda Sanitaria Locale ASL AL, Via Venezia 6, Alessandria, Piemonte, 15121, Italy. vittorio.demicheli@libero.it, vdemicheli@aslal.it.

Editorial group: Cochrane Acute Respiratory Infections Group.

Publication status and date: Edited (no change to conclusions), published in Issue 2, 2020.

Citation: Demicheli V, Jefferson T, Ferroni E, Rivetti A, Di Pietrantonj C. Vaccines for preventing influenza in healthy adults. Cochrane Database of Systematic Reviews 2018, Issue 2. Art. No.: CD001269. DOI: 10.1002/14651858.CD001269.pub6.

Copyright @ 2020 The Cochrane Collaboration. Published by John Wiley \& Sons, Ltd.

\section{A B S T R A C T}

\section{Background}

The consequences of influenza in adults are mainly time off work. Vaccination of pregnant women is recommended internationally. This is an update of a review published in 2014. Future updates of this review will be made only when new trials or vaccines become available. Observational data included in previous versions of the review have been retained for historical reasons but have not been updated due to their lack of influence on the review conclusions.

\section{Objectives}

To assess the effects (efficacy, effectiveness, and harm) of vaccines against influenza in healthy adults, including pregnant women.

\section{Search methods}

We searched the Cochrane Central Register of Controlled Trials (CENTRAL; 2016, Issue 12), MEDLINE (January 1966 to 31 December 2016), Embase (1990 to 31 December 2016), the WHO International Clinical Trials Registry Platform (ICTRP; 1 July 2017), and ClinicalTrials.gov (1 July 2017), as well as checking the bibliographies of retrieved articles.

\section{Selection criteria}

Randomised controlled trials (RCTs) or quasi-RCTs comparing influenza vaccines with placebo or no intervention in naturally occurring influenza in healthy individuals aged 16 to 65 years. Previous versions of this review included observational comparative studies assessing serious and rare harms cohort and case-control studies. Due to the uncertain quality of observational (i.e. non-randomised) studies and their lack of influence on the review conclusions, we decided to update only randomised evidence. The searches for observational comparative studies are no longer updated.

\section{Data collection and analysis}

Two review authors independently assessed trial quality and extracted data. We rated certainty of evidence for key outcomes (influenza, influenza-like illness (ILI), hospitalisation, and adverse effects) using GRADE.

\section{Main results}

We included 52 clinical trials of over 80,000 people assessing the safety and effectiveness of influenza vaccines. We have presented findings from 25 studies comparing inactivated parenteral influenza vaccine against placebo or do-nothing control groups as the most relevant to decision-making. The studies were conducted over single influenza seasons in North America, South America, and Europe between 1969 and 2009. We did not consider studies at high risk of bias to influence the results of our outcomes except for hospitalisation. 
Inactivated influenza vaccines probably reduce influenza in healthy adults from $2.3 \%$ without vaccination to $0.9 \%$ (risk ratio (RR) $0.41,95 \%$ confidence interval $(\mathrm{CI}) 0.36$ to $0.47 ; 71,221$ participants; moderate-certainty evidence), and they probably reduce ILI from $21.5 \%$ to $18.1 \%$ (RR $0.84,95 \% \mathrm{Cl} 0.75$ to $0.95 ; 25,795$ participants; moderate-certainty evidence; 71 healthy adults need to be vaccinated to prevent one of them experiencing influenza, and 29 healthy adults need to be vaccinated to prevent one of them experiencing an ILI). The difference between the two number needed to vaccinate (NNV) values depends on the different incidence of ILI and confirmed influenza among the study populations. Vaccination may lead to a small reduction in the risk of hospitalisation in healthy adults, from $14.7 \%$ to $14.1 \%$, but the $\mathrm{Cl}$ is wide and does not rule out a large benefit (RR 0.96, 95\% Cl 0.85 to 1.08; 11,924 participants; low-certainty evidence). Vaccines may lead to little or no small reduction in days off work ( -0.04 days, $95 \% \mathrm{Cl}-0.14$ days to 0.06 ; low-certainty evidence). Inactivated vaccines cause an increase in fever from $1.5 \%$ to $2.3 \%$.

We identified one RCT and one controlled clinical trial assessing the effects of vaccination in pregnant women. The efficacy of inactivated vaccine containing $\mathrm{pH} 1 \mathrm{~N} 1$ against influenza was $50 \%$ (95\% Cl $14 \%$ to $71 \%)$ in mothers (NNV 55), and $49 \%(95 \% \mathrm{Cl} 12 \%$ to $70 \%)$ in infants up to 24 weeks (NNV 56). No data were available on efficacy against seasonal influenza during pregnancy. Evidence from observational studies showed effectiveness of influenza vaccines against ILI in pregnant women to be $24 \%$ (95\% Cl $11 \%$ to $36 \%$, NNV 94), and against influenza in newborns from vaccinated women to be $41 \%$ (95\% Cl 6\% to $63 \%$, NNV 27 ).

Live aerosol vaccines have an overall effectiveness corresponding to an NNV of 46 . The performance of one- or two-dose whole-virion 1968 to 1969 pandemic vaccines was higher (NNV 16) against ILI and (NNV 35) against influenza. There was limited impact on hospitalisations in the 1968 to 1969 pandemic (NNV 94). The administration of both seasonal and 2009 pandemic vaccines during pregnancy had no significant effect on abortion or neonatal death, but this was based on observational data sets.

\section{Authors' conclusions}

Healthy adults who receive inactivated parenteral influenza vaccine rather than no vaccine probably experience less influenza, from just over $2 \%$ to just under $1 \%$ (moderate-certainty evidence). They also probably experience less ILI following vaccination, but the degree of benefit when expressed in absolute terms varied across different settings. Variation in protection against ILI may be due in part to inconsistent symptom classification. Certainty of evidence for the small reductions in hospitalisations and time off work is low. Protection against influenza and ILI in mothers and newborns was smaller than the effects seen in other populations considered in this review.

Vaccines increase the risk of a number of adverse events, including a small increase in fever, but rates of nausea and vomiting are uncertain. The protective effect of vaccination in pregnant women and newborns is also very modest. We did not find any evidence of an association between influenza vaccination and serious adverse events in the comparative studies considered in this review. Fifteen included RCTs were industry funded (29\%).

\section{PLAIN LANGUAGE SUMMARY}

\section{Vaccines to prevent influenza in healthy adults}

\section{Review aim}

The aim of this Cochrane Review, first published in 1999, was to summarise research that looks at the effects of immunising healthy adults with influenza vaccines during influenza seasons. We used information from randomised trials comparing vaccines with dummy vaccines or nothing. We focused on the results of studies looking at vaccines based on inactivated influenza viruses, which are developed by killing the influenza virus with a chemical and are given by injection through the skin. We evaluated the effects of vaccines on reducing the number of adults with confirmed influenza and the number of adults who had influenza-like symptoms such as headache, high temperature, cough, and muscle pain (influenza-like illness, or ILI). We also evaluated hospital admission and harms arising from the vaccines. Observational data included in previous versions of the review have been retained for historical reasons but have not been updated due to their lack of influence on the review conclusions.

\section{What was studied in this review?}

Over 200 viruses cause ILI, which produces the same symptoms (fever, headache, aches, pains, cough, and runny nose) as influenza. Without laboratory tests, doctors cannot distinguish between ILI and influenza because both last for days and rarely cause serious illness or death. The types of virus contained in influenza vaccines are usually those that are expected to circulate in the following influenza seasons, according to recommendations of the World Health Organization (seasonal vaccine). Pandemic vaccine contains only the virus strain that is responsible of the pandemic (i.e. the type $\mathrm{A}$ H1N1 for the 2009 to 2010 pandemic).

\section{Main results}

We found 52 clinical trials of over 80,000 adults. We were unable to determine the impact of bias on about $70 \%$ of the included studies due to insufficient reporting of details. Around $15 \%$ of the included studies were well designed and conducted. We focused on reporting of results from 25 studies that looked at inactivated vaccines. Injected influenza vaccines probably have a small protective effect against influenza and ILI (moderate-certainty evidence), as 71 people would need to be vaccinated to avoid one influenza case, and 29 would need 
to be vaccinated to avoid one case of ILI. Vaccination may have little or no appreciable effect on hospitalisations (low-certainty evidence) or number of working days lost.

We were uncertain of the protection provided to pregnant women against ILI and influenza by the inactivated influenza vaccine, or this was at least very limited.

The administration of seasonal vaccines during pregnancy showed no significant effect on abortion or neonatal death, but the evidence set was observational.

\section{Key messages}

Inactivated vaccines can reduce the proportion of healthy adults (including pregnant women) who have influenza and ILI, but their impact is modest. We are uncertain about the effects of inactivated vaccines on working days lost or serious complications of influenza during influenza season.

\section{How up to date is this review?}

The evidence is current to 31 December 2016. 


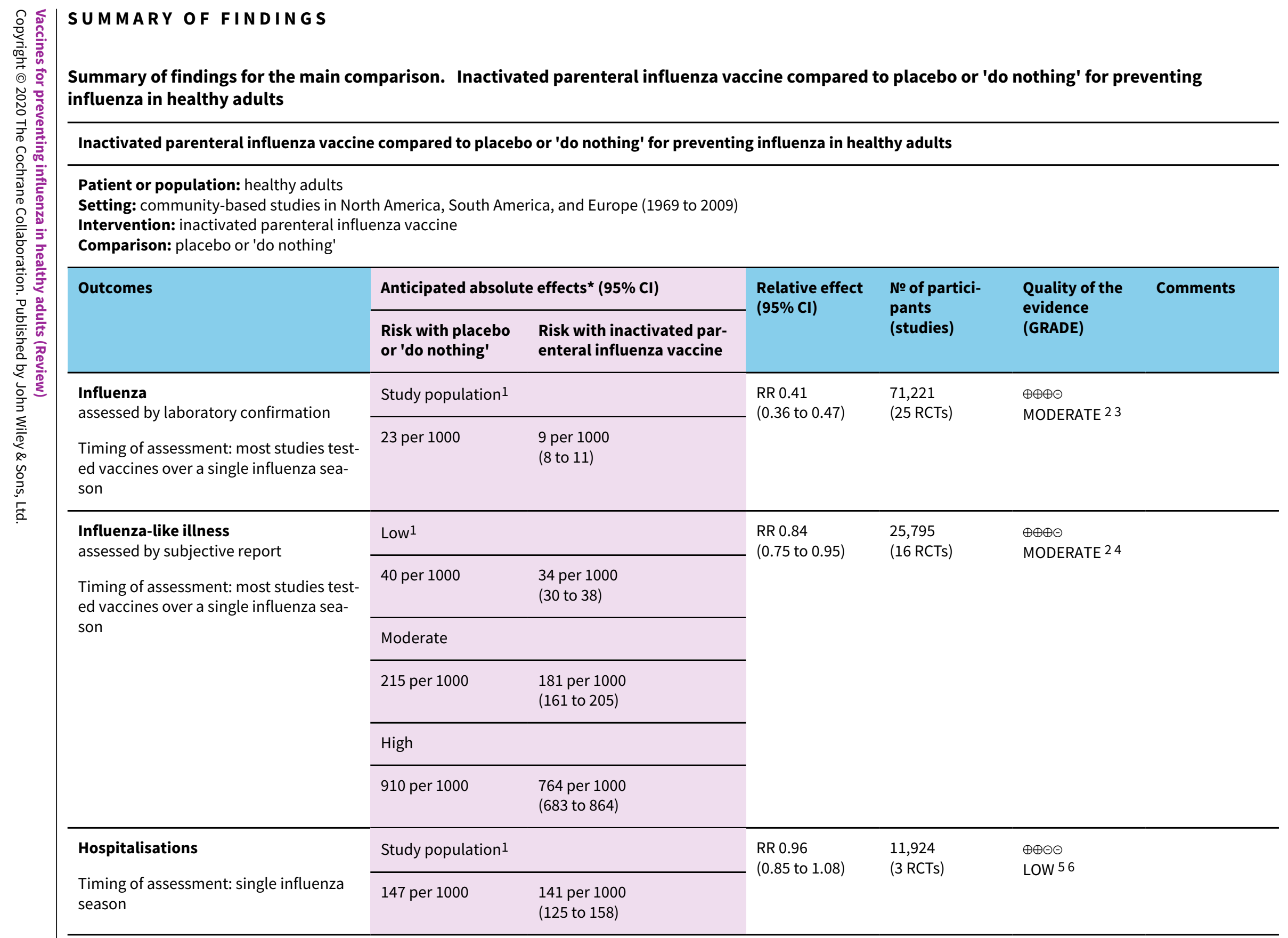

Patient or population: healthy adults

Comparison: placebo or 'do nothing' 


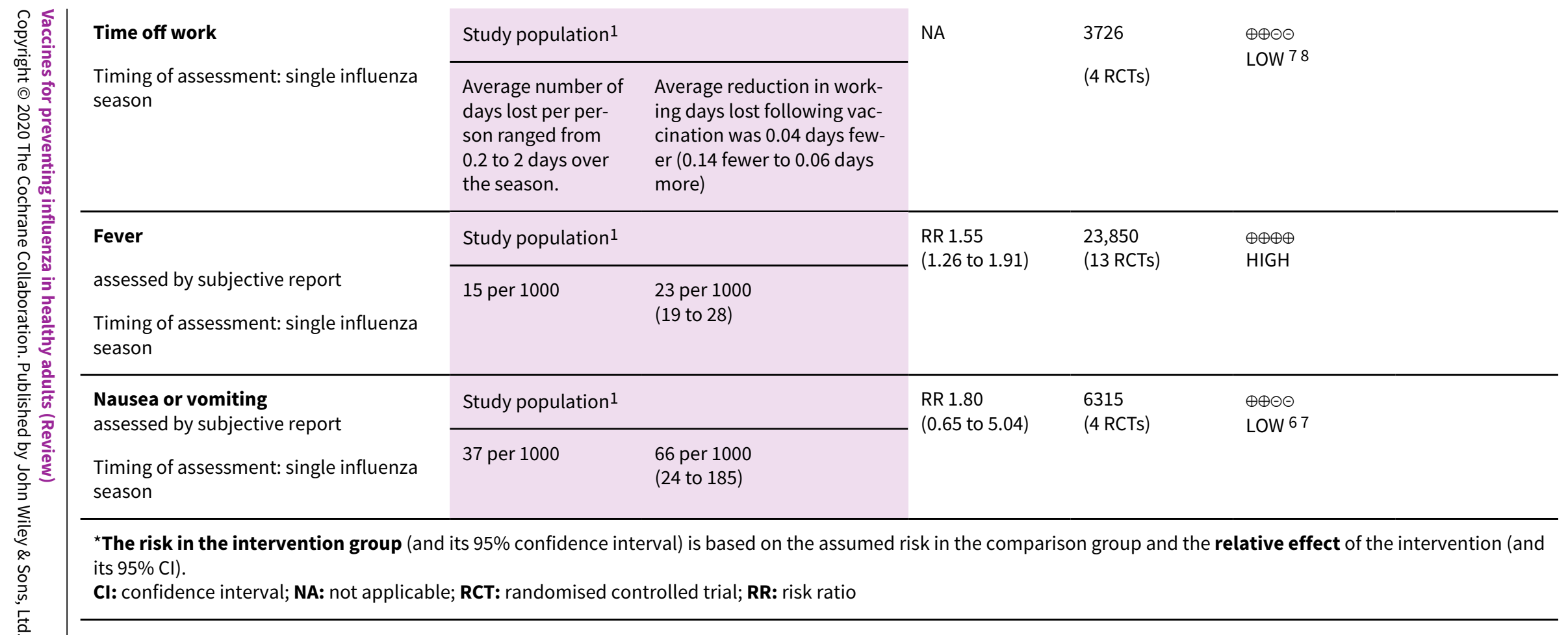

\section{GRADE Working Group grades of evidence}

High quality: We are very confident that the true effect lies close to that of the estimate of the effect.

Moderate quality: We are moderately confident in the effect estimate: the true effect is likely to be close to the estimate of the effect, but there is a possibility that it is substantially different.

Low quality: Our confidence in the effect estimate is limited: the true effect may be substantially different from the estimate of the effect.

Very low quality: We have very little confidence in the effect estimate: the true effect is likely to be substantially different from the estimate of effect.

${ }^{1}$ Control group risk calculated as the sum of events over total sample size from the control groups. For the outcome of influenza-like illness, control group risk was stratified as low, moderate (or median), and high due to variation in risk groups across the studies. For the remaining outcomes, the control group risk was taken as aggregate.

2 Sensitivity analysis by excluding studies with two or more domains at unclear risk of bias did not meaningfully alter the direction, size, or precision of effect. We are confident that bias is unlikely to exaggerate the intervention effect because the absolute reduction in influenza and relative reduction in the risk of influenza-like illness are small with vaccination.

${ }^{3}$ Downgraded one level due to serious indirectness. Uncertainty over definition, surveillance and testing of influenza in older trials.

${ }^{4}$ Downgraded one level for serious inconsistency. There is discordance between the direction and size of effects across the studies. Different definitions of influenza-like illness across the studies could explain why there is variation in the event rates across the control arms.

5 Downgraded one level due to serious risk of bias. Meta-analysis heavily influenced by a large study with high risk of bias across several domains.

6Downgraded one level due to serious imprecision. Confidence interval includes meaningful reduction and increase in effect.

${ }^{7}$ Downgraded one level due to serious risk of bias. Effect is influenced by studies judged to be at unclear risk of bias. 
Downgraded one level due to serious inconsistency. Direction and magnitude of effect differed across the studies $(12=82 \%)$. Wide confidence interval reflects the range of study effect sizes.

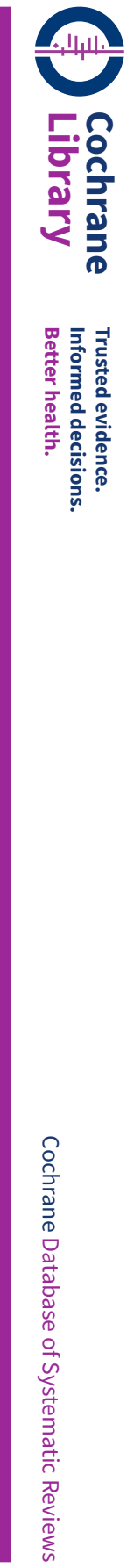




\section{B A C K G R O U N D}

\section{Description of the condition}

Viral respiratory disease imposes a heavy burden on society. The majority of viral respiratory disease (influenza-like illness (ILI)) is caused by many different agents that are not clinically distinguishable from one another. A variable proportion of ILI ( $7 \%$ to $15 \%$ on average) is caused by influenza viruses and is known as influenza (Jefferson 2009a).

Influenza is an acute respiratory infection caused by a virus of the Orthomyxoviridae family. Three serotypes are known (A, B, and C). Influenza causes an acute febrile illness with myalgia, headache, and cough. Although the median duration of the acute illness is three days, cough and malaise can persist for weeks. Complications of influenza include otitis media, pneumonia, secondary bacterial pneumonia, exacerbations of chronic respiratory disease, and bronchiolitis in children. Additionally, influenza can cause a range of non-respiratory complications, including febrile convulsions, Reye's syndrome, and myocarditis (Treanor 2016; Wiselka 1994). Efforts to prevent or minimise the impact of seasonal influenza in the second part of the 20th century were centred on the use of vaccines. Due to the yearly changes in viral antigenic configuration and the lack of carry-over protection from year to year, a new vaccination campaign needs to be organised annually, with a huge scientific and logistic effort to ensure production and delivery of the vaccines.

\section{Description of the intervention}

There are currently three types of influenza vaccines:

1. whole-virion vaccines, which consist of complete viruses that have been 'killed' or inactivated, so that they are not infectious but retain their strain-specific antigenic properties;

2. subunit vaccines, which are made of surface antigens ( $H$ and $N$ ) only; and

3. split-virion vaccines, in which the viral structure is broken up by a disrupting agent.

These vaccines contain both surface and internal antigens. In addition, a variety of non-European manufacturers produce live attenuated vaccines. Whole-virion vaccines are traditionally thought to be less well tolerated due to the presence of a lipid stratum on the surface of the viral particles (a remnant of the host cell membrane coating the virion, when budding from the host cell).

The US Food and Drug Administration (FDA) recently approved a new recombinant vaccine (Flublok) consisting of purified haemagglutinin proteins produced in inset cells for use in adults aged between 18 and 49 years with a known history of egg allergy (ACIP 2015).

Influenza vaccines are produced worldwide. Periodic antigenic drifts and shifts pose problems for vaccine production and procurement, as a new vaccine closely matching the circulating antigenic configuration must be produced and procured for the beginning of each new influenza 'season'. To achieve this, the World Health Organization (WHO) has established a worldwide surveillance system, allowing the identification and isolation of viral strains circulating the different parts of the globe. Sentinel practices recover viral particles from the nasopharynx of patients with influenza-like symptoms, and the samples are sent swiftly to the laboratories of the national influenza centres (110 laboratories in 79 countries). When new strains are detected, the samples are sent to one of the four WHO reference centres (London, Atlanta, Tokyo, and Melbourne) for antigenic analysis. Information on the circulating strain is then sent to the WHO, which in February of each year recommends through a committee the strains to be included in the vaccine for the forthcoming 'season'. Individual governments may or may not follow the WHO recommendations. Australia, New Zealand, and more recently South Africa have followed their own recommendations for vaccine content. Surveillance and early identification thus play a central part in the composition of the vaccine.

\section{How the intervention might work}

Vaccines work by simulating an infection and stimulating the body to produce antibodies against the threat and activate other defence mechanisms. Every vaccination campaign has stated aims against which the effects of the campaign must be measured. Perhaps the most detailed document presenting the rationale for a comprehensive preventive programme was that by the US Advisory Committee on Immunization Practice (ACIP), published in 2006 (ACIP 2006). The document identified 11 categories of people at high risk of complications from influenza, among which are healthy adults 50 to 65 years of age and healthcare workers. The rationale for policy choices rests on the heavy burden that influenza imposes on the populations and on the benefits accruing from vaccinating them. Reductions in cases and complications (such as excess hospitalisations, absence from work, mortality, and healthcare contacts) and the interruption of transmission are the principal arguments for extending vaccination to healthy adults aged 50 to 65 years (ACIP 2006).

The ACIP 2015 document update recommends routine vaccination for all people aged six months and older without contraindications. It underlines the importance of focusing vaccination efforts, when vaccination supplies are limited, on healthy adults who are at increased risk of developing severe complications from influenza, such as:

- people aged 50 years or over;

- women who are or who will be pregnant during the influenza season;

- healthcare personnel;

- household contacts and caregivers of children aged below five years and adults aged 50 years or over, with particular emphasis on vaccinating contacts of children younger than six months of age; and

- household contacts and caregivers of people with medical conditions that put them at higher risk of severe complications from influenza (ACIP 2010; ACIP 2015; Grohskopf 2016).

Pregnant women are included among priority recipients for seasonal influenza immunisation in many countries due to the risk of influenza-associated morbidity during pregnancy and the possible adverse neonatal outcomes associated with maternal influenza infections (AIH 2013; DoH 2015; NACI 2014; STIKO 2010), and based on evidence that vaccination of pregnant women protects their newborns from influenza and influenza-related hospitalisations ( $\mathrm{NACl} 2014$ ). 
Inactivated influenza vaccine may be administered at any stage of pregnancy, whereas live vaccine is not licensed for use during pregnancy as the available data about safety and efficacy in mothers and babies are very limited (ACIP 2010; DoH 2015).

The European Medicines Agency (EMA) recently made changes to the registration of seasonal, pre-pandemic, and pandemic influenza vaccines (EMA 2014; Wijnans 2016). The changes were introduced in 2014, triggered by the realisation that antibody responses are not sufficient predictors of field protection, as our reviews have consistently shown over the years. Most of the data for influenza vaccines included in our reviews are from registered vaccines, and yet the field protection afforded is modest or negligible. In addition, the methods of standardisation of antibody titres were lacking. The new rules for adults and the elderly require demonstration of non-inferiority of antibody response (immunogenicity) by a candidate seasonal influenza vaccine compared to an established one. In addition, whenever a demonstration of clinical efficacy is necessary (see Appendix 1), the EMA encourages minimisation of the use of placebo and encourages the use of active controls (such a non-influenza vaccines) with ILI (and relevant polymerase chain reaction (PCR) results) as a primary endpoint. Clinical effectiveness should be tested by carrying out (preferably prospective) cohort studies or nested so-called test-negative case-control studies following the European Centre for Disease Prevention and Control (ECDC) protocol (Kissling 2009a; Kissling 2009b).

Harms surveillance is now required with a follow-up of at least six months duration and in the general elderly population a database of at least 3000 people exposed to the vaccine. Enhanced vaccine vigilance data should be collected as soon as possible at the beginning of the vaccination campaign each year.

\section{Why it is important to do this review}

Due to the unique production cycle of influenza vaccines (they are tested using surrogate outcomes - antibody stimulation - ahead of each influenza 'season'), past performance is probably the only reliable way to predict future performance.

An accurate assessment of the effects (efficacy, effectiveness, and safety profile) of influenza vaccines is essential to allow rational choice between alternative strategies. This review with its two companion reviews, Demicheli 2014 and Jefferson 2012, are longrunning reviews. They are among the most consistently accessed in the Cochrane Database of Systematic Reviews, confirming the importance of the topic and interest in it. Periodic updates, some stretching back almost two decades, have allowed us to include an increasing number of studies on the effects of influenza vaccines and monitor their impact on our reviews (Table 1 ).

The reviews are not methodologically homogeneous, as their methods reflect the history and development of Cochrane Reviews. In particular, the inclusion of observational studies, which was initially favoured for the assessment of harms, has been a source of discussion. In this review, randomised evidence represents $44 \%$ of studies considered. To enhance the relevance of the review to decision-makers, in the Jefferson 2007 update, we included comparative non-randomised studies reporting evidence of serious or rare harms (or both).
Historically, observational studies have been of poor methodological quality, often reporting conflicting or paradoxical results, preventing the drawing of firm conclusions. However, inclusion of particular study types and increasing size of the data sets has not led to a change in the conclusion of the reviews, while leading to a greatly increased workload. This is the main reason why the authors, the review group, and the Cochrane editors have decided to stabilise all three reviews, that is not to carry out routine updates of the observational data set and update the randomised data set if certain conditions are fulfilled in the future.

For the same reason we have retained the observational content of this review and its companions as historical evidence of the life cycle of the reviews.

Since the 2014 update of this review (Jefferson 2014), we have included evidence about influenza vaccination in pregnant women and newborns.

We plan to update the randomised evidence in this review if any or all of these conditions are fulfilled in the future:

- a trial assessing the clinical effects of the evolution of current technologies becomes available;

- a new type of vaccine is developed; or

- a new credible causal paradigm for influenza is formulated.

For an overview of the three reviews, see the covering editorial at https://community.cochrane.org/news/why-have-three-longrunning-cochrane-reviews-influenza-vaccines-been-stabilised.

\section{O B J E C T IVES}

To assess the effects (efficacy, effectiveness, and harm) of vaccines against influenza in healthy adults, including pregnant women.

We defined 'effects' as follows:

1. efficacy as the capacity of the vaccines to prevent influenza A or $B$ and its complications;

2. effectiveness as the capacity of the vaccines to prevent influenza-like illness and its consequences; and

3. harm as any harmful event potentially associated with exposure to influenza vaccines.

\section{METHODS}

\section{Criteria for considering studies for this review \\ Types of studies}

Any randomised controlled trial (RCT) or quasi-RCT comparing influenza vaccines in humans with placebo or no intervention, or comparing types, doses, or schedules of influenza vaccine. We only considered studies assessing protection from exposure to naturally occurring influenza.

To enhance the relevance of the review to decision-makers, in the Jefferson 2007 update, we included comparative non-randomised studies if they reported evidence on the association between influenza vaccines and serious adverse effects, such as GuillainBarré syndrome or oculo-respiratory syndromes, or if they reported effectiveness or efficacy data for vaccine administration during pregnancy. 
We defined as RCTs studies in which it appeared that the individuals (or other experimental units) included in the study were definitely or possibly assigned prospectively to one of two (or more) alternative forms of health care using random allocation. A study was quasi-randomised when it appeared that the individuals (or other experimental units) followed in the study were definitely or possibly assigned prospectively to one of two (or more) alternative forms of health care using some quasi-random method of allocation (such as by alternation, date of birth, or case record number).

\section{Types of participants}

Healthy individuals aged 16 to 65 years, irrespective of influenza immune status. We excluded studies considering more than $25 \%$ of individuals outside this age range. We also included pregnant women together with their newborns.

\section{Types of interventions}

Live, attenuated, or killed vaccines, or fractions thereof, administered by any route, irrespective of antigenic configuration.

\section{Types of outcome measures}

\section{Primary outcomes}

\section{Clinical}

1. Numbers and seriousness (complications and working days lost) of symptomatic influenza and influenza-like illness (ILI) cases occurring in vaccine and placebo groups.

\section{Harms}

1. Number and seriousness of adverse effects (systemic and severe). Systemic adverse effects included cases of malaise, nausea, fever, arthralgia, rash, headache and more generalised and serious signs, such as neurological harms.

2. Maternal outcomes and outcomes related to the course of pregnancy. These included abortion (spontaneous, internal, foetal death, and stillbirth), preterm birth (less than 37 weeks), and maternal death.

3. Neonatal outcomes: congenital malformations (minor and major), neonatal death.

\section{Secondary outcomes}

1. Local adverse effects including induration, soreness, and redness at the site of inoculation.

\section{Search methods for identification of studies}

\section{Electronic searches}

We searched the Cochrane Central Register of Controlled Trials (CENTRAL; 2016, Issue 12) searched 31 December 2016 via the Cochrane Library), which contains the Cochrane Acute Respiratory Infections Group's Specialised Register; MEDLINE (PubMed) (January 1966 to 31 December 2016); Embase (Elsevier) (1990 to 31 December 2016); WHO International Clinical Trials Registry Platform (ICTRP; www.who.int/ictrp/en, 1 July 2017); and ClinicalTrials.gov (www.clinicaltrials.gov, 1 July 2017). See Appendix 2 for the search strategies used to identify trials.

See Appendix 3 for search strategies used prior to this 2017 update to identify observational studies. See Appendix 4 for strategies used in the 2010 update, and Appendix 5 for the MEDLINE search strategy used in 2004.

\section{Searching other resources}

In order to identify further trials, we read the bibliographies of retrieved articles and handsearched the journal Vaccine from its first issue to the end of 2009. The results of the handsearches are included in CENTRAL. In order to locate unpublished trials for the first edition of this review, we wrote to manufacturers and first or corresponding trial authors of studies in the review.

\section{Data collection and analysis}

\section{Selection of studies}

Two review authors (AR, CDP) independently excluded all initially identified and retrieved articles not fulfilling the inclusion criteria. In the case of disagreement, one review author (VD) acted as arbitrator. We recorded the selection process in sufficient detail to complete a PRISMA flow diagram and 'Characteristics of excluded studies' table (Moher 2009).

\section{Data extraction and management}

Two review authors (AR, CDP) performed data extraction using a data extraction form (Appendix 6). We checked and entered the data into Review Manager 5 software (RevMan 2014). We extracted data on the following:

- methodological quality of studies;

- study design (Appendix 7);

- description of setting;

- characteristics of participants;

- description of vaccines (content and antigenic match);

- description of outcomes;

- publication status;

- date of study;

- location of study.

One review author (CDP) carried out statistical analyses.

We assumed an ILI case (specific definition) to be the same as a 'flulike illness' according to a predefined list of symptoms (such as the Centers for Disease Control and Prevention (CDC) case definition for surveillance) or 'upper respiratory illness' according to a predefined list of symptoms.

The laboratory confirmations of influenza cases we found were:

1. virus isolation from culture;

2. four-fold antibody increase (haemagglutinin) in acute- or convalescent-phase sera;

3. four-fold antibody increase (haemagglutinin) in postvaccination- or postepidemic-phase sera.

\section{Assessment of risk of bias in included studies}

\section{Experimental studies (trials)}

Two review authors (CDP, AR) independently assessed the methodological quality of the included studies using criteria from the Cochrane Handbook for Systematic Reviews of Interventions (Higgins 2011). In case of disagreement, one review author (VD) acted as arbitrator in assigning quality judgements. 
We classified studies according to the following key domains for assessing risk of bias (Higgins 2011).

\section{Random sequence generation}

- Low risk of bias: e.g. a table of random numbers or computergenerated random numbers.

- High risk of bias: e.g. alternation, date of birth, day of the week, or case record number.

- Unclear risk of bias: if insufficient information was provided.

\section{Allocation concealment}

- Low risk of bias: e.g. numbered or coded identical containers were administered sequentially; an onsite computer system that could only be accessed after entering the characteristics of an enrolled participant; or serially numbered, opaque, sealed envelopes, or sealed envelopes that were not sequentially numbered.

- High risk of bias: e.g. an open table of random numbers.

- Unclear risk of bias: if insufficient information was provided.

\section{Blinding}

- Low risk of bias: if adequate double-blinding (e.g. placebo vaccine) or single-blinding (i.e. blinded outcome assessment) was used.

- High risk of bias: if there was no blinding.

- Unclear risk of bias: if insufficient information was provided.

\section{Incomplete outcome data}

Number of losses to follow-up:

- Low risk of bias: no missing data or the proportion of missing data compared with the observed event risk was not enough to have a clinically relevant impact on the intervention effect estimate.

- High risk of bias: when the proportion of missing data compared with observed event risk was large enough to induce clinically relevant bias in the intervention effect estimate.

- Unclear risk of bias: if insufficient information was provided.

\section{Non-experimental studies}

We carried out quality assessment of non-randomised studies in relation to the presence of potential confounders, which could make interpretation of the results difficult. We evaluated the quality of case-control (prospective and retrospective) and cohort studies using the appropriate Newcastle-Ottawa Scales (NOS) (Appendix 8).

Using quality at the analysis stage as a means of interpreting the results, we assigned 'Risk of bias' categories (Higgins 2011):

- Low risk of bias: plausible bias unlikely to seriously alter the results.

- Unclear risk of bias: plausible bias that raises some doubt about the results.

- High risk of bias: plausible bias that seriously weakens confidence in the results.

\section{Measures of treatment effect}

We used the risk ratio (RR) and its 95\% confidence interval (CI) as the summary measure. We calculated vaccine efficacy (or effectiveness) as VE $=1$ - RR, expressed as a percentage, for cohort and RCT/controlled clinical trial (CCT) studies. For case-control studies we adopted an odds ratio (OR) with $95 \%$ Cls.

To enhance relevance to everyday practice, we also expressed the summary measure of the most reliable and significant comparisons (those from RCTs with influenza cases as an outcome by age group) as a risk difference (RD). This is a measure of absolute efficacy of the vaccines, which incorporates significant information such as the incidence in the control arm and allows the calculation of its reciprocal, the number needed to treat for an additional beneficial outcome (NNTB), or in this case, the number needed to vaccinate (NNV).

The NNV expresses the number of children needed to be vaccinated to prevent one case of influenza. The NNV can be computed as 1/RD. Since meta-analysis estimates from RD are affected by statistical heterogeneity, we preferred to compute the NNV from the RD between assumed and corresponding risks. We used aggregate or median of the control group risks, giving a formula of: $1 /$ (control event rate (CER) - CER*RR).

We conducted quantitative synthesis of the evidence from observational studies using adjusted estimates, when these were available; in some cases we also used original data (unadjusted data) in order to compare meta-analysis results from adjusted and unadjusted estimates.

We calculated hospital admission rates as the proportion of cases hospitalised for respiratory causes. We considered complications as the proportion of cases complicated by bronchitis, pneumonia, or otitis. We also considered working days lost due to episodes of sickness absence regardless of cause. Only five trials used working days lost as an outcome measure, of which four trials measured the work absence in terms of the difference in the average number of days lost in two arms of the trial (Analysis 1.7). These studies presented a standard error value measured accordingly. The remainder expressed work absence in terms of rate ratio, which does not allow the recalculation of the correct estimate of the standard error (aa Nichol 1999a). We therefore excluded this study from the pooled analysis.

We presented local symptoms separately from systemic symptoms. We have considered individual harms in the analysis, as well as a combined endpoint (any or highest symptom). We used all data included in the analysis as presented by the authors in the primary study, regardless of the number of dropouts. We decided on this approach (complete-case scenario) because the majority of the included studies did not attempt to use an intention-to-treat analysis or mention the reasons for the loss to follow-up, and they did not contain detailed information to allow estimations of the real number of participants.

\section{Unit of analysis issues}

Several trials included more than one active vaccine arm. Where several active arms from the same trial were included in the same analysis, we split the placebo group equally between the different arms, so that the total number of participants in a single analysis did not exceed the actual number in the trials. 
We found four different definitions of the 'epidemic period'.

1. Interval between the first and the last virus isolation in the community.

2. Interval during which the influenza virus was recovered from more than a stated percentage of ill participants.

3. Period during which an increase of respiratory illness of more than a stated percentage was recorded.

4. Winter period, taken as a proxy for the epidemic period.

We included data regardless of the definition of epidemic period used in the primary study. When data were presented for the epidemic period and the entire follow-up period, we considered those that occurred during the former.

\section{Dealing with missing data}

For the first publication of this review (Demicheli 1999), we wrote to the trial authors and manufacturers to identify possible unpublished studies and missing data. The response was disappointing, and we desisted from any further attempts. Our analysis relies on existing data. Whenever possible we used the intention-to-treat population.

\section{Assessment of heterogeneity}

We calculated the $1^{2}$ statistic for each pooled estimate to assess the impact on statistical heterogeneity. The $1^{2}$ statistic can be interpreted as the proportion of total variation among effect estimates that is due to heterogeneity rather than sampling error, and it is intrinsically independent from the number of studies. When the $1^{2}$ statistic is less than $30 \%$, there is little concern about statistical heterogeneity (Higgins 2011). We used randomeffects models throughout to take into account the between-study variance in our findings (Higgins 2011). Variance is to be expected in influenza vaccine trials, as there are unpredictable systematic differences between trials regarding the circulating strains, degree of antigenic matching of the vaccine, type of vaccine, and the levels of immunity presented by different populations in different settings. Not all studies reported sufficient details to enable a full analysis of the sources of heterogeneity, but we were able to take into account vaccine matching and circulating strain.

\section{Assessment of reporting biases}

Due to the limited number of studies in each comparison or subgroup, assessment of publication bias was not applicable, since the evidence presented in this review originated mainly from published data. For this reason, our results could be affected by publication bias.

The overall quality of the retrieved studies was poor and was affected by poor reporting or limited descriptions of the studies' designs. A detailed description is provided in the Risk of bias in included studies section of the review.

The main problems with influenza vaccine studies are their poor quality and discrepancies between the data presented, their conclusions, and the authors' recommendations.

\section{Data synthesis}

We calculated all meta-analyses using a random-effects model due to expected variation in the efficacy and effectiveness of viral strain matching, and seasonal variation in virulence of the circulating influenza virus. We summarised evidence from non-randomised studies (cohort and case-control) according to Higgins 2011.

\section{Subgroup analysis and investigation of heterogeneity}

We carried out subgroup analyses according to the degree of matching with that year's World Health Organization (WHO) recommended content and with circulating viruses ("WHO recommended and matching" when known). WHO recommendations on the content of vaccines have been published since 1973. Different dosages and schedules of the vaccine and the presence of different adjuvants were not compared. We pooled data from the arms of trials comparing only vaccine composition or dosage in the analysis. We checked compliance of the study vaccine with the official antigenic content and potency recommendations by reviewing the WHO records whenever possible. In case of uncertainty due to ambiguity in the wording used (in the oldest trials), we took into account the opinion given by the trial authors. We classified the compliance of a live attenuated vaccine with the recommendations according to the antigenic comparability of the wild strains.

Since the degree of matching between vaccine and circulating strains could affect the effectiveness/efficacy of the vaccine, we analysed the data in separate subgroups according to this parameter. For serious adverse events, whenever possible we analysed data from pregnant women and the general population in separate subgroups. When case-control studies reported safety outcomes, whenever possible we performed analyses in separate subgroups according to time since exposure. Finally, we carried out a separate analysis of trials carried out during the 1968 to 1969 (H3N2) pandemic and the 2009 to 2010 (H1N1) pandemic.

\section{Sensitivity analysis}

As it was not possible to identify all sources of heterogeneity, we decided to carry out a sensitivity analysis by applying fixed-effect and random-effects models to assess the impact of heterogeneity on our results. In order to assess the robustness of our conclusions, we performed a sensitivity analysis by excluding studies judged to be at high risk of bias for one domain or unclear risk of bias for two or more domains. We restricted sensitivity analyses to Summary of findings for the main comparison outcomes (see below). Historical versions of this review compared the results from the crude data with those from the adjusted data from observational studies (historical versions of this review only).

\section{GRADE and 'Summary of findings' table}

We restricted our focus in the 'Summary of findings' tables to the comparison of inactivated parenteral influenza vaccine with placebo or do nothing, which we regarded as the most commonly adopted strategy. We created a Summary of findings for the main comparison using the following outcomes: ILI, influenza, hospitalisations, time off work, fever, and nausea/vomiting. We used the five GRADE considerations (study limitations, consistency of effect, imprecision, indirectness, and publication bias) to assess the quality of a body of evidence as it relates to the studies that contribute data to the meta-analyses for the prespecified outcomes (Atkins 2004). We used methods and recommendations described in Section 8.5 and Chapter 12 of the Cochrane Handbook for Systematic Reviews of Interventions (Higgins 2011), employing GRADEpro GDT software (GRADEpro GDT 2014). We used the results 
from randomised studies and justified all decisions to down- or upgrade the quality of studies using footnotes, making comments to aid the reader's understanding of the review where necessary.

\section{RESULTS}

\section{Description of studies}

\section{Results of the search}

The first publication of this review contained 20 trials (Demicheli 1999). In the second publication we included five additional trials (Demicheli 2004), and the third publication included 48 trials in total (Jefferson 2007). The fourth published update, Jefferson 2010, included two new trials (aa Beran 2009a; aa Beran 2009b), and excluded three new trials (Belongia 2009; Chou 2007; Khazeni 2009). The fourth update included 41 new study reports and excluded 63 new trials (Jefferson 2014). In this 2016 update we have included 20 new studies, excluded 21 new trials, and added two further trials (three data sets). One was newly identified (aa Mc Bride 2016a; aa Mc Bride 2016b), and one was included from the 'awaiting assessment' category (aa Treanor 2011).
Some of the included studies had more than two arms, comparing different vaccines, routes of administration, schedules, or dosages, or reported data from different settings and epidemic seasons. We split these studies into substudies (data sets). For the remainder of this review, the term 'study report' refers to the original study report, while the word 'data set' refers to the substudy; these substudies could refer either to different study arms, different influenza seasons, or different study designs. Risk of bias may be independently assessed for each substudy (or data set) study design.

More information about the division of study reports into data sets is given in the Characteristics of included studies table. In this 2016 updated review, we included a total of 160 studies (137 data sets), while we no longer updated searches for observational comparative studies (Figure 1). Trial register searches identified 18 completed trials with one or more corresponding publications, reporting methods and study design. All 18 trials had been identified and dealt with appropriately in our searches of journal publication databases. 
Figure 1. Study flow diagram.

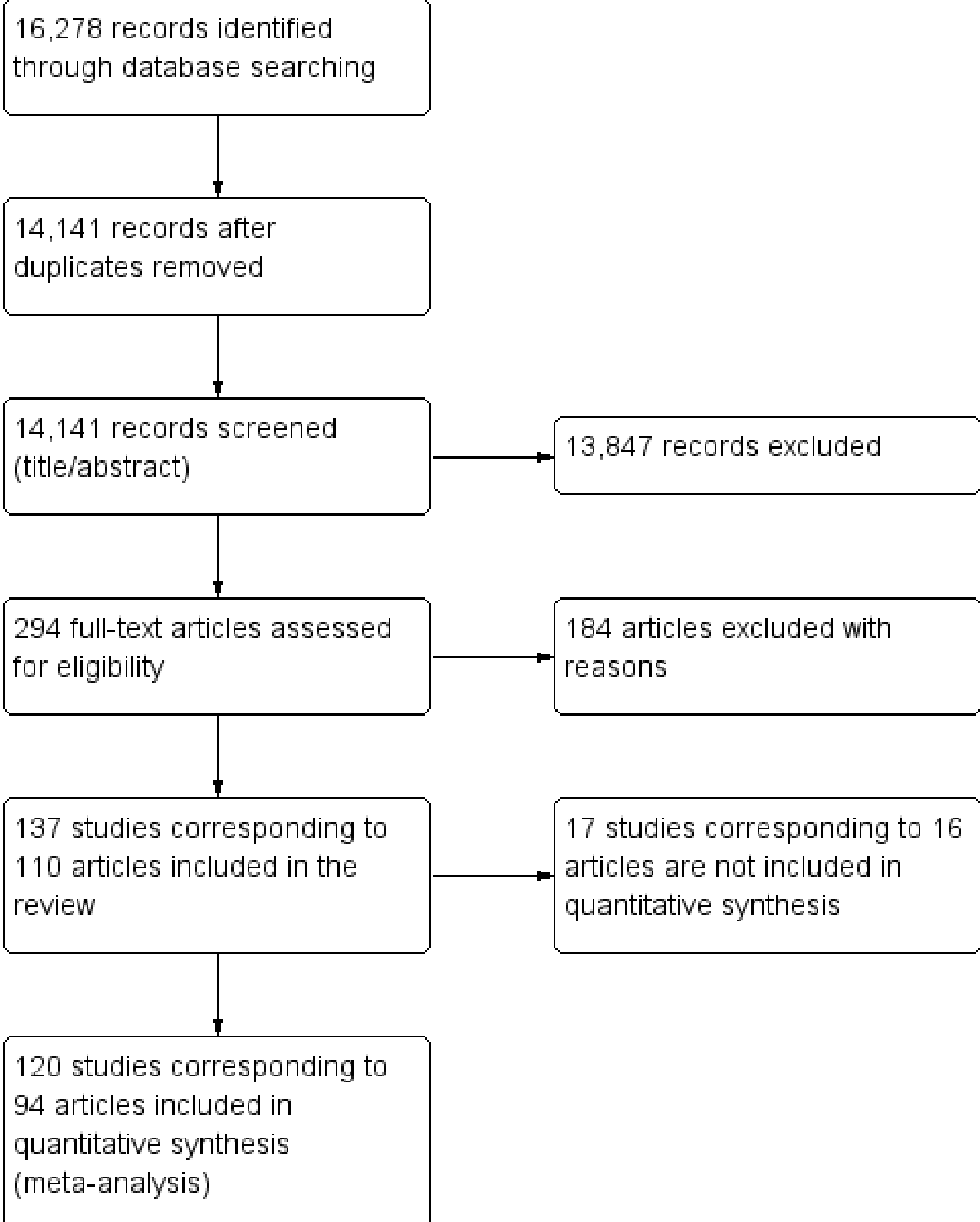




\section{Included studies}

We coded each trial on the basis of study design and the type of data contributed to the review as follows. The letter preceding the study represents the study design: (a) denotes RCTs, (b) denotes case-control studies, and (c) denotes cohort studies. The second letter indicates the contribution to the evidence in the data set: (a) efficacy/effectiveness or (b) harms. So, for example, a case-control study contributing safety or harms data is coded as (bb), and a trial contributing efficacy/effectiveness data is coded as (aa). A (p) code has been added to refer to the studies on vaccination during pregnancy.

\section{Seasonal vaccines: efficacy or effectiveness}

1. RCTs on inactivated parenteral vaccine: $(22$ studies/32 data sets) (aa Barrett 2011; aa Beran 2009a; aa Beran 2009b; aa Bridges 2000a; aa Bridges 2000b; aa Eddy 1970; aa Frey 2010; aa Hammond 1978; aa Jackson 2010a; aa Jackson 2010b; aa Keitel 1988a; aa Keitel 1988b; aa Keitel 1997a; aa Keitel 1997b; aa Keitel 1997c; aa Leibovitz 1971; aa Mcbride 2016a; aa Mcbride 2016b; aa Mesa Duque 2001; aa Mixéu 2002; aa Monto 2009; aa Nichol 1995; aa Ohmit 2006; aa Ohmit 2008; aa Powers 1995a; aa Powers 1995b; aa Powers 1995c; aa Tannock 1984; aa Treanor 2011; aa Weingarten 1988; aa Zhilova 1986a; aa Zhilova 1986b).

2. RCTs on live aerosol vaccine: (8 studies/12 data sets) (aa Edwards 1994a; aa Edwards 1994b; aa Edwards 1994c; aa Edwards 1994d; aa Monto 1982; aa Monto 2009; aa Nichol 1999a; aa Ohmit 2006; aa Ohmit 2008; aa Rytel 1977; aa Zhilova 1986a; aa Zhilova 1986b).

3. RCTs on inactivated aerosol vaccine: (one study/one data set) (aa Langley 2011).

\section{Seasonal vaccines: safety (local and systemic harms)}

1. RCTs on inactivated parenteral vaccine: $(21$ studies/22 data sets) (aa Barrett 2011; aa Bridges 2000a; aa Bridges 2000b; aa Frey 2010; aa Jackson 2010a; aa Mesa Duque 2001; aa Monto 2009; aa Nichol 1995; aa Ohmit 2006; aa Ohmit 2008; aa Powers 1995a; aa Tannock 1984; aa Treanor 2011; aa Weingarten 1988; ab Caplan 1977; ab El'shina 1996; ab Forsyth 1967; ab Goodeve 1983; ab Pyrhönen 1981; ab Rocchi 1979a; ab Saxen 1999; ab Scheifele 2003).

2. RCTs on live aerosol vaccine: (13 studies/14 data sets) (aa Monto 1982; aa Nichol 1999a; aa Ohmit 2006; aa Ohmit 2008; aa Rytel 1977; ab Atmar 1990; ab Betts 1977a; ab Evans 1976; ab Hrabar 1977; ab Keitel 1993a; ab Keitel 1993b; ab Lauteria 1974; ab Miller 1977; ab Rocchi 1979b).

3. RCTs on inactivated aerosol vaccine: (three studies/three data sets) (aa Langley 2011; ab Boyce 2000; ab Langley 2005).

We could not introduce two studies with live aerosol vaccine, ab Reeve 1982 and ab Spencer 1977, (each one a data set) into the harms analysis (secondary effects) because the data did not allow for quantitative analysis (systemic and local harms were reported given as cumulative in ab Spencer 1977 and data were not clearly reported in ab Reeve 1982).

\section{Administration during pregnancy - efficacy/effectiveness in mothers}

1. Seasonal trivalent inactivated vaccine containing pH1N1 RCTs: (one study/one data set) (paa Madhi 2014).
2. 2009 to $\mathbf{2 0 1 0}$ pandemic: inactivated vaccine - CCTs: (one study/one data set) (paa Ma 2014).

3. Seasonal inactivated vaccine - cohort studies: (three studies/ three data sets) (pca Ahrens 2014; pca Black 2004; pca Hulka 1964).

4. 2009 to 2010 pandemic: inactivated vaccines - cohort studies: (one study/one data set) (pca Yamada 2012).

\section{Administration during pregnancy - efficacy/effectiveness in} newborns

1. Seasonal trivalent inactivated vaccine containing pH1N1 RCTs: (one study/one data set) (paa Madhi 2014).

2. Seasonal inactivated vaccine - cohort studies on effectiveness (ILI): (three studies/three data sets) (pca Black 2004; pca Eick 2011; pca France 2006).

3. Seasonal inactivated vaccine - cohort studies on efficacy (laboratory-confirmed): (one study/one data set) (pca Eick 2011).

4. Seasonal inactivated vaccine - case-control on effectiveness (ILI): (two studies/two data sets) (pba Benowitz 2010; pba Poehling 2011).

Administration during pregnancy - pregnancy-related outcomes (abortion, congenital malformation, prematurity, neonatal death)

1. Seasonal inactivated vaccine - cohort studies: (seven studies/ seven data sets) (pca Ahrens 2014; pca Black 2004; pca Munoz 2005; pcb Dodds 2012; pcb Nordin 2014; pcb Omer 2011; pcb Sheffield 2012).

2. $\mathbf{2 0 0 9}$ to $\mathbf{2 0 1 0}$ pandemic: inactivated vaccine - cohort studies: (14 studies/14 data sets) (pcb Beau 2014; pcb Cleary 2014; pcb Fell 2012; pcb Håberg 2013; pcb Heikkinen 2012; pcb Källén 2012; pcb Launay 2012; pcb Lin 2012; pcb Ludvigsson 2013; pcb Oppermann 2012; pcb Pasternak 2012; pcb Richards 2013; pcb Rubinstein 2013; pcb Trotta 2014).

3. Seasonal trivalent inactivated vaccine containing pH1N1 cohort studies: (two studies/two data sets) (pcb Chambers 2013; pcb Louik 2013).

4. Seasonal inactivated vaccine - case-control: (one study/one data set) (pbb Irving 2013).

We did not introduce one study in the quantitative synthesis because it is the only study on the $\mathrm{A} / \mathrm{NJ} / 8 / 76$ vaccine ( $p c b$ Deinard 1981). We also did not include the retrospective cohort study of pcb Toback 2012 in the analysis because it did not contain useful outcomes. Results of one cohort study was not included in the analysis as it was only commented on (pcb Cantu 2013).

\section{Administration during pregnancy - severe harms}

One included cohort study assessed the association between seasonal vaccine exposure during pregnancy and the following harms within 42 days from administration: Guillain-Barré syndrome, demyelinating diseases, and immune thrombocytopenic purpura (pcb Nordin 2013).

\section{Severe harms - general population}

Guillain-Barré syndrome

1. 2009 to $\mathbf{2 0 1 0}$ pandemic - case-control: (two studies/six data sets) (bb Dieleman 2011a; bb Dieleman 2011b; bb Dieleman 
2011c; bb Dieleman 2011d; bb Dieleman 2011e; bb GrimaldiBensouda 2011).

2. Seasonal inactivated vaccine - case-control: (one study/one data set) (bb Galeotti 2013).

3. Seasonal inactivated vaccine - cohort studies: (two studies/ four data sets) (cb Kaplan 1982; cb Lasky 1998).

We did not introduce one cohort study assessing the association between the $\mathrm{A} / \mathrm{NJ} / 8 / 76$ vaccine and Guillain-Barré syndrome into the analysis (cb Shonberger 1979).

Demyelinating diseases (optic neuritis or multiple sclerosis)

1. Seasonal inactivated vaccine - case-control: (four studies/four data sets) (bb DeStefano 2003; bb Hernan 2004; bb Payne 2006; bb Zorzon 2003).

2. $\mathbf{2 0 0 9}$ to $\mathbf{2 0 1 0}$ pandemic - cohort study: (one study/one data set) (cb Moro 2013).

Immune thrombocytopenic purpura

1. Seasonal inactivated vaccine - case-control: (two studies/two data sets) (bb Garbe 2012; bb Grimaldi-Bensouda 2012).

Other serious adverse events

1. Oculo-respiratory syndrome: randomised cross-over trial (one study) (ab Scheifele 2003) and one case-control study (bb Rouleau 2014).

2. Respiratory function: RCT (ab Atmar 1990).

3. Cutaneous melanoma: case-control (bb Mastrangelo 2000).

4. Bell's palsy: case-control (bb Mutsch 2004).

5. Cardiac arrest: case-control (bb Siscovick 2000).

6. Acute myocardial infarction: case-control (bb Maclntyre 2013)

7. Rheumatoid arthritis: case-control (bb Ray 2011).
8. Neurological and autoimmune disorders: three cohort studies (cb Bardage 2011; cb O'Flanagan 2014; cb Persson 2014) and one case-control (bb Dauvilliers 2013).

9. Other serious adverse events: cohort study (cb Baxter 2012).

\section{Pandemic vaccine: efficacy or effectiveness}

1. RCT on inactivated parenteral vaccine: (four studies/seven data sets) (aa Eddy 1970; aa Mogabgab 1970a; aa Mogabgab 1970b; aa Waldman 1969a; aa Waldman 1969b; aa Waldman 1972b; aa Waldman 1972d).

2. RCT on inactivated aerosol vaccine: (two studies/four data sets) (aa Waldman 1969c; aa Waldman 1969d; aa Waldman 1972a; aa Waldman 1972c).

3. RCT on live aerosol vaccine (one study/one data set) (aa Sumarokow 1971).

\section{Excluded studies}

We excluded 183 studies (see Characteristics of excluded studies table).

\section{Risk of bias in included studies}

Out of the 137 included studies (substudy or data set), we classified $16.1 \%(22 / 137)$ as at low risk of bias (12 RCTs, two case-control, eight cohort studies); $17.5 \%(24 / 137)$ as at high risk of bias (seven RCTs, three case-control, 14 cohorts); and $66.4 \%$ (91/137) either did not present sufficient information in one or more key domains or, although presenting a low risk of bias in a specific domain, scored at high risk of bias in one or more items used in the quality evaluation. Table 2 shows the summary quality assessment of all included studies, and graphical displays of the quality assessment are presented in Figure 2 and Figure 3. We have highlighted that each 'paper' could include more than one study (data set), and these different studies required separate quality assessment. The funding source can be referred only to a single paper. 
Figure 2. 'Risk of bias' graph: review authors' judgements about each risk of bias item presented as percentages across all included studies.

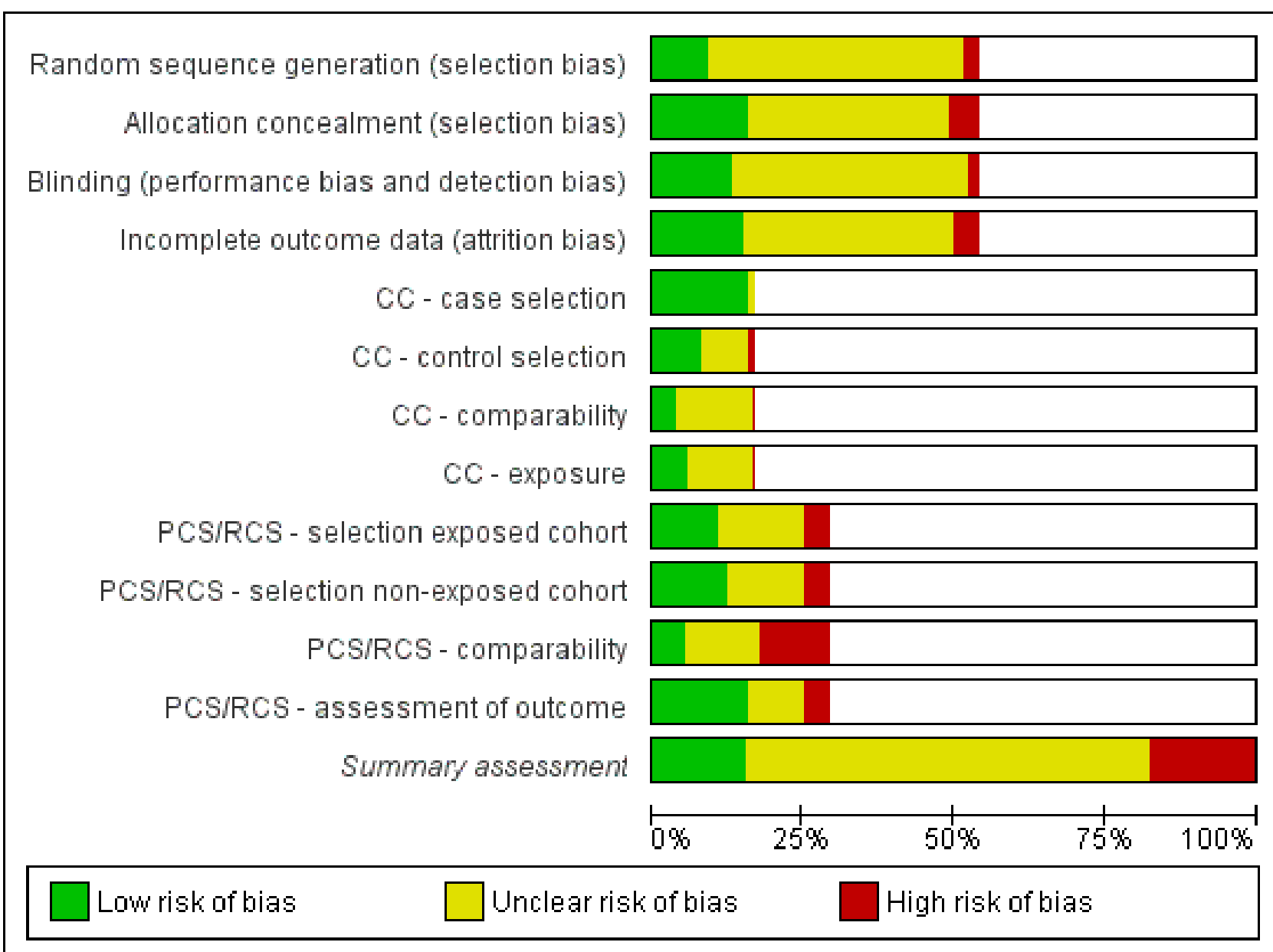


Figure 3. 'Risk of bias' summary: review authors' judgements about each risk of bias item for each included study.

\begin{tabular}{|c|c|c|c|c|c|c|c|c|c|c|c|c|c|}
\hline & 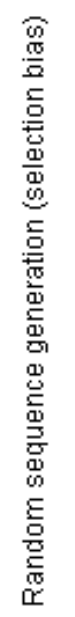 & 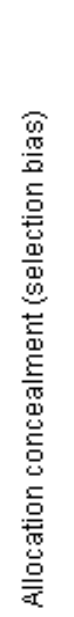 & 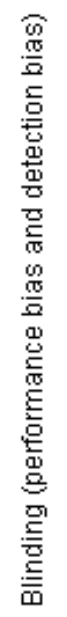 & 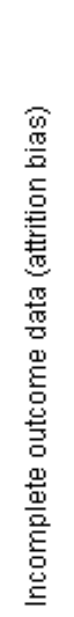 & 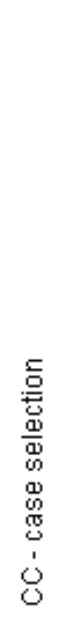 & 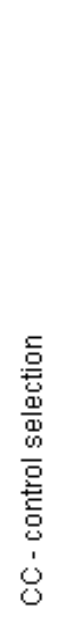 & 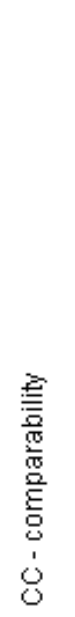 & 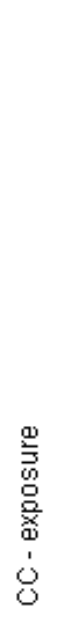 & 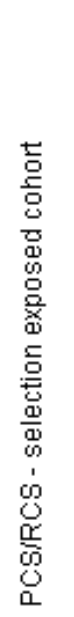 & 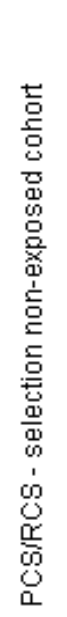 & 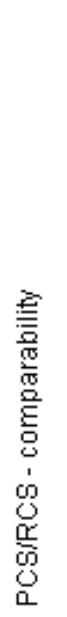 & 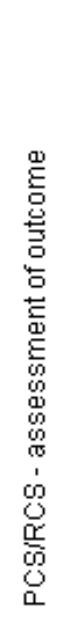 & 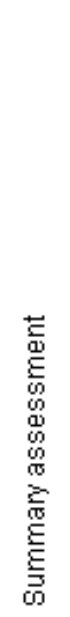 \\
\hline aa Barrett 2011 & $\odot$ & $\odot$ & $\odot$ & + & & & & & & & & & + \\
\hline aa Beran 2009a & + & $?$ & $?$ & + & & & & & & & & & $?$ \\
\hline aa Beran 2009b & $?$ & $?$ & $?$ & $\odot$ & & & & & & & & & $?$ \\
\hline aa Bridges 2000a & $?$ & + & + & + & & & & & & & & & + \\
\hline aa Bridges $2000 \mathrm{~b}$ & $?$ & $\odot$ & $\odot$ & + & & & & & & & & & + \\
\hline aa Eddy 1970 & $O$ & $O$ & $\Theta$ & 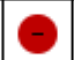 & & & & & & & & & $\Theta$ \\
\hline aa Edwards 1994a & $?$ & $\odot$ & + & $\odot$ & & & & & & & & & $?$ \\
\hline aa Edwards 1994b & $?$ & + & $\odot$ & - & & & & & & & & & $?$ \\
\hline aa Edwards $1994 \mathrm{c}$ & $?$ & + & + & - & & & & & & & & & $?$ \\
\hline aa Edwards 1994d & $?$ & + & + & - & & & & & & & & & $?$ \\
\hline aa Frey 2010 & $?$ & + & $?$ & + & & & & & & & & & $?$ \\
\hline aa Hammond 1978 & 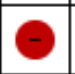 & O & $?$ & $?$ & & & & & & & & & $\theta$ \\
\hline aa Jackson $2010 a$ & ? & $?$ & + & + & & & & & & & & & $?$ \\
\hline aa Jackson 2010 b & $?$ & $?$ & $\odot$ & + & & & & & & & & & $?$ \\
\hline aa Keitel 1988a & $?$ & $?$ & $?$ & $?$ & & & & & & & & & $?$ \\
\hline aa Keitel 1988b & $?$ & $?$ & $?$ & $?$ & & & & & & & & & $?$ \\
\hline aa Keitel 1997a & $?$ & $?$ & $?$ & $?$ & & & & & & & & & $?$ \\
\hline aa Keitel 1997b & + & $?$ & $?$ & $?$ & & & & & & & & & $?$ \\
\hline aa Keitel $1997 \mathrm{c}$ & $?$ & $?$ & $?$ & $?$ & & & & & & & & & $?$ \\
\hline aa Langley 2011 & \begin{tabular}{|l|}
$?$ \\
\end{tabular} & + & + & + & & & & & & & & & + \\
\hline aa Leibovitz 1971 & $?$ & 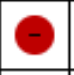 & $?$ & $?$ & & & & & & & & & - \\
\hline aa Mcbride 2016a & + & + & $?$ & $\odot$ & & & & & & & & & $\odot$ \\
\hline
\end{tabular}


Figure 3. (Continued)

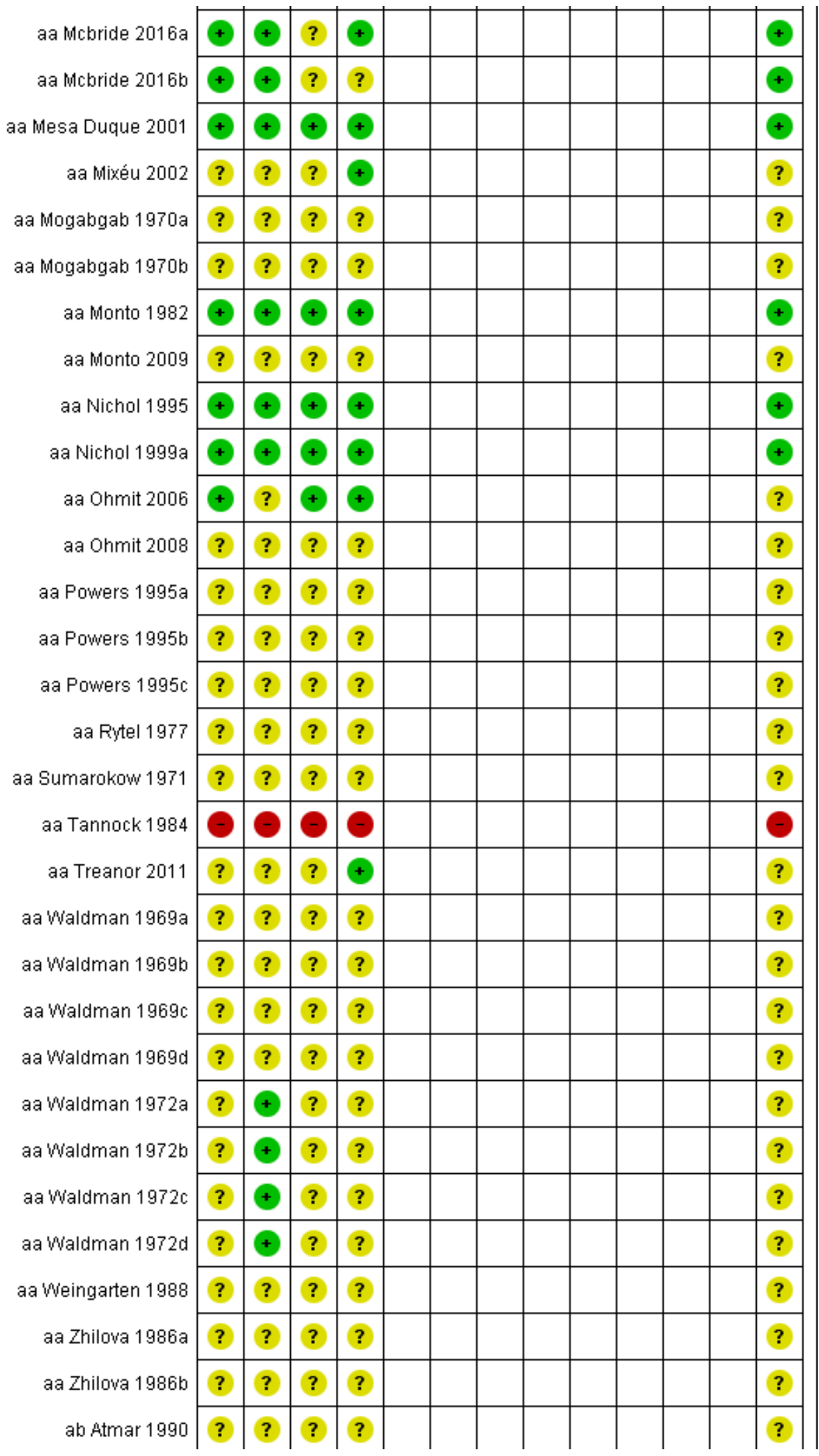


Figure 3. (Continued)

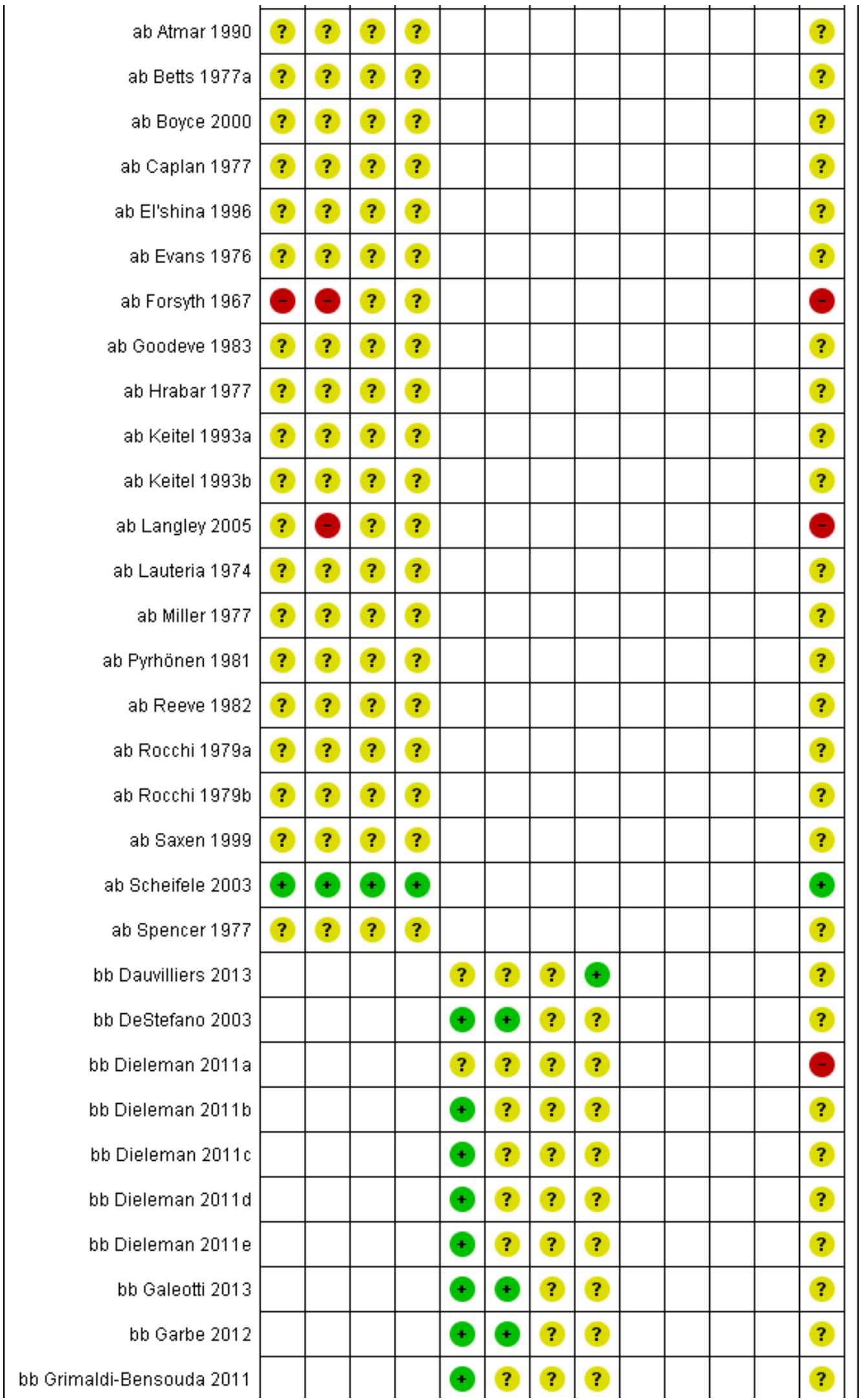


Figure 3. (Continued)

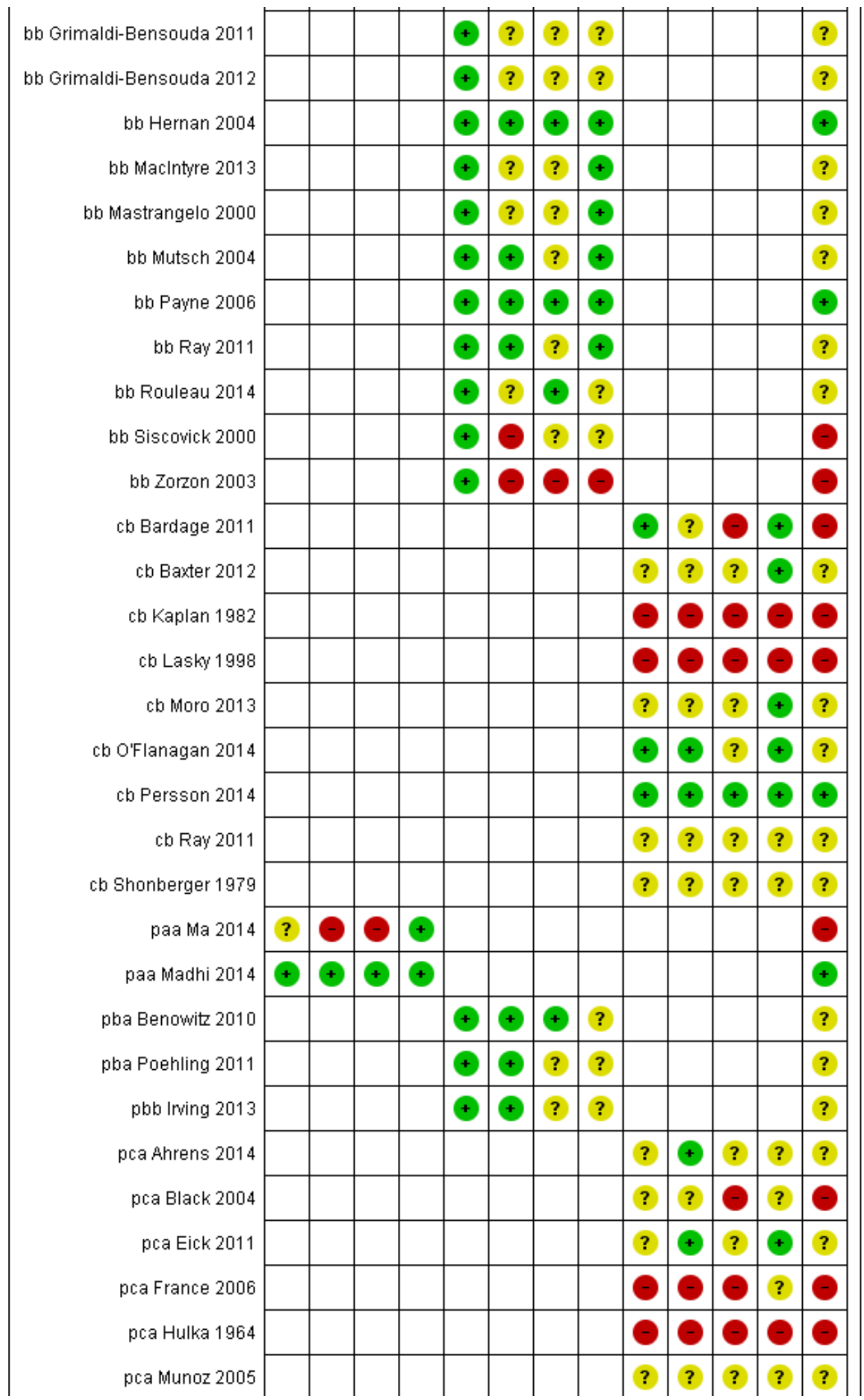


Figure 3. (Continued)

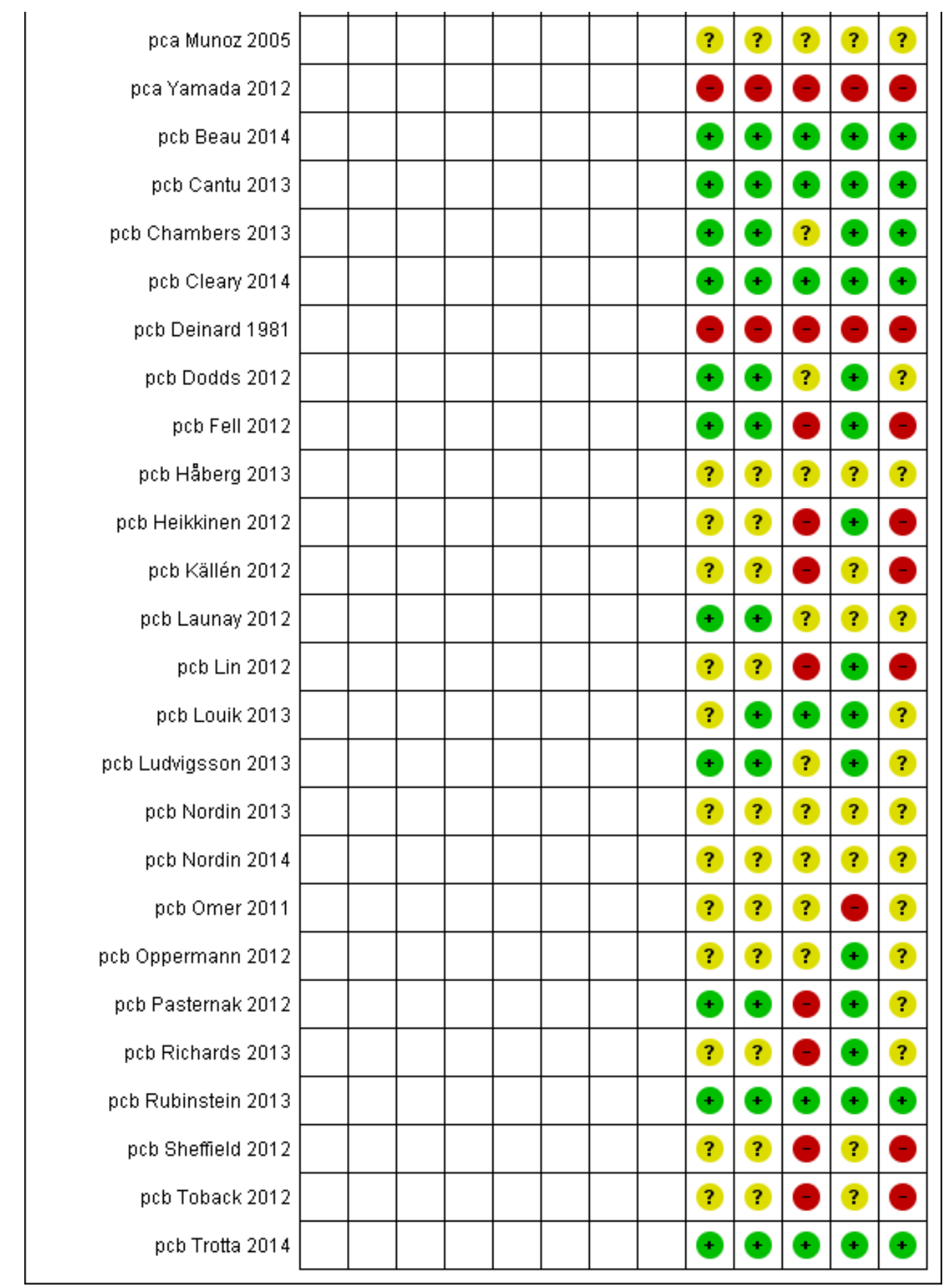

\section{Allocation}

In the included trials allocation concealment was adequate (low risk of bias) in 21 studies (28.4\%), inadequate (high risk of bias) in seven studies (9.5\%), and unclear (unclear risk of bias) in 46 studies $(62.2 \%)$.

\section{Blinding}

We judged blinding as at low risk of bias in $17 \mathrm{RCTs} / \mathrm{CCTs}$ (23\%), high risk of bias in three RCTs/CCTs (4.1\%), and unclear in 54 RCTs/CCTs (73\%).

\section{Incomplete outcome data}

The majority of the included RCTs/CCTs reported insufficient information about loss to follow-up (64 studies; 86.5\%). 


\section{Selective reporting}

The assessment of selective reporting bias presents several difficulties and would require review of the original study protocols for the included studies, which are mainly unavailable.

\section{Other potential sources of bias}

Few studies reported information on influenza circulation in the surrounding community, making interpretation of the results and assessment of their generalisability difficult.

It is now known that industry funding of influenza vaccine studies determines publication in high-prestige journals and higher citation rates than other types of funding. In addition, industry funding is associated with optimistic conclusions, but the quality of the majority of influenza vaccine studies is low, irrespective of funding (Table 3). A previously cited review showed a complex web of interrelationships between these variables (Jefferson 2009b), but the impact of this on policymaking is unknown.

\section{Case-control studies - quality assessment}

- Case selection (definition/representativeness): case identification is mainly performed by means of registers maintained at several healthcare organisations (HMO, Kaiser Permanente) or by hospital or GP (general practice) registers. A further case ascertainment is conducted by specialists in order to verify the agreement with the chosen case definition. In studies assessing vaccine efficacy, cases were identified using a laboratory test performed on all participants having symptoms. For 21 out of $23(91 \%)$, we classified case selection and definition as at low risk of bias.

- Control selection (definition): controls were selected from within the same registers used for case identification or from among participants living in the same catchment area of the hospitals in which the cases were identified. We classified control selection and definition as at low risk of bias for 10 out of 23 studies (43.4\%), and unclear risk of bias for 11 out of 23 $(47.8 \%)$.

- Comparability: the most frequent method used to ensure comparability between cases and controls consisted of matching for age, gender, and index date (onset of symptoms for cases and GP visit for controls). Less frequently matching was also done for other possible parameters, such as the number of GP visits within a certain time interval, or by resorting to the use of a propensity score or multivariate models in order to reduce the impact of other possible confounders. Nevertheless, many studies (18 out of $23(78.3 \%)$ ) provided insufficient information to judge how comparable cases and controls effectively are.

- Exposure ascertainment (same method of ascertainment for cases and controls/non-response rate): for studies based on healthcare organisations or insurance registers, assessment of vaccine exposure was certified in the same registers. In other studies vaccine exposure was ascertained with a structured interview, and less frequently also with the recovering of the vaccination records. In many studies (15 out of 23 (65.2\%)), ascertainment of the vaccine exposure was not fully reliable. For 7 out of 23 studies (30.4\%), we judged exposure ascertainment as at low risk of bias.

\section{Cohort studies - quality assessment}

- Selection exposed cohort (definition/representativeness): the majority of the studies were retrospective and used a data linkage method to select the exposed cohort. In 20 out of 40 studies (50\%), this procedure was insufficiently described.

- Selection non-exposed cohort (definition/ascertainment): most of the studies were based on record linkage and the identification of the non-exposed cohort was done by considering the absence of vaccination records. However, insufficient detail was provided, therefore we classified such studies as at unclear risk of bias (18 out of $40(45 \%))$.

- Comparability: in most of the included cohort studies matching procedures for the most probable confounders were applied using a multivariate model to ensure comparability between exposed and unexposed cohorts. A propensity score procedure was also sometimes used. Therefore in some studies only a few confounders were used to ensure comparability between exposed and non-exposed cohorts. We classified seven studies as at low risk of bias (17.5\%).

- Assessment of outcome (demonstration that outcome of interest was not present at the start of the study/ whether follow-up was long enough for outcomes to occur/ adequacy of follow-up of cohorts): outcomes of interest were generally documented in the registries used to identify the study population, and consequently were almost always retrospectively assessed, thus we classified 11 out of 40 (27.5\%) as at low risk of bias.

\section{Effects of interventions}

See: Summary of findings for the main comparison Inactivated parenteral influenza vaccine compared to placebo or 'do nothing' for preventing influenza in healthy adults

We constructed the Data and analyses tables according to the following criteria.

1. Inactivated parenteral influenza vaccine versus placebo or 'do nothing' (Comparison 01).

2. Live aerosol influenza vaccine versus placebo or 'do nothing' (Comparison 02).

3. Inactivated aerosol influenza vaccine versus placebo or 'do nothing' (Comparison 03).

4. Inactivated parenteral influenza vaccine versus placebo or 'do nothing' administered during pregnancy (Comparison 04).

5. Inactivated parenteral influenza vaccine versus placebo - cohort studies (Comparison 05).

6. Inactivated parenteral influenza vaccine versus placebo - casecontrol studies (Comparison 06).

7. Serious adverse events: Guillain-Barré syndrome - cohort studies (Comparison 07).

8. Serious adverse events: Guillain-Barré syndrome - case-control studies (Comparison 08).

9. Serious adverse events: demyelinating diseases (multiple sclerosis, optic neuritis) - cohort studies (Comparison 09).

10.Serious adverse events: demyelinating diseases (multiple sclerosis, optic neuritis) - case-control studies (Comparison 10).

11.Serious adverse events: immune thrombocytopenic purpura cohort studies (Comparison 11). 
12.Serious adverse events: immune thrombocytopenic purpura case-control studies (Comparison 12).

13.1968 to 1969 pandemic: inactivated polyvalent parenteral influenza vaccine versus placebo (Comparison 13).

14.1968 to 1969 pandemic: inactivated monovalent parenteral influenza vaccine versus placebo (Comparison 14).

15.1968 to 1969 pandemic: inactivated polyvalent aerosol influenza vaccine versus placebo (Comparison 15)

16.1968 to 1969 pandemic: inactivated monovalent aerosol influenza vaccine versus placebo (Comparison 16).

17.1968 to 1969 pandemic: live aerosol influenza vaccine versus placebo (Comparison 17).

Evidence from RCTs/CCTs on vaccine efficacy/effectiveness in the general population is reported in Analyses 1 to 3 . Evidence from RCTs/CCTs on vaccine efficacy/effectiveness in pregnancy is reported in Comparison 4. Evidence from observational studies in pregnancy is reported in Analyses 5 and 6.

Studies investigating the association between influenza vaccination and Guillain-Barré syndrome were included in Comparison 7 (cohort on seasonal vaccine) and Comparison 8 (case-control on H1N1 vaccine). In Comparison 8, we stratified studies according to three different exposure definitions according to the time between vaccination to onset of symptoms (any time, within seven weeks, over seven weeks). We have presented evidence for the association between seasonal vaccine and Guillain-Barré syndrome from cohort studies in Comparison 7.

Studies investigating the association between influenza vaccination and multiple sclerosis and optic neuritis are included in Analyses 9 and 10 (cohort and case-control studies - demyelinating diseases).

Studies investigating the association between influenza vaccination and immune thrombocytopenic purpura are included in Analyses 11 and 12 (cohort and case-control studies - immune thrombocytopenic purpura).

We have constructed a 'Summary of findings' table for key outcomes (see Summary of findings for the main comparison).

\section{Inactivated parenteral influenza vaccine versus placebo or 'do nothing' (Comparison 01)}

Inactivated parenteral vaccines probably have $59 \%$ efficacy in preventing confirmed influenza (risk ratio (RR) $0.41,95 \%$ confidence interval $(\mathrm{Cl}) 0.36$ to $0.47 ; 71,221$ participants; 25 studies, moderate-certainty evidence) (Analysis 1.1). Based on the control group risk of $2.3 \%, 71$ healthy adults need to be vaccinated in order to prevent one of them experiencing influenza. The effects were very similar when matching was absent or unknown. Since heterogeneity was very low $\left(I^{2}=17 \%\right.$ for Analysis $1.2 .1 ; I^{2}=14 \%$ for Analysis 1.1.2), there were no differences when comparing the estimates obtained by using a fixed-effect model with those from a random-effects model. Restricting the analysis to studies at low risk of bias did not affect the direction or size of effect (see Table 4).

Inactivated parenteral vaccines probably have $16 \%$ effectiveness in preventing ILI (RR $0.84,95 \% \mathrm{Cl} 0.75$ to $0.95 ; 25,795$ participants; 16 studies; moderate-certainty evidence) (Analysis 1.2). There was wide variation in the control group risks, with risk differences in low-, moderate-, and high-risk groups of $0.6 \%, 3.4 \%$, and $14.6 \%$.
Based on the median (i.e. moderate risk) control group risk of $21.5 \%, 29$ healthy adults need to be vaccinated to prevent one adult experiencing an ILI. For low- and high-risk control group the corresponding NNVs were 167 and 7, respectively. Sensitivity analysis by risk of bias did not change the size or direction of effect (Table 4).

Results across the subgroups by matching criteria were very similar $(12=0 \%)$

Based on the results from a single study (aa Bridges 2000b), physician visits appear $42 \%$ less frequent (95\% Cl $9 \%$ to $63 \%$ ) in participants immunised with vaccines prepared with strains matching circulating viruses (Analysis 1.3.1), whereas there were no significant results when the degree of matching was unknown or absent (RR 1.28, 95\% Cl 0.90 to 1.83; Analysis 1.3.2). The overall effect was also not significant ( $\mathrm{RR} 0.87,95 \% \mathrm{Cl} 0.40$ to 1.89 ) (Analysis 1.3). Even though the two data sets of aa Bridges $2000 \mathrm{~b}$ showed very high heterogeneity ( $12=87 \%)$, no difference arose when comparing the results from the fixed-effect with the random-effects model.

We observed a similar conflicting result when analysing the effect of inactivated vaccine administration on days of illness (Analysis 1.4), when the estimate (mean difference (MD)) obtained in goodmatch conditions was compared with unknown or absent degree of matching. As a consequence of the high overall heterogeneity $(12=87 \%)$, the result obtained from the fixed-effect model analysis (MD $-0.31,95 \% \mathrm{Cl}-0.54$ to -0.07 ) differed from the result of the application of a random-effects model (MD $-0.21,95 \% \mathrm{Cl}-0.98$ to 0.56).

There seemed to be no effect on the time an antibiotic or drug was prescribed (Analysis 1.5; Analysis 1.6).

Four trials evaluated time off work, estimating that vaccination may save around 0.04 working days per person over a single influenza season. This result was affected by high levels of heterogeneity (12 = $82 \%)$ but did not change depending on whether a fixed-effect (MD $-0.04,95 \% \mathrm{Cl}-0.06$ to -0.01 ) or random-effects model (MD $-0.04,95 \%$ $\mathrm{Cl}-0.14$ to 0.06 ) (Analysis 1.7) was used. We rated the evidence as of low certainty.

Vaccination may have a small effect on hospitalisation (Analysis 1.8), but the $\mathrm{Cl}$ was wide and does not rule out a large reduction in hospitalisation ( $\mathrm{RR} 0.96,95 \% \mathrm{Cl} 0.85$ to 1.08 ; low-certainty evidence). We found no evidence for cases of pneumonia.

\section{Harms}

Live parenteral influenza vaccines increase fever from $1.5 \%$ to 2.33\% (RR $1.55,95 \%$ Cl 1.26 to $1.91 ; 23,850$ participants; 13 studies; high-certainty evidence) (Analysis 1.11.2). The rate of nausea or vomiting was low in the trials (4\% in unvaccinated population versus $7 \%$ with vaccines), although we rated this evidence as low certainty due to wide $\mathrm{Cls}$ and possible impact of bias (see Table 4) (RR $1.80,95 \% \mathrm{Cl} 0.65$ to $5.04 ; 6315$ participants; 4 trials) (Analysis 1.11.5).

Local tenderness and soreness were more than three times as common among parenteral vaccine recipients than among those in the placebo group (RR 3.13, 95\% Cl 2.44 to 4.02) (Analysis 1.10.1). There were also increases in erythema (RR $2.59,95 \% \mathrm{Cl} 1.77$ to 3.78; Analysis 1.10.2) and induration (RR $4.28,95 \% \mathrm{Cl} 1.25$ to 14.67 ) but not in arm stiffness. The combined local effects endpoint was 
significantly higher for those receiving the vaccine (RR $2.44,95 \% \mathrm{Cl}$ 1.82 to 3.28 ; Analysis 1.10 .5 ).

Myalgia was significantly associated with vaccination (RR 1.74, 95\% $\mathrm{Cl} 1.41$ to 2.14 ) (Analysis 1.11.1), fatigue or indisposition (RR 1.19, $95 \% \mathrm{Cl} 1.05$ to 1.36 ) (Analysis 1.11.4), and malaise (RR 1.51, 95\% $\mathrm{Cl} 1.18$ to 1.92) (Analysis 1.11.6). The combined endpoint was not increased (RR 1.16, 95\% Cl 0.87 to 1.53; Analysis 1.11.7).

\section{Live aerosol influenza vaccine versus placebo or 'do nothing' (Comparison 02)}

Live aerosol vaccines have an overall efficacy of 53\% (95\% Cl 38\% to $65 \%)$, and the NNV is $39(95 \% \mathrm{Cl} 32$ to 54$)$. Neither content nor matching appeared to affect their performance significantly. The vaccines have an effectiveness against ILI of $10 \%(95 \% \mathrm{Cl} 4 \%$ to $16 \%$; NNV 46, 95\% Cl 29 to 115), and content and matching appeared not to affect their performance significantly (Analysis 2.2).

No evidence was available on complications (e.g. bronchitis, otitis media, pneumonia).

The effectiveness of the aerosol vaccines against ILI (with no clear definition) was significant only for vaccines with absent or unknown matching (37\%, 95\% Cl 20\% to $51 \%$ ), and the NNV was 69 (95\% Cl 23 to 46) (Analysis 2.3).

The conclusions of this comparison were unaffected by analysis using either the fixed-effect or random-effects models.

\section{Harms}

Significantly more recipients experienced local symptoms after vaccine administration than after placebo administration (Analysis 2.4).

- Upper respiratory infection (RR $1.66,95 \% \mathrm{Cl} 1.22$ to 2.27 ).

- Cough (RR $1.51,95 \% \mathrm{Cl} 1.08$ to 2.10 ).

- Coryza (RR $1.56,95 \% \mathrm{Cl} 1.26$ to 1.94 ).

- Sore throat (RR 1.66, 95\% Cl 1.49 to 1.86 ).

- Combined endpoint (any or highest symptom) (RR 1.56, 95\% Cl 1.31 to 1.87 ).

There was no significant increase in systemic harms (combined endpoint: any or highest symptom RR $1.40,95 \% \mathrm{Cl} 0.82$ to 2.38 ), although rates of myalgia (RR $2.47,95 \% \mathrm{Cl} 1.26$ to 4.85 ) and headache (RR $1.54,95 \% \mathrm{Cl} 1.09$ to 2.18 ) were higher in the vaccine group than in the placebo group (Analysis 2.5).

\section{Inactivated aerosol influenza vaccine versus placebo or 'do nothing' (Comparison 03)}

We could include no RCTs assessing the effectiveness of inactivated aerosol vaccines in preventing ILI; the only available evidence comes from studies carried out during the 1968 to 1969 pandemic (Analyses 12 to 16).

The efficacy of inactivated aerosol vaccine in preventing laboratory-confirmed influenza (Analysis 3.1.1) was assessed in one RCT (aa Langley 2011), whose results do not show a statistically significant protective effect (RR $0.38,95 \% \mathrm{Cl} 0.14$ to 1.02 ).

\section{Harms}

None of the trials on inactivated aerosol vaccines reported significant harms.

\section{Inactivated parenteral influenza vaccine versus placebo or 'do nothing' administered during pregnancy (Comparison 04)}

In this analysis, we considered the results of one RCT (at low risk of bias) and one CCT (at high risk of bias) assessing the effect of vaccination during pregnancy on the prevention of influenza and ILI in both mother and newborns.

Vaccination with trivalent inactivated vaccine containing $\mathrm{pH} 1 \mathrm{~N} 1$ was weakly protective against influenza (RCT data only) in mothers within 24 weeks after delivery (RR $0.50,95 \% \mathrm{Cl} 0.29$ to 0.86 ; vaccine efficacy (or effectiveness) (VE) 50\%, $95 \% \mathrm{Cl} 14 \%$ to $71 \%$; NNV 55 , $95 \% \mathrm{Cl} 39$ to 198; Analysis 4.1), as well as among children born from a vaccinated mother until their first 24 weeks of life (VE 49\%, $95 \% \mathrm{Cl} 12 \%$ to $70 \%$; NNV 56, $95 \% \mathrm{Cl} 39$ to 230; Analysis 4.3). Vaccination with monovalent pandemic or trivalent inactivated vaccine containing $\mathrm{pH} 1 \mathrm{~N} 1$ did not confer significant protection against ILI, either in mothers (RR $0.96,95 \% \mathrm{Cl} 0.79$ to 1.16 ; Analysis 4.2) or in newborns (RR 1.02, 95\% Cl 0.94 to 1.09; Analysis 4.4).

\section{Inactivated parenteral influenza vaccine versus placebo - cohort studies (Comparison 05)}

Based on unadjusted data from a cohort study (high risk of bias), 2009/2010 H1N1 monovalent pandemic vaccines (Analysis 5.1.1) provide a significant protective effect against ILI in pregnant women (RR $0.11,95 \% \mathrm{Cl} 0.06$ to 0.21 ; VE $89 \%, 95 \% \mathrm{Cl} 79 \%$ to 94\%; NNV 54, $95 \% \mathrm{Cl} 51$ to 61 ). Seasonal inactivated vaccine is not effective against ILI (RR $0.54,95 \% \mathrm{Cl} 0.24$ to 1.18 ; Analysis 5.1.2). Sensitivity analysis performed using the fixed-effect model showed statistical significance, even for a modest protective effect (RR 0.76, $95 \% \mathrm{Cl} 0.64$ to 0.89 ; NNV 94, $95 \% \mathrm{Cl} 63$ to 205 ; VE 24\%, $95 \% \mathrm{Cl} 11 \%$ to $36 \%)$.

The effectiveness of vaccination with seasonal inactivated vaccine during pregnancy for preventing ILI in newborns was not statistically significant, as the results are based on two cohort studies using either hazard ratio (HR) or RR adjusted estimates (Analysis 5.2.1 and Analysis 5.3.1, respectively). Efficacy against confirmed influenza (Analysis 5.3.2) is modest but has statistical significance (adjusted RR $0.59,95 \% \mathrm{Cl} 0.37$ to 0.94 ; NNV $27,95 \% \mathrm{Cl}$ 18 to 185 ; VE $41 \%, 95 \% \mathrm{Cl} 6 \%$ to $63 \%)$.

Vaccination with the $2009 / 2010$ H1N1 monovalent pandemic vaccine during pregnancy may not be associated with a higher risk of abortion (Analysis 5.4.1 and Analysis 5.4.2), congenital malformation (Analysis 5.4.3), or neonatal death (Analysis 5.4.9). From a meta-analysis of seven cohort studies, preterm deliveries (before 37 weeks of gestation) occurred with slightly less frequency among women who were immunised with monovalent pandemic $\mathrm{H} 1 \mathrm{~N} 1$ vaccine during pregnancy compared to unvaccinated women (Analysis 5.4 .5 , adjusted $\mathrm{OR} 0.84,95 \% \mathrm{Cl} 0.76$ to 0.93 ). This result was not confirmed by two other cohort studies, which found no significant association (Analysis 5.4.6, adjusted HR 1.11, 95\% Cl 0.46 to 2.68 ) or by two other cohort studies that separately analysed vaccine administration during the first trimester of gestation with that during the second or third trimester (Analysis 5.4.6; Analysis 5.4.7; Analysis 5.4.8). 
Cases of neonatal death and abortion were observed less frequently among women immunised with seasonal influenza vaccine (Analysis 5.5.1 and Analysis 5.5.4, both unadjusted estimates). We found no statistically relevant association between seasonal influenza vaccine exposure during pregnancy and prematurity or congenital malformations (Analysis 5.5.2; Analysis 5.5.3; Analysis 5.5.4). Two other cohort studies did not find any statistically significant association between exposure to seasonal trivalent inactivated vaccine containing $\mathrm{pH} 1 \mathrm{~N} 1$ and prematurity, whatever the trimester of gestation (Analysis 5.6). This finding was confirmed by one other retrospective cohort study, which was not included in the analysis (pcb Cantu 2013, adjusted RR 1.2, 95\% Cl 0.9 to 1.6$)$.

The results of pcb Deinard 1981 are based on the follow-up results of 189 pregnant women immunised with monovalent pandemic $\mathrm{A} /$ New Jersey/8/76 (either in split- or whole-virus formulation) and 517 pregnant women who did not receive vaccination. The time of observation was extended up to the first eight weeks of life of the newborns. No statistically different incidence of maternal pregnancy outcomes or infant deaths was observed between vaccinated and unvaccinated groups. Statistical analysis (Chi ${ }^{2}$ test) showed no relation between immunisation history and presence of anomalities at the eighth week of life. We did not include this cohort study in the analysis, as the vaccine studied is no longer in use.

\section{Inactivated parenteral influenza vaccine versus placebo - case- control studies (Comparison 06)}

This analysis only included studies assessing the effect of vaccination against influenza during pregnancy. The incidence of $\mathrm{ILI}$ in pregnant women who were immunised with inactivated seasonal vaccine during pregnancy was not statistically different when compared with that observed among unvaccinated pregnant women (Analysis 6.1.1). However, the results of the analysis became statistically significant in sensitivity analysis using the fixed-effect model, leading us to conclude that the results of this comparison were affected by the model used to perform the analysis.

One further case-control study did not find a statistically significant association between exposure to seasonal inactivated vaccine in pregnancy and abortion cases (Analysis 6.2.1).

One retrospective cohort study attempted to assess the effect of live attenuated vaccine during pregnancy based on data from a health insurance database during six subsequent influenza seasons ( $p c b$ Toback 2012). A total of 834,999 pregnant women were identified, of whom 138 received live attenuated vaccine at any time during pregnancy. Claims for hospitalisation or visits to the emergency department within 42 days after immunisation were searched for but all observed events were considered to be related to a normal physiological pregnancy and not to immunisation. The system used (claim data) would be unable to detect birth outcomes.

\section{Serious adverse events: Guillain-Barré syndrome - cohort studies (Comparison 07)}

Two cohort studies performed during two subsequent epidemic seasons investigated the possible association between exposure to seasonal inactivated vaccine in healthy adults and GuillainBarré syndrome onset within six weeks following immunisation. No significant association was found (Analysis 7.1.1). Administration of seasonal inactivated vaccine during pregnancy was not associated with Guillain-Barré syndrome onset within six weeks from immunisation (Analysis 7.1.2).

The cohort of cb Shonberger 1979 was the first study that compared Guillain-Barré syndrome cases by vaccination status and the national incidence in vaccinated and unvaccinated national cohorts after the suspension of the National Influenza Immunization Program in the winter of 1976 to 1977. At that time the monovalent inactivated swine vaccine $\mathrm{A} / \mathrm{New}$ Jersey/8/76 had been administered. The attributable risk from vaccination was just below one case of Guillain-Barré syndrome in every 100,000 vaccinations. We did not include this cohort study in the analysis as the vaccine studied is no longer in use.

\section{Serious adverse events: Guillain-Barré syndrome - case- control studies (Comparison 08)}

In an analysis performed using the mean of unadjusted data relative to six data sets, exposure to monovalent H1N1 pandemic inactivated vaccine resulted in an apparent statistically significant association with Guillain-Barré syndrome onset when administration took place within six weeks before symptoms occurred (odds ratio (OR) 2.22, 95\% Cl 1.14 to 4.31; Analysis 8.1.1). It should thus be taken into account that only one out of the six data sets showed a statistically significant association between vaccine exposure and Guillain-Barré syndrome onset (bb Dieleman 2011e). When we performed a sensitivity analysis excluding this data set from the pooled estimate, the result was no longer significant. When the analysis was performed for vaccine exposure that occurred at any time before disease onset, there was no significant association (Analysis 8.1.2).

The analyses performed by pooling authors' estimates adjusted for several confounders (i.e. receipt of other vaccines, family history of autoimmune diseases, physician consultation during the previous year, and use of antibiotic, antiviral, or antipyretic agents) did not show a statistical association for exposure within six weeks (Analysis 8.2.1) before disease onset or for exposure at any time (Analysis 8.2.2).

Data from one other case-control study confirmed that immunisation with seasonal inactivated vaccine is not significantly associated with the onset of Guillain-Barré syndrome within six weeks after inoculation (Analysis 8.3) (bb Galeotti 2013).

\section{Serious adverse events: demyelinating diseases (multiple sclerosis, optic neuritis) - cohort studies (Comparison 09)}

In one cohort study the authors attempted to assess whether there was an association between exposure to inactivated trivalent seasonal influenza vaccine during pregnancy and several pathologies (e.g. Guillain-Barré syndrome, demyelinating diseases, immune thrombocytopenic purpura) within six weeks after immunisation. Unadjusted estimates were calculated for an association with demyelinating diseases by using the number of cases observed among exposed and unexposed hemi-cohorts, and indicated that there was no association (Analysis 9.1.2).

One cohort study assessed the safety of the H1N1 vaccine. No statistical association was found between vaccination with $\mathrm{H} 1 \mathrm{~N} 1$ monovalent pandemic vaccine and demyelinating diseases. 
Serious adverse events: demyelinating diseases (multiple sclerosis, optic neuritis) - case-control studies (Comparison 10)

An association between exposure to seasonal inactivated vaccine and demyelinating diseases (including both multiple sclerosis and optic neuritis case definitions) in a healthy adult population was not statistically significant when we pooled unadjusted data from four case-control studies (OR $0.96,95 \% \mathrm{Cl} 0.79$ to 1.17 ) (Analysis 10.1). Also, when we analysed adjusted data for each of the case definitions separately, the estimates remained nonstatistically significant for multiple sclerosis (Analysis 10.2) and for optic neuritis (Analysis 10.3).

\section{Serious adverse events: immune thrombocytopenic purpura -} cohort studies (Comparison 11)

One cohort study aimed to assess whether there was an association between exposure to inactivated trivalent seasonal influenza vaccine during pregnancy and several pathologies (e.g. Guillain-Barré syndrome, demyelinating diseases, immune thrombocytopenic purpura) within six weeks after immunisation. Neither the unadjusted (Analysis 11.2.2) nor adjusted estimates (Analysis 11.1.2) for an association with immune thrombocytopenic purpura were statistically significant.

\section{Serious adverse events: immune thrombocytopenic purpura - case-control studies (Comparison 12)}

Data analysis of two case-control studies did not show a statistically significant association between immune thrombocytopenic purpura and seasonal influenza vaccine in any of the time frames considered (i.e. less than two months, six or 12 months between immunisation and disease onset), or when the data were pooled together (Analysis 12.2) (bb Garbe 2012; bb Grimaldi-Bensouda 2012). We drew the same conclusions when analysis was performed using estimates adjusted for confounders (Analysis 12.1), and a sensitivity analysis carried out using either a random-effects or fixed-effect model did not change our conclusions, providing further confirmation of them. It should be observed that no data sets included in this comparison, with the exception of bb Garbe 2012, showed a statistical association between disease and influenza vaccination. It is possible that the ages of the participants (cases and controls) were different in these two studies, and that some elderly participants may have been included. Unlike bb Grimaldi-Bensouda 2012, the case-control study bb Garbe 2012 considered as exposed those cases that were immunised up until 28 days before immune thrombocytopenic purpura onset.

\section{Serious and rare harms}

\section{Oculo-respiratory syndrome}

On the basis of one randomised trial in 651 healthy adults aged around 45, trivalent split inactivated vaccine caused mild oculo-respiratory syndrome in people with no previous history of oculo-respiratory syndrome (ab Scheifele 2003). Oculo-respiratory syndrome was defined as bilateral conjunctivitis, facial swelling (lip, lid, or mouth), difficulty in breathing and chest discomfort (including cough, wheeze, dysphagia, or sore throat). Oculorespiratory syndrome (attributable risk $2.9 \%, 95 \% \mathrm{Cl} 0.6$ to 5.2 ), hoarseness $(1.3 \%, 95 \% \mathrm{Cl} 0.3$ to 1.3$)$, and coughing $(1.2 \%, 95 \% \mathrm{Cl}$ 0.2 to 1.6$)$ occurred within six days of vaccination. The association did not appear to be specific to any type of trivalent inactivated vaccine. One register-based case-control study carried out in
Quebec showed an increased risk (adjusted OR 2.71, 95\% Cl 1.80 to 4.08) of oculo-respiratory syndrome during the first four weeks of the 2009 pandemic vaccination campaign (monovalent, AS03adjuvanted $\mathrm{pH} 1 \mathrm{~N} 1$ vaccine) (bb Rouleau 2014).

\section{Bell's palsy}

One case-control study and case series based in the Germanspeaking regions of Switzerland assessed the association between an intranasal inactivated virosomal influenza vaccine and Bell's palsy (bb Mutsch 2004). Two hundred and fifty cases that could be evaluated (from an original 773 cases identified) were matched to 722 controls. All were aged around 50 . The study reported a massive increase in risk (adjusted OR 84, 95\% Cl 20.1 to 351.9) within 1 to 91 days from vaccination. Despite the many limitations of this study (case attrition: 187 cases could not be identified; ascertainment bias: physicians picked controls for their own cases; confounding by indication: different vaccine exposure rate between controls and the reference population), it is unlikely that such a large OR could have been affected significantly by systematic error. The authors called for larger pre-licence harms trials, given the rarity of Bell's palsy. On the basis of this study the vaccine was withdrawn from sale.

\section{Rheumatoid arthritis}

One case-control study used the register of the Northern California Kaiser Permanente Health Plan (NCKPHP) in order to identify cases of rheumatoid arthritis diagnosed during a three-year period (1 January 1997 to 31 December 1999) among members of NCKPHP for at least two years (i.e. since 1 January 1995) and aged between 15 and 59 (bb Ray 2011). After reviewing clinical cards, 415 cases of definite or probable rheumatoid arthritis were included with 1245 randomly selected controls matched for age within one year and for a categorical utilisation variable based on the number of clinic visits during the year prior to the rheumatoid arthritis symptom onset date (none, one to two, three to five, six to nine, or 10+ visits). The Kaiser Immunisation Tracking System and chart review were used to determine vaccination status of cases and controls. Different time intervals between immunisation and rheumatoid arthritis onset were considered for analysis: 90, 180, 365 , and 730 days. No significant association between vaccination and rheumatoid arthritis could be determined for any time interval, even after adjustment for confounders (sex, race, and exact number of utilisation visits). The authors of this study performed a data analysis by using a person-time cohort design, in which vaccinated cases contributed to the unexposed follow-up time until they were immunised and to the exposed follow-up time thereafter. Unlike case-control analysis, person-time cohort analysis was performed by excluding cases who showed symptoms in 1996. Even if a significant association for exposure to vaccine occurred within 180 and 365 days before disease onset (OR adjusted for race, sex, and number of clinic visits $1.36,95 \% \mathrm{Cl} 1.03$ to 1.80 and 1.34 , $95 \% \mathrm{Cl} 1.06$ to 1.69 , respectively), the authors note that it is very difficult to estimate with sufficient precision the true onset date of rheumatoid arthritis, as the first symptoms could already be present for some time before people present for medical care. This is the most important limitation of this study and could have significantly affected the estimates.

\section{Neurological and autoimmune disorders}

The study of cb Bardage 2011 was a large, prospective cohort study carried out in a Stockholm population ( $\mathrm{n}=$ 
$1,945,024)$ during the vaccination campaign with monovalent $A$ (H1N1) pandemic vaccine Pandemrix (GlaxoSmithKline, containing adjuvants $\mathrm{ASO} 3$ and squalene) to evaluate the presence of an association between Pandemrix and neurological and/or autoimmune diseases (Guillain-Barré syndrome, multiple sclerosis, Bell's palsy, narcolepsy, polyneuropathy, an/hypoaesthesia, paraesthesia, rheumatological disease and inflammatory bowel disease). During the first 45 days, participants with high-risk conditions were preferentially vaccinated; vaccination was then offered to the remainder of the population in a second phase of the campaign (see Characteristics of included studies' table for more details).

The analysis of the HR adjusted for age, sex, socioeconomic status, and healthcare consumption (number of hospital admissions and visits to specialist care one year before the pandemic period) showed that in participants immunised during the early phase of the campaign, there was a significantly increased risk of Bell's palsy (HR $1.34,95 \% \mathrm{Cl} 1.11$ to 1.64), paraesthesia (HR 1.25, 95\% $\mathrm{Cl} 1.10$ to 1.41 ), and inflammatory bowel disease (HR 1.25, 95\% $\mathrm{Cl} 1.04$ to 1.50$)$. For the participants vaccinated in the late phase of the campaign ( $>45$ days), HR estimates showed there was no statistically different incidence in the investigated diseases between vaccinated and unvaccinated participants.

A further stratification was performed considering the time since first vaccination (six weeks or less and more than six weeks), which showed that in participants immunised during the first phase of the campaign, an increased incidence of Bell's palsy and paraesthesia was most pronounced, as well as within six weeks of vaccination (HR $1.74,95 \% \mathrm{Cl} 1.16$ to 2.59 for Bell's palsy and $\mathrm{HR} 1.60,95 \% \mathrm{Cl} 1.25$ to 2.05 for paraesthesia) and thereafter (HR $1.26,95 \% \mathrm{Cl} 1.01$ to 1.57 for Bell's palsy and HR $1.17,95 \% \mathrm{Cl} 1.02$ to 1.34 for paraesthesia). An increased risk of inflammatory bowel disease among those vaccinated in the early phase was only observed more than six weeks after vaccination (HR $1.29,95 \% \mathrm{Cl} 1.06$ to 1.58). Formal tests to determine whether risks differed further between those within and more than six weeks from vaccination were only statistically significant for paraesthesia $(P=0.005)$. In participants immunised during the second phase of the campaign, polyneuropathy was significantly more common within six weeks of immunisation (HR $1.79,95 \% \mathrm{Cl} 1.16$ to 2.77 ).

The study by cb Persson 2014 consisted of an extension of the Bardage study to more Swedish regions, namely the healthcare regions of Skåne and Västra Götaland and the counties of Kalmar, Östergötland, Värmland, and Norrbotten. The study included over 5.8 million participants, corresponding to about $61 \%$ of the whole Swedish population in 2009. In all, 207 cases of narcolepsy were confirmed, with the exclusion of eight cases with prodromal conditions during the last five years. The overall risk of narcolepsy after immunisation with Pandemrix assessed by Cox regression after adjusting for age, gender, county, education, income, number of hospital admissions and ambulatory care visits, pregnancy status, and presence of other diagnoses was not statistically relevant in the population aged above 20 years (HR 1.35, 95\% Cl 0.93 to 1.95). A significant association was instead found in those aged below 20 (HR 2.92, $95 \% \mathrm{Cl} 1.78$ to 4.79 ), in whom most cases of narcolepsy had occurred $(n=126)$.

A population-based cohort study carried out in Ireland identified only three cases of narcolepsy in the whole Irish adult population aged above 20 years during the pandemic season 2009 to 2010; two of them received Pandremix and one did not (cb O'Flanagan 2014). The risk estimate was extremely imprecise and did not allow us to draw any conclusions (RR 20.4, 95\% Cl 1.8 to 225). One case-control study (bb Dauvilliers 2013), performed across the institutions of 14 French expert orphan disease narcolepsy centres, identified 25 narcolepsy cases and 73 matched controls (age, sex, and geographical location) in the study population aged at least 18 years. An association between exposure to $\mathrm{H} 1 \mathrm{~N} 1$ vaccination and narcolepsy-catalepsy (crude OR $4.7,95 \% \mathrm{Cl} 2.1$ to 13.9) was found and was also confirmed after the performance of a sensitivity analysis and adjusting for smoking habits and family history of excessive daytime sleepiness (OR 4.1, 95\% Cl 1.4 to 12.2).

\section{Cutaneous melanoma}

A case-control study assessed the association between influenza vaccines and cutaneous melanoma in 99 cases and 104 controls (bb Mastrangelo 2000). The authors reported a protective effect of repeated influenza vaccination on risk of cutaneous melanoma (OR $0.43,95 \% \mathrm{Cl} 0.19$ to 1.00 ). The study was at high risk of bias due to the selective nature of cases (all patients in the authors' hospital), attrition bias (four cases and four controls eliminated due to "failure to collaborate"), recall bias (up to five years' exposure data were based on patients' recollection), and ascertainment bias (non-blinded exposure survey).

\section{Primary cardiac arrest}

A case-control study assessed the association between influenza vaccination the previous year and the risk of primary cardiac arrest (i.e. occurring in people with no previous history of cardiac disease) in 360 cases and 418 controls (bb Siscovick 2000). The authors concluded that vaccination is protective against primary cardiac arrest (OR $0.51,95 \% \mathrm{Cl} 0.33$ to 0.79 ). The difficulty of case ascertainment ( $77 \%$ of potential cases had no medical examiner report and/or autopsy) and recall bias (spouses provided exposure data for 304 cases, while 56 survivor cases provided data jointly with their spouses) make the conclusions of this study unreliable. It is impossible to judge the reliability of this study because of a lack of detail on the circulation of influenza in the study areas in the 12 months preceding cardiac arrest (the causal hypothesis is based on the effects of influenza infection on the oxygen supply to the myocardium through lung infection and inflammation).

\section{Acute myocardial infarction}

One case-control study performed in Australia assessed whether exposure to influenza vaccine provides protection against acute myocardial infarction in an adult population aged over 40 (bb MacIntyre 2013). Cases of acute myocardial infarction admitted to the cardiology unit of a tertiary hospital in Sydney during three consecutive epidemic seasons (2008, 2009, and 2010) were compared to unmatched controls attending the orthopaedic or ophthalmic outpatient clinics during the same time period with respect to their exposure to influenza vaccine (176 cases and 72 controls aged below 64 were included). From multivariate analysis, after adjusting for several confounders, influenza vaccination did not confer significant protection against acute myocardial infarction in an adult population aged between 40 and 64 years (OR $0.55,95 \% \mathrm{Cl} 0.27$ to 1.15$)$.

\section{Pulmonary function}

A double-blind, placebo-controlled randomised trial in 72 healthy volunteers aged around 26 assessed the effects of different types 
of live attenuated cold recombinant influenza vaccination on pulmonary function (data on 17 asthmatics were not extracted) (ab Atmar 1990). The authors reported several non-significant drops in lung function up to seven days postinoculation and a higher incidence of ILI (17/46 versus 4/26) in the vaccinated arms.

\section{Other serious adverse events}

The study of $\mathrm{cb}$ Baxter 2012 is a large, retrospective cohort performed among members of Kaiser Permanente Health Plans of Northern California, Hawaii, and Colorado aged between 18 and 59 years, who were immunised with live attenuated, inactivated influenza vaccine or who did not receive vaccination. The study retrospectively investigated the occurrence of adverse events (see Characteristics of included studies' table for more details) during five subsequent epidemics, but did not identify any unexpected serious risks when the live attenuated vaccine was used in approved populations.

\section{Vaccines for the 1968 to 1969 (H3N2) influenza pandemic (Comparisons 13 to 17 )}

Five studies yielded 12 data sets (aa Eddy 1970; aa Mogabgab 1970a; aa Mogabgab 1970b; aa Sumarokow 1971; aa Waldman 1969a; aa Waldman 1969b; aa Waldman 1969c; aa Waldman 1969d; aa Waldman 1972a; aa Waldman 1972b; aa Waldman 1972c; aa Waldman 1972d). As one would expect, vaccine performance was poor when the content did not match the pandemic strain (Analysis 13.1; Analysis 13.2). However, one- or two-dose monovalent wholevirion (i.e. containing dead complete viruses) vaccines achieved a VE of $65 \%$ (95\% Cl $52 \%$ to $75 \%$ ) protection against ILI (NNV 16, 95\% $\mathrm{Cl} 14$ to 20 ), a VE of $93 \%$ (95\% Cl $69 \%$ to $98 \%$ ) with NNV 35 (95\% $\mathrm{Cl} 33$ to 47$)$ protection against influenza, and a VE of $65 \%(95 \% \mathrm{Cl}$ $6 \%$ to $87 \%$ ) with NNV $94(95 \% \mathrm{Cl} 70$ to 1022$)$ against hospitalisation (Analysis 14.1; Analysis 14.2; Analysis 14.3).

Approximately half a working day and half a day of illness were saved (Analysis 14.5; Analysis 14.6), but no effect was observed on pneumonia (Analysis 14.4). All comparisons except for ILI were based on a single study (Analysis 14.4). The large effect on ILI is coherent with the high proportion of these illnesses caused by influenza viruses in a pandemic (i.e. the gap between the efficacy and effectiveness of the vaccines is narrow). Aerosol polyvalent or monovalent vaccines had a modest effect.

\section{DISCUSSION}

\section{Summary of main results}

In healthy adults live parenteral vaccines probably reduce influenza from $2.3 \%$ to $1 \%$, based on a vaccine efficacy of $59 \%$ (moderatecertainty evidence). This corresponds with an NNV of 71 . Live parenteral vaccine effectiveness against ILI was lower (16\%), with a NNV of 29 based on an assumed control group risk of $21.5 \%$. We found greater variation in control group risks of ILI compared with influenza (Summary of findings for the main comparison). Low and high control group risks (4\% and $91 \%$ ) corresponded to NNVs 167 and 7, respectively. The overall efficacy of inactivated vaccines in preventing influenza is $59 \%(95 \% \mathrm{Cl} 51 \%$ to $66 \%$ ) with a NNV of 77 . When vaccine content matches the circulating strain the efficacy is $59 \%(95 \% \mathrm{Cl} 53 \%$ to $64 \%)$. Based on results of a single study (aa Bridges 2000b), physician visits appear to be $42 \%$ less frequent in participants immunised with vaccines prepared with strains matching circulating viruses, whereas no significant differences were found when the degree of matching was unknown or absent (RR $1.28,95 \% \mathrm{Cl} 0.90$ to 1.83). The overall effect was again not significant (RR $0.87,95 \% \mathrm{Cl} 0.40$ to 1.89 ). There seems to be no effect on the time an antibiotic or a drug is prescribed. Four trials evaluated time off work, estimating that vaccination saves on average around 0.04 working days. This result was affected by high levels of heterogeneity and changes depending on whether a fixedeffect (MD - $0.04,95 \% \mathrm{Cl}-0.06$ to -0.01 ) or random-effects model (MD $-0.04,95 \% \mathrm{Cl}-0.14$ to 0.06$)$ was used.

Live aerosol vaccines have an overall effectiveness against ILI of $10 \%(95 \% \mathrm{Cl} 4 \%$ to $16 \%)$ and a NNV of 46 . Content and matching appear not to affect their performance significantly. The overall efficacy against influenza is $53 \%(95 \% \mathrm{Cl} 38 \%$ to $65 \%)$ and the NNV is 39. Again, neither content nor matching appear to affect their performance significantly. Many more recipients administered vaccine experienced local symptoms than did those administered placebo.

One RCT assessed the efficacy of inactivated aerosol vaccine in preventing influenza (Analysis 3.1.1) (aa Langley 2011). The results did not show a statistically significant protective effect (RR 0.38 , $95 \% \mathrm{Cl} 0.14$ to 1.02 ).

One RCT investigated the effects of influenza vaccine administration in pregnant women and their newborns (paa Madhi 2014). A trivalent inactivated vaccine containing $\mathrm{pH} 1 \mathrm{~N} 1$ was weakly protective against confirmed influenza in both mothers (RR 0.50, $95 \% \mathrm{Cl} 0.29$ to 0.86 ; VE 50\%, 95\% Cl 14\% to $71 \%$; NNV 55, 95\% Cl 39 to 198 ; Analysis 4.1.1) and children (RR $0.51,95 \% \mathrm{Cl} 0.30$ to 0.88 ; VE $49 \%, 95 \% \mathrm{Cl} 12 \%$ to $70 \%$; NNV 56; Analysis 4.3.1). Protection against ILI was not statistically significant. The rest of the evidence on vaccination during pregnancy was based on observational studies (case-control and cohort studies); the effectiveness of vaccination with seasonal inactivated parenteral vaccine during pregnancy for preventing ILI in newborns was not statistically significant. The evidence comes from two cohort studies using either HR or RR adjusted estimates. Pooled data from three cohort studies (two of them at high risk of bias) show a modest effect of vaccination against ILI in pregnant women when the fixed-effect model is applied to the analysis (NNV 92, 95\% Cl 63 to 201). One cohort study showed a modest protective effect against influenza in newborns of vaccinated mothers (NNV 27, 95\% Cl 18 to 185).

Pooled analysis of three RCTs and one CCT showed that immunisation with at least one dose of the 1968 to 1969 pandemic monovalent inactivated whole-virion vaccines achieved a VE of $65 \%$ ( $95 \% \mathrm{Cl} 52 \%$ to $75 \%$ ) against ILI (NNV $16,95 \% \mathrm{Cl} 14$ to 20 ). One RCT showed that the efficacy of the 1968 to 1969 pandemic monovalent inactivated whole-virion vaccines in preventing influenza was $93 \%$ (95\% Cl $69 \%$ to $98 \%$; NNV 35). One other RCT showed an efficacy of $65 \%(95 \% \mathrm{Cl} 6 \%$ to $87 \%)$; NNV $94(95 \% \mathrm{Cl} 70$ to 1022$)$ in preventing hospitalisation. One CCT provided evidence on the effect of immunisation with the 1968 to 1969 pandemic monovalent inactivated whole-virion vaccines on working days lost and days of illness: approximately half a working day and half a day of illness were saved (MD $-0.45,95 \% \mathrm{Cl}-0.60$ to -0.30 ). The effect of this vaccine on prevention of pneumonia was not statistically significant (RR $0.59,95 \% \mathrm{Cl} 0.05$ to 6.51 ).

Based on evidence from observational studies, administration of either seasonal inactivated vaccine or monovalent $\mathrm{H} 1 \mathrm{~N} 1$ pandemic vaccine during pregnancy is not associated with an increased risk of 
abortion, congenital malformation, prematurity, or neonatal death, but $\mathrm{Cls}$ are wide.

We found no evidence of an association between seasonal inactivated vaccines and Guillain-Barré syndrome or H1N1 pandemic vaccine and Guillain-Barré syndrome.

There was no evidence of an association between exposure to seasonal inactivated influenza vaccine and other serious adverse events (multiple sclerosis, optic neuritis, and immune thrombocytopenic purpura).

\section{Overall completeness and applicability of evidence}

A number of issues should be taken into consideration when interpreting the results of this review.

1. Methods of vaccine standardisation have changed significantly.

2. Recent vaccines present significant differences in purity when compared with older ones.

3. Different doses and schedules were pooled in the analysis.

Taken alone, this review shows that according to randomised evidence, inactivated vaccines have a small effect in preventing the symptoms of influenza and getting people back to work more quickly. Looking at the NNVs for influenza and ILI for inactivated parenteral vaccine, it seems that effectiveness against ILI is higher than efficacy against laboratory-confirmed influenza (NNV-ILI 29; NNV-influenza 71). These paradoxical results show an apparently higher non-specific effectiveness and a lower specific efficacy. This reflects different rates of $\mathrm{ILI}$ and confirmed influenza among the study populations in the respective outcomes. The percentage of unvaccinated participants who developed ILI symptoms was $21.5 \%$, whilst $2.3 \%$ participants in the unvaccinated arms of the trials developed laboratory-confirmed influenza.

\section{Quality of the evidence}

We rated the quality of the evidence for ILI and influenza as moderate; hospitalisation, time off work, and increased risk of nausea or vomiting as low; and fever as high (Summary of findings for the main comparison). The impact of bias varied across the outcomes, leading us to downgrade the quality of evidence for hospitalisation, time off work, and nausea. For other outcomes, analyses drew more heavily on studies at low risk of bias or where the likely impact of bias across the studies was small. Variation in the definition of ILI led us to present stratified risk and to downgrade for inconsistency given some discordance in the direction of effect and high statistical heterogeneity. Our decision to downgrade the quality of the evidence for influenza due to indirectness reflects our uncertainty in the methods to ascertain the outcome in older studies and the impact this has on the applicability of the evidence to current settings. The data for hospitalisation was dominated by the aa Leibovitz 1971 study in Analysis 1.8. Whilst the overall direction of effect indicated a small reduction in absolute terms with the vaccine, we are unable to rule out there being no effect of the intervention. The $\mathrm{Cl}$ for the effect on nausea/vomiting was wide, although this may reflect the incorporation of variation in the study results, rather than low power.

\section{Potential biases in the review process}

The conclusions of this review regarding the safety profile of inactivated vaccines are uncertain, which is a reflection of the size of the evidence base.

An earlier review of 274 influenza vaccine studies in all age groups (which included most of the studies in this review) showed an inverse relationship between risk of bias and the direction of study conclusions. Conclusions favourable to the use of influenza vaccines were associated with a higher risk of bias. The authors of studies in this review made claims and drew conclusions that were unsupported by the data they presented. In addition, industryfunded studies are more likely to have favourable conclusions, to be published in significantly higher-impact factor journals, and to have higher citation rates than non-industry-funded studies. This difference is not explained by either their size or methodological quality (Jefferson 2009b). Any interpretation of the body of evidence in this review should be made with these findings in mind.

Additional care should be taken when interpreting the results of observational studies in pregnancy, as the possible presence and effects of immortal time bias were not analysed in previous versions of this review. Immortal time bias occurs when a time-dependent exposure (in this case vaccination) is not included appropriately in an analysis of a survival outcome. The term 'immortal time bias' is used because in observational studies patients must survive sufficiently long to receive treatment; hence, they are immortal by definition before exposure. This type of bias, sometimes referred to as time-dependent bias, is not generally a problem in randomised studies, as treatment (including placebo) is usually given at the beginning of the study. Conversely, in observational studies, exposure to the vaccine has usually taken place before study commencement, with a resulting exposure misclassification. Such bias can affect study conclusions (Jones 2016).

\section{Agreements and disagreements with other studies or reviews}

\section{Systematic reviews estimating the efficacy of influenza vaccination}

DiazGranados 2012 performed a meta-analysis that included RCTs on seasonal inactivated or live attenuated influenza vaccines with influenza (with either polymerase chain reaction (PCR) or serological confirmation of infection) as the efficacy outcome. The meta-analysis included 30 studies in children and adults. The authors provided efficacy estimates (RR with $95 \% \mathrm{Cl}$ ) stratified by the degree of matching between the vaccine and circulating strains (good, poor, no matching, matching) and by strain type (A H1N1, A H3N2, B). DiazGranados 2012 estimated that in an adult population the efficacy of inactivated vaccine against laboratory-confirmed influenza is 59\% (95\% Cl 50\% to 66\%). The efficacy estimate for live attenuated vaccine is $39 \%(95 \% \mathrm{Cl} 16 \%$ to $55 \%)$.

The systematic review by Osterholm 2012 included evidence of the efficacy of both live attenuated and inactivated vaccines in preventing laboratory-confirmed influenza infection assessed exclusively by either PCR or a positive culture. Considering studies carried out in adults only, the pooled estimate of efficacy from six studies (eight data sets) was $59 \%$ (95\% CI 51\% to $67 \%)$. Even though three RCTs estimating the efficacy of live attenuated vaccines were included, the authors did not perform an analysis because none 
of the single estimates was statistically significant. Observational studies were also included and discussed.

\section{Systematic reviews assessing the efficacy/effectiveness and/or safety issues of influenza vaccines when administered during pregnancy}

The review by Skowronski 2009 is the first comprehensive publication in which evidence for the effectiveness and safety aspects of vaccination during pregnancy has been exhaustively discussed. In the first part of the paper, the authors consider the burden of disease during pregnancy, the risk of death, and the influenza-related risk for the foetus and summarise how the US Advisory Committee on Immunization Practice (ACIP) recommendations have changed over the last four decades. The available evidence on protection (in mother and newborns) and vaccination safety issues are descriptively illustrated, discussed, and compared with the statements in the current vaccination policies reported. In the authors' opinion, immunisation against influenza at any stage of pregnancy may be warranted during pandemics or for women with comorbidity. Seasonal immunisation with trivalent inactivated vaccine may be warranted in pregnancy, without potential complications during the second half of the pregnancy. Finally, the available evidence is insufficient to recommend standard routine vaccination in the early stages of pregnancy.

\section{Systematic reviews of evidence of severe harms}

Farez 2011 evaluated the risk of developing multiple sclerosis or experiencing relapsing multiple sclerosis following immunisation with several vaccinations, including influenza. Meta-analysis performed by pooling the results of four case-control studies would exclude an increased risk of developing multiple sclerosis following influenza vaccine administration (OR $0.97,95 \% \mathrm{Cl} 0.77$ to 1.23 ) (bb DeStefano 2003; bb Hernan 2004; bb Payne 2006; bb Zorzon 2003).

\section{Other issues}

Toback 2012 provided evidence supporting the introduction of a new quadrivalent live attenuated influenza vaccine (Q-LAIV, already licensed in the USA, where it will was available for the 2013 to 2014 season) containing two different $B$ strains of different lineage (B/Yamagata/16/88 and B/Victoria/2/87). This evidence comes from two RCTs comparing immunogenicity and local and systemic reactions after administration of either Q-LAIV, trivalent inactivated, or trivalent live attenuated vaccines. One RCT was performed in adults, the other in a paediatric population. The presence of two $B$ strains would not significantly affect the antibody response against each $B$ strain. Local and systemic adverse events induced by Q-LAIV administration did not differ significantly from those recorded after administration of other vaccines already in use.

In summary, the conclusions of the cited reviews are broadly comparable with ours, but the results are reported using relative effects-based estimates. In addition, none of the reviews have identified effects of the vaccines on important outcomes such as complications, hospitalisations, and deaths. These findings are also similar to ours.

\section{AUTHORS' CONCLUSIONS}

\section{Implications for practice}

Healthy adults who receive inactivated parenteral influenza vaccine rather than no vaccine probably have a $1 \%$ lower risk of experiencing influenza over a single influenza season $(2.3 \%$ versus $1 \%$, moderate-certainty evidence) and probably have a 3.4\% lower risk of experiencing influenza-like illness (ILI) $(21.5 \%$ versus $18.1 \%$, moderate-certainty evidence). The numbers needed to vaccinate (NNVs) for influenza and ILI were 71 and 29 , respectively, reflecting high rates of ILI in the control groups for many of the trials. The NNV of 29 conceals variation in the absolute reduction in ILI for low- and high-risk groups, and the degree of benefit may vary at least in part due to inconsistent symptom classification. Extrapolatiing these effects to settings other than those of the studies is challenging due to uncertain methods for confirming influenza and variation in the absolute reductions in ILI following vaccination.

We found low-certainty evidence that hospitalisation rates and time off work may be comparable between vaccinated and unvaccinated adults, although the confidence interval around the effect for hospital admission is wide and there was substantial variation in the direction of effect on time off work. Vaccines increase the risk of a number of adverse events, including a small increase in fever, but the effect on nausea or vomiting is less clear.

\section{Implications for research}

When a new vaccination or preventive technology becomes available, an adequately powered, publicly funded, high-quality, placebo-controlled trial run over several seasons should be undertaken. New insights on the role of viruses and other agents in the genesis of influenza and ILI are also needed.

\section{ACKNOWLEDGEMENTS}

The first publication of this review was funded by the UK Ministry of Defence; the 2004 update was supported by the two Italian Local Health Authorities in which two of the review authors were employed; and the 2007 update was funded by the same Local Health Authorities and the UK's Department of Health Cochrane Incentive Scheme. The 2010 update was not funded. The 2014 update was supported by the two Italian Local Health Authorities in which three of the review authors were employed.

This 2016 update was supported by the National Institute for Health Research (NIHR), via Cochrane Incentive Award funding to the Cochrane Acute Respiratory Infections Group. The views and opinions expressed therein are those of the authors and do not necessarily reflect those of the Systematic Reviews Programme, NIHR, National Health Service (NHS), or the Department of Health.

Professor Jon Deeks designed and carried out statistical analyses in earlier versions of the review. The review authors wish to acknowledge Daniela Rivetti, Ghada A Bawazeer and Lubna AlAnsary as previous authors.

The authors gratefully acknowledge the following people for commenting on previous drafts: Theresa Wrangham, Ann Fonfa, Brian Hutchison, Alan Hampson, James Irlam, Andy Oxman, Barbara Treacy, Gabriella Morandi, Kathie Clark, Hans van der Wouden, Nelcy Rodriguez, Leonard Leibovici, Mark Jones, Jeanne 
Lenzer, Janet Wale, Clare Jeffrey, Robert Ware, Maryann Napoli and Roger Damoiseaux.
For this 2016 update, we acknowledge help and comments received from Liz Dooley, Toby Lasserson, Ann Fonfa, Ross Andrews, Elaine Beller, and Roderick Venekamp. We thank Lisa Winer for copyediting this update. 


\section{RE F E R E N C E S}

\section{References to studies included in this review}

\section{aa Barrett 2011 \{published data only\}}

* Barrett PN, Berezuk G, Fritsch S, Aichinger G, Hart MK, ElAmin W, et al. Efficacy, safety, and immunogenicity of a Verocell-culture-derived trivalent influenza vaccine: a multicentre, double-blind, randomised, placebo-controlled trial. Lancet 2011;377(9767):751-9.

Ehrlich HJ, Berezuk G, Fritsch S, Aichinger G, Singer J, Portsmouth D, et al. Clinical development of a Vero cell culture-derived seasonal influenza vaccine. Vaccine 2011;30(29):4377-86.

Ehrlich HJ, Singer J, Berezuk G, Fritsch S, Aichinger G, Hart MK, et al. A cell culture-derived influenza vaccine provides consistent protection against infection and reduces the duration and severity of disease in infected individuals. Clinical Infectious Diseases 2012;54(7):946-54.

\section{aa Beran 2009a \{published data only\}}

Beran J, Wertzova V, Honegr K, Kaliskova E, Havlickova M, Havlik J, et al. Challenge of conducting a placebo-controlled randomized efficacy study for influenza vaccine in a season with low attack rate and a mismatched vaccine $B$ strain: a concrete example. BMC Infectious Diseases 2009;9:2. [DOI: 10.1186/1471-2334-9-2]

\section{aa Beran 2009b \{published data only\}}

Beran J, Vesikari T, Wertzova V, Karvonen A, Honegr K, Lindblad N, et al. Efficacy of inactivated split-virus influenza vaccine against culture-confirmed influenza in healthy adults: a prospective, randomized, placebo-controlled trial. Journal of Infectious Diseases 2009;200:1861-9.

\section{aa Bridges 2000a \{published data only\}}

Buxton Bridges C, Thompson VV, Meltzer MI, Reeve GR, Talamonti VJ, Cox NJ, et al. Effectiveness and cost benefit of influenza vaccination of healthy working adults, a randomized controlled trial. JAMA 2000;284(13):1655-63.

\section{aa Bridges 2000b \{published data only\}}

Buxton Bridges C, Thompson VV, Meltzer MI, Reeve GR, Talamonti VJ, Cox NJ. Effectiveness and cost benefit of influenza vaccination of healthy working adults, a randomized controlled trial. JAMA 2000;284(13):1655-63.

\section{aa Eddy 1970 \{published data only\}}

Eddy TS, Davies NA. The effect of vaccine on a closed epidemic of Hong Kong influenza. South African Medical Journal 1970;44(8):214-6.

\section{aa Edwards 1994a \{published data only\}}

Edwards KM, Dupont WD, Westrich MK, Plummer WD Jr, Palmer PS, Wright PF. A randomized controlled trial of cold adapted and inactivated vaccines for the prevention of influenza A disease. Journal of Infectious Diseases 1994;169(1):68-76. aa Edwards 1994b \{published data only\}

Edwards KM, Dupont WD, Westrich MK, Plummer WD Jr, Palmer PS, Wright PF. A randomized controlled trial of cold adapted and inactivated vaccines for the prevention of influenza A disease. Journal of Infectious Diseases 1994;169(1):68-76.

\section{aa Edwards 1994c \{published data only\}}

Edwards KM, Dupont WD, Westrich MK, Plummer WD Jr, Palmer PS, Wright PF. A randomized controlled trial of cold adapted and inactivated vaccines for the prevention of influenza A disease. Journal of Infectious Diseases 1994;169(1):68-76.

\section{aa Edwards 1994d \{published data only\}}

Edwards KM, Dupont WD, Westrich MK, Plummer WD Jr, Palmer PS, Wright PF. A randomized controlled trial of cold adapted and inactivated vaccines for the prevention of influenza A disease. Journal of Infectious Diseases 1994;169(1):68-76.

\section{aa Frey 2010 \{published data only\}}

Frey S, Vesikari T, Szymczakiewicz-Multanowska A, Lattanzi M, Izu A, Groth N, et al. Clinical efficacy of cell culture-derived and egg-derived inactivated subunit influenza vaccines in healthy adults. Clinical Infectious Diseases 2010;51(9):997-1004.

aa Hammond 1978 \{published data only\}

Hammond ML, Ferris AA, Faine S. Effective protection against influenza after vaccination with subunit vaccine. Medical Journal of Australia 1978;1(6):301-3.

aa Jackson 2010a \{published data only\}

Jackson LA, Gaglani MJ, Keyserling HL, Balser J, Bouveret N, Fries L, et al. Safety, efficacy, and immunogenicity of an inactivated influenza vaccine in healthy adults: a randomised, placebo-controlled trial over two influenza seasons. BMC Infectious Diseases 2010;10:71.

aa Jackson 2010b \{published data only\}

Jackson LA, Gaglani MJ, Keyserling HL, Balser J, Bouveret N, Fries L, et al. Safety, efficacy, and immunogenicity of an inactivated influenza vaccine in healthy adults: a randomised, placebo-controlled trial over two influenza seasons. BMC Infectious Diseases 2010;10:71.

aa Keitel 1988a \{published data only\}

Keitel WA, Cate TR, Couch RB. Efficacy of sequential annual vaccination with inactivated influenza virus vaccine. American Journal of Epidemiology 1988;127(2):353-64.

aa Keitel 1988b \{published data only\}

Keitel WA, Cate TR, Couch RB. Efficacy of sequential annual vaccination with inactivated influenza virus vaccine. American Journal of Epidemiology 1988;127(2):353-64. 
aa Keitel 1997a \{published data only\}

Keitel WA, Cate TR, Couch RB, Huggins LL, Hess KR. Efficacy of repeated annual immunization with inactivated influenza virus vaccines over a five year period. Vaccine 1997;15(10):1114-22.

\section{aa Keitel 1997b \{published data only\}}

Keitel WA, Cate TR, Couch RB, Huggins LL, Hess KR. Efficacy of repeated annual immunization with inactivated influenza virus vaccines over a five year period. Vaccine 1997;15(10):1114-22.

\section{aa Keitel 1997c \{published data only\}}

Keitel WA, Cate TR, Couch RB, Huggins LL, Hess KR. Efficacy of repeated annual immunization with inactivated influenza virus vaccines over a five year period. Vaccine 1997;15(10):1114-22.

\section{aa Langley 2011 \{published data only\}}

Langley JM, Aoki F, Ward BJ, McGeer A, Angel JB, Stiver G, et al. A nasally administered trivalent inactivated influenza vaccine is well tolerated, stimulates both mucosal and systemic immunity, and potentially protects against influenza illness. Vaccine 2011;29(10):1921-8.

\section{aa Leibovitz 1971 \{published data only\}}

Leibovitz A, Coultrip RL, Kilbourne ED, Legters LJ, Smith CD, Chin J, et al. Correlated studies of a recombinant influenzavirus vaccine. IV. Protection against naturally occurring influenza in military trainees. Journal of Infectious Diseases 1971;124(5):481-7.

\section{aa Mcbride 2016a \{published data only\}}

Mcbride WJ, Abhayaratna WP, Barr I, Booy R, Carapetis J, Carson S, et al. Efficacy of a trivalent influenza vaccine against seasonal strains and against 2009 pandemic H1N1: a randomized, placebo-controlled trial. Vaccine 2016;34(41):4991-7.

\section{aa Mcbride 2016b \{published data only\}}

Mcbride WJ, Abhayaratna WP, Barr I, Booy R, Carapetis J, Carson S, et al. Efficacy of a trivalent influenza vaccine against seasonal strains and against 2009 pandemic H1N1: a randomized, placebo-controlled trial. Vaccine 2016;34(41):4991-7.

\section{aa Mesa Duque 2001 \{published data only\}}

Mesa Duque SS, Moreno AP, Hurtado G, Arbelàaz Montoya MP. Effectiveness of an influenza vaccine in a working population in Colombia [Effectividad de una vacuna anti gripal en una poblaciòn laboral colombiana]. Pan American Journal of Public Health 2001;10(4):232-9.

\section{aa Mixéu 2002 \{published data only\}}

Mixéu MA, Vespa GN, Forleo-Neto E, Toniolo-Neto J, Alves PM. Impact of influenza vaccination on civilian aircrew illness and absenteeism. Aviation, Space, and Environmental Medicine 2002;73(9):876-80.

\section{aa Mogabgab 1970a \{published data only\}}

Mogabgab WJ, Leiderman E. Immunogenicity of 1967 polyvalent and 1968 Hong Kong influenza vaccines. JAMA 1970;211(10):1672-6.

\section{aa Mogabgab 1970b \{published data only\}}

Mogabgab WJ, Leiderman E. Immunogenicity of 1967 polyvalent and 1968 Hong Kong influenza vaccines. JAMA 1970;211(10):1672-6.

\section{aa Monto 1982 \{published data only\}}

Monto AS, DeWolfe Miller F, Maassab HF. Evaluation of an attenuated, cold recombinant influenza $B$ virus vaccine. Journal of Infectious Diseases 1982;145(1):57-64.

aa Monto 2009 \{published data only\}

Monto AS, Ohmit SE, Petrie JG, Johnson E, Truscon R, Teich E, et al. Comparative efficacy of inactivated and live attenuated influenza vaccines. New England Journal of Medicine 2009;361(13):1260-7.

aa Nichol 1995 \{published data only\}

Nichol KL, Lind A, Margolis KL, Murdoch M, McFadden R, Hauge M. The effectiveness of vaccination against influenza in healthy, working adults. New England Journal of Medicine 1995;333(14):889-93.

\section{aa Nichol 1999a \{published data only\}}

Nichol KL, Mendelman PM, Mallon KP, Jackson LA, Gorse GJ, Belshe RB, et al. Effectiveness of live attenuated intranasal influenza virus vaccine in healthy working adults, a randomized controlled trial. JAMA 1999;282(2):137-44.

aa Ohmit 2006 \{published data only (unpublished sought but not used)\}

Ohmit SE, Victor JC, Rotthoff JR, Teich ER, Truscon RK, Baum LL, et al. Prevention of antigenically drifted influenza by inactivated and live attenuated vaccines. New England Journal of Medicine 2006;355(24):2513-22.

\section{aa Ohmit 2008 \{published data only\}}

Ohmit SE, Victor JC, Teich ER, Truscon RK, Rotthoff JR, Newton DW, et al. Prevention of symptomatic seasonal influenza in 2005-2006 by inactivated and live attenuated vaccines. Journal of Infectious Diseases 2008;198(3):312-7.

\section{aa Powers 1995a \{published data only\}}

Powers DC, Smith GE, Anderson EL, Kennedy DJ, Hackett CS, Wilkinson $\mathrm{BE}$, et al. Influenza A virus vaccines containing purified recombinant $\mathrm{H} 3$ hemagglutinin are well tolerated and induce protective immune responses in healthy adults. Journal of Infectious Diseases 1995;171(6):1595-9.

\section{aa Powers 1995b \{published data only\}}

Powers DC, Smith GE, Anderson EL, Kennedy DJ, Hackett CS, Wilkinson $\mathrm{BE}$, et al. Influenza A virus vaccines containing purified recombinant $\mathrm{H} 3$ hemagglutinin are well tolerated and induce protective immune responses in healthy adults. Journal of Infectious Diseases 1995;171(6):1595-9.

\section{aa Powers 1995c \{published data only\}}

Powers DC, Smith GE, Anderson EL, Kennedy DJ, Hackett CS, Wilkinson BE, et al. Influenza A virus vaccines containing purified recombinant $\mathrm{H} 3$ hemagglutinin are well tolerated and induce protective immune responses in healthy adults. Journal of Infectious Diseases 1995;171:1595-9. 
aa Rytel 1977 \{published data only\}

Rytel MW, Jackson LJ, Niebojewski RA, Haagensen JL, Rosenkranz MA. Field trial of live attenuated influenza A/ B ("Alice"/R-75) vaccine. American Journal of Epidemiology 1977;105(1):49-55.

\section{aa Sumarokow 1971 \{published data only\}}

Sumarokow AA, Popov VF, Nefedova LA, Salmin LV, Lazorenko NF. A study of live influenza vaccines in a controlled trial. Zhumal Mikrobiologii Epidemiologii Immunobiologii 1971;48(2):46-52.

\section{aa Tannock 1984 \{published data only\}}

Tannock GA, Bryce DA, Hensley MJ, Saunders NA, Gillett RS, Kennedy WS. Responses to one or two doses of a deoxycholate subunit influenza vaccine in a primed population. Vaccine 1984;2(1):100-5

\section{aa Treanor 2011 \{published data only\}}

Treanor JJ, El Sahly H, King J, Graham I, Izikson R, Kohberger R, et al. Protective efficacy of a trivalent recombinant hemagglutinin protein vaccine (FluBlok) against influenza in healthy adults: a randomised, placebo-controlled trial. Vaccine 2011;29(44):7733-9.

\section{aa Waldman 1969a \{published data only\}}

Waldman RH, Bond JO, Levitt LP, Hartwig EC, Prather EC, Baratta RL, et al. An evaluation of influenza immunization. Bulletin of the World Health Organization 1969;41(3):543-8.

\section{aa Waldman 1969b \{published data only\}}

Waldman RH, Bond JO, Levitt LP, Hartwig EC, Prather EC, Baratta RL, et al. An evaluation of influenza immunization. Bulletin of the World Health Organization 1969;41(3):543-8.

\section{aa Waldman 1969c \{published data only\}}

Waldman RH, Bond JO, Levitt LP, Hartwig EC, Prather EC, Baratta RL, et al. An evaluation of influenza immunization. Bulletin of the World Health Organization 1969;41(3):543-8.

\section{aa Waldman 1969d \{published data only\}}

Waldman RH, Bond JO, Levitt LP, Hartwig EC, Prather EC, Baratta RL, et al. An evaluation of influenza immunization. Bulletin of the World Health Organization 1969;41(3):543-8.

\section{aa Waldman 1972a \{published data only\}}

Waldman RH, Coggins WJ. Influenza immunization: field trial on a university campus. Journal of Infectious Diseases 1972;126(3):242-8.

\section{aa Waldman 1972b \{published data only\}}

Waldman RH, Coggins WJ. Influenza immunization: field trial on a university campus. Journal of Infectious Diseases 1972;126(3):242-8.

\section{aa Waldman 1972c \{published data only\}}

Waldman RH, Coggins WJ. Influenza immunization: field trial on a university campus. Journal of Infectious Diseases 1972;126(3):242-8 aa Waldman 1972d \{published data only\}

Waldman RH, Coggins WJ. Influenza immunization: field trial on a university campus. Journal of Infectious Diseases 1972;126(3):242-8

aa Weingarten 1988 \{published data only\}

Weingarten S, Staniloff H, Ault M, Miles P, Bamberger M, Meyer RD. Do hospital employees benefit from the influenza vaccine?. Journal of General Internal Medicine 1988;3(1):32-7.

aa Zhilova 1986a \{published data only\}

Zhilova GP, Ignat'eva GS, Orlov VA, Malikova EV, Maksakova VL. Results of a study of the effectiveness of simultaneous immunization against influenza with live and inactivated vaccines (1980 - 1983). Voprosy Virusologii 1986;31(1):40-4.

aa Zhilova 1986b \{published data only\}

Zhilova GP, Ignat'eva GS, Orlov VA, Malikova EV, Maksakova VL. Results of a study of the effectiveness of simultaneous immunization against influenza with live and inactivated vaccines (1980 - 1983). Voprosy Virusologii 1986;31(1):40-4.

\section{ab Atmar 1990 \{published data only\}}

Atmar RL, Bloom K, Keitel W, Couch RB, Greenberg SB. Effect of live attenuated, cold recombinant $(C R)$ influenza virus vaccines on pulmonary function in healthy and asthmatic adults. Vaccine 1990;8(3):217-24.

\section{ab Betts 1977a \{published data only\}}

Betts RF, Douglas RG Jr, Roth FK, Little JW 3rd. Efficacy of live attenuated influenza A/Scotland/74 (H3N2) virus vaccine against challenge with influenza A/Victoria/3/75 (H3N2) virus. Journal of Infectious Diseases 1977;136(6):746-53.

\section{ab Boyce 2000 \{published data only\}}

Boyce TG, Hsu HH, Sannella EC, Coleman-Dockery SD, Baylis E, Zhu Y, et al. Safety and immunogenicity of adjuvanted and unadjuvanted subunit influenza vaccines administered intranasally to healthy adults. Vaccine 2000;19(2-3):217-26.

\section{ab Caplan 1977 \{published data only\}}

Caplan ES, Hughes TP, O'Donnel S, Levine MM, Hornick RB. Reactogenicity and immunogenicity of parenteral monovalent influenza A/Victoria/3/75 (H3N2) virus vaccine in healthy adults. Journal of Infectious Diseases 1977;136(Suppl):484-90.

\section{ab El'shina 1996 \{published data only\}}

El'shina GA, Masalin IM, Shervali VI, Gorbunov MA, Lonskaia NI, Agafonova LV, et al. The trivalent polymer-subunit influenza vaccine Grippol studied in a controlled epidemiological trial. Voenno-Meditsinskii Zhurnal 1996;317(8):57-60.

\section{ab Evans 1976 \{published data only\}}

Evans AE, Letley E, Ferris RD, Freestone DS. WRL 105 strain live attenuated influenza vaccine; comparison of one and two dose schedules. Journal of Hygiene 1976;77(3):327-32.

\section{ab Forsyth 1967 \{published data only\}}

Forsyth JR. An assessment of oil adjuvant and aqueous influenza vaccines. I. Reactions to the vaccines. Journal of Hygiene 1967;65(4):485-95. 


\section{ab Goodeve 1983 \{published data only\}}

Goodeve A, Potter CW, Clark A, Jennings R, Schild GC, Yetts R. A graded-dose study of inactivated, surface antigen influenza $B$ vaccine in volunteers: reactogenicity, antibody response and protection to challenge virus infection. Journal of Hygiene 1983;90(1):107-15.

\section{ab Hrabar 1977 \{published data only\}}

Hrabar A, Vodopija I, Andre FE, Mitchell JR, Maassab HF, Hennessy AV, et al. A placebo-controlled dose-response study of the reactogenicity and immunogenicity of a coldadapted recombinant A/Victoria/3/75 (H3N2) live influenza virus candidate vaccine in healthy volunteers. Developments in Biological Standardization 1977;39:53-60.

\section{ab Keitel 1993a \{published data only\}}

Keitel WA, Couch RB, Quarles JM, Cate TR, Baxter B, Maassab HF. Trivalent attenuated cold-adapted influenza virus vaccine: reduced viral shedding and serum antibody responses in susceptible adults. Journal of Infectious Diseases 1993;167(2):305-11.

\section{ab Keitel 1993b \{published data only\}}

Keitel WA, Couch RB, Quarles JM, Cate TR, Baxter B, Maassab HF. Trivalent attenuated cold-adapted influenza virus vaccine: reduced viral shedding and serum antibody responses in susceptible adults. Journal of Infectious Diseases 1993;167(2):305-11.

\section{ab Langley 2005 \{published data only\}}

Langley JM, Halperin SA, McNeil S, Smith B, Jones T, Burt D, et al. Safety and immunogenicity of a Proteosome-trivalent inactivated influenza vaccine, given nasally to healthy adults. Vaccine 2005;24(10):1601-8.

\section{ab Lauteria 1974 \{published data only\}}

Lauteria SF, Kantzler GB, High PC, Lee JD, Waldman RH. An attenuated influenza virus vaccine: reactogenicity, transmissibility, immunogenicity, and protective efficacy. Journal of Infectious Diseases 1974;130(4):380-3.

\section{ab Miller 1977 \{published data only\}}

Miller LW, Togo Y, Hornick RB. Clinical and serologic effects of live attenuated serum inhibitor-resistant influenza B vaccine in seronegative adults. Journal of Medical Virology 1977;1(3):193-9.

\section{ab Pyrhönen 1981 \{published data only\}}

Pyrhönen S, Suni J, Romo M. Clinical trial of a subunit influenza vaccine. Scandinavian Journal of Infectious Diseases 1981;13:95-9.

\section{ab Reeve 1982 \{published data only\}}

Reeve P, Pibermann M, Bachmayer H, Liehl E, Moritz A, Ganzinger $U$, et al. Studies in man with a cold-recombinant live influenza B virus vaccine. Journal of Medical Virology 1982;9(1):1-9.

\section{ab Rocchi 1979a \{published data only\}}

Rocchi G, Ragona G, Piga C, Pelosio A, Volpi A, Vella S, et al. Influenza vaccination with live-attenuated and inactivated virus-vaccines during an outbreak of disease. Journal of Hygiene 1979;83(3):383-90.

ab Rocchi 1979b \{published data only\}

Rocchi G, Ragona G, Piga C, Pelosio A, Volpi A, Vella S, et al. Influenza vaccination with live-attenuated and inactivated virus-vaccines during an outbreak of disease. Journal of Hygiene 1979;83(3):383-90.

ab Saxen 1999 \{published data only\}

Saxen $\mathrm{H}$, Virtanen M. Randomized, placebo-controlled double blind study on the efficacy of influenza immunization on absenteeism of health care workers. Pediatric Infectious Disease Journal 1999;18(9):779-83.

\section{ab Scheifele 2003 \{published data only\}}

Scheifele DW, Duval B, Russell ML, Warrington R, DeSerres G, Skowronski DM, et al. Ocular and respiratory symptoms attributable to inactivated split influenza vaccine: evidence from a controlled trial involving adults. Clinical Infectious Diseases 2003;36(7):850-7.

\section{ab Spencer 1977 \{published data only\}}

Spencer MJ, Cherry JD, Powell KR. Clinical trial with "R-75" strain live, attenuated, serum inhibitor-resistant intranasal influenza B vaccine. Journal of Clinical Microbiology 1977;5(6):584-7.

\section{bb Dauvilliers 2013 \{published data only\}}

Dauvilliers Y, Arnulf I, Lecendreux M, Monaca Charley C, Franco $P$, Drouot $X$, et al. Increased risk of narcolepsy in children and adults after pandemic $\mathrm{H} 1 \mathrm{~N} 1$ vaccination in France. Brain 2013;139(Pt 8):2486-96.

\section{bb DeStefano 2003 \{published data only\}}

DeStefano F, Verstraeten T, Jackson LA, Okoro CA, Benson P, Black SB, et al. Vaccinations and risk of central nervous system demyelinating diseases in adults. Archives of Neurology 2003;60(4):504-9.

\section{bb Dieleman 2011a \{published data only\}}

Dieleman J, Romio S, Johansen K, Weibel D, Bonhoeffer J, Sturkenboom M, et al. Guillain-Barré syndrome and adjuvanted pandemic influenza $A(H 1 N 1) 2009$ vaccine: multinational casecontrol study in Europe. BMJ 2012;343:d3908.

\section{bb Dieleman 2011b \{published data only\}}

Dieleman J, Romio S, Johansen K, Weibel D, Bonhoeffer J, Sturkenboom M, et al. Guillain-Barré syndrome and adjuvanted pandemic influenza A (H1N1) 2009 vaccine: multinational casecontrol study in Europe. BMJ 2012;343:d3908.

\section{bb Dieleman 2011c \{published data only\}}

Dieleman J, Romio S, Johansen K, Weibel D, Bonhoeffer J, Sturkenboom M, et al. Guillain-Barré syndrome and adjuvanted pandemic influenza A (H1N1) 2009 vaccine: multinational casecontrol study in Europe. BMJ 2012;343:d3908.

bb Dieleman 2011d \{published data only\}

Dieleman J, Romio S, Johansen K, Weibel D, Bonhoeffer J, Sturkenboom M, et al. Guillain-Barré syndrome and adjuvanted 
pandemic influenza A (H1N1) 2009 vaccine: multinational casecontrol study in Europe. BMJ 2012;343:d3908.

\section{bb Dieleman 2011e \{published data only\}}

Dieleman J, Romio S, Johansen K, Weibel D, Bonhoeffer J, Sturkenboom M, et al. Guillain-Barré syndrome and adjuvanted pandemic influenza A (H1N1) 2009 vaccine: multinational casecontrol study in Europe. BMJ 2012;343:d3908.

\section{bb Galeotti 2013 \{published data only\}}

Galeotti F, Massari M, D'Alessandro R, Beghi E, Chiò A, Logroscino G, et al. Risk of Guillain-Barré syndrome after 2010-2011 influenza vaccination. European Journal of Epidemiology 2013;28(5):433-44.

\section{bb Garbe 2012 \{published data only\}}

Garbe E, Andersohn F, Bronder E, Klimpel A, Thomae M, Kurtal $H$. Association between drug use and acute immune thrombocytopenia in adults: results from the Berlin casecontrol surveillance study. Pharmacoepidemiology and Drug Safety 2011;20:S147.

Garbe E, Andersohn F, Bronder E, Salama A, Klimpel A, Thomae M, et al. Drug-induced immune thrombocytopaenia: results from the Berlin Case-Control Surveillance Study. European Journal of Clinical Pharmacology 2012;68(5):821-32.

bb Grimaldi-Bensouda 2011 \{published data only\} Grimaldi-Bensouda L, Alpérovitch A, Besson G, Vial C, Cuisset JM, Papeix C, et al. Guillain-Barre syndrome, influenzalike illnesses, and influenza vaccination during seasons with and without circulating A/H1N1 viruses. American Journal of Epidemiology 2011;174(3):326-35.

\section{bb Grimaldi-Bensouda 2012 \{published data only\}}

Grimaldi-Bensouda L, Michel M, Aubrun E, Leighton P, Viallard JF, Adoue D, et al. A case-control study to assess the risk of immune thrombocytopenia associated with vaccines. Blood 2012;120(25):4938-44.

Grimaldi-Bensouda L, Michel M, Viallard J-F, Adoue D, Magy-Bertrand N, Khellaf M, et al. A multicenter casecontrol prospective study to assess the risk of immune thrombocytopenia (ITP) associated with vaccines in adults using the PGRx-ITP registry. Blood 2011;118(21):1169.

\section{bb Hernan 2004 \{published data only\}}

Hernan MA, Jick SS, Olek MJ, Jick H. Recombinant hepatitis B vaccine and the risk of multiple sclerosis: a prospective study. Neurology 2004;63(5):838-42.

\section{bb MacIntyre 2013 \{published data only\}}

MacIntyre CR, Heywood AE, Kovoor P, Ridda I, Seale H, Tan T, et al. Ischaemic heart disease, influenza and influenza vaccination: a prospective case control study. Heart 2013;99(24):1843-8.

\section{bb Mastrangelo 2000 \{published data only\}}

Mastrangelo G, Rossi CR, Pfahlberg A, Marzia V, Barba A, Baldo $M$, et al. Is there a relationship between influenza vaccinations and risk of melanoma? A population-based case-control study. European Journal of Epidemiology 2000;16(9):777-82.

\section{bb Mutsch 2004 \{published data only\}}

Mutsch M, Zhou W, Rhodes P, Bopp M, Chen RT, Linder T, et al. Use of the inactivated intranasal influenza vaccine and the risk of Bell's palsy in Switzerland. New England Journal of Medicine 2004;350(9):896-903.

\section{bb Payne 2006 \{published data only\}}

Payne DC, Rose CE Jr, Kerrison J, Aranas A, Duderstadt S, McNeil MM. Anthrax vaccination and risk of optic neuritis in the United States military, 1998-2003. Archives of Neurology 2006;63(6):871-5.

\section{bb Ray 2011 \{published data only\}}

Ray P, Black S, Shinefield H, Dillon A, Carpenter D, Lewis E, et al. Vaccine Safety Datalink Team. Risk of rheumatoid arthritis following vaccination with tetanus, influenza and hepatitis $B$ vaccines among persons $15-59$ years of age. Vaccine 2011;29(38):6592-7.

\section{bb Rouleau 2014 \{published data only\}}

Rouleau I, De Serres G, Skowronski DM, Drolet JP, Lemire C, Toth E, et al. Risk factors associated with anaphylaxis and other allergic-like events following receipt of 2009 monovalent AS03adjuvanted pandemic influenza vaccine in Quebec, Canada. Vaccine 2014;32(28):3480-7.

\section{bb Siscovick 2000 \{published data only\}}

Siscovick DS, Raghunathan TE, Lin D, Weinmann S, Arbogast P, Lemaitre RN, et al. Influenza vaccination and the risk of primary cardiac arrest. American Journal of Epidemiology 2000;152(7):674-7.

\section{bb Zorzon 2003 \{published data only\}}

Zorzon M, Zivadinov R, Nasuelli D, Dolfini P, Bosco A, Bratina A, et al. Risk factors of multiple sclerosis: a case-control study. Neurological Sciences 2003;24(4):242-7.

\section{cb Bardage 2011 \{published data only\}}

Bardage C, Persson I, Ortqvist A, Bergman U, Ludvigsson JF, Granath F. Neurological and autoimmune disorders after vaccination against pandemic influenza $A(\mathrm{H} 1 \mathrm{~N} 1)$ with a monovalent adjuvanted vaccine: population based cohort study in Stockholm, Sweden. BMJ 2011;343:d5956.

\section{cb Baxter 2012 \{published data only\}}

Baxter R, Toback SL, Sifakis F, Hansen J, Bartlett J, Aukes L, et al. A postmarketing evaluation of the safety of Ann Arbor strain live attenuated influenza vaccine in adults 18-49 years of age. Vaccine 2012;30(20):3053-60.

\section{cb Kaplan 1982 \{published data only\}}

Kaplan JE, Katona P, Hurwitz ES, Schonberger LB. GuillainBarre syndrome in the United States, 1979-1980 and 1980-1981. Lack of an association with influenza vaccination. JAMA 1982;248(6):698-700.

\section{cb Lasky 1998 \{published data only\}}

Lasky T, Terracciano GJ, Magder L, Koski CL, Ballesteros M, Nash D, et al. The Guillain-Barre syndrome and the 1992-1993 and 1993-1994 influenza vaccines. New England Journal of Medicine 1998;339(25):1797-802. 
cb Moro 2013 \{published data only\}

Moro ML, Nobilio L, Voci C, Di Mario S, Candela S, Magrini N. A population based cohort study to assess the safety of pandemic influenza vaccine Focetria in Emilia-Romagna region, Italy - part two. Vaccine 2013;31(10):1438-46.

\section{cb O'Flanagan 2014 \{published data only\}}

O'Flanagan D, Barret AS, Foley M, Cotter S, Bonner C, Crowe $C$, et al. Investigation of an association between onset of narcolepsy and vaccination with pandemic influenza vaccine, Ireland April 2009-December 2010. Eurosurveillance 2014;19(17):15-25.

\section{cb Persson 2014 \{published data only\}}

Persson I, Granath F, Askling J, Ludvigsson JF, Olsson T, Feltelius N. Risks of neurological and immune-related diseases, including narcolepsy, after vaccination with Pandemrix: a population- and registry-based cohort study with over 2 years of follow-up. Journal of Internal Medicine 2014;275(2):172-90.

\section{cb Ray 2011 \{published data only\}}

Ray P, Black S, Shinefield H, Dillon A, Carpenter D, Lewis E, et al. Risk of rheumatoid arthritis following vaccination with tetanus, influenza and hepatitis B vaccines among persons $15-59$ years of age. Vaccine 2011;29(38):6592-7.

\section{cb Shonberger 1979 \{published data only\}}

Schonberger LB, Bregman DJ, Sullivan-Bolyai JZ, Keenlyside RA, Ziegler DW, Retailliau HF, et al. Guillain-Barre syndrome following vaccination in the National Influenza Immunization Program, United States, 1976 - 1977. American Journal of Epidemiology 1979;110(2):105-23.

paa Ma 2014 \{published data only\}

Ma F, Zhang L, Jiang R, Zhang J, Wang H, Gao X, et al. Prospective cohort study of the safety of an influenza A (H1N1) vaccine in pregnant Chinese women. Clinical and Vaccine Immunology 2014;21(9):1282-7.

\section{paa Madhi 2014 \{published data only\}}

Madhi SA, Cutland CL, Kuwanda L, Weinberg A, Hugo A, Jones $S$, et al. Influenza vaccination of pregnant women and protection of their infants. New England Journal of Medicine 2014;371(10):918-31.

\section{pba Benowitz 2010 \{published data only\}}

Benowitz I, Esposito DB, Gracey KD, Shapiro ED, Vázquez M. Influenza vaccine given to pregnant women reduces hospitalization due to influenza in their infants. Clinical Infectious Diseases 2010;51(12):1355-61.

\section{pba Poehling 2011 \{published data only\}}

Poehling KA, Szilagyi PG, Staat MA, Snively BM, Payne DC, Bridges $C B$, et al. Impact of maternal immunization on influenza hospitalizations in infants. American Journal of Obstetrics and Gynecology 2001;204(Suppl 6):141-8.

\section{pbb Irving 2013 \{published data only\}}

Irving SA, Kieke BA, Donahue JG, Mascola MA, Baggs J, Destefano $F$, et al. Trivalent inactivated influenza vaccine and spontaneous abortion. Obstetrics and Gynecology 2013;121(1):159-65.

pca Ahrens 2014 \{published data only\}

Ahrens KA, Louik C, Kerr S, Mitchell AA, Werler MM. Seasonal influenza vaccination during pregnancy and the risks of preterm delivery and small for gestational age birth. Paediatric and Perinatal Epidemiology 2014;28(6):498-509.

pca Black 2004 \{published data only\}

Black SB, Shinefield HR, France EK, Fireman BH, Blatt ST, Shay D. Effectiveness of influenza vaccine during pregnancy in preventing hospitalizations and outpatient visits for respiratory illness in pregnant women and their infants. American Journal of Perinatology 2004;21(6):333-9.

pca Eick 2011 \{published data only\}

Eick AA, Uyeki TM, Klimov A, Hall H, Reid R, Santosham M, et al. Maternal influenza vaccination and effect on influenza virus infection in young infants. Archives of Pediatrics and Adolescent Medicine 2011;165(2):104-11.

\section{pca France 2006 \{published data only\}}

France EK, Smith-Ray R, McClure D, Hambidge S, Xu S, Yamasaki $\mathrm{K}$, et al. Impact of maternal influenza vaccination during pregnancy on the incidence of acute respiratory illness visits among infants. Archives of Pediatrics and Adolescent Medicine 2006;160(12):1277-83.

pca Hulka 1964 \{published data only\}

Hulka JF. Effectiveness of polyvalent influenza vaccine in pregnancy. Report of a controlled study during an outbreak of Asian influenza. Obstetrics and Gynecology 1964;23:830-7.

\section{pca Munoz 2005 \{published data only\}}

Munoz FM, Greisinger AJ, Wehmanen OA, Mouzoon ME, Hoyle JC, Smith FA, et al. Safety of influenza vaccination during pregnancy. American Journal of Obstetrics and Gynecology 2005;192(4):1098-106.

\section{pca Yamada 2012 \{published data only\}}

Yamada T, Yamada T, Morikawa M, Cho K, Endo T, Sato SS, et al. Pandemic (H1N1) 2009 in pregnant Japanese women in Hokkaido. Journal of Obstetrics and Gynaecology Research 2012;38(1):130-6.

\section{pcb Beau 2014 \{published data only\}}

Beau AB, Hurault-Delarue C, Vidal S, Guitard C, Vayssiere C, Petiot D. Pandemic A/H1N1 influenza vaccination during pregnancy: a comparative study using the EFEMERIS database. Vaccine 2014;32(11):1254-8.

\section{pcb Cantu 2013 \{published data only\}}

Cantu J, Biggio J, Jauk V, Wetta L, Andrews W, Tita A. Selective uptake of influenza vaccine and pregnancy outcomes. Journal of Maternal-Fetal and Neonatal Medicine 2013;26(12):1207-11.

pcb Chambers 2013 \{published data only\}

Chambers CD, Johnson D, Xu R, Luo Y, Louik C, Mitchell AA, et al. Risks and safety of pandemic H1N1 influenza vaccine in pregnancy: birth defects, spontaneous abortion, preterm 
delivery, and small for gestational age infants. Vaccine 2013;31(44):5026-32.

\section{pcb Cleary 2014 \{published data only\}}

Cleary BJ, Rice U, Eogan M, Metwally N, McAuliffe F. 2009 $A / H 1 N 1$ influenza vaccination in pregnancy: uptake and pregnancy outcomes - a historical cohort study. European Journal of Obstetrics \& Gynecology and Reproductive Biology 2014;178:163-8.

\section{pcb Deinard 1981 \{published data only\}}

Deinard AS, Ogburn P Jr. A/NJ/8/76 influenza vaccination program: effects on maternal health and pregnancy outcome. American Journal of Obstetrics and Gynecology 1981;140(3):240-5.

\section{pcb Dodds 2012 \{published data only\}}

Dodds L, Macdonald N, Scott J, Spencer A, Allen VM, McNeil S. The association between influenza vaccine in pregnancy and adverse neonatal outcomes. Journal of Obstetrics and Gynaecology Canada 2012;34(8):714-20.

\section{pcb Fell 2012 \{published data only\}}

Fell DB, Sprague AE, Liu N, Yasseen AS 3rd, Wen SW, Smith G, et al. Better Outcomes Registry \& Network (BORN) Ontario. $\mathrm{H} 1 \mathrm{~N} 1$ influenza vaccination during pregnancy and fetal and neonatal outcomes. American Journal of Public Health 2012;102(6):e33-40.

\section{pcb Håberg 2013 \{published data only\}}

Håberg SE, Trogstad L, Gunnes N, Wilcox AJ, Gjessing HK, Samuelsen SO, et al. Risk of fetal death after pandemic influenza virus infection or vaccination. New England Journal of Medicine 2013;368(4):333-40.

* Håberg SE, Trogstad L, Gunnes N, Wilcox AJ, Gjessing HK, Samuelsen SO, et al. Risk of fetal death after pandemic influenza virus infection or vaccination. Obstetrical and Gynecological Survey 2013;68(5):348-9.

\section{pcb Heikkinen 2012 \{published data only\}}

Heikkinen T, Young J, van Beek E, Franke H, Verstraeten T, Weil JG, et al. Safety of MF59-adjuvanted A/H1N1 influenza vaccine in pregnancy: a comparative cohort study. American Journal of Obstetrics \& Gynecology 2012;207(3):177.e1-8.

\section{pcb Källén 2012 \{published data only\}}

Källén B, Olausson PO. Vaccination against H1N1 influenza with Pandemrix during pregnancy and delivery outcome: a Swedish register study. British Journal of Obstetrics and Gynaecology 2012;119(13):1583-90.

\section{pcb Launay 2012 \{published data only\}}

Launay O, Krivine A, Charlier C, Truster V, Tsatsaris V, Lepercq J, et al. Low rate of pandemic A/H1N1 2009 influenza infection and lack of severe complication of vaccination in pregnant women: a prospective cohort study. PLOS ONE 2012;7(12):e52303. pcb Lin 2012 \{published data only\}

Lin TH, Lin SY, Lin CH, Lin RI, Lin HC, Chiu TH, et al. AdimFlu-S influenza $A(H 1 N 1)$ vaccine during pregnancy: the Taiwanese Pharmacovigilance Survey. Vaccine 2012;30(16):2671-5.

pcb Louik 2013 \{published data only\}

Louik C, Ahrens K, Kerr S, Pyo J, Chambers C, Jones KL, et al. Risks and safety of pandemic H1N1 influenza vaccine in pregnancy: exposure prevalence, preterm delivery, and specific birth defects. Vaccine 2013;31(44):5033-40.

pcb Ludvigsson 2013 \{published data only\}

Ludvigsson JF, Zugna D, Cnattingius S, Richiardi L, Ekbom A, Ortqvist $A$, et al. Influenza $H 1 N 1$ vaccination and adverse pregnancy outcome. European Journal of Epidemiology 2013;28(7):579-88.

pcb Nordin 2013 \{published data only\}

Nordin JD, Kharbanda EO, Benitez GV, Nichol K, Lipkind H, Naleway A, et al. Maternal safety of trivalent inactivated influenza vaccine in pregnant women. Obstetrics and Gynecology 2013;121(3):519-25.

pcb Nordin 2014 \{published data only\}

Nordin JD, Kharbanda EO, Vazquez Benitez G, Lipkind H, Vellozzi C, Destefano F. Maternal influenza vaccine and risks for preterm or small for gestational age birth. Journal of Pediatrics 2014;164(5):1051-7.e2.

\section{pcb Omer 2011 \{published data only\}}

Omer SB, Goodman D, Steinhoff MC, Rochat R, Klugman KP, Stoll BJ, et al. Maternal influenza immunization and reduced likelihood of prematurity and small for gestational age births: a retrospective cohort study. PLoS Medicine 2011;8(5):e1000441.

\section{pcb Oppermann 2012 \{published data only\}}

Oppermann M, Fritzsche J, Weber-Schoendorfer C, KellerStanislawski B, Allignol A, Meister R, et al. A(H1N1)v2009: a controlled observational prospective cohort study on vaccine safety in pregnancy. Vaccine 2012;30(30):4445-52.

\section{pcb Pasternak 2012 \{published data only\}}

Pasternak B, Svanström H, Mølgaard-Nielsen D, Krause TG, Emborg HD, Melbye M, et al. Risk of adverse fetal outcomes following administration of a pandemic influenza A (H1N1) vaccine during pregnancy. JAMA 2012;308(2):165-74.

\section{pcb Richards 2013 \{published data only\}}

Richards JL, Hansen C, Bredfeldt C, Bednarczyk RA, Steinhoff MC, Adjaye-Gbewonyo D, et al. Neonatal outcomes after antenatal influenza immunization during the 2009 H1N1 influenza pandemic: impact on preterm birth, birth weight, and small for gestational age birth. Clinical Infectious Diseases 2013;56(9):1216-22.

\section{pcb Rubinstein 2013 \{published data only\}}

Rubinstein F, Micone P, Bonotti A, Wainer V, Schwarcz A, Augustovski F. Influenza A/H1N1 MF59 adjuvanted vaccine in pregnant women and adverse perinatal outcomes: multicentre study. BMJ Online 2013;346(7896):f393. 
pcb Sheffield 2012 \{published data only\}

Sheffield JS, Greer LG, Rogers VL, Roberts SW, Lytle H, McIntire DD, et al. Effect of influenza vaccination in the first trimester of pregnancy. Obstetrics and Gynecology 2012;120(3):532-7.

\section{pcb Toback 2012 \{published data only\}}

Toback SL, Beigi R, Tennis P, Sifakis F, Calingaert B, Ambrose CS. Maternal outcomes among pregnant women receiving live attenuated influenza vaccine. Influenza and Other Respiratory Viruses 2012;6(1):44-51.

\section{pcb Trotta 2014 \{published data only\}}

Trotta F, Da Cas R, Spila Alegiani S, Gramegna M, Venegoni M, Zocchetti $C$, et al. Evaluation of safety of $A / H 1 N 1$ pandemic vaccination during pregnancy: cohort study. $B M J$ 2014;348:g3361.

\section{References to studies excluded from this review}

\section{ab López-Macías 2011a \{published data only\}}

López-Macías C, Ferat-Osorio E, Tenorio-Calvo A, Isibasi A, Talavera J, Arteaga-Ruiz O, et al. Safety and immunogenicity of a virus-like particle pandemic influenza A (H1N1) 2009 vaccine in a blinded, randomised, placebo-controlled trial of adults in Mexico. Vaccine 2011;29(44):7826-34.

\section{ab López-Macías 2011b \{published data only\}}

López-Macías C, Ferat-Osorio E, Tenorio-Calvo A, Isibasi A, Talavera J, Arteaga-Ruiz O, et al. Safety and immunogenicity of a virus-like particle pandemic influenza A (H1N1) 2009 vaccine in a blinded, randomised, placebo-controlled trial of adults in Mexico. Vaccine 2011;29(44):7826-34.

\section{ab Mallory 2010 \{published data only\}}

Mallory RM, Malkin E, Ambrose CS, Bellamy T, Shi L, Yi T, et al. Safety and immunogenicity following administration of a live, attenuated monovalent $2009 \mathrm{H} 1 \mathrm{~N} 1$ influenza vaccine to children and adults in two randomised controlled trials. PLOS ONE 2010;5(10):e13755.

\section{ab Plennevaux 2010 \{published data only\}}

Plennevaux E, Sheldon E, Blatter M, Reeves-Hoché MK, Denis $M$. Immune response after a single vaccination against 2009 influenza A H1N1 in USA: a preliminary report of two randomised controlled phase 2 trials. Lancet 2010;375(9708):41-8.

\section{ab Precioso 2011 \{published data only\}}

Precioso AR, Miraglia JL, Campos LM, Goulart AC, Timenetsky Mdo C, Cardoso MR, et al. A phase I randomised, doubleblind, controlled trial of 2009 influenza A (H1N1) inactivated monovalent vaccines with different adjuvant systems. Vaccine 2011;29(48):8974-81.

\section{ab Treanor 2010 \{published data only\}}

Treanor JJ, Taylor DN, Tussey L, Hay C, Nolan C, Fitzgerald T, et al. Safety and immunogenicity of a recombinant hemagglutinin influenza-flagellin fusion vaccine (VAX125) in healthy young adults. Vaccine 2010;28(52):8268-74. ab Turley 2011 \{published data only\}

Turley CB, Rupp RE, Johnson C, Taylor DN, Wolfson J, Tussey L, et al. Safety and immunogenicity of a recombinant M2e-flagellin influenza vaccine (STF2.4xM2e) in healthy adults. Vaccine 2011;29(32):5145-52.

ab Wacheck 2010 \{published data only\}

Wacheck V, Egorov A, Groiss F, Pfeiffer A, Fuereder T, Hoeflmayer $D$, et al. A novel type of influenza vaccine: safety and immunogenicity of replication-deficient influenza virus created by deletion of the interferon antagonist NS1. Journal of Infectious Diseases 2010;201(3):354-62.

\section{Al-Dabbagh 2013 \{published data only\}}

Al-Dabbagh M, Lapphra K, Scheifele DW, Halperin SA, Langley JM, Cho P, et al. Elevated inflammatory mediators in adults with oculo-respiratory syndrome following influenza immunization: a public health agency of Canada/ Canadian Institutes of Health Research Influenza Research Network (PCIRN) Study. Clinical and Vaccine Immunology 2013;20(8):1108-14.

\section{Ambrosch 1976 \{published data only\}}

Ambrosch F, Balluch H. Studies of the non-specific clinical effectiveness of influenza vaccination. Laryngologie, Rhinologie, Otologie 1976;55:57-61.

\section{Ambrose 2012 \{published data only\}}

Ambrose CS, Wu X. The safety and effectiveness of selfadministration of intranasal live attenuated influenza vaccine in adults. Vaccine 2013;31(6):857-60.

\section{Andersson 2015 \{published data only\}}

Andersson L. Response on the author's reply to the letter to the editor: Contradictory data on type 1 diabetes in a recently published article "Risks of neurological and immunerelated diseases, including narcolepsy, after vaccination with Pandemrix". Journal of Internal Medicine 2015;277(2):272-3.

\section{Aoki 1986 \{published data only\}}

Aoki FY, Sitar DS, Milley EV, Hammond GW, Milley EV, Vermeersch $C$, et al. Potential of influenza vaccine and amantadine to prevent influenza A illness in Canadian forces personnel 1980-83. Military Medicine 1986;151(9):459-65.

\section{Arnou 2010 \{published data only\}}

Arnou R, Eavis P, Pardo JR, Ambrozaitis A, Kazek MP, Weber F. Immunogenicity, large scale safety and lot consistency of an intradermal influenza vaccine in adults aged $18-60$ years: randomized, controlled, phase III trial. Human Vaccines 2010;6(4):346-54

\section{Atmar 1995 \{published data only\}}

Atmar RL, Keitel WA, Cate TR, Quarles JM, Couch RB. Comparison of trivalent cold-adapted recombinant (CR) influenza virus vaccine with monovalent $C R$ vaccines in healthy unselected adults. Journal of Infectious Diseases 1995;172(1):253-7. 
Atmar 2011 \{published data only\}

Atmar RL, Keitel WA, Quarles JM, Cate TR, Patel SM, Nino D, et al. Evaluation of age-related differences in the immunogenicity of a G9 H9N2 influenza vaccine. Vaccine 2011;29(45):8066-72.

\section{Atsmon 2012 \{published data only\}}

Atsmon J, Kate-Ilovitz E, Shaikevich D, Singer Y, Volokhov I, Haim KY, et al. Safety and immunogenicity of multimeric-001 - a novel universal influenza vaccine. Journal of Clinical Immunology 2012;32(3):595-603.

\section{Ausseil 1999 \{published data only\}}

Ausseil F. Immunization against influenza among working adults: the Philippines experience. Vaccine 1999;17(Suppl 1):59-62.

\section{Banzhoff 2001 \{published data only\}}

Banzhoff A, Kaniok W, Muszer A. Effectiveness of an influenza vaccine used in Poland in the 1998-1999 influenza season. Immunological Investigations 2001;30(2):103-13.

\section{Baxter 2010 \{published data only\}}

Baxter R, Ray GT, Fireman BH. Effect of influenza vaccination on hospitalizations in persons aged 50 years and older. Vaccine 2010;28(45):7267-72.

\section{Baxter 2011 \{published data only\}}

Baxter R, Patriarca PA, Ensor K, Izikson R, Goldenthal KL, Cox MM. Evaluation of the safety, reactogenicity and immunogenicity of FluBlok trivalent recombinant baculovirusexpressed hemagglutinin influenza vaccine administered intramuscularly to healthy adults $50-64$ years of age. Vaccine 2011;29(12):2272-8.

\section{Baxter 2012 \{published data only\}}

Baxter R, Lewis N, Bakshi N, Vellozzi C, Klein NP. Recurrent Guillain-Barre syndrome following vaccination. Clinical Infectious Diseases 2012;54(6):800-4.

\section{Baxter 2013 \{published data only\}}

Baxter R, Bakshi N, Fireman B, Lewis E, Ray P, Vellozzi C, et al. Lack of association of Guillain-Barre syndrome with vaccinations. Clinical Infectious Diseases 2013;57(2):197-204.

\section{Belongia 2009 \{published data only\}}

Belongia EA, Kieke BA, Donahue JG, Greenlee RT, Balish A, Foust $A$, et al. Effectiveness of inactivated influenza vaccines varied substantially with antigenic match from the 2004-2005 season to the 2006-2007 season. Journal of Infectious Diseases 2009;199(2):159-67.

\section{Belshe 2001 \{published data only\}}

Belshe RB, Gruber WC. Safety, efficacy and effectiveness of coldadapted, live, attenuated, trivalent, intranasal influenza vaccine in adults and children. Philosophical Transactions of the Royal Society of London 2001;356(1416):1947-51.

\section{Benke 2004 \{published data only\}}

Benke G, Abramson M, Raven J, Thien FCK, Walters EH. Asthma and vaccination history in a young adult cohort. Australian and New Zealand Journal of Public Health 2004;28(4):336-8.

\section{Beran 2013 \{published data only}

Beran JI, Peeters M, Dewe W, Raupachova J, Hobzova L, Devaster JM. Immunogenicity and safety of quadrivalent versus trivalent inactivated influenza vaccine: a randomised, controlled trial in adults. BMC Infectious Diseases 2013;13(1):224.

\section{Betts 1977b \{published data only\}}

Betts RF, Douglas RG Jr. Comparative study of reactogenicity and immunogenicity of influenza A/New Jersey/8/76 (Hsw1N1) virus vaccines in normal volunteers. Journal of Infectious Diseases 1977;136(Suppl):443-9.

Beyer 1996 \{published data only\}

Beyer WEP, Palache AM, Kerstens R, Masurel N. Gender differences in local and systemic reactions to inactivated influenza vaccine, established by a meta-analysis of fourteen independent studies. European Journal of Clinical Microbiology \& Infectious Diseases 1996;15(1):65-70.

\section{Carlson 1979 \{published data only\}}

Carlson AJ, Davidson WL, McLean AA, Vella PP, Weibel RE, Woodhour AF, et al. Pneumococcal vaccine: dose, revaccination, and coadministration with influenza vaccine. Proceedings of the Society for Experimental Biology and Medicine 1979;161(4):558-63.

\section{Cate 1977 \{published data only\}}

Cate TR, Couch RB, Kasel JA, Six HR. Clinical trials of monovalent influenza $\mathrm{A} / \mathrm{New}$ Jersey/76 virus vaccines in adults: reactogenicity, antibody response, and antibody persistence. Journal of Infectious Diseases 1977;136(Suppl):450-5.

\section{Chavant 2013 \{published data only\}}

Chavant F, Ingrand I, Jonville-Bera AP, Plazanet C, GrasChampel V, Lagarce L, et al. The PREGVAXGRIP study: a cohort study to assess foetal and neonatal consequences of in utero exposure to vaccination against A (H1N1) v2009 influenza. Drug Safety 2013;36(6):455-65.

\section{Chichester 2012 \{published data only\}}

Chichester JA, Jones RM, Green BJ, Stow M, Miao F, Moonsammy G, et al. Safety and immunogenicity of a plantproduced recombinant hemagglutinin-based influenza vaccine (HAl-05) derived from A/Indonesia/05/2005 (H5N1) influenza virus: a phase 1 randomised, double-blind, placebocontrolled, dose-escalation study in healthy adults. Viruses 2012;4(11):3227-44.

\section{Chlibek 2002 \{published data only\}}

Chlibek R, Beran J, Splino M. Effectiveness of influenza vaccination in healthy adults - a fourfold decrease in influenza morbidity during one influenza season. Epidemiologie, Mikrobiologie, Imunologie 2002;51(2):47-51.

\section{Choe 2011a \{published data only\}}

Choe YJ, Cho H, Song KM, Kim JH, Han OP, Kwon YH, et al. Active surveillance of adverse events following immunization against pandemic influenza A (H1N1) in Korea. Japanese Journal of Infectious Diseases 2011;64(4):297-303. 
Choe 2011b \{published data only\}

Choe YJ, Cho H, Bae GR, Lee JK. Guillain-Barre syndrome following receipt of influenza A (H1N1) 2009 monovalent vaccine in Korea with an emphasis on Brighton Collaboration case definition. Vaccine 2011;29(11):2066-70.

Choe 2011c \{published data only\}

Choe YJ, Cho H, Kim SN, Bae GR, Lee JK. Serious adverse events following receipt of trivalent inactivated influenza vaccine in Korea, 2003-2010. Vaccine 2011;29(44):7727-32.

\section{Chou 2007 \{published data only\}}

Chou CH, Liou WP, Hu KI, Loh CH, Chou CC, Chen YH. Bell's palsy associated with influenza vaccination: two case reports. Vaccine 2007;25:2839-41.

\section{Clover 1991 \{published data only\}}

Clover RD, Crawford S, Glezen WP, Taber LH, Matson CC, Couch RB. Comparison of heterotypic protection against influenza A/Taiwan/86 (H1N1) by attenuated and inactivated vaccines to A/Chile/83-like viruses. Journal of Infectious Diseases 1991;163(2):300-4.

\section{Confavreux 2001 \{published data only\}}

Confavreux C, Suissa S, Saddier P, Bourdes V, Vukusic S. Vaccinations and the risk of relapse in multiple sclerosis. Vaccines in Multiple Sclerosis Study Group. New England Journal of Medicine 2001;344(5):319-26.

\section{Conlin 2013 \{published data only\}}

Conlin AMS, Bukowinski AT, Sevick CJ, DeScisciolo C, CrumCianflone NF. Safety of the pandemic H1N1 influenza vaccine among pregnant U.S. military women and their newborns. Obstetrics and Gynecology 2013;121(3):511-8.

\section{Couch 2012 \{published data only\}}

Couch RB, Patel SM, Wade-Bowers CL, Niño D. A randomised clinical trial of an inactivated avian influenza $A(H 7 N 7)$ vaccine. PLOS ONE 2012;7(12):e49704.

\section{Das Gupta 2002 \{published data only\}}

Das Gupta R, Guest JF. A model to estimate the cost benefit of an occupational vaccination programme for influenza with Influvac in the UK. Pharmacoeconomics 2002;20(7):475-84.

\section{Davidson 2011 \{published data only\}}

Davidson LE, Fiorino AM, Snydman DR, Hibberd PL. Lactobacillus GG as an immune adjuvant for live-attenuated influenza vaccine in healthy adults: a randomized double-blind placebo-controlled trial. European Journal of Clinical Nutrition 2011;65(4):501-7.

\section{Davies 1972 \{published data only\}}

Davies JE, Howell RW, Meichen FW. A clinical trial of inhaled inactivated influenza vaccine. British Journal of Clinical Practice 1972;26(10):469-71.

\section{Davies 1973 \{published data only\}}

Davies JE, Howell RH, Meichen FW. A clinical trial of inhaled inactivated influenza vaccine. British Journal of General Practice 1970;27(6):219-21.

\section{De Serres 2003a $\{$ published data only\}}

De Serres GI, Boulianne N, Duval B, Rochette L, Grenier JL, Roussel R, et al. Oculo-respiratory syndrome following influenza vaccination: evidence for occurrence with more than one influenza vaccine. Vaccine 2003;21(19-20):2346-53.

De Serres 2003b \{published data only\}

De Serres GI, Grenier JL, Toth E, Menard S, Roussel R, Tremblay M, et al. The clinical spectrum of the oculorespiratory syndrome after influenza vaccination. Vaccine 2003;21(19-20):2354-61.

\section{De Serres 2004 \{published data only\}}

De Serres G, Skowronski DM, Guay M, Rochette L, Jacobsen K, Fuller T, et al. Recurrence risk of oculorespiratory syndrome after influenza vaccination: randomized controlled trial of previously affected persons. Archives of Internal Medicine 2004;164(20):2266-72.

De Wals 2012 \{published data only\}

De Wals P, Deceuninck G, Toth E, Boulianne N, Brunet D, Boucher RM, et al. Risk of Guillain-Barre syndrome following $\mathrm{H} 1 \mathrm{~N} 1$ influenza vaccination in Quebec. JAMA 2012;308(2):175-81.

Dolin 1977 \{published data only\}

Dolin R, Wise TG, Mazur MH, Tuazon CU, Ennis FA. Immunogenicity and reactogenicity of influenza $A / N e w$ Jersey/76 virus vaccines in normal adults. Journal of Infectious Diseases 1977;136(Suppl):435-42.

\section{Dominguez 2012 \{published data only\}}

Dominguez A, Castilla J, Godoy P, Delgado-Rodriguez M, Martin V, Saez M, et al. Effectiveness of pandemic and seasonal influenza vaccines in preventing pandemic influenza-associated hospitalization. Vaccine 2012;30(38):5644-50.

\section{Duffy 2014 \{published data only\}}

Duffy J, Weintraub E, Vellozzi C, DeStefano F. Narcolepsy and influenza A (H1N1) pandemic 2009 vaccination in the United States. Neurology 2014;83(20):1823-30.

Eames 2012 \{published data only\}

Eames KT, Brooks-Pollock E, Paolotti D, Perosa M, Gioannini C, Edmunds WJ. Rapid assessment of influenza vaccine effectiveness: analysis of an internet-based cohort. Epidemiology and Infection 2012;140(7):1309-15.

Edmonson 1970 \{published data only\}

Edmonson KW, Graham SM, Warburton MF. A clinical trial of influenza vaccine in Canberra. Medical Journal of Australia 1970;4:6-13.

\section{Eick-Cost 2012 \{published data only\}}

Eick-Cost AA, Tastad KJ, Guerrero AC, Johns MC, Lee S, MacIntosh VH, et al. Effectiveness of seasonal influenza vaccines against influenza-associated illnesses among US military personnel in 2010-11: a case-control approach. PLOS ONE 2012;7(7):e41435. 
El'shina 1998 \{published data only\}

El'shina GA, Gorbunov MA, Shervarli VI, Lonskaia NI, Pavlova LI, Khaitov RM, et al. Evaluation of the effectiveness of influenza trivalent polymer subunit vaccine "Grippol". Zhumal Mikrobiologii Epidemiologii Immunobiologii 1998;3:40-3.

Englund 1993 \{published data only\}

Englund JA, Mbawuike IN, Hammill H, Holleman MC, Baxter BD, Glezen WP. Maternal immunization with influenza or tetanus toxoid vaccine for passive antibody protection in young infants. Journal of Infectious Diseases 1993;168(3):647-56.

Finklea 1969 \{published data only\}

Finklea JF, Sandifer SH, Peck FB, Manos JP. A clinical and serologic comparison of standard and purified bivalent inactivated influenza vaccines. Journal of Infectious Diseases 1969;120(6):708-12.

\section{Fisher 2012 \{published data only\}}

Fisher BM, Van Bockern J, Hart J, Lynch AM, Winn VD, Gibbs RS, et al. Pandemic influenza A H1N1 2009 infection versus vaccination: a cohort study comparing immune responses in pregnancy. PLoS ONE 2012;7(3):e33048.

\section{Foy 1981 \{published data only\}}

Foy HM, Cooney MK, Allan ID, Frost F, Blumhagen JM, Fox JP. Influenza $B$ virus vaccines in children and adults: adverse reactions, immune response, and observations in the field. Journal of Infectious Diseases 1981;143(5):700-6.

Frank 1981 \{published data only\}

Frank AL, Webster RG, Glezen WP, Cate TR. Immunogenicity of influenza A/USSR (H1N1) subunit vaccine in unprimed young adults. Journal of Medical Virology 1981;7(2):135-42.

\section{Freestone 1976 \{published data only\}}

Freestone DS. Clinical trials with intranasally administered WRL 105 strain live influenza vaccine in volunteers. Developments in Biological Standardization 1976;33:207-12.

\section{Gerstoft 2001 \{published data only\}}

Gerstoft J. Influenza vaccination of healthy adults. Ugeskrift for Laeger 2001;163(19):2615-7.

\section{Greenbaum 2002 \{published data only\}}

Greenbaum E, Furst A, Kiderman A, Stewart B, Levy R, Schlesinger $\mathrm{M}$, et al. Mucosal [SIgA] and serum [IgG] immunologic responses in the community after a single intranasal immunization with a new inactivated trivalent influenza vaccine. Vaccine 2002;20(7-8):1232-9.

\section{Greene 2013 \{published data only\}}

Greene SK, Rett MD, Vellozzi C, Li L, Kulldorff M, Marcy SM, et al. Guillain-Barre syndrome, influenza vaccination, and antecedent respiratory and gastrointestinal infections: a case-centered analysis in the Vaccine Safety Datalink, 2009-2011. PLOS ONE 2013;8(6):e67185.

\section{Gross 1999 \{published data only\}}

Gross PA, Sperber SJ, Donabedian A, Dran S, Morchel G, Cataruozolo $P$, et al. Paradoxical response to a novel influenza virus vaccine strain: the effect of prior immunization. Vaccine 1999;17(18):2284-9.

\section{Grotto 1998 \{published data only\}}

Grotto I, Mandel Y, Green MS, Varsano N, Gdalevich M, Ashkenazi I, et al. Influenza vaccine efficacy in young, healthy adults. Clinical Infectious Diseases 1998;26(4):913-7.

\section{Gruber 1994 \{published data only\}}

Gruber WC, Campbell PW, Thompson JM, Reed GW, Roberts B, Wright PF. Comparison of live attenuated and inactivated influenza vaccines in cystic fibrosis patients and their families: results of a 3-year study. Journal of Infectious Diseases 1994;169(2):241-7.

\section{Gwini 2011 \{published data only\}}

Gwini SM, Coupland CA, Siriwardena AN. The effect of influenza vaccination on risk of acute myocardial infarction: selfcontrolled case-series study. Vaccine 2011;29(6):1145-9.

\section{Haber 2004 \{published data only\}}

Haber P, DeStefano F, Angulo FJ, Iskander J, Shadomy SV, Weintraub $\mathrm{E}$, et al. Guillain-Barre syndrome following influenza vaccination. JAMA 2004;292(20):2478-81.

\section{Haigh 1973 \{published data only\}}

Haigh W, Howell RW, Meichen FW. A comparative trial of influenza immunization by inhalation and hypojet method. The Practitioner 1973;211:365-70.

\section{Halperin 2002 \{published data only\}}

Halperin SA, Smith B, Mabrouk T, Germain M, Trepanier P, Hassell T, et al. Safety and immunogenicity of a trivalent, inactivated, mammalian cell culture-derived influenza vaccine in healthy adults, seniors, and children. Vaccine 2002;20(7-8):1240-7.

\section{Hambidge 2011 \{published data only\}}

Hambidge SJ, Ross C, McClure D, Glanz J. Trivalent inactivated influenza vaccine is not associated with sickle cell hospitalizations in adults from a large cohort. Vaccine 2011;29(46):8179-81.

\section{Heinonen 1973 \{published data only\}}

Heinonen OP, Shapiro S, Monson RR, Hartz SC, Rosenberg L, Slone D. Immunization during pregnancy against poliomyelitis and influenza in relation to childhood malignancy. International Journal of Epidemiology 1973;2(3):229-35.

\section{Hellenbrand 2012 \{published data only\}}

Hellenbrand W, Jorgensen P, Schweiger B, Falkenhorst G, Nachtnebel M, Greutelaers B, et al. Prospective hospital-based case-control study to assess the effectiveness of pandemic influenza $\mathrm{A}(\mathrm{H} 1 \mathrm{~N} 1)$ pdm09 vaccination and risk factors for hospitalization in 2009-2010 using matched hospital and testnegative controls. BMC Infectious Diseases 2012;12:127.

Hobson 1970 \{published data only\}

Hobson D, Baker FA, Chivers CP, Reed SE, Sharp D. A comparison of monovalent Hong Kong influenza virus vaccine with vaccines 
containing only pre-1968 Asian strains in adult volunteers. Journal of Hygiene 1970;68(3):369-78.

\section{Hobson 1973 \{published data only\}}

Hobson D, Baker FA, Curry RL, Beare AS, Massey PM. The efficacy of live and inactivated vaccines of Hong Kong influenza virus in an industrial community. Journal of Hygiene 1973;71(4):641-7.

\section{Hoskins 1973 \{published data only\}}

Hoskins TW, Davies JR, Allchin A, Miller CL, Pollock TM. Controlled trial of inactivated influenza vaccine containing the $\mathrm{A} /$ Hong Kong strain during an outbreak of influenza due to the A/England/42/72 strain. Lancet 1973;2(7821):116-20.

\section{Hoskins 1976 \{published data only\}}

Hoskins TW, Davies JR, Smith AJ, Allchin A, Miller CL, Pollock TM. Influenza at Christ's Hospital: March, 1974. Lancet 1976;1(7951):105-8.

\section{Hoskins 1979 \{published data only\}}

Hoskins TW, Davies JR, Smith AJ, Miller CL, Allchin A. Assessment of inactivated influenza $A$ vaccine after three outbreaks of influenza A at Christ's Hospital. Lancet 1979;1(8106):33-5.

\section{Howell 1967 \{published data only\}}

Howell RW. Long term efficacy of oil-adjuvant influenza vaccine in an industrial population. British Journal of Industrial Medicine 1967;24:66-70

\section{Huang 2011 \{published data only\}}

Huang WT, Chen WC, Teng HJ, Huang WI, Huang YW, Hsu CW. Adverse events following pandemic A (H1N1) 2009 monovalent vaccines in pregnant women - Taiwan, November 2009-August 2010. PLOS ONE 2011;6(8):e23049.

\section{Hurwitz 1983 \{published data only\}}

Hurwitz ES, Holman RC, Nelson DB, Schonberger LB. National surveillance for Guillain-Barré syndrome: January 1978-March 1979. Neurology 1983;33(2):150-7.

\section{Jackson 2011 \{published data only\}}

Jackson LA, Patel SM, Swamy GK, Frey SE, Creech CB, Munoz FM, et al. Immunogenicity of an inactivated monovalent $2009 \mathrm{H} 1 \mathrm{~N} 1$ influenza vaccine in pregnant women. Journal of Infectious Diseases 2011;204(6):854-63.

\section{Janjua 2012 \{published data only\}}

Janjua NZ, Skowronski DM, De Serres G, Dickinson J, Crowcroft NS, Taylor M, et al. Estimates of influenza vaccine effectiveness for 2007-2008 from Canada's sentinel surveillance system: cross-protection against major and minor variants. Journal of Infectious Diseases 2012;205(12):1858-68.

\section{Jianping 1999 \{published data only\}}

Jianping H, Xin F, Changshun L, Bo Z, Linxiu G, Wei X, et al. Assessment of effectiveness of Vaxigrip. Vaccine 1999;17(Suppl 1):57-8.

\section{Jimenez-Jorge 2012 \{published data only\}}

Jimenez-Jorge S, de Mateo S, Pozo F, Casas I, Garcia Cenoz M, Castilla J, et al. Early estimates of the effectiveness of the 2011/12 influenza vaccine in the population targeted for vaccination in Spain, 25 December 2011 to 19 February 2012. Euro Surveillance 2012;17(12):20129.

Keitel 2001 \{published data only\}

Keitel WA, Cate TR, Nino D, Huggins LL, Six HR, Quarles JM, et al. Immunization against influenza: comparison of various topical and parenteral regimens containing inactivated and/or live attenuated vaccines in healthy adults. Journal of Infectious Diseases 2001;183(2):329-32.

Kelly 2012 \{published data only\}

Kelly HA, Sullivan SG, Grant KA, Fielding JE. Moderate influenza vaccine effectiveness with variable effectiveness by match between circulating and vaccine strains in Australian adults aged 20-64 years, 2007-2011. Influenza and Other Respiratory Viruses 2012;7(5):729-37.

\section{Khazeni 2009 \{published data only\}}

Khazeni N, Hutton D, Garber A, Hupert N, Owens D. Effectiveness and cost-effectiveness of vaccination against pandemic influenza (H1N1) 2009. Annals of Internal Medicine 2009;151:829-39.

\section{Kiderman 2001 \{published data only\}}

Kiderman A, Furst A, Stewart B, Greenbaum E, Morag A, Zakay-Rones Z. A double-blind trial of a new inactivated, trivalent, intra-nasal anti-influenza vaccine in general practice: relationship between immunogenicity and respiratory morbidity over the winter of 1997-98. Journal of Clinical Virology 2001;20(3):155-61.

\section{Kim 2012 \{published data only\}}

Kim J-H, Cho H-Y, Hennessey KA, Lee HJ, Bae GR, Kim HC. Adverse events following immunization (AEFI) with the novel influenza $A(H 1 N 1) 2009$ vaccine: findings from the national registry of all vaccine recipients and AEFI and the passive surveillance system in South Korea. Japanese Journal of Infectious Diseases 2012;65(2):99-104.

\section{Kissling 2012 \{published data only\}}

Kissling E, Valenciano M. Early estimates of seasonal influenza vaccine effectiveness in Europe among target groups for vaccination: results from the I-MOVE multicentre case-control study, 2011/12. Euro Surveillance 2012;17(15):20146.

\section{Kunz 1977 \{published data only\}}

Kunz C, Hofmann H, Bachmayer H, Liehl E, Moritz AJ. Clinical trials with a new influenza subunit vaccine in adults and children. Developments in Biological Standardization 1977;39:297-302

\section{Langley 2004 \{published data only\}}

Langley JM, Faughnan ME. Prevention of influenza in the general population. Canadian Medical Association Journal 2004;171(10):1213-22 


\section{Lavallee 2014 \{published data only\}}

Lavallee PC, Labreuche J, Fox KM, Lavados P, Mattle H, Steg PG, et al. Influenza vaccination and cardiovascular risk in patients with recent TIA and stroke. Neurology 2014;82(21):1905-13.

\section{Lee 2011 \{published data only\}}

Lee GM, Greene SK, Weintraub ES, Baggs J, Kulldorff M, Fireman $\mathrm{BH}$, et al. $\mathrm{H} 1 \mathrm{~N} 1$ and seasonal influenza vaccine safety in the Vaccine Safety Datalink project. American Journal of Preventive Medicine 2011;41(2):121-8.

\section{Leeb 2011 \{published data only\}}

Leeb A, Carcione D, Richmond PC, Jacoby P, Effler PV. Reactogenicity of two 2010 trivalent inactivated influenza vaccine formulations in adults. Vaccine 2011;29(45):7920-4.

\section{Leroux-Roels 2010a \{published data only\}}

Leroux-Roels I, Roman F, Forgus S, Maes C, De Boever F, Drame M, et al. Priming with AS03 A-adjuvanted H5N1 influenza vaccine improves the kinetics, magnitude and durability of the immune response after a heterologous booster vaccination: an open non-randomised extension of a double-blind randomised primary study. Vaccine 2010;28(3):849-57.

\section{Leroux-Roels 2010b \{published data only\}}

Leroux-Roels I, Vets E, Freese R, Seiberling M, Weber F, Salamand C, et al. Corrigendum to: "Seasonal influenza vaccine delivered by intradermal microinjection: a randomised controlled safety and immunogenicity trial in adults" by Leroux-Roels et al. [Vaccine 26 (2008) 6614-6619]. Vaccine 2010;28(50):8033.

\section{Liem 1973 \{published data only\}}

Liem KS, Marcus EA, Jacobs J, Strik RV. The protective effect of intranasal immunization with inactivated influenza virus vaccine. Postgraduate Medical Journal 1973;49(569):175-9.

\section{Lind 2014 \{published data only\}}

Lind A, Ramelius A, Olsson T, Arnheim-Dahlstrom L, Lamb F, Khademi M, et al. A/H1N1 antibodies and TRIB2 autoantibodies in narcolepsy patients diagnosed in conjunction with the Pandemrix vaccination campaign in Sweden 2009-2010. Journal of Autoimmunity 2014;50:99-106.

\section{Liu 2012 \{published data only\}}

Liu N, Sprague AE, Yasseen AS, Fell DB, Wen SW, Smith GN, et al. Vaccination patterns in pregnant women during the 2009 H1N1 influenza pandemic: a population-based study in Ontario, Canada. Canadian Journal of Public Health 2012;103(5):e353-8.

Louik 2013 \{published data only\}

Louik C, Chambers C, Jacobs D, Rice F, Johnson D, Mitchell AA. Influenza vaccine safety in pregnancy: can we identify exposures?. Pharmacoepidemiology and Drug Safety 2013;22(1):33-9.

\section{Mackenzie 1975 \{published data only\}}

Mackenzie JS, Mackenzie I, Lloyd J, Dent V. Comparative trials of live attenuated and detergent split influenza virus vaccines. Journal of Hygiene 1975;75(3):425-43.

\section{Mackenzie 2012 \{published data only\}}

Mackenzie IS, Macdonald TM, Shakir S, Dryburgh M, Mantay BJ, Mcdonnell P, et al. Influenza H1N1 (swine flu) vaccination: a safety surveillance feasibility study using self-reporting of serious adverse events and pregnancy outcomes. British Journal of Clinical Pharmacology 2012;73(5):801-11.

Mair 1974 \{published data only\}

Mair HJ, Sansome DAW, Tillett HE. A controlled trial of inactivated monovalent influenza $A$ vaccines in general practice. Journal of Hygiene 1974;73:317-27.

\section{Maynard 1968 \{published data only\}}

Maynard JE, Dull HB, Hanson ML, Feltz ET, Berger R, Hammes L. Evaluation of monovalent and polyvalent influenza vaccines during an epidemic of type $A 2$ and $B$ influenza. American Journal of Epidemiology 1968;87(1):148-57.

McCarthy 2004 \{published data only\} McCarthy MW, Kockler DR. Trivalent intranasal influenza vaccine, live. Annals of Pharmacotherapy 2004;38(12):2086-93.

\section{Mendelman 2001 \{published data only\}}

Mendelman PM, Cordova J, Cho I. Safety, efficacy and effectiveness of the influenza virus vaccine, trivalent, types $A$ and $B$, live, cold-adapted (CAIV-T) in healthy children and healthy adults. Vaccine 2001;19(17-19):2221-6.

\section{Merelli 2000 \{published data only\}}

Merelli E, Casoni F. Prognostic factors in multiple sclerosis: role of intercurrent infections and vaccinations against influenza and hepatitis B. Neurological Sciences 2000;21(4 Suppl 2):853-6.

\section{Meyers 2003a \{published data only\}}

Meyers DG. Could influenza vaccination prevent myocardial infarction, stroke and sudden cardiac death?. American Journal of Cardiovascular Drugs 2003;3(4):241-4.

\section{Meyers 2003b \{published data only\}}

Meyers DG. Myocardial infarction, stroke, and sudden cardiac death may be prevented by influenza vaccination. Current Atherosclerosis Reports 2003;5(2):146-9.

Micheletti 2011 \{published data only\}

Micheletti F, Moretti U, Tridente G, Zanoni G. Consultancy and surveillance of post-immunisation adverse events in the Veneto region of Italy for 1992-2008. Human Vaccines 2011;7(Suppl):234-9.

\section{Monto 2000 \{published data only\}}

Monto AS. Preventing influenza in healthy adults: the evolving story. JAMA 2000;284(13):1699-701.

\section{Montplaisir 2014 \{published data only\}}

Montplaisir J, Petit D, Quinn M-J, Ouakki M, Deceuninck G Desautels $A$, et al. Risk of narcolepsy associated with inactivated adjuvanted (AS03) A/H1N1 (2009) pandemic influenza vaccine in Quebec. PLoS ONE 2014;9(9):e108489. 


\section{Moro 2011 \{published data only\}}

Moro PL, Broder K, Zheteyeva Y, Revzina N, Tepper N, Kissin D, et al. Adverse events following administration to pregnant women of influenza A (H1N1) 2009 monovalent vaccine reported to the Vaccine Adverse Event Reporting System. American Journal of Obstetrics \& Gynecology 2011;205(5):473.e1-9.

\section{Morris 1975 \{published data only\}}

Morris CA, Freestone DS, Stealey VM, Oliver PR. Recombinant WRL 105 strain live attenuated influenza vaccine.

Immunogenicity, reactivity, and transmissibility. Lancet 1975;2(7927):196-9.

\section{Mostow 1977 \{published data only\}}

Mostow SR, Eichkoff TC, Chelgren GA, Retailliau HF, Castle M. Studies of inactivated influenza virus vaccines in hospital employees: reactogenicity and absenteeism. Journal of Infectious Diseases 1977;136 Suppl:S533-8.

\section{Muennig 2001 \{published data only\}}

Muennig PA, Khan K. Cost-effectiveness of vaccination versus treatment of influenza in healthy adolescents and adults. Clinical Infectious Diseases 2001;33(11):1879-85.

\section{Murray 1979 \{published data only\}}

Murray DL, Imagawa DT, Okada DM, St Geme JW Jr. Antibody response to monovalent $A / \mathrm{New}$ Jersey/8/76 influenza vaccine in pregnant women. Journal of Clinical Microbiology 1979;10(2):184-7.

\section{Nazareth 2013 \{published data only\}}

Nazareth I, Tavares F, Rosillon D, Haguinet F, Bauchau V. Safety of AS03-adjuvanted split-virion H1N1 (2009) pandemic influenza vaccine: a prospective cohort study. BMJ Open 2013;3(2):e001912.

\section{Nichol 1996 \{published data only\}}

Nichol KL, Margolis KL, Lind A, Murdoch M, McFadden R, Hauge $M$, et al. Side effects associated with influenza vaccination in healthy working adults. Archives of Internal Medicine 1996;156(14):1546-50.

\section{Nichol 1999b \{published data only\}}

Nichol KL. Clinical effectiveness and cost effectiveness of influenza vaccination among healthy working adults. Vaccine 1999;17(Suppl 1):67-73.

\section{Nichol 2001 \{published data only\}}

Nichol KL. Live attenuated influenza virus vaccines: new options for the prevention of influenza. Vaccine 2001;19(31):4373-7.

\section{Nichol 2003 \{published data only\}}

Nichol KL, Mallon KP, Mendelman PM. Cost benefit of influenza vaccination in healthy, working adults: an economic analysis based on the results of a clinical trial of trivalent live attenuated influenza virus vaccine. Vaccine 2003;21(17-8):2207-17.

\section{Nichol 2004 \{published data only\}}

Nichol KL, Mendelman P. Influence of clinical case definitions with differing levels of sensitivity and specificity on estimates of the relative and absolute health benefits of influenza vaccination among healthy working adults and implications for economic analyses. Virus Research 2004;103(1-2):3-8.

Omon 2011 \{published data only\}

Omon E, Damase-Michel C, Hurault-Delarue C, Lacroix I, Montastruc JL, Oustric S, et al. Non-adjuvanted 2009 influenza A (H1N1)v vaccine in pregnant women: the results of a French prospective descriptive study. Vaccine 2011;29(52):9649-54.

Petrie 2011 \{published data only\}

Petrie JG, Ohmit SE, Johnson E, Cross RT, Monto AS. Efficacy studies of influenza vaccines: effect of end points used and characteristics of vaccine failures. Journal of Infectious Diseases 2011;203(9):1309-15.

Phillips 2013 \{published data only\}

Phillips CJ, Woolpert T, Sevick C, Faix D, Blair PJ, CrumCianflone NF. Comparison of the effectiveness of trivalent inactivated influenza vaccine and live, attenuated influenza vaccine in preventing influenza-like illness among US military service members, 2006-2009. Clinical Infectious Diseases 2013;56(1):11-9.

\section{Phonrat 2013 \{published data only\}}

Phonrat B, Pitisuttithum P, Chamnanchanunt S, Puthavathana P, Ngaosuwankul N, Louisirirotchanakul S, et al. Safety and immune responses following administration of $\mathrm{H} 1 \mathrm{~N} 1$ live attenuated influenza vaccine in Thais. Vaccine 2013;31(11):1503-9.

\section{Pleguezuelos 2012 \{published data only\}}

Pleguezuelos O, Robinson S, Stoloff GA, Caparrós-Wanderley W. Synthetic influenza vaccine (FLU-v) stimulates cell mediated immunity in a double-blind, randomised, placebo-controlled Phase I trial. Vaccine 2012;30(31):4655-60.

Puig-Barbera 2012 \{published data only\}

Puig-Barbera J, Diez-Domingo J, Arnedo-Pena A, Ruiz-Garcia M, Perez-Vilar S, Mico-Esparza JL, et al. Effectiveness of the 2010-2011 seasonal influenza vaccine in preventing confirmed influenza hospitalizations in adults: a case-case comparison, case-control study. Vaccine 2012;30(39):5714-20.

\section{Puleston 2010 \{published data only\}}

Puleston RL, Bugg G, Hoschler K, Konje J, Thornton J, Stephenson I, et al. Observational study to investigate vertically acquired passive immunity in babies of mothers vaccinated against H1N1v during pregnancy. Health Technology Assessment 2010;14(55):1-82.

Pyhala 2001 \{published data only\}

Pyhala R, Haanpaa M, Kleemola M, Tervahauta R, Visakorpi R, Kinnunen L. Acceptable protective efficacy of influenza vaccination in young military conscripts under circumstances of incomplete antigenic and genetic match. Vaccine 2001;19(23-4):3253-60.

Reynales 2012 \{published data only\}

Reynales H, Astudillo P, de Valliere S, Hatz C, Schlagenhauf P, Rath $B$, et al. A prospective observational safety study on MF59 
adjuvanted cell culture-derived vaccine, Celtura during the $A$ / H1N1 (2009) influenza pandemic. Vaccine 2012;30(45):6436-43.

\section{Rimmelzwaan 2000 \{published data only\}}

Rimmelzwaan GF, Nieuwkoop N, Brandenburg A, Sutter G, Beyer WE, Maher D, et al. A randomized, double blind study in young healthy adults comparing cell mediated and humoral immune responses induced by influenza ISCOM vaccines and conventional vaccines. Vaccine 2000;19(9-10):1180-7.

\section{Rocchi 1979c \{published data only\}}

Rocchi G, Carlizza L, Andreoni M, Ragona G, Piga C, Pelosio A, et al. Protection from natural infection after live influenza virus immunization in an open population. Journal of Hygiene 1979;82(2):231-6.

\section{Rowhani-Rahbar 2012 \{published data only\}}

Rowhani-Rahbar A, Klein NP, Lewis N, Fireman B, Ray P, Rasgon $\mathrm{B}$, et al. Immunization and Bell's palsy in children: a case-centered analysis. American Journal of Epidemiology 2012;175(9):878-85

\section{Ruben 1972 \{published data only\}}

Ruben FL, Jackson GG. A new subunit influenza vaccine: acceptability compared with standard vaccines and effect of dose on antigenicity. Journal of Infectious Diseases 1972;125(6):656-64.

\section{Ruben 1973 \{published data only\}}

Ruben FL, Akers LW, Stanley ED, Jackson GG. Protection with split and whole virus vaccines against influenza. Archives of Internal Medicine 1973;132(4):568-71.

\section{Safranek 1991 \{published data only\}}

Safranek TJ, Lawrence DN, Kurland LT, Culver DH, Wiederholt WC, Hayner NS, et al. Reassessment of the association between Guillain-Barre syndrome and receipt of swine influenza vaccine in 1976-1977: results of a twostate study. Expert Neurology Group. American Journal of Epidemiology 1991;133(9):940-51.

\section{Sarateanu 1980 \{published data only\}}

Sarateanu DE, Ehrengut W, Pressler K, Peukert M, Schenk KD. Serological response to whole, split and subunit influenza vaccines of persons with and without immunological experience towards influenza A/U.S.S.R. $90 / 77$ virus. Comparative Immunology, Microbiology and Infectious Diseases 1980;3(1-2):225-36.

\section{Scheifele 2013 \{published data only\}}

Scheifele DW, Dionne M, Ward BJ, Cooper C, Vanderkooi OG, Li Y, et al. Safety and immunogenicity of 2010-2011 A/H1N1pdm09containing trivalent inactivated influenza vaccine in adults previously given AS03-adjuvanted H1N1 2009 pandemic vaccine: results of a randomised trial. Human Vaccines and Immunotherapy 2013;9(1):136-43.

\section{Schonberger 1981 \{published data only\}}

Schonberger LB, Hurwitz ES, Katona P, Holman RC, Bregman DJ. Guillain-Barré syndrome: its epidemiology and associations with influenza vaccination. Annals of Neurology 1981;9(Suppl):31-8.

Schwartz 1996 \{published data only\}

Schwartz K. Influenza vaccine for healthy adults. Journal of Family Practice 1996;42(4):351-2.

\section{Simpson 2012 \{published data only\}}

Simpson CR, Ritchie LD, Robertson C, Sheikh A, McMenamin J. Effectiveness of $\mathrm{H} 1 \mathrm{~N} 1$ vaccine for the prevention of pandemic influenza in Scotland, UK: a retrospective observational cohort study. Lancet Infectious Diseases 2012;12(9):696-702.

Sipilä 2015 \{published data only\}

Sipilä JO, Soilu-Hanninen M. The incidence and triggers of adult-onset Guillain-Barre syndrome in southwestern Finland 2004-2013. European Journal of Neurology 2015;22 (2):292-8.

\section{Skowronski 2002 \{published data only\}}

Skowronski DM, Strauss B, Kendall P, Duval B, De Serres G. Low risk of recurrence of oculorespiratory syndrome following influenza revaccination. Canadian Medical Association Journal 2002;167(8):853-8.

\section{Skowronski 2003 \{published data only\}}

Skowronski DM, De Serres G, Scheifele D, Russell ML, Warrington R, Davies HD, et al. Randomized, double-blind, placebo-controlled trial to assess the rate of recurrence of oculorespiratory syndrome following influenza vaccination among persons previously affected. Clinical Infectious Diseases 2003;37(8):1059-66.

\section{Smith 1977a \{published data only\}}

Smith AJ, Davies JR. The response to inactivated influenza A (H3N2) vaccines: the development and effect of antibodies to the surface antigens. Journal of Hygiene 1977;78:363-75.

\section{Smith 1977b \{published data only\}}

Smith CD, Leighton HA, Shiromoto RS. Antigenicity and reactivity of influenza $A / \mathrm{New}$ Jersey/8/76 virus vaccines in military volunteers at Fort Ord, California. Journal of Infectious Diseases 1977;136(Suppl):460-5.

\section{Song 2011 \{published data only\}}

Song JY, Cheong HJ, Woo HJ, Wie SH, Lee JS, Chung MH, et al. Immunogenicity and safety of trivalent inactivated influenza vaccine: a randomised, double-blind, multi-center, phase 3 clinical trial in a vaccine-limited country. Journal of Korean Medical Science 2011;26(2):191-5.

\section{Souayah 2011 \{published data only\}}

Souayah N, Michas-Martin PA, Nasar A, Krivitskaya N, Yacoub HA, Khan H, et al. Guillain-Barré syndrome after Gardasil vaccination: data from Vaccine Adverse Event Reporting System 2006-2009. Vaccine 2011;29(5):886-9.

\section{Spencer 1975 \{published data only\}}

Spencer MJ, Cherry JD, Powell KR, Sumaya CV, Garakian AJ. Clinical trials with Alice strain, live attenuated, serum inhibitorresistant intranasal influenza A vaccine. Journal of Infectious Diseases 1975;132(4):415-20. 
Spencer 1979 \{published data only\}

Spencer MJ, Cherry JD, Powell KR, Sumaya CV. A clinical trial with Alice/R-75 strain, live attenuated serum inhibitor-resistant intranasal bivalent influenza A/B vaccine. Medical Microbiology and Immunology 1979;167(1):1-9.

Steinhoff 2012 \{published data only\}

Steinhoff MC, Omer SB, Roy E, El Arifeen S, Raqib R, Dodd C, et al. Neonatal outcomes after influenza immunization during pregnancy: a randomised controlled trial. Canadian Medical Association Journal 2012;184(6):645-53.

\section{Sumaya 1979 \{published data only\}}

Sumaya CV, Gibbs RS. Immunization of pregnant women with influenza $A / N e w$ Jersey/76 virus vaccine: reactogenicity and immunogenicity in mother and infant. Journal of Infectious Diseases 1979;140(2):141-6.

Talaat 2010 \{published data only\}

Talaat KR, Greenberg ME, Lai MH, Hartel GF, Wichems CH, Rockman S, et al. A single dose of unadjuvanted novel $2009 \mathrm{H} 1 \mathrm{~N} 1$ vaccine is immunogenic and well tolerated in young and elderly adults. Journal of Infectious Diseases 2010;202(9):1327-37.

\section{Tavares 2011 \{published data only\}}

Tavares F, Nazareth I, Monegal JS, Kolte I, Verstraeten T, Bauchau V. Pregnancy and safety outcomes in women vaccinated with an AS03-adjuvanted split virion H1N1 (2009) pandemic influenza vaccine during pregnancy: a prospective cohort study. Vaccine 2011;29(37):6358-65.

\section{Taylor 1969 \{published data only\}}

Taylor PJ, Miller CL, Pollock TM, Perkins FT, Westwood MA. Antibody response and reactions to aqueous influenza vaccine, simple emulsion vaccine and multiple emulsion vaccine. $A$ report to the Medical Research Council Committee on influenza and other respiratory virus vaccines. Journal of Hygiene 1969;67(3):485-90.

\section{Taylor 2012 \{published data only\}}

Taylor DN, Treanor JJ, Sheldon EA, Johnson C, Umlauf S, Song $\mathrm{L}$, et al. Development of VAX128, a recombinant hemagglutinin $(\mathrm{HA})$ influenza-flagellin fusion vaccine with improved safety and immune response. Vaccine 2012;30(39):5761-9.

\section{Thompson 2014 \{published data only\}}

Thompson MG, Li DK, Shifflett P, Sokolow LZ, Ferber JR, Kurosky S, et al. Effectiveness of seasonal trivalent influenza vaccine for preventing influenza virus illness among pregnant women: a population-based case-control study during the 2010-2011 and 2011-2012 influenza seasons. Clinical Infectious Diseases 2014;58(4):449-57.

\section{Tokars 2012 \{published data only\}}

Tokars JI, Lewis P, Destefano F, Wise M, Viray M, Morgan O, et al. The risk of Guillain-Barré syndrome associated with influenza a (H1N1) 2009 monovalent vaccine and 2009-2010 seasonal influenza vaccines: results from self-controlled analyses. Pharmacoepidemiology and Drug Safety 2012;21(5):546-52.

\section{Treanor 2001 \{published data only\}}

Treanor JJ, Wilkinson BE, Masseoud F, Hu-Primmer J, Battaglia R, O'Brien D, et al. Safety and immunogenicity of a recombinant hemagglutinin vaccine for $\mathrm{H} 5$ influenza in humans. Vaccine 2001;19(13-4):1732-7.

\section{Treanor 2002 \{published data only\}}

Treanor J, Keitel W, Belshe R, Campbell J, Schiff G, Zangwill K, et al. Evaluation of a single dose of half strength inactivated influenza vaccine in healthy adults. Vaccine 2002;20(7-8):1099-105.

\section{Treanor 2012 \{published data only\}}

Treanor JJ, Talbot HK, Ohmit SE, Coleman LA, Thompson MG, Cheng PY, et al. Effectiveness of seasonal influenza vaccines in the United States during a season with circulation of all three vaccine strains. Clinical Infectious Diseases 2012;55(7):951-9.

\section{Tsai 2010 \{published data only\}}

Tsai T, Kyaw MH, Novicki D, Nacci P, Rai S, Clemens R. Exposure to MF59-adjuvanted influenza vaccines during pregnancy - a retrospective analysis. Vaccine 2010;28(7):1877-80.

Tsatsaris 2011 \{published data only\}

Tsatsaris V, Capitant C, Schmitz T, Chazallon C, Bulifon S, Riethmuller $\mathrm{D}$, et al. Maternal immune response and neonatal seroprotection from a single dose of a monovalent nonadjuvanted 2009 influenza $A(\mathrm{H} 1 \mathrm{~N} 1)$ vaccine: a single-group trial. Annals of Internal Medicine 2011;155(11):733-41.

\section{Tyrrell 1970 \{published data only\}}

Tyrrell DA, Buckland R, Rubenstein D, Sharpe DM. Vaccination against Hong Kong influenza in Britain, 1968-9. A report to the Medical Research Council Committee on Influenza and other Respiratory Virus Vaccines. Journal of Hygiene 1970;68(3):359-68.

\section{Vesikari 2012 \{published data only\}}

Vesikari T, Forstén A, Herbinger KH, Cioppa GD, Beygo J, Borkowski A, et al. Safety and immunogenicity of an MF59adjuvanted $\mathrm{A} / \mathrm{H} 5 \mathrm{~N} 1$ pre-pandemic influenza vaccine in adults and the elderly. Vaccine 2012;30(7):1388-96.

\section{Warren-Gash 2013 \{published data only\}}

Warren-Gash C, Geretti AM, Hamilton G, Rakhit RD, Smeeth L, Hayward AC. Influenza-like illness in acute myocardial infarction patients during the winter wave of the influenza $A$ H1N1 pandemic in London: a case-control study. BMJ Open 2013;3(5):e002604.

\section{Warshauer 1976 \{published data only\}}

Warshauer DM, Minor TE, Inhorn SL, Reed CE, Dick EC. Use of an inhibitor-resistant live attenuated influenza vaccine in normal and asthmatic adults. 14th Congress of the International Association of Biological Standardization, Douglas, Isle of Man 1975. Developments in Biological Standardization 1975;33:184-90. 
Wilde 1999 \{published data only\}

Wilde JA, McMillan JA, Serwint J, Butta J, O'Riordan MA, Steinhoff MC. Effectiveness of influenza vaccine in health care professionals. JAMA 1999;281(10):908-13.

\section{Williams 1973 \{published data only\}}

Williams MC, Davignon L, McDonald JC, Pavilanis PV, Boudreault A, Clayton AJ. Trial of aqueous killed influenza vaccine in Canada, 1968-69. WHO Bulletin 1973;49:333-40.

\section{Williams 2011 \{published data only\}}

Williams SE, Pahud BA, Vellozzi C, Donofrio PD, Dekker CL, Halsey N, et al. Causality assessment of serious neurologic adverse events following $2009 \mathrm{H} 1 \mathrm{~N} 1$ vaccination. Vaccine 2011;29(46):8302-8.

\section{Wise 2012 \{published data only\}}

Wise ME, Viray M, Sejvar JJ, Lewis P, Baughman AL, Connor W, et al. Guillain-Barre syndrome during the 2009-2010 H1N1 influenza vaccination campaign: population-based surveillance among 45 million Americans. American Journal of Epidemiology 2012;175(11):1110-9.

\section{Wood 1999 \{published data only\}}

Wood SC, Alexseiv A, Nguyen VH. Effectiveness and economical impact of vaccination against influenza among a working population in Moscow. Vaccine 1999;17(Suppl 3):81-7.

\section{Wood 2000 \{published data only\}}

Wood SC, Nguyen VH, Schmidt C. Economic evaluations of influenza vaccination in healthy working-age adults. Employer and society perspective. Pharmacoeconomics 2000;18(2):173-83.

\section{Xu 2012 \{published data only\}}

Xu R, Luo Y, Chambers C. Assessing the effect of vaccine on spontaneous abortion using time-dependent covariates Cox models. Pharmacoepidemiology and Drug Safety 2012;21(8):844-50.

\section{Yang 2012 \{published data only\}}

Yang Z, Wang S, Li W, Li C, Dong J, Li F, et al. The long-term immunogenicity of an inactivated split-virion 2009 pandemic influenza A H1N1 vaccine: randomized, observer-masked, single-center clinical study. Results in Immunology 2012;2:184-9.

\section{Yeager 1999 \{published data only\}}

Yeager DP, Toy EC, Baker B 3rd. Influenza vaccination in pregnancy. American Journal of Perinatology 1999;16(6):283-6.

\section{Yih 2012 \{published data only\}}

Yih WK, Lee GM, Lieu TA, Ball R, Kulldorff M, Rett M, et al. Surveillance for adverse events following receipt of pandemic 2009 H1N1 vaccine in the Post-Licensure Rapid Immunization Safety Monitoring (PRISM) System, 2009-2010. American Journal of Epidemiology 2012;175(11):1120-8.

\section{Zaman 2008 \{published data only\}}

Zaman K, Roy E, Arifeen SE, Rahman M, Raqib R, Wilson E, et al. Effectiveness of maternal influenza immunization in mothers and infants. New England Journal of Medicine 2008;359(15):1555-64.

\section{Additional references}

\section{ACIP 2006}

Centers for Disease Control and Prevention. Prevention and control of influenza: recommendations of the Advisory Committee on Immunization Practices (ACIP). Recommendations and Reports: Morbidity and Mortality Weekly Report 2006;55:1-41.

\section{ACIP 2010}

Fiore AE, Uyeki TM, Broder K, Finelli L, Euler GL, Singleton JA, et al. Prevention and control of influenza with vaccines: recommendations of the Advisory Committee on Immunization Practices (ACIP), 2010. Morbidity and Mortality Weekly Report 2010;59(RR-8):1-62.

\section{ACIP 2015}

Advisory Committee on Immunization Practices. Prevention and control of influenza with vaccines: recommendations of the Advisory Committee on Immunization Practices, United States, 2015-16 influenza season. Morbidity and Mortality Weekly Report 2015;64(30):818-25.

\section{AlH 2013}

Australian Technical Advisory Group on Immunisation (ATAGI). The Australian Immunisation Handbook. 10th Edition. Australian Government: National Health and Medical Research Council, 2013.

\section{Atkins 2004}

Atkins D, Best D, Briss PA, Eccles M, Falck-Ytter Y, Flottorp S, et al. GRADE Working Group. Grading quality of evidence and strength of recommendations. BMJ 2004;328(7454):1490.

\section{Demicheli 2014}

Demicheli V, Jefferson T, Al-Ansary LA, Ferroni E, Rivetti A, Di Pietrantonj $C$. Vaccines for preventing influenza in healthy adults. Cochrane Database of Systematic Reviews 2014, Issue 3. [DOI: 10.1002/14651858.CD001269.pub5]

\section{DiazGranados 2012}

DiazGranados CA, Denis M, Plotkin S. Seasonal influenza vaccine efficacy and its determinants in children and non-elderly adults: a systematic review with meta-analyses of controlled trials. Vaccine 2012;31(1):49-57.

\section{DoH 2015}

Department of Health, UK. Influenza. Immunisation Against Infectious Diseases: The Green Book. Vol. 19, Department of Health, UK, 2015:18-9.

\section{EMA 2014}

Committee for Medicinal Products for Human Use. Guideline on influenza vaccines. Non-clinical and clinical module. European Medicines Agency, 2014, EMA/CHMP/VWP/457259/2014. [web:: http://www.ema.europa.eu/docs/en_GB/document_library/ Scientific_guideline/2016/07/WC500211324.pdf] 


\section{Farez 2011}

Farez MF, Correale J. Immunizations and risk of multiple sclerosis: systematic review and meta-analysis. Journal of Neurology 2011;258(7):1197-206.

\section{GRADEpro GDT 2014 [Computer program]}

GRADE Working Group, McMaster University. GRADEpro GDT. Hamilton (ON): GRADE Working Group, McMaster University, 2014.

\section{Grohskopf 2016}

Grohskopf LA, Sokolow LZ, Broder KR, Olsen SJ, Karron RA, Jernigan DB, et al. Prevention and control of seasonal influenza with vaccines. Morbidity and Mortality Weekly Report 2016;65(5):1-54. [DOI: 10.15585/mmwr.rr6505a1]

\section{Higgins 2011}

Higgins JP, Green S (editors). Cochrane Handbook for Systematic Reviews of Interventions Version 5.1.0 (updated March 2011). The Cochrane Collaboration, 2011. Available from handbook.cochrane.org.

\section{Jefferson 2009a}

Jefferson TO. Mistaken identity: seasonal influenza versus influenza-like illness. Clinical Evidence 2009;329:1-4.

\section{Jefferson 2009b}

Jefferson T, Di Pietrantonj C, Debalini MG, Rivetti A, Demicheli V. Relation of study quality, concordance, take home message, funding, and impact in studies of influenza vaccines: systematic review. BMJ 2009;338:b354. [DOI: 10.1136/bmj.b354]

\section{Jefferson 2012}

Jefferson T, Rivetti A, Di Pietrantonj C, Demicheli V, Ferroni E. Vaccines for preventing influenza in healthy children. Cochrane Database of Systematic Reviews 2012, Issue 8. [DOI: 10.1002/14651858.CD004879.pub4]

\section{Jones 2016}

Jones M, Fowler R. Immortal time bias in observational studies of time to event outcomes. Journal of Critical Care 2016;36:195-9.

\section{Kissling 2009a}

Kissling E, Moren A, Valenciano M. Protocol for case-control studies to measure pandemic and seasonal influenza vaccine effectiveness in the European Union and European Economic Area Member States. ECDC Technical Document. Paris: European Centre for Disease Prevention and Control, 2009. [web: http://ecdc.europa.eu/en/publications/ Publications/0907_TED_Influenza_AH1N1_Measuring Influenza_Vaccine_Effectiveness_Protocol_Case_Control_ Studies.pdf last accessed on May 25th, 2017]

\section{Kissling 2009b}

Kissling E, Moren A, Valenciano M. Protocol for cohort database studies to measure pandemic and seasonal influenza vaccine effectiveness in the European Union and European Economic Area Member States. ECDC Technical Document. Paris: European Centre for Disease Prevention and Control, 2009. [web: http://ecdc.europa.eu/en/publications/
Publications/0907_TER_Influenza_AH1N1_Measuring _ Influenza_Vaccine_Effectiveness_Protocol_Cohort_Database_ Studies.pdf Last accessed on May 25th, 2017]

\section{Moher 2009}

Moher D, Liberati A, Tetzlaff J, Altman DG, The PRISMA Group. Preferred reporting items for systematic reviews and metaanalyses: The PRISMA Statement. BMJ 2009;339:2535.

\section{NACI 2014}

National Advisory Committee on Immunization (NACI). An Advisory Committee Statement (ACS) National Advisory Commitee on Immunization: statement on seasonal influenza vaccine for 2014-2015. Canada Communicable Diseases Report, 2014. http:/www.phac-aspc.gcca/naci-ccni/assets/pdf/flugrippe-eng.pdf (accessed September 3, 2015).

\section{Osterholm 2012}

Osterholm MT, Kelley NS, Sommer A, Belongia EA. Efficacy and effectiveness of influenza vaccines: a systematic review and meta-analysis. Lancet Infectious Diseases 2012;12:36-44.

\section{RevMan 2014 [Computer program]}

Nordic Cochrane Centre, The Cochrane Collaboration. Review Manager 5 (RevMan 5). Version 5.3. Copenhagen: Nordic Cochrane Centre, The Cochrane Collaboration, 2014.

\section{Skowronski 2009}

Skowronski DM, De Serres G. Is routine immunisation warranted in early pregnancy?. Vaccine 2009;27:4754-70.

\section{STIKO 2010}

Ständigen Impfkommission (STIKO) am Robert Koch-Institut. Recommendations update for immunisation against influenza [Änderung der Empfehlungen zur Impfung gegen Influenza]. Epidemiologisches Bulletin 2010;31:299-308.

\section{Toback 2012}

Toback SL, Levin MJ, Block SL, Belshe RB, Ambrose CS, Falloon J. Quadrivalent Ann Arbor strain liveattenuated influenza vaccine. Expert Reviews Vaccines 2012;11(11):1293-303.

\section{Treanor 2016}

Treanor JJ. Influenza vaccination. New England Journal of Medicine 2016;375(13):1261-8. [DOI: 10.1056/NEJMcp1512870]

\section{Wijnans 2016}

Wijnans $L$, Voordouw B. A review of the changes to the licensing of influenza vaccines in Europe. Viruses 2016;10(1):2-8.

\section{Wiselka 1994}

Wiselka M. Influenza: diagnosis, management and prophylaxis. BMJ 1994;308:1341-5. 


\section{References to other published versions of this review}

\section{Demicheli 1998}

Demicheli V, Jefferson T. Influenza vaccines in healthy adults. Cochrane Database of Systematic Reviews 1998, Issue 4. [DOI: 10.1002/14651858.CD001269]

\section{Demicheli 1999}

Demicheli V, Rivetti D, Deeks JJ, Jefferson TO. Vaccines for preventing influenza in healthy adults. Cochrane Database of Systematic Reviews 1999, Issue 4. [DOI: 10.1002/14651858.CD001269]

\section{Demicheli 2001}

Demicheli V, Rivetti D, Deeks JJ, Jefferson TO. Vaccines for preventing influenza in healthy adults. Cochrane Database of Systematic Reviews 2001, Issue 4. [DOI: 10.1002/14651858.CD001269]

\section{Demicheli 2004}

Demicheli V, Rivetti D, Deeks JJ, Jefferson TO. Vaccines for preventing influenza in healthy adults. Cochrane

\section{CHARACTERISTICS OF STUDIES}

Characteristics of included studies [ordered by study ID]
Database of Systematic Reviews 2004, Issue 3. [DOI: 10.1002/14651858.CD001269.pub2]

\author{
Jefferson 2007 \\ Jefferson TO, Rivetti D, Di Pietrantonj C, Rivetti A, Demicheli V. \\ Vaccines for preventing influenza in healthy adults. \\ Cochrane Database of Systematic Reviews 2007, Issue 2. [DOI: \\ 10.1002/14651858.CD001269.pub3]
}

\section{Jefferson 2010 \\ Jefferson TO, Di Pietrantonj C, Rivetti A, Bawazeer GA, Al- Ansary LA, Feroni E. Vaccines for preventing influenza in healthy adults. Cochrane Database of Systematic Reviews 2010, Issue 7. [DOI: 10.1002/14651858.CD001269.pub4] \\ Jefferson 2014 \\ Jefferson T, Di Pietrantonj C, Rivetti A, Bawazeer GA, Al- Ansary LA, Ferroni E. Vaccines for preventing influenza in healthy adults. Cochrane Database of Systematic Reviews 2014, Issue 3. [DOI: 10.1002/14651858.CD001269.pub5]}

* Indicates the major publication for the study

aa Barrett 2011

Dethods $\begin{aligned} & \text { Double-blind, placebo-controlled, multicentric RCT performed at } 36 \text { centres in the USA assessing effec- } \\ & \text { tiveness, reactogenicity, and antibodies responses of a Vero cell-derived, trivalent, split influenza vac- } \\ & \text { cine }\end{aligned}$

Participants Healthy adults aged 18 to 48 years recruited at 36 centres throughout the USA.

Individuals were excluded if they belonged to a CDC risk category for complications of influenza illness, had a history of surgical or functional asplenia, had been treated with any blood product or immune globulin in the previous 90 days, had a history of allergy to vaccine components, had received a live vaccine within 4 weeks or an inactivated vaccine within 2 weeks of study entry, or had dermatological disorders or tattoos that would obscure the assessment of injection-site reactions. Individuals were not specifically excluded because of egg allergy. Immunisation in previous seasons was not judged to be an exclusion criterion.

Interventions

Inactivated, Vero cell-derived, trivalent split influenza vaccine containing $15 \mu \mathrm{g}$ haemagglutinin of the following strains, which were recommended by WHO for the season 2008 to 2009 in the Northern Hemisphere:

\section{A-H1N1: A/Brisbane/59/2007}

A-H3N2: A/Uruguay/716/2007 (A/Brisbane/10/2007-like) (A/H3N2)

B: B/Florida/4/2006

The vaccine was manufactured by Baxter AG, Vienna. Vaccine strains were egg-derived wild type strains provided by the National Institute for Biological Standard and Control. Placebo consisted of phosphate-buffered saline.

Participants were randomly allocated to receive one $0.5 \mathrm{~mL}$ dose of either vaccine or placebo into the deltoid muscle. Vaccinations were performed between 1 and 15 December 2008. 
aa Barrett 2011 (Continued)

Outcomes
Safety: participants were provided with a diary card, on which they had to record their temperature daily for the first 7 days following immunisation and to report fever and other adverse events for 21 days after immunisation. Participants returned for a final study visit 166 to 194 days after vaccination for a physical examination and final assessment of adverse events.

Serological: the first serum samples were presumably collected before vaccine administration (this is not well described in any of the 3 reports), and the second 18 to 24 days later. Haemagglutination-inhibiting titres and GMT against vaccine strains were assessed by Focus Diagnostics (Cypress, CA, USA). Haemagglutination-inhibiting assays were done in triplicate with egg-derived antigen. Titres of less than $1: 10$ were expressed as $1: 5$ and judged to be negative.

Effectiveness: during the visit at days 18 to 24 after immunisation, participants were instructed to return to the clinic within 48 hours after the onset of symptoms of an influenza-like illness, should they have fever with cough, sore throat, muscle ache, headache, fatigue, nausea, or bloodshot eyes, or any 2 of these symptoms without fever. At every visit for an influenza-like illness until 15 May 2009, nasopharyngeal swabs were obtained for culturing and typing viruses.

Nasopharyngeal swab specimens were sent to BioAnalytical Research (Lake Success, NY, USA), for culture using Rapid R-Mix (Diagnostic Hybrids, Athens, $\mathrm{OH}, \mathrm{USA}$ ) and traditional culture methods, and for virus typing with RT-PCR analyses. Influenza type A/H1N1 or A/H3N2 isolates were sent to the laboratory of the Influenza Division, National Center for Immunization and Respiratory Diseases, CDC, Atlanta, GA, USA, for analyses of $\mathrm{HI}$ using ferret antiserum to assess the antigenic relatedness of the isolate to the vaccine strains.

Notes Industry funded

\section{Risk of bias}

\begin{tabular}{lll} 
Bias & Authors' judgement & Support for judgement \\
\hline $\begin{array}{ll}\text { Random sequence genera- } \\
\text { tion (selection bias) }\end{array}$ & Low risk & $\begin{array}{l}\text { "Individuals were randomly assigned by use of a centralised telephone sys- } \\
\text { tem" }\end{array}$
\end{tabular}

"Randomisation was done in blocks, with block sizes greater than two"

\begin{tabular}{ll}
\hline $\begin{array}{l}\text { Allocation concealment } \\
\text { (selection bias) }\end{array}$ & Low risk \\
& $\begin{array}{l}\text { "The allocation sequence was generated by Baxter, using an interactive voice } \\
\text { response system with the random number generator algorithm of Wichmann } \\
\text { and Hill, as modified by Mcleod" }\end{array}$
\end{tabular}

Blinding (performance Low risk bias and detection bias) All outcomes

\begin{abstract}
"At each study site, an investigator, subinvestigator, or study nurse who was masked to treatment allocation was designated to vaccinate participants, and was then prohibited from participation in data collection or the study. To ensure masking, the participants were enrolled by investigators who were not involved in the randomisation process.
\end{abstract}

Because the syringes containing the test and the control products were different in appearance both studies employed an observational blinding procedure such that study personnel who administered vaccinations were not involved in recording or reviewing study data"

Both efficacy and safety estimates were calculated on ITT study population. We know that all treated participants (3623 to influenza vaccine and 3620 to placebo) had been included in the safety analysis, whereas 3619 and 3617 had been considered for the effectiveness estimate calculation (i.e. those vaccinated and with at least 21 days' follow-up after immunisation). Participants in the per-protocol population (those who completed the study without major protocol deviations) were 3316 and 3318 in the vaccine and placebo arms, respectively. 
aa Barrett 2011 (Continued)

Reasons for non-inclusion in the per-protocol population were not specified for 150 vaccine and 135 placebo recipients.

\begin{tabular}{ll}
\hline Summary assessment Low risk $\quad$ Low risk of bias \\
\hline
\end{tabular}

\section{aa Beran 2009a}

Randomised, double-blind, placebo-controlled study conducted in the Czech Republic during the 2005
to 2006 influenza season. This was defined retrospectively as starting the first week with 2 culture-con-
firmed cases in the study area and ending the last week with 1 culture-confirmed case in the study area.
Randomisation was generated by GSK (sponsor) using the SAS program, in a 2:1 blocking scheme us-
ing a minimisation procedure (with no explanation of why such a method or the ratio was used). The
allocation concealment method was not explicitly mentioned. However, the authors mentioned that
placebo and vaccine treatments were indistinguishable in appearance and that blinding to treatment
assignment was maintained until study analysis.

Participants Self referred healthy adults $(n=6203)$, predominately Caucasian (understood to be white) $(99.8 \%)$, aged between 18 and 64 years (mean $35+13$ years) of both genders (TIV group: female $55.3 \%$, placebo group: female 54.2\%) and with no history of influenza vaccination within the last 3 influenza seasons. A subset of participants who were randomly selected for vaccine safety and reactogenicity were given a calibrated thermometer and a diary card to record symptoms. The method of selection of this subset was not explained. Use of antimicrobial/influenza antiviral therapy seemed to be allowed but was not quantified.

Interventions TIV vaccine: $0.5 \mathrm{~mL}$ single dose by IM injection or placebo (normal saline). Use of more than 1 lot was not reported.

TIV contained haemagglutinin antigens of:

- A/New Caledonia/20/99 (H1N1) IVR-116 virus as an A/New Caledonia/20/99-like strain;

- $\mathrm{A} /$ New York/55/2004 (H3N2) X-157 virus as an A/California/7/2004-like strain;

- B/Jiangsu/10/2003 virus as a B/Shanghai/361/2002-like strain.

2 modes of surveillance were used.

- Passive: started on the day of vaccination, participants self report of ILI symptoms through a toll-free number.

- Active: started 2 weeks after vaccination day: a biweekly telephone contact of the participants by someone (not clear who) for ILI symptoms.

- It is not clear if the surveillance included the entire cohort or just a subset, or why the authors carried out harms surveillance using the 2 surveillance methods already in place.

\section{Serological}

Blood samples were collected for the specified subset and were tested/analysed at GSK Biologicals SSW Dresden, Germany.

Blood sample obtained prior to vaccination and at 21 days following vaccination. Serum samples were stored at $-20^{\circ} \mathrm{C}$ until blinded analyses were conducted.

A haemagglutination-inhibition test was done using chicken red blood cells with the 3 virus strains present in the TIV used as antigens. The serum titre was expressed as the reciprocal of the highest dilution that showed complete inhibition of haemagglutination.

Serology was not a primary outcome in this study.

\section{Effectiveness}

Incidence of culture-confirmed ILI (primary outcome, reported as the attack rate in the efficacy cohort) 
Nasal and throat swab collected by a nurse on the same day.

Swab samples were stored at $28^{\circ} \mathrm{C}$ and transferred within 5 days of the onset of ILI symptoms.

Sample sent to the National Reference Laboratory for Influenza (NRL, Prague, Czech Republic) for conventional influenza virus culture using MDCK cells.

Confirmation of influenza A or B was determined using the following:

- haemagglutination assay with turkey and guinea pig erythrocytes;

- haemagglutination inhibition to identify virus type, subtype, and drift variant;

- direct immunoperoxidase assay using anti-influenza $A$ and anti-influenza B nucleoprotein antibodies.

There were 814 reported ILI episodes, only 46 gave positive culture.

\section{Clinical}

Incidence of ILI symptoms (secondary outcome, reported as attack rate in the ATP cohort)

Influenza-like illness was defined as fever (oral temperature greater or equal to $37.8^{\circ} \mathrm{C}$ ) plus cough and/ or sore throat. An ILI episode was defined as the period from the first day of ILI symptoms until the last day of ILI symptoms. A new episode was taken into account only after the complete resolution of the previous one. To count as a separate episode at least 7 days free of any symptoms should pass.

Number of events was 370 reported events (254 in TIV and 120 in placebo).

Number of participants reporting at least 1 event (240 in TIV and 113 in placebo) was used to calculate the attack rate.

Reasons to exclude from the ATP cohort included:

- protocol violation (inclusion/exclusion criteria): seems that the selected subset have certain criteria but not mentioned by the authors;

- underlying medical condition: not specified what? Or why not excluded from the efficacy cohort as well since participants are reported to be healthy;

- forbidden by the protocol: protocol not clear;

- participants not exposed during the influenza season: unclear what this means (did the participant travel after getting the study treatment?).

\section{Immunogenicity}

Blood sample obtained prior to vaccination and at 21 days following vaccination. Performed only for a subset of participants, not all efficacy cohort.

\section{Safety}

Data on SAEs began at the receipt of vaccine/placebo and continued until the end of the study. However, safety was solicited from a subset of participants (no mention of method used to randomly select them, no justification for not collecting SAEs from all participants, especially with the presence of 2 surveillance methods).

\section{Reactogenicity}

Defined as the presence and intensity of the following symptoms within 4 days of vaccination: pain, redness, and swelling (found to occur more in the TIV group), other general symptoms of fatigue, fever, headache, muscle aches, shivering, and joint pain (found to occur more in the TIV group).

The intensities of adverse events were recorded according to a standard 0 to 3 grade scale: "absent", "easily tolerated", "interferes with normal activity", and "prevents normal activity". 
aa Beran 2009a (Continued)

Industry funded

\title{
Risk of bias
}

\begin{tabular}{lll}
\hline Bias & Authors' judgement & Support for judgement \\
\hline $\begin{array}{ll}\text { Random sequence genera- } \\
\text { tion (selection bias) }\end{array}$ & Low risk & $\begin{array}{l}\text { "A randomisation list was generated by the sponsor by SAS program and used } \\
\text { to number the vaccine and placebo treatments"; "A randomization blocking } \\
\text { scheme }(2: 1) \text { was employed to ensure that balance between treatments was } \\
\text { maintained." }\end{array}$ \\
& &
\end{tabular}

\begin{tabular}{ll}
\hline $\begin{array}{l}\text { Allocation concealment } \\
\text { (selection bias) }\end{array}$ & $\begin{array}{l}\text { No explicit description of the method of concealment, authors only mentioned } \\
\text { that treatments were numbered and that they were indistinguishable in ap- } \\
\text { pearance. }\end{array}$
\end{tabular}

\begin{tabular}{lll}
\hline $\begin{array}{l}\text { Blinding (performance } \\
\text { bias and detection bias) }\end{array}$ & Unclear risk & $\begin{array}{l}\text { Authors reported that the blinding assignment was maintained until study } \\
\text { analysis. }\end{array}$
\end{tabular}

All outcomes

Authors mentioned that the treatments were indistinguishable in appearance.

\begin{tabular}{|c|c|c|}
\hline $\begin{array}{l}\text { Incomplete outcome data } \\
\text { (attrition bias) } \\
\text { All outcomes }\end{array}$ & Low risk & $\begin{array}{l}\text { Exclusion of allocated participants from the analysis of the trial: } \\
\text { a) did the report mention explicitly the exclusion of allocated participants } \\
\text { from the analysis of trial results? Yes; } \\
\text { b) if so did the report mention the reason(s) for exclusion? Yes. Details were re- } \\
\text { ported in the study flow chart. }\end{array}$ \\
\hline Summary assessment & Unclear risk & Unclear risk of bias \\
\hline
\end{tabular}

aa Beran 2009b

Methods

\begin{abstract}
A randomised, double-blind, placebo-controlled study conducted during the 2006 to 2007 influenza season at 15 centres located in the Czech Republic and Finland. The protocols and study documents were approved by the ethics committee of each country. Participants were randomised to receive 1 dose of TIV (lot 1 or lot 2 of Fluarix) or placebo (normal saline solution) at the first study visit (day 0 ) by intramuscular injection. Each $0.5 \mathrm{~mL}$ dose of TIV contained $15 \mathrm{mg}$ of each of the haemagglutinin antigens of strains A/New Caledonia/20/99(H1N1) IVR-116, A/Wisconsin/67/2005(H3N2), and B/ Malaysia/2506/2004 (from the Victoria lineage).
\end{abstract}

From the day of vaccination, passive and active surveillance (biweekly contact) to detect ILI cases. For each case of suspected ILI, a nasal and throat swab specimen (composed of a swab of both nasal sinuses and a second swab of the throat) was collected for culture (as much as possible on the same day as the ILI report and, at the latest, 5 days after the ILI onset). Each participant was provided with a calibrated thermometer to measure temperature and a diary card to record temperatures and symptoms during the ILI episode. Blinded analysis was carried out at GSK Biologicals in Dresden, Germany.

Blood samples for the evaluation of influenza vaccine immunogenicity were obtained from the randomly selected, planned subset of an estimated 500 participants just prior to vaccination and 21 to 28 days later. Frozen aliquots of culture supernatants from positive viral cultures were sent to J Treanor's laboratory (University of Rochester Vaccine Evaluation Unit Influenza Serology Laboratory, Rochester, NY, USA) for identification of virus-matching isolates by conventional haemagglutination-inhibition testing (using $\mathrm{H} 1$ and $\mathrm{H} 3$ antisera from the $\mathrm{CDC}$ and $\mathrm{B} /$ Malaysia antiserum from the WHO).

Participants

Eligible participants were self referred women or men who were between 18 and 64 years of age and had no significant clinical disease at the time of vaccination.

WHO provided written informed consent. 
aa Beran 2009b (Continued)

Interventions
Intervention 1 dose of TIV (lot 1 or lot 2 of Fluarix), IM injection, at the first day of the study (day 0 )

Each $0.5 \mathrm{~mL}$ dose of TIV contained $15 \mathrm{mg}$ of each of the haemagglutinin antigens of strains $\mathrm{A} / \mathrm{New} /$ Caledonia/20/99(H1N1) IVR-116, A/Wisconsin/67/2005(H3N2), and B/Malaysia/2506/2004 (from the Victoria lineage).

Comparator placebo (normal saline solution), IM injection, at the first day of the study (day 0)

Serological (only carried out for the TIV group)

Effectiveness

Evaluate efficacy of TIV versus placebo in the prevention of culture-confirmed influenza A and/or B due to strains antigenically matched to the vaccine (their primary objective)

Secondary objectives were evaluation of TIV in the prevention of:

- culture-confirmed influenza due to strains antigenically matched to the vaccine for each of the 2 vaccine lots;

- culture-confirmed influenza A and/or B attributable to any influenza A or B strain;

- ILI, which was less stringently defined as at least 1 systemic symptom (fever or myalgia, or both) and 1 respiratory symptom (cough or sore throat, or both).

Safety vaccine reactogenicity and immunogenicity in a random subset of participants by obtaining blood samples prior to vaccination and 21 to 28 days later. However, no harms data were reported.

Notes The authors concluded that TIV is efficacious against culture-confirmed influenza in healthy adults. Industry funded

\section{Risk of bias}

\begin{tabular}{lll}
\hline Bias & Authors' judgement & Support for judgement \\
\hline $\begin{array}{l}\text { Random sequence genera- } \\
\text { tion (selection bias) }\end{array}$ & Unclear risk & No details provided. \\
\hline $\begin{array}{l}\text { Allocation concealment } \\
\text { (selection bias) }\end{array}$ & Unclear risk & No details provided. \\
\hline
\end{tabular}

Blinding (performance Unclear risk There is no mention of appearance of the injection content.

bias and detection bias)

All outcomes

Incomplete outcome data Low risk

(attrition bias)

All outcomes

Summaryassessment Unclear risk Unclear risk of bias

\section{aa Bridges 2000a}

$\begin{array}{ll}\text { Methods } & \text { Randomised controlled trial, double-blind, conducted in the USA during the } 1997 \text { to } 1998 \text { influenza } \\ \text { season. Follow-up lasted from November to March. Influenza period was defined as the period dur- } \\ \text { ing which clinical specimens collected from ill participants yielded influenza viruses (8 December } 1997 \\ \text { through } 2 \text { March 1998) and lasted } 12 \text { weeks. Volunteers were randomly allocated to receive vaccine } \\ \text { or placebo using a table of random numbers. Pharyngeal swab and paired sera were collected from ill } \\ \text { people. }\end{array}$


aa Bridges 2000a (Continued)

Participants

1184 healthy factory employees: 595 treated and 589 placebo. Age of participants was 18 to 64 .

Commercial trivalent, inactivated, intramuscular vaccine. Schedule and dose were not indicated. Vaccine composition was: A/Johannesburg/82/96, A/Nanchang/933/95, and B/Harbin/7/94. Placebo was sterile saline for injection. Vaccine was recommended but did not match the circulating strain.

Outcomes

Influenza-like illness, influenza, days ill, physician visits, times any drug was prescribed, times antibiotic was prescribed, working days lost, admissions, adverse effects. They were defined as follows: influenza-like illness: fever $=37.7^{\circ} \mathrm{C}$ with cough or sore throat); upper respiratory illness: cough with sore throat or fever $=37.7^{\circ} \mathrm{C}$. Local adverse effects were arm soreness and redness. Systemic adverse effects were: fever, sore throat, coryza, myalgia, headache, and fatigue, but authors reported no data. Surveillance was passive.

Notes

For analysis we chose the influenza-like illness definition. Intention-to-treat analysis was performed. Systemic adverse effects were not reported. Circulating strain was A/Sidney/5/97-like.

Government funded

\section{Risk of bias}

\begin{tabular}{lll}
\hline Bias & Authors' judgement & Support for judgement \\
\hline $\begin{array}{l}\text { Random sequence genera- } \\
\text { tion (selection bias) }\end{array}$ & Unclear risk & Insufficient description \\
\hline $\begin{array}{l}\text { Allocation concealment } \\
\text { (selection bias) }\end{array}$ & Low risk & Adequate \\
\hline $\begin{array}{l}\text { Blinding (performance } \\
\text { bias and detection bias) } \\
\text { All outcomes }\end{array}$ & Low risk & Adequate \\
\hline $\begin{array}{l}\text { Incomplete outcome data } \\
\text { (attrition bias) } \\
\text { All outcomes }\end{array}$ & Low risk & Attrition reasons for the whole cohort are provided by the participant flow. \\
\hline $\begin{array}{l}\text { Summary assessment } \\
\text { L }\end{array}$ & Low risk & Low risk of bias \\
\hline
\end{tabular}

aa Bridges 2000b

$\begin{array}{ll}\text { Methods } & \text { Randomised controlled trial, double-blind, conducted in the USA during the } 1998 \text { to } 1999 \text { influenza sea- } \\ \text { son. Follow-up lasted from November to March. The influenza period was defined as the period dur- } \\ \text { ing which clinical specimens collected from ill participants yielded influenza viruses (4 January } 1998 \\ \text { through } 14 \text { March 1999) and lasted } 10 \text { weeks. Pharyngeal swabs and paired sera were collected from ill } \\ \text { people. }\end{array}$

Participants 1191 healthy factory employees: 587 treated and 604 placebo. Age of participants was 19 to 64.

Interventions

Commercial trivalent, inactivated, intramuscular vaccine. Schedule and dose were not indicated. Vaccine composition was: A/Beijing/262/95, A/Sydney/5/97, and B/Harbin/7/94. Placebo was sterile saline for injection. Vaccine was recommended and matched circulating strain.

Outcomes

Influenza-like illness, influenza, days ill, physician visits, times any drug was prescribed, times antibiotic was prescribed, working days lost, admissions, adverse effects. They were defined as follows: influenza-like illness: fever $=37.7^{\circ} \mathrm{C}$ with cough or sore throat; upper respiratory illness: cough with sore throat or fever $=37.7^{\circ} \mathrm{C}$. Local adverse effects were arm soreness and redness. Systemic adverse effects 
aa Bridges 2000b (Continued)

were: fever, sore throat, coryza, myalgia, headache, and fatigue, but authors reported no data. Surveillance was passive.
For analysis we chose the influenza-like illness definition. Intention-to-treat analysis was performed. Systemic adverse effects were not reported. Circulating strain was A/Sydney/5/97-like and B/Beijing/184/93-like.

Government funded

\section{Risk of bias}

\begin{tabular}{|c|c|c|}
\hline Bias & Authors' judgement & Support for judgement \\
\hline $\begin{array}{l}\text { Random sequence genera- } \\
\text { tion (selection bias) }\end{array}$ & Unclear risk & $\begin{array}{l}\text { Volunteers were randomly allocated to receive vaccine or placebo using a ta- } \\
\text { ble of random numbers. }\end{array}$ \\
\hline $\begin{array}{l}\text { Allocation concealment } \\
\text { (selection bias) }\end{array}$ & Low risk & Adequate \\
\hline $\begin{array}{l}\text { Blinding (performance } \\
\text { bias and detection bias) } \\
\text { All outcomes }\end{array}$ & Low risk & Placebo was sterile saline for injection. Probably adequate \\
\hline $\begin{array}{l}\text { Incomplete outcome data } \\
\text { (attrition bias) } \\
\text { All outcomes }\end{array}$ & Low risk & Attrition reasons for the whole cohort are provided by the participant flow. \\
\hline Summary assessment & Low risk & Low risk of bias \\
\hline
\end{tabular}

aa Eddy 1970

Controlled clinical trial, single-blind, conducted in South Africa during the 1969 influenza season. Fol-
low-up lasted from May to July. The first clinical case of influenza appeared on 21 May 1969 and the last
6 weeks later. The epidemic period lasted 6 weeks. The control participants were selected by drawing a
1 -in-4 systematic sample from a ranked list of the personnel numbers.
Participants 1758 healthy male black African employees: 1254 treated and 413 placebo. Age of participants was 18 to 65 .

Monovalent inactivated parenteral vaccine. Schedule and dose were single injection, $1 \mathrm{~mL}$. Vaccine
composition was: A2/Aichi/2/68 (Hong Kong variant). Placebo was sterile water. Vaccine was recom-
mended and matched circulating strain.
Outcomes Influenza-like illness, working days lost, days ill. Influenza-like illness was not defined; case features were generically described in results section. All ill people were admitted to hospital until recovery. Surveillance was passive.
Notes The word 'double-blinding' was not used, but the control group received an injection of "dummy vac- cine". Poor reporting, poor-quality study. Circulating strain was A2/Hong Kong/68 virus. Efficacy data only were extracted.
Industry funded

\section{Risk of bias}

Bias Authors' judgement Support for judgement


aa Eddy 1970 (Continued)

Random sequence genera- High risk Systematic selection
tion (selection bias)

\begin{tabular}{lll}
\hline $\begin{array}{l}\text { Allocation concealment } \\
\text { (selection bias) }\end{array}$ & High risk & Inadequate \\
\hline $\begin{array}{l}\text { Blinding (performance } \\
\text { bias and detection bias) } \\
\text { All outcomes }\end{array}$ & High risk & No descriptions \\
\hline $\begin{array}{l}\text { Incomplete outcome data } \\
\text { (attrition bias) }\end{array}$ & High risk & Insufficient description \\
All outcomes & & \\
\hline
\end{tabular}

\begin{tabular}{lll}
\hline Summaryassessment & High risk & High risk of bias \\
\hline
\end{tabular}

Randomised controlled trial, double-blind, conducted in the USA during the 1986 to 1987 influenza sea-
son. Follow-up lasted the whole epidemic period. The epidemic period in any study year started on
the day that the first influenza A virus isolate was obtained in Nashville and ended on the day that the
last isolate was obtained and lasted 8 weeks. Participants were recruited from 7 organisations and as-
signed to 1 of the study groups using a permuted block randomisation scheme that was stratified by
treatment centre and age group. Sealed randomisation envelopes contained vaccine codes. Pharyn-
geal swab and paired sera were collected from ill people.

Participants 1311 healthy children and adults of metropolitan Nashville: 872 treated and 439 placebo. Age of participants was 1 to $65.85 \%$ of participants were older than 16 .

Interventions

Bivalent, live, cold-adapted, aerosol-administered influenza A vaccine and the commercial inactivated intramuscularly administered influenza vaccine. Schedule and dose were: single-dose; cold-adapted 107 to $107.6 \mathrm{pfu} / \mathrm{mL}$; inactivated $15 \mu \mathrm{g}$ each strain. Vaccine composition was: cold-adapted: Texas/1/85 $\mathrm{H} 1 \mathrm{~N} 1$ and Bethesda/1/85 H3N2; inactivated: Chile/1/83 H1N1 and Mississippi/1/85 H3N2. Placebo was allantoic fluid. Vaccine was recommended but did not match circulating strain.

Outcomes

Influenza-like illness, influenza. They were defined as follows: fever of abrupt onset with at least 1 of the following: chills, headache, malaise, myalgia, cough, pharyngitis, or other respiratory complaints (only participants who presented for culture were considered); throat culture. Surveillance was passive.

\section{Notes} Influenza B strain contained in the commercial and monovalent vaccines was not described. Strains
used yearly to develop cold-adapted and inactivated vaccines were antigenically comparable. Since
cold-adapted influenza B vaccines were not sufficiently characterised to include in the study, the au-
thors used monovalent inactivated influenza B vaccine in all participants in the cold-adapted arm and
as placebo in the control group inactivated arm. Only the cold-adapted comparison was included in the
analysis. The circulating strain was Taiwan/1/86. Effectiveness data only were extracted. Government funded

\begin{tabular}{lll}
\hline Risk of bias & \\
\hline Bias & Authors' judgement & Support for judgement \\
\hline $\begin{array}{l}\text { Random sequence genera- } \\
\text { tion (selection bias) }\end{array}$ & Unclear risk & $\begin{array}{l}\text { Insufficient description: "permutated block randomization scheme that was } \\
\text { stratified by treatment centre and age group" }\end{array}$ \\
\hline
\end{tabular}


aa Edwards 1994a (Continued)
Allocation concealment
Low risk
Adequate: participants and clinical staff were kept unaware of the assigned (selection bias) vaccine group through the use of sealed randomisation envelopes that con- tained vaccines codes.

\begin{tabular}{lll}
\hline $\begin{array}{l}\text { Blinding (performance } \\
\text { bias and detection bias) } \\
\text { All outcomes }\end{array}$ & Low risk & \\
\hline $\begin{array}{l}\text { Incomplete outcome data } \\
\text { (attrition bias) }\end{array}$ & High risk & Insufficient description \\
All outcomes & & \\
\hline
\end{tabular}

aa Edwards 1994b

$\begin{array}{ll}\text { Methods } & \text { Randomised controlled trial, double-blind, conducted in the USA during the } 1987 \text { to } 1988 \text { influenza sea- } \\ \text { son. Follow-up lasted the whole epidemic period. The epidemic period in any study year started on } \\ \text { the day that the first influenza A virus isolate was obtained in Nashville and ended on the day that the } \\ \text { last isolate was obtained and lasted } 14 \text { weeks. Participants were recruited from } 7 \text { organisations and as- } \\ \text { signed to } 1 \text { of the study groups using a permuted block randomisation scheme that was stratified by } \\ \text { treatment centre and age group. Sealed randomisation envelopes contained vaccine codes. Pharyn- } \\ \text { geal swab and paired sera were collected from ill people. }\end{array}$

\begin{tabular}{ll} 
Participants & $\begin{array}{l}1561 \text { healthy children and adults of metropolitan Nashville: } 1029 \text { treated and } 532 \text { placebo. Age of par- } \\
\text { ticipants was } 1 \text { to } 65.85 \% \text { of participants were older than } 16 .\end{array}$ \\
\hline Interventions & Bivalent, live, cold-adapted, aerosol-administered influenza A vaccine and the commercial inactivat- \\
& ed intramuscularly administered influenza vaccine. Schedule and dose were: single dose; cold-adapt- \\
& ed 107 to $107.6 \mathrm{pfu} / \mathrm{mL} ;$ inactivated $15 \mu \mathrm{g}$ each strain. Vaccine composition was: cold-adapted: Kawasa- \\
ki/9/86 H1N1 and Bethesda/1/85 H3N2; inactivated: Taiwan/1/86 H1N1 and Leningrad/360/86 H3N2. \\
Placebo was allantoic fluid. Vaccine was recommended but did not match the circulating strain.
\end{tabular}

Outcomes Influenza-like illness, influenza. They were defined as follows: fever of abrupt onset with at least 1 of the following: chills, headache, malaise, myalgia, cough, pharyngitis, or other respiratory complaints (ILI symptoms retrospectively reported were considered); 4-fold antibody rise between postvaccination and spring sera. Surveillance was passive.

Notes Influenza B strain contained in the commercial and monovalent vaccines was not described. Strains used yearly to develop cold-adapted and inactivated vaccines were antigenically comparable. Since cold-adapted influenza B vaccines were not sufficiently characterised to include in the study, the authors used monovalent inactivated influenza B vaccine in all participants in the cold-adapted arm and as placebo in the control group inactivated arm. Only the cold-adapted comparison was included in the analysis. The circulating strain was Sichuan/2/87 (H3N2) (antigen drift from vaccine strain) and B/Victo$\mathrm{ria} / 2 / 87$.

Effectiveness data only were extracted.

\section{Risk of bias}

\begin{tabular}{lll}
\hline Bias & Authors' judgement & Support for judgement \\
\hline $\begin{array}{l}\text { Random sequence genera- } \\
\text { tion (selection bias) }\end{array}$ & Unclear risk & $\begin{array}{l}\text { Insufficient description: "permutated block randomization scheme that was } \\
\text { stratified by treatment centre and age group" }\end{array}$ \\
\hline $\begin{array}{l}\text { Allocation concealment } \\
\text { (selection bias) }\end{array}$ & Low risk & $\begin{array}{l}\text { Adequate: participants and clinical staff were kept unaware of the assigned } \\
\text { vaccine group through the use of sealed randomisation envelopes that con- } \\
\text { tained vaccines codes. }\end{array}$ \\
\hline
\end{tabular}


aa Edwards 1994b (Continued)
Blinding (performance
Low risk
Adequate
bias and detection bias)
All outcomes

\begin{tabular}{lll}
\hline $\begin{array}{l}\text { Incomplete outcome data } \\
\text { (attrition bias) }\end{array}$ & High risk & \\
All outcomes & \\
\hline Summary assessment & Unclear risk & Unclear \\
\hline
\end{tabular}

\title{
aa Edwards 1994c
}

Randomised controlled trial, double-blind, conducted in the USA during the 1988 to 1989 influenza sea-
son. Follow-up lasted the whole epidemic period. The epidemic period in any study year started on
the day that the first influenza A virus isolate was obtained in Nashville and ended on the day that the
last isolate was obtained and lasted 11 weeks. Participants were recruited from 7 organisations and as-
signed to 1 of the study groups using a permuted block randomisation scheme that was stratified by
treatment centre and age group. Sealed randomisation envelopes contained vaccine codes. Pharyn-
geal swab and paired sera were collected from ill people.

Participants $\quad 1676$ healthy children and adults of metropolitan Nashville: 1114 treated and 562 placebo. Age of participants was 1 to $65.85 \%$ of participants were older than 16 .

\begin{abstract}
Interventions Bivalent, live, cold-adapted, aerosol-administered influenza A vaccine and the commercial inactivated intramuscularly administered influenza vaccine. Schedule and dose were: single dose; cold-adapted 107 to $107.6 \mathrm{pfu} / \mathrm{mL}$; inactivated $15 \mu \mathrm{g}$ each strain. Vaccine composition was: cold-adapted: Kawasaki/9/86 H1N1 and Los Angeles/2/87 H3N2; inactivated: Taiwan/1/86 H1N1 and Sichuan/2/87 H3N2. Placebo was allantoic fluid. Vaccine was recommended and matched circulating strain.
\end{abstract}

Influenza-like illness, influenza. They were defined as follows: fever of abrupt onset with at least 1 of
the following: chills, headache, malaise, myalgia, cough, pharyngitis, or other respiratory complaints
(ILI symptoms retrospectively reported were considered); 4 -fold antibody rise between postvaccina-
tion and spring sera. Surveillance was passive.

Notes Influenza B strain contained in the commercial and monovalent vaccines was not described. Strains used yearly to develop cold-adapted and inactivated vaccines were antigenically comparable. Since cold-adapted influenza B vaccines were not sufficiently characterised to include in the study, the authors used monovalent inactivated influenza $B$ vaccine in all participants in the cold-adapted arm and as placebo in the control group inactivated arm. Only the cold-adapted comparison was included in the analysis. The circulating strain was Taiwan/1/86 (H1N1) and B/Yamata/16/88. Effectiveness data only were extracted.

\section{Risk of bias}

\begin{tabular}{lll}
\hline Bias & Authors' judgement & Support for judgement \\
\hline $\begin{array}{l}\text { Random sequence genera- } \\
\text { tion (selection bias) }\end{array}$ & Unclear risk & $\begin{array}{l}\text { Insufficient description: "permutated block randomization scheme that was } \\
\text { stratified by treatment centre and age group" }\end{array}$ \\
\hline $\begin{array}{l}\text { Allocation concealment } \\
\text { (selection bias) }\end{array}$ & Low risk & $\begin{array}{l}\text { Adequate: participants and clinical staff were kept unaware of the assigned } \\
\text { vaccine group through the use of sealed randomisation envelopes that con- } \\
\text { tained vaccines codes. }\end{array}$ \\
\hline $\begin{array}{l}\text { Blinding (performance } \\
\text { bias and detection bias) } \\
\text { All outcomes }\end{array}$ & Low risk & Adequate \\
\hline \hline
\end{tabular}


aa Edwards 1994c (Continued)

Incomplete outcome data High risk Insufficient description
(attrition bias)

All outcomes

Summaryassessment Unclear risk Unclear

\section{aa Edwards 1994d}

Randomised controlled trial, double-blind, conducted in the USA during the 1989 to 1990 influenza sea-
son. Follow-up lasted the whole epidemic period. The epidemic period in any study year started on
the day that the first influenza A virus isolate was obtained in Nashville and ended on the day that the
last isolate was obtained and lasted 11 weeks. Participants were recruited from 7 organisations and as-
signed to 1 of the study groups using a permuted block randomisation scheme that was stratified by
treatment centre and age group. Sealed randomisation envelopes contained vaccine codes. Pharyn-
geal swab and paired sera were collected from ill people.

Participants 1507 healthy children and adults of metropolitan Nashville: 999 treated and 508 placebo. Age of participants was 1 to $65.85 \%$ of participants were older than 16 .

Interventions

Bivalent, live, cold-adapted, aerosol-administered influenza A vaccine and the commercial inactivated intramuscularly administered influenza vaccine. Schedule and dose were: single dose; cold-adapted 107 to $107.6 \mathrm{pfu} / \mathrm{mL}$; inactivated $15 \mu \mathrm{g}$ each strain. Vaccine composition was: Kawasaki/9/86 H1N1 and Los Angeles/2/87 H3N2; inactivated: Taiwan/1/86 H1N1 and Shanghai/11/87 H3N2. Placebo was allantoic fluid. Vaccine was recommended and matched circulating strain.

Outcomes

Influenza-like illness, influenza. They were defined as follows: fever of abrupt onset with at least 1 of the following: chills, headache, malaise, myalgia, cough, pharyngitis, or other respiratory complaints (ILI symptoms retrospectively reported were considered); 4-fold antibody rise between postvaccination and spring sera. Surveillance was passive.

Notes
Influenza B strain contained in the commercial and monovalent vaccines was not described. Strains
cold-adapted influenza B vaccines were not sufficiently characterised to include in the study, the au-
thors used monovalent inactivated influenza B vaccine in all participants in the cold-adapted arm and
as placebo in the control group inactivated arm. Only the cold-adapted comparison was included in the
analysis. The circulating strain was Shanghai/11/87 (H3N2). Effectiveness data only were extracted.

\section{Risk of bias}

\begin{tabular}{lll} 
Bias & Authors' judgement & Support for judgement \\
\hline $\begin{array}{l}\text { Random sequence genera- } \\
\text { tion (selection bias) }\end{array}$ & Unclear risk & $\begin{array}{l}\text { Insufficient description: "permutated block randomization scheme that was } \\
\text { stratified by treatment centre and age group" }\end{array}$ \\
\hline
\end{tabular}

\begin{tabular}{|c|c|c|}
\hline $\begin{array}{l}\text { Allocation concealment } \\
\text { (selection bias) }\end{array}$ & Low risk & $\begin{array}{l}\text { Adequate: participants and clinical staff were kept unaware of the assigned } \\
\text { vaccine group through the use of sealed randomisation envelopes that con- } \\
\text { tained vaccines codes. }\end{array}$ \\
\hline
\end{tabular}

\begin{tabular}{|c|c|c|}
\hline $\begin{array}{l}\text { Blinding (performance } \\
\text { bias and detection bias) } \\
\text { All outcomes }\end{array}$ & Low risk & Adequate \\
\hline
\end{tabular}

\begin{tabular}{l}
\hline Incomplete outcome data High risk \\
(attrition bias) \\
All outcomes
\end{tabular}


aa Edwards 1994d (Continued)

Summaryassessment Unclear risk Unclear

aa Frey 2010

Methods Randomised, controlled, multicentre, observer-blind trial assessing effectiveness, immunogenicity, and safety of both CCIV and TIV containing the strain recommended by WHO for the current season (2007 to 2008)

Participants were recruited at 56 centres in the USA, Finland, and Poland.
Major exclusion criteria: health condition for which inactivated vaccine is recommended, employ-
ment prone to influenza transmission, influenza vaccination or laboratory-confirmed influenza within 6
months of enrolment, history of Guillain-Barré syndrome, a temperature of $37.8^{\circ} \mathrm{C}$ and/or acute illness
within 3 days of enrolment, and pregnancy or breastfeeding.
A total of 11,404 participants were randomised: 11,382 were vaccinated and $10,844(95 \%)$ completed
the study.

Interventions Individuals aged 18 to 49 years were randomised equally, with use of an interactive voice response system, to receive a single dose of CCIV, TIV, or placebo.

Both CCIV and TIV (Novartis Vaccines and Diagnostics) contained $15 \mu \mathrm{g}$ of haemagglutinin per $0.5 \mathrm{~mL}$ dose of each of the following virus strains:

A/Solomon Islands/3/2006 (H1N1)-like

A/Wisconsin/67/2005 (H3N2)-like

B/Malaysia/2506/2004-like

Preparations were administered in the deltoid muscle of the non-dominant arm. Only the vaccine administrator had access to the randomisation code.

\section{Outcomes}

\section{Safety}

Study participants were monitored for 30 minutes after vaccination for immediate reactions. Participants recorded the occurrence, duration, and severity of local injection site and systemic reactions for 7 days after vaccination. Solicited reactions were graded as follows: mild, no limitation of normal daily activities; moderate, some limitation; or severe, unable to perform normal daily activities. Unsolicited reactions were recorded for 21 days after vaccination. Serious adverse events were monitored for the entire study (9 months).

\section{Effectiveness}

Influenza surveillance began 21 days after vaccination. Participants had to report to investigators the occurrence of influenza-like illness symptoms (fever $37.8^{\circ} \mathrm{C}$ plus sore throat or cough, as well as body aches, chills, headache, and runny or stuffy nose). An active survey was also performed by means of weekly phone calls.

Participants reporting influenza-like illness symptoms underwent clinical evaluations; nasal and throat specimens were obtained for laboratory confirmation of influenza virus. Specimens were targeted for collection within 24 hours after symptom onset, with a window of 120 hours. Specimens were cultured on RhMK and tested by PCR.

Each study participant was observed during the 6-month study surveillance period or for 6 months after vaccination, whichever was longer. Study duration was around 9 months.

\section{Immunogenicity}

It was assessed on the first 1045 participants enrolled at USA sites and randomised 8:25:2 to receive CCIV, TIV, or placebo. Serum samples were collected at baseline and 3 weeks after immunisation for seroprotection, seroconversion, and GMT determination.

\section{Notes}

Financial support: "Novartis Vaccines was the funding source and was involved in all stages of the study conduct and analysis"

Potential conflicts of interest: "M.L., A.I., N.G., and S.H. are employees of Novartis Vaccines and Diagnostics. T.V. has received consultancy fees from Medlmmune and speaker fees from Medlmmune, Novartis, and Crucell in relation to meetings on influenza vaccination. S.F. and A.S.-M.: no conflicts"

\section{Risk of bias}


aa Frey 2010 (Continued)

\begin{tabular}{|c|c|c|}
\hline Bias & Authors' judgement & Support for judgement \\
\hline $\begin{array}{l}\text { Random sequence genera- } \\
\text { tion (selection bias) }\end{array}$ & Unclear risk & No description \\
\hline $\begin{array}{l}\text { Allocation concealment } \\
\text { (selection bias) }\end{array}$ & Low risk & $\begin{array}{l}\text { "Individuals ... were randomised equally, with use of an interactive voice re- } \\
\text { sponse system, to receive a single dose of CCIV, TIV, or placebo." }\end{array}$ \\
\hline $\begin{array}{l}\text { Blinding (performance } \\
\text { bias and detection bias) } \\
\text { All outcomes }\end{array}$ & Unclear risk & $\begin{array}{l}\text { "This randomized, placebo-controlled, observer-blind trial evaluated ..." } \\
\text { "Only the vaccine administrator had access to the randomization code." } \\
\text { No information about the appearance of the preparation is provided in the } \\
\text { text. }\end{array}$ \\
\hline $\begin{array}{l}\text { Incomplete outcome data } \\
\text { (attrition bias) } \\
\text { All outcomes }\end{array}$ & Low risk & $\begin{array}{l}\text { Flow of participants during the study is reported and described. Loss to fol- } \\
\text { low-up amounts to about } 5 \% \text { at study end and is balanced through the } 3 \text { arms. }\end{array}$ \\
\hline Summary assessment & Unclear risk & Unclear \\
\hline
\end{tabular}

aa Hammond 1978

\begin{tabular}{|c|c|}
\hline Methods & $\begin{array}{l}\text { Controlled clinical trial, double-blinded conducted in Australia during the } 1976 \text { influenza season. Fol- } \\
\text { low-up lasted the whole epidemic period. Epidemic influenza was defined by virus isolation and serol- } \\
\text { ogy tests and lasted from middle of April to middle of August } 1976 \text { ( } 17 \text { weeks). Coded, identical-looking } \\
\text { vials were sequentially administered to enrolled participants. A throat swab was collected from ill peo- } \\
\text { ple. Serological confirmation was performed on all participants. }\end{array}$ \\
\hline
\end{tabular}

Participants 225 medical students or staff members: 116 treated and 109 placebo. Age of participants was not indicated.

\begin{tabular}{ll}
\hline Interventions & Trivalent parenteral subunit vaccine. Schedule and dose were: single dose; vaccine composition was: \\
& $250 \mathrm{IU}$ of A/Victoria/3/75, $250 \mathrm{IU}$ of $\mathrm{A} / \mathrm{Scotland} / 840 / 74$, and $300 \mathrm{IU}$ of $\mathrm{B} / \mathrm{Hong} \mathrm{Kong} / 8 / 73$. Placebo was \\
diphtheria and tetanus toxoids. Vaccine was recommended and matched circulating strain.
\end{tabular}

$\begin{array}{ll}\text { Outcomes } & \text { Influenza-like illness, influenza. Clinical illnesses were not defined. Influenza was defined as respiratory } \\ \text { illness that was associated with the isolation of influenza virus, a 4-fold or greater rise in antibody titre } \\ \text { occurring between postvaccination and postepidemic sera, or both. Surveillance was active. }\end{array}$

Notes Clinical illness was not defined, and data were included in the analysis as "clinical cases without clear
definition". Circulating strain was A/Vic/3/75-like. Efficacy data only were extracted.

Government funded

\section{Risk of bias}

\begin{tabular}{lll}
\hline Bias & Authors' judgement & Support for judgement \\
\hline $\begin{array}{l}\text { Random sequence genera- } \\
\text { tion (selection bias) }\end{array}$ & High risk & Alternate \\
\hline $\begin{array}{l}\text { Allocation concealment } \\
\text { (selection bias) }\end{array}$ & High risk & No description \\
\hline $\begin{array}{l}\text { Blinding (performance } \\
\text { bias and detection bias) }\end{array}$ & Unclear risk & No description
\end{tabular}


aa Hammond 1978 (Continued)

All outcomes

\begin{tabular}{lll}
$\begin{array}{l}\text { Incomplete outcome data } \\
\text { (attrition bias) } \\
\text { All outcomes }\end{array}$ & Unclear risk & No description \\
\hline Summary assessment & High risk & No description \\
\hline
\end{tabular}

\section{aa Jackson 2010a}

$\begin{array}{ll}\text { Methods } & \begin{array}{l}\text { Randomised, multicentre, double-blind, placebo-controlled trial assessing the effectiveness and safety } \\ \text { of a trivalent inactivated vaccine in preventing confirmed influenza. The study was performed during } 2 \\ \text { influenza seasons (2005 to } 2006 \text { and } 2006 \text { to 2007) in the USA. }\end{array}\end{array}$

Participants

Healthy adults aged between 18 and 49 years without significant acute or chronic medical or psychiatric illness. Individuals with cancer; systolic blood pressure $\geq 140 \mathrm{mmHg}$, diastolic blood pressure $\geq$ $90 \mathrm{mmHg}$; belonging to a risk group for which routine influenza vaccination is recommended (chronic pulmonary, cardiovascular, renal, hepatic, haematological, or metabolic disorders; immunosuppressive illness, recent/ongoing receipt of immunosuppressive therapy, immunoglobulin, other vaccines, or with HIV infection were excluded. Participants enrolled for the first season were not included in the second season.

In season I (2005 to 2006), 3514 participants were recruited at 37 centres from 17 September 2005 onwards.

In season II (2006 to 2007), 4144 participants were recruited at 44 centres from 16 October 2006 onwards.

Interventions

Recruited participants were randomised at the beginning of each season to receive 1 dose of trivalent inactivated split influenza vaccine (FluLaval, a trademark of the GlaxoSmithKline group of companies; manufactured by ID Biomedical Corporation of Quebec, Canada) or saline placebo injection. Each $0.5 \mathrm{~mL}$ dose of TIV contained $15 \mu \mathrm{g}$ of HA antigen of each recommended influenza strain.

For season I (2005 to 2006) antigens were:

A/New Caledonia/20/1999 (H1N1)

A/New York/55/2004 (H3N2, A/California/7/2004-like)

B/Jiangsu/10/2003 (B/Shanghai/361/2002-like)

Outcomes

\section{Effectiveness}

During the influenza seasons, participants were instructed to report symptoms meeting the ILI definition by using a toll-free, study-specific phone number within 48 hours from their onset and to record them together with temperature. Influenza-like illness symptoms were moreover solicited by weekly outbound phone contact. Visits from nurses were dispatched to participants who filled ILI definition within 24 hours after symptoms onset, and nasopharyngeal and oropharyngeal swabs for viral culture were drawn. During season I surveillance for influenza was conducted between 14 November 2005 and 30 April 2006; during season II between 13 November and 30 April.

Primary effectiveness study endpoint was:

$\mathrm{VMCCl}$ (vaccine-matched, culture-confirmed influenza). The case definition required the presence of ILI, defined as symptoms that interfered with normal daily activities and that included cough and at least 1 additional symptom from among fever (oral temperature $>37.7^{\circ} \mathrm{C} / 99.9^{\circ} \mathrm{F}$ ), headache, myalgia and/or arthralgia, chills, rhinorrhoea/nasal congestion, and sore throat. Participants meeting the definition for ILI and with concurrent isolation from a nasopharyngeal swab of an influenza A and/or B virus isolate antigenically matching a vaccine strain for the relevant year were considered to be cases of VM$\mathrm{CCl}$.

Secondary effectiveness endpoints were:

$\mathrm{CCI}$ (culture-confirmed influenza illness). ILI with any influenza A or B virus isolate by culture.

$\mathrm{LCl}$ (laboratory-confirmed influenza illness). 1 or both of CCI or ILI with a 4-fold increase in $\mathrm{HI}$ serum antibody titres to a circulating influenza virus strain between day 21 ( \pm 4 days) postvaccination and final visit specimens obtained after the end of the influenza season. 


\section{Immunogenicity}

Serum samples were collected from study participants at day 0,21 , and about 4 weeks after the end of the surveillance period.

Immunogenicity was assessed determining GMT, seroconversion and seroprotection rate between samples collected at day 21 and at day 0 on a randomly selected subset of participants.

\section{Safety}

Local and systemic reactions (events) occurred within 3 days after immunisation. Participants were observed for the first 30 minutes following immunisation. Participants recorded further reactions occurring no later than 8 days following vaccination by means of an interactive voice response system. The following symptoms were reported (3 days).

- Fever (at least $37.5^{\circ} \mathrm{C}$ )

- Injection site pain/soreness

- Injection site redness

- Injection site swelling

- Myalgia or arthralgia, or both

- Headache

- Tiredness

- Chills

- Malaise

- Red eyes

- Swelling of the face

- Cough

- Chest tightness or difficulty in breathing

- Sore throat, hoarseness, or pain on swallowing

Participants with at least 1 vaccine reactogenicity event Data were provided pooled for the 2 study seasons. Unsolicited spontaneous adverse events, for which follow-up was extended for at least 135 days following immunisation.

\section{Pregnancy outcomes}

Pregnancies

Spontaneous abortion

Full-term birth

Per-protocol: participants who received the treatment to which they were randomised, responded to $\geq 1$ postvaccination active surveillance telephone calls, and had no major protocol deviations considered to affect the efficacy or immunogenicity data (determined before unblinding) (for effectiveness estimates).

Intention-to-immunise: the per-protocol set plus participants with protocol deviations and treatment errors and analysed as randomised.

The safety set included participants who received any study treatment and had any postvaccination safety data. If an incorrect treatment was conclusively documented, participants in the safety set were analysed based on the treatment they had actually received.

Funding source was pharmaceutical.

"GSK Biologicals was the funding source and was involved in all stages of the study conduct and analysis. GSK Biologicals also took in charge all costs associated with the development and the publishing of this manuscript. The corresponding author had full access to the data, and final responsibility for submission of the manuscript for publication"

\section{Risk of bias}

\begin{tabular}{lll}
\hline Bias & Authors' judgement & Support for judgement \\
\hline $\begin{array}{l}\text { Random sequence genera- } \\
\text { tion (selection bias) }\end{array}$ & Unclear risk & $\begin{array}{l}\text { "Treatment allocation was determined by blocked, stratified randomization } \\
\text { with a 1:1 distribution to TIV or placebo; randomization was stratified by study }\end{array}$ \\
\hline
\end{tabular}


center, age (18-34 and 35-49 years), and the subject's report of previous recent receipt (within $\leq 2$ years) of TIV."

\begin{tabular}{|c|c|c|}
\hline $\begin{array}{l}\text { Allocation concealment } \\
\text { (selection bias) }\end{array}$ & Unclear risk & $\begin{array}{l}\text { Insufficient description of allocation concealment: "Each study center had a } \\
\text { pre-determined sequence of randomization numbers which were allocated se- } \\
\text { quentially to eligible participants. Participants were allocated equally among } 3 \\
\text { different vaccine lots" }\end{array}$ \\
\hline
\end{tabular}

$\begin{array}{ll}\text { Blinding (performance } & \text { Low risk Clinic staff (excluding the nurse giving the vaccine), were blinded to the treat- } \\ \text { bias and detection bias) } & \text { ment group until the study was complete." }\end{array}$

All outcomes

\begin{tabular}{lll}
\hline $\begin{array}{l}\text { Incomplete outcome data } \\
\text { (attrition bias) }\end{array}$ & Low risk & \\
All outcomes & \\
\hline Summary assessment & Unclear risk & Unclear \\
\hline
\end{tabular}

\section{aa Jackson 2010b}

\begin{tabular}{ll}
\hline Methods & See aa Jackson 2010a (the following data refer to the second study season) \\
\hline Participants & $\begin{array}{l}\text { In season II (2006 to 2007), } 4144 \text { participants were recruited at } 44 \text { centres from } 16 \text { October } 2006 \text { on- } \\
\text { wards. }\end{array}$ \\
\hline Interventions & $\begin{array}{l}\text { Recruited participants were randomised at the beginning of each season to receive } 1 \text { dose of trivalent } \\
\text { inactivated split influenza vaccine (FluLaval, a trademark of the GlaxoSmithKline group of companies; } \\
\text { manufactured by ID Biomedical Corporation of Quebec, Canada) or saline placebo injection. } \\
\text { Each } 0.5 \text { mL dose of TIV contained } 15 \text { Hg of haemagglutinin antigen of each recommended influenza } \\
\text { strain } \\
\text { Antigens for season II (2006 to 2007) were: } \\
\text { A/New Caledonia/20/1999 (H1N1) virus } \\
\text { A/Wisconsin/67/2005 (H3N2) } \\
\text { B/Malaysia/2506/2004 }\end{array}$ \\
\hline Outcomes & See aa Jackson 2010a \\
\hline Notes & See aa Jackson 2010a
\end{tabular}

\section{Risk of bias}

\begin{tabular}{lll}
\hline Bias & Authors' judgement & Support for judgement \\
\hline $\begin{array}{l}\text { Random sequence genera- } \\
\text { tion (selection bias) }\end{array}$ & Unclear risk & See aa Jackson 2010a \\
\hline $\begin{array}{l}\text { Allocation concealment } \\
\text { (selection bias) }\end{array}$ & Unclear risk & See aa Jackson 2010a \\
\hline $\begin{array}{l}\text { Blinding (performance } \\
\text { bias and detection bias) } \\
\text { All outcomes }\end{array}$ & Low risk & See aa Jackson 2010a \\
\hline $\begin{array}{l}\text { Incomplete outcome data } \\
\text { (attrition bias) } \\
\text { All outcomes }\end{array}$ & Low risk & See aa Jackson 2010a \\
\hline \hline
\end{tabular}


aa Jackson 2010b (Continued)

Summary assessment Unclear risk See aa Jackson 2010a

aa Keitel 1988a

Methods Randomised controlled trial, double-blind, conducted in the USA during the 1983 to 1984 influenza season. Follow-up lasted the whole epidemic period. Influenza period was defined as the interval during which community surveillance recovered influenza viruses from $10 \%$ or more of people with febrile respiratory illness per calendar week (from 8 January to 17 March 1984) and lasted 9 weeks. Volunteers were randomly allocated to receive vaccine or placebo using a table of random numbers according to prior vaccination experience. Specimens for culture and acute-convalescent blood specimens were obtained from ill people. At spring time volunteers were asked to record any illness that occurred during the epidemic period, and blood specimens were collected.

Participants 598 healthy employees working in the Texas Medical Center in Houston, Texas, or in surrounding industrial companies: 300 treated and 298 placebo. Age of participants was 30 to 60.

Trivalent, killed, whole, intramuscularly administered vaccine. Schedule and dose were: single dose; 15
$\mu \mathrm{g}$ of haemagglutinin of each influenza strain. Vaccine composition was: A/Philippines/2/82 (H3N2), A/
Brazil/11/78 (H1N1), and B/Singapore/222/79. Placebo was sterile saline for injection. Vaccine was rec-
ommended but did not match the circulating strain.

Outcomes

Outcomes were: ILI, influenza. Illnesses were classified as "any", "flu-like" (lower respiratory or systemic illness, or both), and "febrile" (oral temperature of $37.8^{\circ} \mathrm{C}$ or higher). Laboratory confirmation was based on culture and/or 4-fold or greater rise in antibody titre occurring between postvaccination (pre-epidemic), acute, convalescent and/or spring (postepidemic) sera.

Notes Influenza-like illness and influenza were detected in 3 groups: first vaccinated, multivaccinated, and placebo. Febrile illnesses were included in the analysis; the first 2 groups' cases were combined. Circulating strain was A/Victoria/7/83 (H1N1) and B/USSR/100/83. Efficacy data only were extracted.

Government funded

\begin{tabular}{lll}
\hline Risk of bias & & \\
\hline Bias & Authors' judgement & Support for judgement \\
\hline $\begin{array}{l}\text { Random sequence genera- } \\
\text { tion (selection bias) }\end{array}$ & Unclear risk & No description \\
\hline $\begin{array}{l}\text { Allocation concealment } \\
\text { (selection bias) }\end{array}$ & Unclear risk & Unclear \\
\hline $\begin{array}{l}\text { Blinding (performance } \\
\text { bias and detection bias) } \\
\text { All outcomes }\end{array}$ & Unclear risk & No description \\
\hline $\begin{array}{l}\text { Incomplete outcome data } \\
\text { (attrition bias) }\end{array}$ & Unclear risk & No description \\
$\begin{array}{l}\text { All outcomes } \\
\text { Summary assessment }\end{array}$ & Unclear risk & No description \\
\hline
\end{tabular}


aa Keitel 1988b

Methods
Randomised controlled trial, double-blind, conducted in the USA during the 1984 to 1985 influenza sea-
ing which community surveillance recovered influenza viruses from $10 \%$ or more of people with febrile
respiratory illness per calendar week (from 6 January to 9 March 1985 ) and lasted 9 weeks. Volunteers
were randomly allocated to receive vaccine or placebo using a table of random numbers according to
prior vaccination experience. Specimens for culture and acute-convalescent blood specimens were ob-
tained from ill people. At spring time volunteers were asked to record any illness that occurred during
the epidemic period, and blood specimens were collected.

Participants

697 healthy employees working in the Texas Medical Center in Houston, Texas, or in surrounding industrial companies: 456 treated and 241 placebo. Age of participants was 30 to 60 .

Interventions

Trivalent, killed, whole, intramuscularly administered vaccine. Schedule and dose were: single dose; 15 $\mu \mathrm{g}$ of haemagglutinin of each influenza strain. Vaccine composition was: A/Philippines/2/82 (H3N2), A/ Chile/1/83 (H1N1), and B/USSR/100/83. Placebo was sterile saline for injection.

Outcomes

Outcomes were: ILI, influenza. Illnesses were classified as "any", "flu-like" (lower respiratory or systemic illness, or both), and "febrile" (oral temperature of $37.8^{\circ} \mathrm{C}$ or higher). Laboratory confirmation was based on culture and/or 4-fold or greater rise in antibody titre occurring between postvaccination (pre-epidemic), acute, convalescent and/or spring (postepidemic) sera. Surveillance was passive.

\begin{tabular}{ll}
\hline Notes Government funded \\
\hline
\end{tabular}

\section{Risk of bias}

\begin{tabular}{|c|c|c|}
\hline Bias & Authors' judgement & Support for judgement \\
\hline $\begin{array}{l}\text { Random sequence genera- } \\
\text { tion (selection bias) }\end{array}$ & Unclear risk & No description \\
\hline $\begin{array}{l}\text { Allocation concealment } \\
\text { (selection bias) }\end{array}$ & Unclear risk & Unclear \\
\hline $\begin{array}{l}\text { Blinding (performance } \\
\text { bias and detection bias) } \\
\text { All outcomes }\end{array}$ & Unclear risk & No description \\
\hline $\begin{array}{l}\text { Incomplete outcome data } \\
\text { (attrition bias) } \\
\text { All outcomes }\end{array}$ & Unclear risk & No description \\
\hline Summary assessment & Unclear risk & No description \\
\hline
\end{tabular}

aa Keitel 1997a

Randomised controlled trial, double-blind, conducted in the USA during the 1985 to 1986 influenza sea-
son. Follow-up lasted the whole epidemic period. The influenza period was defined by viral surveil-
lance. Volunteers were randomly allocated to receive vaccine or placebo using a table of random num-
bers according to prior vaccination experience. Specimens for culture and acute-convalescent blood
specimens were obtained from ill people. At spring time, volunteers were asked to record any illness
that occurred during the epidemic period, and blood specimens were collected.

Participants 830 healthy employees working in the Texas Medical Center in Houston, Texas, or in surrounding industrial companies: 577 treated and 253 placebo. Age of participants was 30 to 60 . 
aa Keitel 1997a (Continued)

Interventions
Trivalent, killed, whole, intramuscularly administered vaccine. Schedule and dose were: single dose; $15 \mu \mathrm{g}$ of haemagglutinin of each influenza strain. Vaccine composition was: A/Philippines/2/82 (H3N2), $\mathrm{A} / \mathrm{Chile} / 1 / 83$ (H1N1), and B/USSR/100/83. Placebo was sterile saline for injection. Vaccine was recommended but did not match the circulating strain.

ILI, influenza. Illnesses were classified as "any", "flu-like" (lower respiratory or systemic illness, or both),
and "febrile" (oral temperature of $37.8^{\circ} \mathrm{C}$ or higher). Laboratory confirmation was based on culture
and/or 4-fold or greater rise in antibody titre occurring between postvaccination (pre-epidemic), acute,
convalescent and/or spring (postepidemic) sera. Surveillance was active.

Notes

Influenza-like illness and influenza cases were detected in 3 groups: first vaccinated, multivaccinated, and placebo. Febrile illnesses were included in the analysis; the first 2 groups' cases were combined. Circulating strains were B/Ann Arbor/1/86, A/Mississippi/1/85.

Efficacy data only were extracted.

Government funded

\section{Risk of bias}

\begin{tabular}{lll}
\hline Bias & Authors' judgement & Support for judgement \\
\hline $\begin{array}{l}\text { Random sequence genera- } \\
\text { tion (selection bias) }\end{array}$ & Unclear risk & No description \\
\hline $\begin{array}{l}\text { Allocation concealment } \\
\text { (selection bias) }\end{array}$ & Unclear risk & Unclear \\
\hline $\begin{array}{l}\text { Blinding (performance } \\
\text { bias and detection bias) } \\
\text { All outcomes }\end{array}$ & Unclear risk & No description \\
\hline $\begin{array}{l}\text { Incomplete outcome data } \\
\text { (attrition bias) }\end{array}$ & Unclear risk & No description \\
All outcomes & & \\
\hline $\begin{array}{l}\text { Summary assessment } \\
\end{array}$ & Unclear risk & No description \\
\hline
\end{tabular}

aa Keitel 1997b

$\begin{array}{ll}\text { Methods } & \text { Randomised controlled trial, double-blind, conducted in the USA during the } 1986 \text { to } 1987 \text { influenza } \\ \text { season. Follow-up lasted the whole epidemic period. Influenza period was defined by viral surveil- } \\ \text { lance. Specimens for culture and acute-convalescent blood specimens were obtained from ill people. } \\ \text { At spring time, volunteers were asked to record any illness that occurred during the epidemic period, } \\ \text { and blood specimens were collected. }\end{array}$

\begin{tabular}{ll} 
Participants & $\begin{array}{l}940 \text { healthy employees working in the Texas Medical Center in Houston, Texas, or in surrounding indus- } \\
\text { trial companies: } 723 \text { treated and } 217 \text { placebo. Age of participants was } 30 \text { to } 60 .\end{array}$ \\
\hline Interventions & $\begin{array}{l}\text { Trivalent, killed, whole, intramuscularly administered vaccine. Schedule and dose were: } 2 \text { doses; } 15 \\
\mu g \text { of haemagglutinin of each influenza strain. Vaccine composition was: A/Mississippi/1/85/H3N2), A/ } \\
\text { Chile/1/83 (H1N1), and B/Ann Arbor/1/86 plus A/Taiwan } / 1 / 86 \text { (H1N1). Placebo was sterile saline for in- } \\
\text { jection. Vaccine was recommended but did not match the circulating strain. }\end{array}$
\end{tabular}

\section{Outcomes}

ILI, influenza. Illnesses were classified as "any", "flu-like" (lower respiratory or systemic illness, or both), and "febrile" (oral temperature of $37.8^{\circ} \mathrm{C}$ or higher). Laboratory confirmation was based on culture 
aa Keitel 1997b (Continued)

and/or 4-fold or greater rise in antibody titre occurring between postvaccination (pre-epidemic), acute, convalescent and/or spring (postepidemic) sera. Surveillance was passive.

Notes

Influenza-like illness and influenza cases were detected in 3 groups: first vaccinated, multivaccinated, and placebo. Febrile illnesses were included in the analysis; the first 2 groups' cases were combined.

Circulating strain was A/Taiwan/1/86. Effectiveness data only were extracted.

\section{Risk of bias}

\begin{tabular}{|c|c|c|}
\hline Bias & Authors' judgement & Support for judgement \\
\hline $\begin{array}{l}\text { Random sequence genera- } \\
\text { tion (selection bias) }\end{array}$ & Low risk & $\begin{array}{l}\text { Volunteers were randomly allocated to receive vaccine or placebo using a ta- } \\
\text { ble of random numbers according to prior vaccination experience. }\end{array}$ \\
\hline $\begin{array}{l}\text { Allocation concealment } \\
\text { (selection bias) }\end{array}$ & Unclear risk & Insufficent information available to judge \\
\hline $\begin{array}{l}\text { Blinding (performance } \\
\text { bias and detection bias) } \\
\text { All outcomes }\end{array}$ & Unclear risk & Described as double-blind, but no further details available. \\
\hline $\begin{array}{l}\text { Incomplete outcome data } \\
\text { (attrition bias) } \\
\text { All outcomes }\end{array}$ & Unclear risk & No description \\
\hline Summary assessment & Unclear risk & No description \\
\hline
\end{tabular}

aa Keitel 1997c

Methods Randomised controlled trial, double-blind, conducted in the USA during the 1987 to 1988 influenza season. Follow-up lasted the whole epidemic period. Influenza period was defined by viral surveillance. Volunteers were randomly allocated to receive vaccine or placebo using a table of random numbers according to prior vaccination experience. Specimens for culture and acute-convalescent blood specimens were obtained from ill people. At spring time, volunteers were asked to record any illness that occurred during the epidemic period, and blood specimens were collected.

\begin{tabular}{ll} 
Participants & $\begin{array}{l}934 \text { healthy employees working in the Texas Medical Center in Houston, Texas, or in surrounding indus- } \\
\text { trial companies: } 789 \text { treated and } 145 \text { placebo. Age of participants was } 30 \text { to } 60 .\end{array}$ \\
\hline Interventions & $\begin{array}{l}\text { Trivalent, killed, whole, intramuscularly administered vaccine. Schedule and dose were: single dose; } 15 \\
\mu g \text { of haemagglutinin of each influenza strain. Vaccine composition was: A/Leningrad/360/86 (H3N2), } \\
\text { A/Taiwan/1/86 (H1N1), B/Ann Arbor/1/86. Placebo was sterile saline for injection. Vaccine was recom- } \\
\text { mended but did not match the circulating strain. }\end{array}$
\end{tabular}

Outcomes ILI, influenza. Illnesses were classified as "any", "flu-like" (lower respiratory or systemic illness, or both), and "febrile" (oral temperature of $37.8^{\circ} \mathrm{C}$ or higher). Laboratory confirmation was based on culture and/or 4-fold or greater rise in antibody titre occurring between postvaccination (pre-epidemic), acute, convalescent and/or spring (postepidemic) sera. Surveillance was passive.

Notes Influenza-like illness and influenza cases were detected in 3 groups: first vaccinated, multivaccinated, and placebo. Febrile illnesses were included in the analysis; the first 2 groups' cases were combined. Circulating strains were A/Sichuan/1/87, B/Victoria/2/87. Effectiveness data only were extracted.

\section{Risk of bias}

Bias Authors' judgement Support for judgement


aa Keitel 1997c (Continued)

Random sequence genera- Unclear risk No description tion (selection bias)

\begin{tabular}{lll}
\hline $\begin{array}{l}\text { Allocation concealment } \\
\text { (selection bias) }\end{array}$ & Unclear risk & Unclear \\
\hline $\begin{array}{l}\text { Blinding (performance } \\
\text { bias and detection bias) }\end{array}$ & Unclear risk & No description \\
All outcomes & \\
\hline
\end{tabular}

Incomplete outcome data
(attrition bias)

All outcomes

Summaryassessment Unclear risk No description

aa Langley 2011

Methods Randomised, placebo-controlled trial assessing the protective efficacy of a nasally administered meningococcal outer membrane protein adjuvanted trivalent influenza vaccine (OMP-TIV) against laboratory-confirmed influenza infection during the 2003 to 2004 influenza season in Canada in healthy adults.

Participants

Healthy adults aged 18 to 64 years who gave informed consent were eligible to participate (1349 were enrolled at 28 sites in Canada). Exclusion criteria: belonging to a group for which annual influenza vaccination is recommended; presence of significant acute or chronic, uncontrolled medical or psychiatric illness; pregnancy; infection with HIV, hepatitis B, or hepatitis C virus; chronic use of any medication or product for symptoms of rhinitis or nasal congestion or any chronic nasopharyngeal complaint or use of such product within 7 days prior to immunisation; asthma; symptoms or diagnosis suggesting gag reflex impairment or predisposition to aspiration; use of systemic glucocorticosteroids or immunosuppressive medications; receipt of investigational drugs in the prior month; presence of febrile or upper respiratory tract illness on the day of immunisation; and known hypersensitivity to mercurials or chicken eggs.

Interventions The vaccine contains equal parts of 3 monovalent egg-grown, formalin-inactivated influenza antigens formulated with OMPs of Neisseria meningitidis serogroup B strain 8047.

The vaccine tested in this study contained HA from each:

- A/New Caledonia/20/99 (H1N1)

- A/Panama/2007/99 (H3N2)

- B/Shangdong/7/97 (H1N1) (recommended for the 2003 to 2004 season)

Vaccine was tested in 2 formulations: 1 containing $75 \pm 15 \mu \mathrm{g} / \mathrm{mL}$ of HA from each of the 3 influenza strains and 1 with $150 \pm 30 \mu \mathrm{g} \mathrm{HA} / \mathrm{mL}$. Both formulations are sterile, colourless to yellowish opalescent, and preserved with $0.01 \%$ thimerosal.

The placebo control was sterile phosphate-buffered isotonic saline with $0.01 \%$ thimerosal and was colourless.

Participants $(n=1348)$ were randomised to 1 of the following 3 regimens:

- Arm 1: meningococcal OMP-adjuvanted TIV with $15 \mu \mathrm{g}$ of each HA antigen on days 0 and $14(n=455)$

- Arm 2: meningococcal OMP-adjuvanted TIV with $30 \mu \mathrm{g}$ of each HA antigen on day 0 and saline placebo on day $14(n=450)$

- Control: saline placebo on days 0 and $14(n=443)$ 
aa Langley 2011 (Continued)

Vaccine and placebo were administered by means of a VP3/100 nasal spray pump (Valois of America, Greenwich, CT, USA) with the participant in a sitting position, administering $0.10 \mathrm{~mL}$ of preparation in each nostril $(0.20 \mathrm{~mL}$ in all).

\section{Outcomes}

\section{Safety}

Participants were monitored for 30 minutes after the immunisation on days 0 and 14 for any immediate adverse events and then completed a questionnaire that graded selected complaints as 0 (none), grade 1 (mild), grade 2 (moderate), or grade 3 (severe). From days 0 to 7 , participants self monitored evening oral temperature and completed a written memory aid of reactogenicity. On days $3,7,17$, and 21 participants reported the maximum oral temperature and severity score in the previous days via an interactive voice response system. A clinic visit for participant assessment was initiated if symptom complaints exceeded grade 2. Prior to the day 14 dose participants were questioned about interim adverse events, and a physical exam was performed. Coding for adverse events was according to Medical Dictionary for Regulatory Activities (MeDRA, Chantilly, VA) version 6.1. The following outcomes were reported:

- Burning or stinging in the nose

- Burning or stinging in the throat

- Itching in the nose, throat, or eyes

- Shortness of breath

- Lightheadedness or dizziness

- New rash or a rash becoming itchy

- Feverishness: temperature $\left({ }^{\circ} \mathrm{C}\right)<37.8 ; 37.8$ to $38.2 ; 38.3$ to $38.9 ; \geq 39.0$

\section{Immunogenicity}

Blood and nasal mucus samples were collected on days 0 and 28 for haemagglutinin inhibition reciprocal titres and salivary secretory $\lg A(\operatorname{sg} A)$ measurement, respectively.

\section{Effectiveness}

Telephone contacts with participants were made every 2 weeks to solicit adverse events and identify ILI. Spontaneous illness reports were received via toll-free telephone call centre and reported to investigators. If the participant illness included at least 2 of the illness criteria and was severe enough to impede normal daily activities, then a nurse visit was initiated. The nurse verified symptoms, collected nose and throat swabs, and recorded the participant's temperature. Samples were cultured on MDCK cells, and a multiplex RT-PCR test was used to detect influenza A and B viruses (viruses A were subsequently subtyped by another RT-PCR assay). The primary outcome measure for efficacy was $\mathrm{CCl}$ defined as fever (oral temperature $>37.8^{\circ} \mathrm{C}$ ) and cough and at least 1 of the following: sore throat, runny nose or nasal congestion, muscle or joint ache, headache, fatigue or chills (with symptoms sufficient to impede normal daily activities), and a positive nose and throat swab culture for influenza A or B virus.

A co-primary endpoint measure was a positive culture, defined as positive nose and throat swab culture for influenza A or B virus and at least 2 of the following 8 symptoms: fever, cough, sore throat, runny nose or nasal congestion, muscle or joint ache, headache, fatigue, or chills.

The secondary outcome measure, ILI with evidence of influenza infection, required laboratory confirmation of influenza by either a positive culture for influenza A or B virus, or positive RT-PCR for influenza A or B virus, or a 4-fold rise in reciprocal titre for a circulating influenza strain between days 28 and 180 and fever and cough and at least 1 of sore throat, runny nose or nasal congestion, muscle or joint ache, headache, fatigue, or chills. participant who received at least 1 dose of test article $(n=1348,455$ in arm 1, 450 in arm 2, 443 in control arm).

For effectiveness estimates of culture positive and ILI, evaluable participants were used, i.e. those who had a complete regimen (i.e. 1 dose of placebo in the placebo group, at least 1 dose of $30 \mu \mathrm{g}, 2$ doses of $15 \mu \mathrm{g}, \mathrm{n}=1347)$.

A total of 1326 participants completed the study (452 in arm 1, 442 in arm 2, 432 in control arm).

Industry funded 
aa Langley 2011 (Continued)

Risk of bias

\begin{tabular}{|c|c|c|}
\hline Bias & Authors' judgement & Support for judgement \\
\hline $\begin{array}{l}\text { Random sequence genera- } \\
\text { tion (selection bias) }\end{array}$ & Unclear risk & "The study was double-blind, randomised and placebo controlled." \\
\hline $\begin{array}{l}\text { Allocation concealment } \\
\text { (selection bias) }\end{array}$ & Low risk & $\begin{array}{l}\text { "Subjects were assigned centrally within blocks and stratified within each site } \\
\text { by age } \leq 49 \text { and }>49 \text { years, and history of prior influenza immunization within } 2 \\
\text { years." }\end{array}$ \\
\hline $\begin{array}{l}\text { Blinding (performance } \\
\text { bias and detection bias) } \\
\text { All outcomes }\end{array}$ & Low risk & $\begin{array}{l}\text { "Neither the subject nor the site study team (staff performing clinical safety or } \\
\text { efficacy evaluations and investigators) were aware of patient assignment. One } \\
\text { research nurse at each site was responsible for randomization, maintenance of } \\
\text { the treatment log, test article preparation and administration." } \\
\text { "This staff member did not perform any safety or efficacy observations and } \\
\text { could not reveal treatment assignment to participants or other study staff." } \\
\text { "Both lots are sterile, colorless to yellowish opalescent and preserved with } \\
0.01 \% \text { thimerosal. The placebo control was sterile phosphate-buffered isoton- } \\
\text { ic saline with } 0.01 \% \text { thimerosal, and was colorless." }\end{array}$ \\
\hline $\begin{array}{l}\text { Incomplete outcome data } \\
\text { (attrition bias) } \\
\text { All outcomes }\end{array}$ & Low risk & About $98 \%$ of the initially enrolled participants completed the study. \\
\hline Summary assessment & Low risk & Low risk of bias \\
\hline
\end{tabular}

\section{aa Leibovitz 1971}

Methods

Controlled clinical trial conducted in the USA during the 1969 to 1970 influenza season. The study period was 30 January to 18 May. Follow-up lasted first 7 weeks of training. Influenza was detected from 11 February to 13 May and lasted 6 weeks. Participants were allocated to vaccine or control group according to the last non-zero digit of the Social Security number. Blinding was not mentioned. Specimens for culture and acute-convalescent blood specimens were obtained from people hospitalised with acute respiratory disease.

Participants 9616 military trainees: 1682 treated and 7934 placebo. Age of participants was 18 to 20.

Interventions

Monovalent inactivated, experimental, intramuscularly administered vaccine. Schedule and dose were: single dose, 556 CCA. Recombinant virus derived from HK/Aichi/68 and A0/PR8/34 was compared against no vaccination. Vaccine was not recommended but matched circulating strain.

Outcomes

Outcomes were: hospitalisation for upper respiratory infection (without definition), hospitalisation for influenza. Laboratory confirmation was based on culture and/or 4-fold or greater rise in antibody titre occurring between acute and convalescent sera. Surveillance was passive.

Notes Recruitment and immunisation period overlapped outbreak period. Most of the illnesses were due to adenovirus. Illnesses during the first 1 or 2 weeks after vaccination were not excluded, but the authors stated that this fact did not affect the results. Efficacy data only were extracted.

Government funded

\section{Risk of bias}


aa Leibovitz 1971 (Continued)

Random sequence genera- Unclear risk Unclear tion (selection bias)

Allocation concealment High risk Inadequate
(selection bias)

Blinding (performance Unclear risk Unclear

bias and detection bias)

All outcomes

Incomplete outcome data Unclear risk Unclear
(attrition bias)
All outcomes

\begin{tabular}{lll}
\hline Summaryassessment & High risk & Unclear \\
\hline
\end{tabular}

aa Mcbride 2016a

\begin{tabular}{|c|c|}
\hline Methods & Randomised placebo controlled trial \\
\hline Participants & Healthy adults aged 18 to 64 \\
\hline \multirow[t]{3}{*}{ Interventions } & $\begin{array}{l}\text { Trivalent influenza inactivated vaccines containing antigens the } 2 \mathrm{~A} \text { strains and } 1 \mathrm{~B} \text { strain recommend- } \\
\text { ed by WHO in } 2008 \text { and } 2009 \text { for the Southern Hemisphere as follows: }\end{array}$ \\
\hline & $\begin{array}{l}\text { Arm 1: } 15 \mathrm{mg} \text { of haemagglutinin antigens Solomon Islands/3/2006 (H1N1), A/Brisbane/10/2007 (H3N2), } \\
\text { B/Brisbane/3/2007. Fluvax; CSL Limited. } 0.5 \mathrm{~mL} \text { single doses administered intramuscularly into deltoid } \\
\text { muscle. }\end{array}$ \\
\hline & Placebo: $0.5 \mathrm{~mL}$ saline, dibasic sodium phosphate and monobasic sodium phosphate. \\
\hline Outcomes & $\begin{array}{l}\text { Influenza cases laboratory confirmed by viral culture and/or real time RT-PCR were followed up until } 30 \\
\text { November each year. Solicited adverse events for } 4 \text { days, unsolicited adverse events for } 20 \text { days, seri- } \\
\text { ous adverse events for } 180 \text { days. Harms data were not extractable due to different definitions. }\end{array}$ \\
\hline Notes & This record is for the 2008 season. Industry funded \\
\hline
\end{tabular}

\section{Risk of bias}

\begin{tabular}{lll} 
Bias & Authors' judgement & Support for judgement \\
\hline $\begin{array}{l}\text { Random sequence genera- } \\
\text { tion (selection bias) }\end{array}$ & Low risk & "Participants were randomized in a 2:1 ratio to receive a single injection of 0.5 \\
& $\mathrm{~mL}$ IIV3 or placebo, administered intramuscularly into the deltoid muscle." \\
& "The randomization code was prepared by a statistician, employed by CSL \\
& Limited, with the use of SAS software (version 9.1.3; SAS Institute, Cary, NC, \\
& USA ..."
\end{tabular}

\begin{tabular}{ll}
\hline $\begin{array}{l}\text { Allocation concealment } \\
\text { (selection bias) }\end{array}$ & "Tow risk \\
& Limited, with the use of SAS software (version 9.1.3; SAS Institute, Cary, NC, \\
& $\begin{array}{l}\text { USA), using simple block randomization to maintain approximate allocation } \\
\text { balance." }\end{array}$
\end{tabular}

\begin{tabular}{|c|c|c|}
\hline $\begin{array}{l}\text { Blinding (performance } \\
\text { bias and detection bias) } \\
\text { All outcomes }\end{array}$ & Unclear risk & $\begin{array}{l}\text { "As there was a visual difference between IIV3 and placebo, study personnel } \\
\text { who were involved in the preparation and administration of the study vaccine } \\
\text { had no further involvement in the study conduct. Participants and investiga- }\end{array}$ \\
\hline
\end{tabular}




\section{Incomplete outcome data Low risk}

(attrition bias)

All outcomes
Flow of participants during the study is reported and described. Loss to follow-up amounts to $0.36 \%$ and $0.44 \%$ study end and among vaccine and placebo recipients, respectively, and is balanced through the 2 arms.

Summary assessment Low risk

aa Mcbride 2016b

\begin{tabular}{ll}
\hline Methods & Randomised placebo-controlled trial \\
\hline Participants & Healthy adults aged 18 to 64
\end{tabular}

Trivalent influenza inactivated vaccines containing antigens the 2 A strains and 1 B strain recommend-
ed by WHO in 2008 and 2009 for the Southern Hemisphere as follows:
Arm 1: $15 \mathrm{mg}$ of haemagglutinin antigens A/Brisbane/59/2007 (H1N1), A/Brisbane/10/2007 (H3N2), B/
Florida/4/2006. Fluvax; CSL Limited. $0.5 \mathrm{~mL}$ single doses administered intramuscularly into deltoid
muscle.
Placebo: $0.5 \mathrm{~mL}$ saline, dibasic sodium phosphate and monobasic sodium phosphate.

\begin{tabular}{ll}
\hline Outcomes & Influenza cases laboratory-confirmed by viral culture and/or real time RT-PCR were followed up until \\
& 30 November each year. Solicited adverse events for 4 days, unsolicited adverse events for 20 days, se- \\
rious adverse events for 180 days. Harms data were not extractable due to different definitions.
\end{tabular}

\begin{tabular}{ll}
\hline Notes & This record is for the 2009 season. Industry funded \\
\hline Risk of bias &
\end{tabular}

\begin{tabular}{|c|c|c|}
\hline Bias & Authors' judgement & Support for judgement \\
\hline \multirow[t]{2}{*}{$\begin{array}{l}\text { Random sequence genera- } \\
\text { tion (selection bias) }\end{array}$} & Low risk & $\begin{array}{l}\text { "Participants were randomized in a 2:1 ratio to receive a single injection of } 0.5 \\
\text { mL IIV3 or placebo, administered intramuscularly into the deltoid muscle." }\end{array}$ \\
\hline & & $\begin{array}{l}\text { "The randomization code was prepared by a statistician, employed by CSL } \\
\text { Limited, with the use of SAS software (version 9.1.3; SAS Institute, Cary, NC, } \\
\text { USA ..." }\end{array}$ \\
\hline $\begin{array}{l}\text { Allocation concealment } \\
\text { (selection bias) }\end{array}$ & Low risk & $\begin{array}{l}\text { "The randomization code was prepared by a statistician, employed by CSL } \\
\text { Limited, with the use of SAS software (version 9.1.3; SAS Institute, Cary, NC, } \\
\text { USA), using simple block randomization to maintain approximate allocation } \\
\text { balance." }\end{array}$ \\
\hline $\begin{array}{l}\text { Blinding (performance } \\
\text { bias and detection bias) } \\
\text { All outcomes }\end{array}$ & Unclear risk & $\begin{array}{l}\text { "As there was a visual difference between IIV3 and placebo, study personnel } \\
\text { who were involved in the preparation and administration of the study vaccine } \\
\text { had no further involvement in the study conduct. Participants and investiga- } \\
\text { tional site staff involved in performing study assessments remained blinded to } \\
\text { treatment allocation." }\end{array}$ \\
\hline $\begin{array}{l}\text { Incomplete outcome data } \\
\text { (attrition bias) }\end{array}$ & Unclear risk & $\begin{array}{l}\text { Flow of participants is reported and described. Attrition was } 2.52 \% \text { and } 1.6 \% \\
\text { for the intervention and placebo arms, respectively. In this season (2009) }\end{array}$ \\
\hline All outcomes & & $\begin{array}{l}104 / 5001 \text { and } 40 / 2499 \text { received } \mathrm{H} 1 \mathrm{~N} 1 \text { pandemic vaccine, respectively in the } 2 \\
\text { arms and were excluded from efficacy assessment. }\end{array}$ \\
\hline
\end{tabular}


aa Mcbride 2016b (Continued)

Summary assessment Low risk

aa Mesa Duque 2001

Methods Randomised controlled trial, double-blind, conducted in Colombia during the 1997 influenza season. Follow-up lasted from 15 March to 31 August. Influenza period was not defined. Virological surveillance was not performed.

\begin{tabular}{ll}
\hline Participants & 493 bank employees: 247 treated and 246 placebo. Age of participants was 18 to 60. \\
\hline Interventions & $\begin{array}{l}\text { Subunit inactivated, intramuscularly administered vaccine. Schedule and dose were: single dose. Vac- } \\
\text { cine composition was: A/Wahan/359/95, A/Texas/36/91, and B/Beijing/184/93. Placebo was vitamin C. } \\
\text { Vaccine was recommended and matched circulating strain. }\end{array}$
\end{tabular}

Episodes of clinical illness, WDL, and adverse effects. Clinical disease was defined as upper respirato-
ry illness (fever, sore throat, and cough lasting more than 24 hours) according to ICD-9 codes 381,382,
$460,466,480$ and from 487 to 490 . Local adverse effects were oedema, erythema, pain, and swelling.
Systemic adverse effects were fever, headache, and indisposition within 5 days of vaccination. Surveil-
lance was passive.

\begin{tabular}{|c|c|}
\hline \multirow[t]{2}{*}{ Notes } & $\begin{array}{l}\text { Circulating strains were not isolated from local cases but by WHO and Colombia surveillance system } \\
\text { and matched vaccine components. Working days lost were detected all year round, so they were not in- } \\
\text { cluded in the analysis. Efficacy and safety data were extracted. }\end{array}$ \\
\hline & Government funded \\
\hline
\end{tabular}

\section{Risk of bias}

\begin{tabular}{|c|c|c|}
\hline Bias & Authors' judgement & Support for judgement \\
\hline $\begin{array}{l}\text { Random sequence genera- } \\
\text { tion (selection bias) }\end{array}$ & Low risk & $\begin{array}{l}\text { Volunteers were randomly allocated to receive vaccine or placebo using a ta- } \\
\text { ble of random numbers. }\end{array}$ \\
\hline $\begin{array}{l}\text { Allocation concealment } \\
\text { (selection bias) }\end{array}$ & Low risk & $\begin{array}{l}\text { Given details provided regarding randomisation process and other aspects of } \\
\text { the study design, we believe the allocation concealment was probably ade- } \\
\text { quate. }\end{array}$ \\
\hline $\begin{array}{l}\text { Blinding (performance } \\
\text { bias and detection bias) } \\
\text { All outcomes }\end{array}$ & Low risk & Double-blinding was ensured by pre-labelled, coded, identical-looking vials. \\
\hline $\begin{array}{l}\text { Incomplete outcome data } \\
\text { (attrition bias) } \\
\text { All outcomes }\end{array}$ & Low risk & Adequate \\
\hline Summary assessment & Low risk & Low risk \\
\hline
\end{tabular}

aa Mixéu 2002

$\begin{array}{ll}\text { Methods } & \text { Randomised controlled trial, double-blind, conducted in Brazil during the } 1997 \text { influenza season. Fol- } \\ & \text { low-up lasted } 6 \text { to } 7 \text { months. Influenza period was not defined. Virologic surveillance was not per- } \\ \text { formed. }\end{array}$


aa Mixéu 2002 (Continued)

Participants

813 flight crews of an airline company: 405 vaccinated and 408 given placebo. Age of participants was 18 to 64 .

Interventions

Split trivalent, intramuscularly administered vaccine. Schedule and dose were: single dose. Vaccine composition was: A/Nanchang/933/95, A/Texas/36/91, and B/Harbin/7/94. Placebo was vaccine diluent. Vaccine was recommended and matched circulating strain.

Outcomes ILI, WDL. Clinical illness was defined as follows: fever $>37.6{ }^{\circ} \mathrm{C}$ and cough, headache, $\mathrm{myalgia}$, rhinorrhoea, sore throat lasting at least 24 hours. Surveillance was passive.

Notes

Local and systemic effects were reported together and therefore not included in the review. Only 294 treated participants and 299 controls completed follow-up. Efficacy data were extracted.

Industry funded

\section{Risk of bias}

\begin{tabular}{|c|c|c|}
\hline Bias & Authors' judgement & Support for judgement \\
\hline $\begin{array}{l}\text { Random sequence genera- } \\
\text { tion (selection bias) }\end{array}$ & Unclear risk & Authors did not describe the methods used to ensure randomisation. \\
\hline $\begin{array}{l}\text { Allocation concealment } \\
\text { (selection bias) }\end{array}$ & Unclear risk & Authors did not describe the methods used to ensure randomisation. \\
\hline $\begin{array}{l}\text { Blinding (performance } \\
\text { bias and detection bias) } \\
\text { All outcomes }\end{array}$ & Unclear risk & Authors did not describe the methods used to ensure blinding. \\
\hline $\begin{array}{l}\text { Incomplete outcome data } \\
\text { (attrition bias) } \\
\text { All outcomes }\end{array}$ & Low risk & Low rates of attrition unlikely to affect study results. \\
\hline Summary assessment & Unclear risk & Unclear \\
\hline
\end{tabular}

aa Mogabgab 1970a

Methods $\quad$ Randomised study conducted in the USA during the 1968 to 1969 influenza season. Influenza outbreak lasted 9 weeks, from 9 December to 3 February. Randomisation methods were not described. Laboratory confirmation was obtained (by culture or 4-fold antibody titre increase in acute convalescent sera) for 20 men randomly selected each week from among the ill.

\begin{tabular}{ll}
\hline Participants & 1402 airmen previously unvaccinated: 881 vaccinated and 521 given placebo. Age of participants was \\
& 18 to 21.
\end{tabular}
18 to 21 .

Interventions

Monovalent inactivated parenteral influenza A vaccine. Schedule and dose were: single dose. Vaccine composition was: A2/Aichi 2/68 300 CCA units. Placebo was saline for injection. Vaccine was recommended and matched circulating strain.

Outcomes ILI and influenza, complications and admissions. All respiratory illnesses were classified as febrile (38.3 ${ }^{\circ} \mathrm{C}$ or greater), afebrile, pharyngitis, bronchitis, or pneumonia (complications). Surveillance was passive.

Notes

Cases occurring during the first 15 days after vaccination were not included in the analysis. Circulating strain was A2/Hong Kong. Efficacy data were extracted. 
aa Mogabgab 1970a (Continued)

Government funded

\section{Risk of bias}

\begin{tabular}{lll}
\hline Bias & Authors' judgement & Support for judgement \\
\hline $\begin{array}{l}\text { Random sequence genera- } \\
\text { tion (selection bias) }\end{array}$ & Unclear risk & Unclear \\
\hline $\begin{array}{l}\text { Allocation concealment } \\
\text { (selection bias) }\end{array}$ & Unclear risk & Unclear \\
\hline $\begin{array}{l}\text { Blinding (performance } \\
\text { bias and detection bias) } \\
\text { All outcomes }\end{array}$ & Unclear risk & Unclear \\
\hline $\begin{array}{l}\text { Incomplete outcome data } \\
\text { (attrition bias) } \\
\text { All outcomes }\end{array}$ & Unclear risk & Unclear \\
\hline $\begin{array}{l}\text { Summary assessment } \\
\end{array}$ & Unclear risk & Unclear \\
\hline
\end{tabular}

\section{aa Mogabgab 1970b}

Methods Randomised study conducted in the USA during the 1968 to 1969 influenza season. Influenza outbreak lasted 9 weeks, from 9 December to 3 February. Randomisation methods were not described. Laboratory confirmation was obtained (by culture or 4-fold antibody titre increase in acute convalescent sera) for 20 men randomly selected each week from among the ill.

Participants 1551 airmen previously unvaccinated: 1030 vaccinated and 521 given placebo. Age of participants was 18 to 21 .

$\begin{array}{ll}\text { Interventions } & \text { Polyvalent inactivated influenza A and B vaccine (the } 1967 \text { military formula). Schedule and dose were: } \\ & \text { single dose. Vaccine composition was: A/Swine/33 } 100 \text { CCA units, A/PR8/34 100 CCA units, A1/AA/1/57 } \\ & 100 \text { CCA units, A2/Taiwan 1/64 } 400 \text { CCA units, B/Lee/40 } 100 \text { CCA units, B/Mass 3/66 200 CCA units. Place- } \\ \text { bo was saline for injection. Vaccine was recommended but did not match the circulating strain. }\end{array}$

Outcomes

ILI and influenza cases, complications and admissions. All respiratory illnesses were classified as febrile $\left(38.3^{\circ} \mathrm{C}\right.$ or greater), afebrile, pharyngitis, bronchitis, or pneumonia (complications). Surveillance was passive.

Notes Cases occurring during the first 15 days after vaccination were not included in the analysis. Circulating strain was A2/Hong Kong. Efficacy data were extracted.

Government funded

\section{Risk of bias}

\begin{tabular}{lll}
\hline Bias & Authors' judgement & Support for judgement \\
\hline $\begin{array}{l}\text { Random sequence genera- } \\
\text { tion (selection bias) }\end{array}$ & Unclear risk & Unclear \\
\hline $\begin{array}{l}\text { Allocation concealment } \\
\text { (selection bias) }\end{array}$ & Unclear risk & Unclear \\
\hline
\end{tabular}


aa Mogabgab 1970b (Continued)
Blinding (performance
Unclear risk
Unclear
bias and detection bias)

All outcomes

\begin{tabular}{lll}
\hline $\begin{array}{l}\text { Incomplete outcome data } \\
\text { (attrition bias) }\end{array}$ & Unclear risk \\
All outcomes & & \\
\hline Summary assessment & Unclear risk & Unclear \\
\hline
\end{tabular}

\section{aa Monto 1982}

$\begin{array}{ll}\text { Methods } & \text { Randomised, single-blind study conducted in the USA during the } 1979 \text { to } 1980 \text { influenza season. Fol- } \\ \text { low-up lasted for the whole epidemic period. The epidemic period was defined by first and last isola- } \\ \text { tion (11 February to } 18 \text { March) and lasted } 5 \text { weeks. Each participant was given a serial number that had } \\ \text { previously been assigned randomly by a code to either the vaccine or the placebo group. Specimens for } \\ \text { culture were obtained from ill people. At spring time blood specimens were collected. }\end{array}$

\begin{tabular}{|c|c|}
\hline Participants & 306 students: 154 vaccinated and 152 given placebo. Age of participants was not reported. \\
\hline Interventions & $\begin{array}{l}\text { Monovalent, live attenuated, intranasal influenza B. Schedule and dose were: single dose. Vaccine } \\
\text { composition was: the vaccine virus, cold recombinant, was produced by recombining the attenuated } \\
\text { B/Ann Arbor/ } 1 / 66 \text { with a wild strain B/Hong Kong/8/73. Placebo was vaccine diluent. Vaccine was not } \\
\text { recommended and did not match the circulating strain. }\end{array}$ \\
\hline Outcomes & $\begin{array}{l}\text { Clinical and laboratory confirmed cases and adverse effects. Participants suffered a respiratory illness } \\
\text { if they had at least } 2 \text { respiratory symptoms. Cases were laboratory confirmed if they had an increase in } \\
\text { antibody titre against } 3 \text { influenza B virus antigens, i.e. if there was a } 4 \text {-fold increase from an initial sam- } \\
\text { ple. Side effects were sore throat, coryza, hoarseness, cough, muscle aches, temperature }>100^{\circ} \mathrm{F} \text { oc- } \\
\text { curring during the first } 3 \text { days after vaccination. Surveillance was active. }\end{array}$ \\
\hline \multirow[t]{2}{*}{ Notes } & $\begin{array}{l}\text { Vaccine content was not recommended or matched. Circulating strain was B/Singapore/79-like and B/ } \\
\text { Buenos Aires/79-like. } \\
\text { Efficacy and safety data were extracted. }\end{array}$ \\
\hline & Government funded \\
\hline
\end{tabular}

\section{Risk of bias}

\begin{tabular}{|c|c|c|}
\hline Bias & Authors' judgement & Support for judgement \\
\hline $\begin{array}{l}\text { Random sequence genera- } \\
\text { tion (selection bias) }\end{array}$ & Low risk & Adequate \\
\hline $\begin{array}{l}\text { Allocation concealment } \\
\text { (selection bias) }\end{array}$ & Low risk & Adequate \\
\hline $\begin{array}{l}\text { Blinding (performance } \\
\text { bias and detection bias) } \\
\text { All outcomes }\end{array}$ & Low risk & Adequate \\
\hline $\begin{array}{l}\text { Incomplete outcome data } \\
\text { (attrition bias) } \\
\text { All outcomes }\end{array}$ & Low risk & Adequate \\
\hline Summary assessment & Low risk & Adequate \\
\hline
\end{tabular}


aa Monto 2009

Methods Third epidemic season (2007 to 2008) of aa Ohmit 2006 and aa Ohmit 2008
$\begin{array}{ll}\text { Participants } & \text { A total of } 1952 \text { healthy adults between the ages of } 18 \text { and } 49 \text { years were enrolled. Some had been also } \\ \text { enrolled in the } 2 \text { previous seasons. }\end{array}$

Interventions Newly enrolled participants were recruited from the community around 4 university campuses in Michigan. Allocation methods are the same as for aa Ohmit 2006 and aa Ohmit 2008.

For the 2007 to 2008 season vaccine composition was the following:

- Fluzone (Sanofi Pasteur, inactivated trivalent vaccine intramuscular): $15 \mu \mathrm{g}$ of haemagglutinin from each of the following strains in a $0.5 \mathrm{~mL}$ dose: A/Solomon Islands/3/2006 (H1N1), A/Wisconsin/67/2005 (H3N2), and B/Malaysia/2506/2004 (B/Victoria lineage).

- FluMist (MedImmune, live attenuated vaccine, intranasal): 106.5-7.5 fluorescent focus units of live attenuated influenza virus reassortants of the same strains as used for the inactivated formulation in a $0.2 \mathrm{~mL}$ dose.

\begin{tabular}{ll}
\hline Outcomes & Same outcomes as aa Ohmit 2008 \\
\hline Notes & Funding source - mixed
\end{tabular}

\section{Risk of bias}

Bias Authors' judgement Support for judgement

Random sequence genera- Unclear risk Unclear

tion (selection bias)

Allocation concealment Unclear risk Unclear
(selection bias)

\begin{tabular}{lll}
\hline $\begin{array}{l}\text { Blinding (performance } \\
\text { bias and detection bias) }\end{array}$ & Unclear risk \\
All outcomes & \\
\hline $\begin{array}{l}\text { Incomplete outcome data } \\
\begin{array}{l}\text { (attrition bias) } \\
\text { All outcomes }\end{array}\end{array}$ & Unclear risk \\
\hline Summary assessment & Unclear risk & Unclear \\
\hline
\end{tabular}

\begin{tabular}{ll} 
aa Nichol 1995 & $\begin{array}{l}\text { Randomised controlled trial conducted in the USA during the } 1994 \text { to } 1995 \text { influenza season. Follow-up } \\
\text { lasted from } 1 \text { December } 1994 \text { through to } 31 \text { March } 1995 . \text { Influenza period was not defined. Virological } \\
\text { surveillance was not performed. }\end{array}$ \\
\hline Participants & 841 full-time employed: 419 treated and 422 placebo. Age of participants was 18 to 64. \\
\hline Interventions & $\begin{array}{l}\text { Subvirion, trivalent, parenteral influenza A and B vaccine. Schedule and dose were: single dose; } 15 \mu g \\
\text { each strain. Vaccine composition was: A/Texas/36/91, A/Shangdong/9/93, B/Panama/45/90. Placebo } \\
\text { was vaccine diluent. Vaccine was recommended and matched circulating strain. }\end{array}$ \\
\hline
\end{tabular}


aa Nichol 1995 (Continued)

Outcomes

Cases (symptom-defined), working days lost due to respiratory illness, side effects. Participants were defined as cases if they had at least 1 upper respiratory illness (a sore throat associated with either fever or cough that lasted at least 24 hours). Local adverse effects were defined as arm soreness. Systemic adverse effects were defined as fever, tiredness, "feeling under the weather", muscle ache, headache (within a week after vaccination). Surveillance was active.

Notes

Circulating strain was not indicated. Efficacy and safety data were extracted.

Industry funded

\section{Risk of bias}

\begin{tabular}{lll}
\hline Bias & Authors' judgement & Support for judgement \\
\hline $\begin{array}{l}\text { Random sequence genera- } \\
\text { tion (selection bias) }\end{array}$ & Low risk & $\begin{array}{l}\text { Randomisation was performed according to a computer-generated randomi- } \\
\text { sation schedule. }\end{array}$ \\
\hline $\begin{array}{l}\text { Allocation concealment } \\
\text { (selection bias) }\end{array}$ & Low risk & Probably adequate \\
\hline $\begin{array}{l}\text { Blinding (performance } \\
\text { bias and detection bias) } \\
\text { All outcomes }\end{array}$ & Low risk & Double-blinding was ensured by preloaded, coded, identical-looking syringes. \\
\hline $\begin{array}{l}\text { Incomplete outcome data } \\
\begin{array}{l}\text { (attrition bias) } \\
\text { All outcomes }\end{array}\end{array}$ & Low risk & Adequate \\
\hline $\begin{array}{l}\text { Summary assessment } \\
\text { Low risk }\end{array}$ & Adequate \\
\hline
\end{tabular}

\section{aa Nichol 1999a}

Methods

Randomised controlled trial conducted in the USA during the 1997 to 1998 influenza season. Follow-up lasted from November to March. Site-specific peak outbreak period was defined as weeks including $80 \%$ of the isolates of a specific area. Total outbreak period lasted from 14 December 1997 through to 21 March 1998. Total outbreak period was included in the analysis and lasted 14 weeks. Participants were recruited from 7 organisations and assigned to 1 of the study groups using a permuted block randomisation scheme that was stratified by treatment centre and age group. Sealed randomisation envelopes contained vaccine codes. Influenza virus surveillance was carried out in the area.

Participants 4561 healthy working adults: 3041 treated and 1520 placebo. Age of participants was 18 to 64 .

Interventions

Trivalent, live attenuated influenza $A$ and $B$ vaccine in a single dose. Vaccine composition was: $A /$ Shenzhen/227/95, A/Wuhan/395/95, B/Harbin/7/94-like. Placebo was egg allantoic fluid. Vaccine was recommended but did not match the circulating strain.

Outcomes Clinical cases (symptom-defined), working days lost, and adverse effects. Case definition had 3 specifications: febrile illness (fever for at least 1 day and 2 or more symptoms for at least 2 days: fever, chills, headache, cough, runny nose, sore throat, muscle aches, tiredness); severe febrile illness (3 days of symptoms and 1 day of fever); febrile upper respiratory tract illness (3 days of upper respiratory tract symptoms and 1 day of fever). We chose the febrile illness outcome for analysis. Systemic adverse effects were defined as headache, muscle aches, chills, tiredness, and fever. Surveillance was passive.

\section{Notes}


aa Nichol 1999a (Continued)

sented in a way that allows us to compute the difference in mean days lost but not to compute the standard error. Circulating strain was A/Sydney/5/97-like. Efficacy and safety data were extracted.

Government and industry funded

\section{Risk of bias}

\begin{tabular}{|c|c|c|}
\hline Bias & Authors' judgement & Support for judgement \\
\hline $\begin{array}{l}\text { Random sequence genera- } \\
\text { tion (selection bias) }\end{array}$ & Low risk & Adequate \\
\hline $\begin{array}{l}\text { Allocation concealment } \\
\text { (selection bias) }\end{array}$ & Low risk & Adequate \\
\hline $\begin{array}{l}\text { Blinding (performance } \\
\text { bias and detection bias) } \\
\text { All outcomes }\end{array}$ & Low risk & Adequate \\
\hline $\begin{array}{l}\text { Incomplete outcome data } \\
\text { (attrition bias) } \\
\text { All outcomes }\end{array}$ & Low risk & Adequate \\
\hline Summary assessment & Low risk & Adequate \\
\hline
\end{tabular}

aa Ohmit 2006

Multicentre, randomised, placebo-controlled trial assessing effectiveness of both inactivated and live
attenuated vaccines in preventing laboratory-confirmed influenza in healthy adults aged below 50.

Participants

For enrolment in the first study year (2004 to 2005), participants were recruited at 4 centres ( 2 university and 2 community sites) in Michigan. Participants were healthy adults between the ages of 18 and 46 years; those for whom influenza vaccination was recommended or contraindicated were excluded. In all 1247 were enrolled.

Interventions

After informed consent was obtained and a first serum sample drawn, enrolled participants were randomly allocated to receive 1 dose of the following:

- Inactivated trivalent vaccine (Fluzone, Sanofi Pasteur) containing $15 \mu \mathrm{g}$ of haemagglutinin from each of the following strains: A/New Caledonia/20/99 (H1N1), A/Wyoming/3/2003 (H3N2, A/Fujian/411/2002-like strain), and B/Jiangsu/10/2003 (B/Shanghai/361/2002-like strain (Yamagata lineage)) in each $0.5 \mathrm{~mL}$ dose, as intramuscular injection.

- Placebo saline administered intramuscularly.

- Live attenuated trivalent vaccine (FluMist, MedImmune) containing a 106.5-7.5 median tissue-culture infective dose of live attenuated influenza virus reassortants of the following strains: $A / N e w$ Caledonia/20/99 (H1N1), A/Wyoming/ 3/2003 (H3N2 A/Fujian/411/2002-like strain), and B/Jilin/20/2003 (B/ Shanghai/361/2002-like strain (Yamagata lineage)) in each $0.5 \mathrm{~mL}$ dose.

- Placebo saline administered intranasally.

Identical syringes were filled on site with the inactivated vaccine or matching placebo (physiologic saline) by study nurses who were aware of the intervention assignments. The live attenuated influenza vaccine and matching placebo (physiologic saline) were preloaded in identical nasal spray devices by the manufacturer. Both vaccines were licenced for use in the 2004 to 2005 influenza season.

Participants were randomised to vaccine or placebo in ratio of 5:1 using 4 site-specific randomisation schedules, generated with the use of a random permuted block design with a block size of 12, in order to assign participants sequentially to receive a vaccine or a placebo as they enrolled. 
aa Ohmit 2006 (Continued)

Since the trial was double-blind, the participants and nurses who administered the study vaccine or placebo were unaware of whether the participant was receiving vaccine or placebo but were aware of the route of administration.

Further serum samples were drawn 3 to 5 weeks after vaccine administration (as participants returned diary cards for local and systemic reactions, preseason sample) and during April to May 2005 (postseason sample).

Outcomes

Local and systemic reactions within 7 days from immunisation (self filled questionnaires): fever, chills, runny nose or congestion, cough, sore throat, headache, muscle aches, weakness, abdominal pain, trouble breathing, red eyes, arm soreness, arm redness.

Laboratory-confirmed influenza. Active surveillance was maintained between November 2004 and April 2005. Participants were contacted by phone or email twice monthly. Symptomatic influenza was described as the presence of at least 1 respiratory symptom (cough or nasal congestion) and at least 1 systemic symptom (fever, feverishness, chills, body aches) occurring during influenza activity and at least 2 weeks after administration. Participants were instructed to contact study staff when at least 2 respiratory and systemic symptoms were observed. Throat swab specimens were collected from all participants with symptomatic influenza.

Swabs were cultured for identification, and all isolates were typed according to strain using the fluorescence antibody assay and evaluated for antigenic relatedness to vaccine strains by the Influenza Branch at the CDC. In addition, all throat-swab specimens obtained from participants with symptomatic influenza were tested at the University of Michigan by means of real-time PCR assays using the TaqMan system (Applied Biosystems).

All collected serum samples were tested with the haemagglutination-inhibition assay, with the virus strains present in the vaccines used as antigens and against the circulating type $A$ (H3N2) (A/Califor$\mathrm{nia} / 07 / 2004-$ like) virus and the circulating type B (B/Hawaii/33/2004-like) virus (i.e. Victoria lineage not included in the vaccine).

For effectiveness the following endpoints were used:

On ITT population: laboratory-confirmed influenza: culture-positive or real-time PCR-positive, or both.

On per-protocol population: laboratory-confirmed influenza: serologically positive; serologically or culture-positive. placebo IN = 103).

Per-protocol analyses: limited to participants having the postintervention (preseason) blood specimen collected at least 3 weeks after receipt of a vaccine or a placebo and at least 2 weeks before the beginning of local influenza activity (TIV = 367; placebo IM = 73; LAIV = 363; placebo IN = 73).

Government funded

\section{Risk of bias}

\begin{tabular}{lll}
\hline Bias & Authors' judgement & Support for judgement \\
\hline $\begin{array}{l}\text { Random sequence genera- } \\
\text { tion (selection bias) }\end{array}$ & Low risk & Centralised automated sequence generation \\
\hline $\begin{array}{l}\text { Allocation concealment } \\
\text { (selection bias) }\end{array}$ & Unclear risk & Allocation procedure not described \\
\hline $\begin{array}{l}\text { Blinding (performance } \\
\text { bias and detection bias) } \\
\text { All outcomes }\end{array}$ & Low risk & Blinding apparently successful \\
\hline
\end{tabular}


aa Ohmit 2006 (Continued)

Incomplete outcome data Low risk Active surveillance carried out Participants contacted bi monthly (attrition bias)

All outcomes

Summary assessment Unclear risk Unclear

aa Ohmit 2008

Multicentre, randomised, placebo-controlled trial assessing the effectiveness of both inactivated and
live attenuated vaccines in preventing laboratory-confirmed influenza in healthy adults aged below 50
years. Same methods as aa Ohmit 2006.
years. Same methods as aa Ohmit 2006.

\section{Participants}

For study year 2005 to 2006, healthy men and women aged 18 to 48 years were recruited at 6 study sites (4 university sites and 2 community sites) in Michigan. In all 2058 participants were enrolled. Of these, 972 were already enrolled in the 2004 to 2005 season (see aa Ohmit 2006).

Interventions

Participants who were enrolled in the 2005 to 2006 season were randomised (see aa Ohmit 2006) to receive inactivated vaccine (Fluzone; Sanofi Pasteur), live attenuated vaccine (FluMist; MedImmune), or placebo. Participants already enrolled in the 2004 to 2005 season received the same intervention type (i.e. Fluzone, FluMist, or placebo) as before.

- Fluzone (intramuscularly administered) contained $15 \mathrm{~g}$ haemagglutinin from each of the following strains: A/New Caledonia/20/99 (H1N1), A/New York/55/2004 (H3N2) (A/California/7/2004-like), and B/ Jiangsu/10/2003 (B/Shanghai/361/2002-like).

- FluMist (intranasally administered) was formulated to contain a median tissue-culture infective dose of $10^{6.5}$ to $10^{7.5}$ live attenuated influenza virus reassortants of the same strains.

- Intramuscular or intranasal saline placebo.

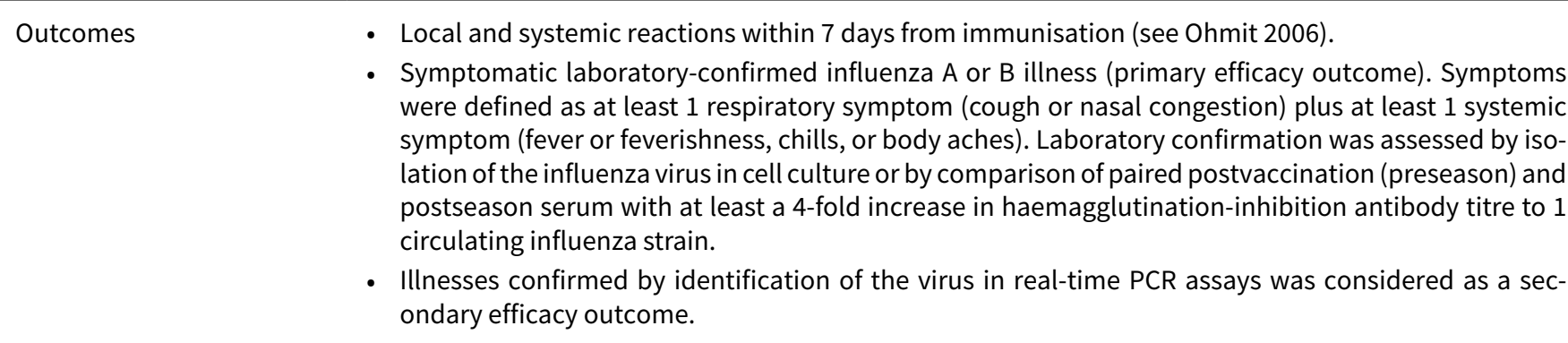

Notes Government funded

\section{Risk of bias}

\begin{tabular}{lll}
\hline Bias & Authors' judgement & Support for judgement \\
\hline $\begin{array}{l}\text { Random sequence genera- } \\
\text { tion (selection bias) }\end{array}$ & Unclear risk & Unclear \\
\hline $\begin{array}{l}\text { Allocation concealment } \\
\text { (selection bias) }\end{array}$ & Unclear risk & Unclear \\
\hline $\begin{array}{l}\text { Blinding (performance } \\
\text { bias and detection bias) } \\
\text { All outcomes }\end{array}$ & Unclear risk & Unclear \\
\hline $\begin{array}{l}\text { Incomplete outcome data } \\
\text { (attrition bias) }\end{array}$ & Unclear risk & Unclear \\
\hline
\end{tabular}


aa Ohmit 2008 (Continued)

All outcomes

Summaryassessment Unclear risk Unclear

aa Powers 1995a

Methods $\quad$ Randomised controlled trial conducted in the USA during the 1993 to 1994 influenza season. Follow-up was not indicated. Influenza period was not defined. Participants were randomly assigned to receive 1 of the following 5 vaccine preparations in a double-blinded manner: $15 \mathrm{mg}$ of rHA0, $15 \mathrm{mg}$ of rHA0 plus alum, $90 \mathrm{mg}$ of $\mathrm{rHAO}$, licensed, and placebo. Spring sera were collected.

Participants $\quad 34$ healthy university students: 26 treated and 8 placebo. Age of participants was 18 to 45 .

Interventions $\quad$ Subvirion licensed trivalent parenteral $A B$ vaccine. Schedule and dose were: single dose; $15 \mu g$ each
strain. Vaccine composition was: A/Texas/36/91 (H1N1), A/Beijing/32/92 (H3N2), and B/Panama/45/90. Placebo was saline for injection. Vaccine was recommended and matched circulating strain.

Outcomes

Clinical and laboratory-confirmed cases and adverse effects. An "influenza-like illness" was defined as the presence of any respiratory symptom(s) for $>=2$ days, accompanied by fever or systemic symptoms of myalgia or chills. Laboratory evidence of influenza A (H3N2) virus infection was defined as either or both of the isolation of virus from nasopharyngeal secretion and $\mathrm{a}>=4$-fold increase in serum $\mathrm{HI}$ antibody titre between the 3-week postvaccination (preseason) specimen and the corresponding postseason specimen collected in the following spring. Local adverse effects were erythema, pain, tenderness, induration, arm stiffness; systemic adverse effects: were headache, generalised myalgia, diarrhoea, nausea, feverishness, temperature $>37.8^{\circ} \mathrm{C}$.

Notes Efficacy and safety data were extracted.

Government funded

\section{Risk of bias}

\begin{tabular}{lll}
\hline Bias & Authors' judgement & Support for judgement \\
\hline $\begin{array}{l}\text { Random sequence genera- } \\
\text { tion (selection bias) }\end{array}$ & Unclear risk & Insufficient information to judge \\
\hline $\begin{array}{l}\text { Allocation concealment } \\
\text { (selection bias) }\end{array}$ & Unclear risk & Insufficient information to judge \\
\hline $\begin{array}{l}\text { Blinding (performance } \\
\text { bias and detection bias) } \\
\text { All outcomes }\end{array}$ & Unclear risk & Insufficient information to judge \\
\hline $\begin{array}{l}\text { Incomplete outcome data } \\
\text { (attrition bias) } \\
\text { All outcomes }\end{array}$ & Unclear risk & Insufficient information to judge \\
\hline $\begin{array}{l}\text { Summary assessment } \\
\end{array}$ & Unclear risk & Unclear \\
\hline
\end{tabular}

\section{aa Powers 1995b}

Methods

Single-blind randomised controlled trial conducted in the USA during the 1974 to 1975 influenza season. Follow-up lasted from winter to spring. A "two-month" epidemic period was described by the au- 
aa Powers 1995b (Continued)

thors with no reference to a definition and lasted 6 weeks. Study participants were randomly assigned into 3 subgroups to receive either 2 doses of the vaccine $(n=47), 1$ dose of vaccine and 1 dose of placebo $(n=48)$, or 2 doses of placebo $(n=48)$ at 14 days apart. 6 -month sera were collected on all study participants.

\begin{tabular}{|c|c|}
\hline Participants & 34 healthy university students: 26 treated and 8 placebo. Age of participants was 18 to 45 . \\
\hline Interventions & $\begin{array}{l}\text { Subvirion monovalent parenteral vaccine. Schedule and dose were: single dose; } 90 \mu \mathrm{g} \text { rHA0. Vaccine } \\
\text { composition was: the recombinant HA vaccine contained HA0 glycoprotein from the influenza A/Bei- } \\
\text { jing/32/92 (H3N2) virus. Placebo was saline for injection. Vaccine was not recommended but matched } \\
\text { circulating strain. }\end{array}$ \\
\hline Outcomes & $\begin{array}{l}\text { Clinical and laboratory-confirmed cases. An "influenza-like illness" was defined as the presence of any } \\
\text { respiratory symptom(s) for }>=2 \text { days, accompanied by fever or systemic symptoms of myalgia or chills. } \\
\text { Laboratory evidence of influenza A (H3N2) virus infection was defined as either or both of the isolation } \\
\text { of virus from nasopharyngeal secretion and a }>=4 \text {-fold increase in serum } \mathrm{HI} \text { antibody titre between the } \\
\text { 3-week postvaccination (preseason) specimen and the corresponding postseason specimen collected } \\
\text { in the following spring. }\end{array}$ \\
\hline
\end{tabular}

Notes

Safety data were not included; effectiveness data were extracted.

\section{Risk of bias}

\begin{tabular}{lll}
\hline Bias & Authors' judgement & Support for judgement \\
\hline $\begin{array}{l}\text { Random sequence genera- } \\
\text { tion (selection bias) }\end{array}$ & Unclear risk & Unclear \\
\hline $\begin{array}{l}\text { Allocation concealment } \\
\text { (selection bias) }\end{array}$ & Unclear risk & Unclear \\
\hline $\begin{array}{l}\text { Blinding (performance } \\
\text { bias and detection bias) }\end{array}$ & Unclear risk & Unclear \\
All outcomes & \\
\hline $\begin{array}{l}\text { Incomplete outcome data } \\
\text { (attrition bias) } \\
\text { All outcomes }\end{array}$ & Unclear risk & Unclear \\
\hline $\begin{array}{l}\text { Summary assessment } \\
\end{array}$ & Unclear risk & Unclear \\
\hline
\end{tabular}

\section{aa Powers 1995c}

\begin{tabular}{ll} 
Methods & $\begin{array}{l}\text { Randomised controlled trial conducted in the USA during the } 1993 \text { to } 1994 \text { influenza season. Follow-up } \\
\text { was not indicated. Influenza period was not defined. Participants were randomly assigned to receive } 1 \\
\text { of the following } 5 \text { vaccine preparations in a double-blinded manner: } 15 \mathrm{mg} \text { of rHA0, } 15 \mathrm{mg} \text { of rHA0 plus } \\
\text { alum, } 90 \mathrm{mg} \text { of } \mathrm{rHA}, \text { licensed, and placebo. Spring sera were collected. }\end{array}$ \\
\hline Participants & 59 healthy university students: 51 treated and 8 placebo. Age of participants was 18 to 45. \\
\hline Interventions & $\begin{array}{l}\text { Subvirion monovalent parenteral vaccine. Schedule and dose were: single dose; } 15 \mu \mathrm{g} \text { rHA0. Vaccine } \\
\text { composition was: the recombinant HA vaccine contained HAO glycoprotein from the influenza A/Bei- } \\
\text { jing/32/92 (H3N2) virus. Placebo was saline for injection. Vaccine was not recommended but matched } \\
\text { circulating strain. }\end{array}$ \\
\hline
\end{tabular}


aa Powers 1995c (Continued)

Outcomes
Clinical and laboratory-confirmed cases. An "influenza-like illness" was defined as the presence of any respiratory symptom(s) for $>=2$ days, accompanied by fever or systemic symptoms of myalgia or chills. Laboratory evidence of influenza A (H3N2) virus infection was defined as either or both of the isolation of virus from nasopharyngeal secretion and $\mathrm{a}>=4$-fold increase in serum $\mathrm{HI}$ antibody titre between the 3-week postvaccination (preseason) specimen and the corresponding postseason specimen collected in the following spring.

Notes Efficacy data only were extracted.

\section{Risk of bias}

\begin{tabular}{lll}
\hline Bias & Authors' judgement & Support for judgement \\
\hline $\begin{array}{l}\text { Random sequence genera- } \\
\text { tion (selection bias) }\end{array}$ & Unclear risk & Unclear \\
\hline $\begin{array}{l}\text { Allocation concealment } \\
\text { (selection bias) }\end{array}$ & Unclear risk & Unclear \\
\hline $\begin{array}{l}\text { Blinding (performance } \\
\text { bias and detection bias) } \\
\text { All outcomes }\end{array}$ & Unclear risk & Unclear \\
\hline $\begin{array}{l}\text { Incomplete outcome data } \\
\text { (attrition bias) } \\
\text { All outcomes }\end{array}$ & Unclear risk & Unclear \\
\hline $\begin{array}{l}\text { Summary assessment } \\
\end{array}$ & Unclear risk & Unclear \\
\hline
\end{tabular}

\section{aa Rytel 1977}

Single-blind randomised controlled trial conducted in the USA during the 1974 to 1975 influenza sea-
son. Follow-up lasted from winter to spring. A "two month" epidemic period was described by the au-
thors with no reference to a definition and lasted 6 weeks. Study participants were randomly assigned
into 3 subgroups to receive either 2 doses of the vaccine $(n=47), 1$ dose of vaccine and 1 dose of place-
bo $(n=48)$, or 2 doses of placebo $(n=48)$ at 14 days apart. 6 -month sera were collected on all study par-
ticipants.

Participants 143 young adult female student nurse volunteers: 95 treated and 48 placebo. Age of participants was
18 to 35 .

Live attenuated, bivalent, intranasal influenza A (containing 107,2 EID50) and B (containing 107,8
EID50) vaccines. Schedule and dose were single or double doses. Vaccine composition was: A/Eng-
land/42/72 (H3N2) and B/Hong Kong/5/72. Placebo was 5\% sucrose. Vaccine was not recommended
and did not match the circulating strain.

Outcomes

Influenza and adverse effects. An influenza case was defined as the presence of an influenza-like illness ( 3 or more symptoms of acute respiratory disease and temperature greater then $37.2^{\circ} \mathrm{C}$ ) and virus isolation and/or 4-fold rise in antibody titre in sera obtained at 30 days and 6 months following immunisation. Local adverse effects were upper respiratory symptoms and cough. These were subdivided into moderate and severe. A definition of general adverse effects (again distinguished between moderate and severe) was not given.

Notes 1 dose and 2 doses were analysed together. Circulating strain was A/PortChalmers/1/73 (H3N2). Efficacy and safety data extracted. 
aa Rytel 1977 (Continued)

Government funded

\section{Risk of bias}

\begin{tabular}{lll}
\hline Bias & Authors' judgement & Support for judgement \\
\hline $\begin{array}{l}\text { Random sequence genera- } \\
\text { tion (selection bias) }\end{array}$ & Unclear risk & Unclear \\
\hline $\begin{array}{l}\text { Allocation concealment } \\
\text { (selection bias) }\end{array}$ & Unclear risk & Unclear \\
\hline $\begin{array}{l}\text { Blinding (performance } \\
\text { bias and detection bias) } \\
\text { All outcomes }\end{array}$ & Unclear risk & Unclear \\
\hline $\begin{array}{l}\text { Incomplete outcome data } \\
\text { (attrition bias) } \\
\text { All outcomes }\end{array}$ & Unclear risk & Unclear \\
\hline $\begin{array}{l}\text { Summary assessment } \\
\end{array}$ & Unclear risk & Unclear \\
\hline
\end{tabular}

\section{aa Sumarokow 1971}

$\begin{array}{ll}\text { Methods } & \text { Field trial conducted in Russia during the } 1968 \text { to } 1969 \text { influenza season. Follow-up lasted the whole } \\ \text { epidemic period. The epidemic period was defined as the period of highest influenza morbidity and } \\ \text { lasted } 11 \text { weeks, from the last } 10 \text { days of January to the first } 10 \text { days of April. Vaccinations were carried } \\ \text { out using coded preparation. Sampling virological and serological survey of ill people was performed. }\end{array}$

Participants 19,887 population: 9945 treated and 9942 placebo. Age of participants was 13 to 25.

Interventions Live allantoic intranasal vaccine. Schedule and dose were: 3 doses. Vaccine composition was not indicated. Placebo was not described. Vaccine was not recommended and did not match the circulating strain.

Outcomes

Clinical cases, deaths, severity of illness. Clinical outcomes were all acute respiratory infections. Laboratory confirmation was obtained on a sample of ill participants by virus isolation or demonstration of seroconversion. Bronchitis, otitis, and pneumonia were considered as complications. Passive surveillance was carried out.

Notes

A first study group with children 3 to 12 years old was excluded. A second study group with participants aged 13 to 25 was included in the analysis. The trial compared 2 live vaccines (allantoic intranasal vaccine and tissue vaccine for oral administration) against placebo. Only intranasal vaccine was included in the analysis. Deaths from flu were not recorded. Circulating strain was A2/Hong Kong/68. Effectiveness data only were extracted.

Government funded

\section{Risk of bias}

\begin{tabular}{lll}
\hline Bias & Authors' judgement & Support for judgement \\
\hline $\begin{array}{ll}\text { Random sequence genera- } \\
\text { tion (selection bias) }\end{array}$ & Unclear risk & Insufficient description
\end{tabular}


aa Sumarokow 1971 (Continued)

\begin{tabular}{lll}
$\begin{array}{l}\text { Allocation concealment } \\
\text { (selection bias) }\end{array}$ & Unclear risk & Insufficient description \\
\hline $\begin{array}{l}\text { Blinding (performance } \\
\text { bias and detection bias) }\end{array}$ & Unclear risk & Insufficient description \\
All outcomes & &
\end{tabular}

\begin{tabular}{lll}
\hline $\begin{array}{l}\text { Incomplete outcome data } \\
\text { (attrition bias) }\end{array}$ & Unclear risk \\
All outcomes & & \\
\hline Summary assessment & Unclear risk & Insufficient description \\
\hline
\end{tabular}

\section{aa Tannock 1984}

$\begin{array}{ll}\text { Methods } & \text { Controlled clinical trial, double-blind, conducted in Australia during the } 1981 \text { influenza season. Fol- } \\ & \text { low-up lasted from winter to spring. Influenza period was not defined. Volunteers were alternatively al- } \\ \text { located to groups in a double-blind manner. 6-month sera were collected. }\end{array}$

\begin{tabular}{|c|c|}
\hline Participants & $\begin{array}{l}88 \text { volunteer staff from Newcastle Hospital and the Commonwealth Steel Corporation: } 56 \text { treated and } \\
32 \text { placebo. Age of participants was } 16 \text { to } 64 \text {. }\end{array}$ \\
\hline Interventions & $\begin{array}{l}\text { Trivalent subunit parenteral vaccine. Schedule and dose were: } 7 \mu g \text { each, } 1 \text { or } 2 \text { doses. Vaccine compo- } \\
\text { sition was: A/Brazil/11/78, A/Bangkok/1/79, B/Singapore/222/79. Placebo was saline for injection. Vac- } \\
\text { cine was recommended and matched circulating strain. }\end{array}$ \\
\hline \multirow[t]{2}{*}{ Notes } & $\begin{array}{l}1 \text { dose and } 2 \text { doses were analysed together; very high dropout. Circulating strain was A/Bangkok/1/79. } \\
\text { Safety data only were extracted. }\end{array}$ \\
\hline & Government funded \\
\hline
\end{tabular}

\section{Risk of bias}

\begin{tabular}{lll}
\hline Bias & Authors' judgement & Support for judgement \\
\hline $\begin{array}{l}\text { Random sequence genera- } \\
\text { tion (selection bias) }\end{array}$ & High risk & Inadequate \\
\hline $\begin{array}{l}\text { Allocation concealment } \\
\text { (selection bias) }\end{array}$ & High risk & Inadequate \\
\hline $\begin{array}{l}\text { Blinding (performance } \\
\text { bias and detection bias) }\end{array}$ & High risk & Inadequate \\
$\begin{array}{l}\text { All outcomes } \\
\text { Incomplete outcome data } \\
\begin{array}{l}\text { Altrition bias) } \\
\text { All outcomes }\end{array}\end{array}$ & High risk & Inadequate \\
\hline
\end{tabular}


aa Tannock 1984 (Continued)
Summary assessment
High risk
Inadequate

aa Treanor 2011

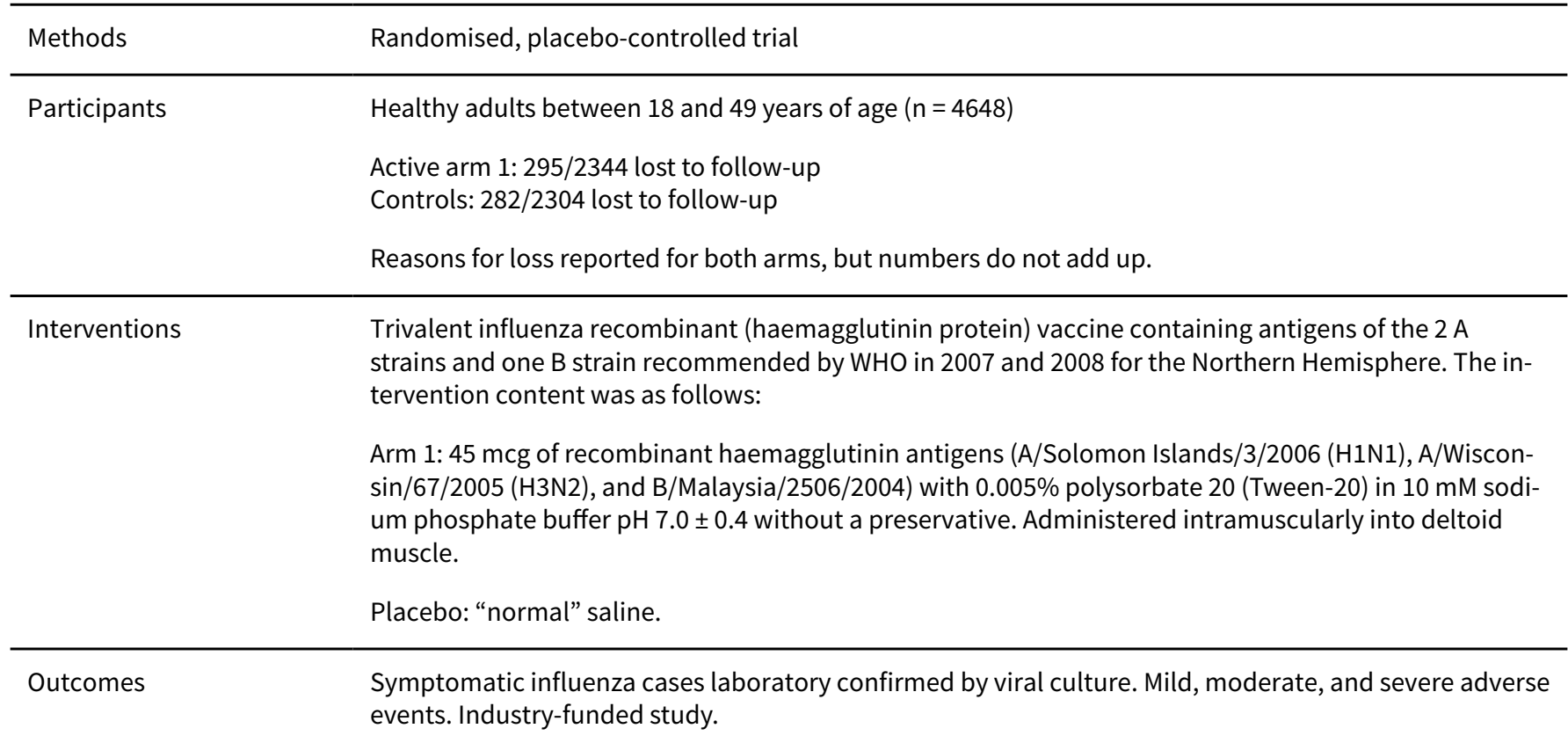

Notes

\section{Risk of bias}

\begin{tabular}{|c|c|c|}
\hline Bias & Authors' judgement & Support for judgement \\
\hline $\begin{array}{l}\text { Random sequence genera- } \\
\text { tion (selection bias) }\end{array}$ & Unclear risk & No description provided \\
\hline $\begin{array}{l}\text { Allocation concealment } \\
\text { (selection bias) }\end{array}$ & Unclear risk & No description provided \\
\hline $\begin{array}{l}\text { Blinding (performance } \\
\text { bias and detection bias) } \\
\text { All outcomes }\end{array}$ & Unclear risk & No description provided \\
\hline $\begin{array}{l}\text { Incomplete outcome data } \\
\text { (attrition bias) } \\
\text { All outcomes }\end{array}$ & Low risk & \\
\hline Summary assessment & Unclear risk & $\begin{array}{l}\text { Loss to follow-up figures do not match, and there are discrepancies between } \\
\text { text and figures. }\end{array}$ \\
\hline
\end{tabular}

aa Waldman 1969a

Methods Randomised controlled trial, double-blind, conducted in the USA during the 1968 to 1969 influenza season. Follow-up lasted the whole epidemic period. The epidemic curve was traced by absenteeism in the 
aa Waldman 1969a (Continued)

local industries and schools and virus isolation and lasted 7 weeks. Randomisation methods were not described. One-half of the volunteers gave serial blood and nasal wash samples.

\begin{tabular}{ll}
\hline Participants & 524 schoolteachers: 465 treated and 118 placebo. Age of participants was not indicated. \\
\hline Interventions & $\begin{array}{l}\text { Monovalent inactivated intramuscular vaccine. Schedule and dose were: } 1 \text { or } 2 \text { doses. Vaccine compo- } \\
\text { sition was: A/Hong Kong/68. Placebo was saline for injection. Vaccine was recommended and matched } \\
\text { circulating strain. }\end{array}$ \\
\hline Outcomes & $\begin{array}{l}\text { Clinical cases and side effects. Clinical case definition was based on the presence of a temperature } \\
>100^{\circ} \mathrm{F} \text { or a feverish feeling plus any } 2 \text { of the following symptoms: sore throat, muscle or joint pain, } \\
\text { cough, stuffy or runny nose. Passive surveillance was carried out. }\end{array}$ \\
\hline Notes & $\begin{array}{l}\text { Data concerning adverse effects were only partially reported by graph. Circulating strain was A2/Hong } \\
\text { Kong/68. Effectiveness data only were extracted. } \\
\text { Government funded }\end{array}$
\end{tabular}

\section{Risk of bias}

\begin{tabular}{lll}
\hline Bias & Authors' judgement & Support for judgement \\
\hline $\begin{array}{l}\text { Random sequence genera- } \\
\text { tion (selection bias) }\end{array}$ & Unclear risk & Unclear \\
\hline $\begin{array}{l}\text { Allocation concealment } \\
\text { (selection bias) }\end{array}$ & Unclear risk & Unclear \\
\hline $\begin{array}{l}\text { Blinding (performance } \\
\text { bias and detection bias) } \\
\text { All outcomes }\end{array}$ & Unclear risk & Unclear \\
\hline $\begin{array}{l}\text { Incomplete outcome data } \\
\text { (attrition bias) } \\
\text { All outcomes }\end{array}$ & Unclear risk & Unclear \\
\hline $\begin{array}{l}\text { Summary assessment } \\
\end{array}$ & Unclear risk & Unclear \\
\hline
\end{tabular}

aa Waldman 1969b

\begin{tabular}{ll}
\hline Methods & Randomised controlled trial, double-blind, conducted in the USA during the 1968 to 1969 influenza sea- \\
son. Follow-up lasted the whole epidemic period. Epidemic curve was traced by absenteeism in the lo- \\
cal industries and schools and virus isolation and lasted 7 weeks. Randomisation methods were not de- \\
scribed. One-half of the volunteers gave serial blood and nasal wash samples.
\end{tabular}

\begin{tabular}{|c|c|}
\hline Participants & 590 schoolteachers: 471 treated and 119 placebo. Age of participants was not indicated. \\
\hline Interventions & $\begin{array}{l}\text { Polyvalent inactivated intramuscular vaccine. Schedule and dose were: } 1 \text { or } 2 \text { doses. Vaccine compo- } \\
\text { sition was: A2/Japan/170/62 150 CCA units, A2/Taiwan/1/64 } 150 \text { CCA units, B/Massachusetts/3/66 } 300 \\
\text { CCA units. Placebo was saline for injection. Vaccine was recommended but did not match the circulat- } \\
\text { ing strain. }\end{array}$ \\
\hline
\end{tabular}
Outcomes
Clinical cases and side effects. Clinical case definition was based on the presence of a temperature
$>100^{\circ} \mathrm{F}$ or a feverish feeling plus any 2 of the following symptoms: sore throat, muscle or joint pain, cough, stuffy or runny nose. Passive surveillance was carried out. 
aa Waldman 1969b (Continued)

Notes

Data concerning adverse effects were only partially reported by graph. Circulating strain was $\mathrm{A} 2 / \mathrm{Hong}$

Kong/68. Efficacy data only were extracted.

Government funded

\section{Risk of bias}

\begin{tabular}{lll}
\hline Bias & Authors' judgement & Support for judgement \\
\hline $\begin{array}{l}\text { Random sequence genera- } \\
\text { tion (selection bias) }\end{array}$ & Unclear risk & Unclear \\
\hline $\begin{array}{l}\text { Allocation concealment } \\
\text { (selection bias) }\end{array}$ & Unclear risk & Unclear \\
\hline $\begin{array}{l}\text { Blinding (performance } \\
\text { bias and detection bias) } \\
\text { All outcomes }\end{array}$ & Unclear risk & Unclear \\
\hline $\begin{array}{l}\text { Incomplete outcome data } \\
\text { (attrition bias) } \\
\text { All outcomes }\end{array}$ & Unclear risk & Unclear \\
\hline $\begin{array}{l}\text { Summary assessment } \\
\end{array}$ & Unclear risk & Unclear \\
\hline
\end{tabular}

aa Waldman 1969c

\begin{tabular}{ll}
\hline Methods & Randomised controlled trial, double-blind, conducted in the USA during the 1968 to 1969 influenza sea- \\
son. Follow-up lasted the whole epidemic period. The epidemic curve was traced by absenteeism in the \\
local industries and schools and virus isolation and lasted 7 weeks. Randomisation methods were not \\
described. One-half of the volunteers gave serial blood and nasal wash samples.
\end{tabular}

\begin{tabular}{ll} 
Participants & 597 schoolteachers: 479 treated and 118 placebo. Age of participants was not indicated. \\
\hline Interventions & $\begin{array}{l}\text { Monovalent inactivated aerosol vaccine. Schedule and dose were: } 1 \text { or } 2 \text { doses. Vaccine composition } \\
\text { was: A/Hong Kong/68. Placebo was saline for injection. Vaccine was recommended and matched circu- } \\
\text { lating strain. }\end{array}$ \\
\hline Outcomes & $\begin{array}{l}\text { Clinical cases and side effects. Clinical case definition was based on the presence of a temperature } \\
>100^{\circ} \mathrm{F} \text { or a feverish feeling plus any } 2 \text { of the following symptoms: sore throat, muscle or joint pain, } \\
\text { cough, stuffy or runny nose. Passive surveillance was carried out. }\end{array}$
\end{tabular}

Notes Data concerning adverse effects were only partially reported by graph. Circulating strain was A2/Hong Kong/68. Efficacy data only were extracted.

Government funded

\section{Risk of bias}

\begin{tabular}{lll}
\hline Bias & Authors' judgement & Support for judgement \\
\hline $\begin{array}{l}\text { Random sequence genera- } \\
\text { tion (selection bias) }\end{array}$ & Unclear risk & Unclear \\
\hline $\begin{array}{l}\text { Allocation concealment } \\
\text { (selection bias) }\end{array}$ & Unclear risk & Unclear \\
\hline
\end{tabular}


aa Waldman 1969c (Continued)
Blinding (performance
Unclear risk
Unclear
bias and detection bias)

All outcomes

\begin{tabular}{lll}
\hline $\begin{array}{l}\text { Incomplete outcome data } \\
\text { (attrition bias) }\end{array}$ & Unclear risk \\
All outcomes & & \\
\hline Summary assessment & Unclear risk & Unclear \\
\hline
\end{tabular}

\section{aa Waldman 1969d}

Randomised controlled trial, double-blind, conducted in the USA during the 1968 to 1969 influenza sea-
son. Follow-up lasted the whole epidemic period. The epidemic curve was traced by absenteeism in the
local industries and schools and virus isolation and lasted 7 weeks. Randomisation methods were not
described. One-half of the volunteers gave serial blood and nasal wash samples.

\begin{tabular}{ll}
\hline Participants & 590 schoolteachers: 471 treated and 119 placebo. Age of participants was not indicated. \\
\hline Interventions & $\begin{array}{l}\text { Polyvalent inactivated aerosol vaccine. Schedule and dose were: } 1 \text { or } 2 \text { doses. Vaccine composition } \\
\text { was: A2/Japan/170/62 } 150 \text { CCA units, A2/Taiwan/1/64 } 150 \text { CCA units, B/Massachusetts/3/66 } 300 \text { CCA } \\
\text { units. Placebo was saline for injection. Vaccine was recommended but did not match the circulating } \\
\text { strain. }\end{array}$ \\
\hline Outcomes & $\begin{array}{l}\text { Clinical cases and side effects. Clinical case definition was based on the presence of a temperature } \\
>100^{\circ} \mathrm{F} \text { or a feverish feeling plus any } 2 \text { of the following symptoms: sore throat, muscle or joint pain, } \\
\text { Cough, stuffy or runny nose. Passive surveillance was carried out. }\end{array}$ \\
\hline Notes & $\begin{array}{l}\text { Data concerning adverse effects were only partially reported by graph. Circulating strain was A2/Hong } \\
\text { Kong/68. Efficacy data only were extracted. } \\
\text { Government funded }\end{array}$
\end{tabular}

\section{Risk of bias}

\begin{tabular}{|c|c|c|}
\hline Bias & Authors' judgement & Support for judgement \\
\hline $\begin{array}{l}\text { Random sequence genera- } \\
\text { tion (selection bias) }\end{array}$ & Unclear risk & Unclear \\
\hline $\begin{array}{l}\text { Allocation concealment } \\
\text { (selection bias) }\end{array}$ & Unclear risk & Unclear \\
\hline $\begin{array}{l}\text { Blinding (performance } \\
\text { bias and detection bias) } \\
\text { All outcomes }\end{array}$ & Unclear risk & Unclear \\
\hline $\begin{array}{l}\text { Incomplete outcome data } \\
\text { (attrition bias) } \\
\text { All outcomes }\end{array}$ & Unclear risk & Unclear \\
\hline Summary assessment & Unclear risk & Unclear \\
\hline
\end{tabular}


aa Waldman 1972a

Methods

Randomised controlled trial, double-blind, conducted in the USA during the 1968 to 1969 influenza season. Follow-up lasted the whole epidemic period. The epidemic curve was traced by absenteeism in the local industries and schools and virus isolation and lasted 7 weeks. Identical-looking, coded vials were used to dispense material. Sampling virological and serological survey of ill people was performed. 2 doses were administered, but as the outbreak occurred mostly between them, only the effectiveness of the first dose was assessed.

Participants 244 volunteer students and staff members: 195 treated and 49 placebo. Age of participants was not indicated.

Interventions

Monovalent A aerosol vaccine. Schedule and dose were: 200 CCA units. Vaccine composition was: A2/ Aichi/1/68. Placebo was saline for injection. Vaccine was recommended and matched circulating strain.

Outcomes

Clinical cases and adverse effects. Clinical cases were defined as febrile respiratory illness with oral temperature higher then $99.5^{\circ} \mathrm{F}$. Local adverse effects were defined as pain and/or tenderness and redness and/or swelling. Systemic adverse effects were defined as general (fever, muscle pain, nausea or vomiting, diarrhoea, and malaise) or respiratory (runny and/or stuffy nose, sore throat, cough, shortness of breath). Passive surveillance was carried out.

Notes Illness during the first 1 or 2 weeks after vaccination was not excluded, but the authors stated that this fact did not affect the results. Circulating strain was A2/Aichi/2/68. Efficacy and safety data were extracted.

Government funded

\section{Risk of bias}

\begin{tabular}{lll}
\hline Bias & Authors' judgement & Support for judgement \\
\hline $\begin{array}{l}\text { Random sequence genera- } \\
\text { tion (selection bias) }\end{array}$ & Unclear risk & Unclear \\
\hline $\begin{array}{l}\text { Allocation concealment } \\
\text { (selection bias) }\end{array}$ & Low risk & Adequate \\
\hline $\begin{array}{l}\text { Blinding (performance } \\
\text { bias and detection bias) }\end{array}$ & Unclear risk & Unclear \\
$\begin{array}{l}\text { All outcomes } \\
\text { Incomplete outcome data }\end{array}$ & Unclear risk & Unclear \\
$\begin{array}{l}\text { (attrition bias) } \\
\text { All outcomes }\end{array}$ & \\
\hline $\begin{array}{l}\text { Summary assessment } \\
\end{array}$ & Unclear risk & Unclear \\
\hline
\end{tabular}

aa Waldman 1972b

\section{Methods}

Randomised controlled trial, double-blind, conducted in the USA during the 1968 to 1969 influenza season. Follow-up lasted the whole epidemic period. The epidemic curve was traced by absenteeism in the local industries and schools and virus isolation and lasted 7 weeks. Identical-looking, coded vials were used to dispense material. Sampling virological and serological survey of ill people was performed. 2 doses were administered, but as the outbreak occurred mostly between them, only the effectiveness of the first dose was assessed.

\section{Participants} 239 volunteer students and staff members: 190 treated and 49 placebo. Age of participants was not indicated. 
aa Waldman 1972b (Continued)

Interventions

Monovalent A subcutaneous vaccine. Schedule and dose were: 200 CCA units. Vaccine composition was: A2/Aichi/1/69. Placebo was saline for injection. Vaccine was recommended and matched circulating strain.

Notes Illness during the first 1 or 2 weeks after vaccination was not excluded, but the authors stated that this fact did not affect the results. Circulating strain was A2/Aichi/2/68. Efficacy and safety data were extracted. Government funded.

\section{Risk of bias}

\begin{tabular}{|c|c|c|}
\hline Bias & Authors' judgement & Support for judgement \\
\hline $\begin{array}{l}\text { Random sequence genera- } \\
\text { tion (selection bias) }\end{array}$ & Unclear risk & Unclear \\
\hline $\begin{array}{l}\text { Allocation concealment } \\
\text { (selection bias) }\end{array}$ & Low risk & Adequate \\
\hline $\begin{array}{l}\text { Blinding (performance } \\
\text { bias and detection bias) } \\
\text { All outcomes }\end{array}$ & Unclear risk & Unclear \\
\hline $\begin{array}{l}\text { Incomplete outcome data } \\
\text { (attrition bias) } \\
\text { All outcomes }\end{array}$ & Unclear risk & Unclear \\
\hline Summary assessment & Unclear risk & Unclear \\
\hline
\end{tabular}

aa Waldman $1972 \mathrm{C}$

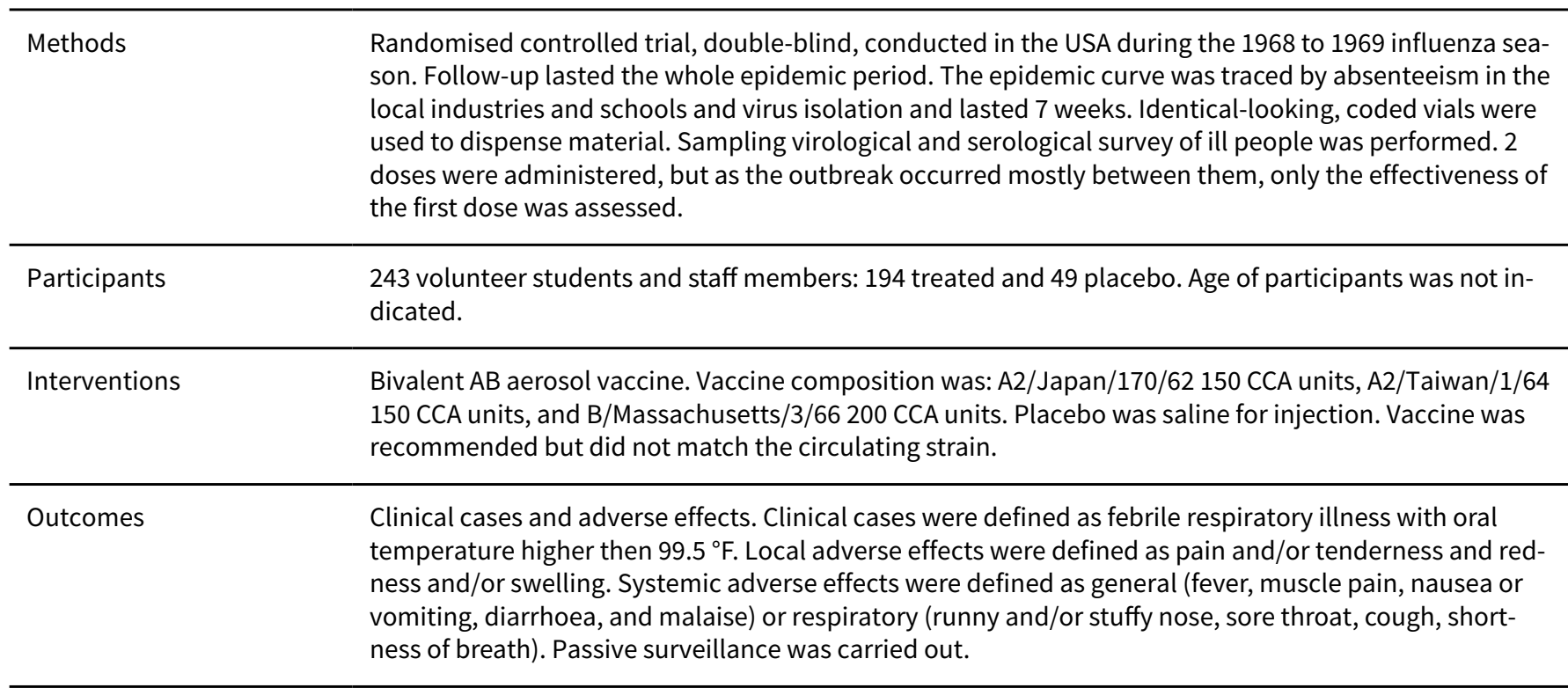


aa Waldman 1972c (Continued)

Notes

Illness during the first 1 or 2 weeks after vaccination was not excluded, but the authors stated that this fact did not affect the results. Circulating strain was A2/Aichi/2/68. Efficacy and safety data were extracted. Government funded.

\section{Risk of bias}

\begin{tabular}{lll}
\hline Bias & Authors' judgement & Support for judgement \\
\hline $\begin{array}{l}\text { Random sequence genera- } \\
\text { tion (selection bias) }\end{array}$ & Unclear risk & Unclear \\
\hline $\begin{array}{l}\text { Allocation concealment } \\
\text { (selection bias) }\end{array}$ & Low risk & Adequate \\
\hline $\begin{array}{l}\text { Blinding (performance } \\
\text { bias and detection bias) } \\
\text { All outcomes }\end{array}$ & Unclear risk & Unclear \\
\hline $\begin{array}{l}\text { Incomplete outcome data } \\
\text { (attrition bias) } \\
\text { All outcomes }\end{array}$ & Unclear risk & Unclear \\
\hline $\begin{array}{l}\text { Summary assessment } \\
\end{array}$ & Unclear risk & Unclear \\
\hline
\end{tabular}

\section{aa Waldman 1972d}

Randomised controlled trial, double-blind, conducted in the USA during the 1968 to 1969 influenza sea-
son. Follow-up lasted the whole epidemic period. The epidemic curve was traced by absenteeism in the
local industries and schools and virus isolation and lasted 7 weeks. Identical-looking, coded vials were
used to dispense material. Sampling virological and serological survey of ill people was performed. 2
doses were administered, but as the outbreak occurred mostly between them, only the effectiveness of
the first dose was assessed.

\begin{tabular}{ll}
\hline Participants & $\begin{array}{l}236 \text { volunteer students and staff members: } 187 \text { treated and } 49 \text { placebo. Age of participants was not in- } \\
\text { dicated. }\end{array}$
\end{tabular}
wan/1/64 150 CCA units, and B/Massachusetts/3/66 200 CCA units. Placebo was saline for injection. Vaccine was recommended but did not match the circulating strain.

$\begin{array}{ll}\text { Outcomes } & \text { Clinical cases and adverse effects. Clinical cases were defined as febrile respiratory illness with oral } \\ & \text { temperature higher then } 99.5^{\circ} \mathrm{F} \text {. Local adverse effects were defined as pain and/or tenderness and red- } \\ & \text { ness and/or swelling. Systemic adverse effects were defined as general (fever, muscle pain, nausea or } \\ & \text { vomiting, diarrhoea, and malaise) or respiratory (runny and/or stuffy nose, sore throat, cough, short- } \\ & \text { ness of breath). Passive surveillance was carried out. }\end{array}$

Notes Illness during the first 1 or 2 weeks after vaccination was not excluded, but the authors stated that this fact did not affect the results. Circulating strain was A2/Aichi/2/68. Efficacy and safety data were extracted. Government funded.

\section{Risk of bias}

\section{Bias Authors' judgement Support for judgement}




\section{aa Waldman 1972d (Continued)}

Random sequence genera- Unclear risk Unclear tion (selection bias)

\begin{tabular}{lll}
\hline $\begin{array}{l}\text { Allocation concealment } \\
\text { (selection bias) }\end{array}$ & Low risk Adequate
\end{tabular}

(etion bias)

Blinding (performance $\quad$ Unclear risk Unclear
bias and detection bias)
All outcomes

\begin{tabular}{lll}
\hline $\begin{array}{l}\text { Incomplete outcome data } \\
\text { (attrition bias) }\end{array}$ & Unclear risk & \\
All outcomes & \\
\hline Summary assessment & Unclear risk & Unclear \\
\hline
\end{tabular}

$\begin{array}{ll}\text { Methods } & \text { Randomised controlled trial, double-blind, conducted in the USA during the } 1985 \text { to } 1986 \text { influenza sea- } \\ \text { son. Follow-up was not indicated. Epidemic influenza was defined according to population surveillance } \\ \text { data (without better explanation), begun in December } 1985 \text { and concluded in February } 1986 . \text { Partici- } \\ \text { pants were assigned using a random number generator to receive either the influenza vaccine or place- } \\ \text { bo. Virological surveillance was not performed. }\end{array}$

\begin{tabular}{|c|c|}
\hline Participants & 179 healthy volunteer hospital employees: 91 treated and 88 placebo. Age of participants was 21 to 65 . \\
\hline Interventions & $\begin{array}{l}\text { Split trivalent intramuscular vaccine. Schedule and dose were: single dose; } 15 \mu \mathrm{g} \text { each strain. Vaccine } \\
\text { composition was: A/Chile/1/83 (H1N1), A/Philippines/2/82 (H3N2), and B/USSR/100/83. Placebo was } \\
\text { saline for injection. Vaccine was recommended but did not match the circulating strain. }\end{array}$ \\
\hline Outcomes & $\begin{array}{l}\text { Clinical cases symptoms defined, WDL regardless of cause, and adverse effects. Influenza illness was } \\
\text { defined by the CDC case definition: a documented temperature greater than } 100^{\circ} \mathrm{F} \text { and at least the } \\
\text { symptoms of cough or sore throat. }\end{array}$ \\
\hline Notes & $\begin{array}{l}\text { Data regarding WDL and adverse effects were not complete and they were not considered. Most of the } \\
\text { influenza infections were caused by type B. } \\
\text { Efficacy data only were extracted. }\end{array}$ \\
\hline & ment fi \\
\hline
\end{tabular}

\section{Risk of bias}

\begin{tabular}{lll}
\hline Bias & Authors' judgement & Support for judgement \\
\hline $\begin{array}{l}\text { Random sequence genera- } \\
\text { tion (selection bias) }\end{array}$ & Unclear risk & Unclear \\
\hline $\begin{array}{l}\text { Allocation concealment } \\
\text { (selection bias) }\end{array}$ & Unclear risk & Unclear \\
\hline $\begin{array}{l}\text { Blinding (performance } \\
\text { bias and detection bias) }\end{array}$ & Unclear risk & Unclear \\
All outcomes & \\
\hline $\begin{array}{l}\text { Incomplete outcome data } \\
\text { (attrition bias) }\end{array}$ & Unclear risk & Unclear \\
\hline
\end{tabular}


aa Weingarten 1988 (Continued)

All outcomes

Summaryassessment Unclear risk Unclear

aa Zhilova 1986a

Methods Semi-randomised, double-blind, placebo-controlled clinical trial conducted in Leningrad, USSR during the 1981 to 1982 influenza season. The study tested the reactogenicity, safety, and effectiveness of an inactivated and a live attenuated vaccine, both administered singly or in combination. Allocation was made on the basis of school classes, and it is unclear whether this is a cluster-randomised or clinical controlled trial. We have opted for the latter, as the text mentions random selection to maintain "equivalence". "Double blind" is mentioned in the text. During January to May 1982 there was a rise in the level of ILI due to influenza and other agents.

Participants 3961 participants were enrolled. Participants were healthy "students" aged 18 to 23 . Numbers in each of the 4 arms are uneven throughout the trial, with no reason provided.

Interventions Inactivated vaccine trivalent (Ministry of Health USSR) by subcutaneous injection $0.2 \mathrm{~mL}$ once (arm 1), or intranasal live "recombinant" "mono" vaccine $0.5 \mathrm{~mL}$ spray 2 to 3 times (Ministry of Health USSR) (arm 2), or combined (arm 3), or subcutaneous and intranasal spray sodium chloride saline placebo (arm 4). The strains contained were $\mathrm{H} 1 \mathrm{~N} 1, \mathrm{H} 3 \mathrm{~N} 2$, and B. Vaccine matching was not good.

\section{Outcomes}

\section{Serological}

Antibody titres - substudy on 1221 participants

\section{Effectiveness}

Influenza-like illness (not defined and from the text it is unclear how many ILI cases were matched to positive laboratory findings)

\section{Safety}

Safety data were not reported in sufficient detail to allow extraction.

Notes

The authors conclude that simultaneous inoculation of the vaccines appeared to produce better humoral antibody responses, especially in the last season. However, the correlation between clinical protection and antibody rises is reported as dubious. The authors make the reasonable point that perhaps live attenuated vaccines work better because they stimulate production of secretory antibodies. This is a poorly reported study. No mention is made of how the placebo could have been correctly used in the schedule (i.e. they should have had 6 arms instead of 4 with subcutaneous placebo, spray placebo administered separately as well as combined; this may be a problem of translation). Efficacy data only were extracted.

Government funded

\section{Risk of bias}

\begin{tabular}{lll}
\hline Bias & Authors' judgement & Support for judgement \\
\hline $\begin{array}{l}\text { Random sequence genera- } \\
\text { tion (selection bias) }\end{array}$ & Unclear risk & Unclear \\
\hline $\begin{array}{l}\text { Allocation concealment } \\
\text { (selection bias) }\end{array}$ & Unclear risk & Unclear \\
\hline $\begin{array}{l}\text { Blinding (performance } \\
\text { bias and detection bias) } \\
\text { All outcomes }\end{array}$ & Unclear risk & Unclear \\
\hline
\end{tabular}


aa Zhilova 1986a (Continued)

Incomplete outcome data Unclear risk Unclear

(attrition bias)

All outcomes

Summaryassessment Unclear risk Unclear

aa Zhilova 1986b

Methods Semi-randomised, double-blind, placebo-controlled clinical trial conducted in Leningrad, USSR during the 1982 to 1983 influenza season. The study tested the reactogenicity, safety, and effectiveness of an inactivated and a live attenuated vaccine, both administered singly or in combination. Allocation was made based on school classes, and it is unclear whether this is a cluster-randomised or clinical controlled trial. We have opted for the latter, as the text mentions random selection to maintain "equivalence". "Double blind" is mentioned in the text. In the season there was an outbreak of A (H3N2) lasting 4 to 5 weeks. However, influenza accounted for only up to $30 \%$ of isolates from ill people.

Participants $\quad 3944$ participants were enrolled. Participants were healthy "students" aged 18 to 23 . Numbers in each of the 4 arms are uneven throughout the trial, with no reason provided.

Interventions

Inactivated vaccine trivalent (Ministry of Health USSR) by subcutaneous injection $0.2 \mathrm{~mL}$ once (arm 1), or intranasal live "recombinant" "mono" vaccine $0.5 \mathrm{~mL}$ spray 2 to 3 times (Ministry of Health USSR) (arm 2), or combined (arm 3), or subcutaneous and intranasal spray sodium chloride saline placebo (arm 4). The strains contained were $\mathrm{H} 1 \mathrm{~N} 1, \mathrm{H} 3 \mathrm{~N} 2$, and B. Vaccine matching was good.

\section{Outcomes}

\section{Serological \\ Antibody titres - substudy on 1221 participants \\ Effectiveness}

Influenza-like illness (not defined and from the text it is unclear how many ILI cases were matched to positive laboratory findings)

\section{Safety}

Safety data were not reported in sufficient detail to allow extraction.

Passive surveillance was carried out.

\section{Notes}

The authors conclude that simultaneous inoculation of the vaccines appeared to produce better humoral antibody responses, especially in the last season. However, the correlation between clinical protection and antibody rises is reported as dubious. The authors make the reasonable point that perhaps live attenuated vaccines work better because they stimulate production of secretory antibodies. This is a poorly reported study. No mention is made of how the placebo could have been correctly used in the schedule (i.e. they should have had 6 arms instead of 4 with subcutaneous placebo, spray placebo administered separately as well combined; this may be a problem of translation). Efficacy data only were extracted. Government funded

\section{Risk of bias}

\begin{tabular}{lll}
\hline Bias & Authors' judgement & Support for judgement \\
\hline $\begin{array}{l}\text { Random sequence genera- } \\
\text { tion (selection bias) }\end{array}$ & Unclear risk & Unclear \\
\hline $\begin{array}{l}\text { Allocation concealment } \\
\text { (selection bias) }\end{array}$ & Unclear risk & Unclear \\
\hline $\begin{array}{l}\text { Blinding (performance } \\
\text { bias and detection bias) } \\
\text { All outcomes }\end{array}$ & Unclear risk & Unclear \\
\hline
\end{tabular}


aa Zhilova 1986b (Continued)
Incomplete outcome data
(attrition bias)
Unclear risk Unclear

All outcomes

Summaryassessment Unclear risk Unclear

\section{ab Atmar 1990}

\begin{tabular}{ll}
\hline Methods & Double-blind, placebo-controlled, randomised trial \\
\hline Participants & 74 healthy volunteers aged 18 to 40 years (data on 17 asthmatics were not extracted) \\
\hline Interventions & $\begin{array}{l}\text { Cold - recombinant vaccine } A(H 1 N 1)(n=16) \text { versus cold }- \text { recombinant vaccine } A(H 3 N 2)(n=13) \text { versus } \\
\text { cold - recombinant vaccine } B(n=17) \text { versus placebo }(n=26) \\
\text { Intranasal }\end{array}$
\end{tabular}

Outcomes
- FEV 1
- FVC
- FEV1/FVC
- Forced expiratory flow rate $25 \%$ to $75 \%$ (FEF 25 to 75 )

\begin{tabular}{|c|c|}
\hline \multirow[t]{2}{*}{ Notes } & $\begin{array}{l}\text { The authors report several non-significant drops in FEV and FVC up to } 7 \text { days' postinoculation and a } \\
\text { higher incidence of ILI (17/46 versus } 4 / 26) \text { in the vaccinated arms. Safety data only were extracted. }\end{array}$ \\
\hline & Government funded \\
\hline
\end{tabular}

\section{Risk of bias}

\begin{tabular}{lll}
\hline Bias & Authors' judgement & Support for judgement \\
\hline $\begin{array}{l}\text { Random sequence genera- } \\
\text { tion (selection bias) }\end{array}$ & Unclear risk & Unclear \\
\hline $\begin{array}{l}\text { Allocation concealment } \\
\text { (selection bias) }\end{array}$ & Unclear risk & Unclear \\
\hline $\begin{array}{l}\text { Blinding (performance } \\
\text { bias and detection bias) }\end{array}$ & Unclear risk & Unclear \\
All outcomes & \\
\hline $\begin{array}{l}\text { Incomplete outcome data } \\
\text { (attrition bias) } \\
\text { All outcomes }\end{array}$ & Unclear risk & Unclear \\
\hline $\begin{array}{l}\text { Summary assessment } \\
\end{array}$ & Unclear risk & Unclear \\
\hline
\end{tabular}

\section{ab Betts 1977a}

\begin{tabular}{ll}
\hline Methods & Randomised controlled trial carried out from April 1976 at Rochester University. Vaccine and placebo \\
were randomly administered in a double-blind manner, but no description of allocation procedure is \\
given. 36 days after immunisation all participants were challenged with wild type virus (A/Victoria/3/75,
\end{tabular}


ab Betts 1977a (Continued)

H3N2), and antibody response was determined from serum and nasal secretions (before vaccination, 36 hours later, and 21 days after challenge, not for analysis).

47 healthy male and female university students with absent or low HI titre (i.e. little or no immunity) to both $\mathrm{A} / \mathrm{Scotland} / 74$ and $\mathrm{A} /$ Victoria/3/75.

\begin{tabular}{ll}
\hline Interventions & $\begin{array}{l}\text { Live attenuated A/Scotland/74 (H3N2) versus placebo, one } 0.5 \mathrm{~mL} \text { dose intranasally. On day } 37 \text { after im- } \\
\text { munisation, participants were challenged with A/Victoria/3/75. }\end{array}$ \\
\hline Outcomes & $\begin{array}{l}\text { A physician examined the participants } 1 \text { day and } 4 \text { days after they received vaccine or placebo. Tem- } \\
\text { perature was observed only } 1 \text { day after. Observed symptoms were: mild sore throat and rhinorrhoea: } \\
\left.\text { vaccine } 4 / 23, \text { placebo } 3 / 24 ; \text { fever (temperature }>37.50^{\circ} \mathrm{C}\right): \text { none had it. }\end{array}$
\end{tabular}

\begin{tabular}{ll}
\hline Notes & Safety data only were extracted. \\
& Industry funded \\
\hline
\end{tabular}

\section{Risk of bias}

\begin{tabular}{|c|c|c|}
\hline Bias & Authors' judgement & Support for judgement \\
\hline $\begin{array}{l}\text { Random sequence genera- } \\
\text { tion (selection bias) }\end{array}$ & Unclear risk & Unclear \\
\hline $\begin{array}{l}\text { Allocation concealment } \\
\text { (selection bias) }\end{array}$ & Unclear risk & Not used \\
\hline $\begin{array}{l}\text { Blinding (performance } \\
\text { bias and detection bias) } \\
\text { All outcomes }\end{array}$ & Unclear risk & Unclear \\
\hline $\begin{array}{l}\text { Incomplete outcome data } \\
\text { (attrition bias) } \\
\text { All outcomes }\end{array}$ & Unclear risk & Unclear \\
\hline Summary assessment & Unclear risk & Unclear \\
\hline
\end{tabular}

\section{ab Boyce 2000}

$\begin{array}{ll}\text { Methods } & \text { Open-label/single-blind randomised controlled trial to assess the safety and immunogenicity of adju- } \\ \text { vanted and unadjuvanted subunit influenza vaccine, prepared with the strains recommended for and } \\ \text { isolated in the } 1997 \text { to } 1998 \text { season. }\end{array}$

Participants

74 healthy adults aged between 10 and 40 years, who did not receive influenza immunisation during the 6 months preceding the trial.

Interventions

1. M-59 adjuvanted subunit trivalent flu vaccine (prepared with A/Bayern/795 H1N1, A/Wuhan/359/95 H3N2, B/Beijing/184/93-like strains, each $15 \mu \mathrm{g} / 0.5 \mathrm{~mL}$ dose)

2. Unadjuvanted vaccine (prepared with the same strains at the same concentrations as the adjuvanted preparation)

3. Placebo (consisting of $0.5 \mathrm{~mL}$ sterile saline)

All preparations were intranasally administered in 2 doses 28 days apart. 24 participants received their first dose of adjuvanted $(n=12)$ or unadjuvanted $(n=12)$ subunit vaccine in an open-label manner. After it was determined that they tolerated the first dose, the randomised phase of the trial $(n=50)$ was 

placebo.

\begin{tabular}{|c|c|c|}
\hline Outcomes & \multicolumn{2}{|c|}{$\begin{array}{l}\text { After each immunisation, participants were observed for } 30 \text { minutes, examined after } 2 \text { days, and then } \\
\text { completed a diary card reporting symptoms that occurred within } 7 \text { days after. Local reactions: nasal } \\
\text { symptoms, unpleasant taste, bloody nasal discharge, sneezing. Systemic reactions: chills, pulmonary, } \\
\text { nausea, malaise, myalgia or arthralgia, urticarial rash, headache, oral temperature }>=38^{\circ} \mathrm{C} \text {, stay at } \\
\text { home, use of analgesic or antipyretic. Data were not given separately for the randomised and open-la- } \\
\text { bel phase of the study. }\end{array}$} \\
\hline Notes & \multicolumn{2}{|c|}{$\begin{array}{l}\text { It was not possible to consider the safety data separately for the } 2 \text { study phases. Safety data only were } \\
\text { extracted. }\end{array}$} \\
\hline & \multicolumn{2}{|l|}{ Industry funded } \\
\hline \multicolumn{3}{|l|}{ Risk of bias } \\
\hline Bias & Authors' judgement & Support for judgement \\
\hline $\begin{array}{l}\text { Random sequence genera- } \\
\text { tion (selection bias) }\end{array}$ & Unclear risk & Unclear \\
\hline $\begin{array}{l}\text { Allocation concealment } \\
\text { (selection bias) }\end{array}$ & Unclear risk & Unclear \\
\hline $\begin{array}{l}\text { Blinding (performance } \\
\text { bias and detection bias) } \\
\text { All outcomes }\end{array}$ & Unclear risk & Unclear \\
\hline $\begin{array}{l}\text { Incomplete outcome data } \\
\text { (attrition bias) } \\
\text { All outcomes }\end{array}$ & Unclear risk & Unclear \\
\hline Summary assessment & Unclear risk & Unclear \\
\hline
\end{tabular}

ab Caplan 1977

$\begin{array}{ll}\text { Methods } & \text { Randomised controlled trial to assess the reactogenicity and safety of monovalent whole-virus and } \\ \text { split-virus vaccines prepared with strain A/Victoria/3/75 from different US manufacturers. }\end{array}$

Participants 208 healthy adult volunteers aged between 18 and 64 years, recruited from the University of Maryland, USA.
Interventions
Monovalent whole-virus vaccine (Merck Sharp \& Dohme, Merrell-National Laboratories) or monovalent split-virus vaccine (Parke-Davis and Company; Wyeth Laboratories) administered in different antigen concentrations $(200,400$, or 800 CCA units) versus placebo. All from A/Victoria75. 1 dose intramuscular- ly.

\begin{tabular}{ll}
\hline Outcomes & $\begin{array}{l}\text { Temperature }>=100^{\circ} \mathrm{F}\left(37.8^{\circ} \mathrm{C}\right) \text {, feverishness, pain or burning, tenderness, malaise or myalgia, nausea } \\
\text { or vomiting, headache, other. 21-day follow-up. Safety outcomes are also given as cumulative \% for } \\
\text { each category: local, systemic, bothersome; febrile; or scores for systemic reactions. }\end{array}$ \\
\hline Notes & Safety data only were extracted. \\
& Government funded \\
\hline
\end{tabular}


ab Caplan 1977 (Continued)

Risk of bias

\begin{tabular}{|c|c|c|}
\hline Bias & Authors' judgement & Support for judgement \\
\hline $\begin{array}{l}\text { Random sequence genera- } \\
\text { tion (selection bias) }\end{array}$ & Unclear risk & Unclear \\
\hline $\begin{array}{l}\text { Allocation concealment } \\
\text { (selection bias) }\end{array}$ & Unclear risk & Unclear \\
\hline $\begin{array}{l}\text { Blinding (performance } \\
\text { bias and detection bias) } \\
\text { All outcomes }\end{array}$ & Unclear risk & Unclear \\
\hline $\begin{array}{l}\text { Incomplete outcome data } \\
\text { (attrition bias) } \\
\text { All outcomes }\end{array}$ & Unclear risk & Unclear \\
\hline Summary assessment & Unclear risk & Unclear \\
\hline
\end{tabular}

ab El'shina 1996

\begin{tabular}{ll}
\hline Methods & Randomised controlled trial \\
\hline Participants & $\begin{array}{l}\text { 432 healthy participants aged between } 18 \text { and } 22 \text { years who had not received any influenza immunisa- } \\
\text { tion during the previous } 2 \text { to } 3 \text { years. }\end{array}$ \\
\hline
\end{tabular}

Interventions

Polymer-subunit influenza vaccine Grippol prepared with the strains A/Victoria/36/88, Wib - 26, B/Panama 45/90. 2 types containing 5 or $2.5 \mu$ g haemagglutinin of each strain, respectively were compared with whole-virion inactivated trivalent vaccine (reference preparation, containing $35 \mu \mathrm{g}$ of haemagglutinin) and placebo (consisting of sterile physiological solution). One $0.5 \mathrm{~mL}$ dose was administered subcutaneously.

Outcomes

After immunisation, participants were placed under medical observation. Fever (48 hours follow-up): weak $\left(37.1\right.$ to $\left.37.5^{\circ} \mathrm{C}\right)$, moderate $\left(37.6\right.$ to $\left.38.5^{\circ} \mathrm{C}\right)$, severe $\left(>38.6^{\circ} \mathrm{C}\right)$. Systemic reactions ( 3 to 4 days follow-up): feeling unwell, sore throat, hyperaemia of nasopharynx, head cold, cough, headache, blocked nose, dizziness, shivering, drowsiness, nausea, hoarseness. Local reaction: all (moderate weak); pain at site of injection.

\begin{tabular}{ll}
\hline Notes & Safety data only were extracted. \\
Government funded
\end{tabular}

\section{Risk of bias}

\begin{tabular}{lll}
\hline Bias & Authors' judgement & Support for judgement \\
\hline $\begin{array}{l}\text { Random sequence genera- } \\
\text { tion (selection bias) }\end{array}$ & Unclear risk & Unclear \\
\hline $\begin{array}{l}\text { Allocation concealment } \\
\text { (selection bias) }\end{array}$ & Unclear risk & Unclear \\
\hline $\begin{array}{l}\text { Blinding (performance } \\
\text { bias and detection bias) }\end{array}$ & Unclear risk & Unclear \\
\end{tabular}


ab El'shina 1996 (Continued)

All outcomes

\begin{tabular}{lll}
\hline $\begin{array}{l}\text { Incomplete outcome data } \\
\text { (attrition bias) }\end{array}$ & Unclear risk & \\
All outcomes & \\
\hline Summary assessment & Unclear risk & Unclear \\
\hline
\end{tabular}

\section{ab Evans 1976}

\begin{tabular}{|c|c|}
\hline Methods & Randomised controlled trial \\
\hline Participants & 162 healthy participants aged 18 to 61 years \\
\hline Interventions & $\begin{array}{l}\text { Bivalent live attenuated vaccine WRL } 105 \text { (recombinant of } \mathrm{A} / \mathrm{Okuda} / 57 \text { and } \mathrm{A} / \text { Finland/4/74) containing } \\
107.0 \text { EID50 virus/0.5 mL dose versus placebo. Both preparations were administered intranasally } 3 \text { to } 4 \\
\text { weeks apart. }\end{array}$ \\
\hline Outcomes & $\begin{array}{l}\text { Reactions to immunisation were observed for } 7 \text { days after each dose. Local symptoms (referable to } \\
\text { the upper respiratory tract, mainly nasal obstruction, nasal discharge, or sore throat) reported as mild, } \\
\text { moderate, or severe. General symptoms (mainly headache, fever, or myalgia). Local and general symp- } \\
\text { toms are further reported in different intensity classes (mild, moderate, severe, lasting for at least } 4 \\
\text { days) reported as mild, moderate, or severe. Use of analgesics. }\end{array}$ \\
\hline \multirow[t]{2}{*}{ Notes } & Safety data only were extracted. \\
\hline & Funding source - mixed \\
\hline
\end{tabular}

\section{Risk of bias}

\begin{tabular}{lll}
\hline Bias & Authors' judgement & Support for judgement \\
\hline $\begin{array}{l}\text { Random sequence genera- } \\
\text { tion (selection bias) }\end{array}$ & Unclear risk & Unclear \\
\hline $\begin{array}{l}\text { Allocation concealment } \\
\text { (selection bias) }\end{array}$ & Unclear risk & Unclear \\
\hline $\begin{array}{l}\text { Blinding (performance } \\
\text { bias and detection bias) } \\
\text { All outcomes }\end{array}$ & Unclear risk & Unclear \\
\hline $\begin{array}{l}\text { Incomplete outcome data } \\
\text { (attrition bias) } \\
\text { All outcomes }\end{array}$ & Unclear risk & Unclear \\
\hline $\begin{array}{l}\text { Summary assessment } \\
\end{array}$ & Unclear risk & Unclear \\
\hline
\end{tabular}

\section{ab Forsyth 1967}

Fethods $\begin{aligned} & \text { From this report, only the first phase of the first trial is of interest to this review, in which administra- } \\ & \text { tion of whole-virus, oil adjuvanted influenza vaccine Invirin (GSK) was compared with placebo in a se- } \\ & \text { mi-randomised allocation. The trial was performed November to December } 1962 .\end{aligned}$




\section{ab Forsyth 1967 (Continued)}

Participants Medical students $(n=380)$ at the Queen's University of Belfast, UK
Trivalent aqueous vaccine (Invirin) one $0.25 \mathrm{~mL}$ dose IM containing strains A/Singapore/1/57, A/England/1/61, B/England/939/59. Placebo (phosphate-buffered saline) was administered as control. Participants born on odd days were given placebo $(n=186)$; those born on even days received the vaccine ( $n$ =194).

\begin{tabular}{lll}
\hline Notes & $\begin{array}{l}\text { Safety data only were extracted. } \\
\text { Government funded }\end{array}$ \\
\hline Risk of bias & Authors' judgement & Support for judgement \\
\hline Bias & High risk & Alternate \\
\hline $\begin{array}{l}\text { Random sequence genera- } \\
\text { tion (selection bias) }\end{array}$ & Not used \\
\hline $\begin{array}{l}\text { Allocation concealment } \\
\text { (selection bias) }\end{array}$ & High risk & Unclear \\
\hline $\begin{array}{l}\text { Blinding (performance } \\
\text { bias and detection bias) } \\
\text { All outcomes }\end{array}$ & Unclear risk & \\
\hline $\begin{array}{l}\text { Incomplete outcome data } \\
\text { (attrition bias) } \\
\text { All outcomes }\end{array}$ & Unclear risk & Unclear \\
\hline $\begin{array}{l}\text { Summary assessment } \\
\text { High risk }\end{array}$ & Unclear \\
\hline
\end{tabular}

\section{ab Goodeve 1983}

\begin{tabular}{ll}
\hline Methods & Randomised controlled trial, double-blind \\
\hline Participants & $\begin{array}{l}119 \text { healthy young adults from the Medical and Science Faculties of Sheffield University, UK, aged } 18 \text { to } \\
19 \text { years without egg allergy }\end{array}$ \\
\hline Interventions & $\begin{array}{l}\text { Purified subunit monovalent B/Hong Kong/73 flu vaccine prepared in } 4 \text { antigen concentrations of } 40, \\
\text { 20, } 10,5 \mu \text { g of HA per each } 0.5 \mathrm{~mL} \text { dose versus saline placebo }(0.5 \mathrm{~mL} \text { dose) subcutaneously adminis- } \\
\text { tered. Participants were divided into } 5 \text { groups of equal dimensions (no further description), each group } \\
\text { received } 1 \text { of the tested coded preparations. Artificial challenge } 1 \text { month later with live attenuated } \\
\text { RB77 virus. }\end{array}$
\end{tabular}


ab Goodeve 1983 (Continued)

Risk of bias

\begin{tabular}{|c|c|c|}
\hline Bias & Authors' judgement & Support for judgement \\
\hline $\begin{array}{l}\text { Random sequence genera- } \\
\text { tion (selection bias) }\end{array}$ & Unclear risk & Unclear \\
\hline $\begin{array}{l}\text { Allocation concealment } \\
\text { (selection bias) }\end{array}$ & Unclear risk & Unclear \\
\hline $\begin{array}{l}\text { Blinding (performance } \\
\text { bias and detection bias) } \\
\text { All outcomes }\end{array}$ & Unclear risk & Unclear \\
\hline $\begin{array}{l}\text { Incomplete outcome data } \\
\text { (attrition bias) } \\
\text { All outcomes }\end{array}$ & Unclear risk & Unclear \\
\hline Summary assessment & Unclear risk & Unclear \\
\hline
\end{tabular}

ab Hrabar 1977

\begin{tabular}{ll}
\hline Methods & Randomised controlled trial, double-blind, carried out during the season 1976 to 1977 \\
\hline Participants & $\begin{array}{l}167 \text { students at the technical school in Zagreb, former Republic of Yugoslavia, without sensitivity to egg } \\
\text { proteins, pregnancy, acute or chronic diseases }\end{array}$ \\
\hline Interventions & $\begin{array}{l}\text { Cold-adapted recombinant A/Victoria/3/75 vaccine administered in } 3 \text { different antigen concentrations } \\
(107.5,106.5,105.5 \mathrm{EID} 50 / 0.5 \mathrm{~mL}) \text { versus placebo. One } 0.5 \mathrm{~mL} \text { dose intranasally. }\end{array}$
\end{tabular}

\section{Outcomes}

Participants were medically examined on each of the successive 5 days after immunisation (lasting for at least 1 day). Throat infection, granular palate, oedematous uvula, fever (no cases) as cases and subject-days. For the following outcomes, authors give the total number of observed cases, without indication of the corresponding arm: malaise, swollen tonsils, fever (1), rhinorrhoea (1), conjunctivitis (7), laryngitis or hoarseness (3), cough (1), swollen tonsils (1), malaise (1). Surveillance was active.

Notes Safety data only were extracted.
Government funded

\section{Risk of bias}

\begin{tabular}{lll}
\hline Bias & Authors' judgement & Support for judgement \\
\hline $\begin{array}{l}\text { Random sequence genera- } \\
\text { tion (selection bias) }\end{array}$ & Unclear risk & Unclear \\
\hline $\begin{array}{l}\text { Allocation concealment } \\
\text { (selection bias) }\end{array}$ & Unclear risk & Unclear \\
\hline $\begin{array}{l}\text { Blinding (performance } \\
\text { bias and detection bias) } \\
\text { All outcomes }\end{array}$ & Unclear risk & Unclear \\
\hline $\begin{array}{l}\text { Incomplete outcome data } \\
\text { (attrition bias) }\end{array}$ & Unclear risk & Unclear \\
\hline
\end{tabular}


ab Hrabar 1977 (Continued)

All outcomes

Summaryassessment Unclear risk Unclear

ab Keitel 1993a

Methods $\quad$ "The two trials (Keitel 1993a and Keitel 1993b) tested three live attenuated vaccines." This paper re-
ports the results of 2 randomised controlled trials carried out in the USA

\begin{tabular}{ll}
\hline Participants & $\begin{array}{l}\text { Healthy volunteers recruited at Texas A\&M University and Texas Medical Center, aged between } 18 \text { and } \\
40 \text { years }\end{array}$ \\
\hline Interventions & $\begin{array}{l}\text { Two } 0.5 \mathrm{~mL} \text { doses of cold-adapted recombinant influenza vaccines, } 1 \text { month apart, containing } 107.1 \\
\text { TCID50 of each strain/dose. } 2 \text { studies were conducted in which } 4 \text { groups were formed }(2 \text { interventions, } \\
2 \text { placebos): } 1) \text { placebo } 1 \text { st and } 2 \text { nd dose. } 2) 1 \text { st: A/Kawasaki/9/86 (H1N1, CR 125) + A/Bethesda/1/85 } \\
(\mathrm{H} 3 \mathrm{~N} 2, \mathrm{CR} 90)+\mathrm{B} / \text { Ann Arbor } / 1 / 86(\mathrm{~B}, \mathrm{CRB} 117)\end{array}$ \\
\hline Outcomes & Mild upper respiratory symptoms. Fever $>=37.8^{\circ} \mathrm{C}$ within 1 week after each inoculation \\
\hline Notes & Safety data only were extracted \\
& Government funded
\end{tabular}

\section{Risk of bias}

\begin{tabular}{lll}
\hline Bias & Authors' judgement & Support for judgement \\
\hline $\begin{array}{l}\text { Random sequence genera- } \\
\text { tion (selection bias) }\end{array}$ & Unclear risk & Unclear \\
\hline $\begin{array}{l}\text { Allocation concealment } \\
\text { (selection bias) }\end{array}$ & Unclear risk & Unclear \\
\hline $\begin{array}{l}\text { Blinding (performance } \\
\text { bias and detection bias) } \\
\text { All outcomes }\end{array}$ & Unclear risk & Unclear \\
\hline $\begin{array}{l}\text { Incomplete outcome data } \\
\text { (attrition bias) } \\
\text { All outcomes }\end{array}$ & Unclear risk & Unclear \\
\hline $\begin{array}{l}\text { Summary assessment } \\
\end{array}$ & Unclear risk & Unclear \\
\hline
\end{tabular}

\section{ab Keitel 1993b}

\begin{tabular}{ll}
\hline Methods & $\begin{array}{l}\text { "The two trials (Keitel 1993a and Keitel 1993b) tested three live attenuated vaccines." This paper re- } \\
\text { ports the results of } 2 \text { randomised controlled trials carried out in the USA }\end{array}$ \\
\hline Participants & $\begin{array}{l}\text { Healthy volunteers recruited at Texas A\&M University and Texas Medical Center, aged between } 18 \text { and } \\
40 \text { years }\end{array}$ \\
\hline Interventions & $\begin{array}{l}\text { "Keitel 1993b tested the CR influenza A/Los Angeles/2/87 (H3N2) CR } 149 \text { with different lots of CR } 125 \\
\text { and CRB } 117 \text { used. CR 125, CR 90, and CR 149 express the hemagglutinin and neuraminidase of wild- } \\
\text { type A/Kawasaki (an A/Taiwan/1/86 [H1N1]-like virus), A/Bethesda (an A/Mississippi/1/86 [H3N2]-l and }\end{array}$ \\
\hline
\end{tabular}


ab Keitel 1993b (Continued)

$\mathrm{A} /$ Los Angeles (an A/Sichuan/2/87 [H3N2]-like virus), respectively, and the internal proteins of coldadapted influenza A/Ann Arbor/6/60 (H2N2). CRB 117 expresses the hemagglutinin and neuraminidase of wild-type influenza B/Ann Arbor/1/ 86 and the internal proteins of cold-adapted influenza B/Ann Arbor/1/66. Placebo was allantoid fluid"

Outcomes Mild upper respiratory symptoms. Fever $>=37.8^{\circ} \mathrm{C}$ within 1 week after each inoculation

Notes See Keitel 1993a. Safety data only were extracted

\section{Risk of bias}

\begin{tabular}{lll}
\hline Bias & Authors' judgement & Support for judgement \\
\hline $\begin{array}{l}\text { Random sequence genera- } \\
\text { tion (selection bias) }\end{array}$ & Unclear risk & Unclear \\
\hline $\begin{array}{l}\text { Allocation concealment } \\
\text { (selection bias) }\end{array}$ & Unclear risk & Unclear \\
\hline $\begin{array}{l}\text { Blinding (performance } \\
\text { bias and detection bias) }\end{array}$ & Unclear risk & Unclear \\
All outcomes & \\
\hline $\begin{array}{l}\text { Incomplete outcome data } \\
\text { (attrition bias) } \\
\text { All outcomes }\end{array}$ & Unclear risk & Unclear \\
\hline $\begin{array}{l}\text { Summary assessment } \\
\end{array}$ & Unclear risk & Unclear \\
\hline
\end{tabular}

ab Langley 2005

\begin{tabular}{|c|c|}
\hline Methods & Randomised controlled trial \\
\hline Participants & Healthy adults aged 18 to 50 years \\
\hline Interventions & $\begin{array}{l}\text { Inactivated } \mathrm{A} / \mathrm{New} \text { Caledonia/20/99 (H1N1) + A/Panama/2007/99 (H3N2) + B/Guangdong/120/2000 non- } \\
\text { covalent associated with outer membrane protein of Neisseria meningitidis. Single nasal dose contain- } \\
\text { ing } 15,30,45 \mu \mathrm{g} \text { versus placebo (phosphate-buffered saline) intranasally administered. }\end{array}$ \\
\hline Outcomes & $\begin{array}{l}\text { Local: within } 7 \text { days, graphic: rhinorrhoea, congestion, itch/burn, nosebleed, red/puffy eyes, sneezing, } \\
\text { sore throat. Systemic: within } 7 \text { days: cough, shortness of breath, headache, muscle/joint aches, poor } \\
\text { appetite, fatigue within } 48 \text { hours, nasal mucosa inflammation, nasal discharge, pharyngeal inflamma- } \\
\text { tion, sinusitis, enlarged cervical/postauricular nodes. }\end{array}$ \\
\hline Notes & $\begin{array}{l}\text { Safety data only were extracted. } \\
\text { Government and industry funded }\end{array}$ \\
\hline
\end{tabular}

\section{Risk of bias}

\begin{tabular}{lll}
\hline Bias & Authors' judgement & Support for judgement \\
\hline $\begin{array}{l}\text { Random sequence genera- } \\
\text { tion (selection bias) }\end{array}$ & Unclear risk & Unclear \\
\hline
\end{tabular}


ab Langley 2005 (Continued)

\begin{tabular}{lll}
$\begin{array}{l}\text { Allocation concealment } \\
\text { (selection bias) }\end{array}$ & High risk & Inadequate \\
\hline $\begin{array}{l}\text { Blinding (performance } \\
\text { bias and detection bias) } \\
\text { All outcomes }\end{array}$ & Unclear risk & Unclear \\
\hline
\end{tabular}

\begin{tabular}{l}
\hline Incomplete outcome data $\quad$ Unclear risk Unclear \\
(attrition bias) \\
All outcomes
\end{tabular}

Summary assessment High risk High risk

\section{ab Lauteria 1974}

Methods Controlled trial. Randomisation procedure was neither described nor mentioned. Participants were paired according to age and sex, 1 participant in each pair received vaccine, the other placebo. Double-blind

\begin{tabular}{ll}
\hline Participants & $\begin{array}{l}37 \text { volunteers aged } 18 \text { to } 24 \text { years, with titre of serum neutralising antibodies to A/Hong Kong/8/68 } \geq \\
1: 16\end{array}$ \\
\hline Interventions & $\begin{array}{l}\text { Live attenuated A/England/8/68 grown in presence of heated equine serum. Two } 0.5 \mathrm{~mL} \text { doses contain- } \\
\text { ing } 104 \text { TCID50 of this strain or placebo (0.85\% sodium chloride) were administered intranasally } 2 \text { to } 3 \\
\text { weeks apart }\end{array}$ \\
\hline Outcomes & $\begin{array}{l}\text { Participants observed for } 4 \text { days, beginning } 24 \text { hours after immunisation. Fever, myalgia, rhinitis, } \\
\text { cough, pharyngitis }\end{array}$ \\
\hline Notes & Safety data only were extracted. \\
Government and industry funded
\end{tabular}

\section{Risk of bias}

\begin{tabular}{lll}
\hline Bias & Authors' judgement & Support for judgement \\
\hline $\begin{array}{l}\text { Random sequence genera- } \\
\text { tion (selection bias) }\end{array}$ & Unclear risk & Unclear \\
\hline $\begin{array}{l}\text { Allocation concealment } \\
\text { (selection bias) }\end{array}$ & Unclear risk & Unclear \\
\hline $\begin{array}{l}\text { Blinding (performance } \\
\text { bias and detection bias) }\end{array}$ & Unclear risk & Unclear \\
All outcomes & \\
\hline $\begin{array}{l}\text { Incomplete outcome data } \\
\text { (attrition bias) } \\
\text { All outcomes }\end{array}$ & Unclear risk & Unclear \\
\hline $\begin{array}{l}\text { Summary assessment } \\
\end{array}$ & Unclear risk & Unclear \\
\hline
\end{tabular}


ab Miller 1977

\begin{tabular}{|c|c|c|}
\hline Methods & \multicolumn{2}{|c|}{ Randomised controlled trial } \\
\hline Participants & \multicolumn{2}{|c|}{43 seronegative healthy adults aged between 22 and 50 years } \\
\hline Interventions & \multicolumn{2}{|c|}{$\begin{array}{l}\text { Live attenuated serum inhibitor resistant flu B vaccine R75 (a recombinant of B/Hong Kong/5/72 with } \\
\text { B/Russia/69) containing } 107.2 \text { EID50 of R75/0.5 mL dose versus placebo (sucrose } 5 \% \text { ). Intranasal, } 2 \text { dos- } \\
\text { es, } 2 \text { weeks apart }\end{array}$} \\
\hline Outcomes & \multicolumn{2}{|c|}{$\begin{array}{l}\text { Participants were interviewed during the } 5 \text { days following each immunisation. Local reaction (defined } \\
\text { as immediate complains and comprising bad taste or burning, lasting for a few moments). Systemic re- } \\
\text { action (consisting essentially of headache and rhinorrhoea) }\end{array}$} \\
\hline Notes & \multicolumn{2}{|c|}{ Safety data only were extracted. } \\
\hline & \multicolumn{2}{|l|}{ Government funded } \\
\hline \multicolumn{3}{|l|}{ Risk of bias } \\
\hline Bias & Authors' judgement & Support for judgement \\
\hline $\begin{array}{l}\text { Random sequence genera- } \\
\text { tion (selection bias) }\end{array}$ & Unclear risk & Unclear \\
\hline $\begin{array}{l}\text { Allocation concealment } \\
\text { (selection bias) }\end{array}$ & Unclear risk & Unclear \\
\hline $\begin{array}{l}\text { Blinding (performance } \\
\text { bias and detection bias) } \\
\text { All outcomes }\end{array}$ & Unclear risk & Unclear \\
\hline $\begin{array}{l}\text { Incomplete outcome data } \\
\text { (attrition bias) } \\
\text { All outcomes }\end{array}$ & Unclear risk & Unclear \\
\hline Summary assessment & Unclear risk & Unclear \\
\hline
\end{tabular}

ab Pyrhönen 1981

\begin{tabular}{ll}
\hline Methods & Randomised controlled trial conducted in the 1976 to 1977 season in Finland \\
\hline Participants & 307 healthy adults \\
\hline Interventions & $\begin{array}{l}1 \text { of the following } 4 \text { preparations was administered to } 1 \text { of the } 4 \text { study arms. "Volunteers were inoculat- } \\
\text { ed with bivalent subunit influenza vaccine containing } \\
1200 \mathrm{IU} \text { of strain A/Victoria/3/75 (H3N2) antigen and } 800 \mathrm{IU} \text { of B/Hongkong/8/73 antigen in } 0.5 \mathrm{ml} \text { of } \\
\text { phosphate or phosphate-buffered saline solution as placebo" }\end{array}$ \\
\hline Outcomes & $\begin{array}{l}\text { Harms assessed by questionnaires filled out by each participant within } 3 \text { days after immunisation. } \\
53 / 151, \text { placebo } 3 / 154 ; \text { tenderness at vaccination site: vaccine } 89 / 151, \text { placebo } 12 / 154 ; \text { tenderness of } \\
\text { axillary glands: vaccine } 6 / 151, \text { placebo } 2 / 154\end{array}$ \\
\hline
\end{tabular}

Notes

Safety data only were extracted

Government funded 
ab Pyrhönen 1981 (Continued)

Risk of bias

\begin{tabular}{lll}
\hline Bias & Authors' judgement & Support for judgement \\
\hline $\begin{array}{l}\text { Random sequence genera- } \\
\text { tion (selection bias) }\end{array}$ & Unclear risk & Unclear \\
\hline $\begin{array}{l}\text { Allocation concealment } \\
\text { (selection bias) }\end{array}$ & Unclear risk & Unclear \\
\hline $\begin{array}{l}\text { Blinding (performance } \\
\text { bias and detection bias) }\end{array}$ & Unclear risk & Unclear \\
All outcomes & \\
\hline $\begin{array}{l}\text { Incomplete outcome data } \\
\text { (attrition bias) } \\
\text { All outcomes }\end{array}$ & Unclear risk & Unclear \\
\hline $\begin{array}{l}\text { Summary assessment } \\
\end{array}$ & Unclear risk & Unclear \\
\hline
\end{tabular}

ab Reeve 1982

\begin{tabular}{ll}
\hline Methods & Randomised controlled trial carried out in Wien \\
\hline Participants & 20 university students aged 20 to 24 years \\
\hline Interventions & $\begin{array}{l}\text { 1st phase: cold-recombinant, live flu vaccine II RB-77 (B/Ann Arbor/1/66 and B/Tecumse/10/77) con- } \\
\text { taining } 107.2 \text { EID50 per } 0.5 \mathrm{~mL} \text { dose versus placebo. } 1 \text { dose intranasally. During this phase, participants } \\
\text { lived under sequestered condition, and close contact between vaccine and placebo recipients was pos- } \\
\text { sible. } 2 \text { nd phase: } 3 \text { weeks after the } 1 \text { st dose all participants were immunised with } 1 \text { dose of the same } \\
\text { vaccine }\end{array}$ \\
\hline
\end{tabular}

Outcomes During the 5 days following immunisation, participants were medically observed and temperature recorded morning and evening. Occurring symptoms were attributed scores ( 0 to 3 ) depending on their severity (no, light, moderate, severe). Fever (oral temperature $>38^{\circ} \mathrm{C}$ ): 0/10, 0/10; sneezing: 1/10, 0/10; stuffy nose: $7 / 10,1 / 10$; running nose: $3 / 10,0 / 10$; afebrile subjective symptoms: $8 / 10,2 / 10$

\begin{tabular}{ll}
\hline Notes & Safety data only were extracted \\
Industry funded
\end{tabular}

\section{Risk of bias}

\begin{tabular}{lll}
\hline Bias & Authors' judgement & Support for judgement \\
\hline $\begin{array}{l}\text { Random sequence genera- } \\
\text { tion (selection bias) }\end{array}$ & Unclear risk & Unclear \\
\hline $\begin{array}{l}\text { Allocation concealment } \\
\text { (selection bias) }\end{array}$ & Unclear risk & Unclear \\
\hline $\begin{array}{l}\text { Blinding (performance } \\
\text { bias and detection bias) } \\
\text { All outcomes }\end{array}$ & Unclear risk & Unclear \\
\hline
\end{tabular}


ab Reeve 1982 (Continued)

Incomplete outcome data Unclear risk Unclear
(attrition bias)

All outcomes

\begin{tabular}{lll}
\hline Summaryassessment $\quad$ Unclear risk & Unclear \\
\hline
\end{tabular}

\section{ab Rocchi 1979a}

\begin{tabular}{|c|c|}
\hline Methods & Cluster-randomised controlled trial carried out during the 1976 to 1977 season \\
\hline Participants & $\begin{array}{l}496 \text { healthy military recruits (aged } 18 \text { to } 20 \text { years) belonging to } 4 \text { different companies from "Scuola Al- } \\
\text { lievi Sottoufficiali" in Viterbo, Italy }\end{array}$ \\
\hline Interventions & $\begin{array}{l}1 \text { of the following } 4 \text { preparations were administered to } 1 \text { of the } 4 \text { study arms: } 1 \text {. live attenuated A/Victo- } \\
\text { ria/3/75; two } 2 \mathrm{~mL} \text { doses ( } 2104.5 \text { EID50/dose) oral. } 2 \text {. Live attenuated recombinant A/Puerto Rico/8/34, } \\
\text { A/Victoria/3/75; two } 0.5 \mathrm{~mL} \text { doses intranasally ( } 107 \text { EID50/dose). } 3 \text {. Inactivated A/Victoria/3/75 (600 } \\
\text { IU), B/Hong Kong/5/72 ( } 300 \mathrm{IU} \text { ) and AlPO4, intramuscular placebo (vaccine diluent) administered in- } \\
\text { tranasally. The } 2 \text { doses were administered } 2 \text { to } 3 \text { weeks apart. }\end{array}$ \\
\hline Outcomes & $\begin{array}{l}\text { Within } 15 \text { days after administration of the } 1 \text { st dose. Malaise, myalgia, headache, sore throat, cough, } \\
\text { fever }>=38.5^{\circ} \mathrm{C} \text {, fever }>=37.5^{\circ} \mathrm{C}, 3 \text { or more symptoms, any symptoms. Surveillance was passive. }\end{array}$ \\
\hline \multirow[t]{2}{*}{ Notes } & $\begin{array}{l}\text { Units of randomisation appear to be companies. No description of manner of allocation is mentioned. } \\
\text { Blind (only for the cases of intranasal administration). Influenza outbreak occurred when the immuni- } \\
\text { sation began (intraepidermic study). } \\
\text { Safety data only were extracted. }\end{array}$ \\
\hline & Government funded \\
\hline
\end{tabular}

\section{Risk of bias}

\section{Bias Authors' judgement Support for judgement}

Random sequence genera- Unclear risk Unclear

tion (selection bias)

\begin{tabular}{lll}
\hline $\begin{array}{l}\text { Allocation concealment } \\
\text { (selection bias) }\end{array}$ & Unclear risk
\end{tabular}

\begin{tabular}{lll}
\hline $\begin{array}{l}\text { Blinding (performance } \\
\text { bias and detection bias) } \\
\text { All outcomes }\end{array}$ & Unclear risk & \\
\hline $\begin{array}{l}\text { Incomplete outcome data } \\
\text { (attrition bias) }\end{array}$ & Unclear risk & Unclear \\
\begin{tabular}{lll} 
All outcomes \\
Summary assessment & Unclear risk & Unclear \\
\hline
\end{tabular}
\end{tabular}

\section{ab Rocchi 1979b}

Methods See ab Rocchi 1979a


ab Rocchi 1979b (Continued)

Participants

Interventions

Outcomes

Notes

Risk of bias

\begin{tabular}{lll}
\hline Bias & Authors' judgement & Support for judgement \\
\hline $\begin{array}{l}\text { Random sequence genera- } \\
\text { tion (selection bias) }\end{array}$ & Unclear risk & Unclear \\
\hline $\begin{array}{l}\text { Allocation concealment } \\
\text { (selection bias) }\end{array}$ & Unclear risk & Not used \\
\hline $\begin{array}{l}\text { Blinding (performance } \\
\text { bias and detection bias) }\end{array}$ & Unclear risk & Unclear \\
All outcomes & \\
\hline $\begin{array}{l}\text { Incomplete outcome data } \\
\text { (attrition bias) }\end{array}$ & Unclear risk & Unclear \\
All outcomes & & \\
\hline $\begin{array}{l}\text { Summary assessment } \\
\end{array}$ & Unclear risk & Unclear \\
\hline
\end{tabular}

ab Saxen 1999

Methods Randomised controlled trial, double-blind, conducted in Finland during the 1996 to 1997 influenza sea-
son. Randomisation methods were not described.

\begin{tabular}{ll}
\hline Participants & 216 healthcare workers: 211 treated and 427 placebo \\
\hline Interventions & $\begin{array}{l}\text { Trivalent inactivated intramuscular vaccine. Schedule and dose were: single dose; } 15 \mu g \text { each strain. } \\
\text { Vaccine composition was: A/Wahan/359/95, A/Singapore/6/86, and B/Beijing/184/93. Placebo was } \\
\text { saline for injection. Vaccine was recommended. }\end{array}$ \\
\hline Outcomes & $\begin{array}{l}\text { Working days lost due to respiratory infections, episodes of respiratory infections, days ill, and antimi- } \\
\text { crobial prescriptions. Respiratory infection was a common cold; febrile ILIs were not deted } \\
\text { adverse effects were defined as local pain. Systemic adverse effects were defined as fever and fatigue. }\end{array}$ \\
\hline Notes & $\begin{array}{l}\text { Efficacy data were not extracted because episodes of respiratory infections were unclearly defined. } \\
\text { Safety data only were extracted. } \\
\text { Government funded }\end{array}$ \\
\hline
\end{tabular}

\section{Risk of bias}

\begin{tabular}{lll}
\hline Bias & Authors' judgement & Support for judgement \\
\hline $\begin{array}{l}\text { Random sequence genera- } \\
\text { tion (selection bias) }\end{array}$ & Unclear risk & Unclear \\
\hline
\end{tabular}


ab Saxen 1999 (Continued)

\begin{tabular}{lll}
$\begin{array}{l}\text { Allocation concealment } \\
\text { (selection bias) }\end{array}$ & Unclear risk & Unclear \\
\hline $\begin{array}{l}\text { Blinding (performance } \\
\text { bias and detection bias) }\end{array}$ & Unclear risk & Unclear \\
All outcomes &
\end{tabular}

\begin{tabular}{l}
\hline Incomplete outcome data Unclear risk Unclear \\
(attrition bias) \\
All outcomes
\end{tabular}

\begin{tabular}{ll}
\hline Summaryassessment $\quad$ Unclear risk & Unclear \\
\hline
\end{tabular}

\section{ab Scheifele 2003}

Rethods
posure to the vaccine and onset of ORS in healthy adults with no previous history of ORS. The trial took
place in 5 centres in Canada in September 2001 and was 1 of the conditions of registration of the vac-
cine, given the high incidence of ORS in the previous season. Centralised randomisation and allocation
of centrally prepared, coded, opaque syringes took place. Cross-over to either vaccine or placebo took
place 5 to 7 days after the initial injection.

\begin{tabular}{ll}
\hline Participants & The study included 651 adults with a mean age of 45.17 participants are unaccounted for. \\
\hline Interventions & Fluviral (Shire) split trivalent containing A/New Caledonia/20/99 (H1N1), A/Panama/2007/99 (H3N2), B/ \\
& Victoria/504/2000 with additional splitting with Triton X-100 splitting agent or saline placebo 0.5 mL. \\
& Additional splitting was necessary to test the hypothesis that large clumps of virions were responsible \\
& for the ORS seen the previous season.
\end{tabular}

Outcomes ORS (bilateral conjunctivitis; facial swelling - lip, lid, or mouth; difficulty in breathing and chest discomfort, including cough, wheeze, dysphagia, or sore throat). Local signs/symptoms (redness, swelling, pain). Follow-up was by phone interview at 24 hours and 6 days after vaccination.

The authors conclude that (mild) ORS is significantly associated with split TIV immunisation (attribut-
able risk $2.9 \%, 0.6$ to 5.2$)$. Other adverse effects associated with TIV are hoarseness (1.3\%, 0.3 to 1.3$)$
and coughing $(1.2 \%, 0.2$ to 1.6$)$. The study is good quality, and the authors' conclusions are robust. It
is extraordinary that no one has looked for these symptoms before, but it may be that the relatively
young age of participants and the hypothesis contributed to this. Safety-only study.
Government funded

\section{Risk of bias}

\begin{tabular}{lll}
\hline Bias & Authors' judgement & Support for judgement \\
\hline $\begin{array}{l}\text { Random sequence genera- } \\
\text { tion (selection bias) }\end{array}$ & Low risk & Block randomisation \\
\hline $\begin{array}{l}\text { Allocation concealment } \\
\text { (selection bias) }\end{array}$ & Low risk & Adequate \\
\hline $\begin{array}{l}\text { Blinding (performance } \\
\text { bias and detection bias) } \\
\text { All outcomes }\end{array}$ & Low risk & Adequate \\
\hline
\end{tabular}


ab Scheifele 2003 (Continued)

Incomplete outcome data Low risk $\quad$ Adequate
(attrition bias)

All outcomes

Summaryassessment Low risk Adequate

ab Spencer 1977

\begin{tabular}{|c|c|}
\hline Methods & Controlled trial, single-blind \\
\hline Participants & $\begin{array}{l}21 \text { pairs of students and employers at the University of California, aged between } 24 \text { and } 50 \text { years who } \\
\text { lived together or worked in close proximity }\end{array}$ \\
\hline Interventions & $\begin{array}{l}\text { Recombinant, live attenuated R } 75 \text { vaccine (B/Hong Kong/ } 5 / 72 \text { and B/Russia/69) containing } 107.5 \mathrm{EID} / \\
\text { dose versus placebo (allantoic fluid). Lyophilised vaccine was supplied by Smith, Kline \& French Labo- } \\
\text { ratories and diluted with } 2.5 \mathrm{~mL} \text { of a } 5 \% \text { sucrose solution just before administration. Both preparations } \\
\text { were administered intranasally ( } 5 \text { drops/nostril). In each pair } 1 \text { individual received vaccine and the oth- } \\
\text { er placebo. A second dose was administered } 14 \text { days apart. }\end{array}$ \\
\hline
\end{tabular}

Outcomes

Any clinical symptoms within 7 days after each immunisation (rhinitis, cough, pharyngitis, headache, malaise, and myalgia were the prominently observed symptoms, but given as aggregates).

\begin{tabular}{ll}
\hline Notes & Reported safety data do not allow quantitative analysis. \\
& Industry funded
\end{tabular}

\section{Risk of bias}

\begin{tabular}{lll}
\hline Bias & Authors' judgement & Support for judgement \\
\hline $\begin{array}{l}\text { Random sequence genera- } \\
\text { tion (selection bias) }\end{array}$ & Unclear risk & Unclear \\
\hline $\begin{array}{l}\text { Allocation concealment } \\
\text { (selection bias) }\end{array}$ & Unclear risk & Unclear \\
\hline $\begin{array}{l}\text { Blinding (performance } \\
\text { bias and detection bias) } \\
\text { All outcomes }\end{array}$ & Unclear risk & Unclear \\
\hline $\begin{array}{l}\text { Incomplete outcome data } \\
\text { (attrition bias) }\end{array}$ & Unclear risk & Unclear \\
All outcomes & & \\
\hline Summary assessment & Unclear risk & Unclear \\
\hline
\end{tabular}

bb Dauvilliers 2013

\begin{tabular}{ll}
\hline Methods & $\begin{array}{l}\text { Case-control study investigating the association between exposure to monovalent, 2009-10 pandemic } \\
\text { H1N1 vaccines and onset of narcolepsy }\end{array}$ \\
\hline Participants & $\begin{array}{l}\text { Cases }(\mathrm{n}=59) \text { : were identified from } 14 \text { French expert orphan disease narcolepsy centres among par- } \\
\text { ticipants referred to } 1 \text { of the participating sleep centres to confirm the diagnosis by polysomnography } \\
\text { as well as the Multiple Sleep Latency Test between } 1 \text { October } 2009 \text { and } 30 \text { April } 2011 \text { (according to the }\end{array}$ \\
\hline
\end{tabular}


bb Dauvilliers 2013 (Continued)

International Classification of Sleep Disorders definition, ICSD 2005). Participating centres identified retrospectively from lists of medical records completed by reference centres for orphan diseases as required by the French government and from hospital statistic databases all their patients with narcolepsy-cataplexy potentially matching the eligibility criteria. All potentially eligible cases were asked to participate, and their clinical history was revised to confirm the diagnosis of narcolepsy-cataplexy following the criteria of the Brighton Collaboration, levels 1 to 3.

Controls $(n=135)$ : were selected among patients from the hospitals to which the participating sleep centres belonged and among healthy volunteers from a national database (Narcobank). Up to 4 controls were matched to each case for sex, age, geographic location.

Only 25 cases and 73 controls were at least 18 years old.

Interventions

Exposure to Pandremix (AS03 adjuvanted) or Panenza (not adjuvanted) monovalent p H1N1 influenza vaccines. Vaccination was ascertained by means of a phone interview, during which other data were also recorded (body mass index, smoking, medical history, history of viral or bacterial infections), and confirmed by vaccination certificates. Date of first disease symptoms was reported.

A sensitivity analysis was carried out considering as index date:

1. the date of narcolepsy-cataplexy diagnosis;

2. the date of Multiple Sleep Latency Test; or

3. the date the first symptoms appeared.

Participants were considered vaccinated if they received vaccination before this latter date (whatever analysis authors performed). Data analysis was performed excluding and including cases for whom symptom onset did occur concomitantly or shortly before vaccination, so that it was not possible to state whether vaccination had effectively been administered before the onset of first symptoms, from analyses 1 and 2. (They remained always included in analysis 3).

Effect estimates were moreover performed considering as exposed those participants who received AS03-adjuvanted pandemic vaccine only.

\begin{tabular}{ll}
\hline Outcomes & Narcolepsy-cataplexy (Brighton Collaboration levels 1 to 3) \\
\hline Notes & Mixed (?) \\
This was not an industry-supported study. This study was funded by grants from the Agence Nationale \\
de Sécurité du Médicament et des Produits de Santé, the European Centre for Disease Prevention and \\
Control, and the PHRC AOM07-138 grant from the French Health Ministry. The funders had no role in \\
study design, data collection and analysis, decision to publish, or preparation of the manuscript. Y \\
Dauvilliers has received funds for speaking and board engagements with UCB, Cephalon, Jazz, No- \\
vartis, and Bioprojet. P Franco has received funds for speaking and board engagements with UCB. \\
MP Ortho has received funds for speaking from Cephalon and board engagements with Bioprojet. C \\
Monaca Charley has received funds for speaking or board engagements, or both with UCB, Novartis, \\
and Cephalon. M Lecendreux has received funds for speaking and board engagements with UCB and \\
Bioprojet.
\end{tabular}

\section{Risk of bias}

\begin{tabular}{lll}
\hline Bias & Authors' judgement & Support for judgement \\
\hline CC - case selection & Unclear risk & $\begin{array}{l}\text { Record linkage. Medical record reviewed, and participants fulfilling levels } 1 \text { to } \\
\text { All outcomes }\end{array}$ \\
& $\begin{array}{l}\text { 3 of Brighton Collaboration definition included as cases. Recruited through } 14 \\
\text { centres across France. It is possible that healthcare professionals were over- } \\
\text { represented. }\end{array}$ \\
\hline
\end{tabular}

CC - control selection Unclear risk Hospital controls
All outcomes

All outcomes

CC - comparability Unclear risk Cases and controls were matched only for age, sex, and geographical area.


bb Dauvilliers 2013 (Continued)

All outcomes

CC - exposure Low risk Vaccination records

All outcomes

Summaryassessment Unclear risk Unclear risk of bias

bb DeStefano 2003

\begin{tabular}{ll}
\hline Methods & Case-control study \\
\hline Participants & Data from Vaccine Safety Datalink (large database of cases of disease following vaccination) in the USA \\
\hline Interventions & Immunisation with influenza and other vaccines assessed by means of medical records. \\
\hline Outcomes & $\begin{array}{l}\text { Cases: physician diagnosis of multiple sclerosis or optic neuritis in medical record } \\
\text { Controls: up to } 3 \text { controls per case were selected from automated HMO member files, at least } 1 \text { year of } \\
\text { HMO enrolment, matched on age (within } 1 \text { year) and gender }\end{array}$ \\
\hline Notes & Rare events (safety) \\
Government funded
\end{tabular}

\section{Risk of bias}

\begin{tabular}{lll}
\hline Bias & Authors' judgement & Support for judgement \\
\hline $\begin{array}{l}\text { CC - case selection } \\
\text { All outcomes }\end{array}$ & Low risk & From HMO registry \\
\hline $\begin{array}{l}\text { CC - control selection } \\
\text { All outcomes }\end{array}$ & Low risk & From HMO registry \\
\hline $\begin{array}{l}\text { CC - comparability } \\
\text { All outcomes }\end{array}$ & Unclear risk & Poor matching \\
\hline $\begin{array}{l}\text { CC - exposure } \\
\text { All outcomes }\end{array}$ & Unclear risk & From registry and telephone interview \\
\hline Summary assessment & Unclear risk & Unclear \\
\hline
\end{tabular}

bb Dieleman 2011a

\begin{tabular}{ll}
\hline Methods & Case-control study \\
\hline Participants & Cases $(n=145)$ : Guillain-Barré syndrome cases (defined according to the Brighton Collaboration defini- \\
& tion) diagnosed in France between 2007 and 2010. \\
& Controls $(n=1080)$ : the dates for control recruitment were matched (by calendar month) to the in- \\
& dex date of the associated case. Additional matching criteria included gender, age (65 years for cases \\
& aged 18 years or more and 61 years for cases younger than 18 years) and place of residence (southern \\
& or northern France).
\end{tabular}
Interventions
Exposure to influenza vaccine. Data about pandemic vaccine analysed separately. 
bb Dieleman 2011a (Continued)

Exposure to virus and occurrence of ILI also tested as risk factor.

Outcomes Association between Guillain-Barré syndrome and influenza vaccine exposure

Notes The study has been financially supported by LA-SER, GSK Biologicals, and Sanofi Pasteur.

\section{Risk of bias}

\begin{tabular}{lll}
\hline Bias & Authors' judgement & Support for judgement \\
\hline $\begin{array}{l}\text { CC - case selection } \\
\text { All outcomes }\end{array}$ & Unclear risk & From different countries \\
\hline $\begin{array}{l}\text { CC - control selection } \\
\text { All outcomes }\end{array}$ & Unclear risk & Not same population, insufficient description \\
\hline $\begin{array}{l}\text { CC - comparability } \\
\text { All outcomes }\end{array}$ & Unclear risk & Matching \\
\hline $\begin{array}{l}\text { CC - exposure } \\
\text { All outcomes }\end{array}$ & Unclear risk & Interview \\
\hline Summary assessment & High risk & High risk of bias \\
\hline
\end{tabular}

bb Dieleman 2011b

\begin{tabular}{|c|c|}
\hline Methods & Case-control study \\
\hline Participants & $\begin{array}{l}\text { Cases ( } n=145 \text { ): Guillain-Barré syndrome cases (defined according to the Brighton Collaboration defini- } \\
\text { tion) diagnosed in France between } 2007 \text { and } 2010 \text {. } \\
\text { Controls ( } n=1080) \text { : the dates for control recruitment were matched (by calendar month) to the index } \\
\text { date of the associated case. Additional matching criteria included gender, age ( } 65 \text { years for cases aged } \\
18 \text { years or more and } 61 \text { years for cases younger than } 18 \text { years), and place of residence (southern or } \\
\text { northern France). }\end{array}$ \\
\hline
\end{tabular}

\begin{tabular}{ll}
\hline Interventions & Exposure to influenza vaccine. Data about pandemic vaccine analysed separately. \\
& Exposure to virus and occurrence of ILI also tested as risk factor.
\end{tabular}

\begin{tabular}{|c|c|c|}
\hline Outcomes & \multicolumn{2}{|c|}{ Association between Guillain-Barré syndrome and influenza vaccine exposure } \\
\hline Notes & \multicolumn{2}{|c|}{ The study has been financially supported by LA-SER, GSK Biologicals, and Sanofi Pasteur. } \\
\hline \multicolumn{3}{|l|}{ Risk of bias } \\
\hline Bias & Authors' judgement & Support for judgement \\
\hline $\begin{array}{l}\text { CC - case selection } \\
\text { All outcomes }\end{array}$ & Low risk & Low \\
\hline $\begin{array}{l}\text { CC - control selection } \\
\text { All outcomes }\end{array}$ & Unclear risk & Not same population \\
\hline $\begin{array}{l}\text { CC - comparability } \\
\text { All outcomes }\end{array}$ & Unclear risk & Matching \\
\hline CC - exposure & Unclear risk & Interview \\
\hline
\end{tabular}


bb Dieleman 2011b (Continued)

All outcomes

Summaryassessment Unclear risk Unclear

bb Dieleman 2011c

\begin{tabular}{ll}
\hline Methods & Case-control study \\
\hline Participants & Cases $(n=145)$ : Guillain-Barré syndrome cases (defined according to the Brighton Collaboration defini- \\
tion) diagnosed in France between 2007 and 2010. & \\
Controls $(n=1080):$ the dates for control recruitment were matched (by calendar month) to the index \\
date of the associated case. Additional matching criteria included gender, age (65 years for cases aged \\
18 years or more and 61 years for cases younger than 18 years), and place of residence (southern or \\
northern France).
\end{tabular}

Interventions

Exposure to influenza vaccine. Data about pandemic vaccine analysed separately.

Exposure to virus and occurrence of ILI also tested as risk factor.

\begin{tabular}{ll}
\hline Outcomes & Association between Guillain-Barré syndrome and influenza vaccine exposure \\
\hline Notes & The study has been financially supported by LA-SER, GSK Biologicals, and Sanofi Pasteur.
\end{tabular}

\section{Risk of bias}

\begin{tabular}{lll}
\hline Bias & Authors' judgement & Support for judgement \\
\hline $\begin{array}{l}\text { CC - case selection } \\
\text { All outcomes }\end{array}$ & Low risk & Low \\
\hline $\begin{array}{l}\text { CC - control selection } \\
\text { All outcomes }\end{array}$ & Unclear risk & Not same population \\
\hline $\begin{array}{l}\text { CC - comparability } \\
\text { All outcomes }\end{array}$ & Unclear risk & Matching \\
\hline $\begin{array}{l}\text { CC - exposure } \\
\text { All outcomes }\end{array}$ & Unclear risk & Interview \\
\hline Summary assessment & Unclear risk & Unclear \\
\hline
\end{tabular}

bb Dieleman 2011d

\begin{tabular}{ll}
\hline Methods & Case-control study \\
\hline Participants & $\begin{array}{l}\text { Cases }(n=145): \text { Guillain-Barré syndrome cases (defined according to the Brighton Collaboration defini- } \\
\text { tion) diagnosed in France between } 2007 \text { and } 2010 .\end{array}$ \\
& $\begin{array}{l}\text { Controls }(n=1080): \text { the dates for control recruitment were matched (by calendar month) to the index } \\
\text { date of the associated case. Additional matching criteria included gender, age (65 years for cases aged } \\
\text { 18 years or more and } 61 \text { years for cases younger than } 18 \text { years), and place of residence (southern or } \\
\text { northern France). }\end{array}$
\end{tabular}

Interventions

Exposure to influenza vaccine. Data about pandemic vaccine analysed separately.

Exposure to virus and occurrence of ILI also tested as risk factor. 
bb Dieleman 2011d (Continued)

\begin{tabular}{|c|c|c|}
\hline Outcomes & \multicolumn{2}{|c|}{ Association between Guillain-Barré syndrome and influenza vaccine exposure } \\
\hline Notes & \multicolumn{2}{|c|}{ The study has been financially supported by LA-SER, GSK Biologicals, and Sanofi Pasteur. } \\
\hline Risk of bias & & \\
\hline Bias & Authors' judgement & Support for judgement \\
\hline $\begin{array}{l}\text { CC - case selection } \\
\text { All outcomes }\end{array}$ & Low risk & Low \\
\hline $\begin{array}{l}\text { CC - control selection } \\
\text { All outcomes }\end{array}$ & Unclear risk & Not same population \\
\hline $\begin{array}{l}\text { CC - comparability } \\
\text { All outcomes }\end{array}$ & Unclear risk & Matching \\
\hline $\begin{array}{l}\text { CC - exposure } \\
\text { All outcomes }\end{array}$ & Unclear risk & Interview \\
\hline Summary assessment & Unclear risk & Unclear \\
\hline
\end{tabular}

bb Dieleman 2011e

\begin{tabular}{|c|c|}
\hline Methods & Case-control study \\
\hline Participants & $\begin{array}{l}\text { Cases ( } n=145) \text { : Guillain-Barré syndrome cases (defined according to the Brighton Collaboration defini- } \\
\text { tion) diagnosed in France between } 2007 \text { and } 2010 \text {. } \\
\text { Controls }(n=1080) \text { : the dates for control recruitment were matched (by calendar month) to the index } \\
\text { date of the associated case. Additional matching criteria included gender, age ( } 65 \text { years for cases aged } \\
18 \text { years or more and } 61 \text { years for cases younger than } 18 \text { years), and place of residence (southern or } \\
\text { northern France). }\end{array}$ \\
\hline
\end{tabular}

$\begin{array}{ll}\text { Interventions } & \text { Exposure to influenza vaccine. Data about pandemic vaccine analysed separately. } \\ & \text { Exposure to virus and occurrence of ILI also tested as risk factor. }\end{array}$

\begin{tabular}{ll}
\hline Outcomes & Association between Guillain-Barré syndrome and influenza vaccine exposure \\
\hline Notes & The study has been financially supported by LA-SER, GSK Biologicals, and Sanofi Pasteur. \\
\hline
\end{tabular}

\section{Risk of bias}

\begin{tabular}{lll}
\hline Bias & Authors' judgement & Support for judgement \\
\hline $\begin{array}{l}\text { CC - case selection } \\
\text { All outcomes }\end{array}$ & Low risk & Low \\
\hline $\begin{array}{ll}\text { CC - control selection } \\
\text { All outcomes }\end{array}$ & Unclear risk & Not same population \\
\hline $\begin{array}{ll}\text { CC - comparability } \\
\text { All outcomes }\end{array}$ & Unclear risk & Matching \\
\hline CC - exposure & & \\
All outcomes & Unclear risk & Interview \\
\hline \hline
\end{tabular}


bb Dieleman 2011e (Continued)
Summary assessment
Unclear risk
Unclear

bb Galeotti 2013

\begin{tabular}{|c|c|}
\hline Methods & Case-control study testing the association between influenza vaccination and Guillain-Barré syndrome \\
\hline \multirow[t]{2}{*}{ Participants } & $\begin{array}{l}\text { Cases ( } n=140 \text { ): adults with Guillain-Barré syndrome defined according to the Brighton Collaboration } \\
\text { definition (levels } 1 \text { to } 3 \text { ) recruited at } 121 \text { neurological centres in } 7 \text { Italian regions and having symptoms } \\
\text { onset between } 1 \text { October } 2010 \text { and } 15 \text { May } 2011 \text {. }\end{array}$ \\
\hline & $\begin{array}{l}\text { Controls }(n=308) \text { : were selected from among patients admitted to the emergency department of the } \\
\text { same hospital as the cases for acute conditions unrelated to chronic diseases (e.g. trauma). Each con- } \\
\text { trol was individually matched to a case for admission date (i.e. the same date as the case or up to } 30 \\
\text { days afterwards), sex, age ( } \pm 5 \text { years), and region of residence. }\end{array}$ \\
\hline
\end{tabular}

\begin{tabular}{ll}
\hline Interventions & $\begin{array}{l}\text { Exposure to influenza vaccination (date and brand of vaccine) was verified by contacting patients' gen- } \\
\text { eral practitioners by telephone. A neurologist (FG) closely verified and queried data quality. }\end{array}$ \\
\hline Outcomes & Guillain-Barré syndrome \\
\hline Notes & $\begin{array}{l}\text { The authors also performed data analysis with a controlled case series design, considering the } 6 \text { weeks } \\
\text { following exposure as the risk time. } \\
\text { Government funded }\end{array}$
\end{tabular}

\section{Risk of bias}

\begin{tabular}{lll}
\hline Bias & Authors' judgement & Support for judgement \\
\hline $\begin{array}{l}\text { CC - case selection } \\
\text { All outcomes }\end{array}$ & Low risk & Consecutive series of cases \\
\hline $\begin{array}{l}\text { CC - control selection } \\
\text { All outcomes }\end{array}$ & Low risk & Hospital control \\
\hline $\begin{array}{l}\text { CC - comparability } \\
\text { All outcomes }\end{array}$ & Unclear risk & Matched analysis only for sex, age, region, admission date \\
\hline $\begin{array}{l}\text { CC - exposure } \\
\text { All outcomes }\end{array}$ & Unclear risk & Unclear if interviewers were blinded to case-control status \\
\hline Summary assessment & Unclear risk & Unclear risk of bias \\
\hline
\end{tabular}

bb Garbe 2012

\begin{tabular}{ll}
\hline Methods & Case-control surveillance study \\
\hline Participants & Cases $(n=169):$ patients 18 years of age or older with a diagnosis of certain or probable immune throm- \\
bocytopenia. Of the included 169 cases, 130 were outpatients and 39 were inpatients. \\
Controls $(n=770): 731$ outpatients and 39 inpatients selected from the same hospitals as the cases. \\
The index date for outpatient controls was defined as the date of hospitalisation or the date of initia- \\
tion of the control disease episode if this preceded hospitalisation. The index date for inpatient con- \\
trols was the date of the interview.
\end{tabular}


bb Garbe 2012 (Continued)

Interventions
Exposure to influenza vaccination during the 28 days preceding the index date. Exposure to other vaccines and drugs was also considered.

\begin{tabular}{lll}
\hline Outcomes & Immune thrombocytopenia \\
\hline Notes & Government funded & \\
\hline Risk of bias & Authors' judgement & Support for judgement \\
\hline Bias & Low risk & Hospital population \\
\hline CC - case selection & & \\
All outcomes & Low risk & Hospital control \\
\hline CC - control selection & & \\
All outcomes & Unclear risk & No matching \\
\hline $\begin{array}{l}\text { CC - comparability } \\
\text { All outcomes }\end{array}$ & & \\
\hline CC - exposure & Unclear risk & Unclear \\
All outcomes & & \\
\hline Summary assessment & Unclear risk & Unclear \\
\hline
\end{tabular}

bb Grimaldi-Bensouda 2011

\begin{tabular}{|c|c|}
\hline Methods & Multicentre, case-control study \\
\hline Participants & $\begin{array}{l}\text { Cases ( } n=104) \text { : Guillain-Barré syndrome cases (Brighton Collaboration definition, levels } 1 \text { to } 3 \text { ). } \\
\text { Controls }(n=1198) \text { : each case was matched to up to } 25 \text { controls on age (plus or minus } 1 \text { year), sex, in- } \\
\text { dex date, and country. Matched controls recruited in the Netherlands, Sweden, the UK, France, and } \\
\text { Denmark. }\end{array}$ \\
\hline
\end{tabular}

\begin{tabular}{|c|c|c|}
\hline Interventions & \multicolumn{2}{|c|}{$\begin{array}{l}\text { Exposure to monovalent pandemic } \mathrm{H} 1 \mathrm{~N} 12009 \text { to } 2010 \text { influenza vaccine during the } 6 \text { months preced- } \\
\text { ing the index date. Vaccination data were obtained from vaccine registries (Denmark and France), from } \\
\text { general practitioner records (UK and Netherlands), and from structured interviews (Sweden). }\end{array}$} \\
\hline Outcomes & \multicolumn{2}{|c|}{ Guillain-Barré syndrome } \\
\hline Notes & \multicolumn{2}{|c|}{ This study was funded by the European Centre for Disease Prevention and Control. } \\
\hline \multicolumn{3}{|l|}{ Risk of bias } \\
\hline Bias & Authors' judgement & Support for judgement \\
\hline $\begin{array}{l}\text { CC - case selection } \\
\text { All outcomes }\end{array}$ & Low risk & Neurological clinic registry \\
\hline $\begin{array}{l}\text { CC - control selection } \\
\text { All outcomes }\end{array}$ & Unclear risk & From the same population using only general practitioner registry \\
\hline $\begin{array}{l}\text { CC - comparability } \\
\text { All outcomes }\end{array}$ & Unclear risk & Poor matching \\
\hline
\end{tabular}


bb Grimaldi-Bensouda 2011 (Continued)

CC - exposure Unclear risk Interview and record linkage
All outcomes

Summaryassessment Unclear risk Unclear

bb Grimaldi-Bensouda 2012

\begin{tabular}{|c|c|}
\hline Methods & Case-control study \\
\hline \multirow[t]{2}{*}{ Participants } & $\begin{array}{l}\text { Cases }(n=198) \text { were people with a diagnosis of immune thrombocytopenia (American Society of } \\
\text { Hematology diagnostic criteria) identified with the collaboration of } 22 \text { university and major regional } \\
\text { hospitals in France participating in the Pharmacoepidemiological General Research on ITP (PGRX-ITP) } \\
\text { registry project. }\end{array}$ \\
\hline & $\begin{array}{l}\text { Controls }(n=878) \text { matched on age ( } 2 \text { years), sex, region of residence (northern or southern France), in- } \\
\text { dex date (date of first symptoms for the cases and date of consultation for the referents } 2 \text { months) from } \\
\text { a random sample. }\end{array}$ \\
\hline Interventions & Exposure to influenza vaccine. Assessed by structured interview and confirmed by vaccination records. \\
\hline Outcomes & Immune thrombocytopenia \\
\hline Notes & Government funded \\
\hline
\end{tabular}

\section{Risk of bias}

\begin{tabular}{lll}
\hline Bias & Authors' judgement & Support for judgement \\
\hline $\begin{array}{l}\text { CC - case selection } \\
\text { All outcomes }\end{array}$ & Low risk & Multicentre registry consecutive series of cases \\
\hline $\begin{array}{l}\text { CC - control selection } \\
\text { All outcomes }\end{array}$ & Unclear risk & Same population using registry from a sample of GPs \\
\hline $\begin{array}{l}\text { CC - comparability } \\
\text { All outcomes }\end{array}$ & Unclear risk & Matching 1:5 \\
\hline $\begin{array}{l}\text { CC - exposure } \\
\text { All outcomes }\end{array}$ & Unclear risk & Structured interview - confirmation by GPs \\
\hline Summary assessment & Unclear risk & Unclear \\
\hline
\end{tabular}

\section{bb Hernan 2004}

\begin{tabular}{ll}
\hline Methods & Case-control study based on the General Practice Research Database (GPRD) \\
\hline Participants & $\begin{array}{l}\text { Cases }(n=163): \text { patients with confirmed diagnosis of multiple sclerosis between } 1 \text { January } 1993 \text { and } 31 \\
\text { December 2000. } \\
\text { Controls }(n=1604) \text { : subjects from the GPRD matched to the cases for age, sex, practice, date of joining } \\
\text { the practice. }\end{array}$
\end{tabular}

Interventions Exposure to vaccinations (also influenza) as shown from medical records


bb Hernan 2004 (Continued)

Outcomes Association between exposure to influenza vaccine and onset of multiple sclerosis

\begin{tabular}{lll}
\hline Notes & Government funded & \\
\hline Risk of bias & & \\
\hline Bias & Authors' judgement & Support for judgement \\
\hline $\begin{array}{ll}\text { CC - case selection } \\
\text { All outcomes }\end{array}$ & Low risk & Nested case-control from GPRD registry \\
\hline $\begin{array}{ll}\text { CC - control selection } \\
\text { All outcomes }\end{array}$ & Low risk & GPRD registry \\
\hline $\begin{array}{l}\text { CC - comparability } \\
\text { All outcomes }\end{array}$ & Low risk & Matched \\
\hline $\begin{array}{l}\text { CC - exposure } \\
\text { All outcomes }\end{array}$ & Low risk & Registry \\
\hline Summary assessment & Low risk & Low \\
\hline
\end{tabular}

bb Maclntyre 2013

Methods

\section{Participants}

Case-control study investigating the protective effect of influenza vaccination against acute myocardial infarction
Cases $(n=275)$ : patients aged $\geq 40$ years of age admitted with an acute myocardial infarction, evolving or recent myocardial infarction to the cardiology unit during the influenza season. Eligible respondents were those able to provide samples within 72 hours of the acute myocardial infarction event, residing in Sydney, Australia, available for follow-up, and provided informed consent. Cases reporting a previous cardiovascular event were eligible. A diagnosis of acute myocardial infarction was defined as a typical rise and gradual fall in troponin or more rapid rise and fall in creatine kinase-MB biochemical markers of myocardial necrosis, with 1 or more of the following: ischaemic symptoms (chest or arm pain, nausea/vomiting, sweating, shortness of breath); development of pathological Q waves on ECG; ECG changes indicative of ischaemia (ST segment elevation or depression); coronary artery intervention; or pathological findings of an acute myocardial infarction. Participants were recruited into the study between 27 June and 20 October 2008; 18 May and 23 October 2009; and 21 June and 28 October 2010.

Controls $(n=284)$ : controls were people aged $\geq 40$ years of age attending the orthopaedic or ophthalmic outpatient clinics during the same time period. Respondents residing in Sydney, available for follow-up, and able to provide informed consent were eligible. Controls were unmatched, except for the same age cut-off and recruitment period, to ensure similar level of exposure to circulating influenza. Controls were excluded if they reported a history of acute myocardial infarction, transient ischaemic attack, or stroke in the previous 12 months. Stable angina was permissible if there had been no worsening of angina or acute myocardial infarction episodes or hospital admissions in the last year. Controls were recruited into the study between 30 June and 31 October 2008; 19 May and 26 October 2009; and 23 June and 29 October 2010.

Interventions Influenza vaccination status was validated for current and previous influenza seasons from hospital and GP records, with GPs contacted via facsimile or telephone. If discrepancies arose between GP and self report, GP-reported vaccination status was considered correct. Self reported vaccination status was considered sufficient in those individuals whose GP could not be contacted. Type and characteristics of the administered vaccines are not provided.

\section{Outcomes}


bb MacIntyre 2013 (Continued)
Notes
Funding source - industry
This work was supported by a grant from GlaxoSmithKline, Belgium. Dr Iman Ridda and Dr Holly Seale are supported by Australian National Health and Medical Research Council Training Fellowships.

\section{Risk of bias}

\begin{tabular}{lll}
\hline Bias & Authors' judgement & Support for judgement \\
\hline $\begin{array}{l}\text { CC - case selection } \\
\text { All outcomes }\end{array}$ & Low risk & $\begin{array}{l}\text { Consecutive series of cases (patients admitted to the cardiology unit during } \\
\text { the influenza season) }\end{array}$ \\
\hline $\begin{array}{l}\text { CC - control selection } \\
\text { All outcomes }\end{array}$ & Unclear risk & $\begin{array}{l}\text { Community controls (patients attending orthopaedic or ophthalmic outpa- } \\
\text { tient clinics during the same period without history of disease) }\end{array}$ \\
\hline $\begin{array}{l}\text { CC - comparability } \\
\text { All outcomes }\end{array}$ & Unclear risk & Unmatched \\
\hline $\begin{array}{l}\text { CC - exposure } \\
\text { All outcomes }\end{array}$ & Low risk & Vaccination certificate, GP records \\
\hline Summary assessment & Unclear risk & Unclear risk of bias \\
\hline
\end{tabular}

\section{bb Mastrangelo 2000}

\begin{tabular}{ll}
\hline Methods & Case-control study assessing the association between influenza vaccines and cutaneous melanoma \\
\hline Participants & 99 cases and 104 controls \\
\hline Interventions & Influenza vaccine exposure is not described. \\
\hline Outcomes & $\begin{array}{l}\text { The authors report a protective effect of repeated influenza vaccination on the risk cutaneous } \\
\text { melanoma (OR 0.43, 95\% Cl } 0.19 \text { to } 1.00) \text {. The study is at high risk of bias due to the selective nature of } \\
\text { cases (all patients in the authors' hospital), attrition bias (4 cases and } 4 \text { controls eliminated because of } \\
\text { "failure to collaborate"), recall bias (up to } 5 \text { years' exposure data were based on patients' recollection), } \\
\text { and ascertainment bias (non-blinded exposure survey). } \\
\text { Rare events (safety) } \\
\text { Government funded }\end{array}$
\end{tabular}

\section{Risk of bias}

\begin{tabular}{lll}
\hline Bias & Authors' judgement & Support for judgement \\
\hline $\begin{array}{ll}\text { CC - case selection } \\
\text { All outcomes }\end{array}$ & Low risk & \\
\hline $\begin{array}{ll}\text { CC - control selection } \\
\text { All outcomes }\end{array}$ & Unclear risk & Insufficient information \\
\hline $\begin{array}{ll}\text { CC - comparability } \\
\text { All outcomes }\end{array}$ & Unclear risk & Insufficient information \\
\hline CC - exposure & Low risk & Low \\
\hline
\end{tabular}


bb Mastrangelo 2000 (Continued)

All outcomes

Summaryassessment Unclear risk Unclear

\title{
bb Mutsch 2004
}

\begin{tabular}{|c|c|}
\hline Methods & $\begin{array}{l}1 \text { case-control study and case series based in the German-speaking regions of Switzerland, which as- } \\
\text { sessed the association between an intranasal inactivated virosomal influenza vaccine and Bell's palsy }\end{array}$ \\
\hline Participants & $\begin{array}{l}250 \text { cases that could be evaluated (from an original } 773 \text { cases identified) were matched to } 722 \text { controls } \\
\text { for age and date of clinic visit. All participants were around age } 50 \text {. }\end{array}$ \\
\hline Interventions & Immunisation with influenza vaccine took place within 91 days before disease onset. \\
\hline Outcomes & Bells' palsy \\
\hline Notes & $\begin{array}{l}\text { The study reports a massive increase in risk (adjusted OR } 84,95 \% \mathrm{Cl} 20.1 \text { to } 351.9 \text { ) within } 1 \text { to } 91 \text { days } \\
\text { since vaccination. Despite the many limitations of the study (case attrition: } 187 \text { cases could not be iden- } \\
\text { tified; ascertainment bias: physicians picked controls for their own cases; confounding by indication: } \\
\text { different vaccine exposure rate between controls and the reference population), it is unlikely that such } \\
\text { a large OR could have been significantly affected by systematic error. The authors called for larger pre- } \\
\text { licence safety trials, given the rarity of Bell's palsy. On the basis of this study the vaccine was withdrawn } \\
\text { commercially. } \\
\text { Rare events (safety) }\end{array}$ \\
\hline & Government funded \\
\hline
\end{tabular}

\section{Risk of bias}

\begin{tabular}{|c|c|c|}
\hline Bias & Authors' judgement & Support for judgement \\
\hline $\begin{array}{l}\text { CC - case selection } \\
\text { All outcomes }\end{array}$ & Low risk & $\begin{array}{l}\text { "All } 4891 \text { primary care physicians, ear, nose, and throat specialists, and neurol- } \\
\text { ogists in the study area were invited twice to report cases of Bell's palsy first di- } \\
\text { agnosed between October } 1,2000 \text {, and April 30, 2001." }\end{array}$ \\
\hline
\end{tabular}

CC - control selection Low risk
All outcomes

All outcomes

\begin{abstract}
Subsequently, the physicians who had reported cases of Bell's palsy were asked to document the date of the visit and information pertinent to the study's inclusion and exclusion criteria and to select from among their patients without Bell's palsy, 3 controls sequentially from their registration log.

Trained study monitors contacted the physicians and reviewed the selection forms regularly to ensure consistency in the selection of controls. At this point, participating physicians had not been made aware of the exposure to be investigated (influenza vaccination).
\end{abstract}

\begin{tabular}{|c|c|c|}
\hline $\begin{array}{l}\text { CC - comparability } \\
\text { All outcomes }\end{array}$ & Unclear risk & $\begin{array}{l}\text { The controls were matched with the case patients according to age (within } 5 \\
\text { years), date of the clinic visit (within } 4 \text { days), and physician. }\end{array}$ \\
\hline
\end{tabular}

$\begin{array}{ll}\text { CC - exposure } & \text { Low risk } \\ \text { All outcomes } & \begin{array}{l}\text { "Physicians were asked to document the dates of administration and the } \\ \text { brand name and type of influenza vaccine (parenteral or intranasal) used dur- } \\ \text { ing the study period. Other vaccine exposures during the study period and the } \\ \text { preceding } 2 \text { months were also documented. Since in all } 43 \text { sentinel cases re- } \\ \text { ported in the study area the onset of Bell's palsy occurred within } 91 \text { days after } \\ \text { intranasal vaccination, we defined the period of } 1 \text { to } 91 \text { days as the postexpo- } \\ \text { sure risk period." }\end{array}\end{array}$


bb Mutsch 2004 (Continued)
Summary assessment
Unclear risk
Unclear

bb Payne 2006

Case-control study assessing the association between influenza and other vaccines (data not extracted
for this review) and optic neuritis.
"A matched case-control study design was used with each optic neuritis case matched to 3 controls
based on sex, deployment during the 18 weeks preceding the diagnosis date, and the military compo-
nent in which the individual served (eg, active or reserve/National Guard). The protocol for this vaccine
postmarketing surveillance investigation was approved by the Centers for Disease Control and Preven-
tion (CDC) Institutional Review Board and reviewed by the Food and Drug Administration and Depart-
ment of Defense"

\begin{tabular}{ll}
\hline Participants & US military personnel aged at least 18 years \\
\hline Interventions & Cases $(n=1131)$ : participants with a diagnosis of optic neuritis between 1 January 1998 and 31 Decem- \\
ber 2003. The following ICD-9 codes were considered: $377.30-32,377.39$. \\
Controls $(n=4524):$ participants were matched to the cases on the basis of sex, deployment during the \\
18 weeks before diagnosis, military component. The study was carried out using data from the Defense \\
Medical Surveillance System (DMSS), a longitudinal surveillance database.
\end{tabular}

\begin{tabular}{ll} 
Outcomes & $\begin{array}{l}\text { Date of case diagnosis was ascertained, and immunisation status (anthrax, smallpox, hepatitis B, in- } \\
\text { fluenza) verified by means of electronic records with respect to } 3 \text { time intervals: 6, 12, and 18 weeks be- } \\
\text { fore onset. For controls, vaccination status was determined for the } 3 \text { intervals before the index date. } \\
\text { Results were focused on the 18-week time interval. }\end{array}$ \\
\hline Notes & Rare events (safety) \\
Government funded
\end{tabular}

\section{Risk of bias}

\begin{tabular}{|c|c|c|}
\hline Bias & Authors' judgement & Support for judgement \\
\hline $\begin{array}{l}\text { CC - case selection } \\
\text { All outcomes }\end{array}$ & Low risk & $\begin{array}{l}\text { "We defined optic neuritis cases as those having a first-time diagnosis of the } \\
\text { following ICD-9-CM codes: optic neuritis, unspecified ( } 377.30) \text {; optic papillitis } \\
\text { ( } 377.31 \text { ); retrobulbar neuritis, acute ( } 377.32) \text {; and optic neuritis, other ( } 377.39) \\
\text { during the period between } 1 \text { January } 1998 \text { and } 31 \text { December 2003." }\end{array}$ \\
\hline
\end{tabular}

\begin{tabular}{|c|c|c|}
\hline $\begin{array}{l}\text { CC - control selection } \\
\text { All outcomes }\end{array}$ & Low risk & $\begin{array}{l}\text { Controls were selected if their DMSS diagnostic records indicated no history of } \\
\text { an optic neuropathy, if they served in the military on the same date of diagno- } \\
\text { sis as their matched case, and if they had at least } 18 \text { weeks of military service } \\
\text { preceding this index date. }\end{array}$ \\
\hline
\end{tabular}

\begin{tabular}{lll}
\hline $\begin{array}{l}\text { CC - comparability } \\
\text { All outcomes }\end{array}$ & Low risk & Matching \\
\hline $\begin{array}{ll}\text { CC - exposure } \\
\text { All outcomes }\end{array}$ & Low risk & $\begin{array}{l}\text { "We ascertained the date of each case's first diagnosis of optic neuritis and de- } \\
\text { termined all vaccinations received during each of the following } 3 \text { prior study } \\
\text { intervals from the electronic record; } 6 \text { weeks ( } 42 \text { days), } 12 \text { weeks (84 days) and } \\
18 \text { weeks (126 days). For each of the } 3 \text { matched controls, we determined all } \\
\text { vaccinations during the } 3 \text { intervals predating their index date." }\end{array}$ \\
& & Low \\
\hline Summary assessment & Low risk & \\
\hline
\end{tabular}


bb Ray 2011

\begin{tabular}{ll}
\hline Methods & Case-control study \\
\hline Participants & $\begin{array}{l}\text { Cases }(n=415): \text { people with diagnosis of definite rheumatoid arthritis based on American College of } \\
\text { Rheumatology criteria. } \\
\text { Controls }(n=1245): \text { matched for age and number of medical visits before index date. }\end{array}$ \\
\hline Interventions & $\begin{array}{l}\text { Exposure to influenza vaccine. Different times intervals before symptom onset were considered ( } 90, \\
\text { System and supplemented by chart reviews. Risk of association was, moreover, also determined for } \\
\text { tetanus and hepatitis B vaccines. }\end{array}$ \\
\hline
\end{tabular}

\title{
Outcomes
}

$\begin{array}{ll}\text { Notes } & \text { This study was funded by the Centers for Disease Control and Prevention Vaccine Safety Datalink } \\ \text { Project. }\end{array}$

\section{Risk of bias}

\begin{tabular}{lll}
\hline Bias & Authors' judgement & Support for judgement \\
\hline $\begin{array}{l}\text { CC - case selection } \\
\text { All outcomes }\end{array}$ & Low risk & $\begin{array}{l}\text { Included as cases the incident cases from the cohort analysis and additional } \\
\text { new onset cases identified from the study population whose symptoms began } \\
\text { during } 1996 .\end{array}$ \\
\hline $\begin{array}{ll}\text { CC - control selection } \\
\text { All outcomes }\end{array}$ & Low risk & Same population \\
\hline $\begin{array}{l}\text { CC - comparability } \\
\text { All outcomes }\end{array}$ & Unclear risk & Poor matching \\
\hline $\begin{array}{l}\text { CC - exposure } \\
\text { All outcomes }\end{array}$ & Low risk & NCKPHP databases \\
\hline \begin{tabular}{l} 
Summary assessment \\
\hline
\end{tabular} & Unclear risk & Unclear \\
\hline
\end{tabular}

bb Rouleau 2014

Methods $\begin{aligned} & \text { Case-control study aiming to identify what exposure factors could be linked to allergic-like events in } \\ & \text { the general adult population }\end{aligned}$

Participants

\begin{abstract}
Cases of allergic-like events were identified starting from the Quebec Adverse Events Surveillance System reviewing allergic-like events that occurred 5 to 8 months after the vaccination campaign (between May and July 2010) and that were classified as "anaphylaxis", ORS, or "allergy". Cases with these diagnoses were contacted and interviewed by trained nurses who used a standardised phone questionnaire to verify the diagnosis by applying the Brighton Collaboration Criteria for Anaphylaxis and the National Advisory Committee on Immunization for ORS (bilateral red eyes, and/or facial swelling, and/or respiratory symptoms (cough, wheeze, chest tightness, difficulty breathing, difficulty swallowing/throat tightness, hoarseness or sore throat) with onset $\leq 24$ hours after influenza vaccination); cases that met neither definition were considered as allergic-like events (immediate or delayed allergic-like events, depending on whether symptoms occurred within 4 hours after vaccination or thereafter, i.e. immediate allergic-like events or delayed allergic-like events).
\end{abstract}


bb Rouleau 2014 (Continued)

Controls were randomly selected from the Pandemic Influenza Vaccination Registry by age and sex matching of each case with 2 controls.

Trained nurses collected by interview data about demographics, personal and family medical conditions, obstetric history (gravida, para, abortus), use of medication within 48 hours of vaccination, the presence of an acute respiratory illness at the time of vaccination (e.g. fever, respiratory infection, or ILI), reported allergy to potential allergenic components of the vaccine (i.e. eggs, fish, shellfish, thimerosal, latex), regular alcohol use, and physical activity.

In all, 471 cases and 849 controls were identified. Of these $36(6 \%)$ and $136(16 \%)$ refused to participate, resulting in 435 cases (50 anaphylaxis, 177 ORS, 97 immediate allergic-like events, and 111 delayed allergic-like events) and 849 controls.

Interventions

A univariate analysis and multivariate logistic regression were performed with the aim of identifying potential risk (aeroallergens, drug allergy, food allergy, dermographism, hypothyroidism, family history, allergy, administration of drugs for obstructive airway disease, healthcare worker as profession, vaccinated in weeks) or protective factors (administration of anti-inflammatory or mineral supplements, being physically active, consumption of alcohol). Separate unconditional regression models were built for each case definition and effect estimate (odds ratio) adjusted for sex and age group.

Outcomes $\quad$ Exposure to vaccination with pandemic, monovalent, AS03-adjuvanted H1N1 vaccine (Arepanrix, GSK) during the first 4 weeks of the immunisation campaign

Notes

Funding source - government

Exposure to vaccination is considered within the first 4 weeks of campaign (i.e. not vaccinated versus vaccinated). Among the group of vaccinated within the 4 campaign weeks, healthcare workers were strongly represented (for this group there was also significant association with all outcomes), which could have introduced a certain recall/selection bias.

\section{Risk of bias}

\begin{tabular}{lll}
\hline Bias & Authors' judgement & Support for judgement \\
\hline $\begin{array}{ll}\text { CC - case selection } \\
\text { All outcomes }\end{array}$ & Low risk & $\begin{array}{l}\text { Review of surveillance register by applying case definition during phone inter- } \\
\text { view. Drawn from a nationwide active surveillance register }\end{array}$ \\
\hline $\begin{array}{l}\text { CC - control selection } \\
\text { All outcomes }\end{array}$ & Unclear risk & Randomly selected from a vaccination registry \\
\hline $\begin{array}{l}\text { CC - comparability } \\
\text { All outcomes }\end{array}$ & Low risk & Possible confounders have been considered for analysis. \\
\hline $\begin{array}{l}\text { CC - exposure } \\
\text { All outcomes }\end{array}$ & Unclear risk & Phone interview \\
\hline Summary assessment & Unclear risk & Unclear risk of bias \\
\hline
\end{tabular}

bb Siscovick 2000

\begin{tabular}{ll} 
Methods & $\begin{array}{l}\text { Study assessing the association between influenza vaccination the previous year and the risk of pri- } \\
\text { mary (i.e. occurring in people with no previous history of cardiac disease) cardiac arrest. Case-control } \\
\text { study on } 360 \text { cases and } 418 \text { controls }\end{array}$ \\
\hline Participants & $\begin{array}{l}\text { Cases: people who had experienced primary cardiac arrest, aged between } 25 \text { and } 74 \text { years. } \\
\text { Controls: healthy people selected randomly from the community, who were matched to the cases for } \\
\text { age and sex. }\end{array}$
\end{tabular}


bb Siscovick 2000 (Continued)

Interventions Immunisation with influenza vaccine, assessed by means of questionnaires

\begin{tabular}{|c|c|}
\hline Outcomes & Cardiac arrest \\
\hline \multirow[t]{3}{*}{ Notes } & $\begin{array}{l}\text { The authors concluded that vaccination is protective against primary cardiac arrest (OR } 0.51,95 \% \mathrm{Cl} \\
0.33 \text { to } 0.79 \text { ). The difficulty of case ascertainment ( } 77 \% \text { of potential cases had no medical report and/ } \\
\text { or autopsy) and recall bias (spouses provided exposure data for } 304 \text { cases, while } 56 \text { survivor cases pro- } \\
\text { vided data jointly with their spouses) make the conclusions of this study unreliable. The reliability of } \\
\text { this study is unclear due to a lack of detail on the circulation of influenza in the study areas in the } 12 \\
\text { months preceding cardiac arrest (the causal hypothesis is based on the effects of influenza infection on } \\
\text { the oxygen supply to the myocardium through lung infection and inflammation). }\end{array}$ \\
\hline & Rare events (safety) \\
\hline & Government funded \\
\hline
\end{tabular}

\section{Risk of bias}

\begin{tabular}{|c|c|c|}
\hline Bias & Authors' judgement & Support for judgement \\
\hline $\begin{array}{l}\text { CC - case selection } \\
\text { All outcomes }\end{array}$ & Low risk & $\begin{array}{l}\text { Cases of out-of-hospital PCA attended by paramedics in King County, Washing- } \\
\text { ton, from October } 1988 \text { to July } 1994 \text { were identified from paramedic incident } \\
\text { reports. Primary cardiac arrest cases were defined by the occurrence of a sud- } \\
\text { den pulseless condition and the absence of evidence of a non-cardiac condi- } \\
\text { tion as the cause of cardiac arrest. }\end{array}$ \\
\hline
\end{tabular}

\begin{tabular}{lll}
\hline $\begin{array}{l}\text { CC - control selection } \\
\text { All outcomes }\end{array}$ & High risk & Selected from the community using random digit dialling \\
\hline $\begin{array}{l}\text { CC - comparability } \\
\text { All outcomes }\end{array}$ & Unclear risk & For each PCA case, 1 to 2 controls, matched for age (within 7 years) and sex \\
\hline CC - exposure & Unclear risk & $\begin{array}{l}\text { "Data on the participants' vaccination status were collected from both case } \\
\text { all outcomes } \\
\text { ipant, information was collected on whether they had received an influenza } \\
\text { vaccination during the previous } 12 \text { months and, if so, when the vaccination } \\
\text { had been given. We did not collect information on whether they had received } \\
\text { influenza vaccination during the years prior to that period." }\end{array}$
\end{tabular}

Summaryassessment High risk

bb Zorzon 2003

\begin{tabular}{ll}
\hline Methods & Case-control study \\
\hline Participants & $\begin{array}{l}\text { Cases }(\mathrm{n}=140): \text { people affected by MS as defined by the International Panel on MS Diagnosis. } \\
\text { Controls }(\mathrm{n}=131): \text { sex- and age-matched to the cases. }\end{array}$ \\
\hline Interventions & $\begin{array}{l}\text { Exposure to influenza vaccination (unspecified). Exposure to many other factors was assessed by } \\
\text { means of face-to-face structured questionnaires. Time of onset after exposure is probably not men- } \\
\text { tioned in the text. }\end{array}$ \\
\hline Outcomes & Multiple sclerosis \\
\hline Notes & "The study was supported by a grant of the University of Trieste, Italy: MPI 60\%, 2001"
\end{tabular}


bb Zorzon 2003 (Continued)

Risk of bias

\begin{tabular}{lll}
\hline Bias & Authors' judgement & Support for judgement \\
\hline $\begin{array}{l}\text { CC - case selection } \\
\text { All outcomes }\end{array}$ & Low risk & Hospital population \\
\hline $\begin{array}{l}\text { CC - control selection } \\
\text { All outcomes }\end{array}$ & High risk & Blood donor population \\
\hline $\begin{array}{l}\text { CC - comparability } \\
\text { All outcomes }\end{array}$ & High risk & Poor matching \\
\hline $\begin{array}{l}\text { CC - exposure } \\
\text { All outcomes }\end{array}$ & High risk & Interview \\
\hline Summary assessment & High risk & High risk \\
\hline
\end{tabular}

\section{cb Bardage 2011}

$\begin{array}{ll}\text { Methods } & \text { Large, prospective, cohort study assessing the possible association between monovalent, pandemic, } \\ \text { H1N1 flu vaccine Pandemrix (GSK) and neurological and/or autoimmune disease }\end{array}$
H1N1 flu vaccine Pandemrix (GSK) and neurological and/or autoimmune disease

Participants

The study population comprised 1,945,024 people and corresponds to all people registered in Stockholm County on 1 October 2009 who had lived in the region since 1 January 1998.

Interventions

Monovalent A (H1N1) pandemic vaccine Pandemrix (GlaxoSmithKline, Middlesex, UK) containing adjuvants $\mathrm{ASO} 3$ and squalene.

H1N1 vaccination campaign was initially targeted at healthcare workers and groups considered to be at high risk of complications from influenza (children with multifunctional disorders; pregnant women; people with chronic heart or lung disease, diabetes mellitus, chronic liver failure, chronic renal failure, or immunosuppression; people with body mass index > 40; people with neuromuscular disease affecting breathing capacity).

For the campaign an apposite register was established (Vaccinera) in which information on the dates of a first and second dose of vaccine, batch number, medical contraindications against vaccination, and chronic conditions defining high-risk patients were recorded.

The vaccination campaign began on 13 October 2009 with 2 phases. During the first 6 weeks (from 13 October through November 2009), participants with a high-risk condition were preferentially vaccinated; the vaccination was then offered to the remainder of the population during the second phase (from December 2009 onwards).

In total, 1,024,019 participants received at least 1 vaccine dose (446,770 during phase I, 577,249 during phase II).

Outcomes

Data on vaccination (Vaccinera database) were linked to data on utilisation of inpatient and specialist health care (admissions to hospital and visits to specialist care in the county, dates, diagnoses, responsible medical departments, and length of hospital stay) contained in the common healthcare registers for Stockholm County Council (GVR) from 1 January 1998 to 31 August 2010.

Neurological and autoimmune diagnoses to consider for follow-up were selected based on indication of the European Medicines Agency and defined by the ICD-10 classification for hospital admissions and visits to specialist care:

- Guillain-Barré syndrome: G61

- Multiple sclerosis (demyelinating disease): G35 (G36.0 + G37.9)

- Bell's paralysis: G51

- Narcolepsy: G47.4 
- Polyneuropathy, unspecified: G62.9

- An/hypoaesthesia: R20.0 + R20.1

- Paraesthesia: R20.2

- Rheumatological disease: M05-M06 + M08

- Inflammatory bowel disease (Crohn's disease and ulcerative colitis): K50-K51

- Insulin-dependent diabetes among individuals born in 1990 and later: E10

Entering diagnoses into the county healthcare database is part of the doctor's routine diagnostic work and therefore depends on patients seeking health care. An active search for adverse events during the study period was not performed.

For each investigated pathology, the prevalent diagnoses were considered (i.e. those registered between 1 January 1998 and 30 September 2009) and the incident diagnoses (i.e. those during or after the pandemic period for unvaccinated people and after a first vaccination for vaccinated people between 1 October 2009 and 31 August 2010).

Since risk groups were prioritised for vaccination, risk estimates analysis data were stratified for the first and second phase of the vaccination campaign (the cut-off point was 45 days from 1 October 2009), considering vaccination as a time-varying covariate and also time since first vaccination (6 weeks).

All but 1 (narcolepsy) of the investigated neurological and autoimmune disorders were significantly more prevalent in those vaccinated in the early phase of the campaign (first 45 days) than in the unvaccinated cohort. Comparing those vaccinated in the late phase ( $>45$ days) with the unvaccinated cohort, the prevalence of the investigated diseases was not statistically relevant, except for inflammatory bowel disease (prevalence OR $1.17,95 \% \mathrm{Cl} 1.12$ to 1.22 ), Guillain-Barré syndrome (OR $0.79,95 \% \mathrm{Cl} 0.67$ to 0.95), and type 1 diabetes (OR $0.77,95 \% \mathrm{Cl} 0.64$ to 0.92 , for those born in 1990 and later).

Government funded

\section{Risk of bias}

\begin{tabular}{lll}
\hline Bias & Authors' judgement & Support for judgement \\
\hline $\begin{array}{ll}\text { PCS/RCS - selection ex- } \\
\text { posed cohort }\end{array}$ & Low risk & Selected group of users \\
All outcomes & \\
\hline
\end{tabular}

PCS/RCS - selection non- Unclear risk Drawn from the same community as the exposed cohort
exposed cohort

All outcomes

\begin{tabular}{|c|c|c|}
\hline $\begin{array}{l}\text { PCS/RCS - comparability } \\
\text { All outcomes }\end{array}$ & High risk & Not assessed \\
\hline $\begin{array}{l}\text { PCS/RCS - assessment of } \\
\text { outcome } \\
\text { All outcomes }\end{array}$ & Low risk & Record linkage \\
\hline Summary assessment & High risk & $\begin{array}{l}\text { "The study is a retrospective datalinkage cohort study, with unclear data qual- } \\
\text { ity" }\end{array}$ \\
\hline
\end{tabular}

\section{cb Baxter 2012}

Methods

Retrospective cohort study in which the incidence of medical attended events (MAEs) that occurred in people immunised with LAIV through several seasons was compared with that observed in 2 matched 
Participants

Participants were members of the Kaiser Permanente (KP) Health Plans in Northern California, Hawaii, and Colorado. Through KP immunisation registries, approximately 20,000 individuals 18 to 49 years of age who were immunised from the 2003 to 2004 to 2007 to 2008 influenza seasons with LAIV as part of routine clinical practice were identified.

Interventions

Intervention hemi-cohort: Live attenuated influenza vaccine vaccine provided by MedImmune. Each annual formulation of the vaccines contained the strains recommended for inclusion by the US Public Health Service. Study participants with high-risk underlying medical conditions such as cancer, organ transplantation, diabetes, endocrine and metabolic disorders, blood disorders, liver disorders, kidney disorders, and cardiopulmonary disorders were identified via automated extraction of healthcare databases and excluded from all analysis cohorts. A total of 21,340 participants 18 to 49 years of age were vaccinated with the Ann Arbor strain LAIV during the 5 study seasons.

Control hemi-cohort 1: unvaccinated $(n=21,340)$. Participants were KP members who participated in the health plan during the same month as the reference LAIV recipients; for the unvaccinated population, the effective vaccination date was the date on which the matched LAIV recipient was vaccinated. Control hemi-cohort 2: trivalent inactivated vaccine purchased by KP for immunisation practices ( $\mathrm{n}=$ 18,316). Participants were KP members vaccinated during the same month as the reference LAIV recipient.

Both controls were matched for region (Northern California, Hawaii, Colorado), birth date (within 1 year), sex, and prior healthcare utilisation ( $\leq 1$ or $>1$ clinic visits during the 180 days before vaccination) $1: 1$ to the participants of the intervention hemi-cohort. For Northern California only, participants were also matched on their specific medical clinic. In the case that a match could not be found within a specific control group, the LAIV recipient was excluded from the cohort comparison.

For self controlled case series analysis, intervals of 3 and 21 days' postvaccination were compared with control intervals from 4 to 42 days' postvaccination (for the 3-day risk interval) and 22 to 42 days' postvaccination (for a 0 to 21 -day risk interval).

\section{Medical attended adverse events}

Based on medical diagnoses found in KP database records and collected from outpatient clinics, emergency departments, and hospital admissions, MAEs occurred in 5 main categories and included events considered to be vaccine associated:

1. Acute respiratory tract events: acute laryngitis, acute laryngotracheitis, acute respiratory failure, acute tracheitis, acute respiratory distress syndrome, asthma, bronchitis, cough, epiglottitis, influenza, influenza with pneumonia, mastoiditis, otitis media, pharyngitis, pneumococcal pneumonia, pneumonia, pulmonary congestion and hypostasis, shortness of breath, sinusitis, tachypnoea, tonsillitis, urinary tract infection, viral pneumonia. Follow-up 42 days

2. Acute gastrointestinal tract events: abdominal pain, acute gastritis, acute gastroenteritis, appendicitis, intestinal obstruction, intussusception, irritable bowel syndrome, mesenteric adenitis, nausea and vomiting, pancreatitis, paralytic ileus, perforation of intestine, peritonitis, persistent vomiting, small bowel obstruction, ulceration of intestine, and volvulus. Follow-up 42 days

3. Asthma and wheezing events: asthma/reactive airway disease, wheezing/shortness of breath. Follow-up 180 days

4. Systemic bacterial infections events: bacteraemia, bacterial meningitis, intracranial and intraspinal abscess, septicaemia, shock: unspecified, shock: endotoxic, and gram-negative shock. Follow-up 42 days

5. Rare diagnoses: potentially related to wild-type influenza infection: encephalitis/encephalopathy, Guillain-Barré syndrome, meningitis, myocarditis, other paralytic syndromes, pericarditis, polymyositis, Reye syndrome, and viral meningitis. Follow-up 42 days

\section{Severe adverse events}

Death, inpatient hospitalisation, persistent or significant disability or incapacity, congenital anomaly/birth defect (in the offspring of a participant), or any life-threatening event. Follow-up from 0 to 42 days' postvaccination 
cb Baxter 2012 (Continued)

Notes
Sources of support: "This study was sponsored by MedImmune, LLC. Authors employed by Medlmmune were involved in the study design, analysis, and interpretation of data, and in the preparation of the manuscript. Authors employed by Kaiser Permanente were involved in the study design, collection, analysis, and interpretation of data, and in the preparation of the manuscript. The Kaiser Permanente Vaccine Study Center was paid for their services in data collection and analysis but authors were not compensated for their work on this manuscript"

\title{
Risk of bias
}

\begin{tabular}{lll}
\hline Bias & Authors' judgement & Support for judgement \\
\hline $\begin{array}{l}\text { PCS/RCS - selection ex- } \\
\text { posed cohort }\end{array}$ & Unclear risk & Selected group of users \\
All outcomes & $\begin{array}{l}\text { Participants were screened for underlying medical conditions and provided } \\
\text { the appropriate vaccine based on the eligibility criteria in each vaccine's pack- } \\
\text { age insert, physician discretion, and patient choice. }\end{array}$ \\
\hline
\end{tabular}

PCS/RCS - selection non- Unclear risk No description of the derivation of the non-exposed cohort
exposed cohort

All outcomes

PCS/RCS - comparability Unclear risk
All outcomes

\begin{abstract}
Matched but not very relevant:
"TIV-vaccinated and unvaccinated participants were matched to LAIV recipients on region (Northern California, Hawaii, Colorado), birth date (within one year), sex, and prior healthcare utilization. Prior utilization was calculated based on the number of clinic visits during the 180 days before vaccination and classified as low ( $\leq 1$ visit) and high (> 1 visit) for matching. In Northern California, participants also were matched on their specific medical clinic, of which there were $48 "$
\end{abstract}

\begin{tabular}{|c|c|c|}
\hline $\begin{array}{l}\text { PCS/RCS - assessment of } \\
\text { outcome } \\
\text { All outcomes }\end{array}$ & Low risk & Record linkage \\
\hline Summary assessment & Unclear risk & Unclear \\
\hline
\end{tabular}

\section{cb Kaplan 1982}

$\begin{array}{ll}\text { Methods } & \text { Surveillance population-based study conducted in the USA during the } 1979 \text { to } 1980 \text { and } 1980 \text { to } 1981 \\ \text { influenza seasons. The study tested the association between influenza vaccination and Guillain-Bar- } \\ \text { ré syndrome. Reports from each case were obtained from neurologists. All case reports were included. } \\ \text { The follow-up period was } 1 \text { September } 1979 \text { to } 31 \text { March } 1980 \text { and } 1 \text { September } 1980 \text { to } 31 \text { March } 1981 .\end{array}$

\begin{tabular}{ll}
\hline Participants & USA (minus Maryland), adult population, 18 years or older \\
\hline Interventions & Seasonal parenteral vaccine \\
\hline Outcomes & $\begin{array}{l}\text { Cases of Guillain-Barré syndrome. Vaccine-associated cases were defined as those with onset within } \\
\text { the 8-week period after influenza vaccination. }\end{array}$ \\
\hline Notes & $\begin{array}{l}\text { Vaccination rates in the population were obtained from a national immunisation survey. } \\
\text { Rare events (safety) } \\
\text { Government funded }\end{array}$ \\
\hline
\end{tabular}


cb Kaplan 1982 (Continued)

\section{Risk of bias}

\begin{tabular}{|c|c|c|}
\hline Bias & Authors' judgement & Support for judgement \\
\hline $\begin{array}{l}\text { PCS/RCS - selection ex- } \\
\text { posed cohort } \\
\text { All outcomes }\end{array}$ & High risk & High risk \\
\hline $\begin{array}{l}\text { PCS/RCS - selection non- } \\
\text { exposed cohort } \\
\text { All outcomes }\end{array}$ & High risk & High risk \\
\hline $\begin{array}{l}\text { PCS/RCS - comparability } \\
\text { All outcomes }\end{array}$ & High risk & High risk \\
\hline $\begin{array}{l}\text { PCS/RCS - assessment of } \\
\text { outcome } \\
\text { All outcomes }\end{array}$ & High risk & High risk \\
\hline Summary assessment & High risk & High risk \\
\hline
\end{tabular}

\section{cb Lasky 1998}

\section{Methods}

Surveillance, population-based study conducted in the USA (4 states: Illinois, Maryland, North Carolina, Washington) during the 1992 to 1993 and 1993 to 1994 influenza seasons. Discharge diagnoses databases were used to identify cases. Hospital charts were reviewed to confirm diagnosis. The follow-up period was 1 September 1992 to 28 February 1993 and 1 September 1993 to 28 February 1994.

\begin{tabular}{ll}
\hline Participants & Approximately 21 million people, 18 years or older \\
\hline Interventions & Seasonal parenteral vaccine \\
\hline Outcomes & $\begin{array}{l}\text { Cases of Guillain-Barré syndrome. Vaccine-associated cases were defined a priori as those with onset } \\
\text { within the 6-week period after influenza vaccination. }\end{array}$ \\
\hline Notes & $\begin{array}{l}\text { Results were stratified by age and adjusted by season and sex. Vaccination rates in population were es- } \\
\text { timated from a random-digit dialling telephone survey. } \\
\text { Rare events (safety) } \\
\text { Government funded }\end{array}$ \\
\hline
\end{tabular}

\section{Risk of bias}

\begin{tabular}{lll}
\hline Bias & Authors' judgement & Support for judgement \\
\hline $\begin{array}{l}\text { PCS/RCS - selection ex- } \\
\text { posed cohort } \\
\begin{array}{ll}\text { All outcomes } \\
\text { PCS/RCS - selection non- }\end{array}\end{array}$ & High risk & High risk risk \\
$\begin{array}{l}\text { exposed cohort } \\
\text { All outcomes }\end{array}$ & High risk \\
\hline $\begin{array}{l}\text { PCS/RCS - comparability } \\
\text { All outcomes }\end{array}$ & High risk & High risk \\
\hline \hline
\end{tabular}


cb Lasky 1998 (Continued)

PCS/RCS - assessment of High risk High risk
outcome

All outcomes

\begin{tabular}{lll}
\hline Summary assessment & High risk & High risk \\
\hline
\end{tabular}

cb Moro 2013

Methods Retrospective cohort study evaluating the association between the administration of monovalent pan-
demic inactivated vaccine $\mathrm{H} 1 \mathrm{~N} 1$ and severe adverse events

Participants were identified within several administrative and medical databases of the Italian region
Emilia Romagna (about 4.4 million individuals). By data linkage participants immunised with Foce-
tria in the 2009 to 2010 season $(n=103,642)$ were identified. From the unvaccinated population $(n=$
$3,967,917)$ a matched unexposed cohort was selected using a propensity score.

\begin{tabular}{ll}
\hline Interventions & Immunisation with MF59-adjuvanted, monovalent H1N1 vaccine Focetria (Novartis Vaccines and Diag- \\
nostics, Siena, Italy)
\end{tabular}

Guillain-Barré syndrome, paralytic syndromes, encephalitis and encephalomyelitis, Bell's palsy, de-
myelinating disease, convulsion, autoimmune hepatitis, vasculitis, immune thrombocytopenia

\begin{tabular}{ll}
\hline Notes Government funded \\
\hline
\end{tabular}

\section{Risk of bias}

\begin{tabular}{lll}
\hline Bias & Authors' judgement & Support for judgement \\
\hline $\begin{array}{l}\text { PCS/RCS - selection ex- } \\
\text { posed cohort } \\
\text { All outcomes }\end{array}$ & Unclear risk & Unclear description of the vaccinated population \\
\hline $\begin{array}{l}\text { PCS/RCS - selection non- } \\
\text { exposed cohort } \\
\text { All outcomes }\end{array}$ & Unclear risk & Using administrative databases \\
\hline $\begin{array}{l}\text { PCS/RCS - comparability } \\
\text { All outcomes }\end{array}$ & Unclear risk & Propensity score \\
\hline $\begin{array}{l}\text { PCS/RCS - assessment of } \\
\text { outcome } \\
\text { All outcomes }\end{array}$ & Low risk & Blind validation process throughout \\
\hline $\begin{array}{l}\text { Summary assessment } \\
\text { Unclear risk }\end{array}$ & Unclear \\
\hline
\end{tabular}

\section{cb O'Flanagan 2014}

\begin{tabular}{ll}
\hline Methods & $\begin{array}{l}\text { Large retrospective, population-based cohort study assessing the possible association between mono- } \\
\text { valent, pandemic, H1N1 flu vaccine Pandemrix (GSK) and narcolepsy }\end{array}$ \\
\hline Participants & $\begin{array}{l}\text { Virtually the whole population of Ireland is included in the study, which consists of } 90,280 \text { children and } \\
\text { adolescents aged below } 20 \text { and 3,325,643 adults. }\end{array}$
\end{tabular}


cb O'Flanagan 2014 (Continued)

Interventions

Exposure to Pandemrix between October 2009 and March 2010. Information on vaccination was collected in 1 of 2 databases, depending on where vaccination was administered: vaccinations performed in general practitioner clinics were registered in the primary care reimbursement service (PCRS) database, and those performed in Health Service Executive mass vaccination clinics in the pandemic data management system (PDMS) database. The number of individuals vaccinated with Pandemrix was extracted from these databases by week of vaccination. The number of unvaccinated individuals was computed by subtracting the number of individuals vaccinated with any pandemic vaccine brand from the total number of individuals reported in the 2011 census.

Level 1: Excessive daytime sleepiness AND/OR suspected cataplexy AND cerebrospinal fluid hypocretin-1 deficiency.

Level 2: Excessive daytime sleepiness AND definite cataplexy AND level 1 or 2 Multiple Sleep Latency Test abnormalities (mean sleep latency $<8$ minutes for adults and $<12$ minutes for children $<16$ years AND/OR at least 2 sleep-onset REM periods).

Level 3: Excessive daytime sleepiness AND level 1 Multiple Sleep Latency Test abnormalities (mean sleep latency $<8$ minutes for adults and $<12$ minutes for children $<16$ years AND at least 2 sleep-onset REM periods).

Narcolepsy cases were identified by means of active case finding by contacting all sleep clinics, neurologists, paediatricians, GPs, psychiatrists, psychologists, and public health nurses in Ireland.

2 experts ( 1 adult and 1 paediatric neurologist who were blinded to the vaccination status of the cases) reviewed the clinical history of narcolepsy cases (medical records and clinical charts) to confirm the diagnosis and classify them using the internationally agreed Brighton Collaboration case definition for narcolepsy. Cases were included in the study if:

- their date of first symptom of narcolepsy recorded in medical files occurred after 1 April 2009 and before 31 December 2010;

- cases or guardians gave oral informed consent;

- they were classified as level 1, 2, or 3 as per the Brighton case definition.

Prevalent cases with onset prior to April 2009 were excluded. The date of first contact with health care for narcolepsy symptoms as retrieved from GP notes and clinical records was used to estimate the onset of narcolepsy in primary analysis.

Notes Funding source - government

\section{Risk of bias}

\begin{tabular}{lll}
\hline Bias & Authors' judgement & Support for judgement \\
\hline $\begin{array}{ll}\text { PCS/RCS - selection ex- } \\
\text { posed cohort }\end{array}$ & Low risk & Virtually the whole Irish population is included. \\
All outcomes & \\
\hline
\end{tabular}

\begin{tabular}{lll}
$\begin{array}{l}\text { PCS/RCS - selection non- } \\
\text { exposed cohort } \\
\text { All outcomes }\end{array}$ & Low risk & Drawn from the same source \\
\hline $\begin{array}{l}\text { PCS/RCS - comparability } \\
\text { All outcomes }\end{array}$ & Unclear risk & Possible confounders have been taken into account. \\
\hline $\begin{array}{l}\text { PCS/RCS - assessment of } \\
\begin{array}{l}\text { outcome } \\
\text { All outcomes }\end{array}\end{array}$ & Low risk & $\begin{array}{l}\text { Clinical information of possible cases were reviewed, and the correspondence } \\
\text { to a standard case definition verified. }\end{array}$ \\
\hline
\end{tabular}


cb O'Flanagan 2014 (Continued)
Summary assessment
Unclear risk
Low risk of bias

cb Persson 2014

$\begin{array}{ll}\text { Methods } & \text { Cohort study. Large prospective, register-based cohort study assessing the possible association be- } \\ \text { tween monovalent, pandemic, H1N1 flu vaccine Pandemrix (GSK) and neurological or autoimmune dis- } \\ \text { ease, or both }\end{array}$

Participants

The present study represents the extension of the cb Bardage 2011 study to the population of more Swedish regions, namely the healthcare regions of Skåne and Västra Götaland and the counties of Kalmar, Östergötland, Stockholm, Värmland, and Norrbotten. Included are 5,845,039 participants, corresponding to about $61 \%$ of the whole Swedish population in 2009.

Interventions

Exposure to Pandemrix between October 2009 and March 2010. Vaccinated participants were registered in vaccination centres and identified by means of a personal identification number (PIN, a 10-digit number attributed to each newborn in Sweden) and linked to vaccination registries. Vaccination data are linked to the National Population Registry by use of the PIN: all individuals registered as vaccinated $(n=3,347,467)$ were exposed, whereas all remaining individuals were assumed not to be vaccinated ( $n$ $=2,497,572$ ).

Personal identification number was also linked to the following databases to obtain further information about participants:

- National Patient Register, Prescribed Drug Register, and Cancer Registry (National Board of Health and Welfare), to identify hospitalisations and non-primary care outpatient visits to identify the outcomes under study;

- Medical Birth Register (National Board of Health and Welfare), to identify pregnancy status at vaccination;

- National Cause of Death Register (National Board of Health and Welfare), to define deaths during follow-up.

Outcomes were selected under consideration of previous influenza safety issues, of the results of the previous study carried out in the Stockholm region only, and identified in the registers by using ICD-10 codes and data about medical drug prescription. Due to the fact that several of the investigated outcomes could have a slow and insidious onset, "prodromal" conditions were identified by linking information present in the registers (date of visits, drug prescriptions, etc.) considering the 5 years preceding the study. Participants who had diagnosis before study start were excluded from risk assessment.

As done in the previous study, risk estimates were stratified for "early" (vaccinated in the first 45 days from the beginning of the campaign) and "late" vaccination (vaccinated after at least 45 days from the beginning of the campaign), as medically "at risk" participants were considered to be priority group for influenza vaccination.

Stratification considering time since vaccination (within/more than 1 year; within 6 weeks/more than 6 weeks) was also carried out.

Association risk between vaccine exposure and outcomes was calculated by means of Cox regression using vaccination as time-dependent variable (i.e. individuals contributed to the unexposed person-time until vaccinated and to the exposed ones thereafter). Hazard risk estimates were adjusted for age (in 5-year bands), gender and county, education and income, number of hospital admissions and ambulatory care visits, pregnancy status, and presence of diagnoses defined by ICD-10 code. 
cb Persson 2014 (Continued)

Vaccination status could not be confirmed for $16 \%$ to $22 \%$ of the Kalmar, Värmland, and Norrbotten participants (corresponding to roughly $2.3 \%$ of the whole vaccinated cohort), because PIN was not available in the database. These participants were considered as unvaccinated.

\section{Risk of bias}

\begin{tabular}{|c|c|c|}
\hline Bias & Authors' judgement & Support for judgement \\
\hline $\begin{array}{l}\text { PCS/RCS - selection ex- } \\
\text { posed cohort } \\
\text { All outcomes }\end{array}$ & Low risk & $\begin{array}{l}\text { The exposed hemi-cohort consists of all people who received the vaccine with- } \\
\text { in } 6 \text { Swedish regions. }\end{array}$ \\
\hline $\begin{array}{l}\text { PCS/RCS - selection non- } \\
\text { exposed cohort } \\
\text { All outcomes }\end{array}$ & Low risk & $\begin{array}{l}\text { Drawn from the same populations as the exposed cohort (all people who did } \\
\text { not receive influenza vaccination) }\end{array}$ \\
\hline $\begin{array}{l}\text { PCS/RCS - comparability } \\
\text { All outcomes }\end{array}$ & Low risk & $\begin{array}{l}\text { Age, gender and county, education and income, number of hospital admis- } \\
\text { sions and ambulatory care visits, pregnancy status, and presence of diagnoses } \\
\text { defined by ICD-10 code. }\end{array}$ \\
\hline $\begin{array}{l}\text { PCS/RCS - assessment of } \\
\text { outcome } \\
\text { All outcomes }\end{array}$ & Low risk & Medical records \\
\hline Summary assessment & Low risk & Low risk of bias \\
\hline
\end{tabular}

cb Ray 2011

Methods See bb Ray 2011. Study data were analysed using a cohort design.

\section{Participants}

Interventions

\section{Outcomes}

Notes Government funded

\section{Risk of bias}

\begin{tabular}{|c|c|c|}
\hline Bias & Authors' judgement & Support for judgement \\
\hline $\begin{array}{l}\text { PCS/RCS - selection ex- } \\
\text { posed cohort } \\
\text { All outcomes }\end{array}$ & Unclear risk & Unclear \\
\hline $\begin{array}{l}\text { PCS/RCS - selection non- } \\
\text { exposed cohort } \\
\text { All outcomes }\end{array}$ & Unclear risk & Unclear \\
\hline $\begin{array}{l}\text { PCS/RCS - comparability } \\
\text { All outcomes }\end{array}$ & Unclear risk & Unclear \\
\hline $\begin{array}{l}\text { PCS/RCS - assessment of } \\
\text { outcome }\end{array}$ & Unclear risk & Unclear \\
\hline
\end{tabular}


cb Ray 2011 (Continued)

All outcomes

Summaryassessment Unclear risk Unclear

\section{cb Shonberger 1979}

$\begin{array}{ll}\text { Methods } & \text { Surveillance, population-based study conducted in the USA during the } 1976 \text { to } 1977 \text { influenza season. } \\ \text { The study tested the association between influenza vaccination and Guillain-Barré syndrome. Neurol- } \\ \text { ogists were directly contacted; physician and hospital records were reviewed. Suspected cases were } \\ \text { reported to the CDC directly by patients or medical personnel and were included only if accepted by a } \\ \text { state health department. Follow-up period was } 1 \text { October } 1976 \text { to } 31 \text { January } 1977 .\end{array}$

\begin{tabular}{ll}
\hline Participants & USA population \\
\hline Interventions & Monovalent A/New Jersey/76 or bivalent A/New Jersey/76 and A/Victoria/75 parenteral vaccine \\
\hline Outcomes & Cases of Guillain-Barré syndrome \\
\hline Notes & $\begin{array}{l}\text { Results were stratified by age group and vaccine type. Vaccination rates in the population were ob- } \\
\text { tained from a national immunisation survey. } \\
\text { Rare events (safety) } \\
\text { Government funded }\end{array}$ \\
\hline
\end{tabular}

\section{Risk of bias}

\begin{tabular}{|c|c|c|}
\hline Bias & Authors' judgement & Support for judgement \\
\hline $\begin{array}{l}\text { PCS/RCS - selection ex- } \\
\text { posed cohort } \\
\text { All outcomes }\end{array}$ & Unclear risk & High risk \\
\hline $\begin{array}{l}\text { PCS/RCS - selection non- } \\
\text { exposed cohort } \\
\text { All outcomes }\end{array}$ & Unclear risk & High risk \\
\hline $\begin{array}{l}\text { PCS/RCS - comparability } \\
\text { All outcomes }\end{array}$ & Unclear risk & High risk \\
\hline $\begin{array}{l}\text { PCS/RCS - assessment of } \\
\text { outcome } \\
\text { All outcomes }\end{array}$ & Unclear risk & High risk \\
\hline Summary assessment & Unclear risk & High risk \\
\hline
\end{tabular}

paa Ma 2014

Methods $\begin{aligned} & \text { Controlled clinical trial. The effect of pandemic influenza vaccine administration during pregnancy } \\ & \text { was assessed by comparing the occurrence and the characteristics of pregnancy outcomes and clinical } \\ & \text { course between vaccinated and non-vaccinated women and assessing the effectiveness of vaccine ad- } \\ & \text { ministration in preventing ILI. }\end{aligned}$

Participants

Healthy pregnant women between the age of 18 and $35(n=226)$ recruited in 4 adjacent villages of Xiangshui, Jiangsu Province, China. The pregnancies ranged from 5 weeks' to 32 weeks' gestation; 122 
paa Ma 2014 (Continued)

women received the $\mathrm{H} 1 \mathrm{~N} 1$ vaccine, whereas 104 formed the control group and did not receive any vaccination. Pregnant women in the control group had to reside in the same or adjacent village/community and have an age difference of $<3$ years compared to the women in the vaccinated group, a gestational age of $<3$ weeks, and the same numbers of pregnancies as those in the vaccinated group.

Interventions Split-virion nonadjuvanted influenza A(H1N1) vaccine (lot 200909008; Shanghai Institute of Biological Products). Each dose contained $15 \mu \mathrm{g}$ of $\mathrm{H} 1 \mathrm{~N} 1$ antigen.

Outcomes

Pregnancy outcomes were recorded by the maternity and child healthcare organisations or midwifery agencies according to routine prenatal and delivery services in the pregnant women's health records (filling out of a unified form on complications during pregnancy and pregnancy outcomes):

- Spontaneous abortion

- Artificial abortion

- Postnatal death

- Premature birth

- Prolonged pregnancy

- Low birth weight

- Delivery mode (eutocia or Caesarean delivery)

- Birth weight $(<3500 \mathrm{~g}$ or $>3500 \mathrm{~g})$

- Apgar score at 1 min (7 to 8 or $>9)$

\section{Effectiveness outcomes}

- Influenza-like illness was defined according to WHO guidelines, which include documented fever (at least $38.0^{\circ} \mathrm{C}$ ) and cough or sore throat. Participants were asked to contact the local vaccination site or the Xiangshui County Center for Disease Control and Prevention once influenza-like symptoms appeared.

This study has been registered at ClinicalTrials.gov under registration no. NCT01842997.

\section{Risk of bias}

\begin{tabular}{lll}
\hline Bias & Authors' judgement & Support for judgement \\
\hline $\begin{array}{l}\text { Random sequence genera- } \\
\text { tion (selection bias) }\end{array}$ & Unclear risk & Not described, only stated that participants were "divided" into 2 groups. \\
\hline $\begin{array}{l}\text { Allocation concealment } \\
\text { (selection bias) }\end{array}$ & High risk & Absent \\
\hline $\begin{array}{l}\text { Blinding (performance } \\
\text { bias and detection bias) } \\
\text { All outcomes }\end{array}$ & High risk & Not blinded \\
\hline $\begin{array}{l}\text { Incomplete outcome data } \\
\text { (attrition bias) } \\
\text { All outcomes }\end{array}$ & Low risk & No loss to follow-up \\
\hline $\begin{array}{l}\text { Summary assessment } \\
\text { High risk }\end{array}$ & High risk of bias \\
\hline
\end{tabular}


paa Madhi 2014

Methods

Randomised controlled trial carried out on pregnant women to assess the effectiveness of trivalent inactivated influenza vaccine administration during pregnancy against confirmed influenza in women and their newborn. The study was carried out during 2 subsequent epidemic seasons (2011 and 2012).

Participants

Pregnant women aged between 18 and 38 years and having an estimated gestation between 20 and 36 weeks who tested negative for HIV were recruited at 4 antenatal clinics of Soweto, South Africa, during 2 consecutive epidemic seasons (March to August 2011 and March to July 2012). In all, 2116 women entered the study: 1062 were allocated to receive vaccine, 1054 to placebo. In addition, 1026 infants born from vaccinated mothers and 1023 infants born from placebo recipients were enrolled.

Interventions

Women enrolled in the study were randomised 1:1 using a computer-generated assignment and a block size of 30 and allocated to 1 of the following treatments:

- Trivalent inactivated influenza vaccine (Vaxigrip, lot number G05831 in 2011 and H7221-2 in 2012; Sanofi Pasteur) containing $15 \mu \mathrm{g}$ each of A/California/7/2009 (A/(H1N1)pdm09), A/Victoria/210/2009 (A/H3N2), and a B/Brisbane/60/2008-like virus (B/Victoria), as recommended by WHO for the Southern Hemisphere in 2011 and 2012

- Placebo consisting of sterile $0.9 \%$ saline solution

Both preparations were administered by study staff in the deltoid muscle in a $0.5 \mathrm{~mL}$ dose and were macroscopically indistinguishable.

Outcomes

Cases of ILI were identified through active surveillance. The following criteria were used to identify cases among mother and infants respectively:

- ILI (mothers): fever $\geq 38^{\circ} \mathrm{C}$ on oral measurement or history of chills, rigors, or feeling feverish; AND

* presence of cough or sore throat or pharyngitis; OR

* presence of myalgia, arthralgia, or headache; OR

* presence of dyspnoea, breathing difficulty, or chest pain when breathing.

- ILI (infants):

* axillary temperature $\geq 37.8^{\circ} \mathrm{C}$ or mother's perception that the infant was feverish, or both, without evidence of a non-respiratory localised source, coupled with at least 1 sign or symptom of acute respiratory infection within the past 72 hours; OR

* at least 2 signs and/or symptoms of acute respiratory illness within the past 72 hours including: respiratory rate of $\geq 60$ and $\geq 50$ breaths per minute in infant 0 to 2 months and 2 to 6 months of age, respectively; difficulty breathing reported by the mother, cough, wheezing, runny or congested nose, cyanosis or oxygen saturation $<90 \%$ on room air, chest wall in-drawing, grunting on expiration, and pus draining from either ear.

Influenza: women and infants with ILI, as well as those presenting or hospitalised at antenatal clinics for any respiratory illness, who underwent PCR test with a positive result for influenza viruses.

Events occurring within the timespans of 24 weeks' postpartum (for women) and the 24th week of age (for infants) have been considered for analysis.

Local and systemic reactions recorded on diary cards during the first week following immunisation

Notes $\quad$ Funding source - industry

Supported by grants from the Bill and Melinda Gates Foundation (OPP1002747), the National Institutes of Health, National Center for Advancing Translational Sciences Colorado Clinical and Translational Sciences Institute (UL1 TR000154, for REDCap), the South African Research Chairs Initiative of the Department of Science and Technology and National Research Foundation in Vaccine-Preventable Diseases, and the Respiratory and Meningeal Pathogens Research Unit of the Medical Research Council.

\section{Risk of bias}


paa Madhi 2014 (Continued)

Random sequence genera- Low risk Computer-generated randomisation lists, in blocks of 30 (15 IIV3, 15 placebo) tion (selection bias) were generated with assignment of a 4-digit study number being done in sequence of enrolment (SAS 9.1, SAS Institute Inc., Cary, NC, USA).

\section{Allocation concealment Low risk} (selection bias)

Block size of 30 were allocated consecutively to the enrolling sites, after which the randomisation forms for that block were provided to the site in sealed, consecutively numbered envelopes with the pre-printed study number and the alphabetical code for vaccine or placebo in the envelope.

Blinding (performance Low risk bias and detection bias)

All outcomes
Both administered preparations were macroscopically indistinguishable.

With the exception of the statistician and the pharmacist, study personnel and study participants were unaware of the group assignments.

\begin{tabular}{lll}
\hline $\begin{array}{l}\text { Incomplete outcome data } \\
\text { (attrition bias) }\end{array}$ & Low risk & \\
All outcomes & \\
\hline Summary assessment & Low risk & Low risk of bias \\
\hline
\end{tabular}

pba Benowitz 2010

\begin{tabular}{ll} 
Methods & $\begin{array}{l}\text { Case-control study assessing the effectiveness of influenza vaccination of pregnant women in prevent- } \\
\text { ing hospitalisation for influenza in their newborns. Study period ranged from October } 2000 \text { to April } \\
2009 .\end{array}$ \\
\hline Participants & $\begin{array}{l}\text { Cases ( } \mathrm{n}=113) \text { : infants below } 12 \text { months hospitalised for influenza between October } 2000 \text { and April } \\
2009 \text { who tested positive for influenza with direct fluorescent antibody (DFA) } \\
\text { Controls ( } \mathrm{n}=192) \text { : participants hospitalised for influenza during the same time interval as the cases but } \\
\text { negative with the DFA test. For each case } 1 \text { or } 2 \text { controls matched for birth date and date of hospitalisa- } \\
\text { tion were randomly selected. }\end{array}$ \\
\hline Interventions & Immunisation with influenza vaccine during pregnancy (until 14 days before delivery) \\
\hline Outcomes & DFA-confirmed influenza \\
\hline Notes & $\begin{array}{l}\text { This study was supported by the National Center for Research Resources, a component of the National } \\
\text { Institutes of Health. }\end{array}$
\end{tabular}

\section{Risk of bias}

\begin{tabular}{lll}
\hline Bias & Authors' judgement & Support for judgement \\
\hline $\begin{array}{ll}\text { CC - case selection } \\
\text { All outcomes }\end{array}$ & Low risk & Infants hospitalised with DFA positive \\
\hline $\begin{array}{l}\text { CC - control selection } \\
\text { All outcomes }\end{array}$ & Low risk & Infant hospitalised with DFA negative \\
\hline $\begin{array}{l}\text { CC - comparability } \\
\text { All outcomes }\end{array}$ & Low risk & Matching \\
\hline $\begin{array}{l}\text { CC - exposure } \\
\text { All outcomes }\end{array}$ & Unclear risk & Structured interview \\
\hline
\end{tabular}


pba Benowitz 2010 (Continued)

Summaryassessment Unclear risk Unclear

pba Poehling 2011

Methods Case-control study assessing the effectiveness of influenza vaccine administered during pregnancy in preventing influenza in newborns under 6 months

Participants Children $(n=1510)$ aged below 6 months who were hospitalised for fever or acute respiratory illness, or both during 7 consecutive epidemic seasons (between 2002 and 2003 and 2008 and 2009). Those with positive laboratory confirmation of influenza were enrolled as cases $(n=151)$; those whose result was negative were enrolled as controls $(n=1359)$.

\begin{tabular}{ll}
\hline Interventions & Influenza vaccination during pregnancy \\
\hline Outcomes & Influenza \\
\hline Notes & $\begin{array}{l}\text { This project was supported the Centers for Disease Control and Prevention, National Institute of Allergy } \\
\text { and Infectious Diseases, and Wachovia Research Fund. } 3 \text { authors had received past funding from indus- } \\
\text { try (of these } 1 \text { was on the Medlmmune Advisory Board and another was a NexBio consultant). } \\
\text { Funding source - mixed }\end{array}$ \\
\hline
\end{tabular}

\section{Risk of bias}

\begin{tabular}{lll}
\hline Bias & Authors' judgement & Support for judgement \\
\hline $\begin{array}{ll}\text { CC - case selection } \\
\text { All outcomes }\end{array}$ & Low risk & Laboratory confirmed \\
\hline $\begin{array}{l}\text { CC - control selection } \\
\text { All outcomes }\end{array}$ & Low risk & Infants without laboratory-confirmed influenza \\
\hline $\begin{array}{l}\text { CC - comparability } \\
\text { All outcomes }\end{array}$ & Unclear risk & No matching, unclear information \\
\hline $\begin{array}{l}\text { CC - exposure } \\
\text { All outcomes }\end{array}$ & Unclear risk & Structured interview \\
\hline Summary assessment & Unclear risk & Unclear \\
\hline
\end{tabular}

pbb Irving 2013

Methods

Case-control study investigating the association between influenza immunisation during pregnancy and spontaneous abortion

\section{Participants}

Cases $(n=243)$ were identified from among the members of 6 Vaccine Safety Datalink organisations. Diagnoses of spontaneous abortion (ICD-9 code 634) and unspecified abortion (ICD-9 codes 637) assigned during the 2005 to 2006 and 2006 to 2007 seasons were reviewed and different diagnoses excluded.

Controls $(n=243)$ were selected from among women who had confirmed intrauterine pregnancy and delivery after the 20th gestational week by frequency-matching of last menstrual period (within 2 weeks) and healthcare organisation. 
pbb Irving 2013 (Continued)

Interventions

Immunisation with influenza vaccine. Participants were considered exposed if they were immunised within 28 days before index date. Analysis considering whether vaccine exposure occurred during or before pregnancy was also performed.

\begin{tabular}{ll}
\hline Outcomes & Spontaneous abortion cases \\
\hline Notes & Government funded
\end{tabular}

\section{Risk of bias}

\begin{tabular}{lll}
\hline Bias & Authors' judgement & Support for judgement \\
\hline $\begin{array}{l}\text { CC - case selection } \\
\text { All outcomes }\end{array}$ & Low risk & Consecutive series of cases from electronic databases \\
\hline $\begin{array}{l}\text { CC - control selection } \\
\text { All outcomes }\end{array}$ & Low risk & From the same population \\
\hline $\begin{array}{l}\text { CC - comparability } \\
\text { All outcomes }\end{array}$ & Unclear risk & Matched by LMP - confounders \\
\hline $\begin{array}{l}\text { CC - exposure } \\
\text { All outcomes }\end{array}$ & Unclear risk & Medical record \\
\hline Summary assessment & Unclear risk & Unclear \\
\hline
\end{tabular}

\section{pca Ahrens 2014}

Methods

Retrospective cohort study. This study was performed by retrospective analysis of data within the Birth Defects Study, an ongoing case-control study investigating the occurrence of neonatal malformations conducted by the Slone Epidemiology Center at Boston University. It includes hospitals serving the areas surrounding Philadelphia and San Diego, Rhode Island, southern New Hampshire, and parts of New York State and Massachusetts. Occurrence of preterm delivery and low birth weight were compared between non-malformed (controls) infants born from vaccinated and unvaccinated mothers.

Participants $\quad$ For the study seasons included in this analysis (from 2006-07 to 2009-10), mothers of live-born, nonmalformed infants were identified; 1619 were included in the study.

Interventions Immunisation with trivalent inactivated vaccine during pregnancy. Within 6 months after delivery, a study nurse conducted a phone interview asking for information about immunisation (and other issues).

Women reporting influenza vaccination during pregnancy were asked to provide a release to allow study staff to obtain their vaccination records, but only $60 \%$ of the women complied with this request.

Reports of seasonal trivalent influenza vaccination were categorised according to the timing of receipt: any time during pregnancy (last menstruation period to day before delivery), first trimester (last menstruation period through 14 weeks), second trimester (greater than 15 through 28 weeks), and third trimester (greater than 29 weeks to day before delivery). Women who reported vaccination with pandemic $\mathrm{H} 1 \mathrm{~N} 1$ vaccine were excluded from the analysis. 334 women were immunised for all seasons considered in the study.

Outcomes

Gestational age at delivery and birth weight were obtained by self report from the mother during the interview. Gestational age was determined by calculating the difference between the last menstruation period and the day of delivery. If the self reported last menstruation period date differed by more than 7 days from the last menstruation period date calculated from the reported ultrasound-determined due date, then the latter last menstruation period date was used to calculate gestational age. If the self 
pca Ahrens 2014 (Continued)

reported last menstruation period date differed by 7 days or less from the last menstruation period calculated from the due date, we chose to use the self reported last menstruation period date because it was a date familiar to the mother and raised less confusion during the course of the interview.

- Small for gestational age, defined as a weight < 10th percentile for gestational age, considering the sex-specific distribution of birth weights of infants born in the US in 1999-2000

- Preterm delivery, defined as live birth before 37 weeks' gestation

ILI: for the last season in study (2009-10), having had ILI symptoms was also ascertained during the interview

Notes

Funding source - government

At the time of manuscript preparation, Katherine Ahrens was a pre-doctoral Boston University Reproductive, Perinatal and Pediatric Epidemiology trainee supported by the National Institutes of Health (Grant T32 HD052458). Data collection for this project has been funded by the Biomedical Advanced Research and Development Authority, Office of the Assistant Secretary for Preparedness and Response, Department of Health and Human Services (Contract No. HHSO100201000038C); the Agency for Healthcare Research and Quality (Grant 1R18HS018463-01); and the National Institutes of Health (Grants 1R01 HD059861 and 2 R01 HD46595). Drs Louik and Mitchell and Mr Kerr receive research support from Novartis Vaccines and Diagnostics (NVD) for an unrelated study of a meningitis vaccine. Dr Mitchell serves as a member of an advisory committee for a pregnancy registry for a multiple sclerosis agent conducted by Biogen Idec and as an unpaid consultant to NVD on matters unrelated to influenza vaccines. Dr Werler has provided consultation for Amgen, Bristol-Meyers Squibb, and Abbott regarding their pregnancy registries for rheumatoid arthritis drugs. These companies do not manufacture influenza vaccines. Dr Ahrens has no conflicts to disclose.

\section{Risk of bias}

\begin{tabular}{lll}
\hline Bias & Authors' judgement & Support for judgement \\
\hline $\begin{array}{l}\text { PCS/RCS - selection ex- } \\
\text { posed cohort }\end{array}$ & Unclear risk & $\begin{array}{l}\text { Description is insufficient. Participants for this study are simply selected from } \\
\text { control population of the Birth Defects Study. }\end{array}$ \\
All outcomes & & \\
\hline
\end{tabular}

\begin{tabular}{|c|c|c|}
\hline $\begin{array}{l}\text { PCS/RCS - selection non- } \\
\text { exposed cohort } \\
\text { All outcomes }\end{array}$ & Low risk & Selected from the same population as the exposed cohort \\
\hline $\begin{array}{l}\text { PCS/RCS - comparability } \\
\text { All outcomes }\end{array}$ & Unclear risk & Not clear whether all possible confounding factors were considered \\
\hline $\begin{array}{l}\text { PCS/RCS - assessment of } \\
\text { outcome } \\
\text { All outcomes }\end{array}$ & Unclear risk & Interview \\
\hline Summary assessment & Unclear risk & Unclear risk of bias \\
\hline
\end{tabular}

\section{pca Black 2004}

\begin{tabular}{ll} 
Methods & $\begin{array}{l}\text { Retrospective cohort study assessing the effectiveness of flu vaccination for the prevention of ILI or } \\
\text { pneumonia in pregnant women and their newborns }\end{array}$ \\
\hline Participants & $\begin{array}{l}\text { All women with live births in Kaiser Permanente Northern California (KPNC) between the November } \\
\text { and February of } 5 \text { subsequent seasons (1997 to } 1998 \text { to } 2001 \text { to } 2002, \mathrm{n}=49,585), \text { excluding cases } \\
\text { lacking birth date information and women who were discharged after the end of the flu season. }\end{array}$
\end{tabular}


pca Black 2004 (Continued)

- All live births in KPNC that occurred during the same time periods as for the mothers ( $n=48,639$ ), again cases lacking gestational age or gender information and infants discharged after the end of the flu season were excluded.

Interventions

Immunisation with flu vaccine (no details about type and composition). Data about immunisation were obtained from the KPNC database. In all, 3707 out of the 49,585 pregnant women included in the study were vaccinated, whereas this was 3652 out of the 48,639 live births.

Outcomes

- Hospitalisation for pneumonia or influenza: at least 1 inpatient stay during the same flu season as delivery or birth with a principal (first) diagnosis of either influenza or pneumonia. To identify these outcomes, the following ICD (9th revision) codes were used to identify inpatient cases: influenza 487 and pneumonia 480, 481, 482, 483, 484, 485, and 486.

- Outpatient visits: at least 1 physician visit during the same flu season as delivery or birth with 1 of the following diagnoses: upper respiratory infection, pharyngitis, otitis media, asthma, bronchial asthma, viral infection, pneumonia, fever, cough or wheezing associated with respiratory illness.

This information was available from the KPNC databases, which include laboratory, hospitalisation, and outpatient utilisation information for their members.

The effect measure (hazard ratio and corresponding 95\% confidence interval) was calculated for ILI visits (including and excluding asthma diagnoses) for the mother and hospitalisation for pneumonia or influenza, ILI visits (excluding otitis media), and otitis media visits in newborns.

- Caesarean section.

- Preterm delivery (<37 weeks).

Notes Government-funded

\section{Risk of bias}

\begin{tabular}{lll}
\hline Bias & Authors' judgement & Support for judgement \\
\hline $\begin{array}{ll}\text { PCS/RCS - selection ex- } \\
\text { posed cohort }\end{array}$ & Unclear risk & $\begin{array}{l}\text { From KPNC databases: the influenza vaccination status of women in the co- } \\
\text { hort was determined through review of the Kaiser Immunization Tracking Sys- } \\
\text { tem datcomes }\end{array}$ \\
\end{tabular}

PCS/RCS - selection non- Unclear risk $\quad$ From KPNC databases
exposed cohort
All outcomes

\begin{tabular}{|c|c|c|}
\hline $\begin{array}{l}\text { PCS/RCS - comparability } \\
\text { All outcomes }\end{array}$ & High risk & No matching \\
\hline $\begin{array}{l}\text { PCS/RCS - assessment of } \\
\text { outcome } \\
\text { All outcomes }\end{array}$ & Unclear risk & $\begin{array}{l}\text { KPNC maintains administrative databases that include laboratory, hospitalisa- } \\
\text { tion, and outpatient utilisation information for their members. }\end{array}$ \\
\hline Summary assessment & High risk & High \\
\hline
\end{tabular}

\section{pca Eick 2011}

$\begin{array}{ll}\text { Methods } & \text { Prospective cohort study carried out in } 6 \text { hospitals located in the Navajo and White Mountain Apache } \\ \text { reservation during } 3 \text { subsequent epidemic seasons (2002 to 2005) }\end{array}$

\footnotetext{
Participants

Mother-infant pairs recruited after delivery at Indian Health Service hospitals on the Navajo or White Mountain Apache reservation, either at the hospital or by home visit.
} 
The enrolment periods for each year were:

- 1 December 2002 to 15 March 2003;

- 1 November 2003 to 8 March 2004;

- 1 November 2004 to 15 March 2005.

Inclusion was restricted to mothers who delivered a healthy infant at 36 weeks or later gestation during the enrolment periods. Eligible infants were aged 2 weeks or younger at enrolment. Overall, 1169 mother-infant pairs were enrolled in the study (241 in 2002 to 2003; 574 in 2003 to 2004; and 354 in 2004 to 2005). Of these, 1160 had at least 1 serum sample and were included.

Interventions Immunisation of the mother with influenza vaccine. Assessed by reviewing of medical record (also in order to obtain information about prenatal visits, illnesses, and birth information, in addition to administration and timing of influenza vaccine) or, if missing, by maternal report at enrolment.

The decision for influenza vaccination was made by the treating clinician and the pregnant woman; personnel had no role in this decision. Altogether 587 children were born from an unvaccinated mother and 573 from a vaccinated mother during the 3 study seasons.

Outcomes Surveillance for all medically attended illnesses in enrolled infants was conducted at Indian Health Service and nearby private facilities through the influenza season, or until the child reached 6 months of age (whichever came first). It also included review of the clinic, emergency department, and inpatient paediatric ward logs. A nasopharyngeal aspirate specimen for viral culture was obtained from infants with ILI within 72 hours of the medical visit.

- Medically attended ILI: defined as a medical visit with at least 1 of the following signs or symptoms reported: fever of $38.0^{\circ} \mathrm{C}$ or higher, diarrhoea, or respiratory symptoms (including cough, runny nose, or difficulty breathing).

- Laboratory-confirmed influenza: the first ILI episode with either:

a. isolation of influenza virus from the nasopharyngeal aspirate specimen;

b. a 4-fold or greater rise in $\mathrm{HI}$ antibody in serum collected at 2 to 3 or 6 months compared with the previous serum specimen, indicating influenza virus infection during the time interval; or

c. a positive rapid influenza diagnostic test result with a medical diagnosis of influenza.

Notes $\quad$ Funding/support: "The study was funded by the National Vaccine Program Office, Department of Health and Human Services, the Office of Minority Women's Health, Centers for Disease Control and Prevention, Aventis-Pasteur, and Evans-Powderject."

Funding source - mixed

\section{Risk of bias}

\begin{tabular}{|c|c|c|}
\hline Bias & Authors' judgement & Support for judgement \\
\hline $\begin{array}{l}\text { PCS/RCS - selection ex- } \\
\text { posed cohort } \\
\text { All outcomes }\end{array}$ & Unclear risk & The study was carried out within Indian reservations. \\
\hline $\begin{array}{l}\text { PCS/RCS - selection non- } \\
\text { exposed cohort } \\
\text { All outcomes }\end{array}$ & Low risk & Derived from the same community as the exposed cohort \\
\hline $\begin{array}{l}\text { PCS/RCS - comparability } \\
\text { All outcomes }\end{array}$ & Unclear risk & $\begin{array}{l}\text { Reported for some parameters only: sex, presence of household smokers, hav- } \\
\text { ing wood or coal stove in the house (more frequent among vaccinated), pres- } \\
\text { ence of other children in day care, infant breast fed (more frequent among vac- } \\
\text { cinated), gestational age, mean birth weight }\end{array}$ \\
\hline
\end{tabular}


pca Eick 2011 (Continued)

PCS/RCS - assessment of Low risk Active surveillance and testing for laboratory confirmation for symptomatic ILI outcome cases

All outcomes

Summaryassessment Unclear risk Unclear

pca France 2006

Methods Retrospective cohort study based on Vaccine Safety Datalink, assessing the effect of influenza vaccination of pregnant women in preventing respiratory illness in newborns. 6 epidemic seasons were considered.

\section{Participants}

Infants who were born before or during the influenza season at 4 MCOs (Kaiser Permanente Colorado, Denver; Kaiser Permanente Northern California, Oakland; Kaiser Permanente Northwest, Portland, Oregon; and Group Health Cooperative, Seattle, Washington) between 1 October 1995 and 30 September 2001 were eligible for study inclusion.

Mother-infant pairs were included in the final study population if:

1. the mothers were aged 18 to 45 years and enrolled in the MCO for longer than 1 year;

2. the infants' gestational age was at least 30 weeks at birth;

3. the infants were continuous MCO members for at least 14 days during the influenza season;

4. the infants had a least 1 outpatient visit during the first 3 months of life.

Interventions

An infant was considered exposed if the mother was vaccinated against influenza during the pregnancy and there were at least 28 days from the vaccination date of the mother to the birth date of the infant. Infants of mothers vaccinated within 27 days of birth were excluded from the primary analysis.

\begin{tabular}{ll}
\hline Outcomes & Medically attended ARI \\
\hline Notes & Government funded \\
\hline
\end{tabular}

\section{Risk of bias}

\begin{tabular}{lll}
\hline Bias & Authors' judgement & Support for judgement \\
\hline $\begin{array}{l}\text { PCS/RCS - selection ex- } \\
\text { posed cohort } \\
\begin{array}{l}\text { All outcomes } \\
\text { PCS/RCS - selection non- }\end{array}\end{array}$ & High risk & From MCO databases \\
$\begin{array}{l}\text { exposed cohort } \\
\text { All outcomes }\end{array}$ & From MCO databases \\
\hline $\begin{array}{l}\text { PCS/RCS - comparability } \\
\text { All outcomes }\end{array}$ & High risk & Poor matching \\
\hline $\begin{array}{l}\text { PCS/RCS - assessment of } \\
\text { outcome } \\
\text { All outcomes }\end{array}$ & Unclear risk & Datalink \\
\hline $\begin{array}{l}\text { Summary assessment } \\
\text { High risk }\end{array}$ & \\
\hline
\end{tabular}


pca Hulka 1964

\begin{tabular}{|c|c|}
\hline Methods & Prospective cohort study assessing the effectiveness of flu vaccination in pregnancy \\
\hline Participants & Pregnant women $(n=544)$ recruited from the "hill" district of Pittsburgh \\
\hline Interventions & $\begin{array}{l}\text { - Polyvalent flu vaccine containing } 200 \text { units of } A 2 \text { antigen } \\
\text { - Placebo } \\
\text { Two } 1 \mathrm{~mL} \text { doses were administered } 1 \text { month apart. }\end{array}$ \\
\hline Outcomes & $\begin{array}{l}\text { - Adverse effects following immunisation (pain, malaise) } \\
\text { - ILI } \\
\text { - Days in bed } \\
\text { Assessed by means of questionnaires/phone interviews after epidemic }\end{array}$ \\
\hline Notes & $\begin{array}{l}\text { Effectiveness follow-up was available for } 59 \% \text { and } 100 \% \text { of participants in the intervention and placebo } \\
\text { arm, respectively. } \\
\text { Funding source - mixed }\end{array}$ \\
\hline
\end{tabular}

\section{Risk of bias}

\begin{tabular}{|c|c|c|}
\hline Bias & Authors' judgement & Support for judgement \\
\hline $\begin{array}{l}\text { PCS/RCS - selection ex- } \\
\text { posed cohort } \\
\text { All outcomes }\end{array}$ & High risk & Unclear \\
\hline $\begin{array}{l}\text { PCS/RCS - selection non- } \\
\text { exposed cohort } \\
\text { All outcomes }\end{array}$ & High risk & Unclear \\
\hline $\begin{array}{l}\text { PCS/RCS - comparability } \\
\text { All outcomes }\end{array}$ & High risk & Unclear - high attrition \\
\hline $\begin{array}{l}\text { PCS/RCS - assessment of } \\
\text { outcome } \\
\text { All outcomes }\end{array}$ & High risk & Interview \\
\hline Summary assessment & High risk & Unclear \\
\hline
\end{tabular}

\section{pca Munoz 2005}

\section{Methods}

Retrospective cohort study based on the electronic database of Kelsey-Seybold Clinic (KSC), a large multispecialty clinic in the metropolitan area of Houston (USA). For the study 5 subsequent flu seasons were considered, from 1998 to 2003, taking into account the time between 1 July and 30 June of each year. Approximately 25 obstetricians and 60 paediatricians provided medical care in KSC locations, and about 2500 deliveries occurred every year during the time considered for the study.

Participants

Exposed cohort $(n=225)$ : women who were immunised with inactivated influenza vaccine within 6 months before delivery and who had an uncomplicated singleton pregnancy, were healthy, had at least 1 prenatal care visit at KSC, and their offspring had at least 1 clinic visit at KSC in their first year of life.

Comparison ( $n=826)$ : for each vaccinated woman, a comparison group was selected by matching (KSC database) 3 to 5 women for maternal age at delivery, month of delivery, and type of insurance (with the 
pca Munoz 2005 (Continued)

exclusion of both Medicaid or self insurance due to small numbers in this clinic population), who had not received influenza vaccine during pregnancy.

Interventions

Influenza vaccines used during the study period were Aventis Pasteur or Wyeth products. For the control group the index date ("pseudo vaccination date") corresponds to the same number of days before delivery as the real vaccination date for a matching vaccinated woman.

\section{Outcomes}

\section{Women}

- Acute respiratory illness: cases recorded at any time, during each flu season and during each epidemic peak of that season diagnosed with the following ICD-9 codes: 079, 460-466, 470-478, 480-487. The peak of influenza activity was the period during which the number of laboratory-confirmed cases included at least $85 \%$ of influenza cases for that season.

- Serious adverse events: hospitalisation (death, cause for hospitalisation, and permanently disabling conditions were also included) within 42 days from immunisation identified by ICD- 9 codes.

Medical diagnoses occurred between vaccination and delivery with an incidence $\geq 2 \%$ among vaccinated women.

\section{Newborns}

- Diagnoses different from a "normal newborn infant" given at discharge and within 2 days from delivery.

- Reason for at least 3 days hospitalisation within 1 week, between 8 and 180 days, and between 6 months and 1 year after delivery.

- Diagnoses reported during ambulatory medical visits during the first 6 months of life.

In the last 2 categories URTI and respiratory infections are also included.

Notes

Little information about characteristics and comparability of the exposed and unexposed cohorts. Outcomes used to assess the effectiveness of vaccination are in some way 'surrogate' and include only hospitalisation and ambulatory diagnoses. The first 2 weeks after vaccination should have been excluded from follow-up for the assessment of effectiveness in mothers.

Government funded

\section{Risk of bias}

\begin{tabular}{|c|c|c|}
\hline Bias & Authors' judgement & Support for judgement \\
\hline $\begin{array}{l}\text { PCS/RCS - selection ex- } \\
\text { posed cohort } \\
\text { All outcomes }\end{array}$ & Unclear risk & $\begin{array}{l}\text { Women were included in the study sample if they had received inactivated in- } \\
\text { fluenza vaccine within } 6 \text { months before delivery of an uncomplicated single- } \\
\text { ton pregnancy and were otherwise healthy, had at least } 1 \text { prenatal care visit at } \\
\mathrm{KSC} \text {, and their offspring had at least } 1 \text { clinic visit at KSC in their first year of life. }\end{array}$ \\
\hline
\end{tabular}

PCS/RCS - selection non- Unclear risk
exposed cohort

$$
\text { All outcomes }
$$

A comparison group was selected by matching of maternal age at delivery, month of delivery, and type of insurance (patients with Medicaid or self insurance were excluded due to the small numbers in this clinic population). For each vaccinated woman, 3 to 5 (ratio 1:3.5) matching healthy women who met all the inclusion criteria but who had not received influenza vaccine during pregnancy were selected.

\begin{tabular}{|c|c|c|}
\hline $\begin{array}{l}\text { PCS/RCS - comparability } \\
\text { All outcomes }\end{array}$ & Unclear risk & Matching \\
\hline $\begin{array}{l}\text { PCS/RCS - assessment of } \\
\text { outcome } \\
\text { All outcomes }\end{array}$ & Unclear risk & $\begin{array}{l}\text { The potential protective effect of the vaccine was estimated by recording the } \\
\text { occurrence of ARIs in vaccinated women from the time of receipt of influenza } \\
\text { vaccine to delivery and in unvaccinated women for the equivalent period of } \\
\text { time. Specifically, the occurrence of ARIs during the peak of the influenza sea- }\end{array}$ \\
\hline
\end{tabular}


pca Munoz 2005 (Continued)

son was compared between the groups. Diagnostic codes for ARI included 079, 460-466, 470-478, 480-487.

\begin{tabular}{lll}
\hline Summary assessment $\quad$ Unclear risk & Unclear \\
\hline
\end{tabular}

\section{pca Yamada 2012}

\begin{tabular}{|c|c|}
\hline Methods & $\begin{array}{l}\text { Questionnaire-based, retrospective cohort study performed at the } 121 \text { obstetrical facilities of Hokkaido } \\
\text { (Japan) }\end{array}$ \\
\hline
\end{tabular}

\begin{tabular}{ll}
\hline Participants & All 121 obstetric facilities in Hokkaido were requested to deliver a 12 -item questionnaire to all postpar- \\
tum women who gave birth between 1 December 2009 and 31 May 2010 during their stay in obstetric \\
facilities. About $1 / 3$ of the women who delivered in Hokkaido during this time answered the question- \\
naire $(n=7535)$.
\end{tabular}

\begin{tabular}{ll}
\hline Interventions & $\begin{array}{l}\text { Influenza vaccination during pregnancy. Out of the } 7535 \text { women who answered the questionnaire, } 4921 \\
\text { received pandemic influenza vaccine. Among them, } 2212 \text { were also reported to have been vaccinated } \\
\text { with seasonal vaccine. A further } 270 \text { (considered as unvaccinated) received seasonal vaccine only. }\end{array}$
\end{tabular}

\begin{tabular}{ll}
\hline Outcomes & $\begin{array}{l}\text { Influenza. Definition was not provided. All information was collected by means of a questionnaire, on } \\
\text { which items about admission to the intensive care unit, intubation or ventilation, and diagnosis of in- } \\
\text { fluenza encephalopathy were also present. }\end{array}$ \\
\hline Notes & Strongly biased \\
& Government funded \\
\hline
\end{tabular}

\section{Risk of bias}

\begin{tabular}{|c|c|c|}
\hline Bias & Authors' judgement & Support for judgement \\
\hline $\begin{array}{l}\text { PCS/RCS - selection ex- } \\
\text { posed cohort } \\
\text { All outcomes }\end{array}$ & High risk & By interview \\
\hline $\begin{array}{l}\text { PCS/RCS - selection non- } \\
\text { exposed cohort } \\
\text { All outcomes }\end{array}$ & High risk & By interview \\
\hline $\begin{array}{l}\text { PCS/RCS - comparability } \\
\text { All outcomes }\end{array}$ & High risk & No matching \\
\hline $\begin{array}{l}\text { PCS/RCS - assessment of } \\
\text { outcome } \\
\text { All outcomes }\end{array}$ & High risk & By interview \\
\hline Summary assessment & High risk & High \\
\hline
\end{tabular}

pcb Beau 2014

$\begin{array}{ll}\text { Methods } & \text { Retrospective cohort study. Pregnancies ending between } 21 \text { October } 2009 \text { (the start of the vaccina- } \\ \text { tion campaign) and } 30 \text { November } 2010 \text { and that had started before } 31 \text { January } 2010 \text { identified using } \\ \text { databases EFEMERIS (database including pregnant women) and CNAMTS (vaccination database): } 1645\end{array}$


pcb Beau 2014 (Continued)

women exposed to $\mathrm{A} / \mathrm{H} 1 \mathrm{~N} 1$ vaccine during pregnancy and 3290 randomly selected who did not receive $\mathrm{A} / \mathrm{H} 1 \mathrm{~N} 1$ (matched for month and year of the start of pregnancy)

Participants

Data about study population come from an extended database (EFEMERIS) collecting and linking information from 4 different sources:

- CPAM (Caisse Primaire d'Assurance Maladie), the French Health Insurance System of Haute-Garonne (South West France): it records all the reimbursed drugs prescribed and dispensed to patients under general state coverage (classified according to the World Health Organization's Anatomical Therapeutic Chemical classification);

- PMI (Protection Maternelle et Infantile), the Mother and Child Protection Center: it contains data about the health of both mother (maternal characteristics, some pathologies during pregnancy) and child (weight, size, Apgar score, neonatal pathologies, psychomotor development, congenital malformations) collected during the compulsory medical examinations at ages 8 days, 9 months and 2 years;

- CDA (Centre de Diagnostic Anténatal), the Antenatal Diagnosis Center centralises data corresponding to all the occurrences of major and minor malformations in the maternities of the region where therapeutic termination has been considered (cause and date of termination);

- PMSI (Programme de médicalisation des systèmes d'information), the French hospital medical information system provides the nature and date of termination (legal termination, stillbirth, and spontaneous abortion) from Toulouse University Hospital Center.

EFEMERIS contains information about 58,171 mother-outcome pairs with women who delivered in Haute-Garonne between 1 July 2004 and 31 December 2010. For the present study, only pregnancies ending between 21 October 2009 (the start of the vaccination campaign) and 30 November 2010 (9 months after the vaccination centres had closed) and that had started before 31 January 2010 were considered $(n=12,120)$.

For each mother-outcome pair exposed to A/H1N1 vaccine during pregnancy ( $n=1645), 2$ mother-outcome pairs were randomly selected from among individuals in the study population who did not receive $A / H 1 N 1$ vaccine during pregnancy but who were matched for month and year of the start of pregnancy $(\mathrm{n}=3290)$.

Interventions

Exposure to monovalent pandemic $\mathrm{H} 1 \mathrm{~N} 1$ vaccine during pregnancy. Vaccination centres were required to report A/H1N1 vaccinations to the French National Health Insurance Fund for Salaried Workers (Caisse National de l'Assurance Maladie des Travailleurs Salariés; CNAMTS)

Exposure was considered at any time during pregnancy. For neonatal pathologies, a subset of babies born from mothers who were immunised within the 3rd pregnancy trimester was also considered.

Authors report that $93 \%$ of the exposed women received the non-adjuvanted Panenza (Sanofi Pasteur), but did not provide any further details.

Outcomes

- All-cause pregnancy loss: any loss occurring during pregnancy (legal and therapeutic termination, spontaneous abortion, stillbirth, and ectopic pregnancy).

- Preterm delivery: birth before 259 days of amenorrhoea/37 completed weeks.

- Small for gestational age: any singleton with a birth weight $<2$ standard deviations from the French reference weight mean, adjusted for gestational age and sex.

- Neonatal pathologies (including respiratory distress, pneumothorax, neonatal jaundice, metabolic disorders, or sepsis): identified from the children's health certificates established on the 8th day, as recorded by the physician during medical consultation; these records provided little detail about the conditions.

Notes Funding source - government

"The EFEMERIS database was funded by the Agence Nationale de Sécurité du Médicament et des produits de santé (ANSM), the CNAMTS, the Mutuelle Générale de l'Education Nationale, the Clinical Research Hospital Program (PHRC) and the Unions régionales des Caisses d'Assurance Maladie. Additional funding has been received from the ANSM for the influenza medication study"

\section{Risk of bias}


pcb Beau 2014 (Continued)

\begin{tabular}{|c|c|c|}
\hline Bias & Authors' judgement & Support for judgement \\
\hline $\begin{array}{l}\text { PCS/RCS - selection ex- } \\
\text { posed cohort } \\
\text { All outcomes }\end{array}$ & Low risk & $\begin{array}{l}\text { The whole cohort of pregnant women between October } 2009 \text { and November } \\
2010 \text { was considered. Those who were vaccinated were the exposed cohort. }\end{array}$ \\
\hline $\begin{array}{l}\text { PCS/RCS - selection non- } \\
\text { exposed cohort } \\
\text { All outcomes }\end{array}$ & Low risk & Drawn from the same source as the exposed cohort \\
\hline $\begin{array}{l}\text { PCS/RCS - comparability } \\
\text { All outcomes }\end{array}$ & Low risk & $\begin{array}{l}\text { Possible confounding factors have been taken into account in data analysis } \\
\text { (adjustment). }\end{array}$ \\
\hline $\begin{array}{l}\text { PCS/RCS - assessment of } \\
\text { outcome } \\
\text { All outcomes }\end{array}$ & Low risk & Secure records \\
\hline Summary assessment & Low risk & Low risk of bias \\
\hline
\end{tabular}

pcb Cantu 2013

Methods Retrospective cohort study. Pregnancy-related outcomes were observed retrospectively among vaccinated and non-vaccinated women who received prenatal care and delivered within Birmingham healthcare system in order to establish if vaccination could represent a risk factor.

Women with singleton pregnancy during 2009-10 pandemic and 2010-11 season who had prenatal visit
between October and end of December of each season at 1 of the 6 prenatal clinics in Birmingham, Al-
abama (USA) without suspected foetal abnormality. Altogether, 1094 vaccinated and 2010 non-vacci-
nated pregnant women were included.

Interventions Vaccination with a pH1N1 virus containing vaccine (not further specified) at any time during pregnancy in pandemic season 2009/10 and in 2010 to 2011 epidemic. Participants immunised exclusively with TIV in 2009/10 season were excluded from the primary analysis.

Vaccination status was ascertained through perinatal record system and vaccination logs. Women who were immunised outside of healthcare system were included if they were able to provide their vaccination date.

The following outcomes were collected and recorded at the time of care at the centres.
- Primary composite outcome: includes miscarriage, stillbirth, preterm birth $<37$ weeks, and neonatal
- Memise
- Miscarriage: defined as delivery prior to 20 weeks
- Birth weight $<2500 \mathrm{~g}$
- Neonatal demise ( 20 weeks)
- Stillbirth: defined as delivery of a non-viable foetus at or after 20 weeks
- Pre-eclampsia
- Small for gestational age: foetal growth less than the 10 th percentile
- Neonatal intensive care unit admission
- Length of maternal stay
- Antiviral (oseltamivir) therapy


pcb Cantu 2013 (Continued)

Results and effect estimates are provided for both seasons pooled.

Study population was limited to women with prenatal visit in the early flu season between 1 October and 31 December each year, when the vast majority of vaccines were given in order to assure that vaccinated and unvaccinated groups had similar exposure periods and avoid potential bias.

\section{Risk of bias}

\begin{tabular}{|c|c|c|}
\hline Bias & Authors' judgement & Support for judgement \\
\hline $\begin{array}{l}\text { PCS/RCS - selection ex- } \\
\text { posed cohort } \\
\text { All outcomes }\end{array}$ & Low risk & $\begin{array}{l}\text { Representative of a pregnant women population belonging to the Birmingham } \\
\text { healthcare system }\end{array}$ \\
\hline $\begin{array}{l}\text { PCS/RCS - selection non- } \\
\text { exposed cohort } \\
\text { All outcomes }\end{array}$ & Low risk & Drawn from the same population as the exposed cohort \\
\hline $\begin{array}{l}\text { PCS/RCS - comparability } \\
\text { All outcomes }\end{array}$ & Low risk & Possible confounders have been taken into account. \\
\hline $\begin{array}{l}\text { PCS/RCS - assessment of } \\
\text { outcome } \\
\text { All outcomes }\end{array}$ & Low risk & Secure record \\
\hline Summary assessment & Low risk & Low risk of bias \\
\hline
\end{tabular}

pcb Chambers 2013

Methods

Prospective cohort study. The study has been carried out within the Organization of Teratology Information Specialists (OTIS) Research Group studies, whose aim is to evaluate the occurrence of pregnancy outcomes following an exposure to a medication or vaccine administered during pregnancy. OTIS services are located in academic institutions or hospitals throughout the US and Canada and provide counselling to about 70,000 callers annually who present with questions about the risks of exposures in pregnancy. Among these women, exposed and not exposed (comparison group) to the agent of interest are included in the study. In the present $\mathrm{pH} 1 \mathrm{~N} 1$ vaccine study, participants were enrolled between October 2009 and April 2012 and were exposed or not exposed to either the monovalent (2009-10 season) or trivalent (2009-12 seasons) pH1N1 vaccine in 1 of 3 influenza seasons. Information about exposure and outcomes of exposed and unexposed cohort were assessed by means of phone questionnaires.

Participants 841 pregnant women exposed to a $\mathrm{pH} 1 \mathrm{~N}$-containing vaccine

191 not exposed pregnant women

Interventions

Information about vaccine type, date of vaccination, and medical setting where immunisation took place are present in the OTIS questionnaires. Participants were further asked to verify provided information on their vaccination record (when available) or to give permission to contact the participant's provider to obtain this information. In cases where the vaccine was given in a non-traditional setting, an attempt was made to determine the specific product used and the date and location of vaccine administration.

The timing of vaccine exposure was divided into 4 categories:

- the 2 weeks between last menstruation period and date of conception;

- from conception to 13 weeks' gestation;

- >13 to 26 weeks' gestation;

- > 26 weeks' gestation. 
pcb Chambers 2013 (Continued)

In the 2009-10 season, some women received the 2009-10 seasonal vaccine (not containing the $\mathrm{pH} 1 \mathrm{~N} 1$ strain) prior to the $\mathrm{pH} 1 \mathrm{~N} 1$ monovalent vaccine becoming available, and were subsequently vaccinated with the monovalent $\mathrm{pH} 1 \mathrm{~N} 1$ vaccine. These women were classified as $\mathrm{pH} 1 \mathrm{~N} 1$ vaccine exposed; however, previous receipt of the non-pandemic vaccine was considered a covariate.

The comparison group consisted of women who received no influenza vaccine of any type throughout their pregnancy. Vaccines were monovalent pH1N1 (unspecified) in 2009-10, and pH1N1-containing TIV in 2010-11 and 2011-12.

Outcomes
from obstetrician, paediatrician, and delivery hospital. Ultrasound dating was used to correct gesta-
tional weeks as necessary using a standard algorithm, or if the LMP was unknown. The following defini-
tions were used:
- Spontaneous abortion: defined as spontaneous pregnancy loss at < 20 gestational weeks
- Preterm delivery: delivery at < 37 completed gestational weeks
- Small for gestational age: defined as < 10th centile for sex and gestational age in live-born infants
using standard US growth charts for full and preterm infants
- Still birth
- Termination
Analysis for the first 3 outcomes was performed considering timing of exposure. Crude and adjusted HH
or OR estimate is provided, other than crude data.
Funding source - government
This project has been funded in whole or in part with Federal funds from the Office of the Assistant Sec-
retary for Preparedness and Response, Biomedical Advanced Research and Development Authority,
Department of Health and Human Services, under Contract No. HHSO100201000029C and the OTIS Col-
laborative Research Group.

\section{Risk of bias}

\begin{tabular}{|c|c|c|}
\hline Bias & Authors' judgement & Support for judgement \\
\hline $\begin{array}{l}\text { PCS/RCS - selection ex- } \\
\text { posed cohort } \\
\text { All outcomes }\end{array}$ & Low risk & $\begin{array}{l}\text { Quite a representative sample of pregnant women enrolled in the OTIS regis- } \\
\text { ters }\end{array}$ \\
\hline $\begin{array}{l}\text { PCS/RCS - selection non- } \\
\text { exposed cohort } \\
\text { All outcomes }\end{array}$ & Low risk & Drawn from the same source as the exposed cohort \\
\hline $\begin{array}{l}\text { PCS/RCS - comparability } \\
\text { All outcomes }\end{array}$ & Unclear risk & Possible confounding factors have been taken into account. \\
\hline $\begin{array}{l}\text { PCS/RCS - assessment of } \\
\text { outcome } \\
\text { All outcomes }\end{array}$ & Low risk & Secure records \\
\hline Summary assessment & Low risk & Low risk of bias \\
\hline
\end{tabular}


pcb Cleary 2014

Methods

Participants
Cohort study. At the time of 2009-10 pandemic, the monovalent pH1N1 was offered to pregnant women and other at-risk groups by the Health Service Executive. Pregnancy outcomes were evaluated in women vaccinated during pregnancy and those not vaccinated during pregnancy.

Women who delivered at the Coombe Women and Infants University Hospital (Dublin) between December 2009 and September 2010 and who reported having been vaccinated $(n=2996)$.

The control consists of women who delivered during the same time interval at the same hospital but who reported not having received influenza vaccination $(n=3898)$.

A second historical control group includes all women who delivered during a time interval (December 2008 to September 2009) before the mass vaccination and the main wave of the 2009-10 pandemic $(n=$ 7044, not considered for the analysis).

Interventions Vaccine exposure was ascertained by means of the delivery suite admission form, which contained the following questions:

1. $\mathrm{H} 1 \mathrm{~N} 1$ vaccine this pregnancy $(\mathrm{Y} / \mathrm{N})$

2. When given (I; II, III trimester)

3. Vaccine used? (Celvapan 1 to 2 doses, Pandremix, unknown)

Any exposure at any time during pregnancy is considered for analysis purposes.

Outcomes

Data on maternal characteristics, medical and obstetric history recorded at the antenatal booking interview, and perinatal outcomes recorded in the delivery suite and neonatal intensive care unit were extracted from electronic hospital records.

- Preterm birth $<37$ weeks

- Spontaneous birth $<37$ weeks

- Very preterm birth $<32$ weeks

- Spontaneous birth $<32$ weeks

- Small for gestational age: birth weight determined to be less than the 10th centile customised for maternal weight, height, gestation, and infant sex, age

- Apgar score $<3$ at 1 min

- Apgar score $<7$ at 5 min

- Admitted to neonatal unit

- Congenital anomaly: ascertained from electronic records of any anomalies identified by midwifery or paediatric staff on the delivery suite or from congenital anomaly, body system or discharge diagnoses fields in the neonatal unit electronic discharge records

- Perinatal death (within 7 days of life)

Notes

Funding source - government

BC was funded by the charity Friends of the Coombe and the School of Pharmacy, Royal College of Surgeons in Ireland.

Exposure: about $56.5 \%$ of vaccinated women reported having received Celvapan (not adjuvanted), $23 \%$ Pandremix (AS03 adjuvanted), and 20\% were unsure about the specific vaccine used.

\section{Risk of bias}

\begin{tabular}{|c|c|c|}
\hline Bias & Authors' judgement & Support for judgement \\
\hline $\begin{array}{l}\text { PCS/RCS - selection ex- } \\
\text { posed cohort } \\
\text { All outcomes }\end{array}$ & Low risk & $\begin{array}{l}\text { All women who delivered at the Coombe Women and Infants University Hospi- } \\
\text { tal between December } 2009 \text { and September } 2010 \text { and received influenza vac- } \\
\text { cine during pregnancy }\end{array}$ \\
\hline $\begin{array}{l}\text { PCS/RCS - selection non- } \\
\text { exposed cohort }\end{array}$ & Low risk & Drawn from the same source as the exposed cohort \\
\hline
\end{tabular}


pcb Cleary 2014 (Continued)

All outcomes

$\begin{array}{ll}\text { PCS/RCS - comparability Low risk } & \text { Characteristics that differ significantly between exposed and not exposed } \\ \text { All outcomes } & \text { group have been taken into account for effect measure calculation. }\end{array}$

\begin{tabular}{lll}
\hline $\begin{array}{l}\text { PCS/RCS - assessment of } \\
\text { outcome }\end{array}$ & Low risk & \\
\begin{tabular}{lll} 
All outcomes & Hospital records \\
\hline Summary assessment & Low risk & Low risk of bias \\
\hline
\end{tabular}
\end{tabular}

\section{pcb Deinard 1981}

$\begin{array}{ll}\text { Methods } & \begin{array}{l}\text { Prospective cohort study assessing the safety of monovalent } \mathrm{A} / \mathrm{NJ} / 8 / 76 \text { vaccine administration during } \\ \text { pregnancy }\end{array}\end{array}$
pregnancy

\begin{tabular}{ll}
\hline Participants & $\begin{array}{l}\text { Pregnant women enrolled at several obstetric clinics (Minneapolis) on the occasion of a prenatal visit } \\
(\mathrm{n}=706)\end{array}$ \\
\hline Interventions & $\begin{array}{l}\text { Flu vaccine containing A/New Jersey/8/76 (split- or whole-virus formulation) administered during the } \\
\text { first, second, or third pregnancy trimester. Vaccine was administered to } 189 \text { women, whereas } 517 \text { acted } \\
\text { as unvaccinated controls. }\end{array}$ \\
\hline Outcomes & $\begin{array}{l}\text { Local and systemic reactions observed and reported after vaccine administration (only the vaccinated } \\
\text { assessed by questionnaire). }\end{array}$ \\
- Pregnancy outcomes: maternal mortality, elective abortion, spontaneous abortion, stillbirth, prema- \\
ture live birth. \\
- Infant outcomes: deaths, major or minor congenital anomalies, abnormalities during the first 8 days \\
of life.
\end{tabular}

Notes $\quad \begin{aligned} & \text { This study should have been performed without external/private/industry funding. } \\ & \text { Government funded }\end{aligned}$

\section{Risk of bias}

Bias Authors' judgement Support for judgement

PCS/RCS - selection ex- High risk

posed cohort

All outcomes

PCS/RCS - selection non- High risk
exposed cohort

All outcomes

PCS/RCS - comparability High risk

All outcomes

PCS/RCS - assessment of High risk
outcome

All outcomes

Summary assessment High risk


pcb Dodds 2012

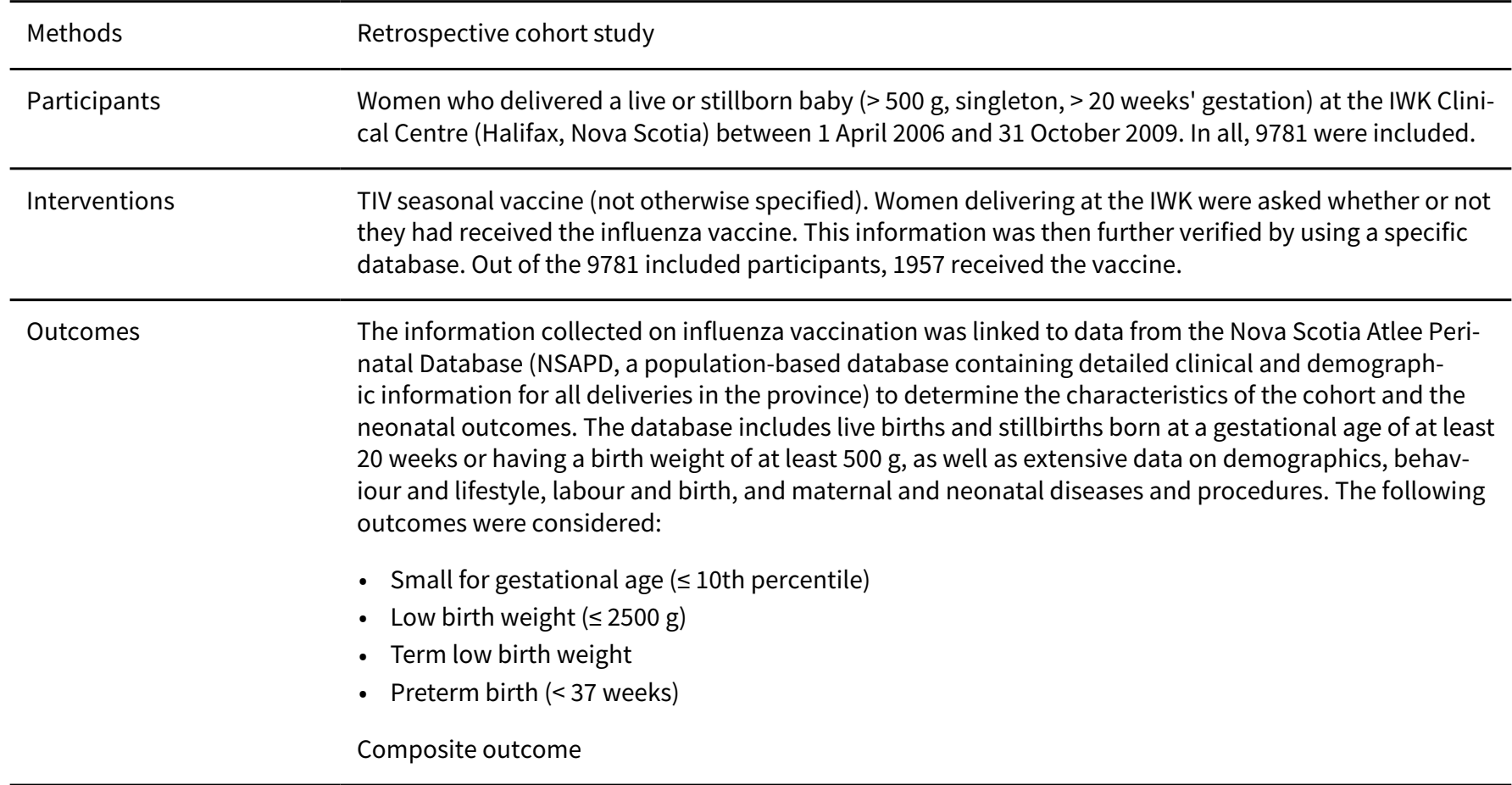

Notes

Funding source - government

This study was funded by grants from the IWK Health Centre and from the Atlee Foundation of the Department of Obstetrics and Gynecology, Dalhousie University.

Data were provided pooled for all 3 seasons and cover the entire years. Data for the time between 1 April 2007 and 31 December 2007 were missed.

\section{Risk of bias}

\begin{tabular}{|c|c|c|}
\hline Bias & Authors' judgement & Support for judgement \\
\hline $\begin{array}{l}\text { PCS/RCS - selection ex- } \\
\text { posed cohort } \\
\text { All outcomes }\end{array}$ & Low risk & About half of deliveries in the region occur in the study health centre. \\
\hline $\begin{array}{l}\text { PCS/RCS - selection non- } \\
\text { exposed cohort } \\
\text { All outcomes }\end{array}$ & Low risk & From the same source as exposed \\
\hline $\begin{array}{l}\text { PCS/RCS - comparability } \\
\text { All outcomes }\end{array}$ & Unclear risk & $\begin{array}{l}\text { It seems that not all possible confounding factors have been taken into ac- } \\
\text { count for calculation of adjusted estimate (only smoking habits } \mathrm{Y} / \mathrm{N} \text { ). Only } \\
\text { those modifying point estimate by }>5 \% \text { were included. }\end{array}$ \\
\hline $\begin{array}{l}\text { PCS/RCS - assessment of } \\
\text { outcome } \\
\text { All outcomes }\end{array}$ & Low risk & Medical database records \\
\hline Summary assessment & Unclear risk & Unclear risk of bias \\
\hline
\end{tabular}


pcb Fell 2012 $\begin{array}{ll}\text { Methods } & \text { Retrospective cohort assessing the safety of pandemic monovalent H1N1 vaccine in pregnant women, } \\ \text { using Ontario's birth record database }\end{array}$

\begin{tabular}{ll}
\hline Participants & Women with singleton birth in 2009 to 2010 season $(\mathrm{n}=55,570)$ \\
\hline Interventions & $\begin{array}{l}\text { Monovalent pandemic H1N1 influenza vaccine. In all, 23,340 pregnant women were also immunised } \\
\text { with seasonal vaccine. }\end{array}$ \\
\hline Outcomes & Frequency of neonatal outcomes in newborns: \\
& - Preterm birth ( $<37$ weeks or $<32$ weeks) \\
& - 5mall for gestational age (below 10th or 3rd percentile) \\
& - Foetal death \\
\hline Notes & "This study was funded by the Canadian Institutes of Health Research (grant 218653)"
\end{tabular}

\section{Risk of bias}

\begin{tabular}{|c|c|}
\hline Bias & Authors' judgement Support for judgement \\
\hline $\begin{array}{l}\text { PCS/RCS - selection ex- } \\
\text { posed cohort } \\
\text { All outcomes }\end{array}$ & Low risk \\
\hline $\begin{array}{l}\text { PCS/RCS - selection non- } \\
\text { exposed cohort } \\
\text { All outcomes }\end{array}$ & Low risk \\
\hline $\begin{array}{l}\text { PCS/RCS - comparability } \\
\text { All outcomes }\end{array}$ & High risk \\
\hline $\begin{array}{l}\text { PCS/RCS - assessment of } \\
\text { outcome } \\
\text { All outcomes }\end{array}$ & Low risk \\
\hline Summary assessment & High risk \\
\hline
\end{tabular}

pcb Heikkinen 2012

\begin{tabular}{ll}
\hline Methods & $\begin{array}{l}\text { Prospective cohort study assessing the safety of pandemic MF-59 adjuvanted influenza vaccine (Foce- } \\
\text { tria) during pregnancy }\end{array}$ \\
\hline Participants & $\begin{array}{l}\text { Pregnant women recruited in midwife practices and hospitals in the Netherlands }(n=4281) \text {, Argenti- } \\
\text { na }(n=239), \text { and Italy }(n=9) \text {. Altogether, } 4508 \text { pregnant women were included: } 2295 \text { were vaccinated } \\
\text { and } 2213 \text { were not immunised. There were } 4522 \text { live births and } 18 \text { intrauterine deaths (2310 born from } \\
\text { vaccinated and } 2213 \text { from unvaccinated mothers). } 3 \text { months' follow-up data were available for } 4385 \text { ba- } \\
\text { bies. }\end{array}$ \\
\hline Interventions & $\begin{array}{l}\text { Monovalent pandemic H1N1, MF-59 adjuvanted flu vaccine Focetria (Novartis Vaccine and Diagnos- } \\
\text { tics, Cambridge, MA, USA). Among the 2295 vaccinated pregnant women, } 1724 \text { received } 2 \text { doses, } 571 \text { re- } \\
\text { ceived } 1 \text { dose. }\end{array}$ \\
\hline Outcomes & Gestational diabetes \\
\hline
\end{tabular}


pcb Heikkinen 2012 (Continued)

- Spontaneous abortion

- Stillbirth

- Live birth

- Low birth weight

- Preterm birth

- Neonatal death

- Congenital malformation

Notes $\quad$ "This study was supported by Novartis Vaccines and Diagnostics"

\section{Risk of bias}

\begin{tabular}{lll}
\hline Bias & Authors' judgement & Support for judgement \\
\hline $\begin{array}{l}\text { PCS/RCS - selection ex- } \\
\text { posed cohort } \\
\text { All outcomes }\end{array}$ & Unclear risk & Unclear \\
\hline $\begin{array}{l}\text { PCS/RCS - selection non- } \\
\text { exposed cohort }\end{array}$ & Unclear risk & Unclear \\
All outcomes & & \\
\hline
\end{tabular}

\section{PCS/RCS - comparability High risk}

All outcomes

PCS/RCS - assessment of Low risk

outcome

All outcomes

\begin{tabular}{lll}
\hline Summaryassessment $\quad$ High risk & Unclear \\
\hline
\end{tabular}

\section{pcb Håberg 2013}

\begin{tabular}{ll}
\hline Methods & $\begin{array}{l}\text { Cohort study assessing the risk of neonatal death following exposure to pandemic monovalent H1N1 } \\
\text { influenza vaccine or influenza virus during pregnancy }\end{array}$ \\
\hline Participants & A total of 113,331 pregnant women \\
\hline Interventions & $\begin{array}{l}\text { Immunisation with pandemic monovalent H1N1 adjuvanted influenza vaccine Pandemrix (GSK) or Cel- } \\
\text { vapan (not adjuvanted) }\end{array}$ \\
\hline Outcomes & Foetal death \\
\hline Notes & Government funded
\end{tabular}

\section{Risk of bias}

\begin{tabular}{lll}
\hline Bias & Authors' judgement & Support for judgement \\
\hline $\begin{array}{l}\text { PCS/RCS - selection ex- } \\
\text { posed cohort } \\
\text { All outcomes }\end{array}$ & Unclear risk & Data link \\
\hline $\begin{array}{l}\text { PCS/RCS - selection non- } \\
\text { exposed cohort }\end{array}$ & Unclear risk & Data link \\
\hline
\end{tabular}


pcb Håberg 2013 (Continued)

All outcomes

PCS/RCS - comparability Unclear risk Multivariate model

All outcomes

\begin{tabular}{lll}
\hline $\begin{array}{l}\text { PCS/RCS - assessment of } \\
\text { outcome } \\
\text { All outcomes }\end{array}$ & Unclear risk & Data link \\
\hline Summary assessment & Unclear risk & Unclear risk of bias \\
\hline
\end{tabular}

pcb Källén 2012 $\begin{array}{ll}\text { Methods } & \text { Retrospective cohort study assessing the effect on newborn outcomes of pandemic squalene adjuvant- } \\ \text { ed H1N1 vaccine }\end{array}$

Participants

The total number of vaccinated women was 18,612 having 18,844 infants (vaccination group, pandemic H1N1 Pandemrix). These women were compared with 136,914 women having 138,931 infants who gave birth after September 2009 and before the end of 2010 (non-vaccinated group) and with 83,298 women having 84,484 infants who gave birth in the year 2009 before October (pre-vaccination group).

\begin{tabular}{ll}
\hline Interventions & $\begin{array}{l}\text { Pandemrix (GlaxoSmithKline; B } \\
\text { California/07/2009, squalene ad }\end{array}$ \\
\hline Outcomes & - Stillbirth \\
- Preterm birth \\
- Low birth weight \\
- Small for gestational age \\
- Congenital malformations
\end{tabular}

Notes $\quad$ "No specific funding was obtained for this study"

\section{Risk of bias}

\begin{tabular}{|c|c|c|}
\hline Bias & Authors' judgement & Support for judgement \\
\hline $\begin{array}{l}\text { PCS/RCS - selection ex- } \\
\text { posed cohort } \\
\text { All outcomes }\end{array}$ & Unclear risk & Unclear \\
\hline $\begin{array}{l}\text { PCS/RCS - selection non- } \\
\text { exposed cohort } \\
\text { All outcomes }\end{array}$ & Unclear risk & Unclear \\
\hline $\begin{array}{l}\text { PCS/RCS - comparability } \\
\text { All outcomes }\end{array}$ & High risk & \\
\hline $\begin{array}{l}\text { PCS/RCS - assessment of } \\
\text { outcome } \\
\text { All outcomes }\end{array}$ & Unclear risk & Unclear \\
\hline Summary assessment & High risk & \\
\hline
\end{tabular}


pcb Launay 2012

\begin{tabular}{ll} 
Methods & $\begin{array}{l}\text { Prospective cohort study assessing the effect of immunisation with pandemic monovalent vaccine dur- } \\
\text { ing pregnancy }\end{array}$ \\
\hline Participants & $\begin{array}{l}\text { Pregnant women }(\mathrm{n}=877) \text { between } 12 \text { and } 35 \text { weeks of gestation, aged at least } 18 \text { years, who were not } \\
\text { vaccinated or infected }\end{array}$ \\
\hline Interventions & Immunisation with pandemic monovalent influenza vaccine \\
\hline Outcomes & Delivery before the 37th gestational week, birth weight, death before or during labour \\
\hline Notes & Government funded
\end{tabular}

Risk of bias

\begin{tabular}{|c|c|c|}
\hline Bias & Authors' judgement & Support for judgement \\
\hline $\begin{array}{l}\text { PCS/RCS - selection ex- } \\
\text { posed cohort } \\
\text { All outcomes }\end{array}$ & Low risk & Low \\
\hline $\begin{array}{l}\text { PCS/RCS - selection non- } \\
\text { exposed cohort } \\
\text { All outcomes }\end{array}$ & Low risk & Low \\
\hline $\begin{array}{l}\text { PCS/RCS - comparability } \\
\text { All outcomes }\end{array}$ & Unclear risk & No information was given about possible confounders \\
\hline $\begin{array}{l}\text { PCS/RCS - assessment of } \\
\text { outcome } \\
\text { All outcomes }\end{array}$ & Unclear risk & Unclear \\
\hline Summary assessment & Unclear risk & Unclear \\
\hline
\end{tabular}

pcb Lin 2012

\begin{tabular}{ll}
\hline Methods & Retrospective cohort study \\
\hline Participants & $\begin{array}{l}\text { A total of } 396 \text { pregnant Taiwanese women were included in the study, of which } 198 \text { received influenza } \\
\text { vaccine during pregnancy }\end{array}$ \\
\hline
\end{tabular}

Interventions Monovalent H1N1 unadjuvanted, inactivated, split-virus vaccine AdimFlu-S (Adimmune Corporation; Taichung, Taiwan) containing $15 \mathrm{~g}$ of New York Medical College X-179A reassortant of the A/California/7/2009 (H1N1)-like strain in $0.5 \mathrm{~mL}$ dose

\begin{tabular}{ll}
\hline Outcomes & Systemic and local adverse events in vaccinated mothers \\
In newborns: \\
- Hyperbilirubinaemia \\
- Contact dermatitis \\
- Upper respiratory tract infection \\
- Seborrhoeic dermatitis \\
- Respiratory distress \\
\hline Notes & Government funded
\end{tabular}


pcb Lin 2012 (Continued)

Risk of bias

\begin{tabular}{lll}
\hline Bias & Authors' judgement & Support for judgement \\
\hline $\begin{array}{l}\text { PCS/RCS - selection ex- } \\
\text { posed cohort } \\
\text { All outcomes }\end{array}$ & Unclear risk & Unclear \\
\hline $\begin{array}{l}\text { PCS/RCS - selection non- } \\
\text { exposed cohort }\end{array}$ & Unclear risk & Unclear \\
$\begin{array}{l}\text { All outcomes } \\
\text { PCS/RCS - comparability }\end{array}$ & High risk \\
$\begin{array}{ll}\text { All outcomes } \\
\text { PCS/RCS - assessment of }\end{array}$ & Low risk \\
$\begin{array}{l}\text { outcome } \\
\text { All outcomes }\end{array}$ & Medical records \\
\hline $\begin{array}{l}\text { Summary assessment } \\
\end{array}$ & High risk \\
\hline
\end{tabular}

\section{pcb Louik 2013}

$\begin{array}{ll}\text { Methods } & \text { Retrospective cohort study. The effect of immunisation with an influenza vaccine containing pH1N1 } \\ \text { during pregnancy on preterm birth was assessed comparing vaccinated and non-vaccinated women }\end{array}$

\section{Participants}

Study population belonged to those enrolled in a large surveillance-based, case-control study carried out by the Slone Epidemiology Center at Boston University. Children-cases with major structural defects were identified from participating hospitals in the areas surrounding Philadelphia and San Diego as well as by means of birth defect registries in New York State and Massachusetts. Controls were normal-formed infants randomly selected within the same study hospitals. For the purposes of the present study, only mothers of controls (without malformations) who delivered during the 2009-10 and 2010-11 seasons are included. Only mothers of singleton, live-born infants, who were immunised not after the 37 th gestation week, were included. Altogether, 951 women were included, 378 of whom received influenza vaccine.

Interventions

Exposure to a pH1N1-containing vaccine during pregnancy within the seasons 2009-10 and 2010-11. Exposure was ascertained by means of a computer-assisted phone interview administered 6 months after delivery and eventually verified by examining the vaccination records. A woman was considered exposed if she had received a pH1N1-containing vaccination. Time of exposure was considered within 1st trimester (until 14th gestation week), 2nd trimester (gestation weeks 15 to 28), and 3rd trimester (from week 29 through delivery). Women whose reported time of exposure could not be attributed to 1 of the trimesters were excluded. Not-exposed participants should have last menstrual date within the range of last menstrual date reported by exposed participants.

\begin{tabular}{ll}
\hline Outcomes & Preterm delivery: defined as delivery at gestational age less than 37 weeks \\
\hline Notes & Funding source - industry \\
& Drs Louik, Chambers, Jones, Schatz, and Mitchell and Mr Kerr receive research support from Novartis \\
& Vaccines and Diagnostics (NVD) for an unrelated study of a meningitis vaccine. Dr Mitchell serves as a \\
& member of an advisory committee for a pregnancy registry for a multiple sclerosis agent conducted \\
& by Biogen-Idec and as an unpaid consultant to NVD on matters unrelated to influenza vaccines. Drs \\
& Chambers and Jones receive support from GlaxoSmithKline Bio for an unrelated study of human papil- \\
& loma virus vaccine. Drs Chambers and Jones receive support for unrelated research projects from var- \\
& ious pharmaceutical companies: Abbott, Amgen, Bristol-Myers Squibb, GlaxoSmithKline, Parr, Pfizer, \\
& Janssen, Roche Genentech, Sanofi Genzyme, Sandoz, and Teva. Dr Schatz has received research sup-
\end{tabular}


pcb Louik 2013 (Continued)

port for projects unrelated to the current study from Aerocrine, Genentech, GlaxoSmithKline, MedImmune, and Merck. Dr Schatz is also a research consultant on subjects unrelated to the current study for

Amgen, Boston Scientific, and GlaxoSmithKline. Ms Pyo and Dr Ahrens have no conflicts to disclose.

\section{Risk of bias}

\begin{tabular}{|c|c|c|}
\hline Bias & Authors' judgement & Support for judgement \\
\hline $\begin{array}{l}\text { PCS/RCS - selection ex- } \\
\text { posed cohort } \\
\text { All outcomes }\end{array}$ & Unclear risk & $\begin{array}{l}\text { Cohort consists of the control population of case-control studies in which case } \\
\text { population is represented by mothers of children born with major defects. }\end{array}$ \\
\hline $\begin{array}{l}\text { PCS/RCS - selection non- } \\
\text { exposed cohort } \\
\text { All outcomes }\end{array}$ & Low risk & Drawn from the same source as the exposed one \\
\hline $\begin{array}{l}\text { PCS/RCS - comparability } \\
\text { All outcomes }\end{array}$ & Low risk & Taken into account \\
\hline $\begin{array}{l}\text { PCS/RCS - assessment of } \\
\text { outcome } \\
\text { All outcomes }\end{array}$ & Low risk & Secure records \\
\hline Summary assessment & Unclear risk & Unclear risk of bias \\
\hline
\end{tabular}

pcb Ludvigsson 2013

Methods Retrospective cohort study. Pregnancy outcomes were compared between women exposed to Pandemrix (monovalent H1N1 pandemic-AS03-adjuvanted influenza vaccine) during pregnancy and nonexposed women.

Participants All live-born single infants in Stockholm County (conceived between February 2009 and January 2010 , $\mathrm{n}=21,087)$

Interventions

Exposure to 1 dose of monovalent pH1N1-AS03-adjuvanted influenza vaccine (Pandemrix) at any time during the pregnancy before the 36th week. Records of vaccination are available from Vaccinera database (vaccination campaign was performed between October 2009 and April 2010, thus about $90 \%$ of the doses were administered before end of 2009). In total, 13,297 women were vaccinated against H1N1 during pregnancy before the 36th week. The non-exposed hemi-cohort consisted of women who did not receive influenza vaccination during pregnancy or who were immunised after the 36 th week $(n=$ 7790).

Different times of exposure during pregnancy (1st or 2 nd - 3rd trimester) were also considered for analysis.

Outcomes

Data about pregnancy outcomes were available in the Obstetrix database, in which information from the 1st antenatal visits ( 8 to 12 gestation weeks) until discharge are collected. For each participant a record is available that can be identified by means of a unique identifier (PIN), which permitted the link between Obstetrix data and those recorded in other archives (Vaccinera among others).

- Birth weight $<2500 \mathrm{~g}$

- Gestational duration < 37 weeks

- Small for gestational age

- Apgar score at $5 \mathrm{~min}<7$

- Caesarean section 
pcb Ludvigsson 2013 (Continued)

Notes Funding source - government

This project was supported by grants from the Swedish Research Council (Medicine), and the Swedish Council for Working Life and Social Research (FAS). JFL was funded by the Swedish Research Council (Medicine), OS was funded by the Swedish Society of Medicine. LR was partially supported by grants from the Compagnia san Paolo/Firms and the Italian Association for Cancer Research.

\section{Risk of bias}

\begin{tabular}{|c|c|c|}
\hline Bias & Authors' judgement & Support for judgement \\
\hline $\begin{array}{l}\text { PCS/RCS - selection ex- } \\
\text { posed cohort } \\
\text { All outcomes }\end{array}$ & Low risk & $\begin{array}{l}\text { The whole birth cohort of infants conceived between February } 2009 \text { and Janu- } \\
\text { ary } 2010 \text { in Stockholm, Sweden, whose mother received the influenza vaccine } \\
\text { during pregnancy }\end{array}$ \\
\hline $\begin{array}{l}\text { PCS/RCS - selection non- } \\
\text { exposed cohort } \\
\text { All outcomes }\end{array}$ & Low risk & $\begin{array}{l}\text { The whole birth cohort of infants conceived between February } 2009 \text { and Jan- } \\
\text { uary } 2010 \text { in Stockholm, Sweden, whose mother did not receive influenza vac- } \\
\text { cine during pregnancy }\end{array}$ \\
\hline $\begin{array}{l}\text { PCS/RCS - comparability } \\
\text { All outcomes }\end{array}$ & Unclear risk & All possible confounders have been taken into account. \\
\hline $\begin{array}{l}\text { PCS/RCS - assessment of } \\
\text { outcome } \\
\text { All outcomes }\end{array}$ & Low risk & Secure records \\
\hline Summary assessment & Unclear risk & Unclear risk of bias \\
\hline
\end{tabular}

pcb Nordin 2013

\begin{tabular}{ll}
\hline Methods & Retrospective cohort study based on data from Vaccine Safety Datalink \\
\hline Participants & $\begin{array}{l}\text { Pregnant women aged between } 14 \text { and } 49 \text { years }(\mathrm{n}=223,898) \text { identified in the Vaccine Safety Datalink, } \\
\text { who were pregnant between } 1 \text { June } 2002 \text { and } 31 \text { July } 2009\end{array}$ \\
\hline Interventions & Immunisation with inactivated trivalent influenza vaccine \\
\hline Outcomes & Demyelinating diseases, neurological events, thrombocytopenia within 42 days after immunisation \\
\hline Notes & Government funded \\
\hline
\end{tabular}

\section{Risk of bias}

\begin{tabular}{|c|c|c|}
\hline Bias & Authors' judgement & Support for judgement \\
\hline $\begin{array}{l}\text { PCS/RCS - selection ex- } \\
\text { posed cohort } \\
\text { All outcomes }\end{array}$ & Unclear risk & $\mathrm{KP}$ registry \\
\hline $\begin{array}{l}\text { PCS/RCS - selection non- } \\
\text { exposed cohort } \\
\text { All outcomes }\end{array}$ & Unclear risk & $\mathrm{KP}$ registry \\
\hline $\begin{array}{l}\text { PCS/RCS - comparability } \\
\text { All outcomes }\end{array}$ & Unclear risk & Matched analysis \\
\hline
\end{tabular}


pcb Nordin 2013 (Continued)
PCS/RCS - assessment of outcome
Unclear risk
$\mathrm{KP}$ registry
All outcomes

\begin{tabular}{lll}
\hline Summaryassessment $\quad$ Unclear risk & Unclear \\
\hline
\end{tabular}

pcb Nordin 2014

\begin{tabular}{ll}
\hline Methods & Retrospective cohort study. Data from 7 Vaccine Safety Datalink sites \\
\hline Participants & $\begin{array}{l}\text { In all, } 57,554 \text { women vaccinated and } 57,554 \text { matched women not vaccinated during pregnancy intro- } \\
\text { duced in data analysis. }\end{array}$
\end{tabular}

\begin{tabular}{ll}
\hline Interventions & $\begin{array}{l}\text { Seasonal trivalent inactivated vaccine. Epidemic seasons 2004-05, 2005-06, 2006-07, 2007-08, 2008-09 } \\
\text { were considered. }\end{array}$
\end{tabular}

\begin{tabular}{ll}
\hline Outcomes & Small for gestational age $(<10$ th or $>5$ th percentile $)$ \\
& Preterm delivery $(<37$ weeks or $<34$ weeks $)$ \\
\hline Notes & Founding source - government \\
& $\begin{array}{l}\text { Data and estimate are pooled for all seasons. This is part of the population of the pcb Nordin } 2013 \\
\text { study. }\end{array}$ \\
\hline
\end{tabular}

\section{Risk of bias}

\begin{tabular}{lll}
\hline Bias & Authors' judgement & Support for judgement \\
\hline $\begin{array}{l}\text { PCS/RCS - selection ex- } \\
\text { posed cohort } \\
\text { All outcomes }\end{array}$ & Unclear risk & KP registry \\
\hline $\begin{array}{l}\text { PCS/RCS - selection non- } \\
\text { exposed cohort }\end{array}$ & Unclear risk & KP registry \\
$\begin{array}{lll}\text { All outcomes } \\
\text { PCS/RCS - comparability }\end{array}$ & Unclear risk & Matched analysis \\
$\begin{array}{l}\text { All outcomes } \\
\text { PCS/RCS - assessment of }\end{array}$ & Unclear risk & KP registry \\
$\begin{array}{l}\text { outcome } \\
\text { All outcomes }\end{array}$ & \\
\hline $\begin{array}{l}\text { Summary assessment } \\
\text { Unclear risk }\end{array}$ & Unclear risk of bias \\
\hline
\end{tabular}

\section{pcb Omer 2011}

\begin{tabular}{ll}
\hline Methods & Retrospective cohort study based on data from the Georgia Pregnancy Risk Assessment Monitoring \\
& System (PRAMS) \\
\hline Participants & $\begin{array}{l}\text { In all, } 4168 \text { pregnant women were included during } 2 \text { consecutive epidemic seasons (2004 to } 2005 \text { and } \\
2005 \text { to } 2006 \text { ), of whom } 578 \text { received influenza vaccination. }\end{array}$ \\
\hline
\end{tabular}


pcb Omer 2011 (Continued)

Interventions Influenza vaccination during pregnancy

\begin{tabular}{ll}
\hline Outcomes & $\begin{array}{l}\text { Small for gestational age and preterm births. Periods with different viral circulation were considered in } \\
\text { the analysis. }\end{array}$ \\
\hline Notes & "The study was partially funded through the Emory University, Global Health Institute Faculty of Dis- \\
tinction Fund award (recipient: SBO). The funders had no role in study design, data collection and \\
analysis, decision to publish, or preparation of the manuscript" \\
Government funded
\end{tabular}

\section{Risk of bias}

\begin{tabular}{lll}
\hline Bias & Authors' judgement & Support for judgement \\
\hline $\begin{array}{l}\text { PCS/RCS - selection ex- } \\
\text { posed cohort } \\
\text { All outcomes }\end{array}$ & Unclear risk & Unclear \\
\hline $\begin{array}{l}\text { PCS/RCS - selection non- } \\
\text { exposed cohort } \\
\text { All outcomes }\end{array}$ & Unclear risk & Unclear \\
\hline $\begin{array}{l}\text { PCS/RCS - comparability } \\
\text { All outcomes }\end{array}$ & Unclear risk & Unclear \\
\hline $\begin{array}{l}\text { PCS/RCS - assessment of } \\
\text { outcome } \\
\text { All outcomes }\end{array}$ & High risk & Interview \\
\hline $\begin{array}{l}\text { Summary assessment } \\
\text { Unclear risk }\end{array}$ & \\
\hline
\end{tabular}

pcb Oppermann 2012

\begin{tabular}{|c|c|}
\hline Methods & $\begin{array}{l}\text { Prospective cohort study based on data from the Institute for Clinical Teratology and Drug Risk Assess- } \\
\text { ment in Pregnancy (D) carried out during the } 2009 \text { to } 2010 \text { pandemic }\end{array}$ \\
\hline Participants & $\begin{array}{l}\text { Pregnant women who received consultation regarding reproductive safety of medical products, } \\
\text { planned pregnancy, and lactation from the Institute for Clinical Teratology and Drug Risk Assessment. } \\
\text { Out of the initial population ( } n=16,788), 323 \text { participants received influenza vaccine and completed } \\
\text { the follow-up. A randomly selected group of } 1329 \text { non-vaccinated women formed the control group. }\end{array}$ \\
\hline Interventions & $\begin{array}{l}\text { - Non-adjuvanted split-virion vaccine CSL H1N1 Pandemic Influenza Vaccine (CSL Biotherapies) ap- } \\
\text { proved by the responsible national authority (Paul-Ehrlich-Institut) in November } 2009 \text { exclusively for } \\
\text { the vaccination of pregnant women (216/323). } \\
\text { - MF59-adjuvanted monovalent H1N1 vaccine (2/32). } \\
\text { - Pandemrix (GlaxoSmithKline) AS03-adjuvanted monovalent split-virion influenza vaccine }(90 / 323) \text {. } \\
\text { - Unknown vaccine (15/323). }\end{array}$ \\
\hline
\end{tabular}

\begin{tabular}{ll}
\hline Outcomes & Abortion, preterm birth, malformations \\
\hline Notes & $\begin{array}{l}\text { "This study was supported by the German Federal Institute for Vaccines and Biomedicines (Paul- } \\
\text { Ehrlich-Institut), Langen, Germany" }\end{array}$ \\
\hline
\end{tabular}

\section{Risk of bias}


pcb Oppermann 2012 (Continued)

\begin{tabular}{|c|c|c|}
\hline Bias & Authors' judgement & Support for judgement \\
\hline $\begin{array}{l}\text { PCS/RCS - selection ex- } \\
\text { posed cohort } \\
\text { All outcomes }\end{array}$ & Unclear risk & Unclear \\
\hline $\begin{array}{l}\text { PCS/RCS - selection non- } \\
\text { exposed cohort } \\
\text { All outcomes }\end{array}$ & Unclear risk & Unclear \\
\hline $\begin{array}{l}\text { PCS/RCS - comparability } \\
\text { All outcomes }\end{array}$ & Unclear risk & Unclear \\
\hline $\begin{array}{l}\text { PCS/RCS - assessment of } \\
\text { outcome } \\
\text { All outcomes }\end{array}$ & Low risk & Low \\
\hline Summary assessment & Unclear risk & Unclear \\
\hline
\end{tabular}

pcb Pasternak 2012

\begin{tabular}{ll} 
Methods & Retrospective cohort study assessing the safety of pandemic H1N1 vaccination \\
\hline Participants & $\begin{array}{l}\text { Danish women who were pregnant during the time interval between November } 2009 \text { and September } \\
2010(\mathrm{n}=58,585) . \text { Of these, } 7062 \text { received influenza vaccine. }\end{array}$ \\
\hline Interventions & $\begin{array}{l}\text { Monovalent, inactivated, AS03-adjuvanted split-virion influenza A (H1N1) pdm09 vaccine (Pandemrix, } \\
\text { Glaxosmithkline Biologicals) }\end{array}$ \\
\hline Outcomes & Abortion cases (retained or spontaneous) \\
\hline Notes & Government funded
\end{tabular}

\section{Risk of bias}

\begin{tabular}{|c|c|c|}
\hline Bias & Authors' judgement & Support for judgement \\
\hline $\begin{array}{l}\text { PCS/RCS - selection ex- } \\
\text { posed cohort } \\
\text { All outcomes }\end{array}$ & Low risk & \\
\hline $\begin{array}{l}\text { PCS/RCS - selection non- } \\
\text { exposed cohort } \\
\text { All outcomes }\end{array}$ & Low risk & \\
\hline $\begin{array}{l}\text { PCS/RCS - comparability } \\
\text { All outcomes }\end{array}$ & High risk & \\
\hline $\begin{array}{l}\text { PCS/RCS - assessment of } \\
\text { outcome } \\
\text { All outcomes }\end{array}$ & Low risk & \\
\hline Summary assessment & Unclear risk & Unclear \\
\hline
\end{tabular}


pcb Richards 2013

$\begin{array}{ll}\text { Methods } & \begin{array}{l}\text { Retrospective cohort study assessing the effect of pandemic H1N1 immunisation during pregnancy on } \\ \text { neonatal outcomes }\end{array}\end{array}$

Participants

Eligible pregnant women were identified by means of electronic medical records from Kaiser Permanente (KP) managed care organisation sites in Georgia and mid-Atlantic states. A total of 3327 thirdtrimester live births to 3236 mothers between 25 May 2009 and 17 April 2010 were included.

\begin{tabular}{ll}
\hline Interventions & Immunisation with H1N1 pandemic vaccine \\
\hline Outcomes & Preterm birth (27 to 36 weeks), low birth weight \\
\hline Notes & Government funded \\
\hline
\end{tabular}

\section{Risk of bias}

\begin{tabular}{lll}
\hline Bias & Authors' judgement & Support for judgement \\
\hline $\begin{array}{l}\text { PCS/RCS - selection ex- } \\
\text { posed cohort } \\
\text { All outcomes }\end{array}$ & Unclear risk & KP registry \\
\hline $\begin{array}{l}\text { PCS/RCS - selection non- } \\
\text { exposed cohort } \\
\text { All outcomes }\end{array}$ & Unclear risk & KP registry \\
\hline $\begin{array}{l}\text { PCS/RCS - comparability } \\
\text { All outcomes }\end{array}$ & High risk & \\
\hline $\begin{array}{l}\text { PCS/RCS - assessment of } \\
\text { outcome } \\
\text { All outcomes }\end{array}$ & Low risk & Possible residual confounding \\
\hline $\begin{array}{l}\text { Summary assessment } \\
\text { Unclear risk }\end{array}$ & Unclear \\
\hline
\end{tabular}

pcb Rubinstein 2013

\begin{tabular}{ll}
\hline Methods & Cohort study \\
\hline Participants & $\begin{array}{l}\text { Women with live-born or stillborn infants of at least } 22 \text { weeks or weighing at least } 500 \mathrm{~g} \text { between } \\
\text { September } 2010 \text { and May } 2011 \text { in } 49 \text { hospitals of the Public Health Sector in major Argentinian cities } \\
\text { were invited to participate in the study. }\end{array}$ \\
\hline
\end{tabular}

Interventions MF-59 adjuvanted pandemic H1N1 vaccine (Focetria). Information about exposure status and vaccination were obtained from documentation and official registry: vaccination chart, hospital vaccine registries, or centralised registry from the Ministry of Health if available.

The non-exposed group consisted of non-vaccinated pregnant women. In total, 7293 vaccinated and 23,195 not vaccinated pregnant women were included.

\section{Outcomes} After participants had signed informed consent form, data from the medical record regarding maternal
characteristics, evolution of the index pregnancy and delivery, and status of the newborn were extract-
ed. Participants then completed a brief survey to complement the information. Authors collected da-
ta on both mothers and newborns up to day 7 after delivery. For cases discharged before day 7 , partici-
pants were contacted by telephone at day 7 to check their health status. During the data collection pe-
riod, a supervisory team visited the participating hospitals weekly to collect and check completion of 
pcb Rubinstein 2013 (Continued)

the forms and to check the quality of the information by reviewing the birth record and clinical record. 2 field supervisors reviewed all forms before information was entered into the database. The following outcomes were considered:

- Preterm delivery (<37 weeks): defined as a newborn with a gestational age of less than 37 weeks according to physical examination at birth.

- Low birth weight $(<2500 \mathrm{~g})$ : newborn weighing less than $2500 \mathrm{~g}$.

- Very low birth weight $(<1500 \mathrm{~g})$ : newborn weighing less than $1500 \mathrm{~g}$.

- Congenital malformations: defined as a newborn presenting with alterations in anatomical development occurring during intrauterine life and diagnosed during gestation or by physical examination within the first 7 days after birth.

- Early neonatal mortality: defined as death of a newborn within 7 days after birth, foetal mortality as intrauterine death of the foetus in a pregnancy over 22 weeks, and perinatal mortality as early neonatal mortality plus foetal mortality.

- Low Apgar scores at 5 minutes: defined as a newborn presenting with an Apgar score of less than 7 at 5 minutes.

- Admission to neonatal intensive care: defined as admission of a newborn to the intensive care unit for a period longer than 48 hours.

Notes

Funding source - industry

This study was funded by an independent research grant from Novartis Argentina SA (Internal Protocol No V111_17TP. 2010). The investigators designed and conducted the study; performed the analysis and interpretation of the data; and are responsible for the results, conclusions, and recommendations.

Sensitivity analysis was performed by excluding those participants for whom vaccination records were not available.

\section{Risk of bias}

\begin{tabular}{lll}
\hline Bias & Authors' judgement & Support for judgement \\
\hline $\begin{array}{ll}\text { PCS/RCS - selection ex- } \\
\text { posed cohort }\end{array}$ & Low risk & $\begin{array}{l}\text { The study was performed in } 49 \text { public hospitals where about } 113,000 \text { deliveries } \\
\text { occur annually (about } 15 \% \text { of overall annual live births in Argentina). This is a } \\
\text { sll outcomes }\end{array}$ \\
\end{tabular}

PCS/RCS - selection non- Low risk Drawn from the same population as the exposed cohort exposed cohort

All outcomes

PCS/RCS - comparability Low risk Possible confounding factors have been taken into account.
All outcomes

PCS/RCS - assessment of Low risk Hospital medical records
outcome
All outcomes

Summary assessment Low risk Low risk of bias

pcb Sheffield 2012

$\begin{array}{ll}\text { Methods } & \text { Retrospective cohort study assessing the safety of seasonal influenza vaccination administered during } \\ \text { pregnancy, covering } 5 \text { subsequent epidemic seasons (from } 2003 \text { to } 2004 \text { to } 2007 \text { to } 2008 \text { ) }\end{array}$


pcb Sheffield 2012 (Continued)
Participants
Women who delivered and received prenatal care at the Southwestern Medical Center of University of Texas and Parkland Health \& Hospital System, Dallas, Texas. In all, 8690 were vaccinated and 76,153 acted as unvaccinated controls.

\begin{tabular}{ll}
\hline Interventions & $\begin{array}{l}\text { Seasonal influenza vaccination was offered to pregnant women from October through March in each } \\
\text { season. }\end{array}$ \\
\hline Outcomes & Estimated gestational age \\
- Birth weight \\
- Major malformations* \\
- Stillbirth* \\
- NeO admission* \\
- Neonatal pneumonia* \\
- Hyperbilirubinaemia \\
*For these outcomes the authors provided effect estimates considering the trimester of administration. \\
This study should have been performed without external/private/industry funding. \\
Government funded
\end{tabular}

\section{Risk of bias}

\begin{tabular}{|c|c|c|}
\hline Bias & Authors' judgement & Support for judgement \\
\hline $\begin{array}{l}\text { PCS/RCS - selection ex- } \\
\text { posed cohort } \\
\text { All outcomes }\end{array}$ & Unclear risk & Unclear \\
\hline $\begin{array}{l}\text { PCS/RCS - selection non- } \\
\text { exposed cohort } \\
\text { All outcomes }\end{array}$ & Unclear risk & Unclear \\
\hline $\begin{array}{l}\text { PCS/RCS - comparability } \\
\text { All outcomes }\end{array}$ & High risk & \\
\hline $\begin{array}{l}\text { PCS/RCS - assessment of } \\
\text { outcome } \\
\text { All outcomes }\end{array}$ & Unclear risk & Unclear \\
\hline Summary assessment & High risk & \\
\hline
\end{tabular}

pcb Toback 2012

$\begin{array}{ll}\text { Methods } & \begin{array}{l}\text { Retrospective cohort study testing the safety of live attenuated influenza vaccine when administered } \\ \text { during pregnancy }\end{array}\end{array}$

Pregnant women $(n=834,999)$ identified by means of a safety database (LifeLink Health Plan Claims
Database, Norwalk, USA) between October 2003 and September 2009. Of these, 138 received immuni-
sation with live attenuated influenza vaccine during their pregnancy.
sation with live attenuated influenza vaccine during their pregnancy.

\begin{tabular}{ll}
\hline Interventions & Live attenuated influenza vaccine \\
\hline Outcomes & Hospitalisation and emergency department visits within 42 days after immunisation \\
\hline
\end{tabular}


pcb Toback 2012 (Continued)

Notes
"This research was funded by MedImmune, LLC, Gaithersburg, MD. As part of a consulting agreement with RTI Health Solutions, MedImmune provided funding to support protocol development, data collection, analysis, and manuscript development activities associated with this manuscript. Editorial assistance in formatting the manuscript for submission was provided by Sue Myers, MSc, and Gerard P. Johnson, PhD, of Complete Healthcare Communications, Inc. (Chadds Ford, PA) and was funded by MedImmune, LLC"

\section{Risk of bias}

\begin{tabular}{lll}
\hline Bias & Authors' judgement & Support for judgement \\
\hline $\begin{array}{l}\text { PCS/RCS - selection ex- } \\
\text { posed cohort } \\
\text { All outcomes }\end{array}$ & Unclear risk & Unclear \\
\hline $\begin{array}{l}\text { PCS/RCS - selection non- } \\
\text { exposed cohort }\end{array}$ & Unclear risk & Unclear \\
All outcomes & & \\
\hline
\end{tabular}

\section{PCS/RCS - comparability High risk}

All outcomes

\begin{tabular}{l}
\hline PCS/RCS - assessment of Unclear risk Unclear \\
outcome \\
All outcomes
\end{tabular}

Summary assessment High risk

pcb Trotta 2014

\begin{tabular}{ll}
\hline Methods & Retrospective cohort study \\
\hline Participants & $\begin{array}{l}\text { Women residing in the Lombardy region, aged at least } 12 \text { and up to } 55 \text { years }(\mathrm{n}=86,171) \text {, whose deliv- } \\
\text { ery took place between } 23 \text { and } 45 \text { weeks of gestation between } 1 \text { October } 2009 \text { and } 30 \text { September } 2010, \\
\text { in public or private institutions as well as at home, identified through the regional birth registry (still- } \\
\text { births were included if the gestational age exceeded } 180 \text { days) }\end{array}$ \\
\hline
\end{tabular}

Interventions MF-59 adjuvanted pandemic H1N1 vaccine. 6426 women received the vaccine during pregnancy.

\section{Outcomes Pregnancy complication}

- pre-eclampsia/eclampsia, gestational diabetes

- in-hospital maternal death (deaths during labour or delivery occurring in a healthcare institution)

- admission to intensive care unit

- type of delivery

\section{Perinatal death}

- stillbirth (delivery of a dead foetus after 180 days of amenorrhoea)

- in-hospital neonatal death

\section{Neonatal outcomes}

- small for gestational age neonates ( $<10$ th centile)

- admission to NICU

- neonatal reanimation 
pcb Trotta 2014 (Continued)

- composite outcome: presence of any of the following: clinical information/diagnoses: very low 5minute Apgar score $(\leq 3)$, acute respiratory distress syndrome, asphyxia, intraventricular haemorrhage, and acute necrotising enterocolitis

- congenital malformations including: nervous system, eye, ear, face, and neck, congenital heart defects, respiratory, orofacial clefts, digestive system, abdominal wall defects, urinary, genital, limb, others. Diagnosis with a code compatible to to ICD-9 in either the medical birth registry or the hospital discharge, according to EUROCAT guideline
Funding source - government

Funding: Only public employees of the national or regional health authorities were involved in conceiving, planning, and conducting the study; no additional funding was received.

\section{Risk of bias}

\begin{tabular}{|c|c|c|}
\hline Bias & Authors' judgement & Support for judgement \\
\hline $\begin{array}{l}\text { PCS/RCS - selection ex- } \\
\text { posed cohort } \\
\text { All outcomes }\end{array}$ & Low risk & $\begin{array}{l}\text { All mothers with singleton pregnancy between } 1 \text { October } 2009 \text { and } 30 \text { Septem- } \\
\text { ber } 2010 \text { in the Italian region of Lombardia }\end{array}$ \\
\hline $\begin{array}{l}\text { PCS/RCS - selection non- } \\
\text { exposed cohort } \\
\text { All outcomes }\end{array}$ & Low risk & From the same population as the exposed cohort \\
\hline $\begin{array}{l}\text { PCS/RCS - comparability } \\
\text { All outcomes }\end{array}$ & Low risk & Possible confounders have been taken into account. \\
\hline $\begin{array}{l}\text { PCS/RCS - assessment of } \\
\text { outcome } \\
\text { All outcomes }\end{array}$ & Low risk & Hospital records \\
\hline Summary assessment & Low risk & Low risk of bias \\
\hline
\end{tabular}

$\mathrm{AE}=$ adverse event

ARI = acute respiratory illness

ATP $=$ according to protocol

$\mathrm{CCA}=$ chicken erythrocyte agglutination

$\mathrm{CCl}=$ culture-confirmed influenza illness

CCIV = cell culture-derived inactivated flu vaccine

$C D C=$ Centers for Disease Control and Prevention

$\mathrm{Cl}=$ confidence interval

DFA $=$ direct fluorescent antibody

ECG = electrocardiogram

$\mathrm{FEF}=$ forced expiratory flow

$F E V 1=$ forced expiratory volume in one second

FVC $=$ forced vital capacity

$\mathrm{GBS}=$ Guillain-Barré syndrome

GMT = geometrical mean titre

$\mathrm{GP}=$ general practitioner

GSK = GlaxoSmithKline

$\mathrm{HA}=$ haemagglutinin

$\mathrm{HAO}=$ full-length uncleaved haemagglutinin

$\mathrm{HI}=$ haemagglutination inhibition

$\mathrm{HMO}=$ health maintenance organisation

$I C D=$ International Classification of Diseases

$\lg \mathrm{A}=$ immunoglobulin $\mathrm{A}$

ILI = influenza-like illness

$I T I=$ intention-to-immunise 
ITT = intention-to-treat

$\mathrm{IM}=$ intramuscular

IN = intranasal

$\mathrm{IU}=$ international units

$\mathrm{KP}=$ Kaiser Permanente

$\mathrm{KSC}=$ Kelsey-Seybold Clinic

LAIV = live attenuated influenza vaccine

$\mathrm{LCl}=$ laboratory-confirmed influenza

$\mathrm{LMP}=$ last menstrual period

$M A E=$ medical attended event

$\mathrm{MCO}=$ managed care organisation

MDCK = Madin-Darby canine kidney cells

$\mathrm{MS}=$ multiple sclerosis

NCKPHP $=$ Northern California Kaiser Permanente Health Plan

$\mathrm{NICU}=$ neonatal intensive care unit

$\mathrm{OMP}=$ outer membrane protein

$\mathrm{OR}=$ odds ratio

ORS = oculo-respiratory syndrome

$\mathrm{PCA}=$ primary cardiac arrest

$\mathrm{PCR}=$ polymerase chain reaction

PCS/RCS = prospective/retrospective cohort study

pfu $=$ plaque-forming units

$\mathrm{PP}=$ per-protocol

$\mathrm{RCT}=$ randomised controlled trial

$\mathrm{rHAO}=$ recombinant uncleaved haemagglutinin glycoprotein

$\mathrm{RhMK}=$ rhesus macaque kidney cells

RT-PCR = reverse transcription polymerase chain reaction

$\mathrm{SAE}=$ serious adverse event

SAS = statistical analysis systems

TIV = trivalent inactivated vaccine

URTI = upper respiratory tract infection

$\mathrm{VMCCl}=$ vaccine-matched, culture-confirmed influenza

WDL $=$ working days lost

WHO $=$ World Health Organization

WRL = Wellcome Research Laboratories (Beckenham, Kent)

Characteristics of excluded studies [ordered by study ID]

Study Reason for exclusion

ab Wacheck 2010

Experimental vaccine; dose escalation study

ab López-Macías 2011a

Experimental vaccine; no outcomes of interest

ab López-Macías 2011b

Experimental vaccine; no outcomes of interest

\begin{tabular}{ll}
\hline ab Mallory 2010 & No outcomes of interest \\
\hline ab Plennevaux 2010 & No outcomes of interest \\
\hline ab Precioso 2011 & No outcomes of interest \\
\hline ab Treanor 2010 & Experimental vaccine \\
\hline ab Turley 2011 & Experimental vaccine; no outcomes of interest \\
\hline Al-Dabbagh 2013 & $\begin{array}{l}\text { No outcomes of interest, differences in cytokine levels between ORS cases and controls after vacci- } \\
\text { nation }\end{array}$ \\
\hline
\end{tabular}




\begin{tabular}{|c|c|}
\hline Study & Reason for exclusion \\
\hline Ambrosch 1976 & Data tables and figure missing \\
\hline Ambrose 2012 & No original data \\
\hline Andersson 2015 & Comment on cb Persson 2014 study \\
\hline Aoki 1986 & $\begin{array}{l}\text { Randomised controlled trial, single-blind. Outcomes were clinical cases and adverse effects. Fol- } \\
\text { low-up data were not reported by arm. }\end{array}$ \\
\hline Arnou 2010 & $\begin{array}{l}\text { Intradermal administration ( } 3 \text { different lots of the same vaccine) versus intramuscular administra- } \\
\text { tion. Serologic response and AE at day } 21 . \text { No adequate placebo/no intervention control }\end{array}$ \\
\hline Atmar 1995 & No outcomes of interest \\
\hline Atmar 2011 & Absence of an adequate control \\
\hline Atsmon 2012 & Experimental vaccine; no outcomes of interest \\
\hline Ausseil 1999 & $\begin{array}{l}\text { No design (average days of sick leave in vaccinated and non-vaccinated participants during } 1996 \\
\text { and } 1997 \text { from staff of an international banking institution) }\end{array}$ \\
\hline Banzhoff 2001 & No design (cohort), no safety outcomes \\
\hline Baxter 2010 & No design: cohort study for effectiveness \\
\hline Baxter 2011 & $\begin{array}{l}\text { A 'head-to-head' trial: "FluBlok (purified HA proteins manufactured in expresSF+ }{ }^{\circledR} \text { insect cells un- } \\
\text { der serum free conditions using a baculovirus expression system (BEVS). Uncleaved HA produced } \\
\text { by this method is referred to as rHA0. Vaccine formulation consisted of } 135 \mathrm{~g} \text { total HA protein ( } 45 \mathrm{~g} \\
\text { each) as determined by single radial immunodiffusion assay (SRID) and included rHA0 derived from } \\
\text { the following influenza strains A/Solomon Islands/03/2006 (H1N1), A/Wisconsin/67/2005 (H3N2), } \\
\text { and B/Malaysia/2506/2004 VS. The same CDC-derived vaccine seed viruses were used for the li- } \\
\text { censed trivalent inactivated vaccine (TIV; Fluzone [2007-2008 formulation; Sanofi Pasteur, Swift- } \\
\text { water, PA), which contained } 15 \mathrm{~g} \text { of each HA [45g total])" }\end{array}$ \\
\hline Baxter 2012 & No design: controlled case series \\
\hline Baxter 2013 & Self controlled time series study \\
\hline Belongia 2009 & Case-control study, no harm assessment \\
\hline Belshe 2001 & No original data \\
\hline Benke 2004 & Questionnaire survey; non-comparative analysis \\
\hline Beran 2013 & $\begin{array}{l}\text { Absence of an adequate control group (quadrivalent versus trivalent inactivated vaccine; low ver- } \\
\text { sus normal adjuvant content) }\end{array}$ \\
\hline Betts $1977 b$ & Trial with swine vaccine (Hsw1N1, A/New Jersey/76) \\
\hline Beyer 1996 & Review \\
\hline Carlson 1979 & No adequate control, no outcome of interest \\
\hline Cate 1977 & Trial with swine vaccine (Hsw1N1, A/New Jersey/76) \\
\hline Chavant 2013 & Absence of a control group; study population consists of vaccinated pregnant women only \\
\hline
\end{tabular}




\begin{tabular}{|c|c|}
\hline Study & Reason for exclusion \\
\hline Chichester 2012 & Experimental vaccine; no outcomes of interest \\
\hline Chlibek 2002 & Not a randomised controlled trial \\
\hline Choe 2011a & No design: cross-sectional study \\
\hline Choe 2011b & No design: case series \\
\hline Choe 2011c & No design: case series \\
\hline Chou 2007 & Case report \\
\hline Clover 1991 & $\begin{array}{l}\text { Randomised controlled trial. More than } 75 \% \text { of the study population was out of the age range stat- } \\
\text { ed in the protocol. }\end{array}$ \\
\hline Confavreux 2001 & Participants are MS cases. \\
\hline Conlin 2013 & $\begin{array}{l}\text { Inadequate comparison and study design: cohort study with pandemic versus seasonal (not ex- } \\
\text { posed) vaccines in women and newborns }\end{array}$ \\
\hline Couch 2012 & Experimental vaccine; no outcomes of interest \\
\hline Das Gupta 2002 & Does not contain effectiveness data \\
\hline Davidson 2011 & $\begin{array}{l}\text { Inadequate comparison: all enrolled participants received LAIV, then were randomised to either } \\
\text { placebo or Lactobacillus rhamnosus GG }\end{array}$ \\
\hline Davies 1972 & Cohort with efficacy outcomes. Experimental and control group were selected separately. \\
\hline Davies 1973 & $\begin{array}{l}\text { Not randomised. Participants volunteered for immunisation, and comparison was made with a } \\
\text { randomly selected non-immunised control group. }\end{array}$ \\
\hline De Serres 2003a & No comparison, absence of adequate control group \\
\hline De Serres 2003b & No control \\
\hline De Serres 2004 & Population at risk of further ORS episodes. \\
\hline De Wals 2012 & No design: self controlled case series for association between H1N1 and GBS \\
\hline Dolin 1977 & Trial with swine vaccine (Hsw1N1, A/New Jersey/76) \\
\hline Dominguez 2012 & No design: case-control study assessing effectiveness in general population \\
\hline Duffy 2014 & Case-centred study \\
\hline Eames 2012 & No design: effectiveness cohort study in general population \\
\hline Edmonson 1970 & Influenza B vaccine was used as control. \\
\hline Eick-Cost 2012 & No design: case-control study assessing effectiveness in general population \\
\hline El'shina 1998 & Major inconsistencies in the study text \\
\hline Englund 1993 & Inadequate comparison (tetanus toxoid vaccine) \\
\hline
\end{tabular}




\section{Study}

Finklea 1969

\section{Reason for exclusion}

Randomised controlled trial, double-blind. 2 bivalent inactivated influenza vaccines with the same viral composition, differing in purification procedures, were compared.

Outcomes were clinical cases and adverse effects.

Raw data about clinical cases were not reported by arm.

Circulating virus showed significant antigenic differences from the $\mathrm{A} 2$ vaccine strain.

\begin{tabular}{ll}
\hline Fisher 2012 & No outcomes of interest (antibody titres only) \\
\hline Foy 1981 & Absence of adequate control \\
\hline Frank 1981 & No usable safety data (scores) \\
\hline Freestone 1976 & Conference proceedings \\
\hline Gerstoft 2001 & Not a randomised controlled trial \\
\hline Greenbaum 2002 & No outcome of interest \\
\hline Greene 2013 & Case-centred study \\
\hline
\end{tabular}

Gross $1999 \quad$ Outcome measures outside inclusion criteria.

Grotto $1998 \quad$ Not a randomised controlled trial

Randomised controlled trial conducted in the USA on 41 cystic fibrosis (CF) patients and 89 family members, recruited through a clinic. Participants were randomly assigned in a double-blinded fashion by family to receive either intranasal, live, cold-adapted influenza A vaccine or the recommended intramuscular trivalent inactivated influenza vaccine.

The study lasted 3 years (from 1989 to 1991). Participants were immunised each fall, staying in the same assigned vaccine group. The live vaccine arm counted $20 \mathrm{CF}$ and 33 family members; the trivalent vaccine arm 21 and 56, respectively.

69 participants (17 CF patients and 52 family members) dropped out. The reasons were stated in the article.

The live vaccine was the same throughout the period: A/Kawasaki/9/86 (H1N1) 107.3 pfu, A/Los Angeles/2/87 107.3 pfu.

The viral strains used in the inactivated vaccines were:

- 1989 to 1990: A/Taiwan/1/86 (H1N1), A/Shanghai/11/87 (H3N2), B/Yagamata/16/88, 15 mg/dose of each

- 1990 to 1991: A/Taiwan/1/86 (H1N1), A/Shanghai/16/89 (H3N2), B/Yagamata/16/88, 15 mg/dose of each

- 1991 to 1992: A/Taiwan/1/86 (H1N1), A/Beijing/353/89 (H3N2), B/Panama/45/90, 15 mg/dose of each

Live vaccine recipients also received monovalent inactivated influenza $B$ vaccine (identical to that contained in the trivalent vaccine) as an intramuscular placebo. Allantoic fluid was the placebo for aerosol administration.

Data were extracted and loaded for family members only.

Outcomes were clinical and laboratory-confirmed cases, working days lost, admissions, deaths, and adverse effects.

Clinical cases were classified as "respiratory illness" or "febrile respiratory illness". Laboratory-confirmed cases were defined by an influenza virus isolation from a throat swab. 


Study Reason for exclusion

Adverse effects were defined as temperature $>38^{\circ} \mathrm{C}$, rhinorrhoea, sore throat, cough, increasing sputum, redness, swelling, chills. Results are expressed as $\%$ of participant-days with symptoms.

Participants were followed throughout the period. Owing to the dropouts, the vaccinated were counted as participant-years: 54 in the live vaccine arm; 56 in the trivalent vaccine arm.

The influenza illness surveillance period for study participants was defined as the interval from the date of the first influenza isolate from the population under routine surveillance to 2 weeks after the last isolate for each year.

Viral strains circulating during the outbreaks were:

- 1989 to 1990: A/Shanghai/11/87 (H3N2)

- 1990 to 1991: A/Beijing/353/89 (H3N2), B/Panama/45/90-like

- 1991 to 1992: A/Beijing/353/89 (H3N2)

We excluded this trial because it was not placebo controlled, and the authors did not specify if the strains used to develop cold-adapted and inactivated vaccines were antigenically comparable or not.

\begin{tabular}{ll}
\hline Gwini 2011 & No design: self controlled case series \\
\hline Haber 2004 & $\begin{array}{l}\text { Analysis of temporal trends of GBS } 1990 \text { to 2003, comparison with temporal trends of non-GBS ad } \\
\text { verse event reports from the Vaccine Adverse Event Reporting System (VAERS) }\end{array}$ \\
\hline Haigh 1973 & $\begin{array}{l}\text { Not randomised: all the volunteers were immunised on a single day, and the intention to allocate } \\
\text { participants randomly was not strictly adhered to }\end{array}$
\end{tabular}

\begin{tabular}{|c|c|}
\hline Halperin 2002 & Outcome measures outside inclusion criteria. \\
\hline Hambidge 2011 & Participants affected by sickle cell crisis. \\
\hline Heinonen 1973 & Control consists of another vaccine. \\
\hline Hellenbrand 2012 & No design: case-control study assessing effectiveness in general population \\
\hline Hobson 1970 & Polyvalent influenza vaccine was used as control. \\
\hline Hobson 1973 & Randomised controlled trial. Clinical outcomes were side effects only. \\
\hline Hoskins 1973 & Influenza B vaccine was used as control. \\
\hline Hoskins 1976 & Not placebo or 'do nothing' controlled \\
\hline Hoskins 1979 & No control group \\
\hline Howell 1967 & Not prospective: appears to be an historical cohort \\
\hline Huang 2011 & Comparison is not adequate (vaccine versus vaccine). \\
\hline Hurwitz 1983 & Report of GBS surveillance 1978 to 1979 , non-comparative study \\
\hline Jackson 2011 & $\begin{array}{l}\text { No adequate control (the same vaccine prepared with different antigenic concentrations was ad- } \\
\text { ministered to each group) }\end{array}$ \\
\hline Janjua 2012 & No design: case-control study assessing effectiveness in general population \\
\hline
\end{tabular}




\begin{tabular}{|c|c|}
\hline Study & Reason for exclusion \\
\hline Jianping 1999 & Not a randomised controlled trial \\
\hline Jimenez-Jorge 2012 & No design: case-control study assessing effectiveness in general population \\
\hline Keitel 2001 & $\begin{array}{l}\text { Efficacy outcome measures outside inclusion criteria. The safety data are presented in a non- } \\
\text { analysable way. }\end{array}$ \\
\hline Kelly 2012 & No design: case-control study assessing effectiveness in general population \\
\hline Khazeni 2009 & Review and cost-effectiveness analysis \\
\hline Kiderman 2001 & Tables and text show inconsistencies that do not allow data extraction. \\
\hline Kim 2012 & Surveillance for adverse events \\
\hline Kissling 2012 & No design: case-control study assessing effectiveness in general population \\
\hline Kunz 1977 & No adequate control \\
\hline Langley 2004 & Review \\
\hline Lavallee 2014 & Review about stroke and vaccination in elderly people \\
\hline Lee 2011 & No design: self controlled case series \\
\hline Leeb 2011 & No design: case series \\
\hline Leroux-Roels 2010a & Absence of an adequate control, serological outcomes only \\
\hline Leroux-Roels 2010b & Absence of an adequate control, serological outcomes only \\
\hline Liem 1973 & $\begin{array}{l}\text { Reported the results of } 9 \text { placebo-controlled clinical trials and } 2 \text { field studies, involving a total of } \\
\text { about 10,000 participants, carried out in several countries to assess the efficacy of killed influenza } \\
\text { spray vaccines. Studies were conducted during the years } 1969 \text { to } 1971 \text {. } \\
\text { Allocation of the participants to the arms of the trials was done according to a predetermined ran- } \\
\text { domisation scheme. } 8 \text { of the studies were double-blind. The field studies were not randomised. The } \\
\text { attack rate for influenza among the population study was very low, and in } 2 \text { of the trials the vacci- } \\
\text { nation procedure started too late, when the outbreak was ongoing. The attack rates, based exclu- } \\
\text { sively on the serologically confirmed cases, are only reported by a graph and deriving the crude da- } \\
\text { ta is impossible. }\end{array}$ \\
\hline
\end{tabular}

\begin{tabular}{ll}
\hline Lind 2014 & Surrogate exposure assessment (antibody level) \\
\hline Liu 2012 & Study to identify variables associated with uptake of influenza vaccination during pregnancy \\
\hline Louik 2013 & Methods for assessing flu vaccine exposure during pregnancy \\
\hline Mackenzie 1975 & No design: allocation is arbitrary, and groups with different characteristics were formed \\
\hline Mackenzie 2012 & Non-comparative design \\
\hline Mair 1974 & Influenza B vaccine was used as control. \\
\hline Maynard 1968 & Influenza B vaccine was used as control. \\
\hline
\end{tabular}




\begin{tabular}{ll}
\hline Study & Reason for exclusion \\
\hline McCarthy 2004 & Review \\
\hline Mendelman 2001 & Does not report original results \\
\hline Merelli 2000 & Review \\
\hline Meyers 2003a & Review \\
\hline Meyers 2003b & Review \\
\hline Micheletti 2011 & Total number of AEs observed after administration of each vaccine type \\
\hline Monto 2000 & Not a randomised controlled trial \\
\hline Montplaisir 2014 & Study population outside age range. \\
\hline Moro 2011 & Non-comparative study \\
\hline Morris 1975 & $\begin{array}{l}\text { Design is unclear: no standard random allocation. Only 25 out of 30 participants seem to have been } \\
\text { immunised, but in the method description } 30 \text { were considered for exposure to natural influenza A/ } \\
\text { Scotland/840/74. 1 of these was excluded prior due to tonsillitis. }\end{array}$ \\
\hline
\end{tabular}

\begin{tabular}{|c|c|}
\hline Mostow 1977 & Outcomes were safety only. Absence of adequate control \\
\hline Muennig 2001 & Not a randomised controlled trial \\
\hline Murray 1979 & Not adequate comparison (pregnant versus non-pregnant women) \\
\hline Nazareth 2013 & Absence of control group, non-comparative \\
\hline Nichol 1996 & Same data as Nichol 1995 (included) \\
\hline Nichol 1999b & Review \\
\hline Nichol 2001 & Not a randomised controlled trial \\
\hline Nichol 2003 & Contains data from previous studies \\
\hline Nichol 2004 & Re-analysis of Nichol 1999 (included) \\
\hline Omon 2011 & Non-comparative study \\
\hline Petrie 2011 & $\begin{array}{l}\text { No new data: reports data from already published and included studies (aa Ohmit 2006, aa Ohmit } \\
\text { 2008, aa Monto 2009) }\end{array}$ \\
\hline Phillips 2013 & Absence of adequate control group \\
\hline Phonrat 2013 & No outcomes of interest \\
\hline Pleguezuelos 2012 & Experimental vaccine; no outcomes of interest \\
\hline Puig-Barbera 2012 & $\begin{array}{l}\text { No design: case-control study assessing effectiveness in general population (also children and el- } \\
\text { derly) }\end{array}$ \\
\hline Puleston 2010 & Not outcomes of interest \\
\hline
\end{tabular}




\begin{tabular}{|c|c|}
\hline Study & Reason for exclusion \\
\hline Pyhala 2001 & Not a randomised controlled trial \\
\hline Reynales 2012 & Safety survey after Celtura (H1N1) administration. Absence of control group \\
\hline Rimmelzwaan 2000 & Outcome measures outside inclusion criteria. \\
\hline Rocchi 1979 c & Very poor reporting, unclear definition, no description of methods \\
\hline Rowhani-Rahbar 2012 & Participants are children \\
\hline Ruben 1972 & Absence of adequate control \\
\hline Ruben 1973 & Both arms contained the same vaccine strains. \\
\hline Safranek 1991 & Reassessment of Schonberger 1979 (included) \\
\hline Sarateanu 1980 & Absence of adequate control \\
\hline Scheifele 2013 & No outcomes of interest \\
\hline Schonberger 1981 & Review of the evidence of the aetiology of GBS, no original data presented \\
\hline Schwartz 1996 & Report about Nichol 1995 (included) \\
\hline Simpson 2012 & No design: cohort and case-control study assessing effectiveness in general population \\
\hline Sipilä 2015 & Ecological study \\
\hline Skowronski 2002 & Non-comparative (survey) \\
\hline Skowronski 2003 & Population at risk of further ORS episodes \\
\hline Smith $1977 a$ & $\begin{array}{l}\text { Reports a small part of the Hoskins trial. It compared illness occurring among a group of vaccinated } \\
\text { boys against non-vaccinated controls that had no part in the trial. }\end{array}$ \\
\hline Smith 1977b & Trial with swine vaccine (Hsw1N1, A/New Jersey/76) \\
\hline Song 2011 & $\begin{array}{l}1 \text { trial is a 'head-to-head' trial (Gc501 versus Fluarix) with serological outcomes only; the other trial } \\
\text { (safety) has no control. }\end{array}$ \\
\hline Souayah 2011 & $\begin{array}{l}\text { Compares the incidence of GBS cases after tetravalent human papillomavirus vaccine with that ob- } \\
\text { served after pneumococcal and flu vaccine administration }\end{array}$ \\
\hline Spencer 1975 & Authors did not report crude data on the clinical outcomes. \\
\hline Spencer 1979 & $\begin{array}{l}\text { Reporting does not make clear the methods used to allocate participants and to conceal alloca- } \\
\text { tion. Clinical outcome data are not reported. }\end{array}$ \\
\hline Steinhoff 2012 & $\begin{array}{l}\text { Inadequate control (23v pneumococcal vaccine administered to the control group). Re-analysis of } \\
\text { Zaman } 2008 \text { data (excluded) }\end{array}$ \\
\hline Sumaya 1979 & No outcomes of interest \\
\hline Talaat 2010 & Data on AEs are not provided in a useful form (bar graphs or cumulatively in the text). \\
\hline
\end{tabular}




\begin{tabular}{|c|c|}
\hline Study & Reason for exclusion \\
\hline Tavares 2011 & Non-comparative \\
\hline Taylor 1969 & No outcomes of interest, rhinovirus vaccine as control \\
\hline Taylor 2012 & Experimental vaccine; no outcomes of interest \\
\hline Thompson 2014 & Test-positive case-control study \\
\hline Tokars 2012 & No design: controlled case series \\
\hline Treanor 2001 & Outcome measures outside inclusion criteria. \\
\hline Treanor 2002 & Outcome measures outside inclusion criteria. \\
\hline Treanor 2012 & No design: case-control study \\
\hline Tsai 2010 & Non-comparative \\
\hline Tsatsaris 2011 & Same vaccine administered in different pregnancy weeks (inadequate comparison). \\
\hline Tyrrell 1970 & $\begin{array}{l}\text { We were unable to include the } 3 \text { studies reported in this paper for the following reasons. } \\
\text { 1. No design, no comparison, no outcomes. } \\
\text { 2. Probable controlled clinical trial, but participants' ages likely out of range (schools). } \\
\text { 3. No design, even if an unvaccinated control group for school } 3 \text { and for the employees of the Impe- } \\
\text { rial Chemical Industries is present. }\end{array}$ \\
\hline Vesikari 2012 & Safety data after dose I (seasonal versus placebo) are not extracted (bar graph). \\
\hline Warren-Gash 2013 & Outside target age; all participants were older than 60 years \\
\hline Warshauer 1976 & Not randomised. Data reporting was not complete. \\
\hline Wilde 1999 & Pneumococcal vaccine was used as control. \\
\hline Williams 1973 & No placebo or 'do nothing' control \\
\hline Williams 2011 & No design: case series \\
\hline Wise 2012 & No design \\
\hline Wood 1999 & Not a randomised controlled trial \\
\hline Wood 2000 & Not a randomised controlled trial \\
\hline Xu 2012 & No original data presented \\
\hline Yang 2012 & No safety data \\
\hline Yeager 1999 & Non-comparative study: absence of a control arm \\
\hline Yih 2012 & No design: controlled case series \\
\hline Zaman 2008 & Inadequate control ( $23 \mathrm{v}$ pneumococcal vaccine administered to the control group) \\
\hline
\end{tabular}


$\mathrm{AE}=$ adverse event

GBS = Guillain-Barré syndrome

LAIV = live attenuated influenza vaccine

$\mathrm{MS}=$ multiple sclerosis

ORS = oculo-respiratory syndrome

pfu = plaque-forming units

DATA AND ANALYSES

Comparison 1. Inactivated parenteral influenza vaccine versus placebo or 'do nothing'

\begin{tabular}{|c|c|c|c|c|}
\hline Outcome or subgroup title & No. of studies & $\begin{array}{l}\text { No. of partici- } \\
\text { pants }\end{array}$ & Statistical method & Effect size \\
\hline 1 Influenza & 25 & 71221 & $\begin{array}{l}\text { Risk Ratio (M-H, Random, 95\% } \\
\mathrm{Cl} \text { ) }\end{array}$ & $0.41[0.36,0.47]$ \\
\hline $\begin{array}{l}1.1 \text { WHO recommended - matching } \\
\text { vaccine }\end{array}$ & 15 & 46444 & $\begin{array}{l}\text { Risk Ratio (M-H, Random, 95\% } \\
\mathrm{Cl})\end{array}$ & $0.41[0.34,0.49]$ \\
\hline $\begin{array}{l}1.2 \text { WHO recommended - vaccine } \\
\text { matching absent or unknown }\end{array}$ & 7 & 15068 & $\begin{array}{l}\text { Risk Ratio (M-H, Random, 95\% } \\
\mathrm{Cl})\end{array}$ & $0.45[0.34,0.59]$ \\
\hline $\begin{array}{l}1.3 \text { Monovalent not WHO recom- } \\
\text { mended - vaccine matching }\end{array}$ & 2 & 9675 & $\begin{array}{l}\text { Risk Ratio (M-H, Random, 95\% } \\
\mathrm{Cl})\end{array}$ & $0.22[0.10,0.52]$ \\
\hline $\begin{array}{l}\text { 1.4 Monovalent not WHO recom- } \\
\text { mended - vaccine matching - high } \\
\text { dose }\end{array}$ & 1 & 34 & $\begin{array}{l}\text { Risk Ratio (M-H, Random, 95\% } \\
\mathrm{Cl})\end{array}$ & $0.11[0.00,2.49]$ \\
\hline 2 Influenza-like illness & 16 & 25795 & $\begin{array}{l}\text { Risk Ratio (M-H, Random, 95\% } \\
\mathrm{Cl})\end{array}$ & $0.84[0.75,0.95]$ \\
\hline $\begin{array}{l}2.1 \text { WHO recommended - matching } \\
\text { vaccine }\end{array}$ & 7 & 4760 & $\begin{array}{l}\text { Risk Ratio (M-H, Random, 95\% } \\
\mathrm{Cl})\end{array}$ & $0.84[0.77,0.91]$ \\
\hline $\begin{array}{l}2.2 \text { WHO recommended - vaccine } \\
\text { matching absent or unknown }\end{array}$ & 7 & 20942 & $\begin{array}{l}\text { Risk Ratio (M-H, Random, 95\% } \\
\mathrm{Cl})\end{array}$ & $0.90[0.69,1.18]$ \\
\hline $\begin{array}{l}2.3 \text { Monovalent not WHO recom- } \\
\text { mended - vaccine matching }\end{array}$ & 1 & 59 & $\begin{array}{l}\text { Risk Ratio (M-H, Random, 95\% } \\
\mathrm{Cl})\end{array}$ & $1.02[0.28,3.70]$ \\
\hline $\begin{array}{l}\text { 2.4 Monovalent not WHO recom- } \\
\text { mended - vaccine matching - high } \\
\text { dose }\end{array}$ & 1 & 34 & $\begin{array}{l}\text { Risk Ratio (M-H, Random, 95\% } \\
\mathrm{Cl} \text { ) }\end{array}$ & $0.46[0.09,2.30]$ \\
\hline 3 Physician visits & 2 & 2308 & $\begin{array}{l}\text { Risk Ratio (M-H, Random, 95\% } \\
\mathrm{Cl})\end{array}$ & $0.87[0.40,1.89]$ \\
\hline $\begin{array}{l}3.1 \text { WHO recommended - matching } \\
\text { vaccine }\end{array}$ & 1 & 1178 & $\begin{array}{l}\text { Risk Ratio (M-H, Random, 95\% } \\
\mathrm{Cl} \text { ) }\end{array}$ & $0.58[0.37,0.91]$ \\
\hline $\begin{array}{l}\text { 3.2 WHO recommended - vaccine } \\
\text { matching absent or unknown }\end{array}$ & 1 & 1130 & $\begin{array}{l}\text { Risk Ratio (M-H, Random, 95\% } \\
\text { Cl) }\end{array}$ & $1.28[0.90,1.83]$ \\
\hline
\end{tabular}




\begin{tabular}{|c|c|c|c|c|}
\hline Outcome or subgroup title & No. of studies & $\begin{array}{l}\text { No. of partici- } \\
\text { pants }\end{array}$ & Statistical method & Effect size \\
\hline 4 Days ill & 3 & 3133 & $\begin{array}{l}\text { Mean Difference (IV, Random, } \\
95 \% \mathrm{CI})\end{array}$ & $-0.21[-0.98,0.56]$ \\
\hline $\begin{array}{l}4.1 \text { WHO recommended - matching } \\
\text { vaccine }\end{array}$ & 2 & 2003 & $\begin{array}{l}\text { Mean Difference (IV, Random, } \\
95 \% \mathrm{CI})\end{array}$ & $-0.58[-0.85,-0.32]$ \\
\hline $\begin{array}{l}4.2 \text { WHO recommended - matching } \\
\text { absent or unknown }\end{array}$ & 1 & 1130 & $\begin{array}{l}\text { Mean Difference (IV, Random, } \\
95 \% \mathrm{CI})\end{array}$ & $0.66[0.16,1.16]$ \\
\hline 5 Times any drugs were prescribed & 2 & 2308 & $\begin{array}{l}\text { Mean Difference (IV, Random, } \\
95 \% \mathrm{CI})\end{array}$ & $-0.01[-0.03,0.01]$ \\
\hline $\begin{array}{l}5.1 \text { WHO recommended - matching } \\
\text { vaccine }\end{array}$ & 1 & 1178 & $\begin{array}{l}\text { Mean Difference (IV, Random, } \\
95 \% \mathrm{CI})\end{array}$ & $-0.02[-0.04,-0.00]$ \\
\hline $\begin{array}{l}5.2 \text { WHO recommended - matching } \\
\text { absent or unknown }\end{array}$ & 1 & 1130 & $\begin{array}{l}\text { Mean Difference (IV, Random, } \\
95 \% \mathrm{CI})\end{array}$ & $0.0[-0.00,0.00]$ \\
\hline 6 Times antibiotic was prescribed & 2 & 2308 & $\begin{array}{l}\text { Mean Difference (IV, Random, } \\
95 \% \mathrm{CI})\end{array}$ & $-0.02[-0.03,-0.01]$ \\
\hline $\begin{array}{l}6.1 \text { WHO recommended - matching } \\
\text { vaccine }\end{array}$ & 1 & 1178 & $\begin{array}{l}\text { Mean Difference (IV, Random, } \\
95 \% \mathrm{CI})\end{array}$ & $-0.02[-0.03,-0.01]$ \\
\hline $\begin{array}{l}6.2 \text { WHO recommended - matching } \\
\text { absent or unknown }\end{array}$ & 1 & 1130 & $\begin{array}{l}\text { Mean Difference (IV, Random, } \\
95 \% \mathrm{CI})\end{array}$ & $-0.01[-0.03,0.01]$ \\
\hline 7 Working days lost & 4 & 3726 & $\begin{array}{l}\text { Mean Difference (IV, Random, } \\
95 \% \mathrm{CI})\end{array}$ & $-0.04[-0.14,0.06]$ \\
\hline $\begin{array}{l}7.1 \text { WHO recommended - matching } \\
\text { vaccine }\end{array}$ & 3 & 2596 & $\begin{array}{l}\text { Mean Difference (IV, Random, } \\
95 \% \mathrm{CI})\end{array}$ & $-0.09[-0.19,0.02]$ \\
\hline $\begin{array}{l}7.2 \text { WHO recommended - matching } \\
\text { absent or unknown }\end{array}$ & 1 & 1130 & $\begin{array}{l}\text { Mean Difference (IV, Random, } \\
95 \% \mathrm{CI})\end{array}$ & $0.09[0.00,0.18]$ \\
\hline 8 Hospitalisations & 3 & 11924 & $\begin{array}{l}\text { Risk Ratio (M-H, Random, 95\% } \\
\mathrm{Cl})\end{array}$ & $0.96[0.85,1.08]$ \\
\hline $\begin{array}{l}8.1 \text { WHO recommended - matching } \\
\text { vaccine }\end{array}$ & 1 & 1178 & $\begin{array}{l}\text { Risk Ratio (M-H, Random, 95\% } \\
\mathrm{Cl})\end{array}$ & $0.0[0.0,0.0]$ \\
\hline $\begin{array}{l}8.2 \text { WHO recommended - vaccine } \\
\text { matching absent or unknown }\end{array}$ & 1 & 1130 & $\begin{array}{l}\text { Risk Ratio (M-H, Random, 95\% } \\
\mathrm{Cl} \text { ) }\end{array}$ & $2.89[0.12,70.68]$ \\
\hline $\begin{array}{l}8.3 \text { Monovalent not WHO recom- } \\
\text { mended - vaccine matching }\end{array}$ & 1 & 9616 & $\begin{array}{l}\text { Risk Ratio (M-H, Random, 95\% } \\
\mathrm{Cl} \text { ) }\end{array}$ & $0.96[0.85,1.08]$ \\
\hline $\begin{array}{l}9 \text { Clinical cases (clinically defined } \\
\text { without clear definition) }\end{array}$ & 3 & 4259 & $\begin{array}{l}\text { Risk Ratio (M-H, Random, 95\% } \\
\mathrm{Cl})\end{array}$ & $0.87[0.72,1.05]$ \\
\hline $\begin{array}{l}\text { 9.1 WHO recommended - matching } \\
\text { vaccine }\end{array}$ & 2 & 2056 & $\begin{array}{l}\text { Risk Ratio (M-H, Random, 95\% } \\
\mathrm{Cl})\end{array}$ & $0.89[0.64,1.25]$ \\
\hline
\end{tabular}




\begin{tabular}{|c|c|c|c|c|}
\hline Outcome or subgroup title & No. of studies & $\begin{array}{l}\text { No. of partici- } \\
\text { pants }\end{array}$ & Statistical method & Effect size \\
\hline $\begin{array}{l}9.2 \text { WHO recommended - vaccine } \\
\text { matching absent or unknown }\end{array}$ & 1 & 2203 & $\begin{array}{l}\text { Risk Ratio (M-H, Random, 95\% } \\
\mathrm{Cl})\end{array}$ & $0.83[0.69,0.99]$ \\
\hline 10 Local harms & 20 & & $\begin{array}{l}\text { Risk Ratio (M-H, Random, 95\% } \\
\mathrm{Cl})\end{array}$ & Subtotals only \\
\hline 10.1 Local - tenderness/soreness & 20 & 35655 & $\begin{array}{l}\text { Risk Ratio (M-H, Random, 95\% } \\
\mathrm{Cl})\end{array}$ & $3.13[2.44,4.02]$ \\
\hline 10.2 Local - erythema & 9 & 29499 & $\begin{array}{l}\text { Risk Ratio (M-H, Random, 95\% } \\
\text { Cl) }\end{array}$ & $2.59[1.77,3.78]$ \\
\hline 10.3 Local - induration & 3 & 7786 & $\begin{array}{l}\text { Risk Ratio (M-H, Random, 95\% } \\
\text { Cl) }\end{array}$ & $4.28[1.25,14.67]$ \\
\hline 10.4 Local - arm stiffness & 1 & 50 & $\begin{array}{l}\text { Risk Ratio (M-H, Random, 95\% } \\
\mathrm{Cl} \text { ) }\end{array}$ & $1.62[0.54,4.83]$ \\
\hline $\begin{array}{l}10.5 \text { Local - combined endpoint (any } \\
\text { or highest symptom) }\end{array}$ & 11 & 12307 & $\begin{array}{l}\text { Risk Ratio (M-H, Random, 95\% } \\
\text { Cl) }\end{array}$ & $2.44[1.82,3.28]$ \\
\hline 11 Systemic harms & 17 & & $\begin{array}{l}\text { Risk Ratio (M-H, Random, 95\% } \\
\text { Cl) }\end{array}$ & Subtotals only \\
\hline 11.1 Systemic - myalgia & 11 & 35008 & $\begin{array}{l}\text { Risk Ratio (M-H, Random, 95\% } \\
\mathrm{Cl})\end{array}$ & $1.74[1.41,2.14]$ \\
\hline 11.2 Systemic - fever & 13 & 23850 & $\begin{array}{l}\text { Risk Ratio (M-H, Random, 95\% } \\
\mathrm{Cl})\end{array}$ & $1.55[1.26,1.91]$ \\
\hline 11.3 Systemic - headache & 14 & 35999 & $\begin{array}{l}\text { Risk Ratio (M-H, Random, 95\% } \\
\mathrm{Cl})\end{array}$ & $1.14[0.99,1.30]$ \\
\hline $\begin{array}{l}11.4 \text { Systemic - fatigue or indisposi- } \\
\text { tion }\end{array}$ & 12 & 35788 & $\begin{array}{l}\text { Risk Ratio (M-H, Random, 95\% } \\
\mathrm{Cl})\end{array}$ & $1.19[1.05,1.36]$ \\
\hline 11.5 Systemic - nausea/vomiting & 4 & 6315 & $\begin{array}{l}\text { Risk Ratio (M-H, Random, 95\% } \\
\mathrm{Cl})\end{array}$ & $1.80[0.65,5.04]$ \\
\hline 11.6 Systemic - malaise & 3 & 26111 & $\begin{array}{l}\text { Risk Ratio (M-H, Random, 95\% } \\
\text { Cl) }\end{array}$ & $1.51[1.18,1.92]$ \\
\hline $\begin{array}{l}11.7 \text { Systemic - combined endpoint } \\
\text { (any or highest symptom) }\end{array}$ & 6 & 2128 & $\begin{array}{l}\text { Risk Ratio (M-H, Random, 95\% } \\
\text { Cl) }\end{array}$ & $1.16[0.87,1.53]$ \\
\hline
\end{tabular}

Analysis 1.1. Comparison 1 Inactivated parenteral influenza vaccine versus placebo or 'do nothing', Outcome 1 Influenza.

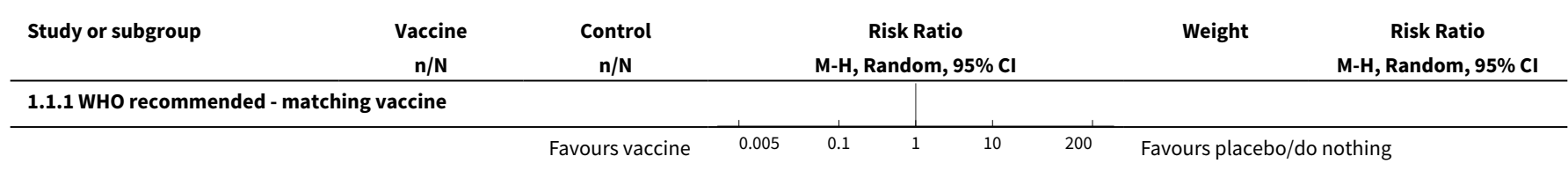




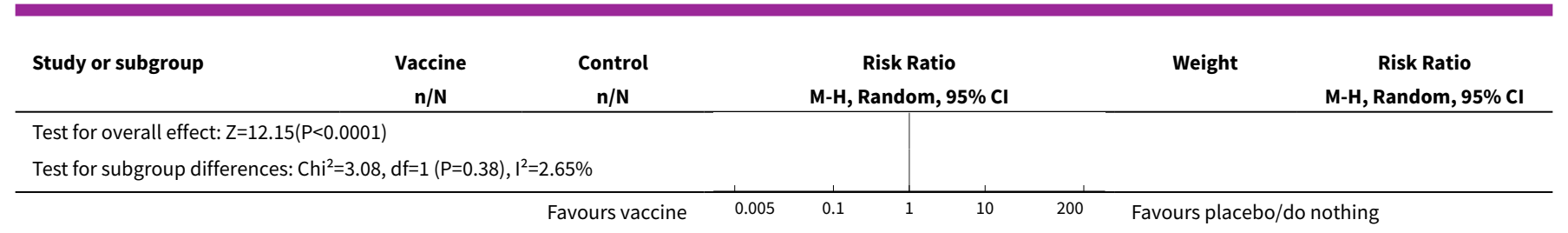

\section{Analysis 1.2. Comparison 1 Inactivated parenteral influenza vaccine versus placebo or 'do nothing', Outcome 2 Influenza-like illness.}

\begin{tabular}{lrr} 
Study or subgroup & $\begin{array}{c}\text { Vaccine } \\
\mathbf{n} / \mathbf{N}\end{array}$ & $\begin{array}{c}\text { Control } \\
\mathbf{n} / \mathbf{N}\end{array}$ \\
\hline 1.2.1 WHO recommended - matching vaccine & \\
aa Mesa Duque 2001 & $194 / 247$ & $225 / 246$ \\
aa Keitel 1997b & $25 / 723$ & $14 / 217$ \\
aa Mixéu 2002 & $86 / 294$ & $98 / 299$ \\
aa Bridges 2000b & $82 / 582$ & $128 / 596$ \\
aa Nichol 1995 & $249 / 409$ & $287 / 416$ \\
aa Keitel 1988b & $13 / 456$ & $9 / 241$ \\
aa Powers 1995a & $4 / 26$ & $2 / 8$ \\
Subtotal (95\% CI) & $\mathbf{2 7 3 7}$ & $\mathbf{2 0 2 3}$
\end{tabular}

Total events: 653 (Vaccine), 763 (Control)

Heterogeneity: $\mathrm{Tau}^{2}=0 ; \mathrm{Chi}^{2}=8.12, \mathrm{df}=6(\mathrm{P}=0.23) ; \mathrm{I}^{2}=26.14 \%$

Test for overall effect: $Z=4(P<0.0001)$

1.2.2 WHO recommended - vaccine matching absent or unknown

aa Bridges 2000a

aa Keitel 1997c

\section{$53 / 789$}

$132 / 554$

aa Keitel 1997a

$41 / 577$

$14 / 145$

aa Keitel 1988a

aa Beran 2009a

aa Weingarten 1988

$15 / 300$

$23 / 253$

$254 / 4011$

$120 / 2003$

$21 / 91$

$19 / 88$

aa Frey 2010

$432 / 7414$

$353 / 3843$

13758

7184

Total events: 977 (Vaccine), 675 (Control)

Heterogeneity: $\mathrm{Tau}^{2}=0.09 ; \mathrm{Chi}^{2}=33.72, \mathrm{df}=6(\mathrm{P}<0.0001) ; \mathrm{I}^{2}=82.21 \%$

Test for overall effect: $\mathrm{Z}=0.78(\mathrm{P}=0.44)$

1.2.3 Monovalent not WHO recommended - vaccine matching

aa Powers $1995 \mathrm{c}$

Subtotal $(95 \% \mathrm{Cl})$

$13 / 51$

51

$2 / 8$

Total events: 13 (Vaccine), 2 (Control)

Heterogeneity: Not applicable

Test for overall effect: $\mathrm{Z}=0.03(\mathrm{P}=0.98)$

1.2.4 Monovalent not WHO recommended - vaccine matching - high dose

aa Powers 1995b

Subtotal $(95 \% \mathrm{Cl})$

$3 / 26$

26

$2 / 8$

Total events: 3 (Vaccine), 2 (Control)

Heterogeneity: Tau $^{2}=0 ; \mathrm{Chi}^{2}=0, \mathrm{df}=0(\mathrm{P}<0.0001) ; \mathrm{I}^{2}=100 \%$

Test for overall effect: $Z=0.94(P=0.34)$
Risk Ratio Weigh

M-H, Random, $95 \% \mathrm{Cl}$

\begin{tabular}{|c|c|c|}
\hline * & $14.6 \%$ & $0.86[0.8,0.93]$ \\
\hline 1 & $2.72 \%$ & $0.54[0.28,1.01]$ \\
\hline * & $9.3 \%$ & $0.89[0.7,1.14]$ \\
\hline * & $8.93 \%$ & $0.66[0.51,0.84]$ \\
\hline$*$ & $13.92 \%$ & $0.88[0.8,0.98]$ \\
\hline & $1.71 \%$ & $0.76[0.33,1.76]$ \\
\hline & $0.57 \%$ & $0.62[0.14,2.76]$ \\
\hline 1 & $51.75 \%$ & $0.84[0.77,0.91]$ \\
\hline
\end{tabular}

$1.17[0.96,1.43]$

$0.7[0.4,1.22]$

$0.78[0.48,1.27]$

$1.06[0.52,2.17]$

$1.06[0.86,1.3]$

$1.07[0.62,1.85]$

$0.63[0.55,0.73]$

$0.9[0.69,1.18]$
$1.02[0.28,3.7]$

$1.02[0.28,3.7]$
$0.77 \%$

$0.77 \%$ 


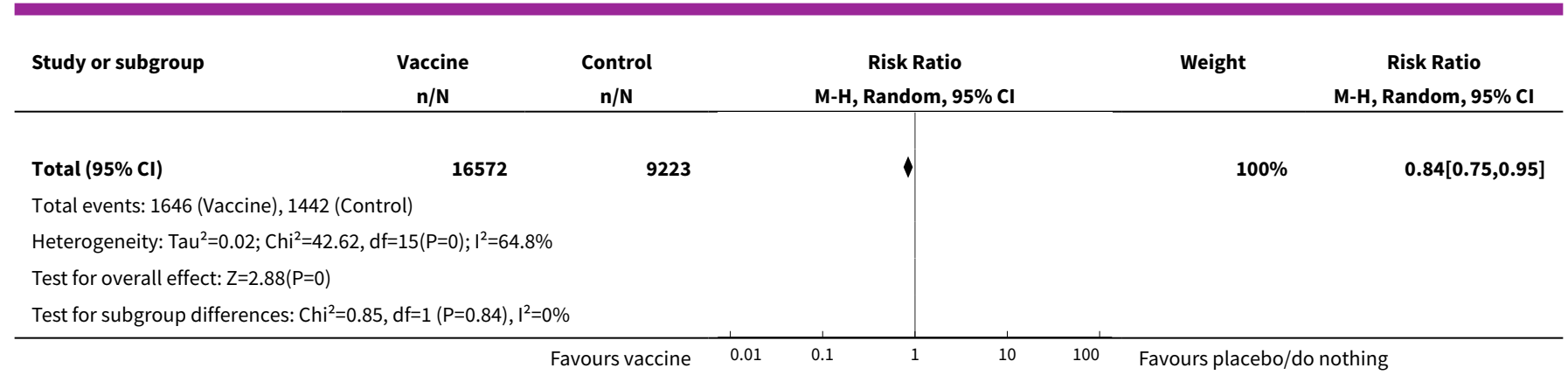

Analysis 1.3. Comparison 1 Inactivated parenteral influenza vaccine versus placebo or 'do nothing', Outcome 3 Physician visits.

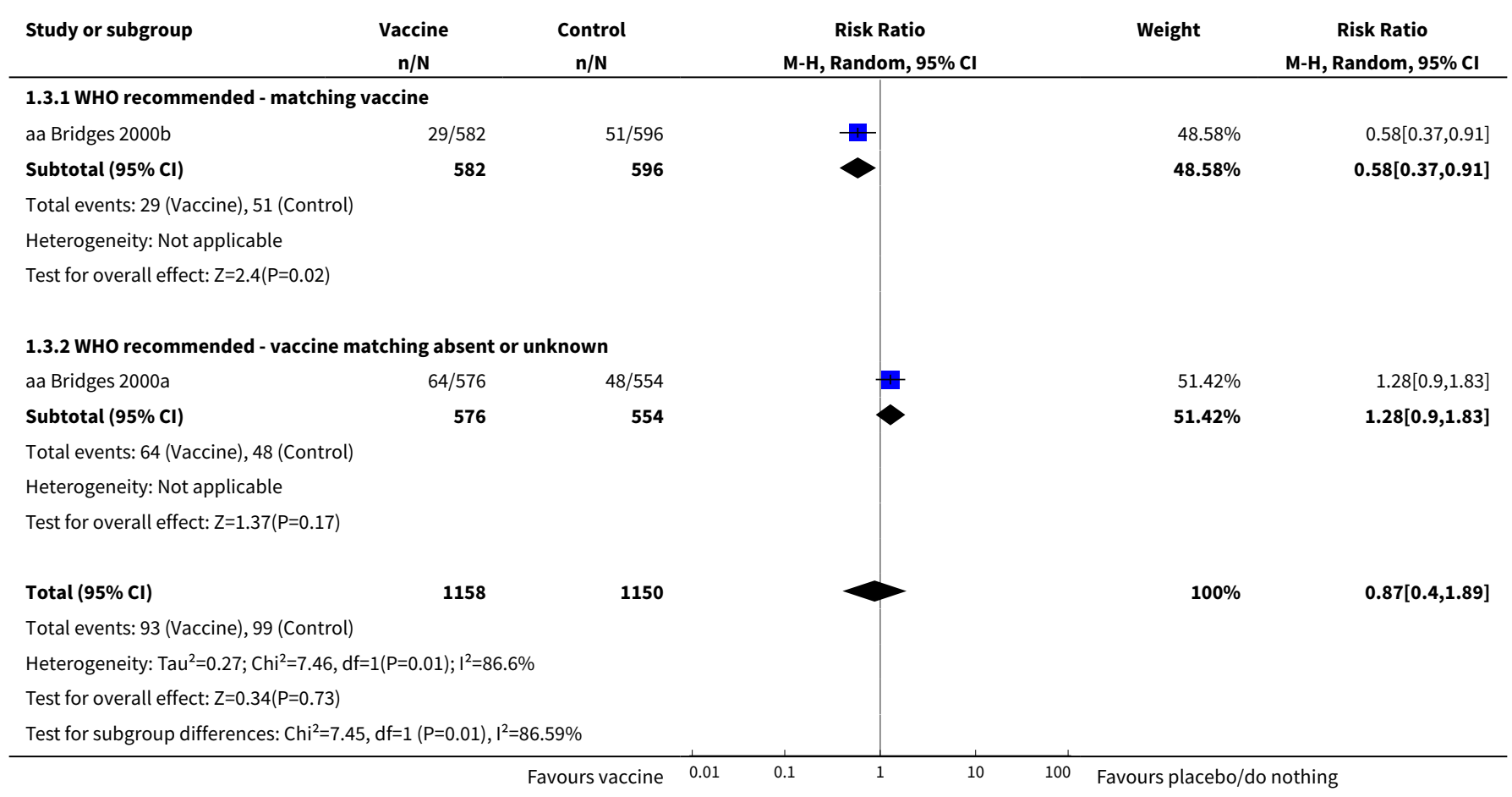

\section{Analysis 1.4. Comparison 1 Inactivated parenteral influenza} vaccine versus placebo or 'do nothing', Outcome 4 Days ill.

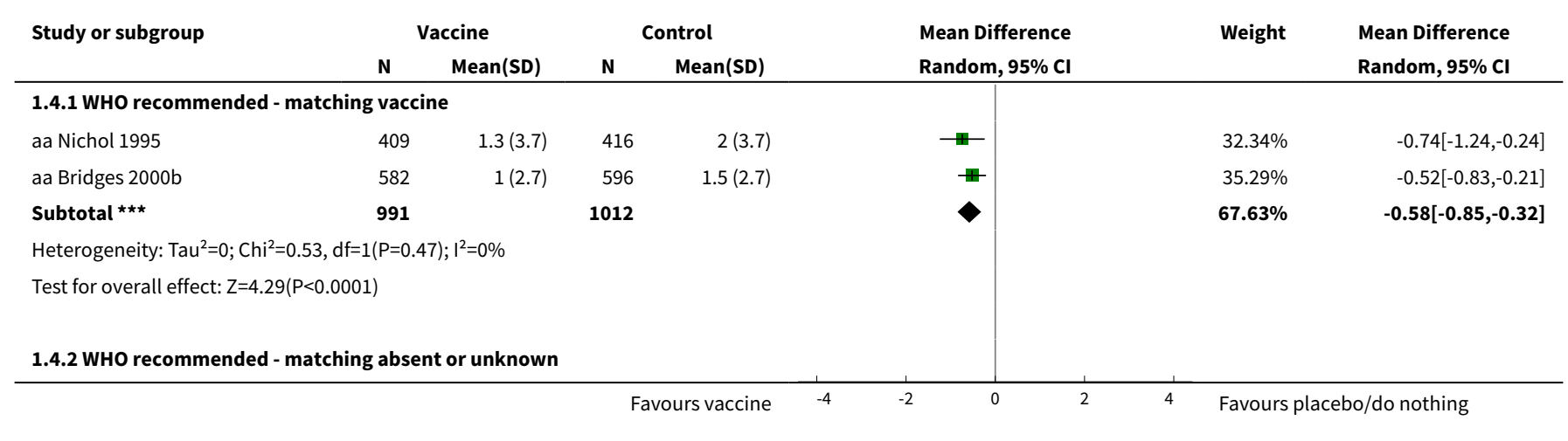




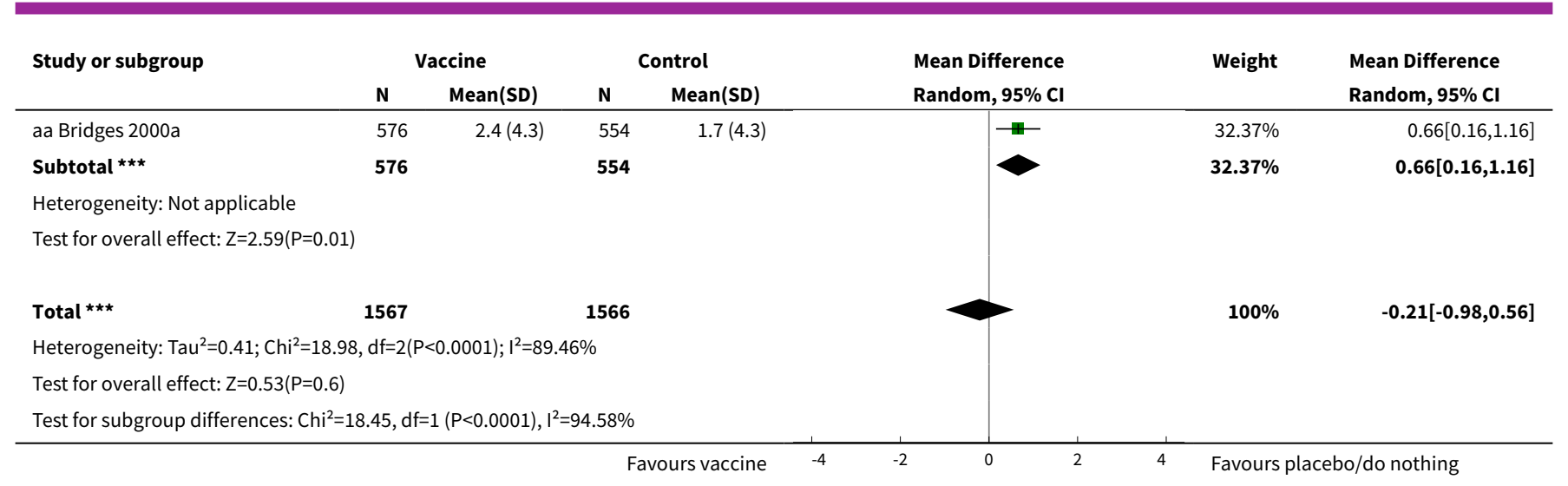

Analysis 1.5. Comparison 1 Inactivated parenteral influenza vaccine versus placebo or 'do nothing', Outcome 5 Times any drugs were prescribed.

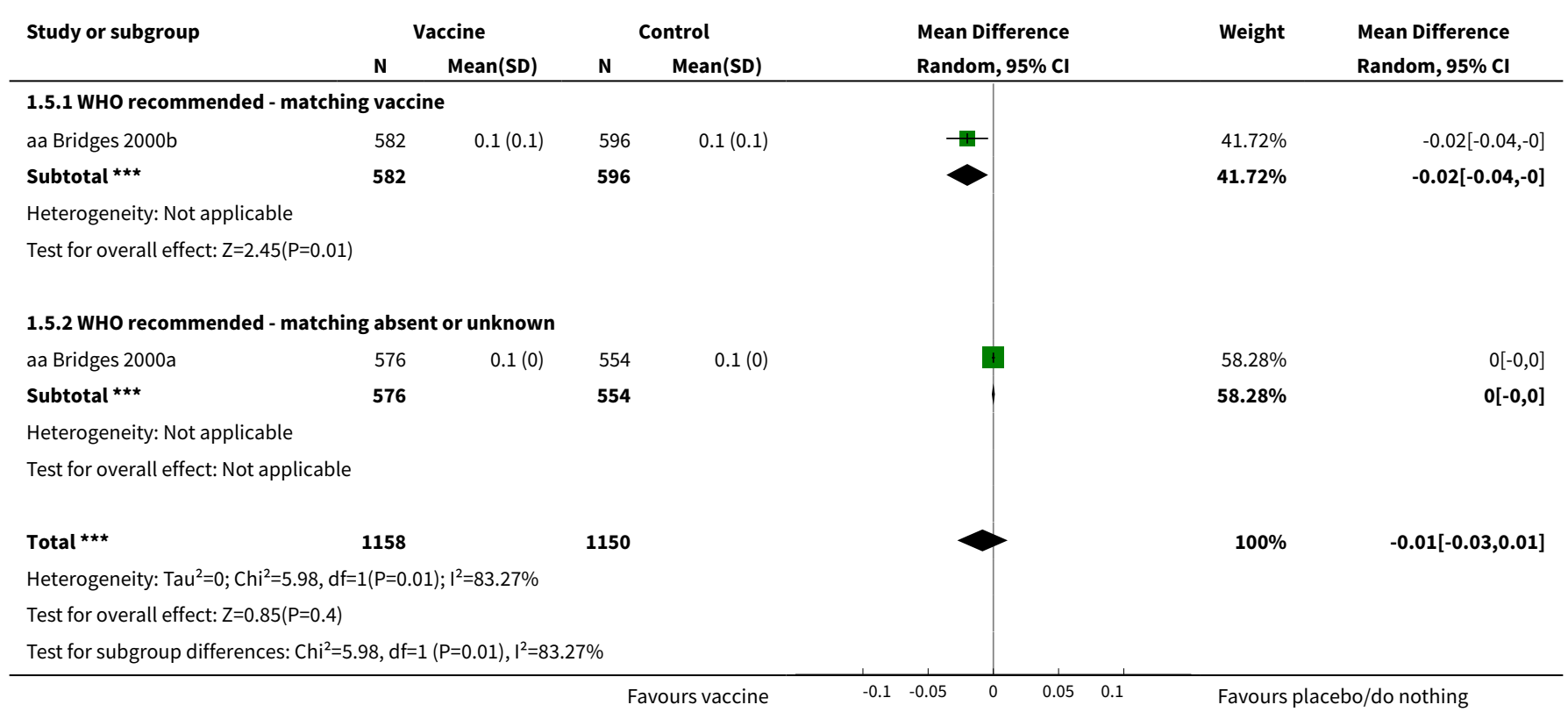

Analysis 1.6. Comparison 1 Inactivated parenteral influenza vaccine versus placebo or 'do nothing', Outcome 6 Times antibiotic was prescribed.

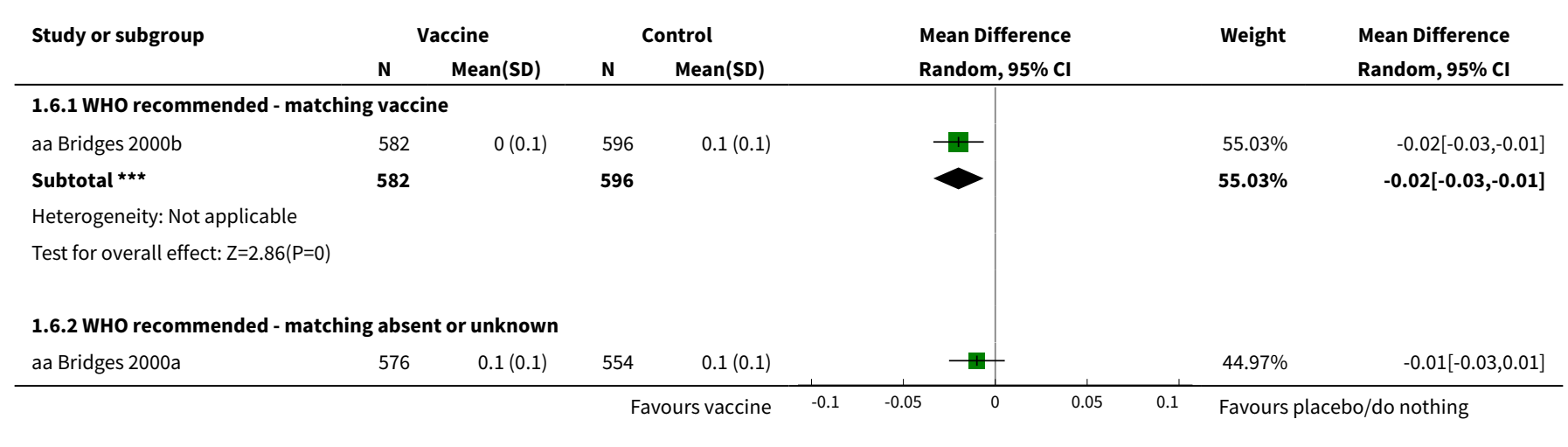




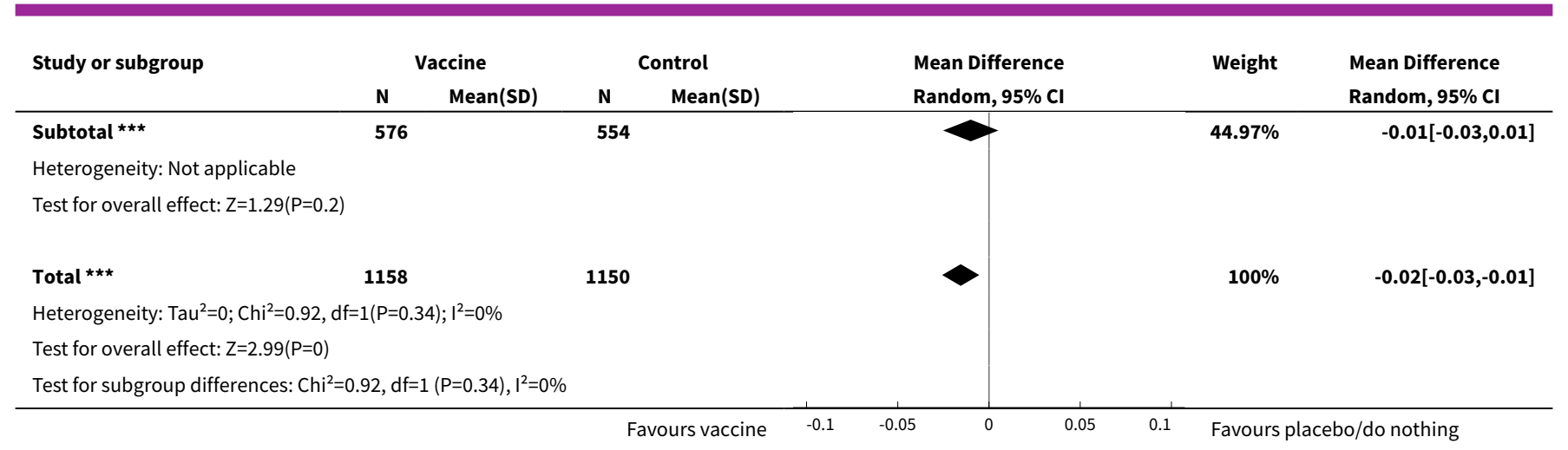

\section{Analysis 1.7. Comparison 1 Inactivated parenteral influenza vaccine versus placebo or 'do nothing', Outcome 7 Working days lost.}

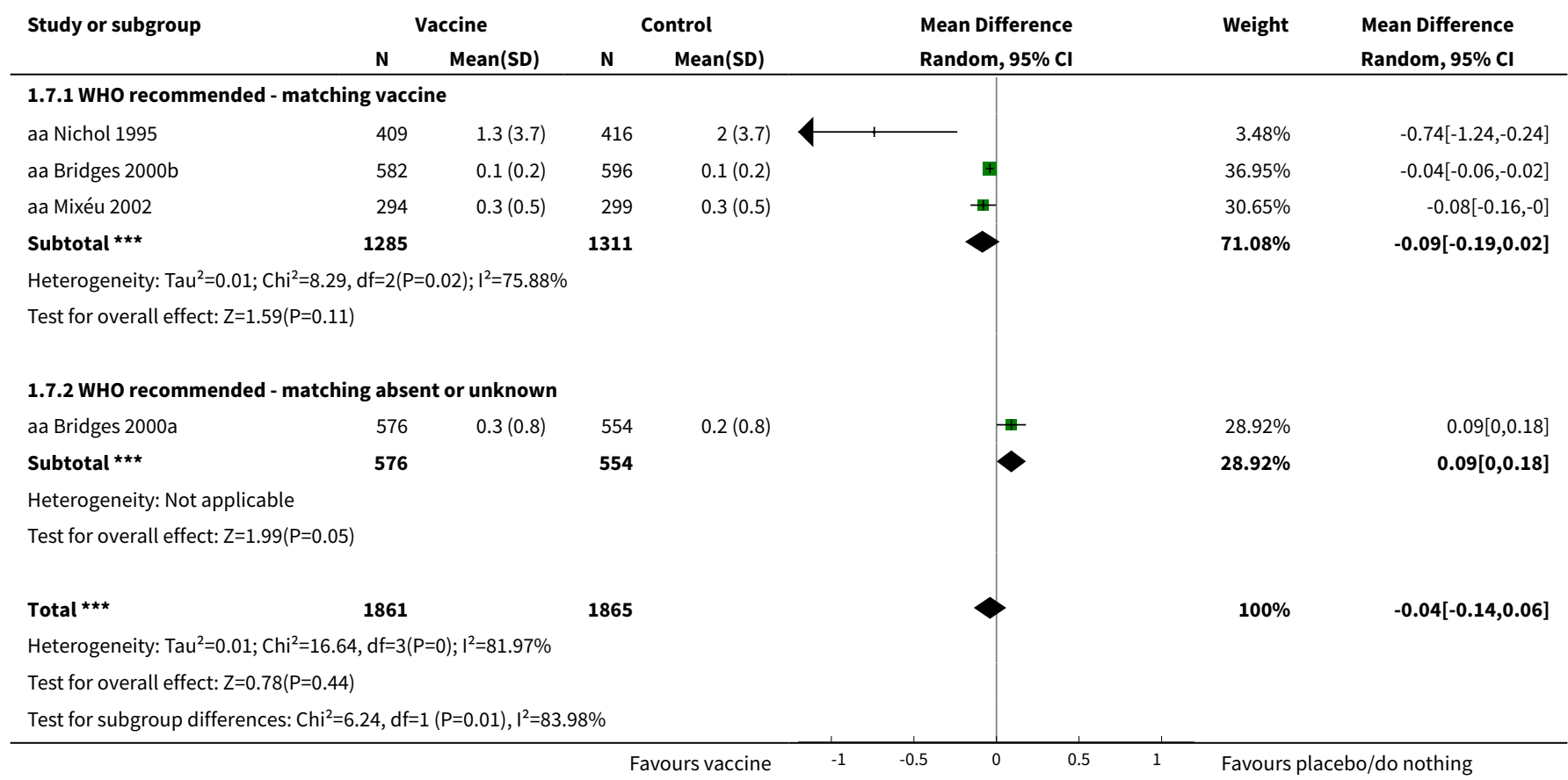

Analysis 1.8. Comparison 1 Inactivated parenteral influenza vaccine versus placebo or 'do nothing', Outcome 8 Hospitalisations.

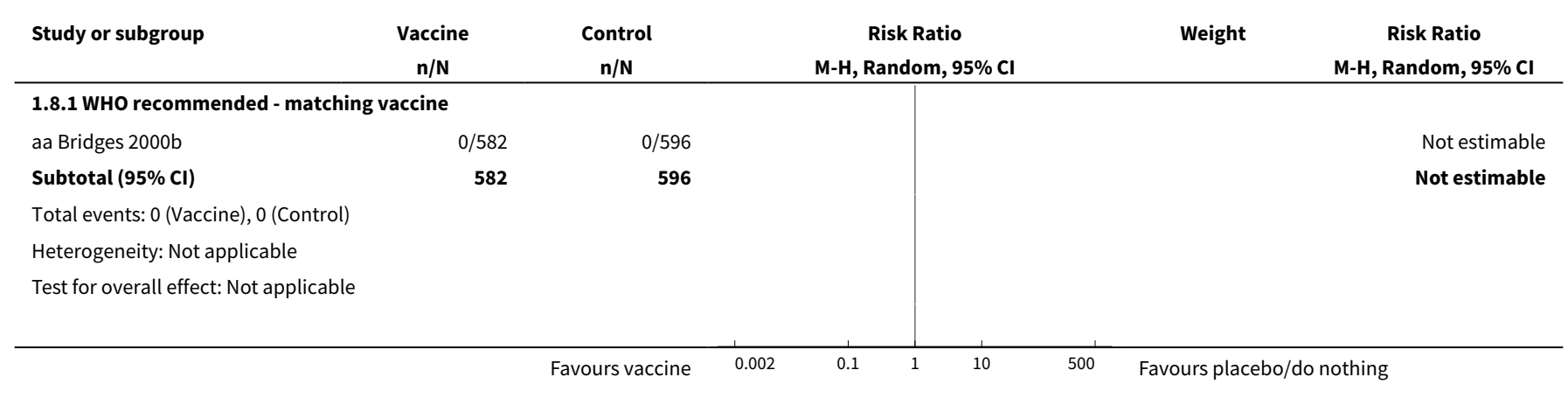




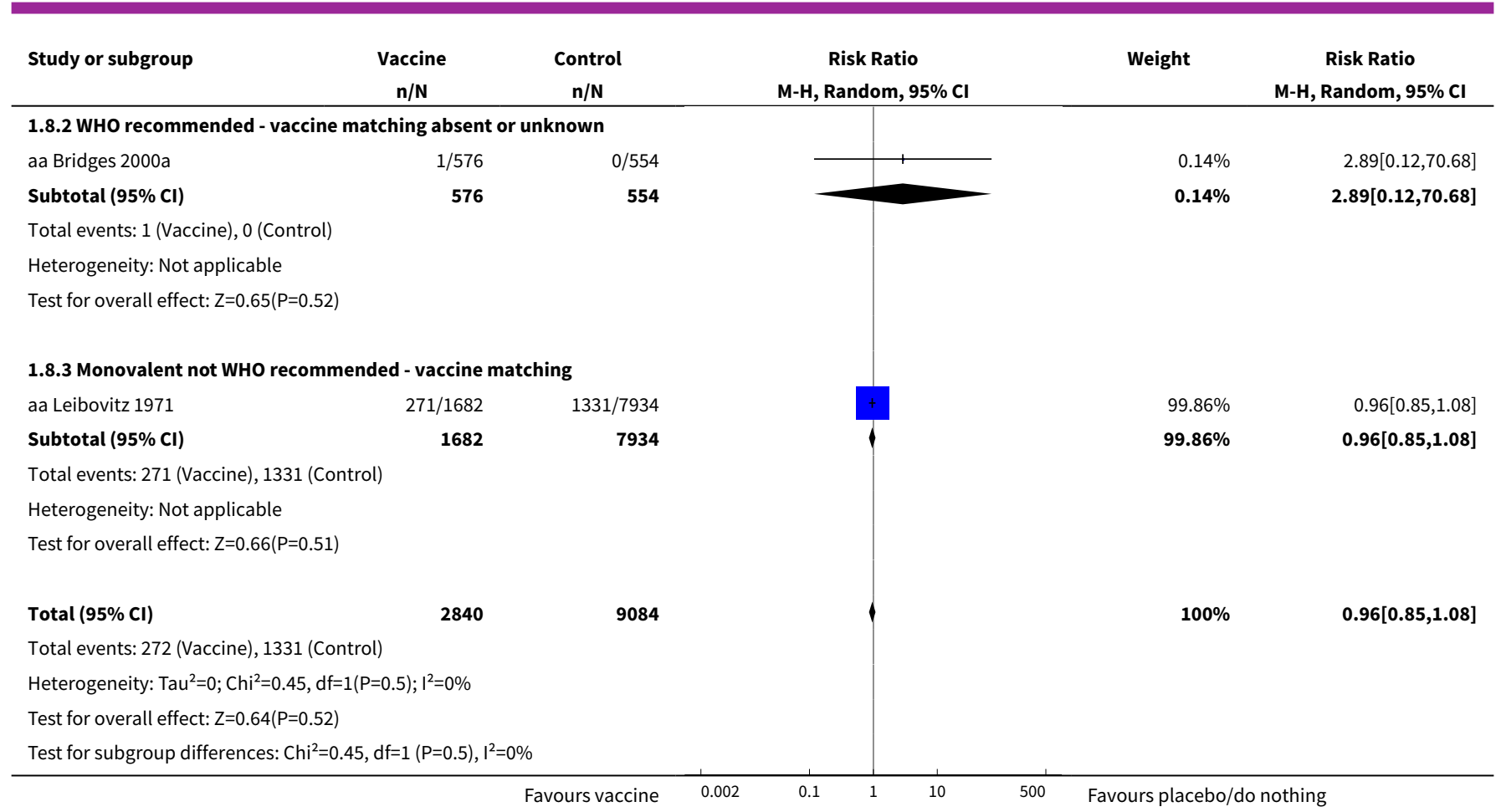

\section{Analysis 1.9. Comparison 1 Inactivated parenteral influenza vaccine versus placebo or 'do nothing', Outcome 9 Clinical cases (clinically defined without clear definition).}

\begin{tabular}{|c|c|c|c|c|c|}
\hline Study or subgroup & $\begin{array}{c}\text { Vaccine } \\
n / N\end{array}$ & $\begin{array}{c}\text { Control } \\
n / N\end{array}$ & $\begin{array}{c}\text { Risk Ratio } \\
\text { M-H, Random, } 95 \% \mathrm{Cl}\end{array}$ & Weight & $\begin{array}{c}\text { Risk Ratio } \\
\text { M-H, Random, } 95 \% \text { CI }\end{array}$ \\
\hline \multicolumn{6}{|c|}{ 1.9.1 WHO recommended - matching vaccine } \\
\hline aa Hammond 1978 & $75 / 116$ & $68 / 109$ & & $34.4 \%$ & $1.04[0.85,1.26]$ \\
\hline aa Zhilova 1986b & $100 / 895$ & $138 / 936$ & 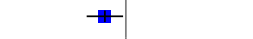 & $29.22 \%$ & $0.76[0.6,0.96]$ \\
\hline Subtotal $(95 \% \mathrm{Cl})$ & 1011 & 1045 & & $63.61 \%$ & $0.89[0.64,1.25]$ \\
\hline \multicolumn{6}{|c|}{ Total events: 175 (Vaccine), 206 (Control) } \\
\hline \multicolumn{6}{|c|}{ Test for overall effect: $Z=0.67(P=0.5)$} \\
\hline \multicolumn{6}{|c|}{ 1.9.2 WHO recommended - vaccine matching absent or unknown } \\
\hline aa Zhilova 1986a & $139 / 818$ & $285 / 1385$ & + & $36.39 \%$ & $0.83[0.69,0.99]$ \\
\hline Subtotal $(95 \% \mathrm{Cl})$ & 818 & 1385 & & $36.39 \%$ & $0.83[0.69,0.99]$ \\
\hline \multicolumn{6}{|c|}{ Total events: 139 (Vaccine), 285 (Control) } \\
\hline \multicolumn{6}{|c|}{ Test for overall effect: $Z=2.05(P=0.04)$} \\
\hline Total $(95 \% \mathrm{Cl})$ & 1829 & 2430 & & $100 \%$ & $0.87[0.72,1.05]$ \\
\hline \multicolumn{6}{|c|}{ Total events: 314 (Vaccine), 491 (Control) } \\
\hline \multicolumn{6}{|c|}{ Heterogeneity: $\mathrm{Tau}^{2}=0.02 ; \mathrm{Chi}^{2}=5.07, \mathrm{df}=2(\mathrm{P}=0.08) ; \mathrm{I}^{2}=60.53 \%$} \\
\hline \multicolumn{6}{|c|}{ Test for overall effect: $Z=1.43(P=0.15)$} \\
\hline Test for subgroup di & $d f=1(P=0.69)$ & & & & \\
\hline
\end{tabular}


Analysis 1.10. Comparison 1 Inactivated parenteral influenza vaccine versus placebo or 'do nothing', Outcome 10 Local harms.

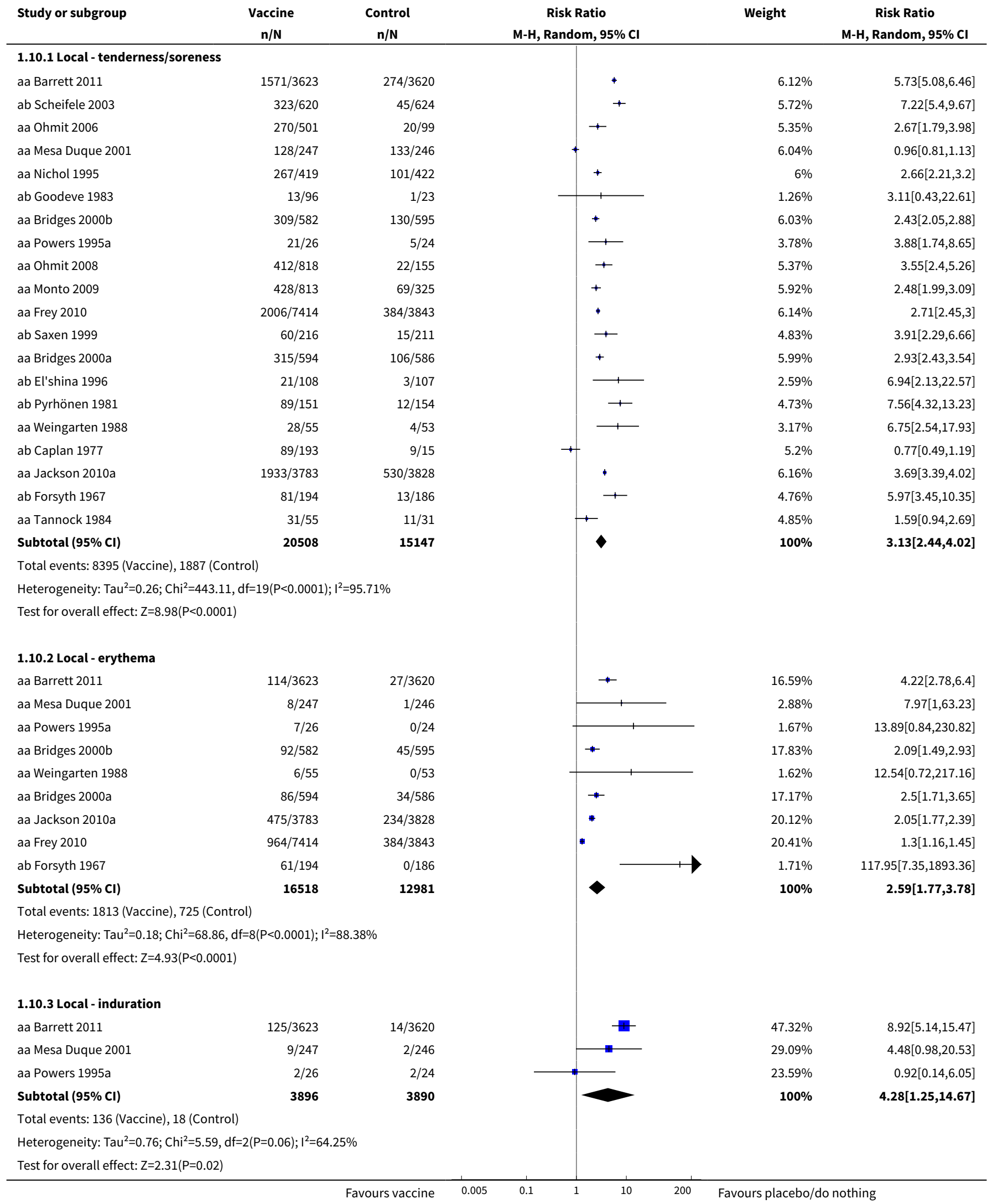




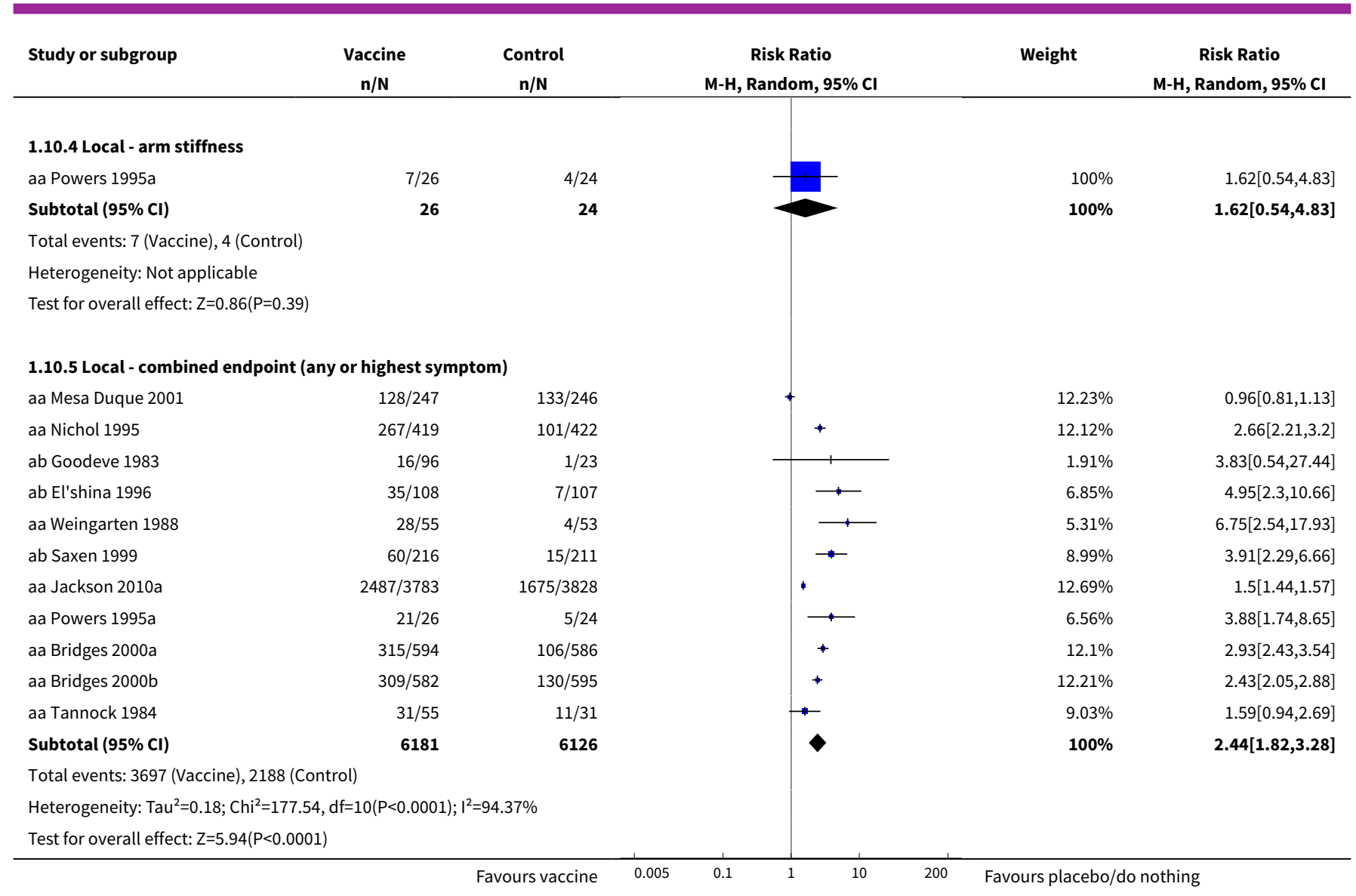

Analysis 1.11. Comparison 1 Inactivated parenteral influenza vaccine versus placebo or 'do nothing', Outcome 11 Systemic harms.

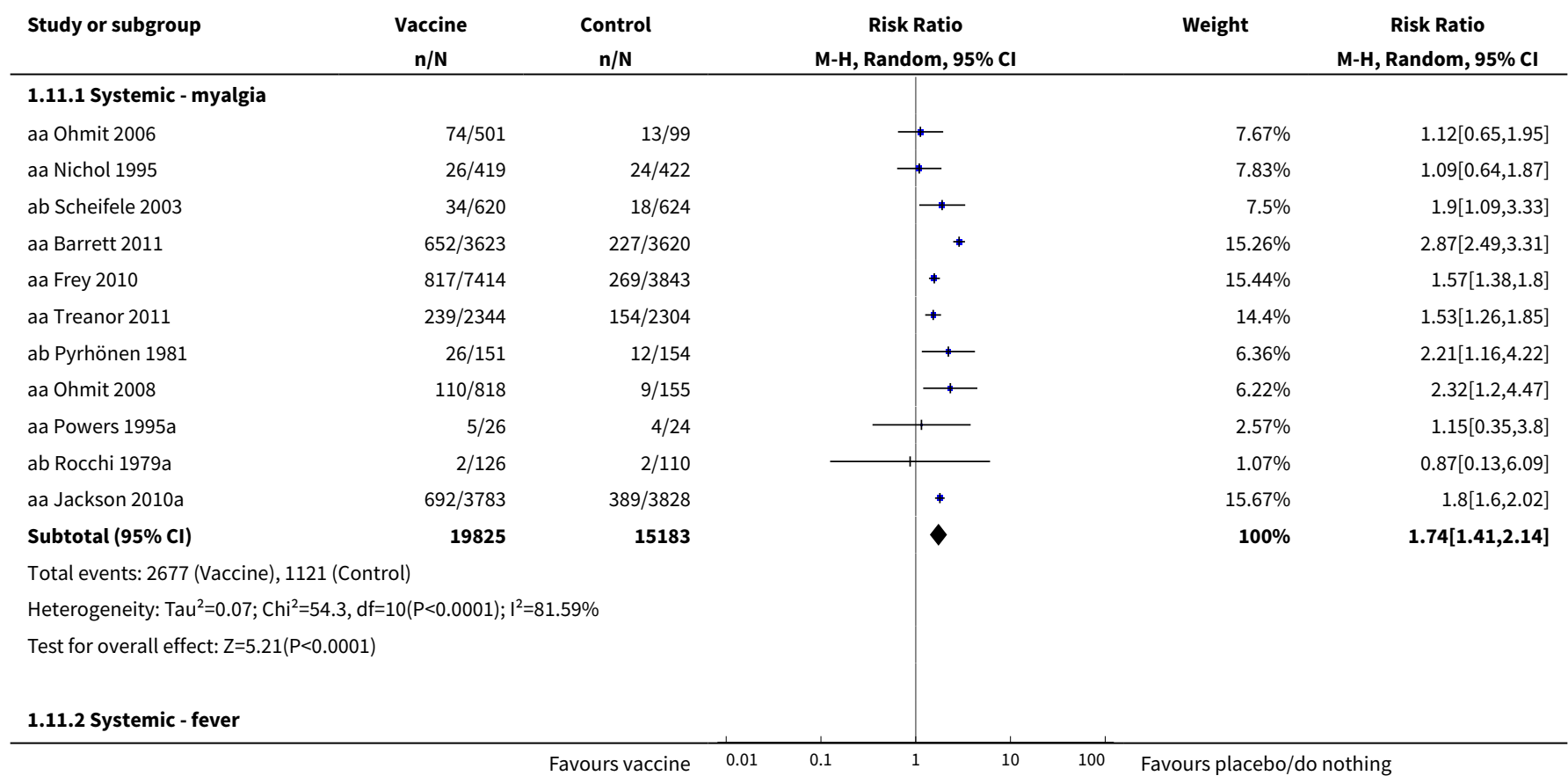




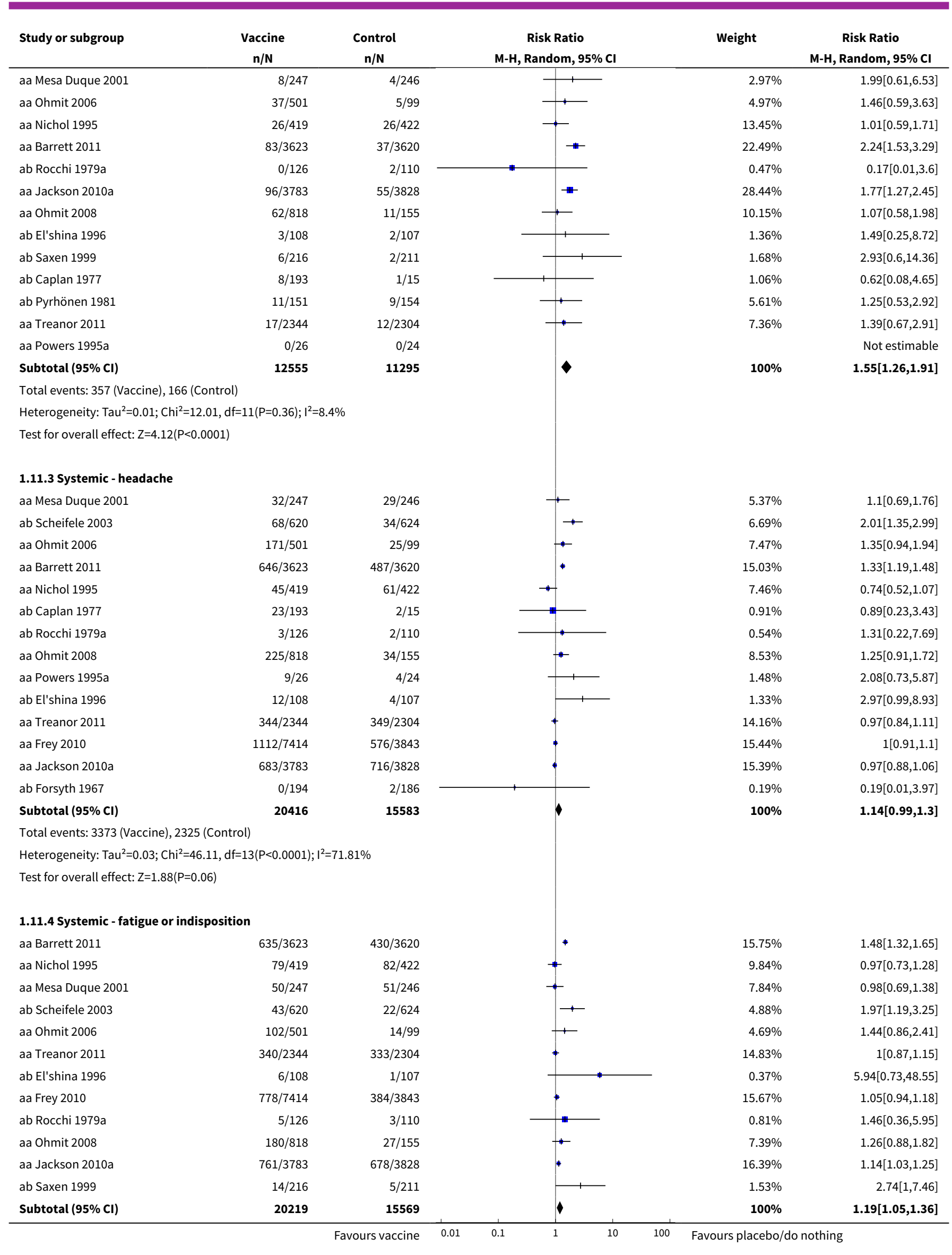




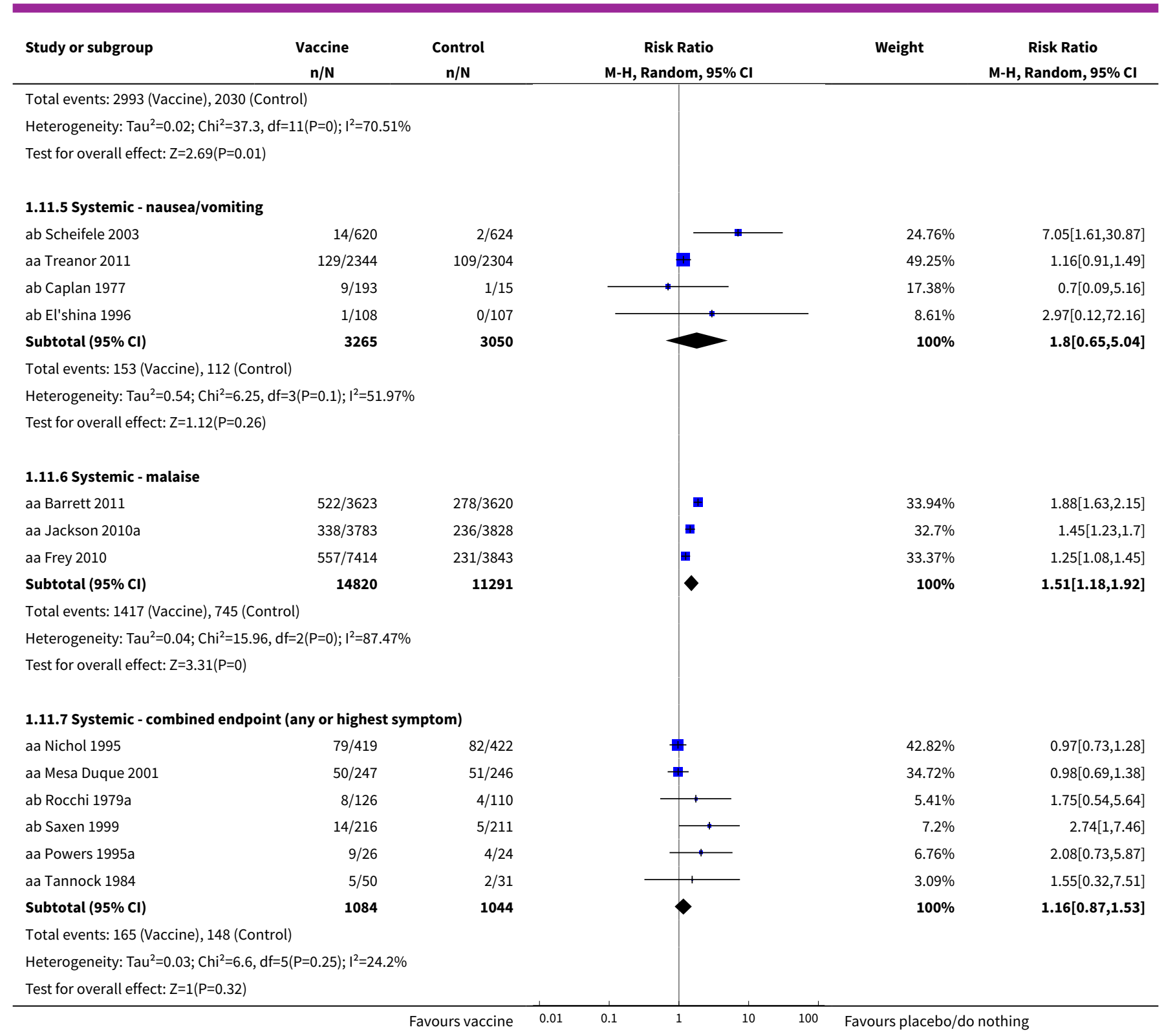

\section{Comparison 2. Live aerosol influenza vaccine versus placebo or 'do nothing'}

\begin{tabular}{lllll}
\hline Outcome or subgroup title & No. of studies & $\begin{array}{l}\text { No. of partici- } \\
\text { pants }\end{array}$ & Statistical method & Effect size \\
\hline 1 Influenza & 9 & 11579 & $\begin{array}{l}\text { Risk Ratio (M-H, Random, 95\% } \\
\mathrm{Cl})\end{array}$ & $0.47[0.35,0.62]$ \\
\hline $\begin{array}{l}1.1 \text { WHO recommended - matching } \\
\text { vaccine }\end{array}$ & 4 & 6584 & $\begin{array}{l}\text { Risk Ratio (M-H, Random, 95\% } \\
\mathrm{Cl})\end{array}$ & $0.55[0.37,0.82]$ \\
\hline $\begin{array}{l}1.2 \text { WHO recommended - vaccine } \\
\text { matching absent or unknown }\end{array}$ & 3 & 4568 & $\begin{array}{l}\text { Risk Ratio (M-H, Random, 95\% } \\
\mathrm{Cl})\end{array}$ & $0.43[0.27,0.68]$ \\
\hline
\end{tabular}




\begin{tabular}{|c|c|c|c|c|}
\hline Outcome or subgroup title & No. of studies & $\begin{array}{l}\text { No. of partici- } \\
\text { pants }\end{array}$ & Statistical method & Effect size \\
\hline $\begin{array}{l}1.3 \text { Non WHO recommended - vac- } \\
\text { cine matching absent or unknown }\end{array}$ & 2 & 427 & $\begin{array}{l}\text { Risk Ratio (M-H, Random, 95\% } \\
\text { Cl) }\end{array}$ & $0.21[0.08,0.56]$ \\
\hline 2 Influenza-like illness & 6 & 12688 & $\begin{array}{l}\text { Risk Ratio (M-H, Random, 95\% } \\
\mathrm{Cl})\end{array}$ & $0.90[0.84,0.96]$ \\
\hline $\begin{array}{l}2.1 \text { WHO recommended - matching } \\
\text { vaccine }\end{array}$ & 2 & 4254 & $\begin{array}{l}\text { Risk Ratio (M-H, Random, 95\% } \\
\mathrm{Cl})\end{array}$ & $0.92[0.76,1.12]$ \\
\hline $\begin{array}{l}2.2 \text { WHO recommended - vaccine } \\
\text { matching absent or unknown }\end{array}$ & 3 & 8150 & $\begin{array}{l}\text { Risk Ratio (M-H, Random, 95\% } \\
\mathrm{Cl})\end{array}$ & $0.89[0.82,0.97]$ \\
\hline $\begin{array}{l}2.3 \text { Non WHO recommended - vac- } \\
\text { cine matching absent or unknown }\end{array}$ & 1 & 284 & $\begin{array}{l}\text { Risk Ratio (M-H, Random, 95\% } \\
\mathrm{Cl})\end{array}$ & $0.92[0.73,1.16]$ \\
\hline $\begin{array}{l}3 \text { Influenza cases (clinically defined } \\
\text { without clear definition) }\end{array}$ & 3 & 23900 & $\begin{array}{l}\text { Risk Ratio (M-H, Random, 95\% } \\
\mathrm{Cl})\end{array}$ & $0.89[0.71,1.11]$ \\
\hline $\begin{array}{l}3.1 \text { WHO recommended - matching } \\
\text { vaccine }\end{array}$ & 1 & 1931 & $\begin{array}{l}\text { Risk Ratio (M-H, Random, 95\% } \\
\mathrm{Cl})\end{array}$ & $0.63[0.49,0.80]$ \\
\hline $\begin{array}{l}3.2 \text { WHO recommended - vaccine } \\
\text { matching absent or unknown }\end{array}$ & 1 & 2082 & $\begin{array}{l}\text { Risk Ratio (M-H, Random, 95\% } \\
\mathrm{Cl})\end{array}$ & $1.05[0.88,1.25]$ \\
\hline $\begin{array}{l}\text { 3.3 Non WHO recommended - vac- } \\
\text { cine matching absent or unknown }\end{array}$ & 1 & 19887 & $\begin{array}{l}\text { Risk Ratio (M-H, Random, 95\% } \\
\mathrm{Cl})\end{array}$ & $0.98[0.92,1.05]$ \\
\hline 4 Local harms & 13 & & $\begin{array}{l}\text { Risk Ratio (M-H, Random, 95\% } \\
\text { Cl) }\end{array}$ & Subtotals only \\
\hline $\begin{array}{l}4.1 \text { Local - upper respiratory infec- } \\
\text { tion symptoms }\end{array}$ & 6 & 496 & $\begin{array}{l}\text { Risk Ratio (M-H, Random, 95\% } \\
\mathrm{Cl})\end{array}$ & $1.66[1.22,2.27]$ \\
\hline 4.2 Local - cough & 6 & 2401 & $\begin{array}{l}\text { Risk Ratio (M-H, Random, 95\% } \\
\mathrm{Cl})\end{array}$ & $1.51[1.08,2.10]$ \\
\hline 4.3 Local - coryza & 2 & 4782 & $\begin{array}{l}\text { Risk Ratio (M-H, Random, 95\% } \\
\mathrm{Cl})\end{array}$ & $1.56[1.26,1.94]$ \\
\hline 4.4 Local - sore throat & 7 & 6940 & $\begin{array}{l}\text { Risk Ratio (M-H, Random, 95\% } \\
\text { Cl) }\end{array}$ & $1.66[1.49,1.86]$ \\
\hline 4.5 Local - hoarseness & 1 & 306 & $\begin{array}{l}\text { Risk Ratio (M-H, Random, 95\% } \\
\text { Cl) }\end{array}$ & $1.21[0.51,2.83]$ \\
\hline $\begin{array}{l}4.6 \text { Local - combined endpoint (any } \\
\text { or highest symptom) }\end{array}$ & 3 & 4921 & $\begin{array}{l}\text { Risk Ratio (M-H, Random, 95\% } \\
\text { Cl) }\end{array}$ & $1.56[1.31,1.87]$ \\
\hline 5 Systemic harms & 7 & & $\begin{array}{l}\text { Risk Ratio (M-H, Random, 95\% } \\
\text { Cl) }\end{array}$ & Subtotals only \\
\hline 5.1 Systemic - myalgia & 4 & 1318 & $\begin{array}{l}\text { Risk Ratio (M-H, Random, 95\% } \\
\text { Cl) }\end{array}$ & $2.47[1.26,4.85]$ \\
\hline
\end{tabular}




\begin{tabular}{|c|c|c|c|c|}
\hline Outcome or subgroup title & No. of studies & $\begin{array}{l}\text { No. of partici- } \\
\text { pants }\end{array}$ & Statistical method & Effect size \\
\hline 5.2 Systemic - fever & 4 & 1318 & $\begin{array}{l}\text { Risk Ratio (M-H, Random, 95\% } \\
\text { Cl) }\end{array}$ & $1.01[0.54,1.92]$ \\
\hline $\begin{array}{l}5.3 \text { Systemic - fatigue or indisposi- } \\
\text { tion }\end{array}$ & 3 & 1018 & $\begin{array}{l}\text { Risk Ratio (M-H, Random, 95\% } \\
\text { Cl) }\end{array}$ & $1.39[0.93,2.07]$ \\
\hline 5.4 Systemic - headache & 2 & 975 & $\begin{array}{l}\text { Risk Ratio (M-H, Random, 95\% } \\
\mathrm{Cl} \text { ) }\end{array}$ & $1.54[1.09,2.18]$ \\
\hline $\begin{array}{l}5.5 \text { Systemic - combined endpoint } \\
\text { (any or highest symptom) }\end{array}$ & 5 & 1018 & $\begin{array}{l}\text { Risk Ratio (M-H, Random, 95\% } \\
\text { Cl) }\end{array}$ & $1.40[0.82,2.38]$ \\
\hline
\end{tabular}

\section{Analysis 2.1. Comparison 2 Live aerosol influenza vaccine versus placebo or 'do nothing', Outcome 1 Influenza.}

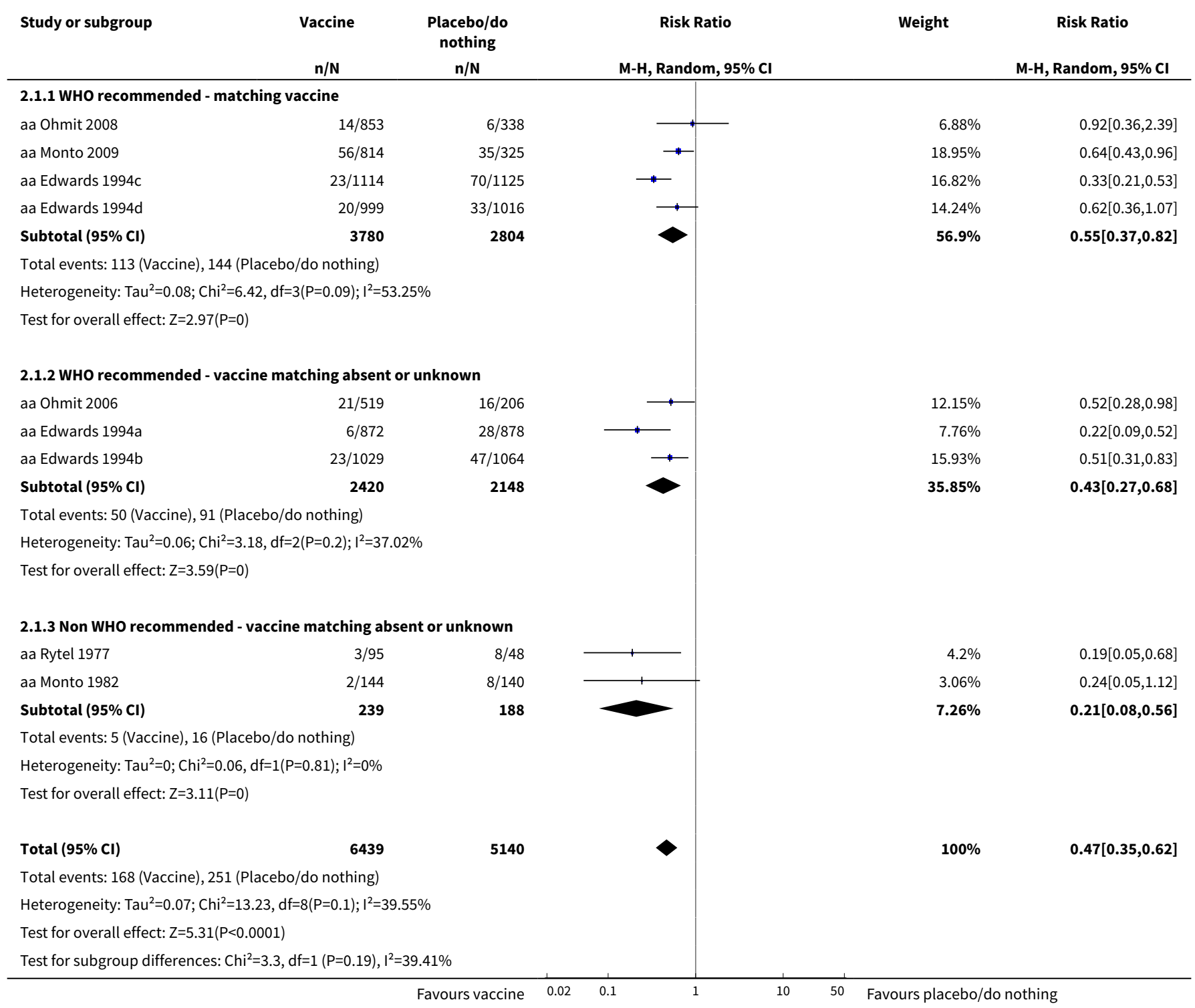


Analysis 2.2. Comparison 2 Live aerosol influenza vaccine versus placebo or 'do nothing', Outcome 2 Influenza-like illness.

\begin{tabular}{|c|c|c|c|c|c|}
\hline \multirow[t]{2}{*}{ Study or subgroup } & Vaccine & $\begin{array}{l}\text { Placebo/do } \\
\text { nothing }\end{array}$ & Risk Ratio & \multirow[t]{2}{*}{ Weight } & \multirow{2}{*}{$\begin{array}{c}\text { Risk Ratio } \\
\text { M-H, Random, } 95 \% \mathrm{CI}\end{array}$} \\
\hline & $n / N$ & $n / N$ & M-H, Random, 95\% Cl & & \\
\hline \multicolumn{6}{|c|}{ 2.2.1 WHO recommended - matching vaccine } \\
\hline aa Edwards 1994c & $201 / 1114$ & $240 / 1125$ & $\rightarrow-$ & $15.75 \%$ & $0.85[0.71,1]$ \\
\hline aa Edwards 1994d & $148 / 999$ & $146 / 1016$ & & $9.97 \%$ & $1.03[0.83,1.27]$ \\
\hline Subtotal $(95 \% \mathrm{Cl})$ & 2113 & 2141 & & $25.73 \%$ & $0.92[0.76,1.12]$ \\
\hline \multicolumn{6}{|c|}{ Heterogeneity: $\operatorname{Tau}^{2}=0.01 ; \mathrm{Chi}^{2}=2.07, \mathrm{df}=1(\mathrm{P}=0.15) ; \mathrm{I}^{2}=51.63 \%$} \\
\hline \multicolumn{6}{|c|}{ Test for overall effect: $Z=0.81(P=0.42)$} \\
\hline \multicolumn{6}{|c|}{ 2.2.2 WHO recommended - vaccine matching absent or unknown } \\
\hline aa Edwards 1994a & $89 / 872$ & $92 / 878$ & $\longrightarrow$ & $5.85 \%$ & $0.97[0.74,1.28]$ \\
\hline aa Edwards 1994b & $208 / 1029$ & $262 / 1064$ & $\rightarrow$ & $17.26 \%$ & $0.82[0.7,0.96]$ \\
\hline Subtotal $(95 \% \mathrm{CI})$ & 4775 & 3375 & 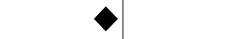 & $65.82 \%$ & $0.89[0.82,0.97]$ \\
\hline \multicolumn{6}{|c|}{ Total events: 1048 (Vaccine), 766 (Placebo/do nothing) } \\
\hline \multicolumn{6}{|c|}{ Heterogeneity: $\mathrm{Tau}^{2}=0 ; \mathrm{Chi}^{2}=1.55, \mathrm{df}=2(\mathrm{P}=0.46) ; \mathrm{I}^{2}=0 \%$} \\
\hline \multicolumn{6}{|c|}{ Test for overall effect: $Z=2.77(P=0.01)$} \\
\hline \multicolumn{6}{|c|}{ 2.2.3 Non WHO recommended - vaccine matching absent or unknown } \\
\hline aa Monto 1982 & $70 / 144$ & $74 / 140$ & $\rightarrow$ & $8.45 \%$ & $0.92[0.73,1.16]$ \\
\hline Subtotal $(95 \% \mathrm{Cl})$ & 144 & 140 & & $8.45 \%$ & $0.92[0.73,1.16]$ \\
\hline \multicolumn{6}{|c|}{ Total events: 70 (Vaccine), 74 (Placebo/do nothing) } \\
\hline \multicolumn{6}{|c|}{ Heterogeneity: $\operatorname{Tau}^{2}=0 ; \mathrm{Chi}^{2}=0, \mathrm{df}=0(\mathrm{P}<0.0001) ; \mathrm{I}^{2}=100 \%$} \\
\hline \multicolumn{6}{|c|}{ Test for overall effect: $Z=0.72(P=0.47)$} \\
\hline Total $(95 \% \mathrm{Cl})$ & 7032 & 5656 & $\boldsymbol{\gamma}$ & $100 \%$ & $0.9[0.84,0.96]$ \\
\hline \multicolumn{6}{|c|}{ Heterogeneity: $\operatorname{Tau}^{2}=0 ; \mathrm{Chi}^{2}=3.76, \mathrm{df}=5(\mathrm{P}=0.58) ; \mathrm{I}^{2}=0 \%$} \\
\hline \multicolumn{6}{|c|}{ Test for overall effect: $Z=3.14(P=0)$} \\
\hline Test for subgroup dif & $d f=1(P=0.92)$, & & & & \\
\hline
\end{tabular}

Analysis 2.3. Comparison 2 Live aerosol influenza vaccine versus placebo or 'do nothing', Outcome 3 Influenza cases (clinically defined without clear definition).

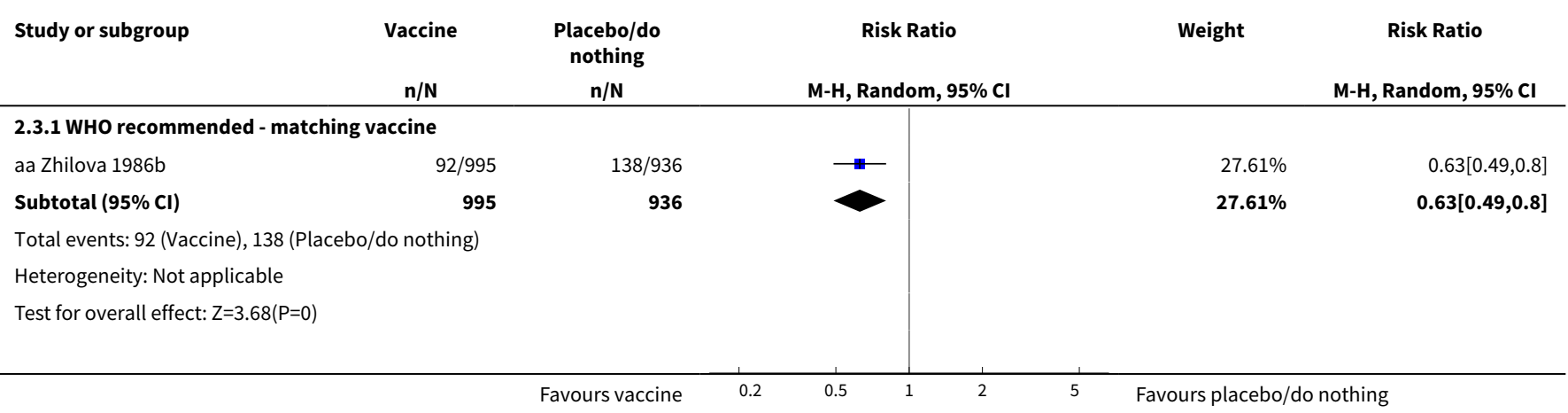




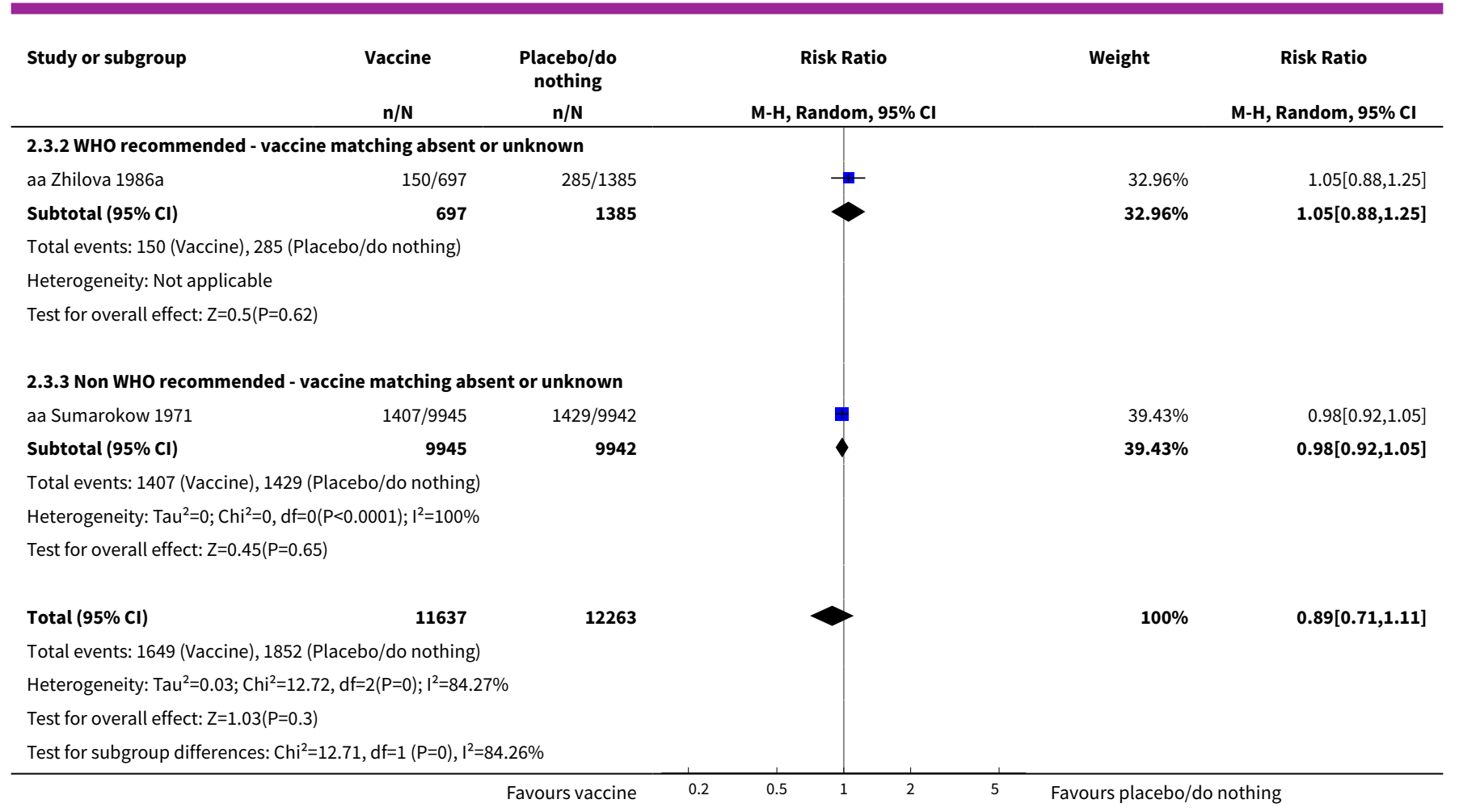

Analysis 2.4. Comparison 2 Live aerosol influenza vaccine versus placebo or 'do nothing', Outcome 4 Local harms.

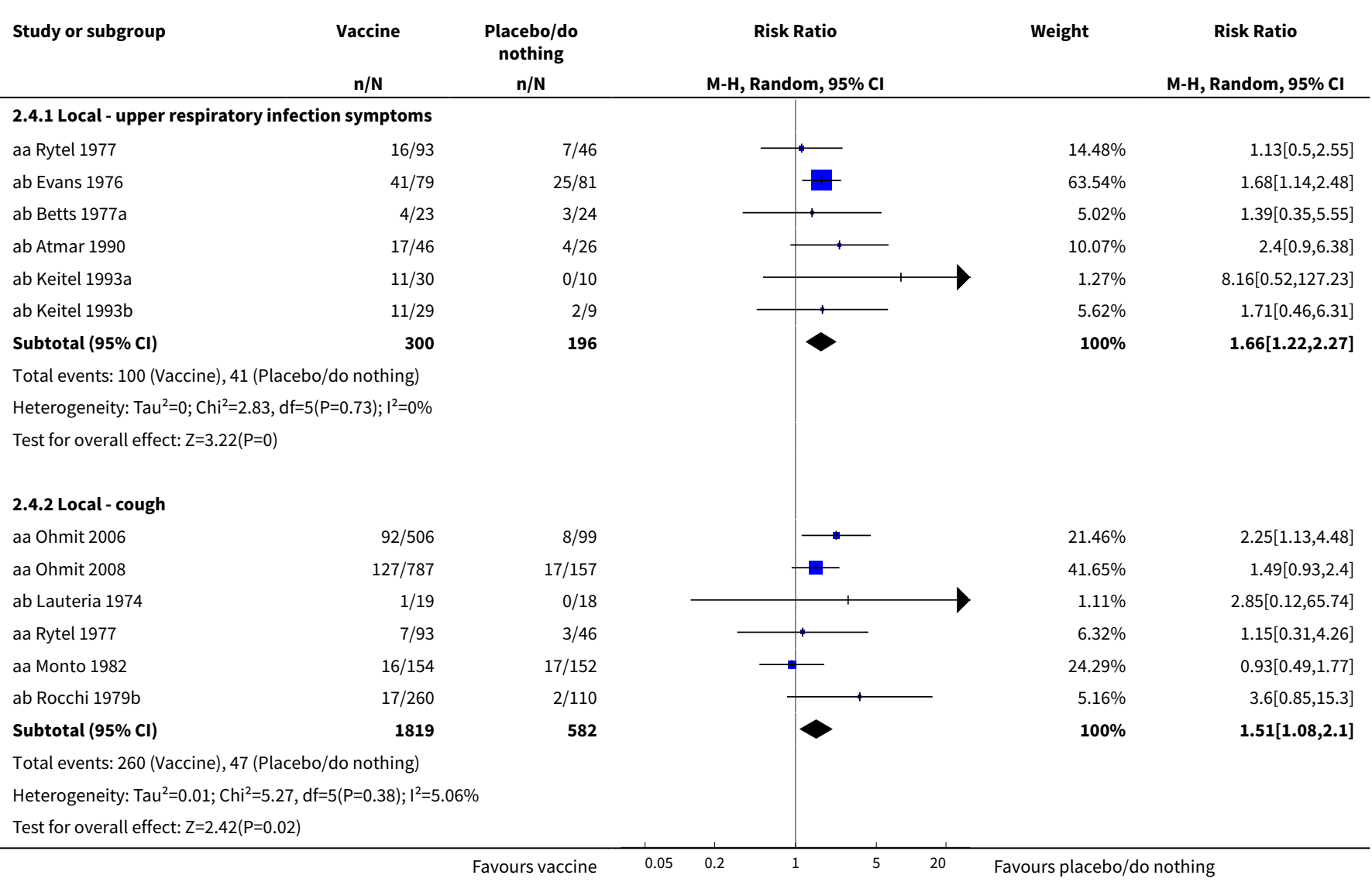




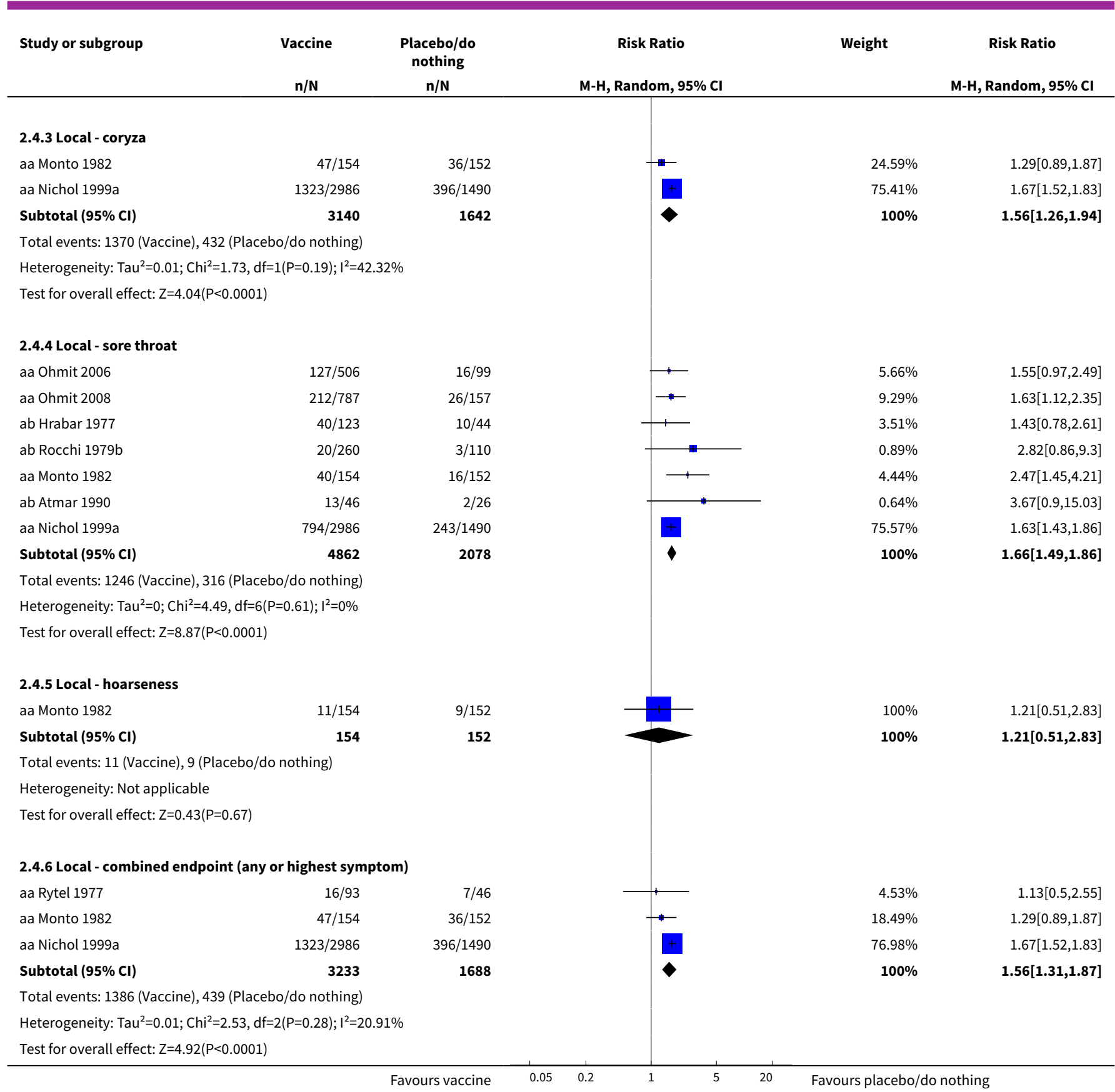

\section{Analysis 2.5. Comparison 2 Live aerosol influenza vaccine versus placebo or 'do nothing', Outcome 5 Systemic harms.}

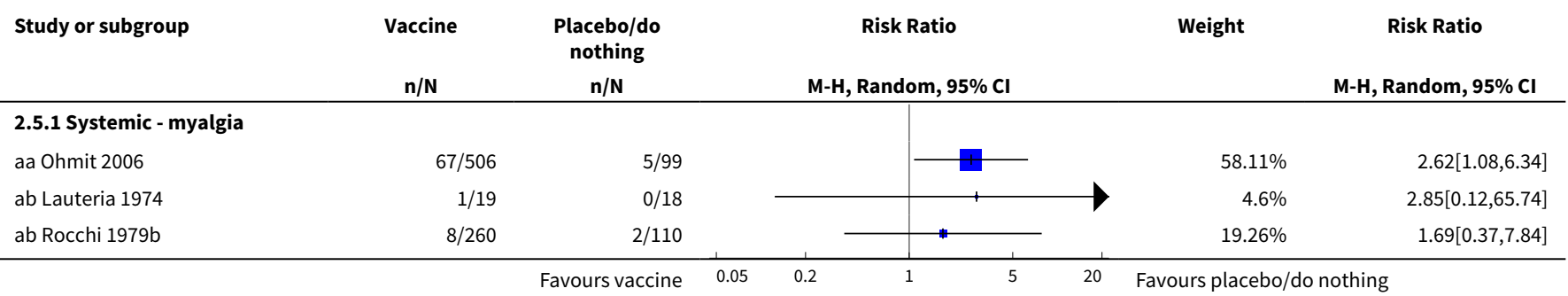




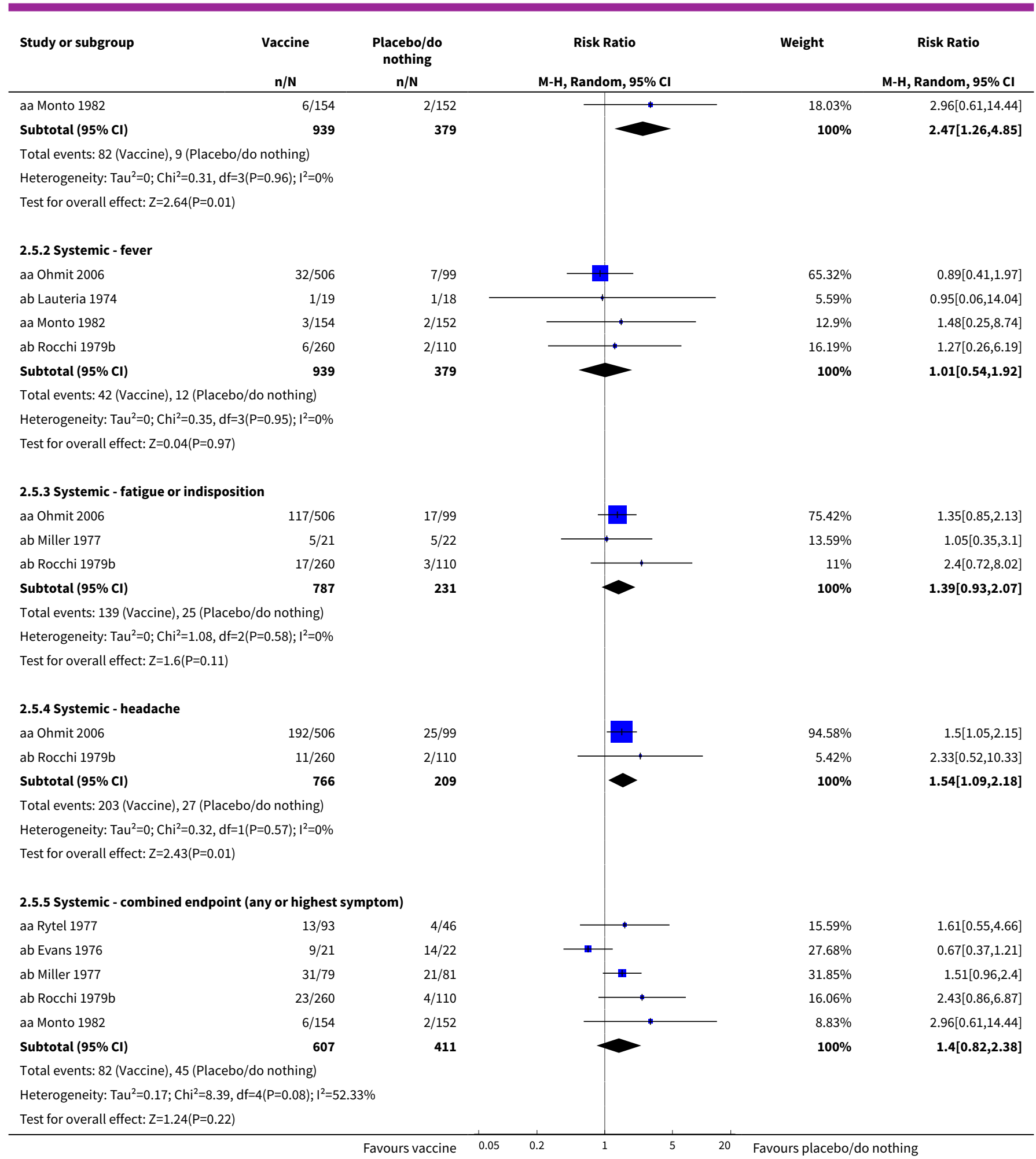


Comparison 3. Inactivated aerosol influenza vaccine versus placebo or 'do nothing'

\begin{tabular}{|c|c|c|c|c|}
\hline Outcome or subgroup title & No. of studies & $\begin{array}{l}\text { No. of partici- } \\
\text { pants }\end{array}$ & Statistical method & Effect size \\
\hline 1 Influenza & 1 & 1348 & Odds Ratio (M-H, Random, 95\% Cl) & $0.38[0.14,1.02]$ \\
\hline $\begin{array}{l}1.1 \text { WHO recommended - vac- } \\
\text { cine matching absent or un- } \\
\text { known }\end{array}$ & 1 & 1348 & Odds Ratio (M-H, Random, 95\% Cl) & $0.38[0.14,1.02]$ \\
\hline $\begin{array}{l}1.2 \text { WHO recommended - } \\
\text { matching vaccine }\end{array}$ & 0 & 0 & Odds Ratio (M-H, Random, 95\% Cl) & $0.0[0.0,0.0]$ \\
\hline 2 Local harms & 3 & 1578 & Risk Ratio (M-H, Random, 95\% Cl) & $0.95[0.71,1.27]$ \\
\hline 2.1 Local - sore throat & 3 & 1500 & Risk Ratio (M-H, Random, 95\% Cl) & $0.85[0.54,1.33]$ \\
\hline $\begin{array}{l}2.2 \text { Local - combined endpoint } \\
\text { (any or highest symptom) }\end{array}$ & 1 & 78 & Risk Ratio (M-H, Random, 95\% Cl) & $1.03[0.71,1.48]$ \\
\hline 3 Systemic harms & 3 & 1880 & Risk Ratio (M-H, Random, 95\% Cl) & $1.07[0.71,1.62]$ \\
\hline 3.1 Systemic - myalgia & 2 & 151 & Risk Ratio (M-H, Random, 95\% Cl) & $0.90[0.36,2.25]$ \\
\hline $\begin{array}{l}3.2 \text { Systemic - fatigue or indis- } \\
\text { position }\end{array}$ & 2 & 151 & Risk Ratio (M-H, Random, 95\% Cl) & $1.40[0.52,3.75]$ \\
\hline 3.3 Systemic - headache & 2 & 151 & Risk Ratio (M-H, Random, 95\% Cl) & $1.52[0.85,2.72]$ \\
\hline 3.4 Systemic - fever & 1 & 1349 & Risk Ratio (M-H, Random, 95\% Cl) & $0.49[0.03,7.80]$ \\
\hline $\begin{array}{l}\text { 3.5 Systemic - combined end- } \\
\text { point (any or highest symp- } \\
\text { tom) }\end{array}$ & 1 & 78 & Risk Ratio (M-H, Random, 95\% Cl) & $0.36[0.12,1.04]$ \\
\hline
\end{tabular}

Analysis 3.1. Comparison 3 Inactivated aerosol influenza vaccine versus placebo or 'do nothing', Outcome 1 Influenza.

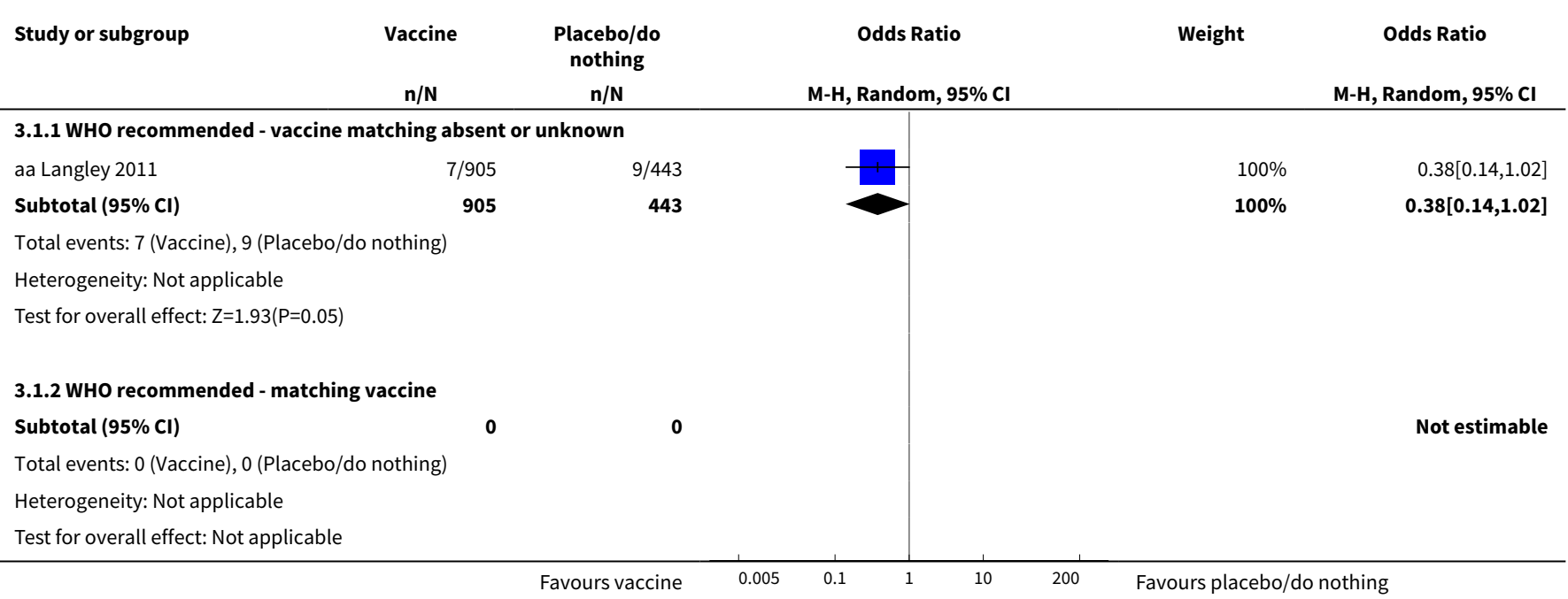




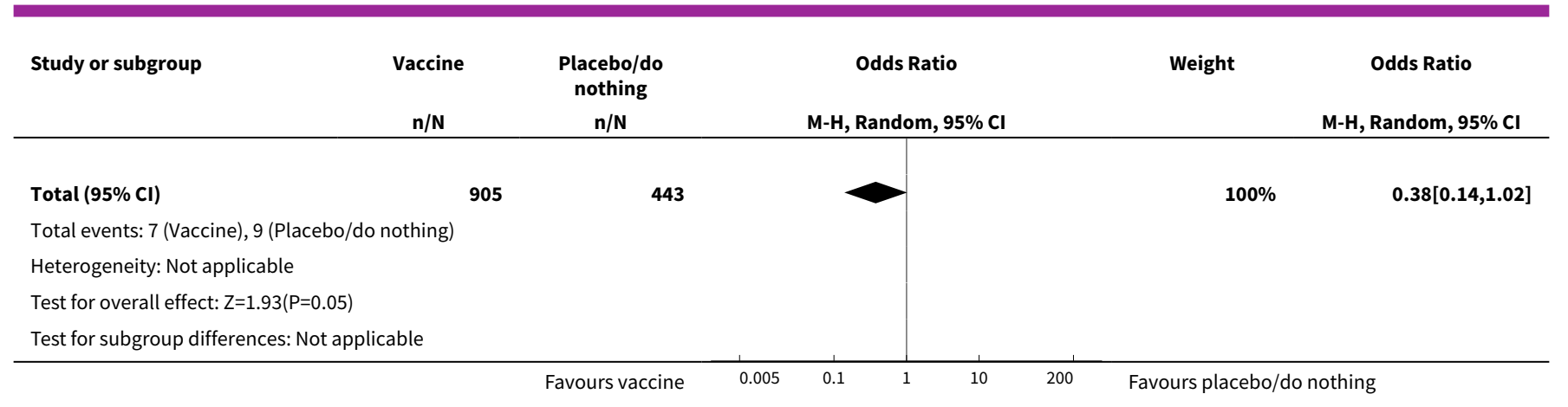

Analysis 3.2. Comparison 3 Inactivated aerosol influenza vaccine versus placebo or 'do nothing', Outcome 2 Local harms.

\begin{tabular}{ccccc} 
Study or subgroup & Vaccine & $\begin{array}{c}\text { Placebo/do } \\
\text { nothing } \\
n / N\end{array}$ & Risk Ratio & Wisk Ratio \\
\hline
\end{tabular}

3.2.1 Local - sore throat

$\begin{array}{lrr}\text { aa Langley } 2011 & 25 / 906 & 14 / 443 \\ \text { ab Boyce 2000 } & 20 / 60 & 4 / 13 \\ \text { ab Langley 2005 } & 10 / 60 & 5 / 18 \\ \text { Subtotal (95\% CI) } & \mathbf{1 0 2 6} & \mathbf{4 7 4}\end{array}$

Total events: 55 (Vaccine), 23 (Placebo/do nothing) Heterogeneity: $\mathrm{Tau}^{2}=0 ; \mathrm{Chi}^{2}=0.82, \mathrm{df}=2(\mathrm{P}=0.66) ; \mathrm{I}^{2}=0 \%$

Test for overall effect: $\mathrm{Z}=0.72(\mathrm{P}=0.47)$

3.2.2 Local - combined endpoint (any or highest symptom)

$\begin{array}{lr}\text { ab Langley } 2005 & 41 / 60 \\ \text { Subtotal (95\% Cl) } & \mathbf{6 0}\end{array}$

\section{Analysis 3.3. Comparison 3 Inactivated aerosol influenza vaccine versus placebo or 'do nothing', Outcome 3 Systemic harms.}

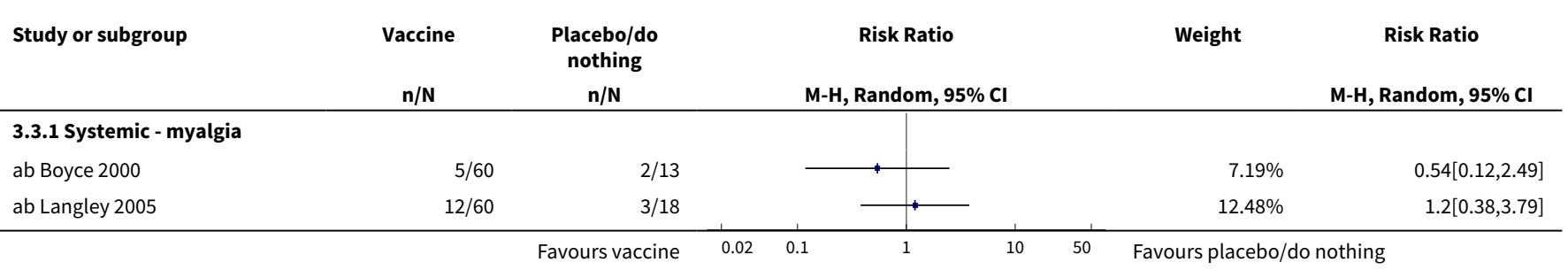




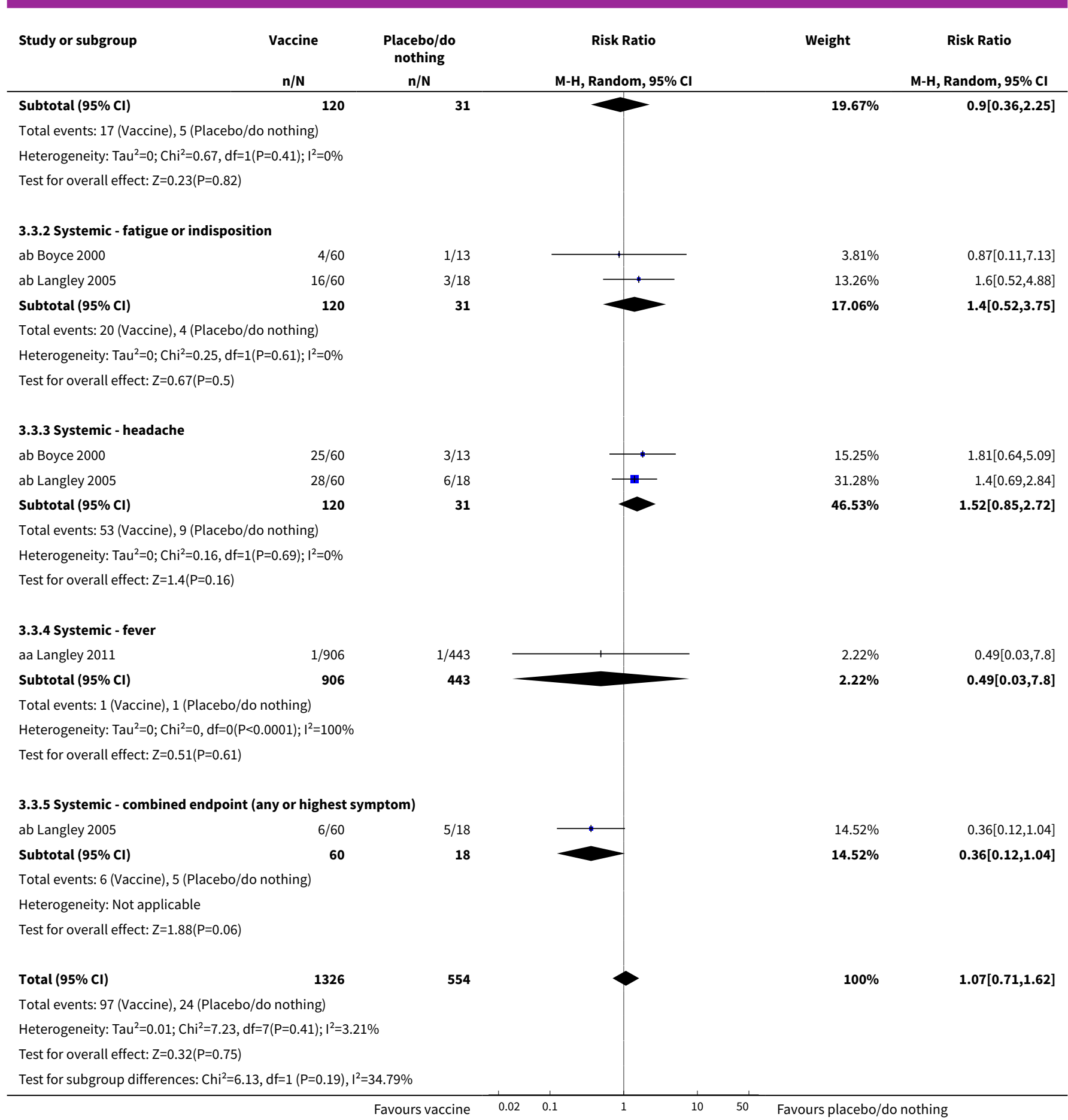

\section{Comparison 4. Inactivated parenteral influenza vaccine versus placebo or 'do nothing' administered during} pregnancy

\begin{tabular}{lllll}
\hline Outcome or subgroup title & No. of studies & $\begin{array}{l}\text { No. of partici- } \\
\text { pants }\end{array}$ & Statistical method & Effect size \\
\hline 1 Influenza in mothers & 1 & Risk Ratio (M-H, Random, 95\% Cl) & Totals not selected \\
\hline
\end{tabular}




\begin{tabular}{|c|c|c|c|c|}
\hline Outcome or subgroup title & No. of studies & $\begin{array}{l}\text { No. of partici- } \\
\text { pants }\end{array}$ & Statistical method & Effect size \\
\hline 1.1 TIV containing $\mathrm{pH} 1 \mathrm{~N} 1$ & 1 & & Risk Ratio (M-H, Random, 95\% Cl) & $0.0[0.0,0.0]$ \\
\hline $\begin{array}{l}2 \text { Influenza-like illness in } \\
\text { mothers }\end{array}$ & 2 & 2342 & Risk Ratio (M-H, Random, 95\% Cl) & $0.62[0.20,1.95]$ \\
\hline 2.1 TIV containing $\mathrm{pH} 1 \mathrm{~N} 1$ & 1 & 2116 & Risk Ratio (M-H, Random, 95\% Cl) & $0.96[0.79,1.16]$ \\
\hline 2.2 Monovalent pH1N1 & 1 & 226 & Risk Ratio (M-H, Random, 95\% Cl) & $0.28[0.08,1.02]$ \\
\hline 3 Influenza in newborn & 1 & & Risk Ratio (M-H, Random, 95\% Cl) & Totals not selected \\
\hline 3.1 TIV containing $\mathrm{pH} 1 \mathrm{~N} 1$ & 1 & & Risk Ratio (M-H, Random, 95\% Cl) & $0.0[0.0,0.0]$ \\
\hline $\begin{array}{l}4 \text { Influenza-like illness in } \\
\text { newborn }\end{array}$ & 1 & & Risk Ratio (M-H, Random, 95\% Cl) & Totals not selected \\
\hline 4.1 TIV containing $\mathrm{pH} 1 \mathrm{~N} 1$ & 1 & & Risk Ratio (M-H, Random, 95\% Cl) & $0.0[0.0,0.0]$ \\
\hline
\end{tabular}

\section{Analysis 4.1. Comparison 4 Inactivated parenteral influenza vaccine versus placebo or 'do nothing' administered during pregnancy, Outcome 1 Influenza in mothers.}

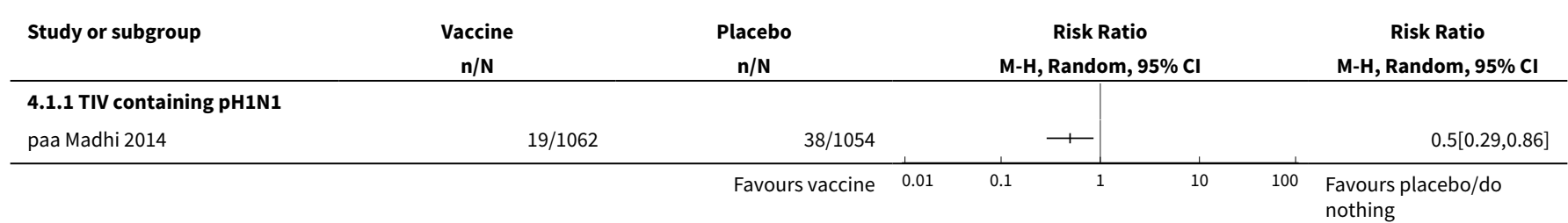

Analysis 4.2. Comparison 4 Inactivated parenteral influenza vaccine versus placebo or 'do nothing' administered during pregnancy, Outcome 2 Influenza-like illness in mothers.

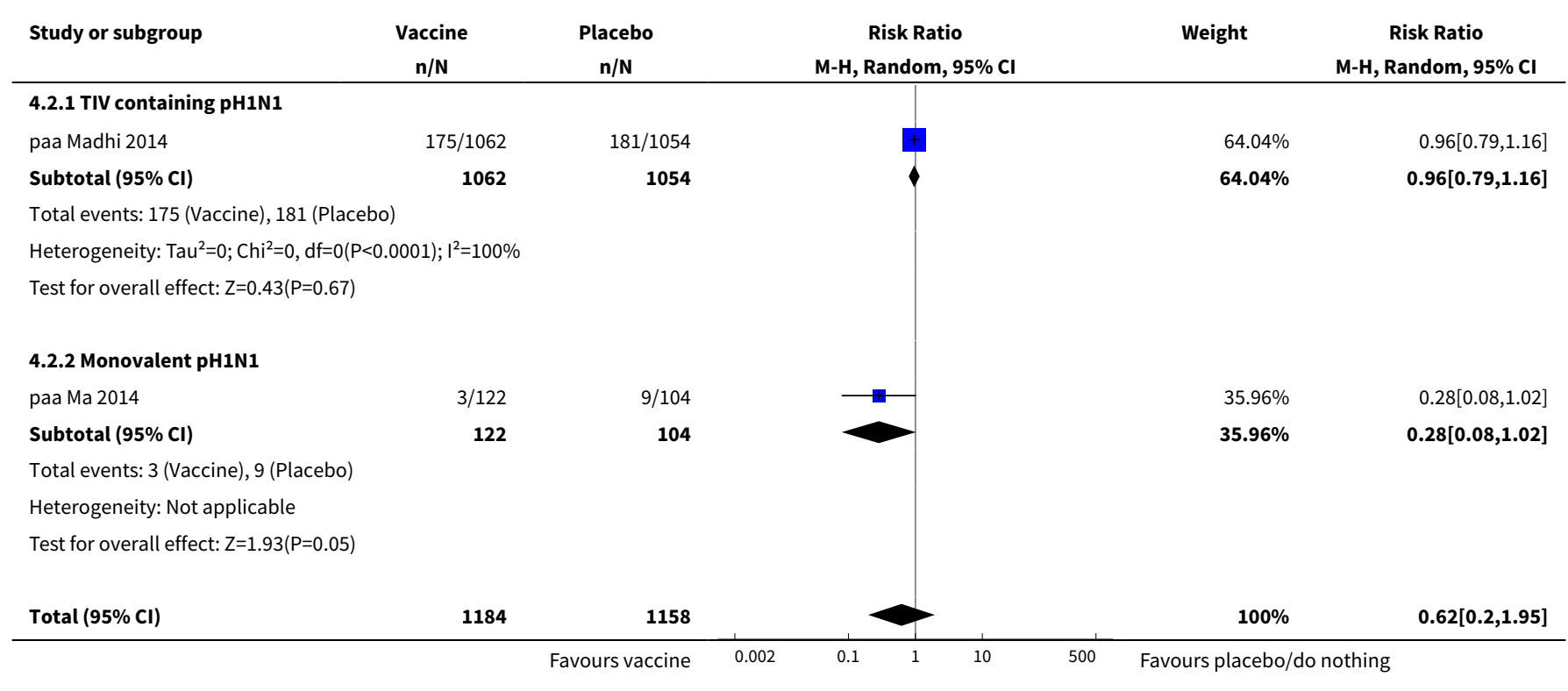




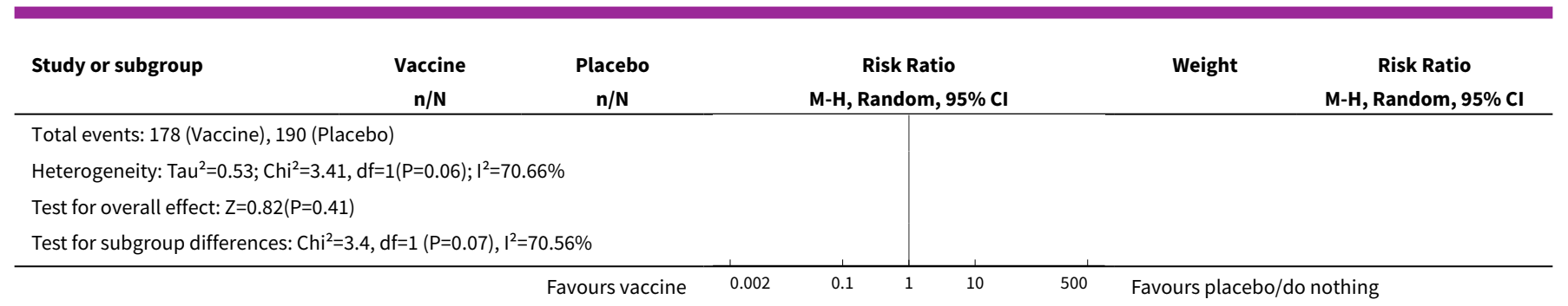

Analysis 4.3. Comparison 4 Inactivated parenteral influenza vaccine versus placebo or 'do nothing' administered during pregnancy, Outcome 3 Influenza in newborn.

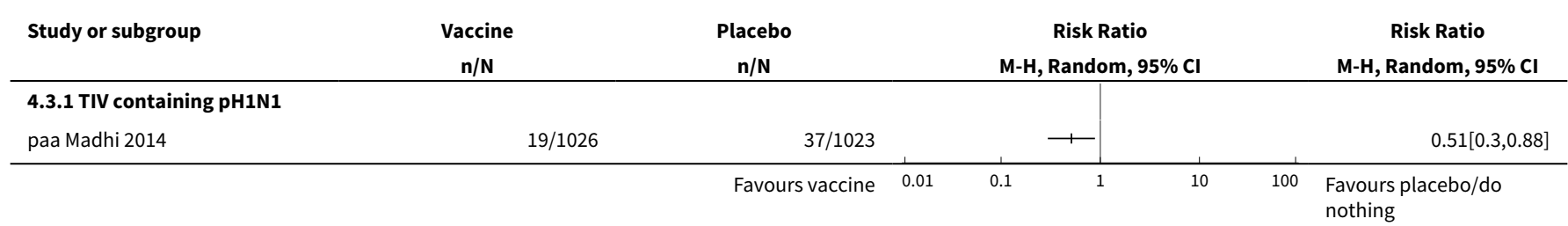

Analysis 4.4. Comparison 4 Inactivated parenteral influenza vaccine versus placebo or 'do nothing' administered during pregnancy, Outcome 4 Influenza-like illness in newborn.

\begin{tabular}{|c|c|c|c|c|}
\hline Study or subgroup & $\begin{array}{c}\text { Vaccine } \\
\mathbf{n} / \mathbf{N}\end{array}$ & $\begin{array}{c}\text { Placebo } \\
\mathbf{n} / \mathbf{N}\end{array}$ & $\begin{array}{c}\text { Risk Ratio } \\
\text { M-H, Random, 95\% Cl }\end{array}$ & $\begin{array}{c}\text { Risk Ratio } \\
\text { M-H, Random, 95\% Cl }\end{array}$ \\
\hline \multicolumn{5}{|l|}{ 4.4.1 TIV containing $\mathrm{pH} 1 \mathrm{~N} 1$} \\
\hline paa Madhi 2014 & $595 / 1026$ & $584 / 1023$ & -1 & $1.02[0.94,1.09]$ \\
\hline & & Favours vaccine & 1 & $\begin{array}{l}\text { Favours placebo/do } \\
\text { nothing }\end{array}$ \\
\hline
\end{tabular}

\section{Comparison 5. Inactivated parenteral influenza vaccine versus placebo - cohort studies}

\begin{tabular}{|c|c|c|c|c|}
\hline Outcome or subgroup title & No. of studies & $\begin{array}{l}\text { No. of partici- } \\
\text { pants }\end{array}$ & Statistical method & Effect size \\
\hline $\begin{array}{l}1 \text { Seasonal inactivated vaccine effectiveness } \\
\text { in mothers - pregnant women }\end{array}$ & 4 & & $\begin{array}{l}\text { Risk Ratio (M-H, Ran- } \\
\text { dom, } 95 \% \mathrm{Cl})\end{array}$ & Subtotals only \\
\hline $\begin{array}{l}1.1 \mathrm{H} 1 \mathrm{~N} 1 \text { - vaccine - effectiveness ILI (unad- } \\
\text { justed data) }\end{array}$ & 1 & 7328 & $\begin{array}{l}\text { Risk Ratio (M-H, Ran- } \\
\text { dom, } 95 \% \mathrm{Cl})\end{array}$ & $0.11[0.06,0.21]$ \\
\hline $\begin{array}{l}\text { 1.2 Seasonal - vaccine - effectiveness ILI - } \\
\text { (unadjusted data) }\end{array}$ & 3 & 50507 & $\begin{array}{l}\text { Risk Ratio (M-H, Ran- } \\
\text { dom, } 95 \% \mathrm{Cl})\end{array}$ & $0.54[0.24,1.18]$ \\
\hline $\begin{array}{l}2 \text { Seasonal inactivated vaccine effectiveness } \\
\text { in newborns - pregnant women }\end{array}$ & 2 & & $\begin{array}{l}\text { Hazard Ratio (Random, } \\
95 \% \mathrm{Cl} \text { ) }\end{array}$ & Subtotals only \\
\hline $\begin{array}{l}2.1 \text { Seasonal vaccine effectiveness ILI (HR } \\
\text { adjusted data) }\end{array}$ & 2 & & $\begin{array}{l}\text { Hazard Ratio (Random, } \\
95 \% \mathrm{Cl} \text { ) }\end{array}$ & $0.96[0.90,1.03]$ \\
\hline $\begin{array}{l}3 \text { Seasonal inactivated vaccine effectiveness } \\
\text { in newborns - pregnant women }\end{array}$ & 1 & & $\begin{array}{l}\text { Risk Ratio (Random, } \\
95 \% \mathrm{Cl} \text { ) }\end{array}$ & Subtotals only \\
\hline
\end{tabular}




\begin{tabular}{|c|c|c|c|c|}
\hline Outcome or subgroup title & No. of studies & $\begin{array}{l}\text { No. of partici- } \\
\text { pants }\end{array}$ & Statistical method & Effect size \\
\hline $\begin{array}{l}\text { 3.1 Seasonal vaccine effectiveness ILI (RR } \\
\text { adjusted data) }\end{array}$ & 1 & & $\begin{array}{l}\text { Risk Ratio (Random, } \\
95 \% \mathrm{Cl} \text { ) }\end{array}$ & $0.92[0.73,1.16]$ \\
\hline $\begin{array}{l}\text { 3.2 Seasonal vaccine efficacy influenza - lab- } \\
\text { oratory-confirmed }\end{array}$ & 1 & & $\begin{array}{l}\text { Risk Ratio (Random, } \\
95 \% \mathrm{Cl} \text { ) }\end{array}$ & $0.59[0.37,0.94]$ \\
\hline $\begin{array}{l}4 \mathrm{H} 1 \mathrm{~N} 1 \text { vaccine - safety - pregnancy-related } \\
\text { outcomes - pregnant women }\end{array}$ & 15 & & $\begin{array}{l}\text { Odds Ratio (Random, } \\
95 \% \mathrm{Cl} \text { ) }\end{array}$ & Subtotals only \\
\hline 4.1 Abortion (OR adjusted data) & 5 & & $\begin{array}{l}\text { Odds Ratio (Random, } \\
95 \% \mathrm{Cl} \text { ) }\end{array}$ & $0.75[0.62,0.90]$ \\
\hline 4.2 Abortion (HR adjusted data) & 3 & & $\begin{array}{l}\text { Odds Ratio (Random, } \\
95 \% \mathrm{Cl} \text { ) }\end{array}$ & $0.81[0.63,1.04]$ \\
\hline $\begin{array}{l}4.3 \text { Congenital malformation (OR adjusted } \\
\text { data) }\end{array}$ & 6 & & $\begin{array}{l}\text { Odds Ratio (Random, } \\
95 \% \mathrm{Cl} \text { ) }\end{array}$ & $1.11[0.99,1.23]$ \\
\hline $\begin{array}{l}\text { 4.4 Prematurity ( }<37 \text { weeks) (OR unadjusted } \\
\text { data) }\end{array}$ & 11 & & $\begin{array}{l}\text { Odds Ratio (Random, } \\
95 \% \mathrm{Cl} \text { ) }\end{array}$ & $0.76[0.67,0.85]$ \\
\hline $\begin{array}{l}\text { 4.5 Prematurity (<37 weeks) (OR adjusted } \\
\text { data) }\end{array}$ & 7 & & $\begin{array}{l}\text { Odds Ratio (Random, } \\
95 \% \mathrm{Cl} \text { ) }\end{array}$ & $0.84[0.76,0.93]$ \\
\hline $\begin{array}{l}\text { 4.6 Prematurity ( }<37 \text { weeks) (HR adjusted } \\
\text { data) }\end{array}$ & 2 & & $\begin{array}{l}\text { Odds Ratio (Random, } \\
95 \% \mathrm{Cl} \text { ) }\end{array}$ & $1.11[0.46,2.68]$ \\
\hline $\begin{array}{l}\text { 4.7 Prematurity }(<37 \text { weeks) vaccination in I } \\
\text { trimester OR adjusted data }\end{array}$ & 2 & & $\begin{array}{l}\text { Odds Ratio (Random, } \\
95 \% \mathrm{Cl} \text { ) }\end{array}$ & $1.08[0.92,1.28]$ \\
\hline $\begin{array}{l}\text { 4.8 Prematurity }(<37 \text { weeks) vaccination in } \\
\text { II/III trimester OR adjusted data }\end{array}$ & 2 & & $\begin{array}{l}\text { Odds Ratio (Random, } \\
95 \% \mathrm{Cl} \text { ) }\end{array}$ & $0.96[0.87,1.06]$ \\
\hline 4.9 Neonatal death (OR adjusted data) & 2 & & $\begin{array}{l}\text { Odds Ratio (Random, } \\
95 \% \mathrm{Cl} \text { ) }\end{array}$ & $1.09[0.40,2.95]$ \\
\hline $\begin{array}{l}5 \text { Seasonal vaccine - safety - pregnancy-re- } \\
\text { lated outcomes - pregnant women }\end{array}$ & 7 & & $\begin{array}{l}\text { Odds Ratio (Random, } \\
95 \% \mathrm{Cl} \text { ) }\end{array}$ & Subtotals only \\
\hline 5.1 Abortion (OR unadjusted data) & 1 & & $\begin{array}{l}\text { Odds Ratio (Random, } \\
95 \% \mathrm{Cl} \text { ) }\end{array}$ & $0.60[0.41,0.86]$ \\
\hline $\begin{array}{l}5.2 \text { Congenital malformation (OR unadjust- } \\
\text { ed data) }\end{array}$ & 2 & & $\begin{array}{l}\text { Odds Ratio (Random, } \\
95 \% \mathrm{Cl} \text { ) }\end{array}$ & $0.55[0.08,3.73]$ \\
\hline 5.3 Prematurity (OR unadjusted data) & 6 & & $\begin{array}{l}\text { Odds Ratio (Random, } \\
95 \% \mathrm{Cl} \text { ) }\end{array}$ & $0.95[0.82,1.10]$ \\
\hline 5.4 Prematurity (OR adjusted data) & 2 & & $\begin{array}{l}\text { Odds Ratio (Random, } \\
95 \% \mathrm{Cl} \text { ) }\end{array}$ & $0.93[0.82,1.06]$ \\
\hline 5.5 Neonatal death (OR unadjusted data) & 1 & & $\begin{array}{l}\text { Odds Ratio (Random, } \\
95 \% \mathrm{Cl} \text { ) }\end{array}$ & $0.55[0.35,0.88]$ \\
\hline
\end{tabular}




\begin{tabular}{|c|c|c|c|c|}
\hline Outcome or subgroup title & No. of studies & $\begin{array}{l}\text { No. of partici- } \\
\text { pants }\end{array}$ & Statistical method & Effect size \\
\hline 6 Seasonal vaccine containing $\mathrm{H} 1 \mathrm{~N} 1$ & 2 & & $\begin{array}{l}\text { Risk Ratio (Random, } \\
95 \% \mathrm{Cl} \text { ) }\end{array}$ & Subtotals only \\
\hline $\begin{array}{l}\text { 6.1 Prematurity ( } 37 \text { weeks) vaccination in I } \\
\text { trimester HR adjusted data }\end{array}$ & 2 & & $\begin{array}{l}\text { Risk Ratio (Random, } \\
95 \% \mathrm{Cl} \text { ) }\end{array}$ & $1.63[0.76,3.47]$ \\
\hline $\begin{array}{l}\text { 6.2 Prematurity ( }<37 \text { weeks) vaccination in II } \\
\text { trimester HR adjusted data }\end{array}$ & 2 & & $\begin{array}{l}\text { Risk Ratio (Random, } \\
95 \% \mathrm{Cl} \text { ) }\end{array}$ & $1.48[0.21,10.64]$ \\
\hline $\begin{array}{l}\text { 6.3 Prematurity ( }<37 \text { weeks) vaccination in } \\
\text { III trimester HR adjusted data }\end{array}$ & 2 & & $\begin{array}{l}\text { Risk Ratio (Random, } \\
95 \% \mathrm{Cl} \text { ) }\end{array}$ & $1.37[0.44,4.25]$ \\
\hline $\begin{array}{l}\text { 6.4 Prematurity ( }<37 \text { weeks) vaccination at } \\
\text { any time during pregnancy HR adjusted data }\end{array}$ & 2 & & $\begin{array}{l}\text { Risk Ratio (Random, } \\
95 \% \mathrm{Cl} \text { ) }\end{array}$ & $1.75[0.57,5.44]$ \\
\hline
\end{tabular}

Analysis 5.1. Comparison 5 Inactivated parenteral influenza vaccine versus placebo - cohort
studies, Outcome 1 Seasonal inactivated vaccine effectiveness in mothers - pregnant women.

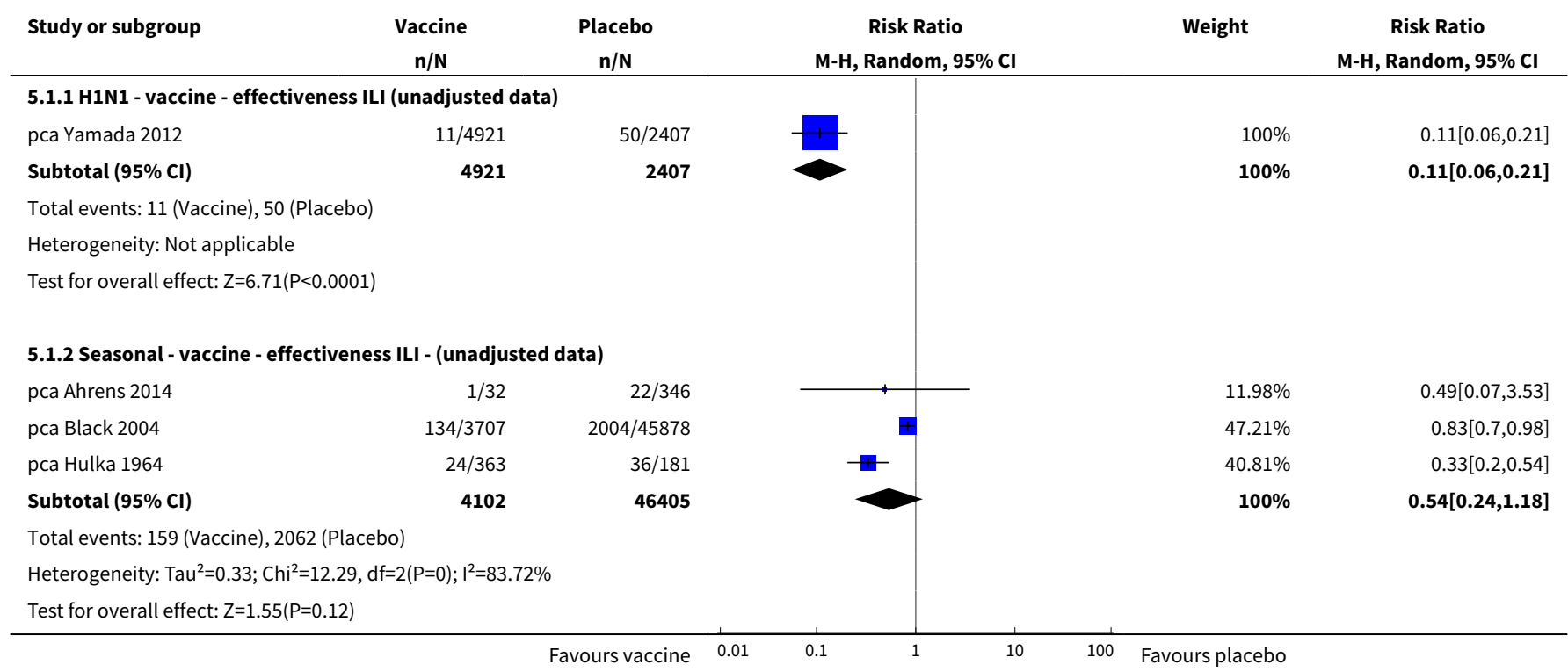

Analysis 5.2. Comparison 5 Inactivated parenteral influenza vaccine versus placebo - cohort studies, Outcome 2 Seasonal inactivated vaccine effectiveness in newborns - pregnant women.

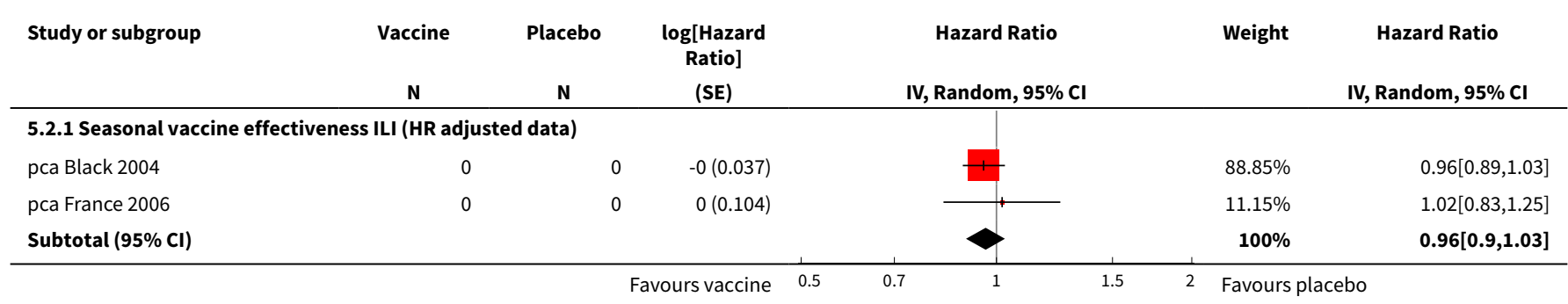




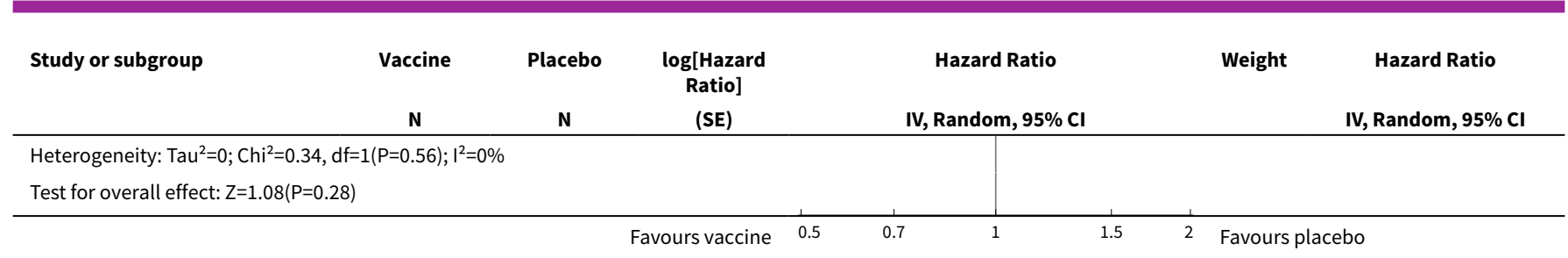

Analysis 5.3. Comparison 5 Inactivated parenteral influenza vaccine versus placebo - cohort studies, Outcome 3 Seasonal inactivated vaccine effectiveness in newborns - pregnant women.

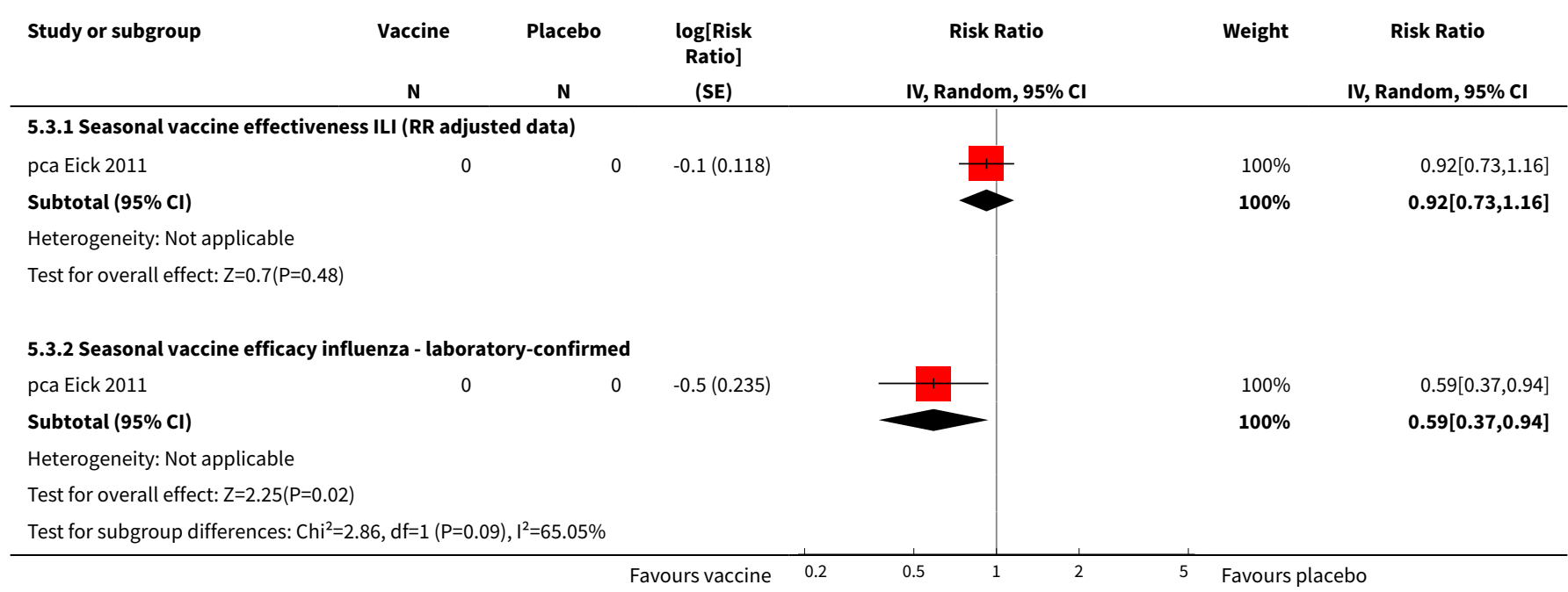

\section{Analysis 5.4. Comparison 5 Inactivated parenteral influenza vaccine versus placebo - cohort studies, Outcome 4 H1N1 vaccine - safety - pregnancy-related outcomes - pregnant women.}

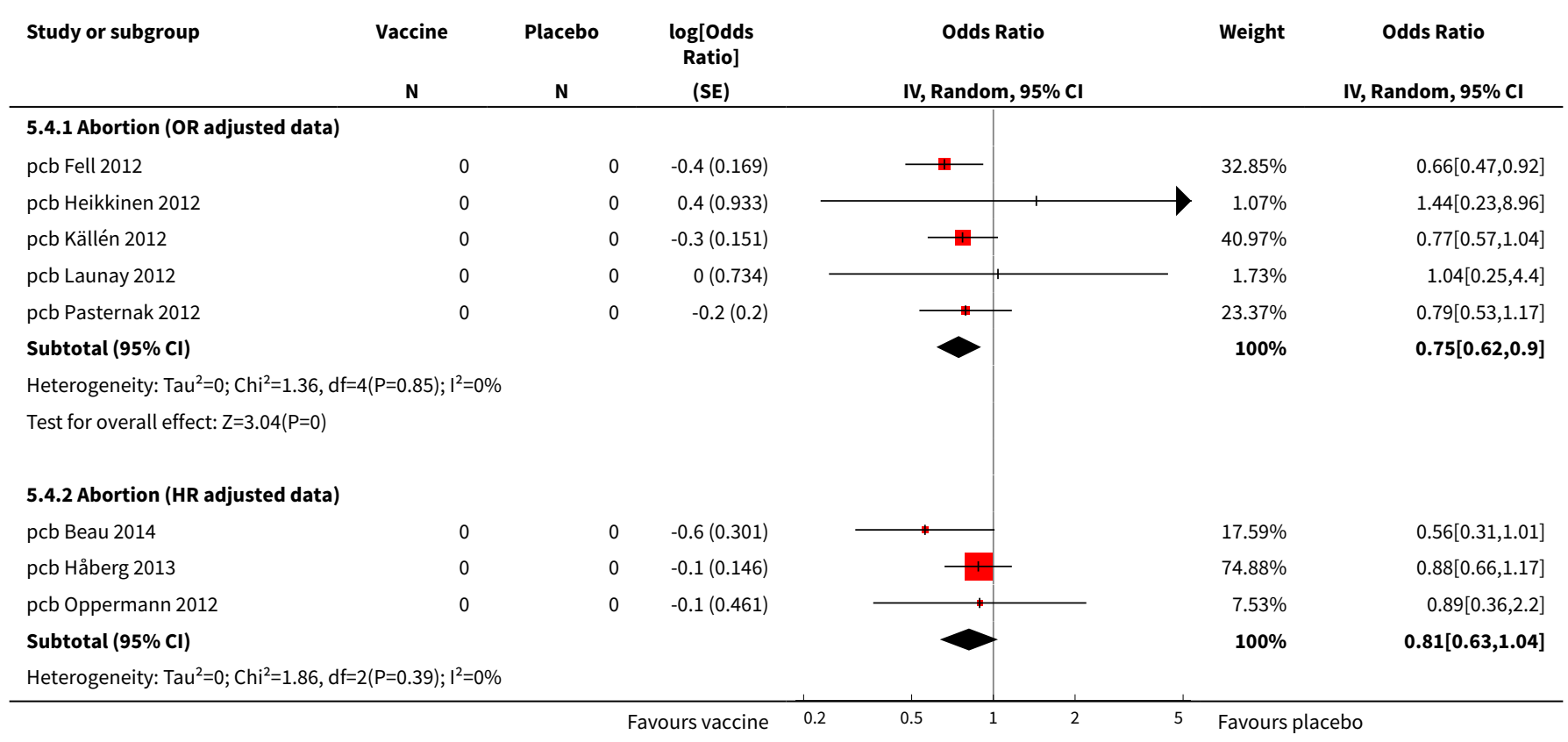




$\begin{array}{ccccc}\text { Study or subgroup } & \text { Vaccine } & \text { Placebo } & \begin{array}{c}\text { log[Odds } \\ \text { Ratio] }\end{array} & \text { Odds Ratio } \\ & \text { N } & \text { N } & \text { (SE) } & \text { IV, Random, } 95 \% \mathrm{Cl}\end{array}$

Test for overall effect: $\mathrm{Z}=1.63(\mathrm{P}=0.1)$

5.4.3 Congenital malformation (OR adjusted data)

pcb Heikkinen 2012

pcb Källén 2012

pcb Launay 2012

pcb Oppermann 2012

pcb Pasternak 2012

pcb Trotta 2014

0
0
0
0
0
0

Subtotal $(95 \% \mathrm{Cl})$

Heterogeneity: $\mathrm{Tau}^{2}=0 ; \mathrm{Chi}^{2}=3.4, \mathrm{df}=5(\mathrm{P}=0.64) ; \mathrm{I}^{2}=0 \%$

Test for overall effect: $Z=1.86(P=0.06)$

\subsubsection{Prematurity ( $<37$ weeks) (OR unadjusted data)}

pcb Beau 2014

pcb Cleary 2014

pcb Fell 2012

pcb Heikkinen 2012

pcb Källén 2012

pcb Launay 2012

pcb Lin 2012

pcb Ludvigsson 2013

pcb Oppermann 2012

pcb Richards 2013

pcb Rubinstein 2013

Subtotal $(95 \% \mathrm{Cl})$

Heterogeneity: Tau $^{2}=0.02 ; \mathrm{Chi}^{2}=46.74, \mathrm{df}=10(\mathrm{P}<0.0001) ; \mathrm{I}^{2}=78.61 \%$

Test for overall effect: $Z=4.75(P<0.0001)$

\subsubsection{Prematurity (<37 weeks) (OR adjusted data)}

pcb Cleary 2014

pcb Fell 2012

pcb Heikkinen 2012

pcb Källén 2012

pcb Ludvigsson 2013

pcb Richards 2013

pcb Rubinstein 2013

Subtotal $(95 \% \mathrm{Cl})$

Heterogeneity: $\mathrm{Tau}^{2}=0.01 ; \mathrm{Chi}^{2}=20.76, \mathrm{df}=6(\mathrm{P}=0) ; \mathrm{I}^{2}=71.09 \%$

Test for overall effect: $Z=3.39(P=0)$

\subsubsection{Prematurity (< 37 weeks) (HR adjusted data)}

pcb Beau 2014

0

pcb Louik 2013

0

Subtotal $(95 \% \mathrm{CI})$

$-0.3(0.109)$

$-0.1(0.038)$

$-0.3(0.155)$

$-0.2(0.056)$

$-0(0.054)$

$-0.5(0.148)$

$-0.2(0.068)$

Heterogeneity: $\operatorname{Tau}^{2}=0.28 ; \mathrm{Chi}^{2}=2.41, \mathrm{df}=1(\mathrm{P}=0.12) ; \mathrm{I}^{2}=58.51 \%$

Test for overall effect: $Z=0.23(P=0.82)$

5.4.7 Prematurity ( $<37$ weeks) vaccination in I trimester OR adjusted data

pcb Ludvigsson 2013

pcb Pasternak 2012

0
0

$$
\begin{array}{r}
-0.2(0.128) \\
-0.3(0.108) \\
-0.1(0.036) \\
-0.3(0.149) \\
-0.4(0.044) \\
-0.1(0.274) \\
-0.6(0.35) \\
-0.2(0.063) \\
-0(0.217) \\
-0.5(0.136) \\
-0.3(0.061)
\end{array}
$$

$-0.3(0.061)$

IV, Random, $95 \%$ C

$1.33[0.88,2.01]$
$1.01[0.83,1.23]$
$2.34[0.52,10.51]$
$0.92[0.58,1.46]$
$1.21[0.6,2.45]$
$1.14[0.99,1.31]$
$\mathbf{1 . 1 1}[0.99, \mathbf{1 . 2 3}]$

$0.81[0.63,1.04]$

$0.71[0.58,0.88]$

$0.91[0.85,0.98]$

$0.74[0.55,0.99]$

$0.65[0.59,0.7]$

$0.93[0.54,1.59]$

$0.54[0.27,1.07]$

$0.81[0.71,0.91]$

$0.98[0.64,1.49]$

$0.6[0.46,0.79]$

$0.74[0.65,0.83]$

$0.76[0.67,0.85]$

$0.72[0.58,0.89]$

$0.95[0.88,1.02]$

$0.75[0.55,1.02]$

$0.86[0.77,0.96]$

$0.99[0.89,1.1]$

$0.63[0.47,0.84]$

$0.79[0.69,0.9]$

$0.84[0.76,0.93]$

$6.45 \%$

$100 \%$

$0.82[0.64,1.06]$

$2.17[0.65,7.22]$

$1.11[0.46,2.68]$ 


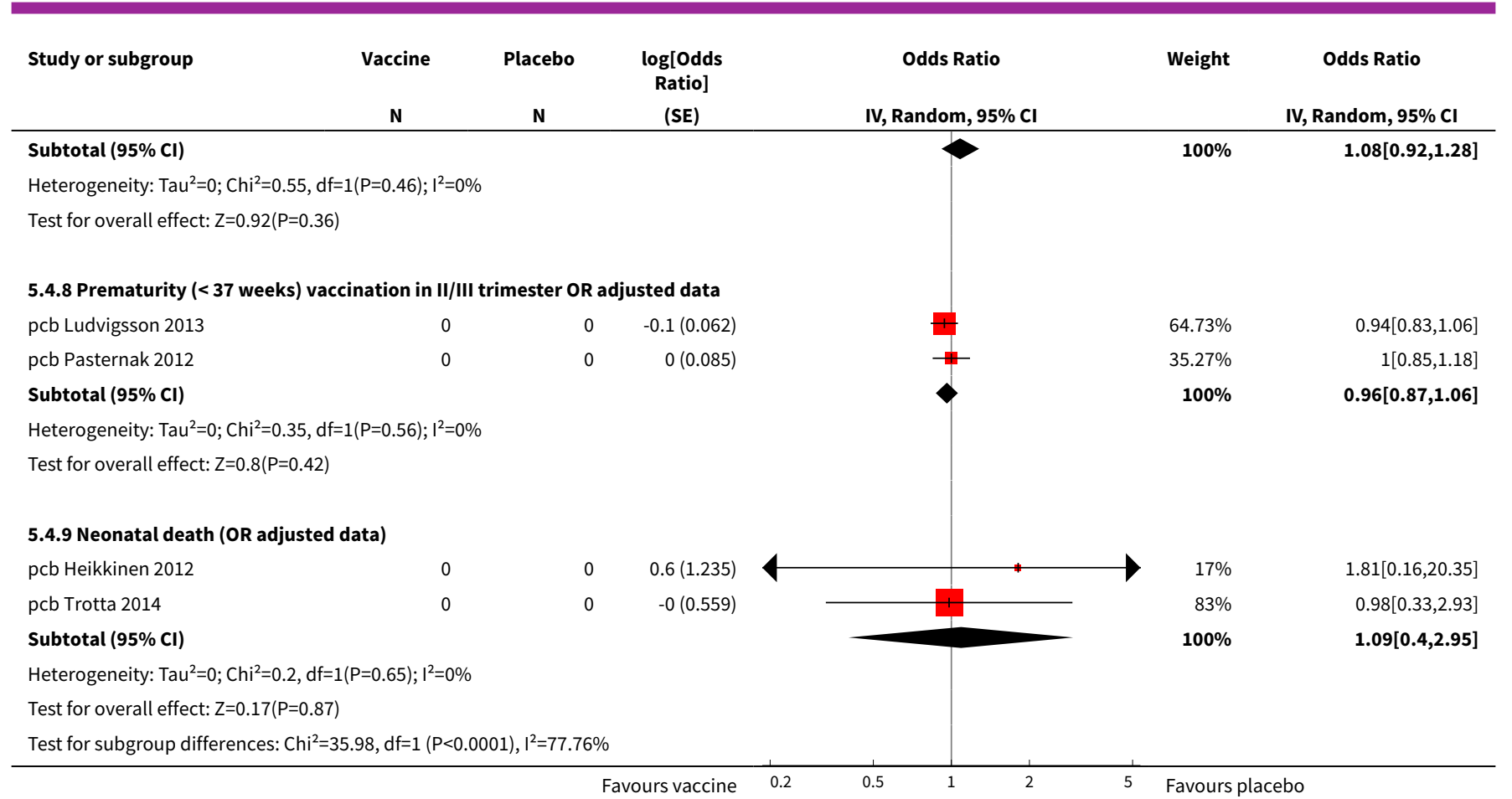

\section{Analysis 5.5. Comparison 5 Inactivated parenteral influenza vaccine versus placebo - cohort studies, Outcome 5 Seasonal vaccine - safety - pregnancy-related outcomes - pregnant women.}

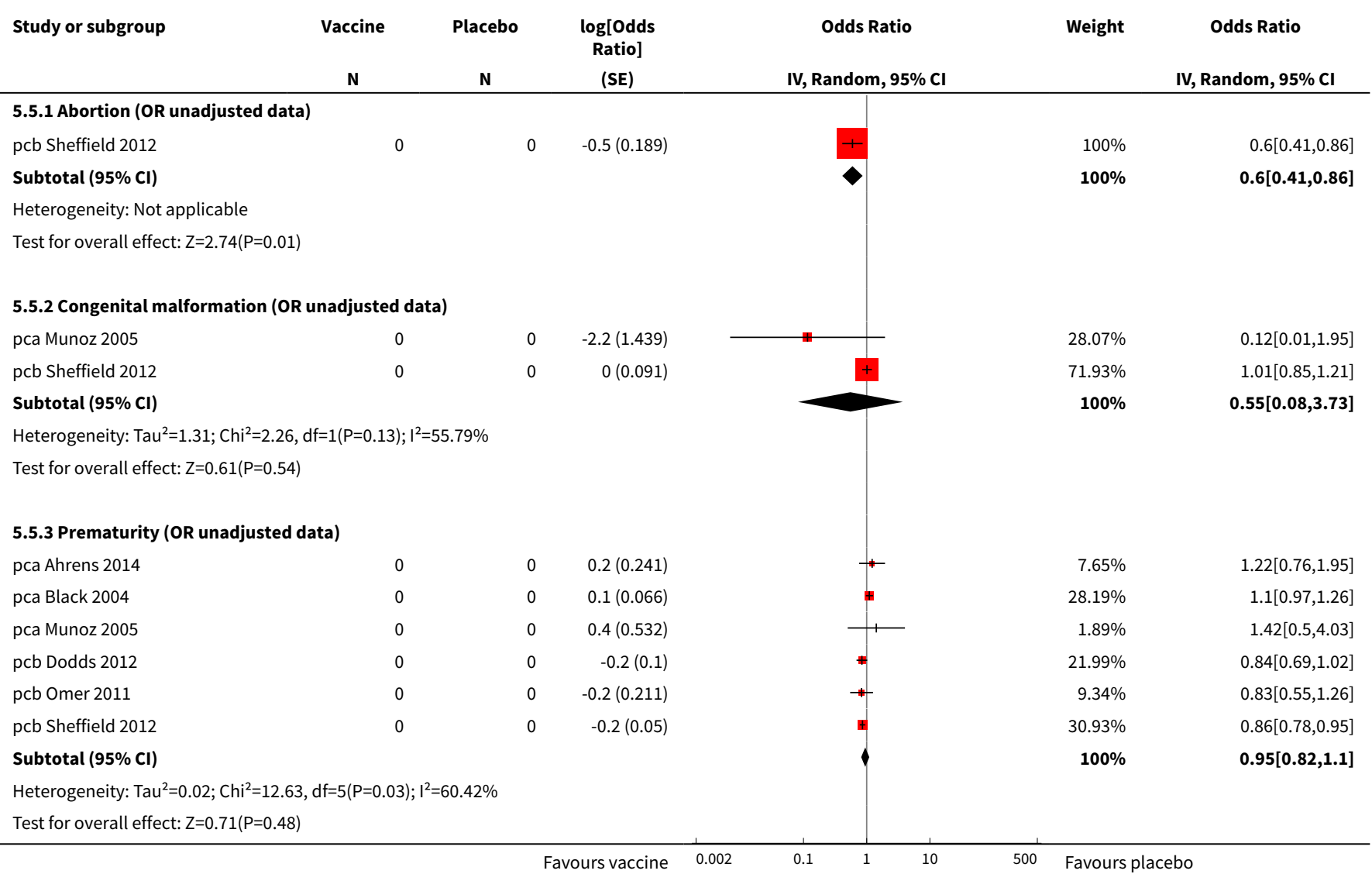




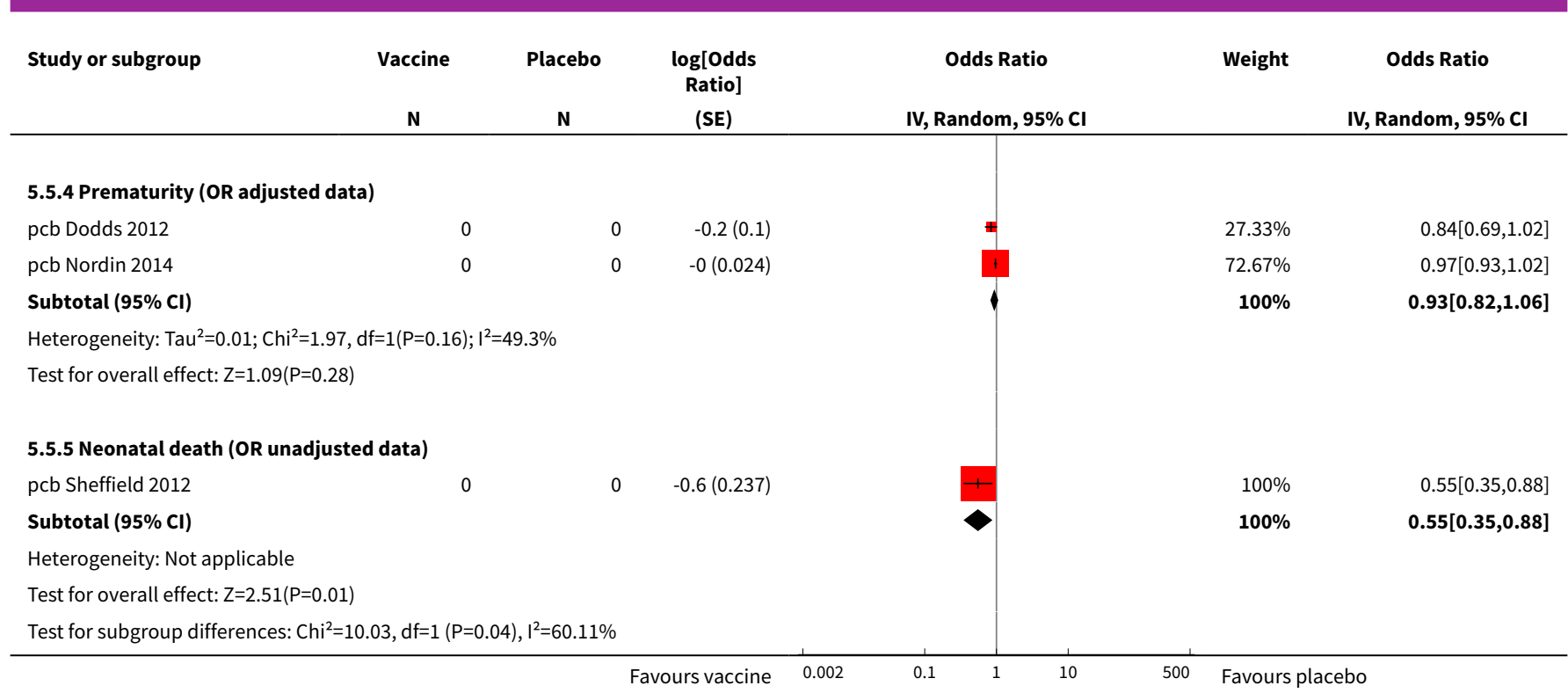

\section{Analysis 5.6. Comparison 5 Inactivated parenteral influenza vaccine versus placebo - cohort studies, Outcome 6 Seasonal vaccine containing H1N1.}

\begin{tabular}{|c|c|c|c|c|c|c|}
\hline Study or subgroup & $\begin{array}{c}\text { Vaccine } \\
\mathbf{N} \\
\end{array}$ & $\begin{array}{c}\text { Placebo } \\
\text { N }\end{array}$ & $\begin{array}{c}\text { log[Risk } \\
\text { Ratio] } \\
\text { (SE) }\end{array}$ & $\begin{array}{c}\text { Risk Ratio } \\
\text { IV, Random, } 95 \% \text { CI }\end{array}$ & Weight & IV, Random, 95\% Cl \\
\hline \multicolumn{7}{|c|}{ 5.6.1 Prematurity ( 37 weeks) vaccination in I trimester HR adjusted data } \\
\hline pcb Chambers 2013 & 0 & 0 & $0.8(0.588)$ & - & $43.06 \%$ & $2.22[0.7,7.03]$ \\
\hline pcb Louik 2013 & 0 & 0 & $0.3(0.511)$ & & $56.94 \%$ & $1.29[0.47,3.52]$ \\
\hline Subtotal $(95 \% \mathrm{Cl})$ & & & & & $100 \%$ & $1.63[0.76,3.47]$ \\
\hline \multicolumn{7}{|c|}{ Test for overall effect: $Z=1.27(P=0.21)$} \\
\hline \multicolumn{7}{|c|}{ 5.6.2 Prematurity ( $<37$ weeks) vaccination in II trimester HR adjusted data } \\
\hline pcb Chambers 2013 & 0 & 0 & $1.4(0.626)$ & +1- & $49.12 \%$ & 4.12[1.21,14.06] \\
\hline pcb Louik 2013 & 0 & 0 & $-0.6(0.567)$ & & $50.88 \%$ & $0.55[0.18,1.67]$ \\
\hline Subtotal $(95 \% \mathrm{Cl})$ & & & & & $100 \%$ & $1.48[0.21,10.64]$ \\
\hline \multicolumn{7}{|c|}{ Test for overall effect: $Z=0.39(P=0.7)$} \\
\hline \multicolumn{7}{|c|}{ 5.6.3 Prematurity (<37 weeks) vaccination in III trimester HR adjusted data } \\
\hline pcb Chambers 2013 & 0 & 0 & $0.4(0.97)$ & - & $35.45 \%$ & $1.47[0.22,9.83]$ \\
\hline pcb Louik 2013 & 0 & 0 & $0.3(0.719)$ & & $64.55 \%$ & $1.32[0.32,5.4]$ \\
\hline Subtotal $(95 \% \mathrm{Cl})$ & & & & & $100 \%$ & $1.37[0.44,4.25]$ \\
\hline \multicolumn{7}{|c|}{ Heterogeneity: $\operatorname{Tau}^{2}=0 ; \mathrm{Chi}^{2}=0.01, \mathrm{df}=1(\mathrm{P}=0.93) ; \mathrm{I}^{2}=0 \%$} \\
\hline \multicolumn{7}{|c|}{ Test for overall effect: $\mathrm{Z}=0.55(\mathrm{P}=0.58)$} \\
\hline \multicolumn{7}{|c|}{$\begin{array}{l}\text { 5.6.4 Prematurity ( }<37 \text { weeks) vaccination at any time during pregnancy HR } \\
\text { adjusted data }\end{array}$} \\
\hline pcb Chambers 2013 & 0 & 0 & $1.2(0.493)$ & 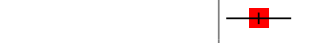 & $45.94 \%$ & $3.28[1.25,8.62]$ \\
\hline pcb Louik 2013 & 0 & 0 & $0(0.366)$ & & $54.06 \%$ & $1.03[0.5,2.11]$ \\
\hline & & & cine & 0.002 & Favours & \\
\hline
\end{tabular}




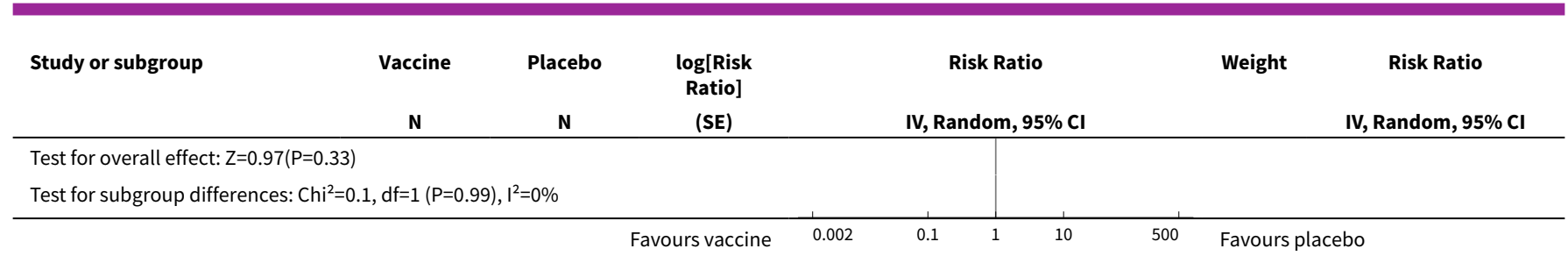

Comparison 6. Inactivated parenteral influenza vaccine versus placebo - case-control studies

\begin{tabular}{|c|c|c|c|c|}
\hline Outcome or subgroup title & No. of studies & $\begin{array}{l}\text { No. of partici- } \\
\text { pants }\end{array}$ & Statistical method & Effect size \\
\hline $\begin{array}{l}1 \text { Effectiveness in newborns - pregnant } \\
\text { women (adjusted data) }\end{array}$ & 2 & & $\begin{array}{l}\text { Odds Ratio (Random, } \\
95 \% \mathrm{Cl} \text { ) }\end{array}$ & $0.24[0.04,1.40]$ \\
\hline $\begin{array}{l}\text { 1.1 Seasonal vaccine - effectiveness - ILI - } \\
\text { pregnant women }\end{array}$ & 2 & & $\begin{array}{l}\text { Odds Ratio (Random, } \\
95 \% \mathrm{Cl} \text { ) }\end{array}$ & $0.24[0.04,1.40]$ \\
\hline $\begin{array}{l}2 \text { Seasonal vaccine safety - pregnancy-re- } \\
\text { lated outcomes (adjusted data) }\end{array}$ & 1 & & $\begin{array}{l}\text { Odds Ratio (Random, } \\
95 \% \mathrm{Cl} \text { ) }\end{array}$ & $0.80[0.36,1.78]$ \\
\hline 2.1 Abortion & 1 & & $\begin{array}{l}\text { Odds Ratio (Random, } \\
95 \% \mathrm{Cl} \text { ) }\end{array}$ & $0.80[0.36,1.78]$ \\
\hline
\end{tabular}

Analysis 6.1. Comparison 6 Inactivated parenteral influenza vaccine versus placebo - casecontrol studies, Outcome 1 Effectiveness in newborns - pregnant women (adjusted data).

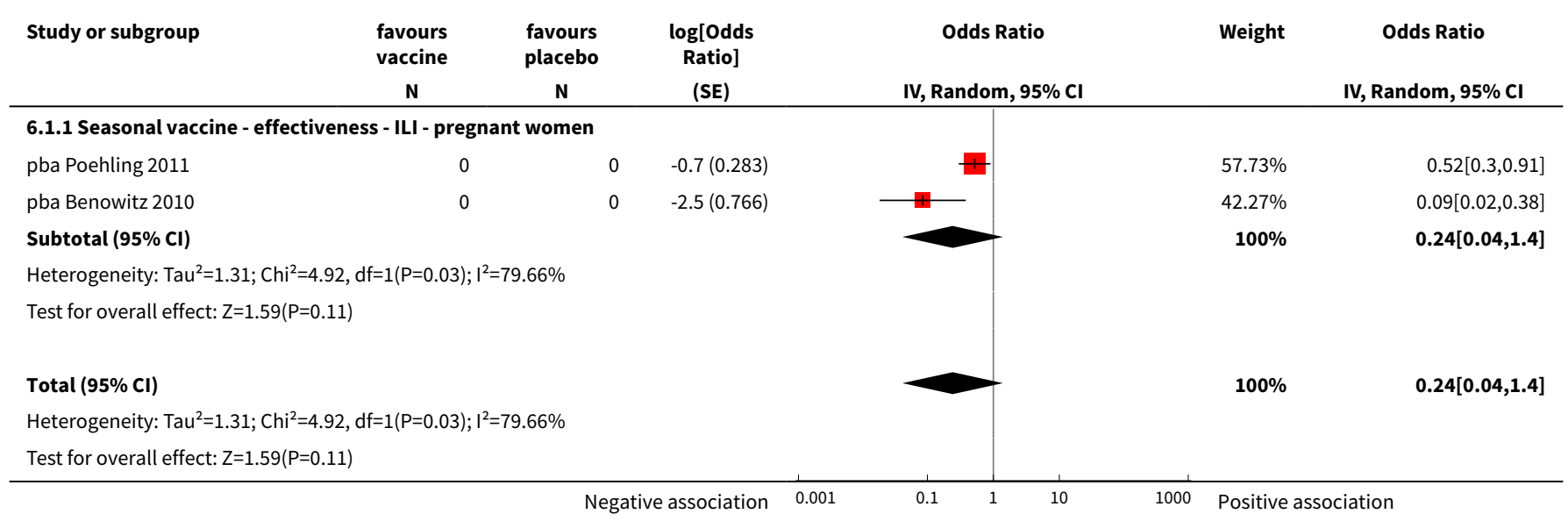


Analysis 6.2. Comparison 6 Inactivated parenteral influenza vaccine versus placebo - casecontrol studies, Outcome 2 Seasonal vaccine safety - pregnancy-related outcomes (adjusted data).

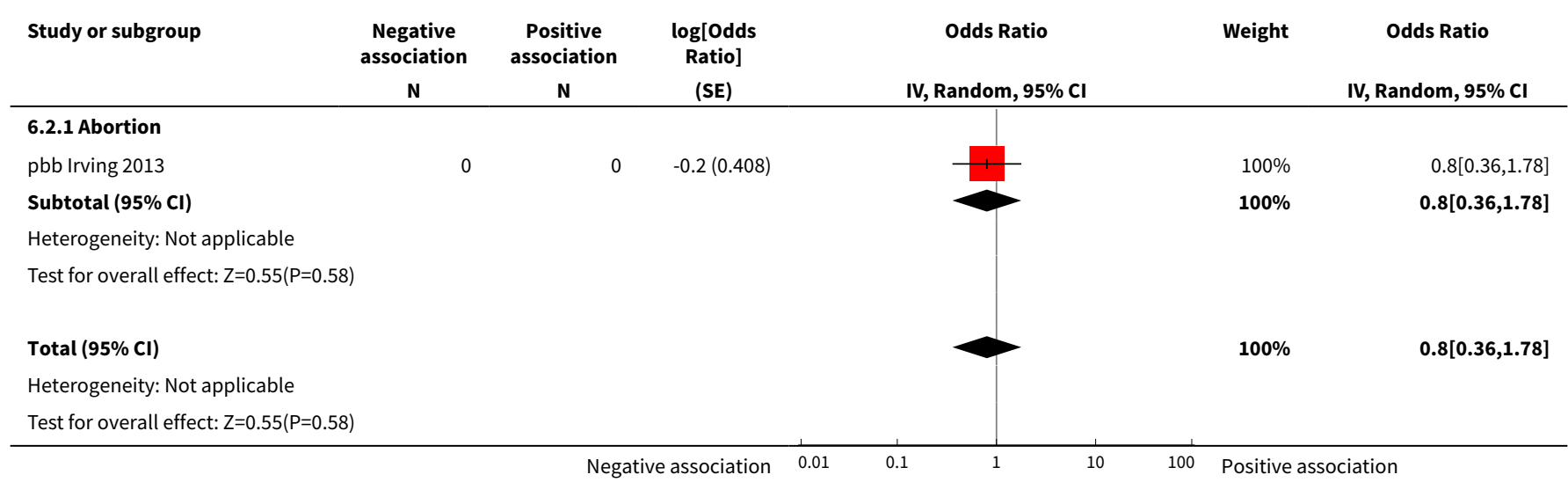

Comparison 7. Serious adverse events: Guillain-Barré syndrome - cohort studies

\begin{tabular}{lllll}
\hline Outcome or subgroup title & No. of studies & $\begin{array}{l}\text { No. of partici- } \\
\text { pants }\end{array}$ & Statistical method & Effect size \\
\hline $\begin{array}{l}\text { 1 Seasonal influenza vaccination and } \\
\text { Guillain-Barré syndrome }\end{array}$ & 3 & & $\begin{array}{l}\text { Risk Ratio (Random, 95\% } \\
\text { Cl) }\end{array}$ & $1.28[0.85,1.93]$ \\
\hline 1.1 General population (adjusted data) & 2 & $\begin{array}{l}\text { Risk Ratio (Random, 95\% } \\
\text { Cl) }\end{array}$ & $1.29[0.83,2.02]$ \\
\hline 1.2 Pregnant women (unadjusted data) & 1 & Risk Ratio (Random, 95\% & $0.65[0.03,15.95]$ \\
\hline
\end{tabular}

Analysis 7.1. Comparison 7 Serious adverse events: Guillain-Barré syndrome - cohort studies, Outcome 1 Seasonal influenza vaccination and Guillain-Barré syndrome.

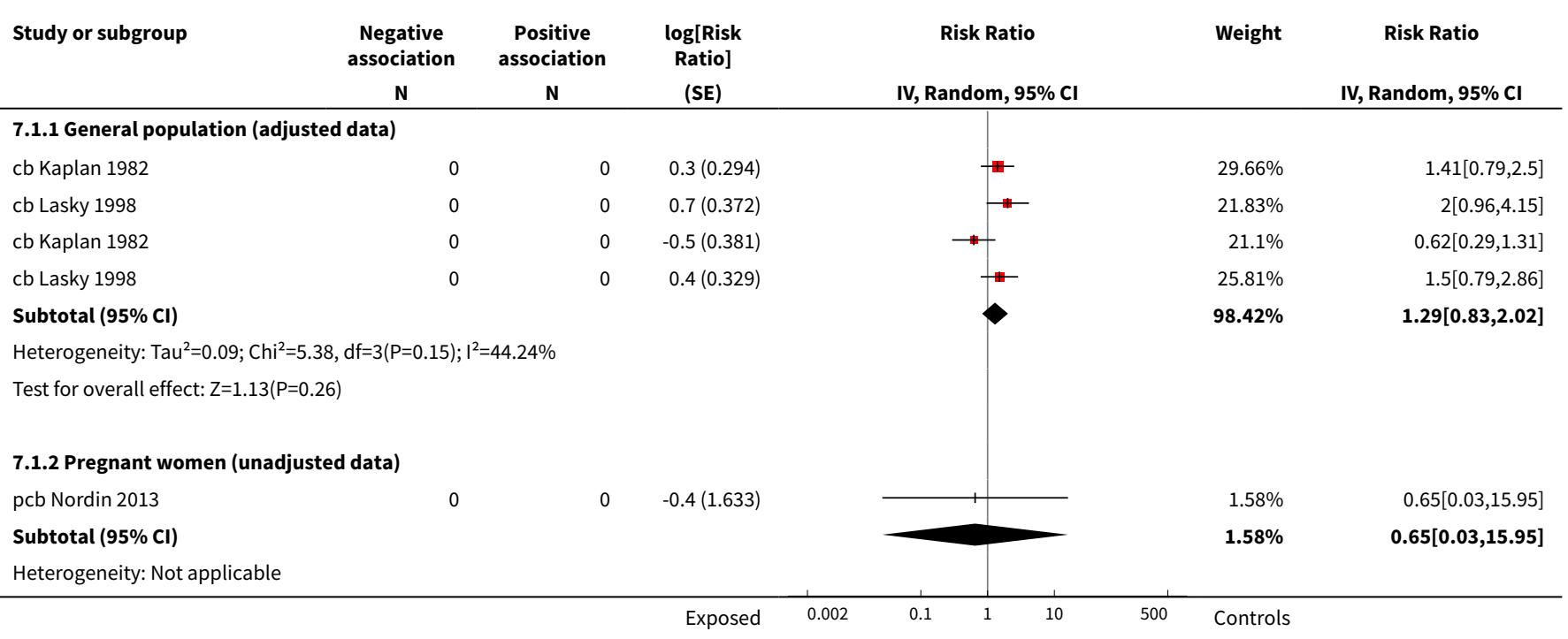




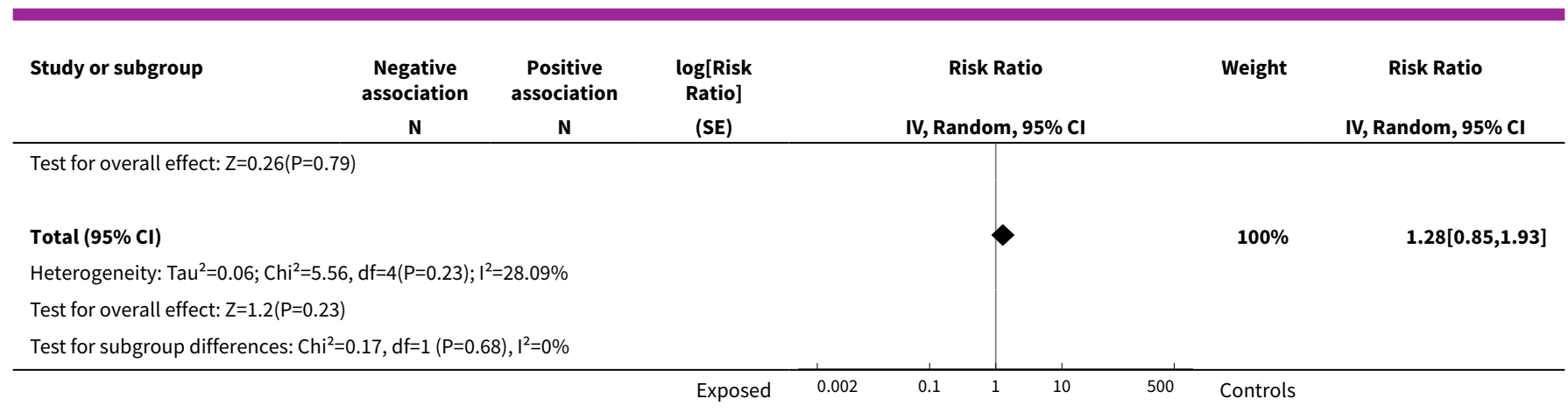

Comparison 8. Serious adverse events: Guillain-Barré syndrome - case-control studies

\begin{tabular}{|c|c|c|c|c|}
\hline Outcome or subgroup title & No. of studies & $\begin{array}{l}\text { No. of partici- } \\
\text { pants }\end{array}$ & Statistical method & Effect size \\
\hline $\begin{array}{l}12009 \text { to } 2010 \mathrm{~A} / \mathrm{H} 1 \mathrm{N1} \text { - general } \\
\text { population (unadjusted data) }\end{array}$ & 6 & & $\begin{array}{l}\text { Odds Ratio (M-H, Random, } \\
95 \% \mathrm{Cl} \text { ) }\end{array}$ & Subtotals only \\
\hline $1.1<7$ weeks & 6 & 1528 & $\begin{array}{l}\text { Odds Ratio (M-H, Random, } \\
95 \% \mathrm{Cl} \text { ) }\end{array}$ & $2.22[1.14,4.31]$ \\
\hline 1.2 At any time & 6 & 1656 & $\begin{array}{l}\text { Odds Ratio (M-H, Random, } \\
95 \% \mathrm{Cl} \text { ) }\end{array}$ & $1.69[0.87,3.29]$ \\
\hline $\begin{array}{l}22009 \text { to } 2010 \mathrm{~A} / \mathrm{H} 1 \mathrm{N1} \text { - general } \\
\text { population (adjusted data) }\end{array}$ & 4 & & Odds Ratio (Random, 95\% Cl) & $0.83[0.39,1.75]$ \\
\hline $2.1<7$ weeks & 4 & & Odds Ratio (Random, 95\% Cl) & $0.92[0.35,2.40]$ \\
\hline $2.2>6$ weeks (i.e. at any time) & 3 & & Odds Ratio (Random, 95\% Cl) & $0.71[0.22,2.32]$ \\
\hline $\begin{array}{l}3 \text { Seasonal influenza vaccination } \\
\text { general population (adjusted data) }\end{array}$ & 1 & & Odds Ratio (Random, 95\% Cl) & $1.38[0.18,10.43]$ \\
\hline
\end{tabular}

Analysis 8.1. Comparison 8 Serious adverse events: Guillain-Barré syndrome - casecontrol studies, Outcome 12009 to 2010 A/H1N1 - general population (unadjusted data).

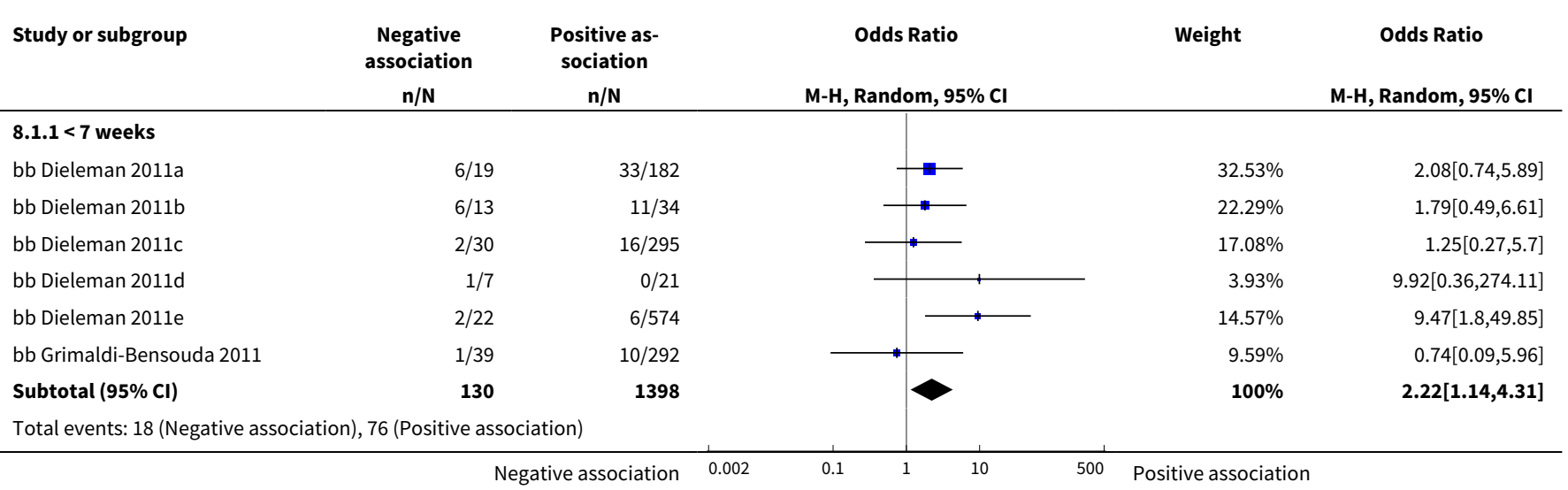




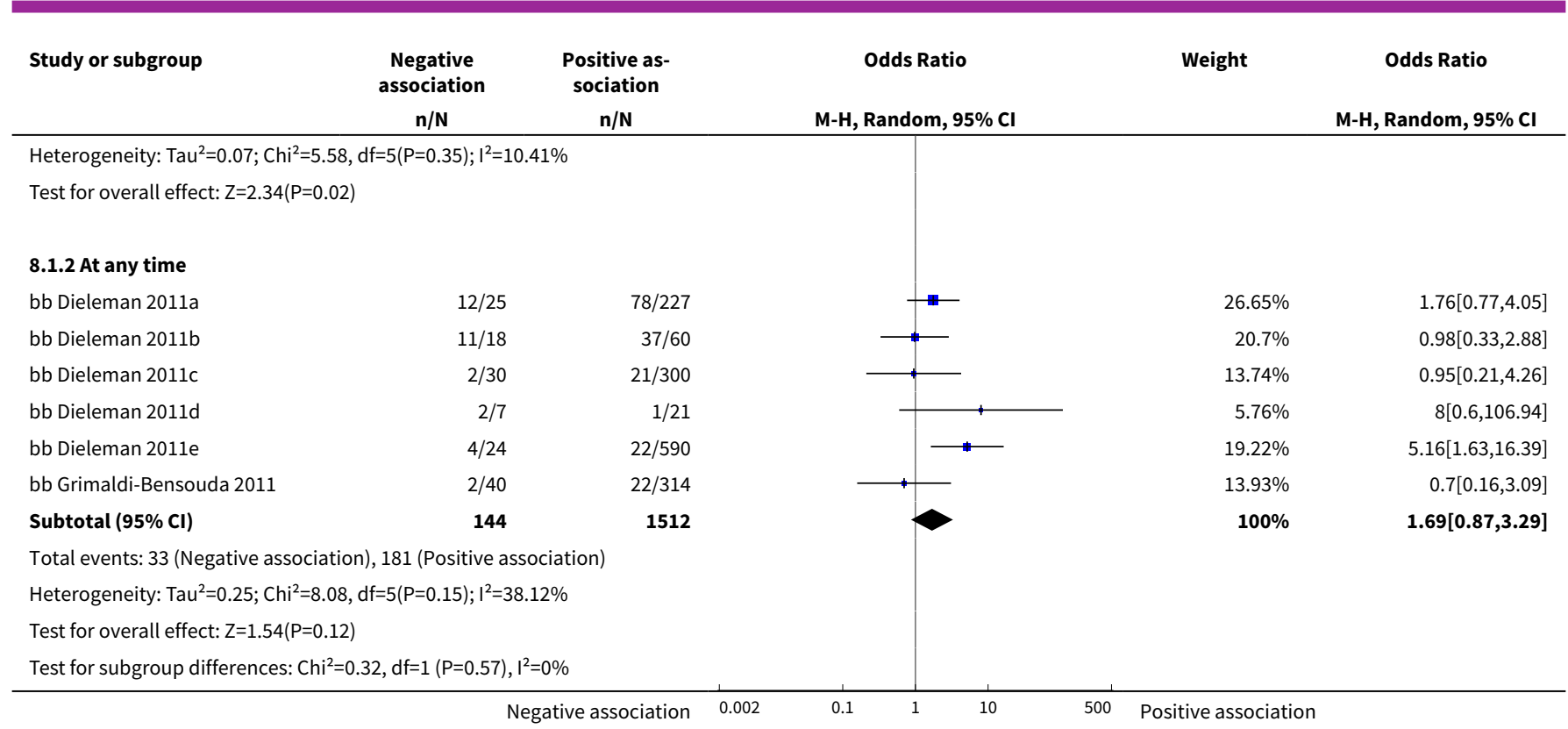

Analysis 8.2. Comparison 8 Serious adverse events: Guillain-Barré syndrome - casecontrol studies, Outcome 22009 to 2010 A/H1N1 - general population (adjusted data).

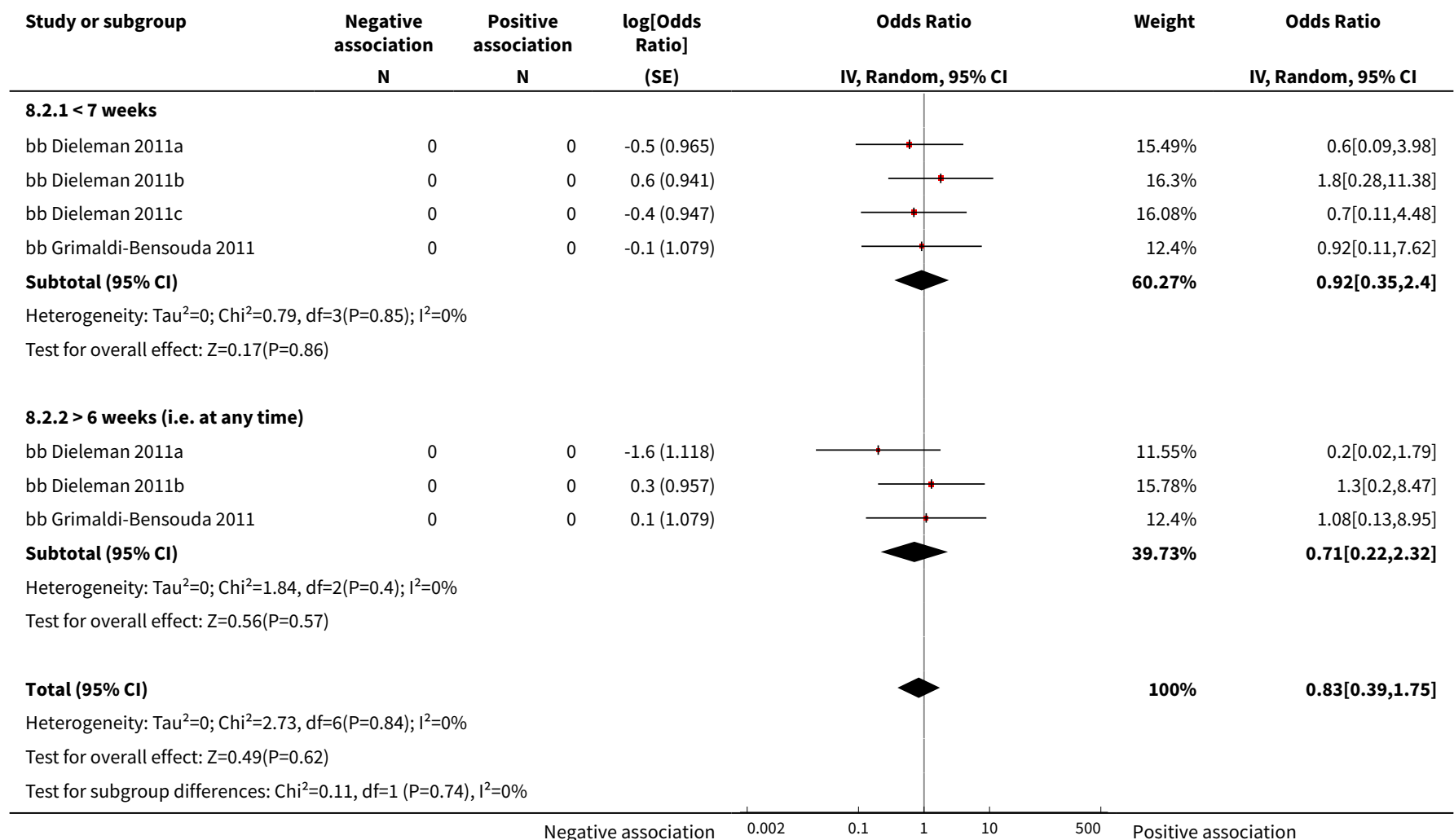


Analysis 8.3. Comparison 8 Serious adverse events: Guillain-Barré syndrome - case-control studies, Outcome 3 Seasonal influenza vaccination general population (adjusted data).

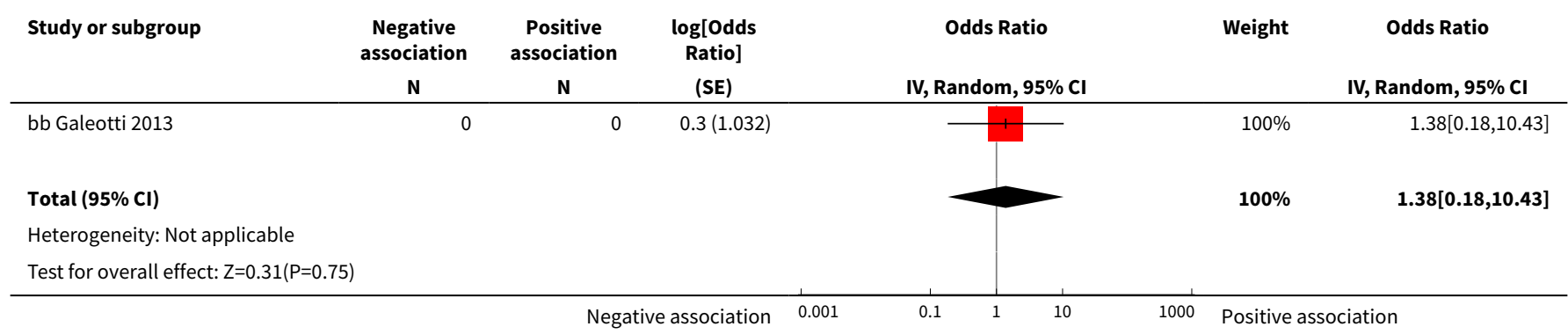

Comparison 9. Serious adverse events: demyelinating diseases (multiple sclerosis, optic neuritis) - cohort studies

\begin{tabular}{|c|c|c|c|c|}
\hline Outcome or subgroup title & No. of studies & $\begin{array}{l}\text { No. of partici- } \\
\text { pants }\end{array}$ & Statistical method & Effect size \\
\hline $\begin{array}{l}1 \text { Influenza vaccination (seasonal) - de- } \\
\text { myelinating diseases (unadjusted data) }\end{array}$ & 1 & 223898 & $\begin{array}{l}\text { Odds Ratio (M-H, Random, } \\
95 \% \mathrm{Cl})\end{array}$ & $0.16[0.02,1.25]$ \\
\hline 1.1 General population & 0 & 0 & $\begin{array}{l}\text { Odds Ratio (M-H, Random, } \\
95 \% \mathrm{Cl})\end{array}$ & $0.0[0.0,0.0]$ \\
\hline 1.2 Pregnant women & 1 & 223898 & $\begin{array}{l}\text { Odds Ratio (M-H, Random, } \\
95 \% \mathrm{Cl})\end{array}$ & $0.16[0.02,1.25]$ \\
\hline $\begin{array}{l}2 \text { Influenza vaccination ( } \mathrm{H} 1 \mathrm{~N} 1 \text { ) - de- } \\
\text { myelinating diseases (unadjusted) }\end{array}$ & 1 & 144252 & $\begin{array}{l}\text { Risk Ratio (M-H, Random, } \\
95 \% \mathrm{Cl})\end{array}$ & $2.06[0.51,8.22]$ \\
\hline
\end{tabular}

\section{Analysis 9.1. Comparison 9 Serious adverse events: demyelinating diseases (multiple sclerosis, optic neuritis) - cohort studies, Outcome 1 Influenza vaccination (seasonal) - demyelinating diseases (unadjusted data).}

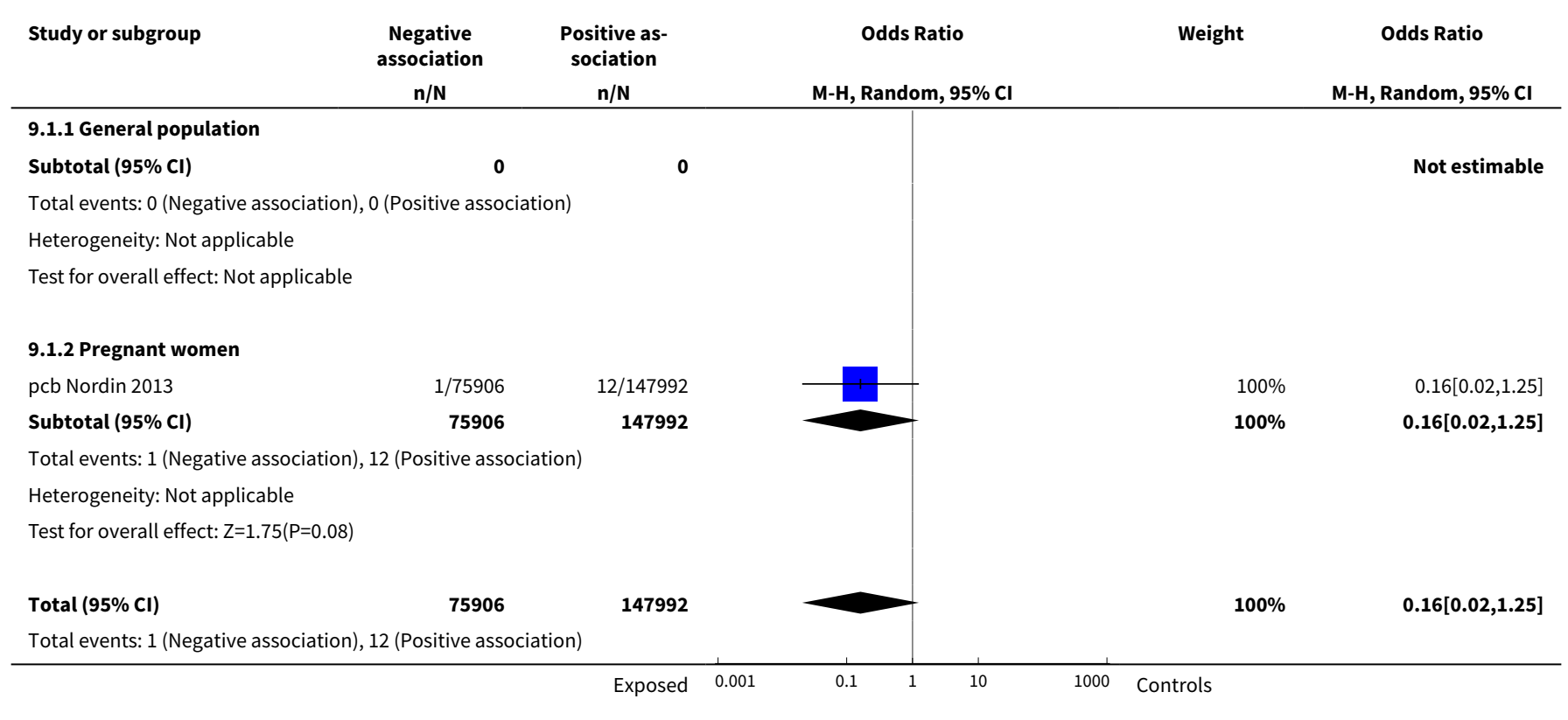




\begin{tabular}{|c|c|c|c|c|c|c|}
\hline Study or subgroup & $\begin{array}{c}\text { Negative } \\
\text { association } \\
\mathbf{n} / \mathbf{N}\end{array}$ & $\begin{array}{c}\text { Positive as- } \\
\text { sociation } \\
n / N\end{array}$ & & Odds Ratio & Weight & $\begin{array}{c}\text { Odds Ratio } \\
\text { M-H, Random, } 95 \% \mathrm{Cl}\end{array}$ \\
\hline \multicolumn{7}{|c|}{ Heterogeneity: Not applicable } \\
\hline \multicolumn{7}{|c|}{ Test for overall effect: $Z=1.75(P=0.08)$} \\
\hline \multicolumn{7}{|c|}{ Test for subgroup differences: Not applicable } \\
\hline
\end{tabular}

Analysis 9.2. Comparison 9 Serious adverse events: demyelinating diseases (multiple sclerosis, optic neuritis) - cohort studies, Outcome 2 Influenza vaccination (H1N1) - demyelinating diseases (unadjusted).

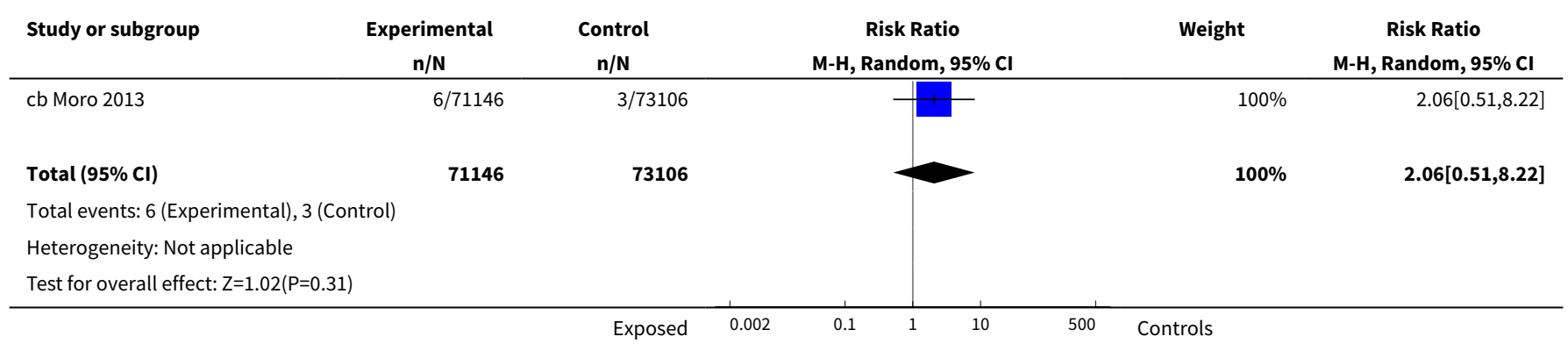

Comparison 10. Serious adverse events: demyelinating diseases (multiple sclerosis, optic neuritis) - case-control studies

\begin{tabular}{lllll}
\hline Outcome or subgroup title & No. of studies & $\begin{array}{l}\text { No. of partici- } \\
\text { pants }\end{array}$ & Statistical method & Effect size \\
\hline $\begin{array}{l}1 \text { Influenza vaccination (seasonal) - general pop- } \\
\text { ulation - demyelinating diseases (unadjusted } \\
\text { data) }\end{array}$ & 8009 & $\begin{array}{l}\text { Odds Ratio (M-H, } \\
\text { Random, 95\% Cl) }\end{array}$ & $0.96[0.79,1.17]$ \\
\hline $\begin{array}{l}2 \text { Influenza vaccination (seasonal) - general pop- } \\
\text { ulation - multiple sclerosis (adjusted data) }\end{array}$ & 2 & & $\begin{array}{l}\text { Odds Ratio (Ran- } \\
\text { dom, 95\% Cl) }\end{array}$ & $0.76[0.54,1.08]$ \\
\hline $\begin{array}{l}3 \text { Influenza vaccination (seasonal) - general pop- } \\
\text { ulation - optic neuritis (adjusted data) }\end{array}$ & 2 & $\begin{array}{l}\text { Odds Ratio (Ran- } \\
\text { dom, 95\% Cl) }\end{array}$ & 1.03 [0.82, 1.30] \\
\hline
\end{tabular}

Analysis 10.1. Comparison 10 Serious adverse events: demyelinating diseases

(multiple sclerosis, optic neuritis) - case-control studies, Outcome 1 Influenza vaccination (seasonal) - general population - demyelinating diseases (unadjusted data).

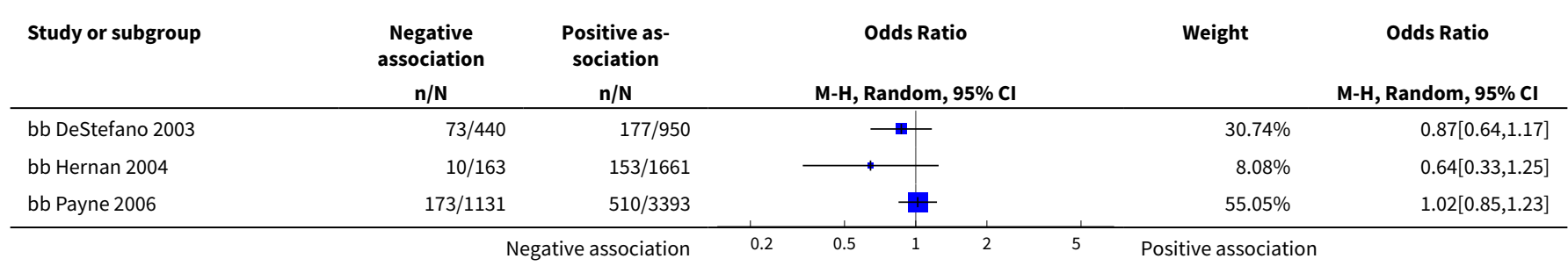




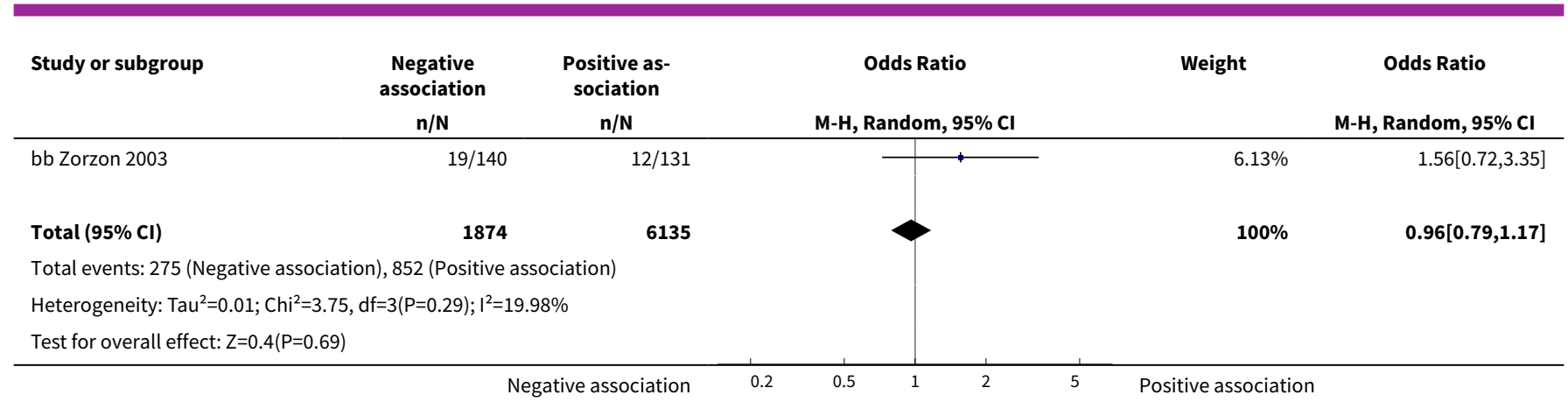

Analysis 10.2. Comparison 10 Serious adverse events: demyelinating diseases (multiple sclerosis, optic neuritis) - case-control studies, Outcome 2 Influenza vaccination (seasonal) - general population - multiple sclerosis (adjusted data).

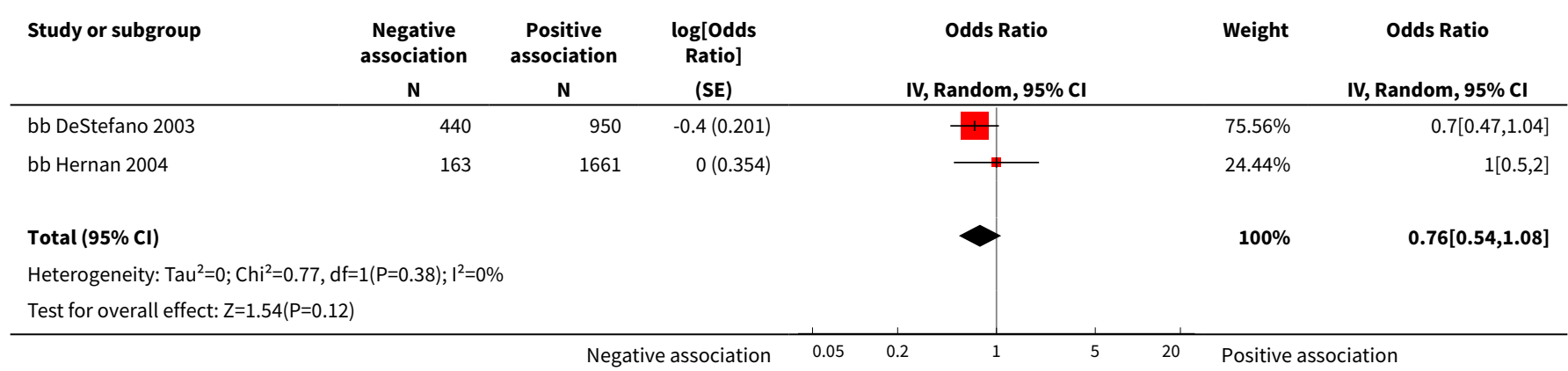

Analysis 10.3. Comparison 10 Serious adverse events: demyelinating diseases (multiple sclerosis, optic neuritis) - case-control studies, Outcome 3 Influenza vaccination (seasonal) - general population - optic neuritis (adjusted data).

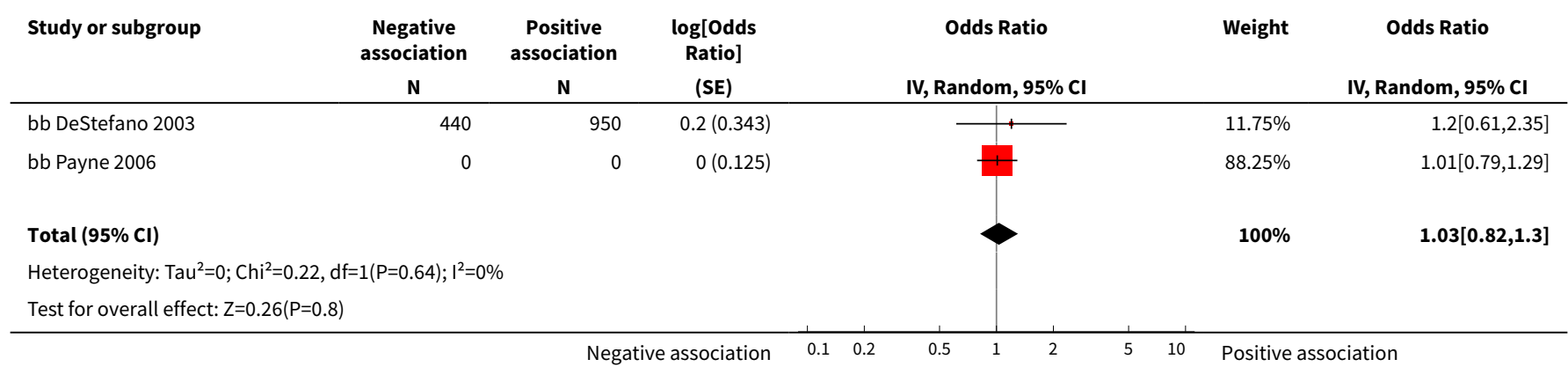

\section{Comparison 11. Serious adverse events: immune thrombocytopenic purpura - cohort studies}

\begin{tabular}{lllll}
\hline Outcome or subgroup title & No. of studies & $\begin{array}{l}\text { No. of partici- } \\
\text { pants }\end{array}$ & Statistical method & Effect size \\
\hline $\begin{array}{l}1 \text { Seasonal influenza vaccine - HR } \\
\text { (adjusted data) }\end{array}$ & 1 & Hazard Ratio (Random, 95\% Cl) & Subtotals only \\
\hline
\end{tabular}




\begin{tabular}{|c|c|c|c|c|}
\hline Outcome or subgroup title & No. of studies & $\begin{array}{l}\text { No. of partici- } \\
\text { pants }\end{array}$ & Statistical method & Effect size \\
\hline 1.1 General population & 0 & & Hazard Ratio (Random, 95\% Cl) & $0.0[0.0,0.0]$ \\
\hline 1.2 Pregnant women & 1 & & Hazard Ratio (Random, 95\% Cl) & $0.90[0.68,1.19]$ \\
\hline $\begin{array}{l}2 \text { Seasonal influenza vaccine (un- } \\
\text { adjusted data) }\end{array}$ & 1 & & $\begin{array}{l}\text { Odds Ratio (M-H, Random, 95\% } \\
\mathrm{Cl} \text { ) }\end{array}$ & Subtotals only \\
\hline 2.1 General population & 0 & 0 & $\begin{array}{l}\text { Odds Ratio (M-H, Random, 95\% } \\
\mathrm{Cl} \text { ) }\end{array}$ & $0.0[0.0,0.0]$ \\
\hline 2.2 Pregnant women & 1 & 223898 & $\begin{array}{l}\text { Odds Ratio (M-H, Random, 95\% } \\
\mathrm{Cl})\end{array}$ & $0.92[0.70,1.20]$ \\
\hline
\end{tabular}

Analysis 11.1. Comparison 11 Serious adverse events: immune thrombocytopenic purpura - cohort studies, Outcome 1 Seasonal influenza vaccine - HR (adjusted data).

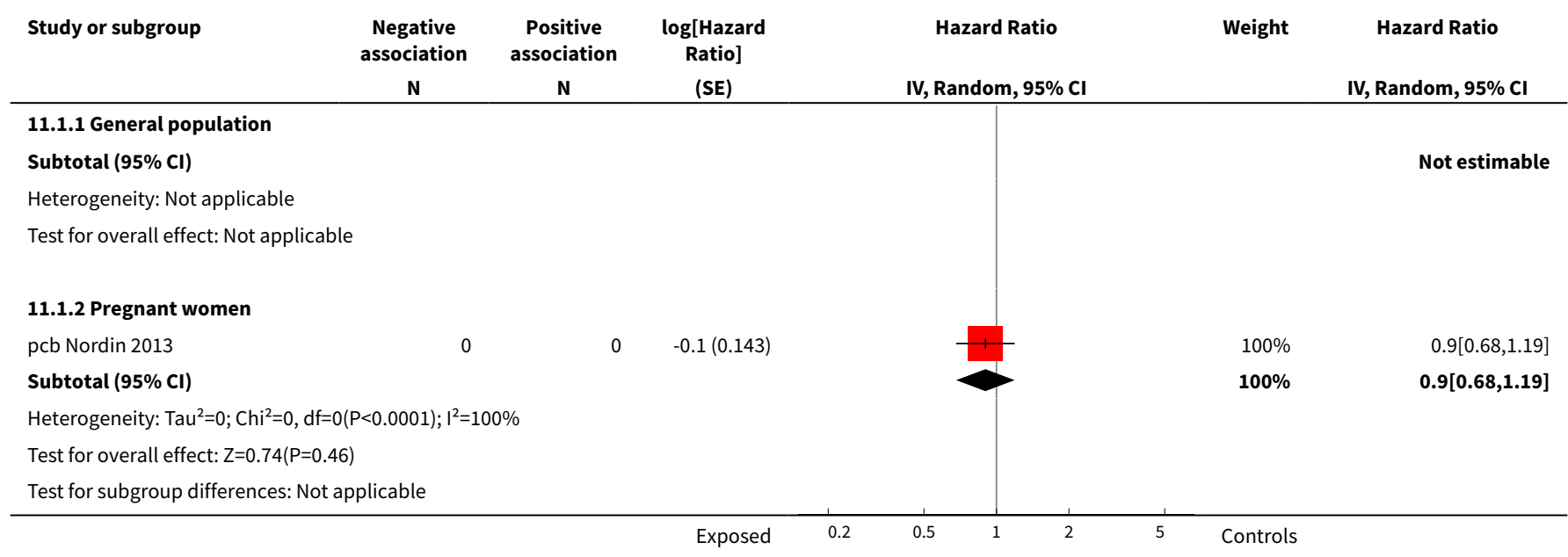

Analysis 11.2. Comparison 11 Serious adverse events: immune thrombocytopenic purpura - cohort studies, Outcome 2 Seasonal influenza vaccine (unadjusted data).

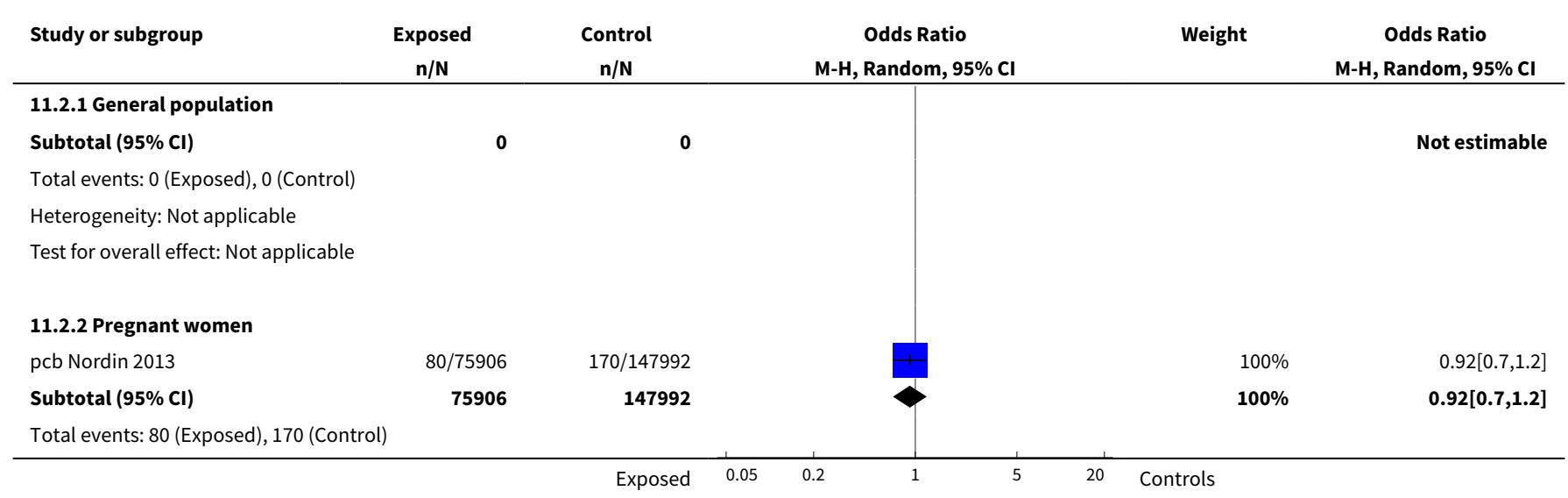




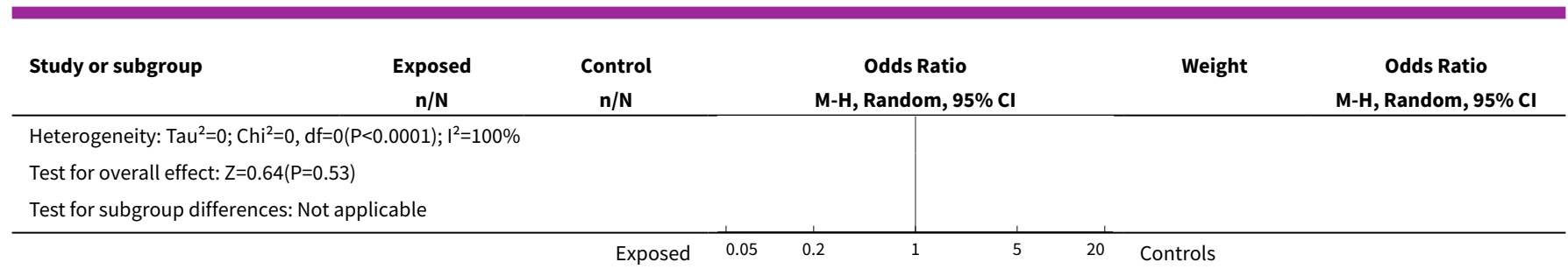

Comparison 12. Serious adverse events: immune thrombocytopenic purpura - case-control studies

\begin{tabular}{|c|c|c|c|c|}
\hline Outcome or subgroup title & No. of studies & $\begin{array}{l}\text { No. of partici- } \\
\text { pants }\end{array}$ & Statistical method & Effect size \\
\hline $\begin{array}{l}1 \text { Seasonal influenza vaccine - } \\
\text { general population (adjusted } \\
\text { data) }\end{array}$ & 2 & & Odds Ratio (Random, 95\% Cl) & Subtotals only \\
\hline $1.1<2$ months & 2 & & Odds Ratio (Random, 95\% Cl) & $1.87[0.43,8.06]$ \\
\hline $1.2<6$ months & 1 & & Odds Ratio (Random, 95\% Cl) & $0.90[0.55,1.47]$ \\
\hline $1.3<12$ months & 1 & & Odds Ratio (Random, 95\% Cl) & $0.70[0.47,1.04]$ \\
\hline $\begin{array}{l}2 \text { Seasonal influenza vaccine - } \\
\text { general population (unadjust- } \\
\text { ed data) }\end{array}$ & 2 & & Odds Ratio (M-H, Random, 95\% Cl) & Subtotals only \\
\hline $2.1<2$ months & 2 & 1926 & Odds Ratio (M-H, Random, 95\% Cl) & $1.72[0.48,6.15]$ \\
\hline $2.2<6$ months & 1 & 1065 & Odds Ratio (M-H, Random, 95\% Cl) & $0.92[0.59,1.43]$ \\
\hline $2.3<12$ months & 1 & 1066 & Odds Ratio (M-H, Random, 95\% Cl) & $0.72[0.50,1.05]$ \\
\hline
\end{tabular}

Analysis 12.1. Comparison 12 Serious adverse events: immune thrombocytopenic purpura case-control studies, Outcome 1 Seasonal influenza vaccine - general population (adjusted data).

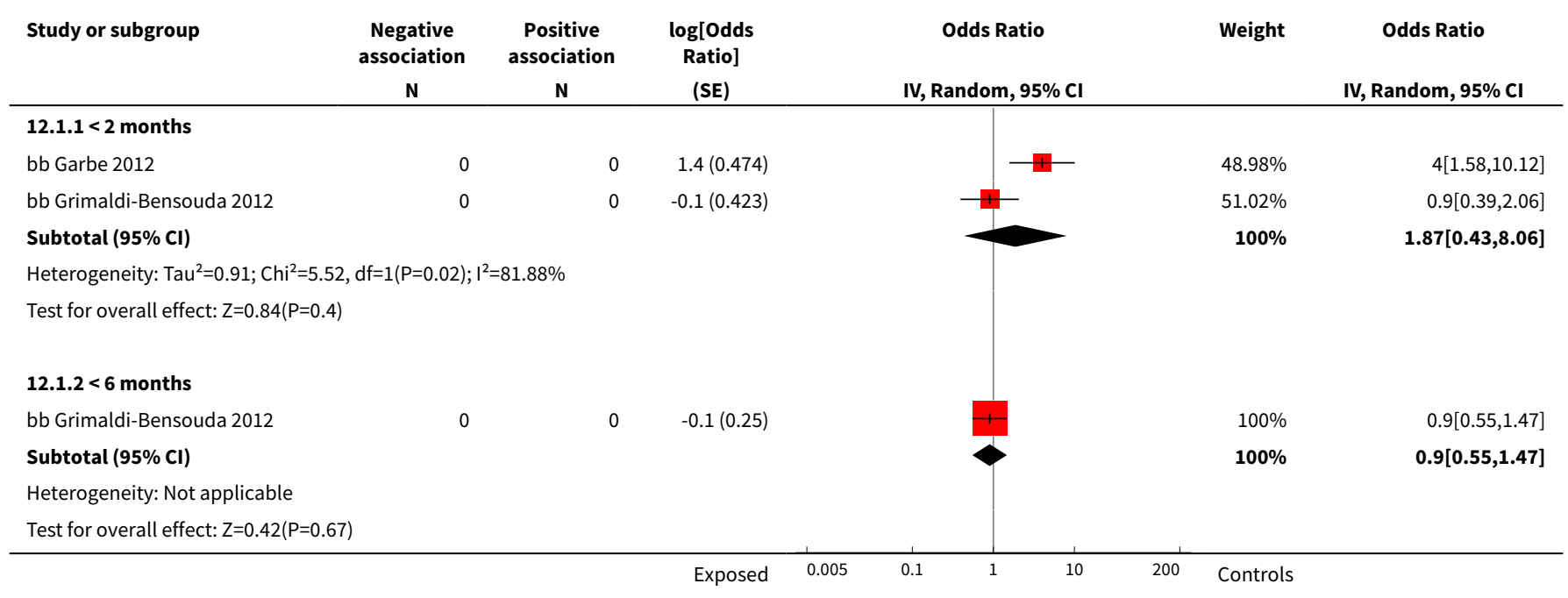




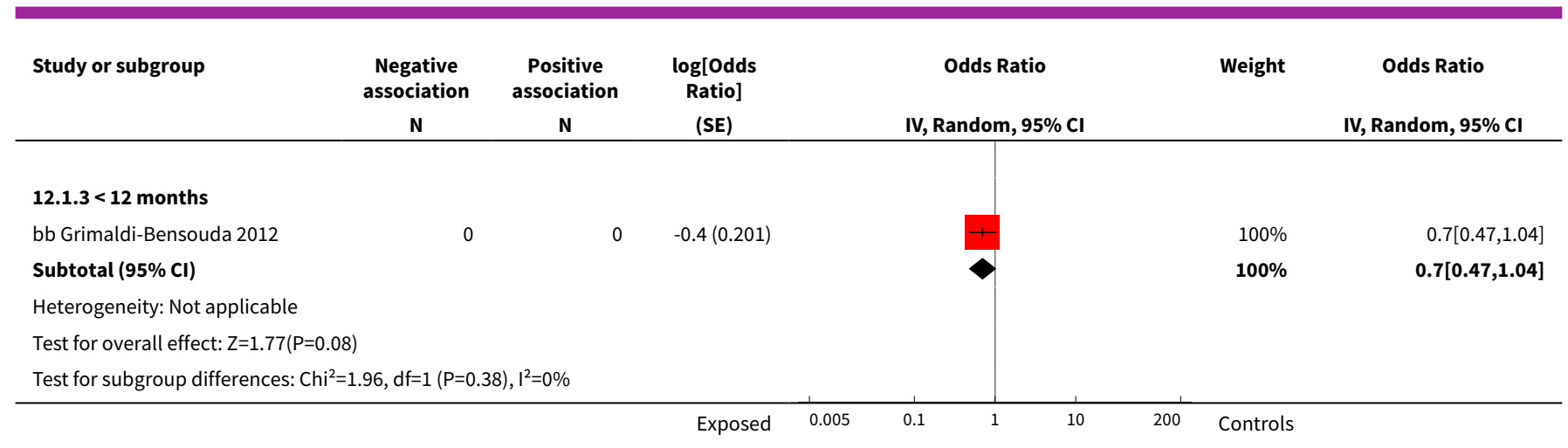

\section{Analysis 12.2. Comparison 12 Serious adverse events: immune thrombocytopenic purpura - case- control studies, Outcome 2 Seasonal influenza vaccine - general population (unadjusted data).}

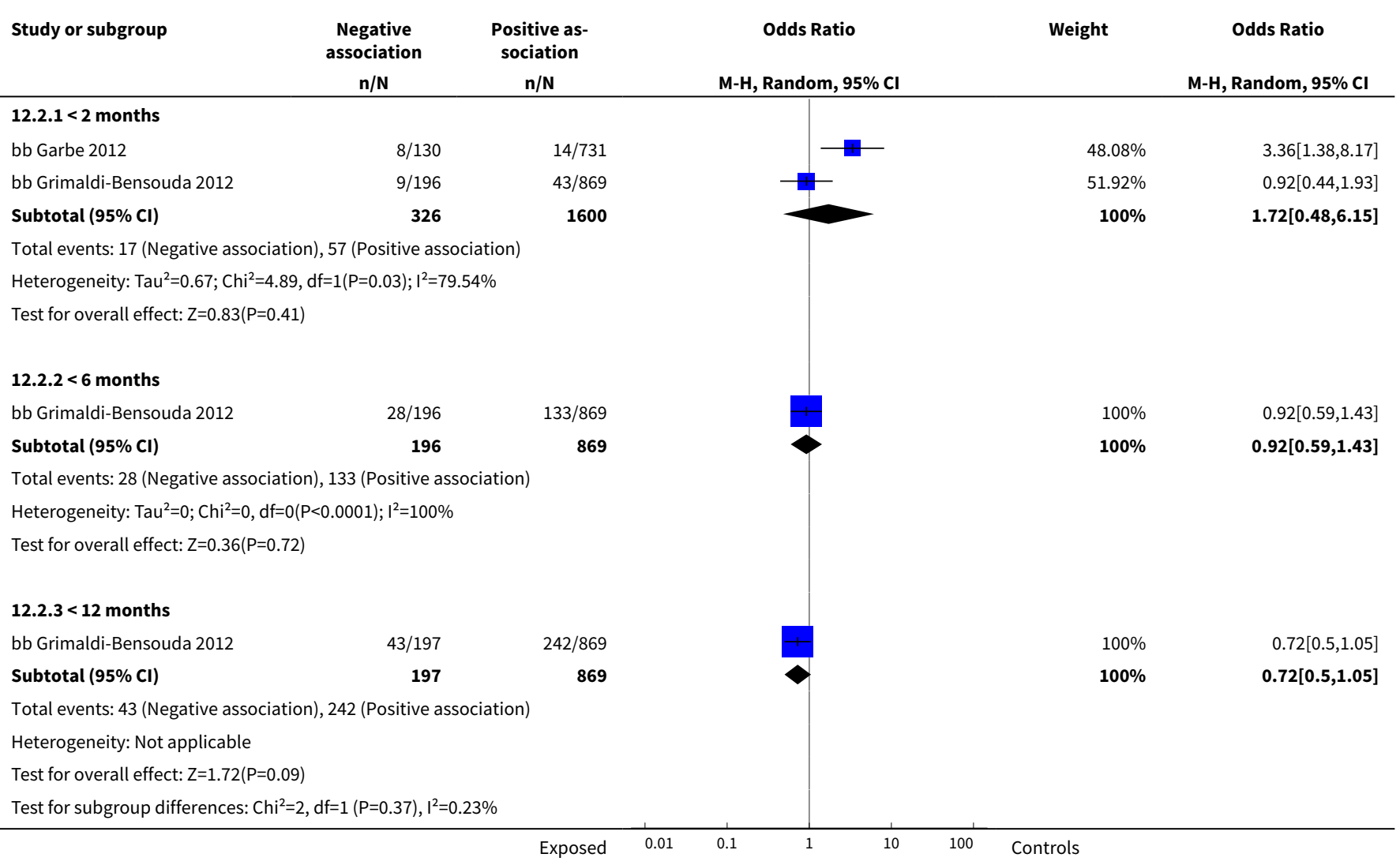

Comparison 13. 1968 to 1969 pandemic: inactivated polyvalent parenteral influenza vaccine versus placebo

\begin{tabular}{lllll}
\hline Outcome or subgroup title & No. of studies & $\begin{array}{l}\text { No. of partici- } \\
\text { pants }\end{array}$ & Statistical method & Effect size \\
\hline 1 Influenza-like illness & 3 & 3065 & $\begin{array}{l}\text { Risk Ratio (M-H, Random, 95\% } \\
\text { Cl) }\end{array}$ & $0.71[0.57,0.88]$ \\
\hline
\end{tabular}




\begin{tabular}{|c|c|c|c|c|}
\hline Outcome or subgroup title & No. of studies & $\begin{array}{l}\text { No. of partici- } \\
\text { pants }\end{array}$ & Statistical method & Effect size \\
\hline $\begin{array}{l}\text { 1.1 Standard recommended par- } \\
\text { enteral - non-matching - } 1 \text { dose }\end{array}$ & 3 & 2715 & $\begin{array}{l}\text { Risk Ratio (M-H, Random, 95\% } \\
\mathrm{CI} \text { ) }\end{array}$ & $0.74[0.57,0.95]$ \\
\hline $\begin{array}{l}\text { 1.2 Standard recommended par- } \\
\text { enteral - non-matching - } 2 \text { doses }\end{array}$ & 1 & 350 & $\begin{array}{l}\text { Risk Ratio (M-H, Random, 95\% } \\
\mathrm{CI} \text { ) }\end{array}$ & $0.66[0.44,0.98]$ \\
\hline 2 Influenza & 1 & 2072 & $\begin{array}{l}\text { Risk Ratio (M-H, Random, 95\% } \\
\text { CI) }\end{array}$ & $0.47[0.26,0.87]$ \\
\hline $\begin{array}{l}\text { 2.1 Standard recommended par- } \\
\text { enteral - non-matching }\end{array}$ & 1 & 2072 & $\begin{array}{l}\text { Risk Ratio (M-H, Random, 95\% } \\
\mathrm{Cl} \text { ) }\end{array}$ & $0.47[0.26,0.87]$ \\
\hline 3 Hospitalisations & 1 & 2072 & $\begin{array}{l}\text { Risk Ratio (M-H, Random, 95\% } \\
\mathrm{Cl} \text { ) }\end{array}$ & $0.83[0.41,1.68]$ \\
\hline $\begin{array}{l}\text { 3.1 Standard recommended par- } \\
\text { enteral - non-matching }\end{array}$ & 1 & 2072 & $\begin{array}{l}\text { Risk Ratio (M-H, Random, 95\% } \\
\mathrm{CI})\end{array}$ & $0.83[0.41,1.68]$ \\
\hline 4 Pneumonia & 1 & 2072 & $\begin{array}{l}\text { Risk Ratio (M-H, Random, 95\% } \\
\mathrm{Cl} \text { ) }\end{array}$ & $1.01[0.14,7.17]$ \\
\hline $\begin{array}{l}\text { 4.1 Standard recommended par- } \\
\text { enteral - non-matching }\end{array}$ & 1 & 2072 & $\begin{array}{l}\text { Risk Ratio (M-H, Random, 95\% } \\
\mathrm{Cl} \text { ) }\end{array}$ & $1.01[0.14,7.17]$ \\
\hline
\end{tabular}

Analysis 13.1. Comparison 131968 to 1969 pandemic: inactivated polyvalent parenteral influenza vaccine versus placebo, Outcome 1 Influenza-like illness.

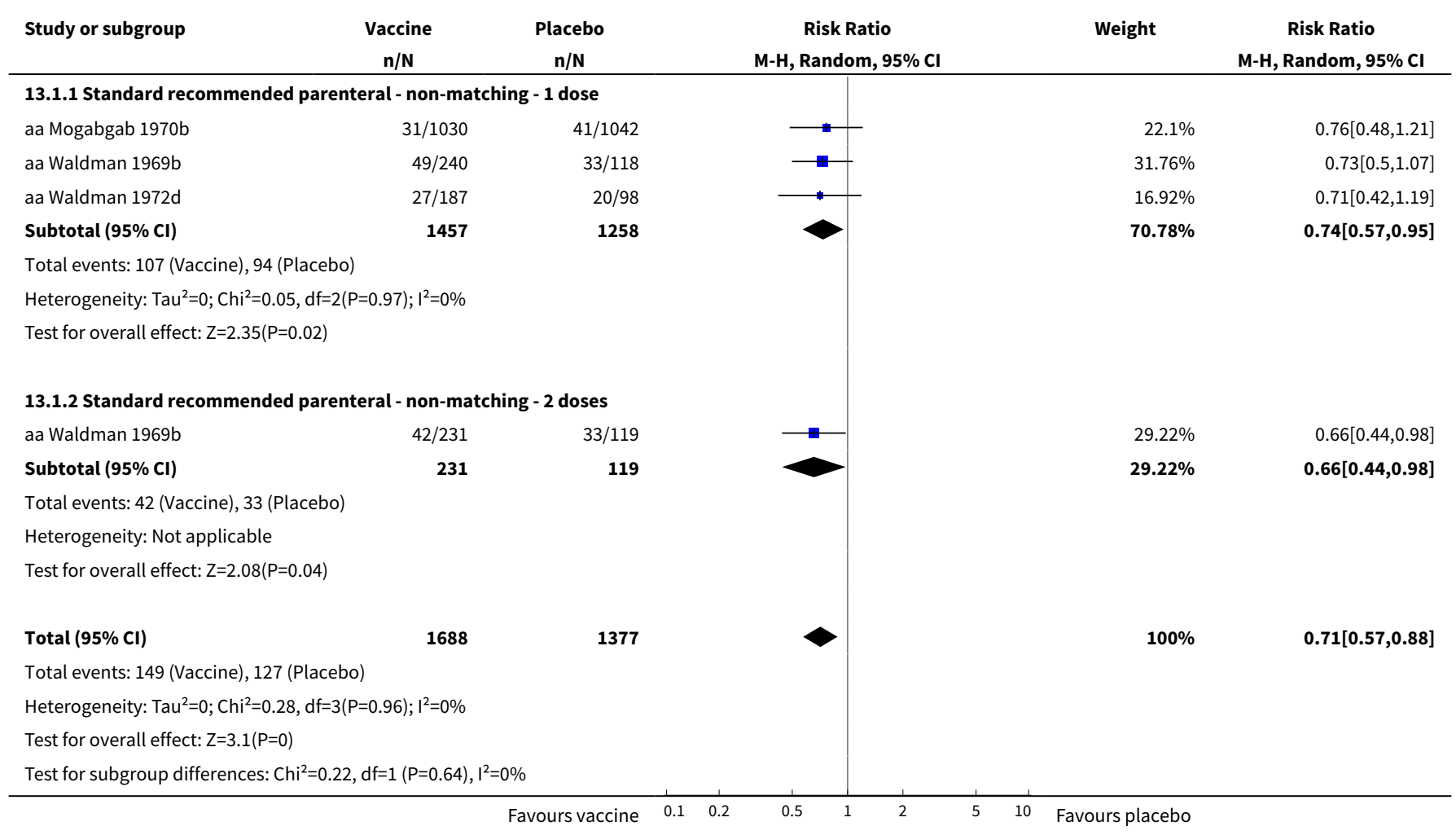


Analysis 13.2. Comparison 131968 to 1969 pandemic: inactivated polyvalent parenteral influenza vaccine versus placebo, Outcome 2 Influenza.

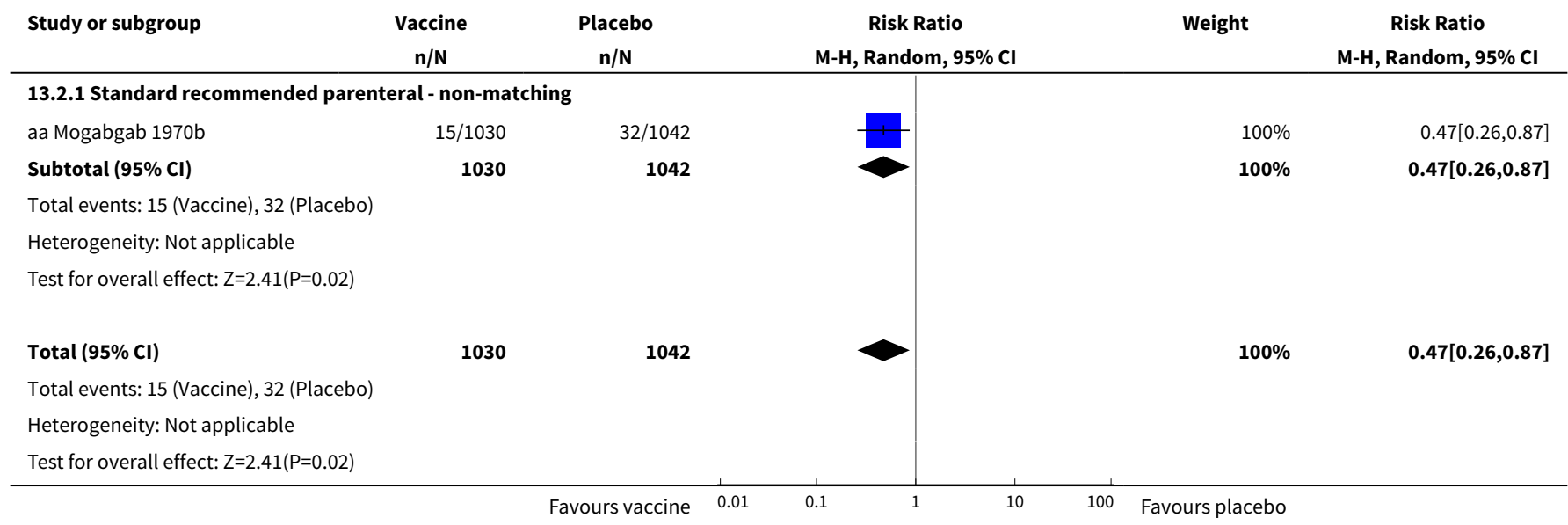

Analysis 13.3. Comparison 131968 to 1969 pandemic: inactivated polyvalent parenteral influenza vaccine versus placebo, Outcome 3 Hospitalisations.

\begin{tabular}{|c|c|c|c|c|c|}
\hline Study or subgroup & $\begin{array}{c}\text { Vaccine } \\
\mathbf{n} / \mathbf{N}\end{array}$ & $\begin{array}{c}\text { Placebo } \\
n / N\end{array}$ & $\begin{array}{c}\text { Risk Ratio } \\
\text { M-H, Random, 95\% Cl }\end{array}$ & Weight & $\begin{array}{c}\text { Risk Ratio } \\
\text { M-H, Random, } 95 \% \text { Cl }\end{array}$ \\
\hline \multicolumn{6}{|c|}{ 13.3.1 Standard recommended parenteral - non-matching } \\
\hline aa Mogabgab 1970b & $14 / 1030$ & $17 / 1042$ & & $100 \%$ & $0.83[0.41,1.68]$ \\
\hline Subtotal $(95 \% \mathrm{Cl})$ & 1030 & 1042 & & $100 \%$ & $0.83[0.41,1.68]$ \\
\hline \multicolumn{6}{|c|}{ Total events: 14 (Vaccine), 17 (Placebo) } \\
\hline \multicolumn{6}{|c|}{ Heterogeneity: Not applicable } \\
\hline \multicolumn{6}{|c|}{ Test for overall effect: $\mathrm{Z}=0.51(\mathrm{P}=0.61)$} \\
\hline Total $(95 \% \mathrm{Cl})$ & 1030 & 1042 & & $100 \%$ & $0.83[0.41,1.68]$ \\
\hline \multicolumn{6}{|c|}{ Total events: 14 (Vaccine), 17 (Placebo) } \\
\hline \multicolumn{6}{|c|}{ Heterogeneity: Not applicable } \\
\hline \multicolumn{6}{|c|}{ Test for overall effect: $\mathrm{Z}=0.51(\mathrm{P}=0.61)$} \\
\hline
\end{tabular}

Analysis 13.4. Comparison 131968 to 1969 pandemic: inactivated polyvalent parenteral influenza vaccine versus placebo, Outcome 4 Pneumonia.

\begin{tabular}{|c|c|c|c|c|c|}
\hline Study or subgroup & $\begin{array}{c}\text { Vaccine } \\
\mathrm{n} / \mathrm{N}\end{array}$ & $\begin{array}{c}\text { Placebo } \\
\mathbf{n} / \mathbf{N}\end{array}$ & $\begin{array}{c}\text { Risk Ratio } \\
\text { M-H, Random, } 95 \% \mathrm{Cl} \\
\end{array}$ & Weight & $\begin{array}{c}\text { Risk Ratio } \\
\text { M-H, Random, } 95 \% \mathrm{Cl} \\
\end{array}$ \\
\hline \multicolumn{6}{|c|}{ 13.4.1 Standard recommended parenteral - non-matching } \\
\hline aa Mogabgab 1970b & $2 / 1030$ & $2 / 1042$ & & $100 \%$ & $1.01[0.14,7.17]$ \\
\hline Subtotal $(95 \% \mathrm{Cl})$ & 1030 & 1042 & & $100 \%$ & $1.01[0.14,7.17]$ \\
\hline \multicolumn{6}{|c|}{ Total events: 2 (Vaccine), 2 (Placebo) } \\
\hline \multicolumn{6}{|c|}{ Heterogeneity: Not applicable } \\
\hline
\end{tabular}




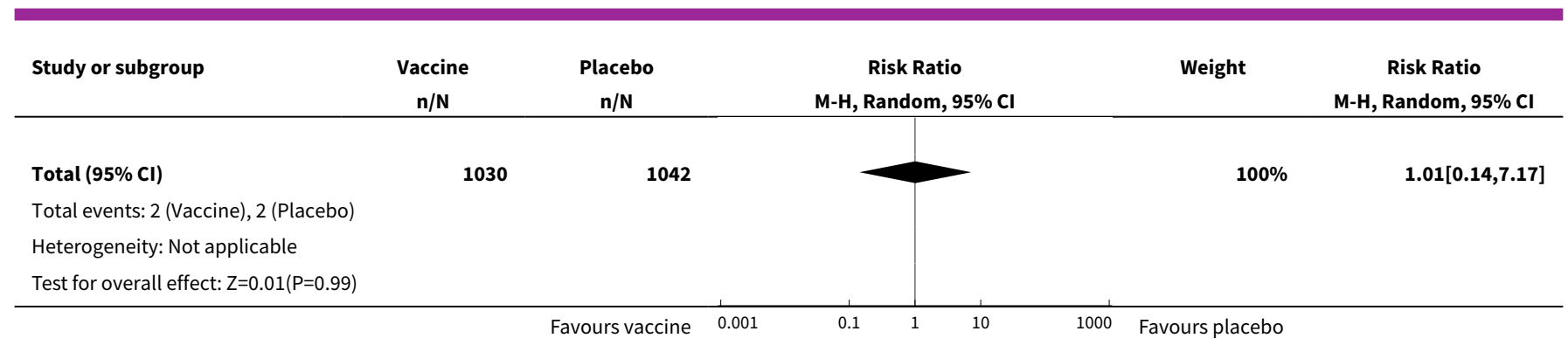

\section{Comparison 14. 1968 to 1969 pandemic: inactivated monovalent parenteral influenza vaccine versus placebo}

\begin{tabular}{|c|c|c|c|c|}
\hline Outcome or subgroup title & No. of studies & $\begin{array}{l}\text { No. of partici- } \\
\text { pants }\end{array}$ & Statistical method & Effect size \\
\hline 1 Influenza-like illness & 4 & 4580 & $\begin{array}{l}\text { Risk Ratio (M-H, Random, 95\% } \\
\text { Cl) }\end{array}$ & $0.35[0.25,0.48]$ \\
\hline $\begin{array}{l}1.1 \text { WHO recommended parenter- } \\
\text { al - matching vaccine - } 1 \text { dose }\end{array}$ & 4 & 4226 & $\begin{array}{l}\text { Risk Ratio (M-H, Random, 95\% } \\
\mathrm{Cl} \text { ) }\end{array}$ & $0.35[0.23,0.53]$ \\
\hline $\begin{array}{l}1.2 \text { WHO recommended parenter- } \\
\text { al - matching vaccine - } 2 \text { doses }\end{array}$ & 1 & 354 & $\begin{array}{l}\text { Risk Ratio (M-H, Random, 95\% } \\
\mathrm{Cl} \text { ) }\end{array}$ & $0.35[0.22,0.57]$ \\
\hline 2 Influenza & 1 & 1923 & $\begin{array}{l}\text { Risk Ratio (M-H, Random, 95\% } \\
\mathrm{Cl} \text { ) }\end{array}$ & $0.07[0.02,0.31]$ \\
\hline $\begin{array}{l}2.1 \text { WHO recommended parenter- } \\
\text { al - matching vaccine }\end{array}$ & 1 & 1923 & $\begin{array}{l}\text { Risk Ratio (M-H, Random, 95\% } \\
\mathrm{Cl} \text { ) }\end{array}$ & $0.07[0.02,0.31]$ \\
\hline 3 Hospitalisations & 1 & 1923 & $\begin{array}{l}\text { Risk Ratio (M-H, Random, 95\% } \\
\mathrm{CI})\end{array}$ & $0.35[0.13,0.94]$ \\
\hline $\begin{array}{l}3.1 \text { WHO recommended parenter- } \\
\text { al - matching vaccine }\end{array}$ & 1 & 1923 & $\begin{array}{l}\text { Risk Ratio (M-H, Random, 95\% } \\
\mathrm{Cl})\end{array}$ & $0.35[0.13,0.94]$ \\
\hline 4 Pneumonia & 1 & 1923 & $\begin{array}{l}\text { Risk Ratio (M-H, Random, 95\% } \\
\mathrm{Cl})\end{array}$ & $0.59[0.05,6.51]$ \\
\hline $\begin{array}{l}\text { 4.1 WHO recommended parenter- } \\
\text { al - matching vaccine }\end{array}$ & 1 & 1923 & $\begin{array}{l}\text { Risk Ratio (M-H, Random, 95\% } \\
\mathrm{Cl})\end{array}$ & $0.59[0.05,6.51]$ \\
\hline 5 Working days lost & 1 & 1667 & $\begin{array}{l}\text { Mean Difference (IV, Random, } \\
95 \% \mathrm{CI} \text { ) }\end{array}$ & $-0.45[-0.60,-0.30]$ \\
\hline $\begin{array}{l}5.1 \text { WHO recommended parenter- } \\
\text { al - matching vaccine }\end{array}$ & 1 & 1667 & $\begin{array}{l}\text { Mean Difference (IV, Random, } \\
95 \% \mathrm{CI} \text { ) }\end{array}$ & $-0.45[-0.60,-0.30]$ \\
\hline 6 Days ill & 1 & 1667 & $\begin{array}{l}\text { Mean Difference (IV, Random, } \\
95 \% \mathrm{CI} \text { ) }\end{array}$ & $-0.45[-0.60,-0.30]$ \\
\hline $\begin{array}{l}6.1 \text { WHO recommended - match- } \\
\text { ing vaccine }\end{array}$ & 1 & 1667 & $\begin{array}{l}\text { Mean Difference (IV, Random, } \\
95 \% \mathrm{CI})\end{array}$ & $-0.45[-0.60,-0.30]$ \\
\hline
\end{tabular}


Analysis 14.1. Comparison 141968 to 1969 pandemic: inactivated monovalent parenteral influenza vaccine versus placebo, Outcome 1 Influenza-like illness.

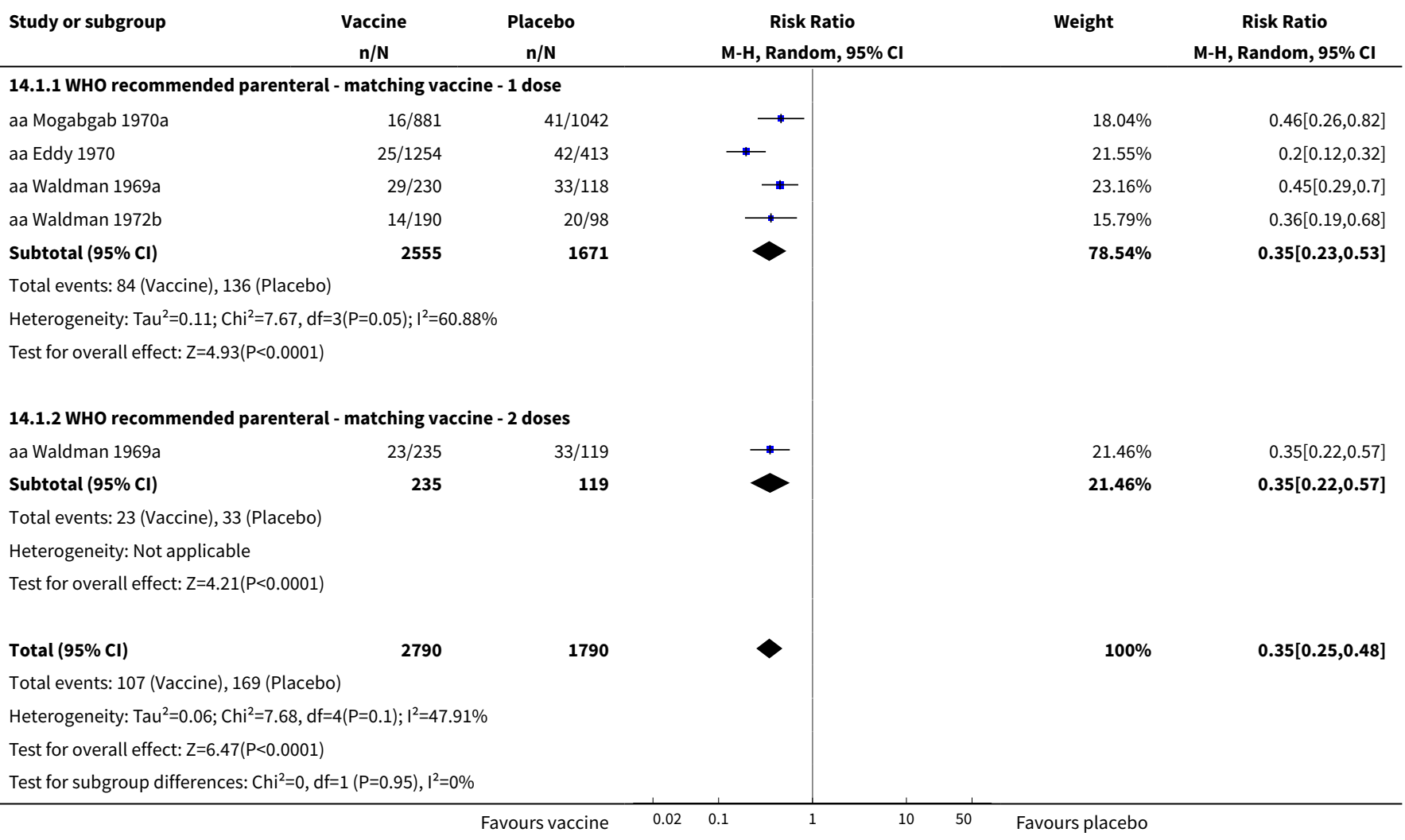

Analysis 14.2. Comparison 141968 to 1969 pandemic: inactivated monovalent parenteral influenza vaccine versus placebo, Outcome $\mathbf{2}$ Influenza.

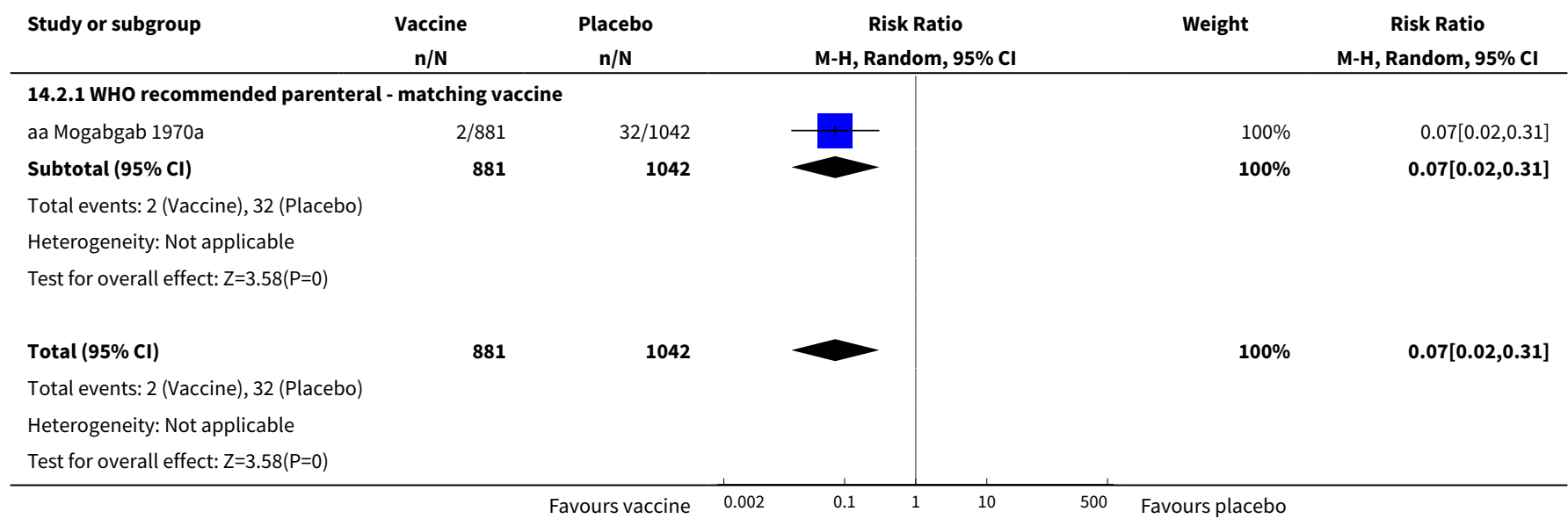


Analysis 14.3. Comparison 141968 to 1969 pandemic: inactivated monovalent parenteral influenza vaccine versus placebo, Outcome 3 Hospitalisations.

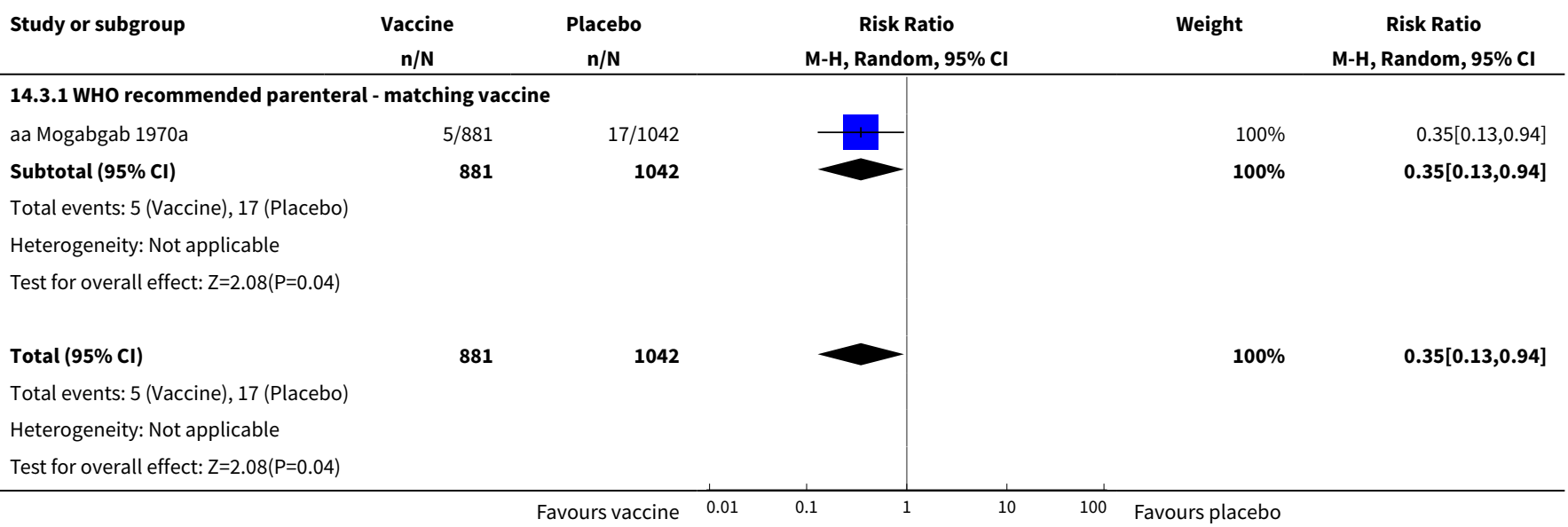

Analysis 14.4. Comparison 141968 to 1969 pandemic: inactivated monovalent parenteral influenza vaccine versus placebo, Outcome 4 Pneumonia.

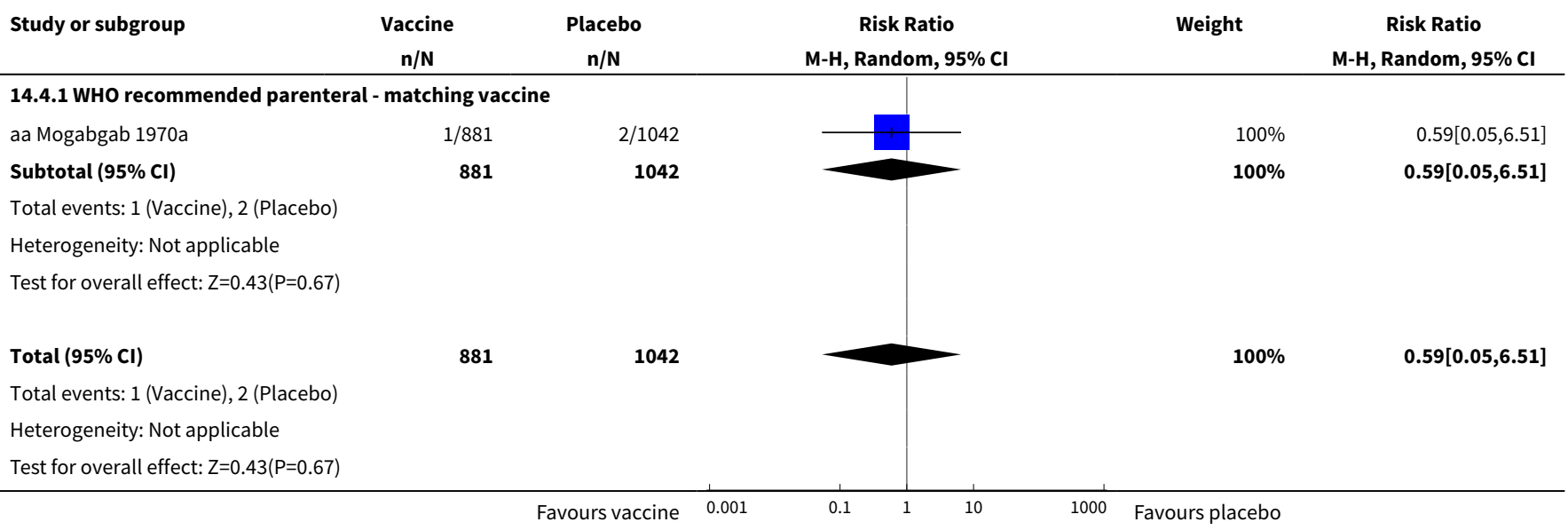

Analysis 14.5. Comparison 141968 to 1969 pandemic: inactivated monovalent parenteral influenza vaccine versus placebo, Outcome 5 Working days lost.

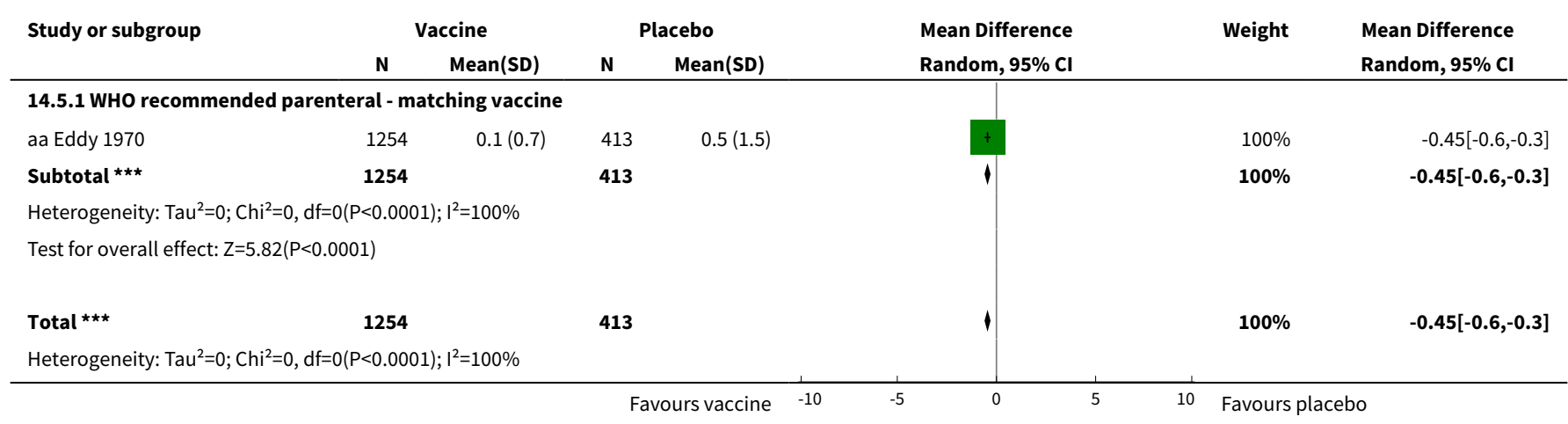




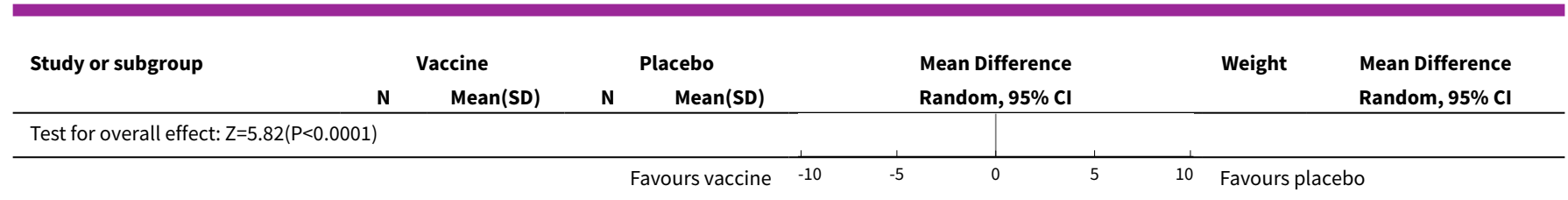

Analysis 14.6. Comparison 141968 to 1969 pandemic: inactivated monovalent parenteral influenza vaccine versus placebo, Outcome 6 Days ill.

\begin{tabular}{|c|c|c|c|c|c|c|c|}
\hline \multirow[t]{2}{*}{ Study or subgroup } & \multicolumn{2}{|c|}{ Vaccine } & \multicolumn{2}{|c|}{ Placebo } & \multirow{2}{*}{$\begin{array}{l}\text { Mean Difference } \\
\text { Random, } 95 \% \mathrm{Cl}\end{array}$} & \multirow[t]{2}{*}{ Weight } & \multirow{2}{*}{$\begin{array}{l}\text { Mean Difference } \\
\text { Random, } 95 \% \mathrm{Cl}\end{array}$} \\
\hline & $\mathbf{N}$ & Mean(SD) & $\mathbf{N}$ & $\operatorname{Mean}(\mathrm{SD})$ & & & \\
\hline \multicolumn{8}{|c|}{ 14.6.1 WHO recommended - matching vaccine } \\
\hline aa Eddy 1970 & 1254 & $0.1(0.7)$ & 413 & $0.5(1.5)$ & & $100 \%$ & $-0.45[-0.6,-0.3]$ \\
\hline Subtotal $* \star \star$ & 1254 & & 413 & & 1 & $100 \%$ & $-0.45[-0.6,-0.3]$ \\
\hline \multicolumn{8}{|c|}{ Heterogeneity: $\operatorname{Tau}^{2}=0 ; \mathrm{Chi}^{2}=0, \mathrm{df}=0(\mathrm{P}<0.0001) ; \mathrm{I}^{2}=100 \%$} \\
\hline \multicolumn{8}{|c|}{ Test for overall effect: $Z=5.82(P<0.0001)$} \\
\hline Total $\star \star \star ~$ & 1254 & & 413 & & 1 & $100 \%$ & $-0.45[-0.6,-0.3]$ \\
\hline \multicolumn{8}{|c|}{ Heterogeneity: $\operatorname{Tau}^{2}=0 ; C h i^{2}=0, d f=0(P<0.0001) ; I^{2}=100 \%$} \\
\hline \multicolumn{8}{|c|}{ Test for overall effect: $Z=5.82(P<0.0001)$} \\
\hline & & & & rs Vaccine & 0 & Favours $\mathrm{P}$ & \\
\hline
\end{tabular}

\section{Comparison 15. 1968 to 1969 pandemic: inactivated polyvalent aerosol influenza vaccine versus placebo}

\begin{tabular}{lllll}
\hline Outcome or subgroup title & No. of studies & $\begin{array}{l}\text { No. of partici- } \\
\text { pants }\end{array}$ & Statistical method & Effect size \\
\hline 1 Influenza-like illness & 2 & 1000 & $\begin{array}{l}\text { Risk Ratio (M-H, Random, } \\
95 \% \mathrm{Cl})\end{array}$ & $0.66[0.46,0.95]$ \\
\hline $\begin{array}{l}1.1 \text { Inactivated polyvalent aerosol vaccine } \\
\text { versus placebo - non-matching - 1 dose }\end{array}$ & 2 & 644 & $\begin{array}{l}\text { Risk Ratio (M-H, Random, } \\
95 \% \mathrm{Cl})\end{array}$ & $0.64[0.32,1.27]$ \\
\hline $\begin{array}{l}1.2 \text { Inactivated polyvalent aerosol vaccine } \\
\text { versus placebo - non-matching - 2 doses }\end{array}$ & 1 & 356 & $\begin{array}{l}\text { Risk Ratio (M-H, Random, } \\
95 \% \mathrm{Cl})\end{array}$ & $0.65[0.44,0.97]$ \\
\hline
\end{tabular}

Analysis 15.1. Comparison 151968 to 1969 pandemic: inactivated polyvalent aerosol influenza vaccine versus placebo, Outcome 1 Influenza-like illness.

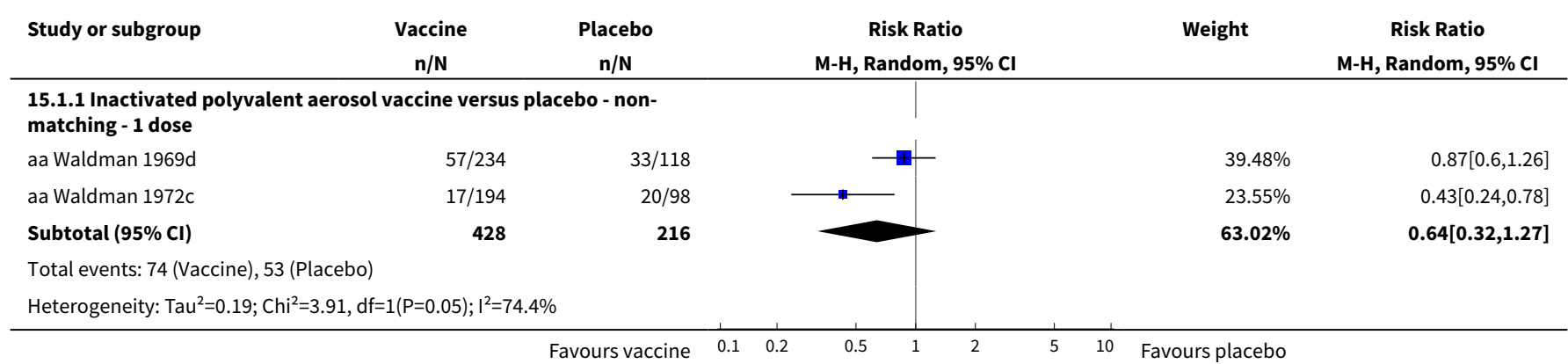




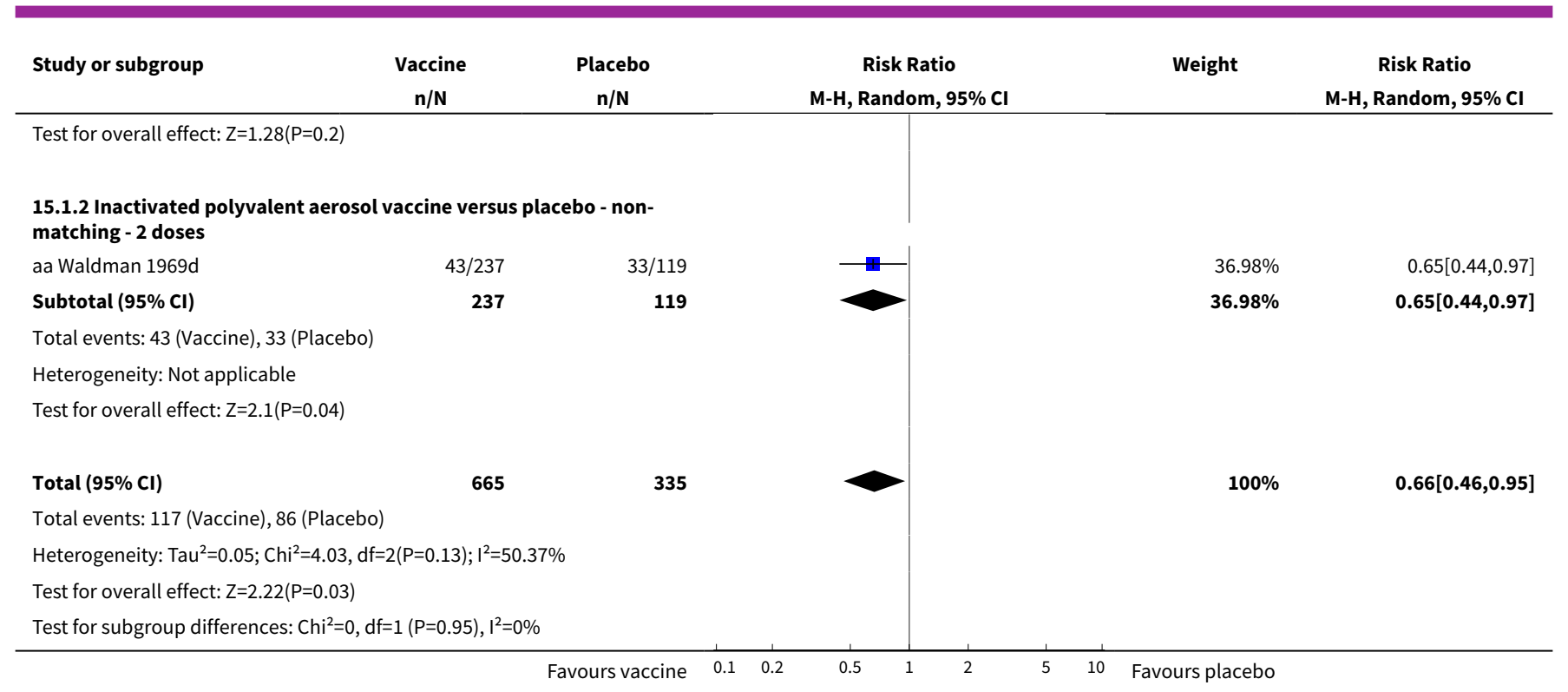

Comparison 16. 1968 to 1969 pandemic: inactivated monovalent aerosol influenza vaccine versus placebo

\begin{tabular}{lllll}
\hline Outcome or subgroup title & No. of studies & $\begin{array}{l}\text { No. of partici- } \\
\text { pants }\end{array}$ & Statistical method & Effect size \\
\hline 1 Influenza-like illness & 2 & 1009 & $\begin{array}{l}\text { Risk Ratio (M-H, Random, } \\
95 \% \mathrm{Cl})\end{array}$ & $0.54[0.32,0.91]$ \\
\hline $\begin{array}{l}1.1 \text { Inactivated monovalent aerosol vac- } \\
\text { cine versus placebo - matching - 1 dose }\end{array}$ & 2 & 650 & $\begin{array}{l}\text { Risk Ratio (M-H, Random, } \\
95 \% \mathrm{Cl})\end{array}$ & $0.49[0.17,1.41]$ \\
\hline $\begin{array}{l}1.2 \text { Inactivated monovalent aerosol vac- } \\
\text { cine versus placebo - matching - 2 doses }\end{array}$ & 1 & 359 & $\begin{array}{l}\text { Risk Ratio (M-H, Random, } \\
95 \% \mathrm{Cl})\end{array}$ \\
\hline
\end{tabular}

Analysis 16.1. Comparison 161968 to 1969 pandemic: inactivated monovalent aerosol influenza vaccine versus placebo, Outcome 1 Influenza-like illness.

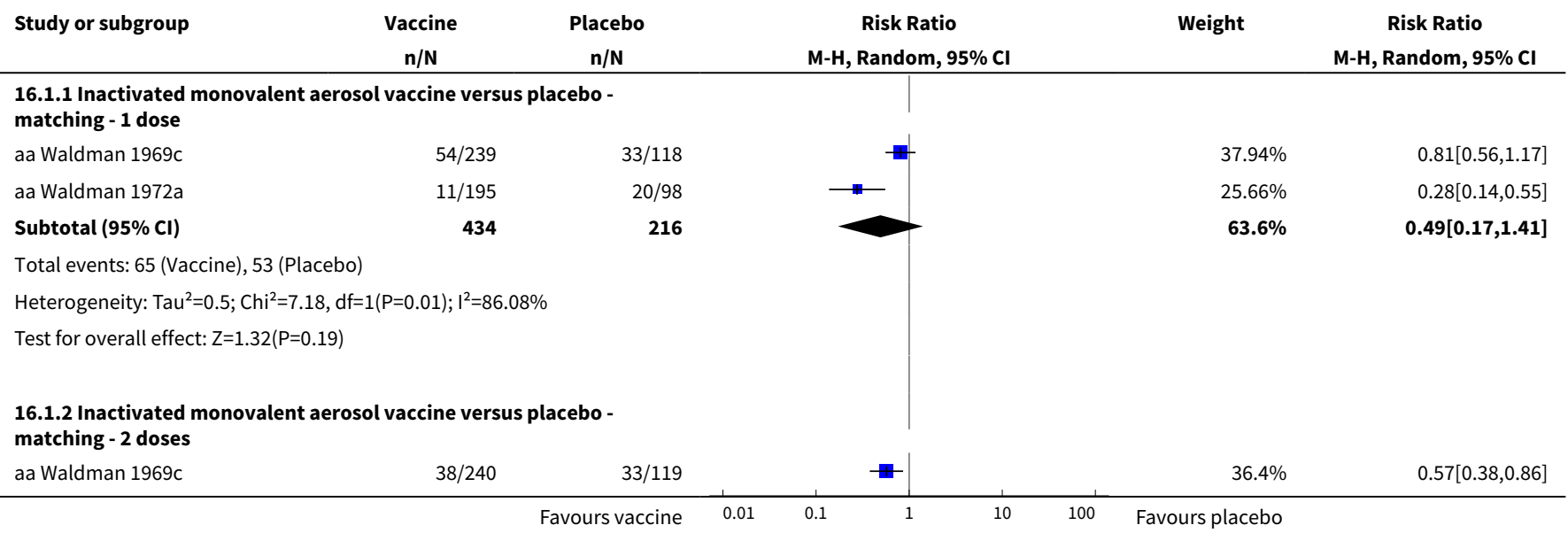




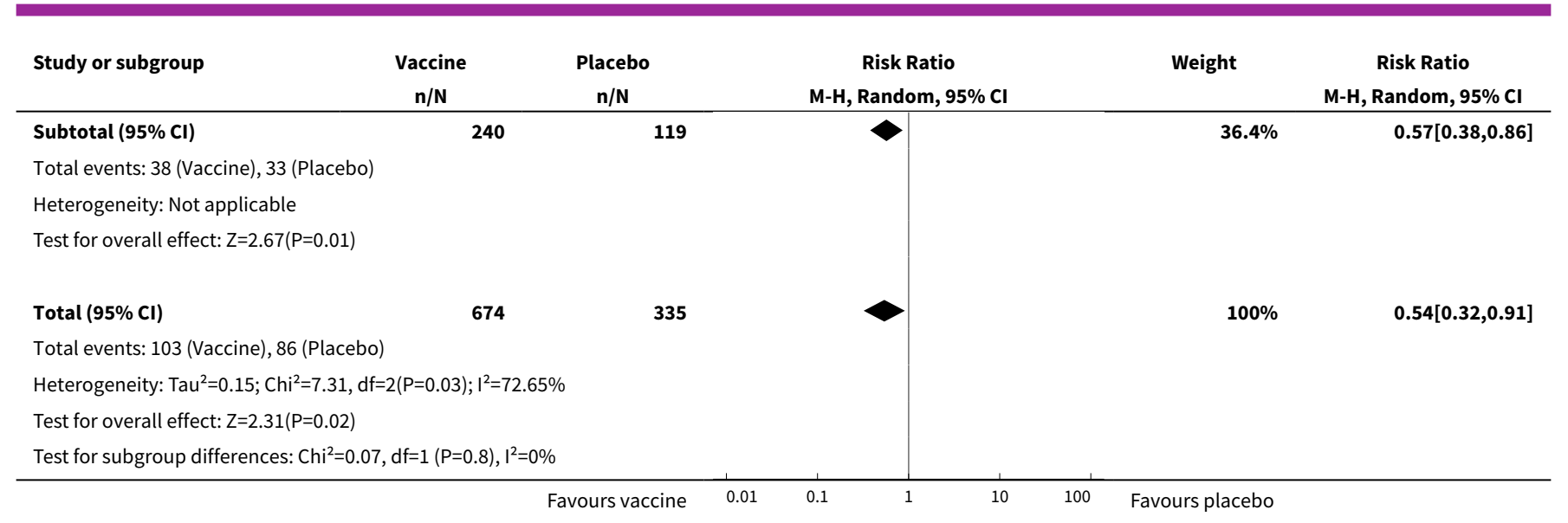

\section{Comparison 17. 1968 to 1969 pandemic: live aerosol influenza vaccine versus placebo}

\begin{tabular}{|c|c|c|c|c|}
\hline Outcome or subgroup title & No. of studies & $\begin{array}{l}\text { No. of partici- } \\
\text { pants }\end{array}$ & Statistical method & Effect size \\
\hline $\begin{array}{l}1 \text { Influenza cases (clinically defined } \\
\text { without clear definition) }\end{array}$ & 1 & 19887 & $\begin{array}{l}\text { Risk Ratio (M-H, Random, 95\% } \\
\mathrm{Cl})\end{array}$ & $0.98[0.92,1.05]$ \\
\hline 1.1 Non-matching & 1 & 19887 & $\begin{array}{l}\text { Risk Ratio (M-H, Random, 95\% } \\
\mathrm{Cl})\end{array}$ & $0.98[0.92,1.05]$ \\
\hline $\begin{array}{l}2 \text { Complications (bronchitis, otitis, } \\
\text { pneumonia) }\end{array}$ & 1 & 19887 & $\begin{array}{l}\text { Risk Ratio (M-H, Random, 95\% } \\
\mathrm{Cl})\end{array}$ & $0.25[0.03,2.24]$ \\
\hline 2.1 Non-matching & 1 & 19887 & $\begin{array}{l}\text { Risk Ratio (M-H, Random, 95\% } \\
\mathrm{Cl} \text { ) }\end{array}$ & $0.25[0.03,2.24]$ \\
\hline
\end{tabular}

Analysis 17.1. Comparison 171968 to 1969 pandemic: live aerosol influenza vaccine versus placebo, Outcome 1 Influenza cases (clinically defined without clear definition).

\begin{tabular}{|c|c|c|c|c|c|}
\hline Study or subgroup & $\begin{array}{c}\text { Vaccine } \\
n / N\end{array}$ & $\begin{array}{c}\text { Placebo } \\
n / N\end{array}$ & $\begin{array}{c}\text { Risk Ratio } \\
\text { M-H, Random, 95\% Cl }\end{array}$ & Weight & $\begin{array}{c}\text { Risk Ratio } \\
\text { M-H, Random, } 95 \% \mathrm{Cl}\end{array}$ \\
\hline \multicolumn{6}{|l|}{ 17.1.1 Non-matching } \\
\hline aa Sumarokow 1971 & $1407 / 9945$ & $1429 / 9942$ & & $100 \%$ & $0.98[0.92,1.05]$ \\
\hline Subtotal (95\% CI) & 9945 & 9942 & & $100 \%$ & $0.98[0.92,1.05]$ \\
\hline \multicolumn{6}{|c|}{ Total events: 1407 (Vaccine), 1429 (Placebo) } \\
\hline \multicolumn{6}{|c|}{ Heterogeneity: $\mathrm{Tau}^{2}=0 ; \mathrm{Chi}^{2}=0, \mathrm{df}=0(\mathrm{P}<0.0001) ; \mathrm{I}^{2}=100 \%$} \\
\hline \multicolumn{6}{|c|}{ Test for overall effect: $Z=0.45(P=0.65)$} \\
\hline Total $(95 \% \mathrm{Cl})$ & 9945 & 9942 & & $100 \%$ & $0.98[0.92,1.05]$ \\
\hline \multicolumn{6}{|c|}{ Total events: 1407 (Vaccine), 1429 (Placebo) } \\
\hline \multicolumn{6}{|c|}{ Heterogeneity: Tau $^{2}=0 ; \mathrm{Chi}^{2}=0, \mathrm{df}=0(\mathrm{P}<0.0001) ; \mathrm{I}^{2}=100 \%$} \\
\hline \multicolumn{6}{|c|}{ Test for overall effect: $Z=0.45(P=0.65)$} \\
\hline
\end{tabular}


Analysis 17.2. Comparison 171968 to 1969 pandemic: live aerosol influenza vaccine versus placebo, Outcome 2 Complications (bronchitis, otitis, pneumonia).

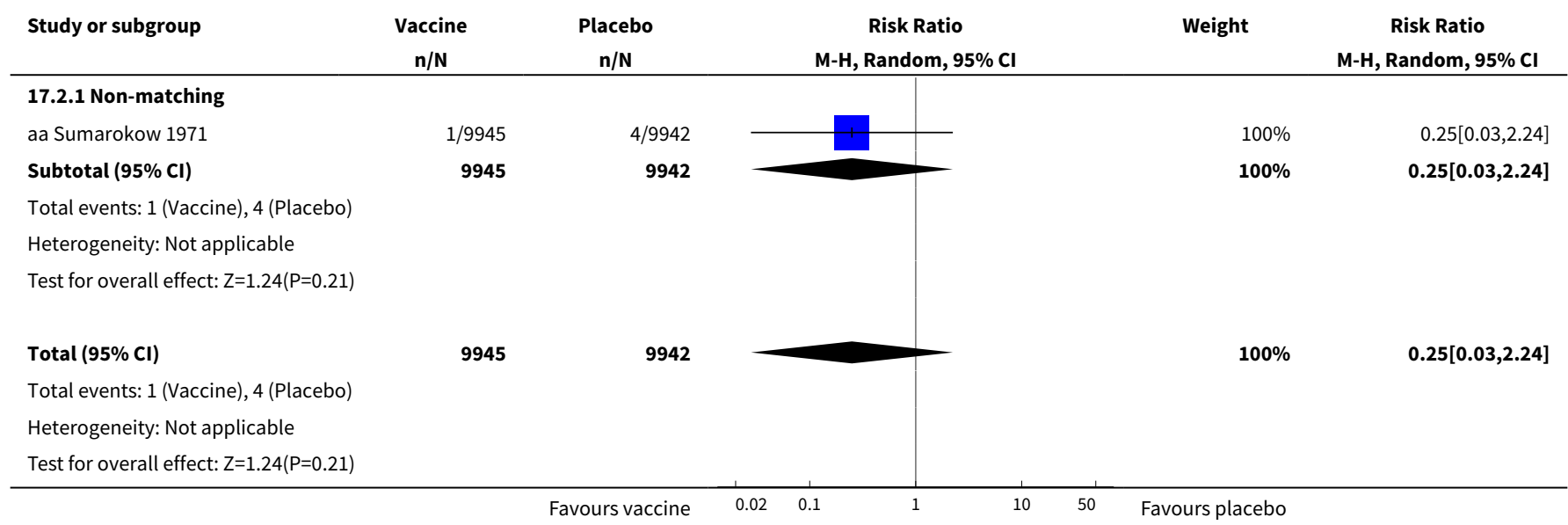

\section{ADDITIONAL TABLES}

Table 1. Studies included in the various versions of this review and their impact on our conclusions

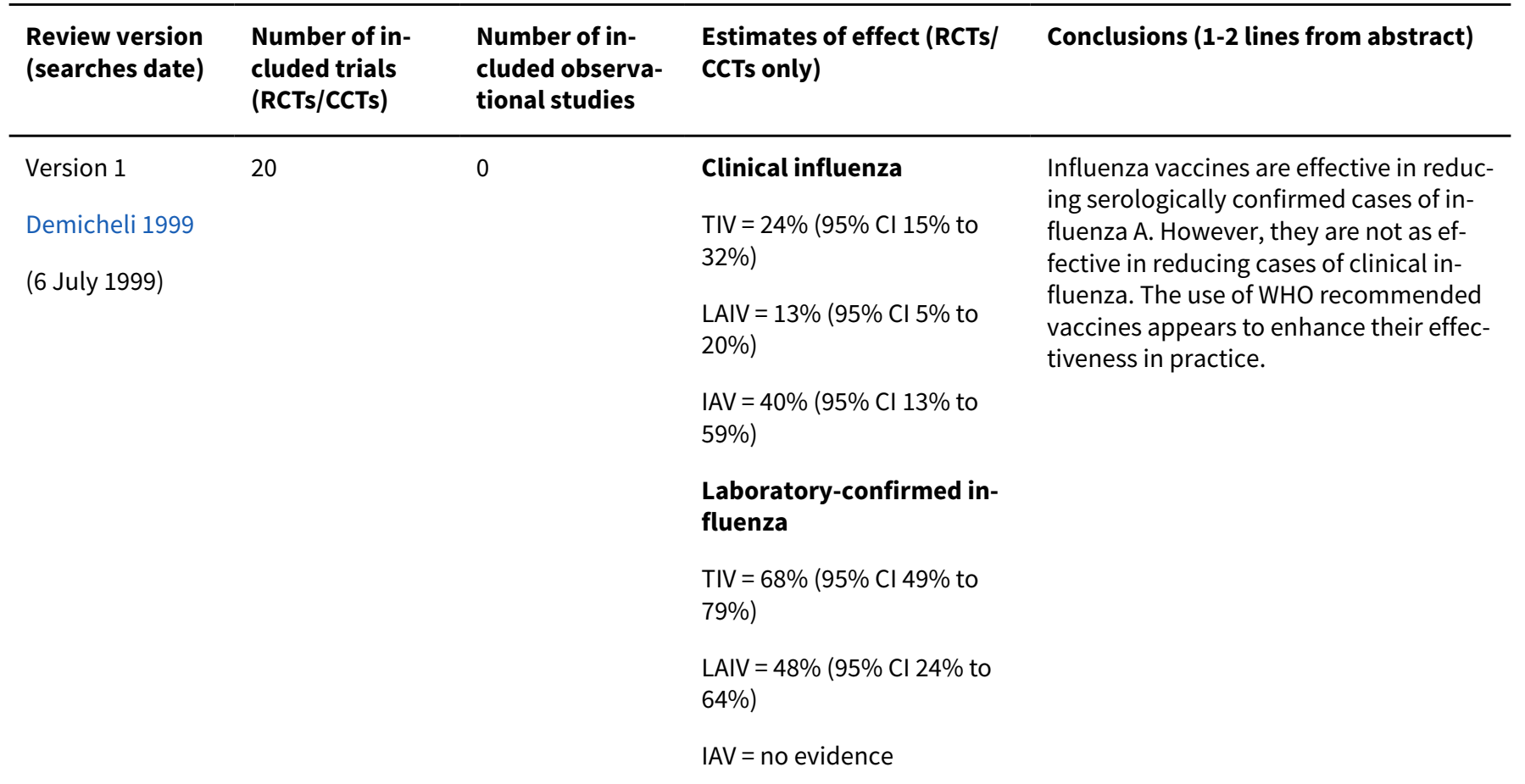

\begin{tabular}{|c|c|c|c|c|}
\hline $\begin{array}{l}\text { Version } 2 \\
\text { Demicheli } 2004 \\
\text { (24 May 2004) }\end{array}$ & 25 & 0 & $\begin{array}{l}\text { Clinical influenza } \\
\text { TIV }=25 \%(95 \% \mathrm{Cl} 13 \% \text { to } \\
35 \%) \\
\text { LAIV }=15 \%(95 \% \mathrm{Cl} 8 \% \text { to } \\
21 \%)\end{array}$ & $\begin{array}{l}\text { Influenza vaccines are effective in re- } \\
\text { ducing serologically confirmed cases of } \\
\text { influenza. However, they are not as ef- } \\
\text { fective in reducing cases of clinical in- } \\
\text { fluenza and number of working days } \\
\text { lost. Universal immunisation of healthy } \\
\text { adults is not supported by the results of } \\
\text { this review. }\end{array}$ \\
\hline
\end{tabular}


Table 1. Studies included in the various versions of this review and their impact on our conclusions (Continued)

\author{
$\mathrm{IAV}=40 \%(95 \% \mathrm{Cl} 13 \%$ to \\ $59 \%)$
}

\title{
Laboratory-confirmed in- fluenza
}

$\mathrm{TIV}=70 \%(95 \% \mathrm{CI} 56 \%$ to

$80 \%)$

LAIV $=48 \%(95 \% \mathrm{CI} 24 \%$ to

$64 \%)$

$\mathrm{IAV}=$ no evidence

\begin{tabular}{|c|c|c|c|}
\hline \multirow{8}{*}{$\begin{array}{l}\text { Version } 3 \\
\text { Jefferson } 2007 \\
\text { (16 February } \\
2007 \text { ) }\end{array}$} & 38 & 10 & ILI \\
\hline & & (for harms only) & $\begin{array}{l}\text { TIV }=30 \%(95 \% \mathrm{Cl} 17 \% \text { to } \\
41 \%)\end{array}$ \\
\hline & & & LAIV = n.s. \\
\hline & & & $I A V=$ n.s. \\
\hline & & & Influenza \\
\hline & & & $\begin{array}{l}\text { TIV }=80 \%(95 \% \mathrm{Cl} 56 \% \text { to } \\
81 \%)\end{array}$ \\
\hline & & & $\begin{array}{l}\text { LAIV }=56 \%(95 \% \mathrm{Cl} 19 \% \text { to } \\
76 \%)\end{array}$ \\
\hline & & & $\mathrm{IAV}=$ no evidence \\
\hline
\end{tabular}

$\begin{array}{llll}\text { Version } 4 & 40 & 10 & \text { ILI }\end{array}$

Jefferson 2010

(15 June 2010)
$\mathrm{TIV}=30 \%(95 \% \mathrm{Cl} 17 \%$ to $41 \%)$

LAIV $=$ n.s.

$\mathrm{IAV}=\mathrm{n} . \mathrm{s}$.

\section{Influenza}

TIV $=73 \%(95 \% \mathrm{CI} 54 \%$ to $84 \%)$

LAIV $=56 \%(95 \% \mathrm{Cl} 19 \%$ to $76 \%)$

$\mathrm{IAV}=$ no evidence
Influenza vaccines are effective in reducing cases of influenza, especially when the content accurately predicts circulating types and circulation is high. However, they are less effective in reducing cases of influenza-like illness and have a modest impact on working days lost. There is insufficient evidence to assess their impact on complications. Wholevirion monovalent vaccines may perform best in a pandemic.
Influenza vaccines have a modest effect in reducing influenza symptoms and working days lost. There is no evidence that they affect complications, such as pneumonia, or transmission.

\begin{tabular}{lll}
\hline $\begin{array}{l}\text { Version } 5 \\
\text { Jefferson 2014 }\end{array}$ & 48 & ILI \\
(4 March 2014) & TIV $=17 \%(95 \% \mathrm{Cl} 11 \%$ to \\
& $23 \%)$ \\
& LAIV = n.s. \\
& IAV = n.s. \\
& Influenza \\
& TIV $=63 \%(95 \% \mathrm{Cl} 55 \%$ to \\
& $69 \%)$
\end{tabular}

Influenza vaccines have a very modest effect in reducing influenza symptoms and working days lost in the general population, including pregnant women. No evidence of association between influenza vaccination and serious adverse events was found in the comparative studies considered in the review. 
Table 1. Studies included in the various versions of this review and their impact on our conclusions (Continued)

LAIV $=45 \%(95 \% \mathrm{Cl} 18 \%$ to

$63 \%$ )

$\mathrm{IAV}=$ n.s.

CCT: controlled clinical trial

$\mathrm{Cl}$ : confidence interval

IAV: inactivated aerosol vaccines

ILI: influenza-like illness

LAIV: live attenuated vaccines

n.s.: not statistically significant

$\mathrm{RCT}$ : randomised controlled trial

TIV: trivalent inactivated vaccines

WHO: World Health Organization

\section{Versions 1 and 2}

Effect estimates are from Comparison 02 (At least one vaccine recommended for that year versus placebo or other vaccine).

A clinically defined case was assumed as any case definition based on symptoms without further specification.

A clinically defined case (specific definition) was defined as:

- 'flu-like illness' according to a predefined list of symptoms (including the Centers for Disease Control and Prevention case definition for surveillance);

- 'upper respiratory illness' according to a predefined list of symptoms.

When more than one definition was given for the same trial, data related to the more specific definition were included.

In Analysis 2.1 from versions 1 and 2, studies with both definitions are included.

Evidence about effectiveness of aerosol inactivated vaccine comes only from studies carried out during the 1968-69 pandemic. From version 3 onwards, specific comparisons have been added.

\section{Versions 3, 4, 5}

Recommended vaccine matching circulating strains.

\section{Version 5}

Out of the 42 included observational studies, 8 assessed efficacy or effectiveness of vaccine, or both, when administered during pregnancy (6 cohort and 2 case-control studies).

\section{Version 6 (current)}

In two new RCTs included in this version, vaccination was performed during pregnancy.

Regarding efficacy/effectiveness of TIV administered in general population, estimates assessed by applying random-effects model were $16 \%(95 \% \mathrm{Cl} 9 \%$ to $23 \%)$ against ILI and $62 \%(95 \% \mathrm{Cl} 52 \%$ to $69 \%)$ against influenza, respectively.

In a previous interim unpublished update before the decision to stabilise the review was made, a further 16 observational studies were included: 3 case-control and 2 cohort studies assessing the safety of influenza vaccine administration in general population, 10 cohort studies assessing the safety of influenza vaccine administration during pregnancy, and one cohort study assessing efficacy/effectiveness of the vaccine administration during pregnancy. In this 2016 updated review, we included a total of 160 studies (137 data sets), while we no longer updated searches for observational comparative studies.

Table 2. Risk of bias in included studies

\begin{tabular}{lllll}
\hline Study design & High risk & Low risk & Unclear risk & Total \\
\hline Case-control & 3 & 2 & 18 & 23 \\
\hline Cohort & 14 & 8 & 18 & 40 \\
\hline RCT/CCT & 7 & 12 & 55 & 74 \\
\hline Total & 24 & 22 & 91 & 137 \\
\hline
\end{tabular}

CCT: controlled clinical trial

$\mathrm{RCT}$ : randomised controlled trial

This table displays the overall methodological quality assessment of the included studies described in the text and represented in extended form (with all items of the tools) in Figure 1. 
Table 3. Funding source of included studies

\begin{tabular}{lllll}
\hline Study design & $\begin{array}{l}\text { Government, institutional, or } \\
\text { public }\end{array}$ & Industry & Mixed & Total \\
\hline Case-control & 14 & 2 & 2 & 18 \\
\hline Cohort & 33 & 5 & 2 & 40 \\
\hline RCT/CCT & 32 & 15 & 5 & 52 \\
\hline Total & 79 & 22 & 9 & 110 \\
\hline
\end{tabular}

CCT: controlled clinical trial

$\mathrm{RCT}$ : randomised controlled trial

Table 4. Sensitivity analysis for 'Summary of findings' table outcomes

\begin{tabular}{lll}
\hline Outcome (analysis) & All studies (primary analysis) & $\begin{array}{l}\text { Studies at low risk of bias (sensitivity } \\
\text { analysis) }\end{array}$ \\
\hline Influenza (Analysis 1.1) & RR 0.41 (0.36 to 0.47) & RR 0.34 (0.25 to 0.45) \\
\hline Influenza-like illness (Analysis 1.2) & RR 0.84 (0.75 to 0.95) & RR 0.82 (0.69 to 0.98) \\
\hline Hospitalisations (Analysis 1.8) & RR 0.96 (0.85 to 1.08$)$ & RR 2.89 (0.12 to 70.68) \\
\hline Fever (Analysis 1.11.2) & RR $1.55(1.26$ to 1.91$)$ & RR $1.59(1$ to 2.53) \\
\hline Nausea/vomiting (Analysis 1.11.5) & RR $1.80(0.65$ to 5.04) & RR $7.05(1.61$ to 30.87)
\end{tabular}

RR: risk ratio

\section{A P P E N D I C S}

\section{Appendix 1. Glossary}

\section{Efficacy}

The impact of an intervention (drug, vaccines, etc.) on a problem or disease in ideal conditions - in this case the capacity of vaccines to prevent or treat influenza and its complications.

\section{Effectiveness}

The impact of an intervention (drug, vaccines, etc.) on a problem or disease in field conditions - in this case the capacity of vaccines to prevent influenza-like illness and its complications.

\section{Influenza}

An acute respiratory infection caused by a virus of the Orthomyxoviridae family. Three serotypes are known (A, B, and C). Influenza causes an acute febrile illness with myalgia, headache, and cough. Although the median duration of the acute illness is three days, cough and malaise can persist for weeks. Complications of influenza include otitis media, pneumonia, secondary bacterial pneumonia, exacerbations of chronic respiratory disease, and bronchiolitis in children. These illnesses may require treatment in a hospital and can be life-threatening, particularly in 'high-risk' people, such as the elderly and people suffering from chronic heart disease. Additionally, influenza can cause a range of non-respiratory complications including febrile convulsions, Reye's syndrome, and myocarditis. The influenza virus is composed of a protein envelope around an RNA core. On the envelope are two antigens: neuraminidase ( $\mathrm{N}$ antigen) and haemagglutinin (H antigen). Haemagglutinin is an enzyme that facilitates the entry of the virus into cells of the respiratory epithelium, while neuraminidase facilitates 
the release of newly produced viral particles from infected cells. The influenza virus has a marked propensity to mutate its external antigenic composition to escape the host's immune defences. Given this extreme mutability, a classification of viral subtype $\mathrm{A}$ based on $\mathrm{H}$ and $\mathrm{N}$ typing has been introduced. Additionally, strains are classified on the basis of antigenic type of the nucleoprotein core (A, B), geographical location of first isolation, strain serial number, and year of isolation. Every item is separated by a slash mark (e.g. A/Wuhan/359/95 (H3N2)). Unless otherwise specified such strains are of human origin. The production of antibodies against influenza beyond a conventional quantitative threshold is called seroconversion. Seroconversion in the absence of symptoms is called asymptomatic influenza.

\section{Influenza-like illness}

An acute respiratory illness caused by scores of different viruses (including influenza A and B) presenting with symptoms and signs that are not distinguishable from those of influenza. Influenza-like illness does not have documented laboratory isolation of the causative agent and is what commonly presents to physicians and patients (also known as 'the flu').

\section{Appendix 2. Search strategies used to identify trials \\ MEDLINE (PubMed)}

\#1 "Influenza, Human"[MeSH]

\#2 "Influenzavirus A"[MeSH]

\#3 "Influenzavirus B"[MeSH]

\#4 influenza*[Text Word] OR flu[Text Word]

\#5 \#1 OR \#2 OR \#3 OR \#4

\#6 "Vaccines"[MeSH]

\#7 "Immunization"[MeSH]

$\# 8$ (vaccin ${ }^{\star}[$ Text Word] OR immuni*[Text Word] OR inocula*[Text Word])

\#9 \#6 OR \#7 OR \#8

\#10 \#5 AND \#10

\#11 "Influenza Vaccines"[MeSH]

\#12 \#10 OR \#11

\#13 "Randomized Controlled Trial" [Publication Type]

\#14 "Controlled Clinical Trial" [Publication Type]

\#15 randomized[Title/Abstract]

\#16 placebo[Title/Abstract]

\#17 "drug therapy" [Subheading]

\#18 randomly[Title/Abstract]

\#19 trial[Title/Abstract]

\#20 groups[Title/Abstract]

\#21 \#13 OR \#14 OR \#15 OR \#16 OR \#17 OR \#18 OR \#19 OR \#20

\#22 ("Animals"[MeSH]) NOT "Humans"[MeSH]

\#23 \#21 NOT \#22

\#24 \#12 AND \#23

\section{Embase (Elsevier)}

\#1 'influenza vaccine'/de

\#2 'influenza'/exp

\#3 'influenza virus a'/exp OR 'influenza virus b'/exp

\#4 flu:ab,ti OR influenza*:ab,ti

\#5 \#2 OR \#3 OR \#4

\#6 'vaccine'/de OR 'acellular vaccine'/de OR 'dna vaccine'/de OR 'inactivated vaccine'/de OR 'live vaccine'/de OR 'subunit vaccine'/de OR 'virus vaccine'/de OR 'virosome vaccine'/de OR 'recombinant vaccine'/de

\#7 'immunization'/de OR 'vaccination'/de OR 'active immunization'/de OR 'immunoprophylaxis'/de OR 'mass immunization'/de

\#8 vaccin*:ab,ti OR immuni*:ab,ti OR inocul*:ab,ti

\#9 \#6 OR \#7 OR \#8

\#10 \#5 AND \#9

\#11 \#1 OR \#10

\#12 'randomized controlled trial'/exp OR 'single blind procedure'/exp OR 'double blind procedure'/exp OR 'crossover procedure'/exp

\#13 random*:ab,ti OR placebo*:ab,ti OR factorial*:ab,ti OR crossover*:ab,ti OR 'cross-over':ab,ti OR 'cross over':ab,ti OR assign*:ab,ti OR allocat ${ }^{\star}:$ ab,ti OR volunteer ${ }^{\star}:$ ab,ti OR ((singl ${ }^{\star}$ OR doubl*) NEAR/3 (blind ${ }^{\star}$ OR mask $\left.\left.^{\star}\right)\right)$ :ab,ti

$\# 14$ \#12 OR \#13

\#15 \#11 AND \#14

\section{WHO ICTRP}


vaccine $^{\star}$ AND influenza

immuni* AND influenza

inocul ${ }^{\star}$ AND influenza

vaccine ${ }^{\star}$ AND flu

immuni* AND flu

inocul ${ }^{*}$ AND flu

\section{ClinicalTrials.gov}

(vaccine $O R$ vaccines $O R$ vaccinate $O R$ vaccination $O R$ vaccinated $O R$ vaccinating $O R$ immunise $O R$ immunised $O R$ immunising $O R$ immunisation OR immunize OR immunized OR immunizing OR immunization) AND (influenza OR influenza OR flu)

(inoculate OR inoculated OR inoculating OR inoculation) AND (influenza OR influenza OR flu)

\section{Appendix 3. Search strategies used to identify observational study searches (prior to the 2017 update) MEDLINE (PubMed)}

\#1 "Influenza Vaccines"[MeSH] OR "Influenza, Human"[MeSH]

\#2 (influenza* [Text Word] OR flu[Text Word]) AND (vaccin*[Text Word] OR immuni*[Text Word] OR inocula*[Text Word])

\#3 \#1 OR \#2

\#4 (randomized controlled trial [pt] OR controlled clinical trial [pt] OR randomized [tiab] OR placebo [tiab] OR drug therapy [sh] OR randomly [tiab] OR trial [tiab] OR groups [tiab])

\#5 ("cross over" OR "crossover" OR "Follow Up") OR ("Cross-Over Studies"[MeSH] OR "Follow-Up Studies"[MeSH] OR "Prospective Studies"[MeSH]) OR ("time series" OR "interrupted time series") OR ("Case-Control Studies"[MeSH] OR (cases[Title/Abstract] AND controls[Title/Abstract])) OR ("Cohort Studies"[MeSH] OR cohort*) OR ("Comparative Study"[Publication Type]) OR ("before after"[Title/ Abstract] OR "before-after"[Title/Abstract] OR "before/after"[Title/Abstract] OR "before and after"[Title/Abstract]) OR (volunteer[Title/ Abstract]) OR (control* [Text Word] AND evaluation[Text Word]) OR (longitudinal[Text Word]) OR (retrospective* [Text Word])

\#6 \#4 OR \#5

\#7 \#3 OR \#6

\section{EMBASE}

\#1 'influenza vaccine' OR ( influenza OR flu AND ( vaccin* OR immuni* OR inoculat* )) OR 'influenza vaccine' /syn OR ('influenza' /exp AND 'vaccine' /exp)

\#2 'case control study' /syn OR 'case control' :de,ab,ti OR ( cases :ab,ti AND controls :ab,ti) OR 'cohort analysis' /syn OR 'cohort study' :de,ab,ti OR 'study cohort' : de,ab,ti OR prospectiv* :ab,ti OR volunteer* :ab,ti OR observational :ab,ti OR 'clinical trial' :it OR 'randomized controlled trial' :it OR 'drug therapy' /exp OR 'drug therapy' :de OR randomized :ab,ti OR randomised :ab,ti OR placebo :ab,ti OR randomly :ab,ti OR trial :ab,ti OR groups :ab,ti

\#3 'clinical trial' :it OR 'randomized controlled trial' :it OR 'randomized controlled trial' /exp OR 'randomization' /exp OR 'single blind procedure' /exp OR 'double blind procedure' /exp OR 'clinical trial' /exp OR 'clinical' NEAR/O 'trial' OR 'clinical trial' OR ( singl ${ }^{\star}$ OR doubl ${ }^{\star}$ OR trebl* OR tripl* AND ( mask* OR blind*)) OR 'placebo' /exp OR placebo^ OR random* OR 'control group' /exp OR 'experimental design' /exp OR 'comparative study' /exp OR 'evaluation study' OR 'evaluation studies' /exp OR 'follow up' /exp OR 'prospective study' /exp OR control* OR prospectiv ${ }^{\star}$ OR volunteer*

\#4 \#2 OR \#3

\#5 \#1 AND \#4

\#6 \#1 AND \#4 AND [embase]/lim

Appendix 4. Search strategies for 2010 update

MEDLINE (PubMed) 
\#1 "Influenza Vaccines"[MeSH] OR ("Influenza, Human/complications"[MeSH] OR "Influenza, Human/epidemiology"[MeSH] OR "Influenza, Human/immunology"[MeSH] OR "Influenza, Human/mortality"[MeSH] OR "Influenza, Human/prevention and control"[MeSH] OR "Influenza, Human/transmission"[MeSH])

\#2 ((influenza vaccin*[Text Word]) OR ((influenza [Text Word] OR flu[Text Word]) AND (vaccin*[Text Word] OR immuni*[Text Word] OR inoculation*[Text Word] OR efficacy[Text Word] OR effectiveness[Text Word])))

\#3 \#1 OR \#2

\#4 randomized controlled trial [pt] OR controlled clinical trial [pt] OR randomized [tiab] OR placebo [tiab] OR drug therapy [sh] OR randomly [tiab] OR trial [tiab] OR groups [tiab]) AND humans [mh]

\#5 ("cross over" OR "crossover" OR "Follow Up") OR ("Cross-Over Studies"[MeSH] OR "Follow-Up Studies"[MeSH] OR "Prospective Studies"[MeSH]) OR ("time series" OR "interrupted time series") OR (placebo* OR random* OR "double blind" OR "single blind" OR clinical trial* OR trial design) OR ("Case-Control Studies"[MeSH] OR (cases[Title/Abstract] AND controls[Title/Abstract])) OR ("Cohort Studies"[MeSH] OR cohort*) OR ("Comparative Study"[Publication Type]) OR ("before after"[Title/Abstract] OR "before-after"[Title/ Abstract] OR "before/after"[Title/Abstract] OR "before and after"[Title/Abstract]) OR (volunteer*[Title/Abstract]) OR (control*[Text Word] AND evaluation[Text Word])

\#6 \#4 OR \#5

\#7 \#3 AND \#6

\section{EMBASE}

\#1 'influenza vaccine' /exp OR 'influenza vaccine' OR ( influenza OR flu AND ( vaccin* OR immuni* OR inoculat*)) OR 'influenza vaccine' / syn OR ( 'influenza' /exp AND 'vaccine' /exp)

\#2 'case control study' /syn OR 'case control' :de,ab,ti OR ( cases :ab,ti AND controls :ab,ti) OR 'cohort analysis' /syn OR 'cohort study' :de,ab,ti OR 'study cohort' : de,ab,ti OR prospectiv* :ab,ti OR volunteer ${ }^{\star}: a b, t i$ OR observational :ab,ti OR 'clinical trial' :it OR 'randomized controlled trial' :it OR 'drug therapy' /exp OR 'drug therapy' :de OR randomized :ab,ti OR randomised :ab,ti OR placebo :ab,ti OR randomly :ab,ti OR trial :ab,ti OR groups :ab,ti

\#3 'clinical trial' :it OR 'randomized controlled trial' :it OR 'drug therapy' /exp OR 'drug therapy' :de OR randomized :ab,ti OR randomised :ab,ti OR placebo :ab,ti OR randomly :ab,ti OR trial :ab,ti OR groups :ab,ti

\#4 'clinical trial' :it OR 'randomized controlled trial' :it OR 'randomized controlled trial' /exp OR 'randomization' /exp OR 'single blind procedure' /exp OR 'double blind procedure' /exp OR 'clinical trial' /exp OR 'clinical' NEAR/0 'trial' OR 'clinical trial' OR ( singl* OR doubl ${ }^{\star}$ OR trebl* OR tripl*AND ( mask* OR blind*)) OR 'placebo' /exp OR placebo* OR random OR 'control group' /exp OR 'experimental design' /exp OR 'comparative study' /exp OR 'evaluation study' OR 'evaluation studies' /exp OR 'follow up' /exp OR 'prospective study' /exp OR control* OR prospectiv* OR volunteer* AND [humans]/lim

\#5 \#2 OR \#3 OR \#4

\#6 \#1 AND \#5

\#7 \#1 AND \#5 AND [humans]/lim AND [embase]/lim

\section{Appendix 5. MEDLINE search strategy for 2004 update}

\section{MEDLINE}

\#1 ("Influenza Vaccine/administration and dosage"[MeSH] OR "Influenza Vaccine/adverse effects"[MeSH] OR "Influenza Vaccine/ contraindications"[MeSH] OR "Influenza Vaccine/immunology"[MeSH] OR "Influenza Vaccine/metabolism"[MeSH] OR "Influenza Vaccine/ radiation effects"[MeSH] OR "Influenza Vaccine/therapeutic use"[MeSH] OR "Influenza Vaccine/toxicity"[MeSH]) OR ("Influenza/ epidemiology"[MeSH] OR "Influenza/immunology"[MeSH] OR "Influenza/mortality"[MeSH] OR "Influenza/prevention and control"[MeSH] OR "Influenza/transmission"[MeSH])

\#2 (influenza vaccin*[Title/Abstract]) OR ((influenza [Title/Abstract] OR flu[Title/Abstract]) AND (vaccin*[Title/Abstract] OR immuni^[Title/ Abstract] OR inoculati ${ }^{\star}$ [Title/Abstract] OR efficacy[Title/Abstract] OR effectiveness[Title/Abstract])

\#3 \#1 OR \#2

\#4 "Randomized Controlled Trial"[Publication Type] OR "Randomized Controlled Trials"[MeSH] OR "Controlled Clinical Trial"[Publication Type] OR "Controlled Clinical Trials"[MeSH] OR "Random Allocation"[MeSH] OR "Double-Blind Method"[MeSH] OR "Single-Blind Method"[MeSH]

\#5 controlled clinical trial*[Title/Abstract] OR randomised controlled trial*[Title/Abstract] OR clinical trial*[Title/Abstract] OR random allocation[Title/Abstract] OR random*[Title/Abstract] OR placebo[Title/Abstract] OR double - blind[Title/Abstract] OR single - blind[Title/ Abstract] OR RCT[Title/Abstract] OR CCT[Title/Abstract] OR allocation[Title/Abstract] OR follow - up[Title/Abstract] 
\#6 \#4 OR \#5

\#7 \#3 AND \#6

\section{Appendix 6. Data extraction form}

PART 1

\section{Background information and description of study}

Reviewer:

Study unique identifier:

Published: $\mathrm{Y} / \mathrm{N}$

Journal: (if applicable)

Year of publication:

Period study conducted:

Abstract/full paper

Country or countries of study:

Number of studies included in this paper:

Funding source (delete non-applicable items):

Government, pharmaceutical, private, unfunded, unclear

Paper/abstract numbers of other studies with which these data are linked:

Reviewer's assessment of study design (delete non-applicable items):

\begin{tabular}{llll}
\hline Study category & Study design & & Cross-over RCT \\
\hline Experimental & RCT/CCT & Cross-sectional \\
\hline $\begin{array}{l}\text { Non-randomised analytical (specifically designed to } \\
\text { assess association) }\end{array}$ & $\begin{array}{l}\text { Prospective/retro- } \\
\text { spective cohort }\end{array}$ & Case-control & $\begin{array}{l}\text { Indirect comparison } \\
\text { (before and after) }\end{array}$ \\
\hline $\begin{array}{l}\text { Non-randomised comparative (not specifically de- } \\
\text { signed to assess association) }\end{array}$ & $\begin{array}{l}\text { Case cross-over/time } \\
\text { series }\end{array}$ & Ecological study & \\
\hline Non-comparative & EXCLUDE & & \\
\hline
\end{tabular}

Does the study present data distributed by age group/occupation/health status?

Subgroup distribution

Yes No

Age group

Occupation 
(Continued)

Health status

\section{Gender}

Risk group

\section{Description of study}

Methods

Participants

Interventions/exposure

Outcomes

Notes

PART 2a

Methodological quality assessment

RCTs and CCTs only

\section{RANDOM SEQUENCE GENERATION}

Selection bias (biased allocation to interventions) due to inadequate generation of a randomised sequence.

Criteria for a judgement of 'Low risk' of bias

The investigators describe a random component in the sequence generation process such as:

- Referring to a random number table

- Using a computer random number generator

- Coin tossing

- Shuffling cards or envelopes

- Throwing dice

- Drawing of lots

- Minimisation*

*Minimisation may be implemented without a random element and this is considered to be equivalent to being random

Criteria for the judgement of 'High risk' of bias
The investigators describe a non-random component in the sequence generation process. Usually, the description would involve some systematic, non-random approach, for example:

- Sequence generated by odd or even date of birth

- Sequence generated by some rule based on date (or day) of admission

- Sequence generated by some rule based on hospital or clinic record number

Other non-random approaches happen much less frequently than the systematic approaches mentioned above and tend to be obvious. They usually involve judgement or some method of non-random categorisation of participants, for example:

- Allocation by judgement of the clinician

- Allocation by preference of the participant 
- Allocation by availability of the intervention

Criteria for the judgement of 'Unclear risk' of bias
Insufficient information about the sequence generation process to permit judgement of 'Low risk' or 'High risk'

\section{ALLOCATION CONCEALMENT}

\section{Selection bias (biased allocation to interventions) due to inadequate concealment of allocations prior to assignment}

Criteria for a judgement of 'Low risk' of bias

Participants and investigators enrolling participants could not foresee assignment because one of the following, or an equivalent method, was used to conceal allocation:

- Central allocation (including telephone, web-based and pharmacy-controlled randomisation)

- Sequentially numbered drug containers of identical appearance

- Sequentially numbered, opaque, sealed envelopes

Criteria for the judgement of 'High risk' of bias

Participants or investigators enrolling participants could possibly foresee assignments and thus introduce selection bias, such as allocation based on:

- Using an open random allocation schedule (e.g. a list of random numbers)

- Assignment envelopes were used without appropriate safeguards (e.g. if envelopes were unsealed or nonopaque or not sequentially numbered)

- Alternation or rotation

- Date of birth

- Case record number

- Any other explicitly unconcealed procedure

Criteria for the judgement of 'Unclear risk' of bias
Insufficient information to permit judgement of 'Low risk' or 'High risk'. This is usually the case if the method of concealment is not described or not described in sufficient detail to allow a definite judgement - for example if the use of assignment envelopes is described, but it remains unclear whether envelopes were sequentially numbered, opaque and sealed

\section{BLINDING OF PARTICIPANTS AND PERSONNEL}

Performance bias due to knowledge of the allocated interventions by participants and personnel during the study

\section{Criteria for a judgement of}

'Low risk' of bias

\section{Any one of the following:}

- No blinding or incomplete blinding, but the review authors judge that the outcome is not likely to be influenced by lack of blinding

- Blinding of participants and key study personnel ensured, and unlikely that the blinding could have been broken

Criteria for the judgement of 'High risk' of bias

\section{Any one of the following:}

- No blinding or incomplete blinding, and the outcome is likely to be influenced by lack of blinding

- Blinding of key study participants and personnel attempted, but likely that the blinding could have been broken, and the outcome is likely to be influenced by lack of blinding

Criteria for the judgement of

'Unclear risk' of bias
Any one of the following:

- Insufficient information to permit judgement of 'Low risk' or 'High risk' 


\section{BLINDING OF OUTCOME ASSESSMENT}

\section{Detection bias due to knowledge of the allocated interventions by outcome assessors}

Criteria for a judgement of

'Low risk' of bias

Any one of the following:

- No blinding of outcome assessment, but the review authors judge that the outcome measurement is not likely to be influenced by lack of blinding

- Blinding of outcome assessment ensured, and unlikely that the blinding could have been broken

Criteria for the judgement of 'High risk' of bias
Any one of the following:

- No blinding of outcome assessment, and the outcome measurement is likely to be influenced by lack of blinding

- Blinding of outcome assessment, but likely that the blinding could have been broken, and the outcome measurement is likely to be influenced by lack of blinding
Any one of the following:

- Insufficient information to permit judgement of 'Low risk' or 'High risk'

- The study did not address this outcome

\section{INCOMPLETE OUTCOME DATA}

Attrition bias due to amount, nature or handling of incomplete outcome data

Criteria for a judgement of

'Low risk' of bias

Any one of the following:

- No missing outcome data

- Reasons for missing outcome data unlikely to be related to true outcome (for survival data, censoring unlikely to be introducing bias)

- Missing outcome data balanced in numbers across intervention groups, with similar reasons for missing data across groups

- For dichotomous outcome data, the proportion of missing outcomes compared with observed event risk not enough to have a clinically relevant impact on the intervention effect estimate

- For continuous outcome data, plausible effect size (difference in means or standardised difference in means) among missing outcomes not enough to have a clinically relevant impact on observed effect size

- Missing data have been imputed using appropriate methods

Criteria for the judgement of

'High risk' of bias
Any one of the following:

- Reason for missing outcome data likely to be related to true outcome, with either imbalance in numbers or reasons for missing data across intervention groups

- For dichotomous outcome data, the proportion of missing outcomes compared with observed event risk enough to induce clinically relevant bias in intervention effect estimate

- For continuous outcome data, plausible effect size (difference in means or standardised difference in means) among missing outcomes enough to induce clinically relevant bias in observed effect size

- 'As-treated' analysis done with substantial departure of the intervention received from that assigned at randomisation 
Criteria for the judgement of 'Unclear risk' of bias

\section{Any one of the following:}

- Insufficient reporting of attrition/exclusions to permit judgement of 'Low risk' or 'High risk' (e.g. number randomised not stated, no reasons for missing data provided)

- The study did not address this outcome

\section{SELECTIVE REPORTING}

\section{Reporting bias due to selective outcome reporting}

Criteria for a judgement of 'Low risk' of bias

Any of the following:

- The study protocol is available and all of the study's prespecified (primary and secondary) outcomes that are of interest in the review have been reported in the prespecified way

- The study protocol is not available but it is clear that the published reports include all expected outcomes, including those that were prespecified (convincing text of this nature may be uncommon)

Criteria for the judgement of 'High risk' of bias

\section{Any one of the following:}

- Not all of the study's prespecified primary outcomes have been reported

- One or more primary outcomes is reported using measurements, analysis methods or subsets of the data (e.g. subscales) that were not prespecified

- One or more reported primary outcomes were not prespecified (unless clear justification for their reporting is provided, such as an unexpected adverse effect)

- One or more outcomes of interest in the review are reported incompletely so that they cannot be entered in a meta-analysis

- The study report fails to include results for a key outcome that would be expected to have been reported for such a study

Criteria for the judgement of Insufficient information to permit judgement of 'Low risk' or 'High risk'. It is likely that the majority of studies will fall into this category

\section{OTHER BIAS}

\section{Bias due to problems not covered elsewhere in the table}

\section{Criteria for a judgement of The study appears to be free of other sources of bias}

'Low risk' of bias

Criteria for the judgement of

'High risk' of bias
There is at least one important risk of bias. For example, the study:

- Had a potential source of bias related to the specific study design used or

- Has been claimed to have been fraudulent or

- Had some other problem

There may be a risk of bias, but there is either:

- Insufficient information to assess whether an important risk of bias exists or

- Insufficient rationale or evidence that an identified problem will introduce bias 


\section{PART 2b}

\section{Description of interventions and outcomes}

RCT and CCT only

Vaccines used

$\begin{array}{lll}\begin{array}{l}\text { Vaccines and compo- } \\ \text { sition }\end{array} & \begin{array}{l}\text { Product and manu- } \\ \text { facturer }\end{array} & \begin{array}{l}\text { Schedule \& dosage and } \\ \text { status }\end{array}\end{array}$

Route of administration

Arm 1
Arm 2
Arm 3
Arm 4
Placebo

Rule: index vaccine goes in the Arm 1 line, placebo in the last line

Status: primary, secondary or tertiary immunisation

\begin{tabular}{llll}
\hline Enrolled & Missing & $\begin{array}{l}\text { Inclusion in } \\
\text { analysis }\end{array}$ \\
\hline
\end{tabular}

\section{Active arm 1}

Active arm 2

\section{Active arm 3}

Active arm 4

\section{Controls}


Outcomes list - effectiveness

\begin{tabular}{lll}
\hline Outcome How defined $\quad$ Description/follow-up/notes & How
\end{tabular}

Outcomes list - safety

\begin{tabular}{lll}
\hline Outcome How defined $\quad$ Description/follow-up/notes
\end{tabular}

Investigators to be contacted for more information? Yes/No

Contact details (principal investigator, fill in only if further contact is necessary):

\section{PART 2c}

\section{Data extraction and manipulation}

(To be used for dichotomous or continuous outcomes)

RCT and CCT only

Comparison

Outcomes $\quad \mathrm{n} / \mathrm{N}$ index arm $\quad \mathrm{n} / \mathrm{N}$ comparator

Notes (for statistical use only)

\section{PART 3a}

\section{Description of interventions and outcomes}

Non-randomised longitudinal studies only

Vaccines used 


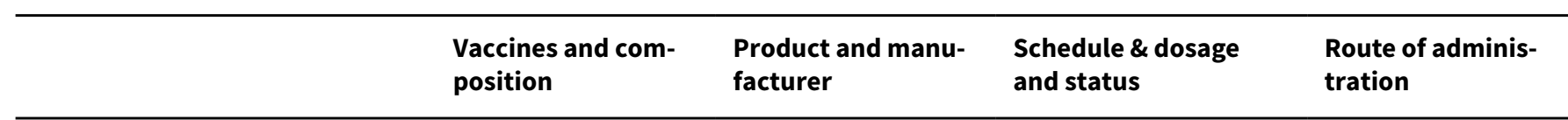

Group 1

Group 2

Group 3

Group 4

Comparator

Rule: index vaccine goes in the Group 1 line, placebo in the last line

Vaccine

Batch numbers

Details of participants

\begin{tabular}{lllll}
\hline & Enrolled & Missing & Reasons & Inclusion in analysis \\
\hline Group 1 & & \\
\hline Group 2 & \\
\hline Group 3 & \\
\hline Group 4 & \\
\hline Comparator
\end{tabular}

Outcomes list - effectiveness 


\begin{tabular}{lll}
\hline Outcome How defined (including length of follow-up) $\quad$ Description/follow-up/notes
\end{tabular}

Investigators to be contacted for more information? Yes/No

Contact details (principal investigator, fill in only if further contact is necessary):

\section{PART 3b}

\section{Data extraction and manipulation}

(To be used for dichotomous outcomes)

Non-randomised longitudinal studies only

Comparison

Outcomes $\quad n / N$ index group n/N comparator

Notes (for statistical use only)

\section{PART 3C}

\section{Description of studies}

Case-control studies only

Event 1

\begin{tabular}{lllll}
\hline How defined & Enrolled & Missing & Reasons & Inclusion in analysis \\
\hline Cases $n=$ & & \\
\hline Controls $n=$ & \\
\hline
\end{tabular}

\section{Exposure}




How defined How ascertained Hotes H H

Vaccine exposure 1

Vaccine exposure 2

\section{Event 2}

\begin{tabular}{llll}
\hline How defined $\quad$ Enrolled & Measonsing $\quad$ Inclusion in analysis \\
\hline
\end{tabular}

Cases $\mathrm{n}=$

Controls $n=$

Exposure

\begin{tabular}{lll}
\hline & How defined How ascertained $\quad$ Notes \\
\hline Vaccine exposure 1 & How \\
\hline
\end{tabular}

Vaccine exposure 2

Notes (for statistical use only)

Part 3d

Data extraction and manipulation

Case-control studies only

\begin{tabular}{lll}
\hline Status & Numerator & Denominator \\
\hline Cases & \\
\hline
\end{tabular}

Control

Notes (for statistical use only)

\section{Appendix 7. Included studies design}

A case-control study is a prospective or retrospective epidemiological study usually used to investigate the causes of disease. Study participants who have experienced an adverse outcome or disease are compared with participants who have not. Any differences in the presence or absence of hypothesised risk factors are noted.

A cohort study is an epidemiological study where groups of individuals are identified who vary in their exposure to an intervention or hazard and who are then followed to assess outcomes. Association between exposure and outcome are then estimated. Cohort studies are best performed prospectively, but can also be undertaken retrospectively if suitable data records are available. 
A randomised controlled trial is any study on humans in which the individuals (or other experimental units) followed in the study were definitely or possibly assigned prospectively to one of two (or more) alternative forms of health care using random allocation.

A quasi-randomised clinical trial is any study on humans in which the individuals (or other experimental units) followed in the study were definitely or possibly assigned prospectively to one of two (or more) alternative forms of health care using some quasi-random method of allocation (such as alternation, date of birth, or case record number).

\section{Appendix 8. Methodological quality of non-randomised studies}

\section{Newcastle-Ottawa quality assessment scale - case-control studies}

Note: a study can be awarded a maximum of one star (i.e.asterisk) for each numbered item within the Selection and Exposure categories. A maximum of two stars can be given for Comparability.

\section{Selection}

1. Is the case definition adequate?
a. Yes, with independent validation*
b. Yes, e.g. record linkage or based on self reports
c. No description

2. Representativeness of the cases

a. Consecutive or obviously representative series of cases*

b. Potential for selection biases or not stated

3. Selection of controls
a. Community controls
b. Hospital controls
c. No description

4. Definition of controls
a. No history of disease (endpoint)*
b. No description of source

\section{Comparability}

1. Comparability of cases and controls on the basis of the design or analysis a. Study controls for ________________ (Select the most important factor)*

b. Study controls for any additional factor ${ }^{\star}$ (This criterion could be modified to indicate specific control for a second important factor)

\section{Exposure}

1. Ascertainment of exposure
a. Secure record (e.g. surgical records)*
b. Structured interview where blind to case/control status*
c. Interview not blinded to case/control status
d. Written self report or medical record only
e. No description

2. Same method of ascertainment for cases and controls
a. Yes*
b. No

3. Non-response rate
a. Same rate for both groups ${ }^{\star}$
b. Non-respondents described
c. Rate different and no designation

\section{Newcastle-Ottawa quality assessment scale - cohort studies}

Note: a study can be awarded a maximum of one star for each numbered item within the Selection and Outcome categories. A maximum of two stars can be given for Comparability. 


\section{Selection}

1. Representativeness of the exposed cohort
a. Truly representative of the average (describe) in the community ${ }^{\star}$
b. Somewhat representative of the average in the community ${ }^{*}$
c. Selected group of users, e.g. nurses, volunteers
d. No description of the derivation of the cohort

2. Selection of the non-exposed cohort
a. Drawn from the same community as the exposed cohort ${ }^{\star}$
b. Drawn from a different source
c. No description of the derivation of the non-exposed cohort

3. Ascertainment of exposure
a. Secure record (e.g. surgical records) ${ }^{\star}$
b. Structured interview *
c. Written self report
d. No description

4. Demonstration that outcome of interest was not present at start of study
a. Yes*
b. No

\section{Comparability}

1. Comparability of cohorts on the basis of the design or analysis
a. Study controls for (select the most important factor)*
b. Study controls for any additional factor ${ }^{\star}$ (This criterion could be modified to indicate specific control for a second important factor)

\section{Outcome}

1. Assessment of outcome
a. Independent blind assessment ${ }^{\star}$
b. Record linkage*
c. Self report
d. No description

2. Was follow-up long enough for outcomes to occur

a. Yes (select an adequate follow-up period for outcome of interest)*

b. No

3. Adequacy of follow-up of cohorts
a. Complete follow-up - all participants accounted for*
b. Participants lost to follow-up unlikely to introduce bias - small number lost -> provided of those lost) ${ }^{\star}$
c. Follow-up rate < ____\% (select an adequate \%) and no description of those lost
d. No statement

\section{FEE D B A C K}

\section{Inconsistency between results and abstract, 6 April 2007}

\section{Summary}

We feel there is some inconsistency between results and abstract of this review regarding off work time.

In the results it states that 0.4 days are saved, but that this result is not statistically significant. In the abstract, however, this difference is labelled significant. Can you help us in understanding this?

I certify that I have no affiliations with or involvement in any organisation or entity with a direct financial interest in the subject matter of my criticisms.

\section{Reply}

The difference is statistically significant as it says in the abstract. In the results the word "statistical" has been used instead of "clinical". Indeed the meaning of the comment was to underline that, although statistically significant, a difference of 0.4 day is clinically inconsistent. 
I certify that I have no affiliations with or involvement in any organisation or entity with a direct financial interest in the subject matter of my criticisms

Vittorio Demicheli

\section{Contributors}

JC van der Wouden

\section{Comments regarding the conclusion, 5 April 2006}

\section{Summary}

Your conclusion is confusing. You write: "Universal immunization of healthy adults is not supported by the results of this review." If so, why the first sentence? You wrote in the Discussion that "serologically confirmed cases of influenza are only part of the spectrum of clinical effectiveness." Furthermore, it would be helpful if you had explained the difference between influenza and influenza-like illness in the abstract. Also, the title of the synopsis is inaccurate. Why say "not enough evidence" when there are so many trials in your review? It should read: Clinical trials do not support the universal recommendation, etc. And "by a quarter" is not going to be understood by the general public. Please put in absolute terms.

I certify that I have no affiliations with or involvement in any organization or entity with a financial interest in the subject matter of my feedback.

\section{Reply}

This comment has been superseded and addressed by the 2006 latest update.

\section{Contributors}

Maryann Napoli

\section{Vaccines for preventing influenza in healthy adults, 13 May 2013}

\section{Summary}

There seems to be an inconsistency in the presentation of the Cochrane Summary: "Vaccines to prevent influenza in healthy adults". The Plain language summary states that "Vaccine use did not affect the number of people hospitalised or working days lost", but under Main Results we read that "Vaccination had a modest effect on time off work and had no effect on hospital admissions". These two claims seem to be at odds regarding working days/time lost.

I agree with the conflict of interest statement below:

I certify that I have no affiliations with or involvement in any organization or entity with a financial interest in the subject matter of my feedback.

Robyn Kath

\section{Reply}

This review has now been updated and both paragraphs have been rewritten.

\section{Contributors}

Vittorio Demicheli

\section{Vaccines for preventing influenza in healthy adults, 15 September 2014}

\section{Summary}

In occupational health, there is a great interest in the effect of vaccination on the number of workdays lost. The abstract reports that vaccination had a modest effect on time off work. The results in the review that I can find for this outcome show a mean difference of 0.04 with a $95 \%$ confidence interval of -0.14 to 0.06 . It depends on whose point of view you take, but I don't think that there is any stakeholder that will rate a 17 minutes decrease in worktime lost a modest effect. In addition, it is not significant. Did I overlook something or is this a mistake?

Best wishes, Jos Verbeek

I agree with the conflict of interest statement below: 
I certify that I have no affiliations with or involvement in any organization or entity with a financial interest in the subject matter of my feedback.

Jos Verbeek

Email Address: jos.verbeek@ttl.fi

Affiliation: Finnish Institute of Occupational Health

Role: Senior Reseacher

\section{Reply}

The text of the abstract resuming the available includible evidence of vaccination on time off work has been amended and the word "modest" has now been replaced with "negligible". In any cases only by applying the fixed model effect the difference results significant.

\section{Contributors}

All Authors

WHAT'S NEW

\begin{tabular}{lll}
\hline Date & Event & Description \\
\hline 10 February 2020 & Amended & Typographic error corrected in Figure 1 \\
\hline
\end{tabular}

\section{H I S T O R Y}

Protocol first published: Issue 4, 1998

Review first published: Issue 4, 1999

\begin{tabular}{lll}
\hline Date & Event & Description \\
\hline 31 December 2016 & New search has been performed & For this update we included 20 new trials (aa Mcbride 2016a; aa \\
& & Mcbride 2016b; aa Treanor 2011; bb Dauvilliers 2013; bb Macln- \\
& tyre 2013; bb Rouleau 2014; cb O'Flanagan 2014; cb Persson \\
& 2014; paa Ma 2014; paa Madhi 2014; pca Ahrens 2014; pcb Beau \\
& 2014; pcb Cantu 2013; pcb Chambers 2013; pcb Cleary 2014; pcb \\
& Dodds 2012; pcb Louik 2013; pcb Ludvigsson 2013; pcb Nordin \\
& 2014; pcb Rubinstein 2013; pcb Trotta 2014).
\end{tabular}

We excluded 21 new trials (Andersson 2015; Atsmon 2012; Baxter 2013; Chavant 2013; Chichester 2012; Couch 2012; Duffy 2014; Greene 2013; Heinonen 1973; Huang 2011; Lavallee 2014; Lind 2014; Liu 2012; Montplaisir 2014; Phonrat 2013; Pleguezuelos 2012; Scheifele 2013; Sipilä 2015; Taylor 2012; Thompson 2014; Warren-Gash 2013).

We excluded 17 trials previously awaiting classification (ab López-Macías 2011a; ab López-Macías 2011b; ab Mallory 2010; ab Plennevaux 2010; ab Precioso 2011; ab Treanor 2010; ab Turley 2011; ab Wacheck 2010; Atsmon 2012; Chichester 2012; Couch 2012; Heinonen 1973; Huang 2011; Phonrat 2013; Pleguezuelos 2012; Scheifele 2013; Taylor 2012; Xu 2012).

\begin{tabular}{lll}
\hline 31 December 2016 & $\begin{array}{l}\text { New citation required but conclusions } \\
\text { have not changed }\end{array}$ & Our conclusions remain unchanged. \\
\hline 15 September 2014 & Feedback has been incorporated & Feedback comment submitted. \\
\hline 4 March 2014 & New search has been performed & $\begin{array}{l}\text { We updated the searches and included 41 new trials (aa Bar- } \\
\text { rett 2011; aa Frey 2010; aa Jackson 2010a; aa Jackson 2010b; }\end{array}$
\end{tabular}




\begin{tabular}{lll}
\hline Date Event $\quad$ Description
\end{tabular}

aa Langley 2011; aa Monto 2009; aa Ohmit 2006; aa Ohmit 2008; bb Dieleman 2011a; bb Dieleman 2011b; bb Dieleman 2011c; bb Dieleman 2011d; bb Dieleman 2011e; bb Galeotti 2013; bb Garbe 2012; bb Grimaldi-Bensouda 2011; bb Grimaldi-Bensouda 2012; bb Hernan 2004; bb Ray 2011; bb Zorzon 2003; cb Bardage 2011; cb Baxter 2012; cb Moro 2013; cb Ray 2011; pba Benowitz 2010; pba Poehling 2011; pbb Irving 2013; pca Black 2004; pca Eick 2011; pca France 2006; pca Hulka 1964; pca Munoz 2005; pca Yamada 2012; pcb Deinard 1981; pcb Fell 2012; pcb Håberg 2013; pcb Heikkinen 2012; pcb Källén 2012; pcb Launay 2012; pcb Lin 2012; pcb Nordin 2013; pcb Omer 2011; pcb Oppermann 2012; pcb Pasternak 2012; pcb Richards 2013; pcb Sheffield 2012; pcb Toback 2012), which corresponded to 47 data sets. We excluded 63 new trials.

\begin{tabular}{ll}
\hline March 2014 & $\begin{array}{l}\text { New citation required and conclusions } \\
\text { have changed }\end{array}$ \\
\hline
\end{tabular}

\begin{tabular}{ll}
\hline 24 May 2013 & Feedback has been incorporated \\
\hline 15 June 2010 & $\begin{array}{l}\text { New citation required but conclusions } \\
\text { have not changed }\end{array}$
\end{tabular}

15 June $2010 \quad$ New search has been performed

\section{New search has been performed}

For this update we added vaccine efficacy/effectiveness and safety evidence on pregnant women.
Feedback comment added to the review.

For this update Eliana Ferroni (EF), Lubna Al Ansary, and Ghada Bawazeer joined as new authors. Carlo Di Pietrantonj (CDP), Alessandro Rivetti (AR), and Tom Jefferson (TJ) remained.

Searches conducted. For this update we screened 3729 titles and identified 44 studies for possible inclusion. We included two new trials, aa Beran 2009a and aa Beran 2009b, and excluded three new trials (Belongia 2009; Chou 2007; Khazeni 2009).

\begin{tabular}{|c|c|c|}
\hline 10 May 2009 & Amended & Contact details updated. \\
\hline 26 April 2008 & Amended & Converted to new review format. \\
\hline 15 April 2007 & Feedback has been incorporated & Feedback comment added to review. \\
\hline \multirow[t]{2}{*}{16 February 2007} & $\begin{array}{l}\text { New citation required and conclusions } \\
\text { have changed }\end{array}$ & $\begin{array}{l}\text { Substantive amendment. For the } 2006 \text { update we included } 30 \\
\text { new studies but tightened up our inclusion criteria, excluding } \\
\text { studies with influenza B vaccine as a control, which did not come } \\
\text { within our comparator rules of placebo or do nothing. Twen- } \\
\text { ty-two of the new included studies were clinical trials evaluating } \\
\text { the efficacy or safety (or both) of different type of influenza vac- } \\
\text { cines. }\end{array}$ \\
\hline & & $\begin{array}{l}\text { We also carried out a subanalysis of the five } 1968 \text { to } 1969 \text { pan- } \\
\text { demic trials (with numerous subtrials) in our data set. Finally, } \\
\text { we included more data ( } 10 \text { studies) on potential serious or rare } \\
\text { harms, looking also at non-randomised evidence. }\end{array}$ \\
\hline 4 April 2006 & Feedback has been incorporated & Feedback comment added to review. \\
\hline 24 May 2004 & New search has been performed & $\begin{array}{l}\text { Searches conducted. In the } 2004 \text { update we included five more } \\
\text { studies not identified by the original searches and updated the } \\
\text { text and references. We also assessed and excluded } 25 \text { more } \\
\text { studies. We used the random-effects model for analysing all the } \\
\text { comparisons and outcomes. The updated results and conclu- } \\
\text { sions of our review did not change significantly much. }\end{array}$ \\
\hline
\end{tabular}

6 July $1997 \quad$ New search has been performed $\quad$ Searches conducted. Review first published Issue 4, 1999.




\section{CONTRIBUTIONS OF AUTHORS}

Carlo Di Pietrantonj (CDP) and Alessandro Rivetti (AR) designed both the 2014 and the 2016 updates.

AR carried out the searches and preliminary screening of references.

$A R$ and CDP applied the inclusion criteria.

$A R$ and CDP extracted data.

CDP checked the data extraction, performed the meta-analysis, and carried out statistical testing.

CDP and AR wrote the final report.

For this 2016 update Tom Jefferson, Alex Rivetti and Vittorio Demicheli updated searches and content. The other authors approved the text.

\section{DECLARATIONSOF INTEREST}

Vittorio Demicheli: none known

Tom Jefferson (TJ) was a co-recipient of a UK National Institute for Health Research grant (HTA - 10/80/01 Update and amalgamation of two Cochrane Reviews: neuraminidase inhibitors for preventing and treating influenza in healthy adults and children (www.nets.nihr.ac.uk/ projects/hta/108001)). TJ receives royalties from his books published by Blackwells and II Pensiero Scientifico Editore, Rome. TJ is occasionally interviewed by market research companies for anonymous interviews about phase I or II pharmaceutical products. In 2011 to 2013, TJ acted as an expert witness in a litigation case related to oseltamivir phosphate (Tamiflu; Roche) and in a labour case on influenza vaccines in healthcare workers in Canada. TJ acted as a consultant for Roche (1997-99), GSK (2001-2), and Sanofi-Synthelabo (2003) for the antirhinoviral pleconaril, which was not approved by the US Food and Drug Administration. TJ was a consultant for IMS Health in 2013 , and in 2014 he was retained as a scientific adviser to a legal team acting on the drug oseltamivir (Tamiflu; Roche). In 2014 to 2015, TJ was a member of two advisory boards for Boerhinger and is in receipt of a Cochrane Methods Innovations Fund grant to develop guidance on the use of regulatory data in Cochrane Reviews. TJ has a potential financial conflict of interest in the investigation of the drug oseltamivir. TJ acted as an expert witness in a legal case involving the drug oseltamivir (Roche) and the vaccine Pandemrix (GSK). TJ was a member of an Independent Data Monitoring Committee for a Sanofi Pasteur clinical trial.

Eliana Ferroni: none known

Alessandro Rivetti: none known

Carlo Di Pietrantonj: none known

\section{SOURCES OF SUPPORT}

\section{Internal sources}

- ASL (Local Health Unit) AL, Piemonte, Italy.

\section{External sources}

- NHS Department of Health Cochrane Incentive Scheme, UK.

This project was supported by the National Institute for Health Research (NIHR), via Cochrane Incentive Award funding to the Cochrane Acute Respiratory Infections Group. The views and opinions expressed therein are those of the authors and do not necessarily reflect those of the Systematic Reviews Programme, NIHR, NHS (National Health Service), or the Department of Health.

- Ministry of Defence, UK.

\section{DIFFERENCES BETWEEN PROTOCOLANDREVIEW}

Evidence about the safety and efficacy/effectiveness of influenza vaccine administration during pregnancy is included in this 2016 update. Previous versions of this review included observational comparative studies assessing serious and rare harms cohort and casecontrol studies. Because of the uncertain quality of observational (i.e. non-randomised) studies and their lack of influence on the review conclusions, we have decided to update only randomised evidence. We have no longer updated the searches for observational comparative studies.

\section{INDEX TERMS}

\section{Medical Subject Headings (MeSH)}

Absenteeism; Drug Industry; Health Status; Hospitalization [statistics \& numerical data]; Influenza A virus; Influenza B virus; Influenza Vaccines [adverse effects] [ ${ }^{\star}$ therapeutic use]; Influenza, Human [ ${ }^{\star}$ prevention \& control] [virology]; Nausea [chemically induced]; Pregnancy Complications, Infectious [prevention \& control] [virology]; Publication Bias; Research Support as Topic; Vomiting [chemically induced] 


\section{MeSH check words}

Adult; Female; Humans; Male; Pregnancy 